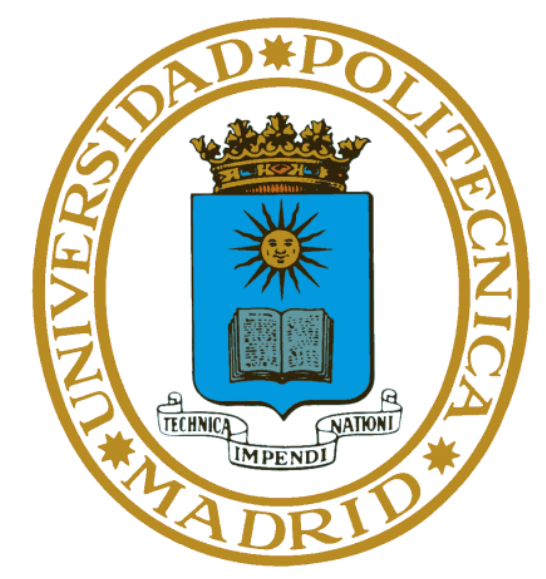

\title{
LINEALIZACIÓN DE AMPLIFICADORES DE POTENCIA DE ALTO RENDIMIENTO SOBRE TECNOLOGÍA GAN MEDIANTE TÉCNICAS EER/ET
}

\author{
TESIS DOCTORAL \\ POR \\ DAVID TENA RAMOS \\ Ingeniero Técnico de Telecomunicación (Sistemas de Telecomunicación) \\ Máster en Ingeniería de Servicios y Sistemas Accesibles para la Sociedad de la información
}




\title{
LINEALIZACIÓN DE AMPLIFICADORES DE POTENCIA DE ALTO RENDIMIENTO SOBRE TECNOLOGÍA GAN MEDIANTE TÉCNICAS EER/ET
}

\author{
TESIS DOCTORAL \\ AUTOR: \\ DAVID TENA RAMOS \\ DIRECTORES: \\ FRANCISCO JAVIER ORTEGA GONZÁLEZ \\ Doctor Ingeniero de Telecomunicación \\ JOSÉ MANUEL PARDO MARTÍN \\ Doctor Ingeniero de Telecomunicación
}

Ingeniero Técnico de Telecomunicación (Sistemas de Telecomunicación)

Máster en Ingeniería de Servicios y Sistemas Accesibles para la Sociedad de la información

2020 
Tribunal nombrado por el Magnífico y Excelentísimo Sr. Rector de la Universidad Politécnica de Madrid

$\begin{array}{ll}\text { PRESIDENTE: } & \text { nombre } \\ & \text { Doctor en / universidad } \\ \text { VOCALES: } & \text { nombre } \\ & \text { Doctor en / universidad } \\ & \text { nombre } \\ & \text { Doctor en / universidad } \\ & \text { nombre } \\ & \text { Doctor en / universidad } \\ \text { SECRETARIO: } & \text { nombre } \\ & \text { Doctor en / universidad } \\ \text { SUPLENTES: } & \text { nombre } \\ & \text { Doctor en / universidad }\end{array}$

Este tribunal acuerda otorgar la calificación de:

EL PRESIDENTE

LOS VOCALES

EL SECRETARIO

Realizado el acto de defensa y lectura de la Tesis en Madrid, a .... de de 2020 
A mi mujer y mi hija

"Bienvenida a casa pequeña gran revolución que con tus pasos marcas un nuevo rumbo en dirección a nuevas montañas que parecen menos altas con cada palabra que nace en tu garganta pequeña gran revolución"

Izal 


\section{AGRADECIMIENTOS}

Mis más sinceros agradecimientos a cuantas personas e instituciones han ayudado en la realización de esta tesis doctoral:

- Al Grupo de Ingeniería de Radio (GIRA), sin el cual este trabajo no habría sido posible.

- Al Doctor Ingeniero Don Francisco Javier Ortega González, tutor y director de esta tesis, porque debido a sus altos conocimientos, consejos y la confianza depositada en mí ha hecho crecer mi motivación durante toda mi etapa en el grupo de investigación.

- Al Doctor Ingeniero Don José Manuel Pardo Martín, codirector de esta tesis, por la facilidad que tiene de transmitir sus conocimientos y resolver los problemas que se hayan presentado.

- Al resto de miembros y exmiembros del grupo GIRA, por tantas horas compartidas en el mismo laboratorio trabajando juntos en un fin común; en especial a Alex por sus ánimos en momentos importantes, a Diego por nuestras conversaciones y anécdotas y a David porque es una persona que siempre presta su ayuda.

- A mi mujer Rocío y mi hija Sofía de las que me he tenido que separar un poco durante la realización de esta tesis (tiempo que debemos recuperar).

- A mis padres María Luisa y Eduardo, por todo su soporte y apoyo durante mi vida estudiantil, pilar fundamental en la consecución de este objetivo.

- A mi suegro Luis, porque a pesar de ponerlo tan difícil al final vemos un rayo de esperanza.

- A mi hermano Javier, por su apoyo moral y por ser el mejor padrino para mi hija.

- A mi familia, por aprovechar juntos los ratos que hemos tenido.

- Por supuesto, a mis amigos, con los que cada fin de semana era un día diferente lleno de alegría y diversión. En especial quisiera agradecer a Marta y a Miguel Ángel por la ayuda prestada en los picos de trabajo.

- Al Centro de Electrónica Industrial por la colaboración conjunta en la investigación relacionada con esta tesis doctoral.

Agradecer también:

- A la Universidad Politécnica de Madrid por su financiación en el programa FPI para la formación de investigadores.

- A todas las empresas ya sean públicas o privadas que han financiado proyectos para el grupo GIRA donde he desempeñado mi trabajo de la mejor manera posible.

- A todas las empresas que han financiado la publicación de los artículos de esta tesis.

En general, un abrazo enorme para todas aquellas personas que de una manera u otra habéis estado relacionados conmigo durante la realización de esta tesis doctoral. 


\section{Resumen}

Los nuevos sistemas de comunicaciones evolucionan con el paso del tiempo hacia esquemas de modulación más complejos con el fin de incrementar la tasa de transmisión de datos y aprovechar el limitado espectro de frecuencia. El objetivo es permitir mayores capacidades de transmisión de datos y ofrecer mayor flexibilidad en las comunicaciones inalámbricas. Sin embargo, este objetivo implica un incremento del ancho de banda de RF y de la relación pico-medio (PAPR) de las señales.

Por consiguiente, el valor de PAPR de las señales se ha convertido en un concepto importante a la hora de diseñar amplificadores de potencia de RF. De ahí que conocer las características de estas señales moduladas deba convertirse en el primer paso antes de realizar el diseño del amplificador de RF.

El amplificador de RF es normalmente el mayor responsable del consumo de potencia en los sistemas. Mejorar su rendimiento contribuirá a reducir significativamente el tamaño y coste de los equipos. Además, estos amplificadores en combinación con arquitecturas linealización de alto rendimiento facilitan el funcionamiento con señales de alta PAPR.

En un amplificador de potencia el principal elemento es el dispositivo activo. La continua investigación en tecnologías de semiconductores conduce a la mejora de las prestaciones de los amplificadores de potencia. Una tecnología que está creciendo mucho en los últimos años es GaN HEMT. Sus características le hacen adecuado para su uso en amplificadores de potencia conmutados. Presenta las ventajas de los dispositivos de Si (alta potencia) así como la de los dispositivos de GaAs (alta frecuencia.

Diferentes técnicas de diseño fueron empleadas con el objetivo de implementar amplificadores de potencia de RF de alta potencia y alto rendimiento. El método load pull con pre-adaptación de impedancias armónicas fue empleado en el diseño de un amplificador clase $\mathrm{F}$ en banda L cuyo prototipo fue capaz de entregar una potencia de salida de $45 \mathrm{~W}$ con un rendimiento del $70 \%$ controlando únicamente los dos primeros armónicos. El método basado en la síntesis de la admitancia de carga fue empleado en el diseño de dos amplificadores clase E subóptimos: el diseño en banda L es capaz de entregar una potencia de salida de $180 \mathrm{~W}$ con un rendimiento del $85 \%$ desde $900 \mathrm{MHz}$ 
hasta $1500 \mathrm{MHz}$; el diseño en banda $\mathrm{S}$ ofrece una potencia de salida de $14 \mathrm{~W}$ con un rendimiento del $79 \%$ en la banda de $2100 \mathrm{MHz}$ a $2600 \mathrm{MHz}$. Según los mejores conocimientos del autor, estos amplificadores superan los diseños publicados en otros trabajos.

Estos amplificadores de RF de alto rendimiento tienen como principal campo de aplicación los sistemas RADAR, donde la señal no presente cambios en la envolvente. Sin embargo, también pueden ser empleados en transmisores que usen señales de alta PAPR intrigándolos con arquitecturas de linealización de alto rendimiento, como pueden ser EER y ET. En ambas, un amplificador de envolvente se encarga de modular el puerto de alimentación del amplificador de RF. El diseño del amplificador de envolvente es un elemento crucial del sistema.

Varios transmisores fueron implementados integrando los amplificadores diseñados en arquitecturas EER o ET. En primer lugar, se diseñó un transmisor EER y ET compuesto por un convertido buck multifase asistido por un regulador lineal y por el amplificador clase $\mathrm{F}$ en banda $\mathrm{L}$ implementado. Un rendimiento del $27 \%$ y unos niveles de ACPR por encima de 30dB fueron obtenidos para una señal de prueba 64QAM. En segundo lugar, un transmisor ET fue diseñado empleando el mismo convertidor buck multifase (sin regulador lineal) y el amplificador clase F (trabajado en su zona lineal). En este caso, un rendimiento del $33 \%$ y un nivel de ACPR superior a $28 \mathrm{~dB}$ fueron alcanzados para la misma señal de prueba. Comparando ambas arquitecturas, la influencia del regulador lineal es patente en el rendimiento y linealidad de los sistemas, aún más al trabajar con amplios anchos de banda de envolvente.

Por último, se propone una nueva técnica EER híbrida consiste básicamente en hacer trabajar al amplificador de RF en un modo mixto en función del nivel de envolvente. Esto se consigue mediante procesado digital de la señal. De esta manera, un transmisor EER híbrido fue implementado a partir de un convertidor buck multifase y el amplificador clase $\mathrm{E}$ en banda L implementado. La técnica EER híbrida solventa algunos de los inconvenientes de la arquitectura EER, principalmente los debidos a bajos niveles de envolvente. Mejoras en el IMD3 de $10 \mathrm{~dB}$ fueron conseguidas a causa de una leve reducción en el rendimiento para una señal de prueba de dos tonos. El sistema también fue probado con una señal OFDM obteniendo una mejora en el EVM del $7 \%$. 


\section{Abstract}

Over the years, new communications systems evolve towards more complex modulation schemes in order to increase the data transmission rate and take advantage of the limited frequency spectrum. The goal is to allow greater data transmission capabilities and offer greater flexibility in wireless communications. However, this objective implies an increase in RF bandwidth and peak-to-average power ratio (PAPR) of the signals.

Therefore, the PAPR value of the signals has become an important point when designing RF power amplifiers. Hence, to know the characteristics of these modulated signals should become the first step before designing the RF amplifier.

The RF amplifier is usually the most responsible for the power consumption in the systems. Improving their performance will contribute to significantly reduce the size and cost of equipments. In addition, these amplifiers in combination with highperformance linearization architectures facilitate operation with high PAPR signals.

The main element In a power amplifier is the active device. Continuous research in semiconductor technologies leads to improved performance of power amplifiers. A technology that is growing a lot in recent years is GaN HEMT. Its features make it suitable for use in switched power amplifiers. It has the advantages of Si devices (high power) as well as GaAs devices (high frequency).

Different design techniques were employed in order to implement high power high efficiency RF power amplifiers. Load pull method with pre-adaptation of harmonic impedances was used in the design of a class $\mathrm{F}$ amplifier in L band whose prototype was able to delivery $45 \mathrm{~W}$ output power with $70 \%$ drain efficiency controlling only the first two harmonics. The method based on the load admittance synthesis was used in the design of two suboptimal class E amplifiers: the L-band design delivers 180W output power with $85 \%$ drain efficiency from $900 \mathrm{MHz}$ to $1500 \mathrm{MHz}$; the S-band design provides $14 \mathrm{~W}$ output power with $79 \%$ drain efficiency from $2100 \mathrm{MHz}$ to $2600 \mathrm{MHz}$. According to the author's best knowledge, these amplifiers exceed published designs in other works. 
RADAR systems are the main field of application of these high-efficiency RF amplifiers, where the signal does not show changes in the envelope. However, they can also be integrated in transmitters that use high PAPR signals in conjunction with high performance linearization architectures, such as EER and ET. In both, an envelope amplifier is responsible to modulate the power port of the RF amplifier. The envelope amplifier design is a crucial element of the system.

Several transmitters were implemented by integrating amplifiers designed in EER or ET architectures. First, an EER transmitter was designed, consisting of a multiphase buck DC-DC converter assisted by a linear regulator and the implemented class F amplifier in the L-band. A $27 \%$ efficiency and ACPR levels above 30dB were achieved for 64QAM test signal. Secondly, an ET transmitter was designed using the same multiphase buck DC-DC converter (without linear regulator) and the class F amplifier (working in its linear zone). In this case, 33\% efficency and ACPR levels greater than $28 \mathrm{~dB}$ were reached for the same test signal. Comparing both architectures, the influence of the linear regulator is clear in efficiency and linearity figures of the systems, even more when working with wideband envelope signals.

Finally, a new hybrid EER technique is proposed, consisting of making the RF amplifier work in a mixed mode depending on the envelope level. This is achieved by digital signal processing. In this way, a hybrid EER transmitter was implemented from a multiphase buck DC-DC converter and the class E amplifier in L-band implemented. The hybrid EER technique solves some of the disadvantages in EER architecture, mainly those due to low envelope levels. IMD3 improvements of $10 \mathrm{~dB}$ were achieved because of a slight reduction in performance for a two-tone test signal. The system was also tested with an OFDM signal obtaining an improvement in the EVM of $7 \%$. 


\section{Índice de contenidos}

1. Capítulo 1: Introducción.......................................................................... 1

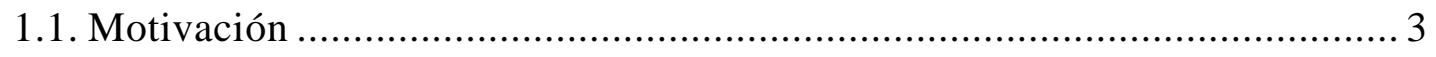

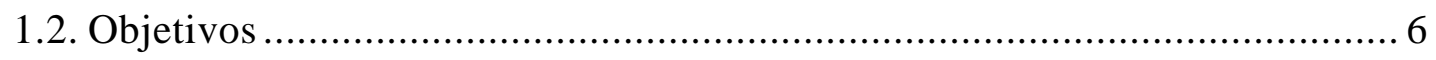

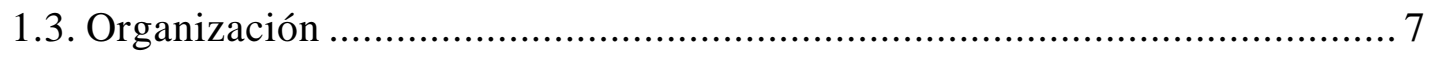

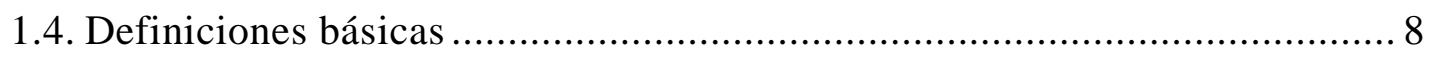

2. Capítulo 2: Estado del arte ..................................................................... 15

2.1. Características de las señales moduladas modernas .........................................17

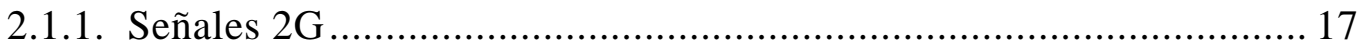

2.1.2. Señales $3 G$......................................................................... 18

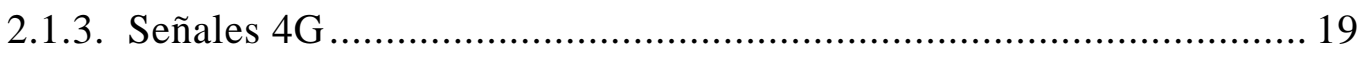

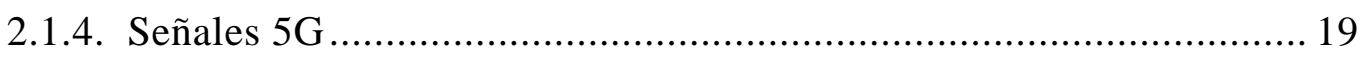

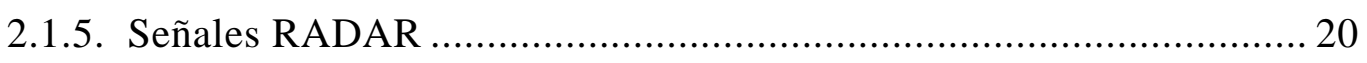

2.2. Dispositivos para PA de alto rendimiento .....................................................22

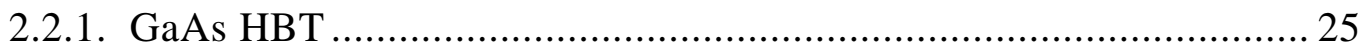

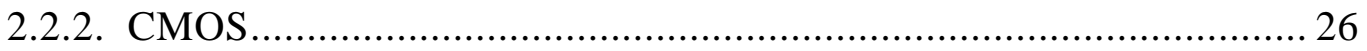

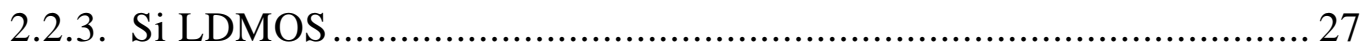

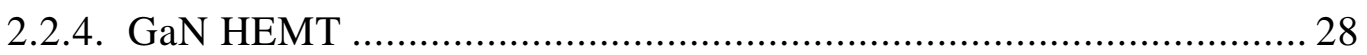

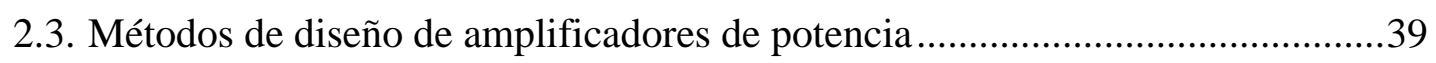

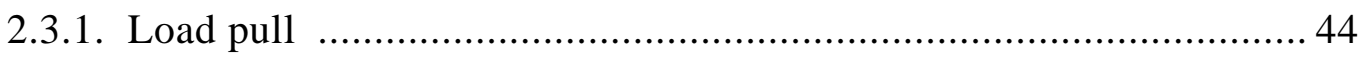

2.3.1.1. Sistemas load pull pasivos ............................................. 45

2.3.1.2. Sistemas load pull activos ............................................. 48

2.3.1.3. Sistemas load-pull multiarmónico .................................... 48

2.3.2. PA en modo de conmutación ......................................................... 51

2.3.2.1. Amplificadores clase D y D-1 ..................................... 52

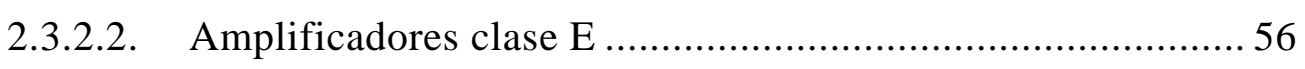

2.3.2.3. Amplificadores clase F y F-1 ........................................ 59

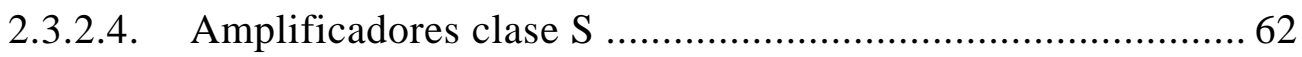

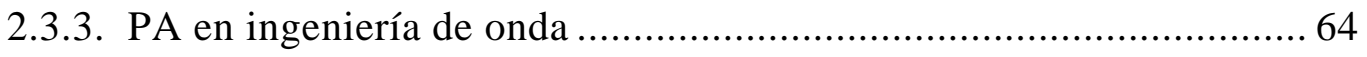

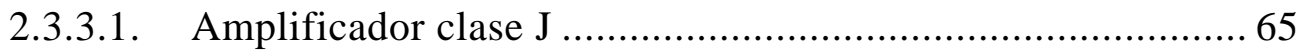

2.3.3.2. Amplificador clase P........................................................ 66 


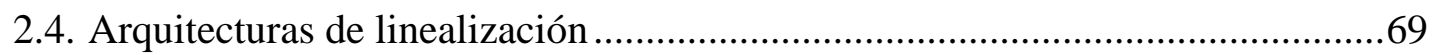

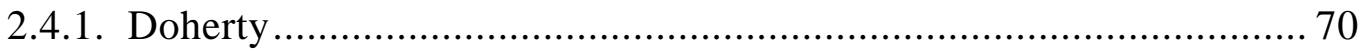

2.4.2. LINC y out-phasing …..................................................... 75

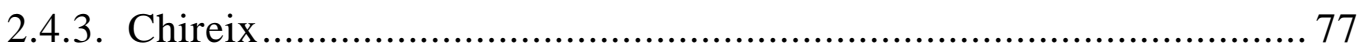

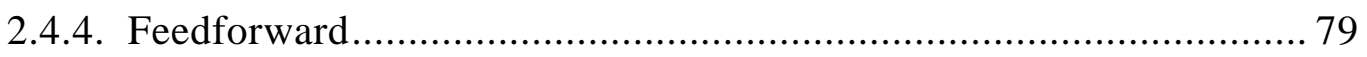

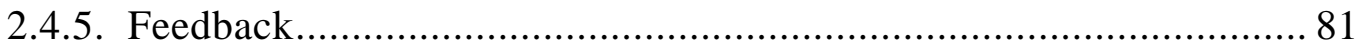

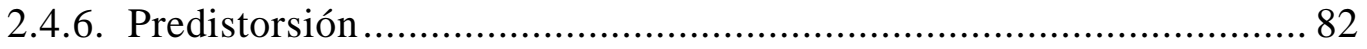

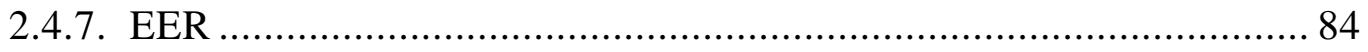

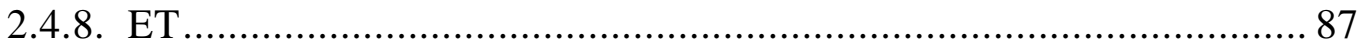

2.4.9. Comparación de las técnicas de linealización ................................. 91

3. Capítulo 3: Diseño de amplificadores de alto rendimiento ..........................93

3.1. Amplificador de potencia clase F en banda L ................................................95

3.1.1. Caracterización del transistor..................................................... 97

3.1.1.1. Capacidades no lineales del transistor ................................ 99

3.1.1.2. Planos de transformación ............................................... 106

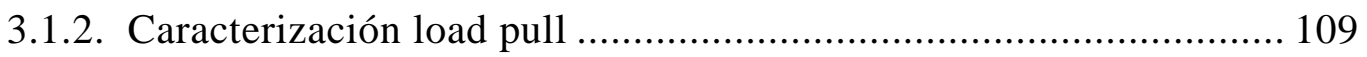

3.1.2.1. Sintonizadores mecánicos y circuitos de preadaptación ...... 109

3.1.2.2. Calibración ................................................................... 113

3.1.2.3. Banco de medidas ...................................................... 121

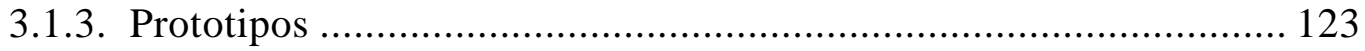

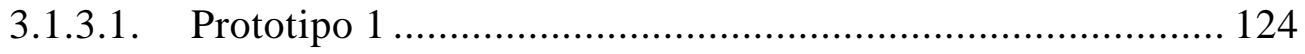

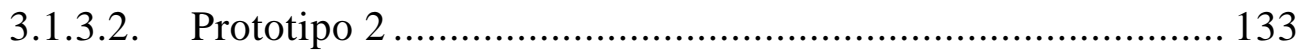

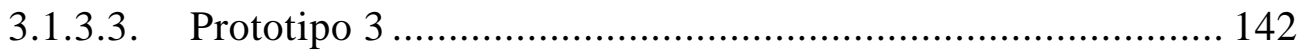

3.2. Amplificador de potencia clase E subóptimo en banda L ...............................149

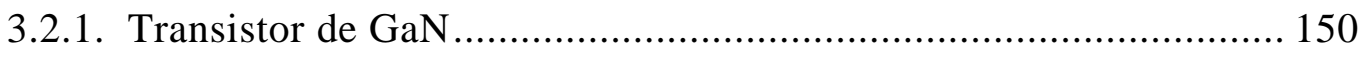

3.2.2. Funcionamiento subóptimo en clase E en banda L ...................... 153

3.2.3. Diseño del amplificador clase E subóptimo .............................. 157

3.2.4. Simulación del amplificador clase E subóptimo en banda L ......... 164

3.2.5. Implementación del amplificador clase E subóptimo en banda L ... 166

3.3. Amplificador de potencia clase E subóptimo en banda $\mathrm{S}$..............................176

3.3.1. Transistor de GaN ................................................................ 177

3.3.2. Diseño del amplificador clase E subóptimo en banda $\mathrm{S}$................ 179

3.3.3. Simulación del amplificador clase E sub-óptimo en banda S ........ 183 
3.3.4. Implementación del amplificador clase E subóptimo en banda S ... 185

4. Capítulo 4: Linealización de amplificadores

4.1. Introducción

4.1.1. Moduladores de envolvente

4.1.1.1. Convertidores conmutados 193

4.1.1.2. Convertidores conmutados asistidos linealmente 196

4.1.2. Generación de señales 197

4.1.3. Alineamiento temporal 198

4.2. Transmisor lineal basado en amplificador clase $\mathrm{F}$ 199

4.2.1. Amplificador de envolvente ................................................. 200

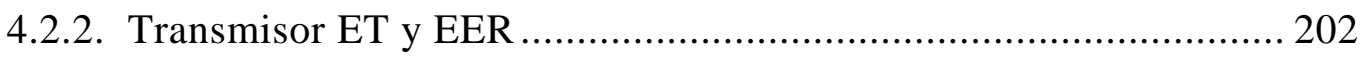

4.2.2.1. Aplicación EER .......................................................... 204

4.2.2.2. Aplicación ET ............................................................. 217

4.2.2.3. Comparación EER y ET …............................................... 225

4.3. Transmisor lineal basado en amplificador clase E ........................................226

4.3.1. Amplificador de envolvente ................................................ 228

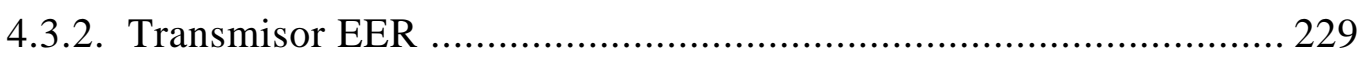

4.3.2.1. Propuesta técnica EER híbrida ....................................... 231

4.3.2.2. Prototipo sistema EER ................................................. 238

5. Capítulo 5: Conclusión ................................................................................. 247

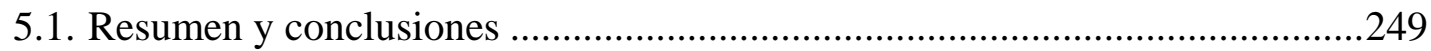

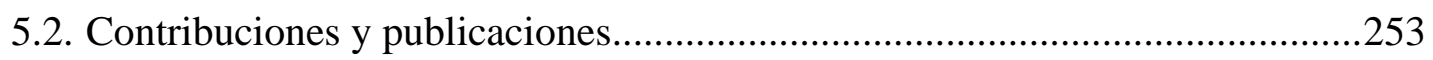

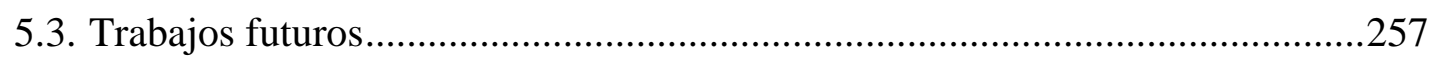

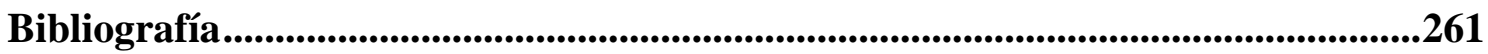




\section{Listado de figuras}

Figura 1.1. Diagrama de bloques de un transmisor de RF

Figura 1.2. Diagrama de bloques de un PA

Figura 2.1. Evolución de la tecnología de amplificadores de RF en comunicaciones inalámbricas en función del incremento de la PAPR de las señales (basada en [9]).

Figura 2.2. Tipos de transmisión y tipos de modulación de señales RADAR

Figura 2.3. Cronología de la tecnología GaN

Figura 2.4. Comparación ilustrativa de las ventajas del GaN respecto al GaAs .30

Figura 2.5. Propiedades y beneficios de la tecnología GAN. 30

Figura 2.6. Comparativa de las propiedades físicas de diferentes semiconductores ....35

Figura 2.7. Limitaciones de los dispositivos de amplificación

Figura 2.8. Rendimiento teórico de topologías y arquitecturas de amplificación respecto a la potencia de salida

Figura 2.9. Caracterización I-V del transistor y regiones de funcionamiento

Figura 2.10. Modelo simplificado de un transistor FET.

Figura 2.11. Sintonizadores de impedancia: manual (izq) y automático (drch)

Figura 2.12. Diagrama de bloques de un sistema load-pull manual. .46

Figura 2.13. Esquema de un sintonizador de impedancia manual .46

Figura 2.14. Representación del funcionamiento de un sintonizador en la carta de Smicth. 
Figura 2.15. Diagrama de bloques de un sistema load pull multiarmónico pasivo 49

Figura 2.16. Diagrama de bloques de un sistema load pull con control de armónicos (etapa de salida) .50

Figura 2.17. Esquema de un SMPA: ZVS o conmutación de tensión nula (a) y ZCS o conmutación de corriente nula (b) 52

Figura 2.18. Esquema de un SMPA clase D en modo tensión y formas de onda de tensión y de corriente

Figura 2.19. Formas de onda ideales de tensión y de corriente de un SMPA clase D ...54

Figura 2.20. Esquema de un SMPA clase D en modo corriente o clase $\mathrm{D}^{-1}$ 55

Figura 2.21. Formas de onda ideales de tensión y de corriente de un SMPA clase $\mathrm{D}^{-1} .55$

Figura 2.22. Esquema de un SMPA clase E básico 56

Figura 2.23. Formas de onda ideales de un SMPA en clase E .57

Figura 2.24. Esquema de un SMPA clase $\mathrm{E}^{-1}$ básico 58

Figura 2.25. Esquema de un SMPA clase F con infinitos resonadores .60

Figura 2.26. Formas de onda ideales de un SMPA en clase F. .60

Figura 2.27. Esquema de un SMPA clase $\mathrm{F}^{-1}$ 61

Figura 2.28. Diagrama de bloques de un SMPA clase $\mathrm{S}$ .63

Figura 2.29. Esquema de un amplificador clase $\mathbf{J}$ .65

Figura 2.30. Formas de onda ideales de un PA en clase J

Figura 2.31. Diagrama de bloques de un PA en clase $\mathrm{P}$ .68

Figura 2.32. Formas de onda ideales de un PA en clase $\mathrm{P}$ .68 
Figura 2.33. Diagrama de bloques de un sistema Doherty clásico .71

Figura 2.34. Comparación del rendimiento de un sistema Doherty y un amplificador clase B

Figura 2.35. Diagrama de bloques de un sistema Doherty asimétrico .72

Figura 2.36. Comparación del rendimiento de un sistema Doherty y un amplificador clase $\mathrm{B}$

Figura 2.37. Diagrama de bloques de un sistema Doherty invertido. . .74

Figura 2.38. Diagrama de bloques de un sistema Doherty multietapa 74

Figura 2.39. Comportamiento teórico del rendimiento de un sistema Doherty multietapa75

Figura 2.40. Diagrama de bloques de un transmisor LINC. .76

Figura 2.41. Diagrama de bloques de un transmisor Chireix .78

Figura 2.42. Efecto de la mejora del lazo feedforward. . .79

Figura 2.43. Diagrama de bloques de un transmisor basado en el lazo feedforward .....80

Figura 2.44. Esquema de un lazo de realimentación 81

Figura 2.45. Concepto de predistorsión .83

Figura 2.46. Diagrama de bloques de un transmisor EER .84

Figura 2.47. Arquitectura de un transmisor EER con modulación de driver parcial .....86

Figura 2.48. Diagrama de bloques de un transmisor ET .88

Figura 3.1. Módulos de evaluación del transistor RF3931 proporcionados por RFMD cuya frecuencia central es $900 \mathrm{MHz}$ (izq) y es $2140 \mathrm{MHz}$ (drch) .96

Figura 3.2. Detalle de la die del transistor RF3931 (izq) y modelo para AWR de la die del dispositivo y de su encapsulado (drch) .96 
Figura 3.3. Impedancias óptimas vistas por el dispositivo polarizado a $V_{D S}=48 \mathrm{~V}$ y $I_{D Q}$ $=130 \mathrm{~mA}[90]$

Figura 3.4. Diagrama de bloques de la red de salida de un amplificador clase F especificando los diferentes planos de referencia de carga

Figura 3.5. Modelo de transistor FET básico (die y encapsulado) y planos de

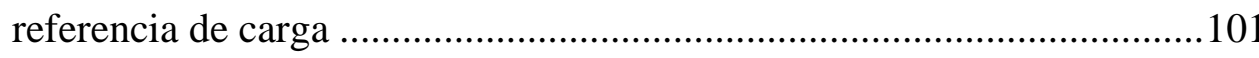

Figura 3.6. Modelo de transistor bajo polarización inversa o pinch-off..... 102

Figura 3.7. Diagrama de bloques del procedimiento de medida de la capacidad de salida equivalente del dispositivo. 103

Figura 3.8. Fotografía del proceso de medida de la capacidad de salida del dispositivo104

Figura 3.9. Modelo de transistor cortocircuitando drenador y fuente 104

Figura 3.10. Diagrama de bloques del procedimiento de medida de la capacidad de entrada equivalente del dispositivo 105

Figura 3.11. Detalle del procedimiento de medida de la capacidad de entrada del dispositivo 105

Figura 3.12. Capacidades equivalentes de entrada $\left(C_{i s s}\right)$ y de salida $\left(C_{\text {out }}\right)$ del transistor RF3931 106

Figura 3.13. Simulación de la transformación de impedancias del P3 al P1 a $2.14 \mathrm{GHz}$ provocada por el encapsulado: entrada (a) y salida (b) 107

Figura 3.14. Simulación de la transformación de impedancias del P3 al P1 a $1.64 \mathrm{GHz}$ provocada por el encapsulado: entrada (a) y salida (b) 108

Figura 3.15. Ejemplo de red de salida con control de armónicos usando líneas de transmisión

Figura 3.16. Constelación de la impedancia vista en el puerto 1 a la frecuencia armónica en función de la longitud eléctrica a la cual se posiciona el resonador armónico

Figura 3.17. Diagrama de bloques del bando de prueba utilizado en la caracterización load pull.......

Figura 3.18. Contornos de ganancia de verificación de la correcta calibración de un sistema load pull

Figura 3.19. Estándares de calibración TRL

Figura 3.20. Fotografía real de los estándares de calibración TRL diseñados 116

Figura 3.21. Comparación de las pérdidas de inserción de un cortocircuito usando calibración TRL y calibración coaxial con extensión de puertos 
Figura 3.22. Comparación de las pérdidas de retorno de un cortocircuito usando calibración TRL y calibración coaxial con extensión de puertos

Figura 3.23. Comparación de las pérdidas de inserción de una línea de $30^{\circ}$ eléctricos usando calibración TRL y calibración coaxial con extensión de puertos 118

Figura 3.24. Comparación de las pérdidas de retorno de una línea de $30^{\circ}$ eléctricos usando calibración TRL y calibración coaxial con extensión de puertos 118

Figura 3.25. Comparación de las pérdidas de inserción de un circuito abierto usando calibración TRL y calibración coaxial con extensión de puertos

Figura 3.26. Comparación de las pérdidas de retorno de un circuito abierto usando calibración TRL y calibración coaxial con extensión de puertos

Figura 3.27. Proceso de medida de la impedancia vista por el dispositivo utilizando los bloques TRL con los que se ha calibrado.

Figura 3.28. Banco de medida implementado para realizar el proceso load pull.

Figura 3.29. Prototipo de amplificador clase F con control de armónicos a través de un útil de pre-adaptación de impedancias a los armónicos (prototipo 1) ......125

Figura 3.30. Transformación de impedancias del P1 al P4 a la entrada (izq) y a la salida (drch) del prototipo 1. En rojo $2 f_{0}$ y en azul $3 f_{0}$, siendo la $f_{0}=1640 \mathrm{MHz} 126$

Figura 3.31. Medida de las impedancias intrínsecas a partir de la transformación de impedancias del P3 al P1 a la entrada (izq) y a la salida (drch) del prototipo 1. En rojo $2 f_{0}$ y en azul $3 f_{0}$, siendo la $f_{0}=1640 \mathrm{MHz}$.

Figura 3.32. Esquema del amplificador de potencia clase $F$ diseñado con el método load pull a partir del útil de pre-adaptación de impedancias (prototipo 1)128

Figura 3.33. Simulación de las formas de onda de drenador en el plano P2 del amplificador de potencia clase $\mathrm{F}$ diseñado (prototipo 1)

Figura 3.34. Simulación del funcionamiento del amplificador clase F (prototipo 2) en función de la frecuencia

Figura 3.35. Simulación del funcionamiento del amplificador clase F (prototipo 1) en función de la potencia de entrada

Figura 3.36. Medidas del funcionamiento del amplificador clase F (prototipo 1) en función de la frecuencia

Figura 3.37. Medidas del funcionamiento del amplificador clase F (prototipo 1) en función de la potencia de entrada.....

Figura 3.38. Detalle de la adaptación/recorte de los pads del dispositivo en la realización de las medidas load pull 
Figura 3.39. Prototipo de amplificador clase F con control de armónicos a través de un útil de pre-adaptación de impedancias a los armónicos (prototipo 2) ......133

Figura 3.40. Detalle de la red de entrada del amplificador clase F (prototipo 2) donde se ve el transformador multisección lineal con una longitud de $\lambda / 4$

Figura 3.41. Fotografía real de los estándares de calibración TRL diseñados con el transformador multisección lineal

Figura 3.42. Transformación de impedancias del P1 al P4 a la entrada (izq) y a la salida (drch) del prototipo 2. En rojo $2 f_{0}$ y en azul $3 f$, siendo la $f_{0}=1640 \mathrm{MHz} 136$

Figura 3.43. Medida de las impedancias intrínsecas a partir de la transformación de impedancias del P3 al P1 a la entrada (izq) y a la salida (drch) del prototipo 2. En rojo $2 f_{0}$ y en azul $3 f_{0}$, siendo la $f_{0}=1640 \mathrm{MHz}$.

Figura 3.44. Esquema del amplificador de potencia clase $\mathrm{F}$ diseñado con el método load pull a partir del útil de pre-adaptación de impedancias (prototipo 2)138

Figura 3.45. Simulación de las formas de onda de drenador en el plano P2 del amplificador de potencia clase $\mathrm{F}$ diseñado (prototipo 2)

Figura 3.46. Simulación del funcionamiento del amplificador clase F (prototipo 2) en función de la frecuencia

Figura 3.47. Simulación del funcionamiento del amplificador clase F (prototipo 2) en función de la potencia de entrada.....

Figura 3.48. Medidas del funcionamiento del amplificador clase F (prototipo 2) en función de la frecuencia 141

Figura 3.49. Medidas del funcionamiento del amplificador clase F (prototipo 2) en función de la potencia de entrada

Figura 3.50. Prototipo de amplificador clase F con control de armónicos (prototipo 3) 142

Figura 3.51. Transformación de impedancias del P1 al P4 a la entrada (izq) y a la salida (drch) del prototipo 3. En rojo $2 f_{0}$ y en azul $3 f_{0}$, siendo la $f_{0}=1640 \mathrm{MHz} 143$

Figura 3.52. Medida de las impedancias intrínsecas a partir de la transformación de impedancias del P3 al P1 a la entrada (izq) y a la salida (drch) del prototipo 3. En rojo $2 f_{0}$ y en azul $3 f_{0}$, siendo la $f_{0}=1640 \mathrm{MHz}$. 144

Figura 3.53. Esquema del amplificador de potencia clase F diseñado por simulación con control de armónicos (prototipo 3)

Figura 3.54. Simulación de las formas de onda de drenador en el plano P2 del amplificador de potencia clase $\mathrm{F}$ diseñado (prototipo 3) 146 
Figura 3.55. Simulación del funcionamiento del amplificador clase F (prototipo 3) en función de la frecuencia

Figura 3.56. Simulación del funcionamiento del amplificador clase F (prototipo 3) en función de la potencia de entrada

Figura 3.57. Medidas del funcionamiento del amplificador clase F (prototipo 3) en función de la frecuencia

Figura 3.58. Medidas del funcionamiento del amplificador clase F (prototipo 3) en función de la potencia de entrada 148

Figura 3.59. Transistor CGH40180PP empleado en el amplificador clase E subóptimo 151

Figura 3.60. Frecuencia crítica de un amplificador clase E en función de la potencia de salida para el transistor CGH40180pp

Figura 3.61. Modelo de la entrada y la salida para un transistor encapsulado 153

Figura 3.62. Formas de onda de un amplificador clase E en modo subóptimo y óptimo154

Figura 3.63. Variación del rendimiento, potencia, tensión y corriente máximas en función de la frecuencia de trabajo normalizada por $f_{\max }$

Figura 3.64. Ángulo óptimo de la admitancia para un amplificador clase E subóptimo trabajando por encima de $f_{\text {max }}$ 156

Figura 3.65. Esquema de la red de salida del amplificador clase E subóptimo en banda $\mathrm{L}$ 158

Figura 3.66. Perfil de admitancia proporcionado en el plano LP1 por la red de salida del amplificador 160

Figura 3.67. Esquema de la red de entrada del amplificador clase E subóptimo en banda $\mathrm{L}$

Figura 3.68. Perfil de ganancia en tensión del plano SP3 al SP1 y pérdidas de retorno en el plano SP3 en función de la frecuencia

Figura 3.69. Esquema completo del amplificador clase E subóptimo balanceado.......164

Figura 3.70. Simulación de la potencia de salida en función de la frecuencia en CW (para una célula del amplificador)

Figura 3.71. Simulación del rendimiento de drenador en función de la frecuencia en CW (para una célula del amplificador)

Figura 3.72. Layout diseñado en Autocad del amplificador balanceado 166

Figura 3.73. Fotografía del amplificador balanceado clase E subóptimo implementado166

Figura 3.74. Banco de medida controlado por ordenador para la realización de las medidas del amplificador clase E subóptimo balanceado 167 
Figura 3.75. Medida de la potencia de salida en función de la frecuencia en CW (para una célula del amplificador)

Figura 3.76. Medida del rendimiento de drenador en función de la frecuencia en CW (para una célula del amplificador)

Figura 3.77. Medida de la PAE en función de la frecuencia en CW (para una célula del amplificador)

Figura 3.78. Medida de la potencia de salida en función de la potencia de entrada en CW (para una célula del amplificador) 170

Figura 3.79. Sintetizador de pulsos para las medidas del amplificador en modo pulsado171

Figura 3.80. Medida de la potencia de salida en función de la frecuencia en modo pulsado (para dos células del amplificador)

Figura 3.81. Medida del rendimiento de drenador en función de la frecuencia en modo pulsado (para dos células del amplificador)

Figura 3.82. Medida de las pérdidas de retorno y de la relación de onda estacionaria de tensión en función de la frecuencia (para dos células del amplificador)..172

Figura 3.83. Diagrama de bloques del método utilizado para caracterizar la distorsión $\mathrm{V}_{\mathrm{DD}}-\mathrm{AM}$ y $\mathrm{V}_{\mathrm{DD}}-\mathrm{PM}$ del amplificador balanceado

Figura 3.84. Caracterización de la conversión $\mathrm{V}_{\mathrm{DD}}-\mathrm{AM}$ y $\mathrm{V}_{\mathrm{DD}} \mathrm{PM}$ del amplificador balanceado

Figura 3.85. Medida de un pulso RADAR de anchura $1 \mathrm{~ms}$ y frecuencia de repetición $100 \mathrm{~Hz}$ a $1300 \mathrm{MHz}$ empleando el amplificador balanceado 174

Figura 3.86. Propuesta de combinación de varios amplificadores balanceados mediante combinadores Wilkinson para duplicar la potencia de salida

Figura 3.87. Nivel de los armónicos en función de la frecuencia del tono empleado en la medida

Figura 3.88. Transistor CGH40010P empleado diseño del amplificador clase E en banda $S$

Figura 3.89. Frecuencia crítica de un amplificador clase E en función de la potencia de salida para el transistor CGH4001P 178

Figura 3.90. Esquema de la red de salida del amplificador clase E subóptimo en banda S.

Figura 3.91. Perfil de admitancia proporcionado por la red de salida del amplificador a la derecha del conmutador equivalente 181 
Figura 3.92. Esquema de la red de entrada del amplificador clase E subóptimo en band S.

Figura 3.93. Perfil de ganancia en potencia y pérdidas de retorno del amplificador en función de la frecuencia

Figura 3.94. Modelo del transistor CGH40010p ofrecido por el fabricante donde se aprecia un acceso al drenador intrínseco 184

Figura 3.95. Formas de onda simuladas del amplificador clase E subóptimo en banda S184

Figura 3.96. Simulación de la potencia de salida y del rendimiento de drenador en función de la frecuencia para el amplificador clase E subóptimo en banda S

Figura 3.97. Fotografía del amplificador clase E subóptimo en banda S implementado186

Figura 3.98. Medida de la potencia de salida y del rendimiento de drenador del amplificador clase E subóptimo en banda $\mathrm{S}$ 187

Figura 3.99. Medida de la relación de onda estacionaria y de la ganancia en potencia del amplificador clase E subóptimo en banda $\mathrm{S}$ 188

Figura 4.1. Diagrama de bloques simplificado de un transmisor de RF formado por un modulador de envolvente y amplificador de RF lineal

Figura 4.2. Funcionamiento de un amplificador de envolvente con tensión de alimentación fija 191

Figura 4.3. Funcionamiento de un amplificador de envolvente con tensión de alimentación modulada en función de la envolvente de la señal

Figura 4.4. Esquema de un convertidor reductor: asíncrono (a) y síncrono (b) . 194

Figura 4.5. Esquema de un convertidor reductor asistido en serie por un amplificador lineal 196

Figura 4.6. Esquema de un convertidor reductor asistido en paralelo por un amplificador lineal.

Figura 4.7. Diagrama de bloques del esquema empleado en la generación de señales198 
Figura 4.8. Ilustración gráfica de como un desalineamiento temporal afecta en el EVM de un sistema EER y de un sistema ET

Figura 4.9. Esquema simplificado del convertidor multifase y multinivel en serie con un regulador lineal. .201

Figura 4.10. Medida del rendimiento estático de convertidor buck multifase [159] ....202

Figura 4.11. Esquema del sistema para aplicaciones ET y EER .203

Figura 4.12. Fotografía del prototipo implementado para aplicaciones ET y EER [159] 204

Figura 4.13. Banco de medida del transmisor para aplicaciones ET y EER 205

Figura 4.14. Diagrama de bloques transmisor EER con convertidor multinivel asistido por amplificador lineal 205

Figura 4.15. Formas de onda de la salida del convertidor buck multifase (canal 1), de la envolvente de salida del convertidor (canal 3) y de la salida de RF (canal 4) de una señal 64QAM con un ancho de banda de RF de $300 \mathrm{KHz}$......206

Figura 4.16. Formas de onda de la salida del convertidor buck multifase (canal 1), de la envolvente de salida del convertidor (canal 3) y de la salida de RF (canal 4) de una señal 64QAM con un ancho de banda de RF de $600 \mathrm{KHz} . . . . . .207$

Figura 4.17. Formas de onda de la salida del convertidor buck multifase (canal 1), de la envolvente de salida del convertidor (canal 3) y de la salida de RF (canal 4) de una señal 64QAM con un ancho de banda de RF de $1200 \mathrm{KHz} . . . .207$

Figura 4.18. Formas de onda de la salida del convertidor buck multifase (canal 1), de la envolvente de salida del convertidor (canal 3) y de la salida de RF (canal 4) de una señal 64QAM con un ancho de banda de RF de $2400 \mathrm{KHz} . . . .208$

Figura 4.19. Formas de onda de la salida del convertidor buck multifase (canal 1), de la envolvente de salida del convertidor (canal 3) y de la salida de RF (canal 4) de una señal 64QAM con un ancho de banda de RF de $4800 \mathrm{KHz} . . . .208$ 
Figura 4.20. Formas de onda del nivel fijo del convertidor buck multifase (canal 1), de la envolvente de salida del convertidor (canal 3) y de la salida de RF (canal 4) de una señal 64QAM con un ancho de banda de RF de $300 \mathrm{KHz} . . . . . .209$

Figura 4.21. Formas de onda del nivel fijo del convertidor buck multifase (canal 1), de la envolvente de salida del convertidor (canal 3) y de la salida de RF (canal 4) de una señal 64QAM con un ancho de banda de RF de $600 \mathrm{KHz}$.......210

Figura 4.22. Formas de onda del nivel fijo del convertidor buck multifase (canal 1), de la envolvente de salida del convertidor (canal 3) y de la salida de RF (canal 4) de una señal 64QAM con un ancho de banda de RF de $1200 \mathrm{KHz} . . . .210$

Figura 4.23. Formas de onda del nivel fijo del convertidor buck multifase (canal 1), de la envolvente de salida del convertidor (canal 3) y de la salida de RF (canal 4) de una señal 64QAM con un ancho de banda de RF de $2400 \mathrm{KHz} . . . .211$

Figura 4.24. Formas de onda del nivel fijo del convertidor buck multifase (canal 1), de la envolvente de salida del convertidor (canal 3) y de la salida de RF (canal 4) de una señal 64QAM con un ancho de banda de RF de $4800 \mathrm{KHz} . . . .211$

Figura 4.25. Espectro de salida normalizado del amplificador EER para una señal 64QAM con un ancho de banda de RF de $300 \mathrm{KHz}$

Figura 4.26. Espectro de salida normalizado del amplificador EER para una señal 64QAM con un ancho de banda de RF de $600 \mathrm{KHz}$

Figura 4.27. Espectro de salida normalizado del amplificador EER para una señal 64QAM con un ancho de banda de RF de $1200 \mathrm{KHz}$

Figura 4.28. Espectro de salida normalizado del amplificador EER para una señal 64QAM con un ancho de banda de RF de $2400 \mathrm{KHz}$

Figura 4.29. Espectro de salida normalizado del amplificador EER para una señal 64QAM con un ancho de banda de RF de $4800 \mathrm{KHz}$ 214

Figura 4.30. Resumen de la linealidad del sistema EER en función del ancho de banda de la señal..... 215 
Figura 4.31. Rendimiento de drenador del transmisor EER en función del ancho de banda de la señal de prueba 216

Figura 4.32. Diagrama de bloques transmisor ET con convertidor multinivel

Figura 4.33. Formas de onda del transmisor ET a la salida del convertidor buck multifase (canal 1) y a la salida de RF (canal 4) para una señal 64QAM con un ancho de banda de RF de $300 \mathrm{KHz}$ .218

Figura 4.34. Formas de onda del transmisor ET a la salida del convertidor buck multifase (canal 1) y a la salida de RF (canal 4) para una señal 64QAM con un ancho de banda de RF de $600 \mathrm{KHz}$

Figura 4.35. Formas de onda del transmisor ET a la salida del convertidor buck multifase (canal 1) y a la salida de RF (canal 4) para una señal 64QAM con un ancho de banda de RF de $1200 \mathrm{KHz}$

Figura 4.36. Formas de onda del transmisor ET a la salida del convertidor buck multifase (canal 1) y a la salida de RF (canal 4) para una señal 64QAM con un ancho de banda de RF de $2400 \mathrm{KHz}$ 220

Figura 4.37. Formas de onda del transmisor ET a la salida del convertidor buck multifase (canal 1) y a la salida de RF (canal 4) para una señal 64QAM con un ancho de banda de RF de $4800 \mathrm{KHz}$

Figura 4.38. Espectro de salida normalizado del amplificador ET para una señal 64QAM con un ancho de banda de RF de $300 \mathrm{KHz}$ 221

Figura 4.39. Espectro de salida normalizado del amplificador ET para una señal 64QAM con un ancho de banda de RF de $600 \mathrm{KHz}$ .221

Figura 4.40. Espectro de salida normalizado del amplificador ET para una señal 64QAM con un ancho de banda de RF de $1200 \mathrm{KHz}$ 222

Figura 4.41. Espectro de salida normalizado del amplificador ET para una señal 64QAM con un ancho de banda de RF de $2400 \mathrm{KHz}$ .222 
Figura 4.42. Espectro de salida normalizado del amplificador ET para una señal 64QAM con un ancho de banda de RF de $4800 \mathrm{KHz}$ .223

Figura 4.43. Resumen de la linealidad del sistema ET en función del ancho de banda de la señal

Figura 4.44. Rendimiento de drenador del transmisor ET en función del ancho de banda de la señal de prueba 224

Figura 4.45. Prototipo amplificador de envolvente 229

Figura 4.46. Esquema de un sistema EER con modulación de excitación completa ...230

Figura 4.47. Esquema de un sistema EER con modulación parcial de excitación 231

Figura 4.48. Esquema del sistema EER híbrido propuesto

Figura 4.49. Formas de onda medidas del sistema EER híbrido para una señal de prueba de dos tonos .234

Figura 4.50. Productos de intermodulación normalizados con respecto a la técnica de modulación de excitación de Raab [83] $\left(E_{k}=0\right)$ para diferentes valores de $E_{0}$ y rendimiento de drenador de la técnica de modulación propuesta en este trabajo. 235

Figura 4.51. Caracterización de la distorsión del transmisor a partir de una señal triangular de baja frecuencia 236

Figura 4.52. Demodulación en amplitud (izq) y en fase (drch) de la señal triangular aplicada al transmisor siendo la frecuencia portadora $1300 \mathrm{MHz}$

Figura 4.53. Conversión $\mathrm{V}_{\mathrm{dd}}-\mathrm{AM}$ del sistema EER a $1200 \mathrm{MHz}$ 237

Figura 4.54. Conversión $\mathrm{V}_{\mathrm{dd}}-\mathrm{PM}$ del sistema EER a $1200 \mathrm{MHz}$ 238

Figura 4.55. Fotografía del prototipo de transmisor EER 238 
Figura 4.56. Filtro paso bajo diseñado (a) y respuesta en frecuencia (b) empleado en la prueba de dos tonos ....................................240

Figura 4.57. Formas de onda de un sistema EER convencional (azul) para una señal de prueba de dos tonos comparadas con las formas de onda de

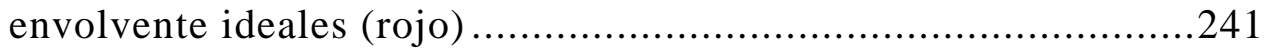

Figura 4.58. Formas de onda del sistema EER híbrido propuesto (azul) para una señal de prueba de dos tonos comparadas con las formas de onda de envolvente ideales (rojo) 241

Figura 4.59. Medida del espectro de un sistema EER convencional y del sistema EER híbrido propuesto para una señal de prueba de dos tonos ....242

Figura 4.60. Filtro paso bajo diseñado (a) y respuesta en frecuencia (b) empleado en la prueba OFDM

Figura 4.61. Medida del espectro de un sistema EER convencional y del sistema EER híbrido propuesto para una señal de prueba OFDM (con predistorsión digital) .244

Figura 4.62. Medida del EVM para el sistema EER convencional (9.95\%) y para el sistema EER híbrido propuesto $(2.86 \%)$ para una señal de prueba OFDM (con predistorsión digital) 245 


\section{Listado de tablas}

Tabla 2.1. Resumen de características de las clases de amplificación .........................69

Tabla 2.2. Comparación de las técnicas de linealización .............................................91

Tabla 3.1. Valores del modelo simplificado para el transistor CGH40180pp ............153

Tabla 3.2. Valores los componentes de la red de salida .........................................159

Tabla 3.3. Valores los componentes de la red de entrada..........................................162

Tabla 3.4. Comparación del amplificador diseñado en este trabajo con respecto al estado del arte de amplificadores de potencia en banda L.........................176

Tabla 3.5. Valores de los componentes de la red de salida .....................................180

Tabla 3.6. Valores de los componentes de la red de entrada.....................................182

Tabla 3.7. Comparación del amplificador diseñado en este trabajo con respecto al estado del arte de amplificadores de potencia en banda S........................188

Tabla 4.1. Resultados experimentales de un sistema EER convencional y del sistema EER híbrido propuesto para una señal de prueba de dos

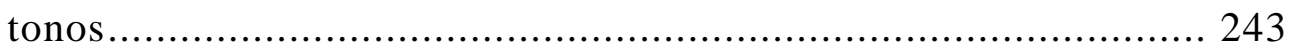

Tabla 4.2. Resultados experimentales de un sistema EER convencional y del sistema EER híbrido propuesto para una señal de prueba OFDM ..246 


\section{Capítulo 1}

\section{Introducción}

Motivación, objetivos y organización de la tesis doctoral 


\section{Capítulo 1}

\section{Introducción}

\section{Motivación, objetivos y organización de la tesis doctoral}

\section{CONTENIDO}

1.1 Motivación

1.2 Objetivos

\subsection{Organización}

1.4 Definiciones básicas

Esta tesis está encaminada hacia el problema de la amplificación con alto rendimiento de señales de radiofrecuencia (radio frequency, RF). En la actualidad, la demanda de grandes tasas de datos con espectros limitados en los enlaces de comunicaciones provoca el empleo de señales con alta relación de potencia pico a promedio (peak-to-average power ratio, PAPR). Tales señales presentan variaciones tanto en la amplitud como en la fase de la señal, lo que dificulta la amplificación eficiente de estas señales de RF.

Por otro lado, la continua investigación en tecnología de semiconductores conduce a la mejora de las prestaciones de los amplificadores de potencia (power amplifier, PA). La fabricación de dispositivos de estado sólido capaces de trabajar a altas temperaturas y a tensiones más altas ofrece considerables beneficios para los sistemas de comunicaciones actuales, entre los que se pueden destacar la reducción del coste y del tamaño.

En este capítulo se define la motivación que ha llevado al desarrollo de esta tesis doctoral junto con los objetivos a lograr. Además, se presenta la estructura de la memoria. Por último, se detallan algunas definiciones básicas de los transmisores de microondas que han sido usadas en el progreso de escritura de esta tesis. 


\subsection{Motivación}

Los nuevos sistemas de comunicación han ido evolucionando hacia esquemas de modulación más complejos con el fin de incrementar la tasa de transmisión de datos y aprovechar el limitado espectro de frecuencias. El objetivo principal es permitir mayores capacidades de transmisión de datos y ofrecer mayor flexibilidad en las comunicaciones inalámbricas. Sin embargo, este objetivo implica un incremento del ancho de banda y de la relación pico-medio de las señales y, por lo tanto, queda limitado por las características del nivel físico de los sistemas.

Generalmente, las señales moduladas capaces de conseguir altas tasas de transmisión de datos se caracterizan por presentar envolventes no constantes. Al variar la envolvente en el tiempo, las señales sufren grandes diferencias entre la potencia media y la de pico. Empleando señales con alta PAPR, se convierte menor cantidad de potencia de DC (Direct Current) en potencia de RF radiada, debido a que normalmente el amplificador alcanza su máximo rendimiento trabajando en la zona de saturación de potencia de salida y se reduce significativamente a medida que la potencia de entrada va disminuyendo, o lo que es lo mismo va aumentando el "back-off'. Además, se corre el riesgo de producir distorsión si se aplican niveles excesivos de potencia al amplificador cuando se trabaja con señales de alta PAPR.

La amplificación de señales moduladas en amplitud requiere mantener una alta linealidad con el objetivo de que la distorsión a la salida se encuentre dentro de los límites permitidos por las especificaciones de los sistemas. Normalmente, la búsqueda de una mayor linealidad implica que el rendimiento del amplificador de potencia se reduzca. Por ello, es necesario buscar un equilibrio entre rendimiento y linealidad cuando se trabaja con señales de alta PAPR. Además, en transmisores multiportadora, como son WCDMA, HSPA y LTE, la PAPR de la señal suele ser mayor que en los sistemas monoportadora.

Por consiguiente, la característica PAPR de las señales se ha convertido en un concepto importante a la hora de diseñar amplificadores de potencia de RF en los sistemas actuales [1]. De ahí que conocer las características de estas señales moduladas debe convertirse en el primer paso a la hora de trabajar en el diseño de amplificadores de potencia. 
Como ya se ha comentado, trabajar en la zona lineal del transistor provoca una reducción de la potencia de salida y del rendimiento medio. Por otro lado, el empleo de modos de amplificación de alto rendimiento, normalmente altamente no lineales, requieren la utilización de otras técnicas para restaurar la linealidad de la señal modulada en amplitud.

El empleo de señales con alta PAPR con el fin de incrementar la eficiencia espectral y aumentar la tasa de datos tiene su contrapartida en el diseño de los amplificadores. Los niveles de potencia de salida con mayor probabilidad se producen a niveles de rendimiento bajos. Este impacto se puede ver claramente si se examina el consumo de potencia de una estación base de alta potencia para comunicaciones móviles. Típicamente, el enlace descendente consta de una etapa de potencia formada por un transmisor clase AB. El consumo de potencia del sistema se encuentra entre 500 y 1000 W para generar una potencia de salida de RF de 60-120W, lo que se traduce en un rendimiento cercano al $12 \%$ [2].

Los operadores de red se enfrentan a un alto coste operativo donde el amplificador de potencia de RF representa el 50-80\% de la potencia total consumida [3]. De esta potencia consumida, normalmente solo el $30 \%$ se convierte en señal de RF trasmitida [4]. Una estación base para una infraestructura 2G/3G/4G normalmente consume entre $2 \mathrm{~kW}$ y $5 \mathrm{~kW}$ [5]. Según el proyecto Opera-Net 2 encaminado a investigar las mejoras en el rendimiento energético de las redes radio móviles [6], solo en Europa se estima que una mejora de un $20 \%$ de rendimiento en el amplificador de potencia de una estación base $3 \mathrm{G}$ resultaría en la reducción anual de 17 kilotoneladas de emisiones de $\mathrm{CO}_{2}$.

En la práctica, la energía empleada se puede calcular en emisiones de $\mathrm{CO}_{2}$. Un kilovatio-hora se puede convertir en aproximadamente $0.658 \mathrm{Kg}$ de emisiones de $\mathrm{CO}_{2}$ [7]. Las redes de comunicaciones móviles son las que más contribuyen en las emisiones de $\mathrm{CO}_{2}$ [8]. Las claves esenciales para enfocar la reducción de las emisiones de $\mathrm{CO}_{2}$ se centran principalmente en reducir el consumo de energía utilizado por las estaciones base, reducir el consumo de energía de los sistemas de refrigeración y reducir el tamaño de los equipos que forman las estaciones base. Todas estas claves se pueden enfocar en una sola: incrementar el rendimiento. 
Por otro lado, la continua investigación en tecnología de semiconductores conduce a la mejora de las prestaciones de los amplificadores de potencia. Los tubos de vacío desaparecieron de los productos electrónicos hace años. Sin embargo, ha habido algunos productos que continúan basándose en esta tecnología para su producción, como pueden ser los tubos de ondas progresivas (TWT) en los satélites.

Uno de estos productos es el horno microondas. El componente principal de un horno de microondas es un magnetrón normalmente basado en un oscilador de tubo de vacío de microondas de alta potencia que opera a $2.45 \mathrm{GHz}$. Esta tecnología tiene problemas de deterioro con el tiempo. En la actualidad ya es posible reemplazar el magnetrón con dispositivos de estado sólido. Hasta ahora había pocos transistores, si es que había alguno, que pudiesen generar cientos de vatios en la frecuencia de microondas. Eso ha cambiado en los últimos años con los avances de la tecnología GaN (Nitruro de Galio) HEMT (High Electron Mobility Transistor) y el avance del LDMOS (Laterally-Diffused Metal-Oxide Semiconductor) de silicio.

La fabricación de dispositivos de estado sólido capaces de trabajar a altas temperaturas y a tensiones más altas ofrece considerables beneficios para los sistemas de comunicaciones actuales, entre los que se pueden destacar la reducción del coste y del tamaño, y para la fabricación de otros productos comerciales, como puede ser el ejemplo del microondas.

En conclusión, un incremento del rendimiento del PA reduce el consumo de potencia de alimentación debido tanto a la etapa de potencia como a los elementos de refrigeración y otro equipamiento adicional y, en consecuencia, también se reducen las emisiones de $\mathrm{CO}_{2}$, el tamaño y peso de los sistemas y se mejora su fiabilidad, además de la reducción del coste de la energía necesaria para su funcionamiento. Este objetivo puede ser conseguido mediante el empleo y la combinación de diferentes arquitecturas (modo de amplificación de alto rendimiento o técnicas de linealización) y/o tecnologías de nuevos semiconductores.

Esta investigación está enfocada en el desarrollo de amplificadores de potencia de alto rendimiento empleando semiconductores de GaN con el fin de abordar las limitaciones de rendimiento en potencia de los sistemas. Para ello, diferentes arquitecturas de linealización fueron empleadas, tales como la técnica de seguimiento de envolvente (ET) y la técnica de restauración y eliminación de envolvente (EER). 
La investigación ha sido realizada en el Grupo de Ingeniería de Radio (GIRA) perteneciente al Centro de Electrónica Industrial (CEI) de la Universidad Politécnica de Madrid (UPM). El desarrollo de esta tesis doctoral ha sido gracias al equipamiento del grupo de investigación y a los proyectos de investigación que además han servido de validación de los prototipos diseñados.

\subsection{Objetivos}

Varios objetivos fueron planteados durante la realización de esta tesis doctoral con el fin de desarrollar amplificadores de potencia de alto rendimiento para aplicaciones ET/EER basados en tecnología GaN.

El primer objetivo es realizar un estudio minucioso del estado del arte dentro del campo de esta investigación. Dentro de este objetivo se pueden distinguir cuatro puntos de investigación. El primero de ellos es referente a las características de las señales de comunicaciones en microondas y su evolución a lo largo del tiempo. El segundo es realizar un estudio de las diferentes tecnologías de semiconductores para fabricar dispositivos con los que diseñar amplificadores de potencia de alto rendimiento. El tercero se basa en explicar los diferentes métodos y técnicas de diseño de amplificadores de potencia de alto rendimiento. Y, por último, poner sobre la mesa las diferentes técnicas de linealización necesarias en los sistemas de comunicaciones modernos.

El segundo objetivo es utilizar las capacidades del grupo de investigación para comprender el efecto de los elementos parásitos de los transistores de GaN en los amplificadores de potencia de alto rendimiento. La capacidad equivalente de salida $\left(C_{\text {out }}\right)$ de un transistor varia de manera no lineal con la tensión de alimentación y, junto con el efecto del encapsulado trabajando a altas frecuencias, es necesario realizar un análisis de la carga presentada al dispositivo vista desde el interior del mismo para conocer la dinámica de funcionamiento del amplificador.

El tercero objetivo es emplear tanto ingeniería en la forma de onda como en la metodología de diseño de amplificadores de potencia basados en GaN. De esta manera se diseñaron, construyeron y probaron varios amplificadores de alto rendimiento con 
diferentes topologías y a diferentes frecuencias. En el diseño de estos amplificadores se optó por dos caminos totalmente diferentes: uno de ellos basado en medidas empíricas a través del método load-pull y el otro basado en el cálculo teórico de la carga que debe ser presentada al dispositivo.

El cuarto objetivo está enfocado en la aplicación de técnicas de linealización como ET o EER para ajustarse a las señales de comunicaciones inalámbricas modernas. Dentro de este objetivo también se incluye la integración de los amplificadores diseñados en el objetivo anterior con el fin de implementar transmisores de alto rendimiento cuya linealidad sea capaz de cumplir las especificaciones de los estándares de comunicaciones.

El último objetivo fue la implementación de un banco de medida automático para la realización de las medidas de los amplificadores y transmisores diseñados. Este banco de medida, controlado a través de Matlab, permite realizar la caracterización de los prototipos de una manera más rápida y exacta, evitando problemas de calentamiento que desvirtúen los resultados en el tiempo.

\subsection{Organización}

Este trabajo está dividido en los siguientes capítulos:

Capítulo 2: presenta los desafíos que exhiben las señales con alta PAPR en el diseño de amplificadores de potencia. Posteriormente, se ofrece una descripción general de las tecnologías de semiconductores emergentes. Por último, se discute sobre el estado de arte de los amplificadores de potencia y de las diferentes arquitecturas de linealización más importantes en los últimos tiempos.

Capítulo 3: desarrolla diversos prototipos diseñados a partir de los métodos discutidos en el capítulo anterior y exhibe los objetivos, el diseño de las redes de adaptación, las simulaciones y los resultados experimentales obtenidos. Además, muestra la implementación de un banco de pruebas automático para la realización de las medidas. 
Capítulo 4: presenta la integración de los amplificadores de alto rendimiento diseñados con alguna de las arquitecturas de linealización presentadas, concretamente, ET y/o EER. Además, compara el funcionamiento de ambas arquitecturas. La integración de unos de los transmisores implementado en este capítulo se realizó en colaboración con el CEI de la UPM. Los transmisores diseñados fueron caracterizados y medidos empleando diferentes señales de prueba, tanto analógicas como digitales.

Capítulo 5: resume los principales puntos de investigación de esta tesis doctoral y las conclusiones más importantes, destacando las contribuciones más relevantes que avalan la calidad del trabajo de investigación realizado. Adicionalmente, se listan todas las publicaciones que han sido publicadas a raíz de este trabajo de investigación y se discute sobre los trabajos de investigación futuros.

\subsection{Definiciones básicas}

El diagrama de bloques de un transmisor de RF polar se puede ver en la Figura 1.1. El amplificador de potencia o etapa de potencia es la fuente principal de consumo de potencia, lo que hace enfocar la mayoría de los trabajos, incluido éste, en este componente clave. Entre los parámetros principales en el diseño de amplificadores de potencia (PAs) se pueden destacar la frecuencia, el ancho de banda, la potencia de salida, la ganancia y el rendimiento.

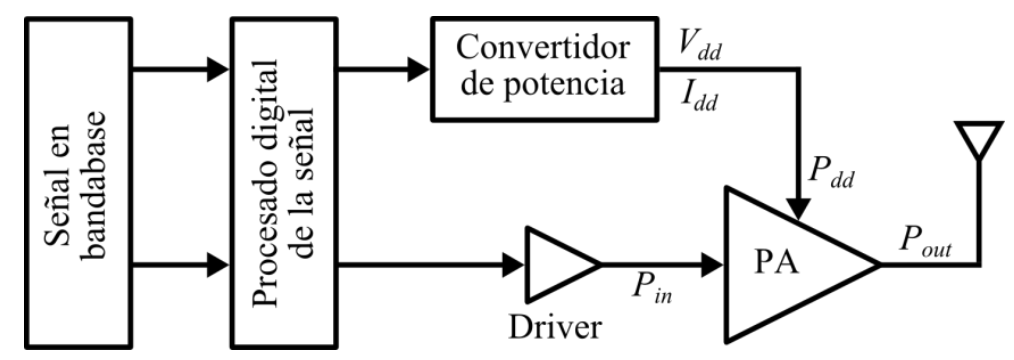

Figura 1.1. Diagrama de bloques de un transmisor de RF

Esta tesis se centra en el diseño de amplificadores de alta potencia en la banda de frecuencias de microondas con el objetivo de obtener altos rendimientos en grandes anchos de banda de RF y funcionamiento lineal. 
A continuación, se van a describir algunas definiciones generales que serán empleadas a lo largo de este trabajo:

Frecuencia fundamental y armónicos: la frecuencia fundamental, $f_{0}$, o frecuencia de portadora (a veces escrita como $f_{c}$ ) se empleará para definir la frecuencia del transmisor; y las frecuencias armónicas, $f_{n}$, se emplearán para denotar las frecuencias que son múltiplo entero de la fundamental. Por ejemplo, $f_{2}=2 f_{0}$ se refiere al segundo armónico.

Potencia de entrada y de salida de RF: $P_{\text {in }}$ y $P_{\text {out }}$ es la potencia media de RF de entrada y de salida respectivamente, siempre referenciada a una carga de $50 \Omega$. La conversión entre la potencia de RF y el valor de pico de la tensión de RF (también llamada tensión de envolvente o modulación de amplitud) se puede obtener como:

$$
P=\frac{\left|V_{r m s}\right|^{2}}{R}=\frac{\left(\frac{\left|V_{p k}\right|^{2}}{\sqrt{2}}\right)}{R}=\frac{\left|V_{p k}\right|^{2}}{2 R}
$$

Ganancia: G es la relación entre la potencia de salida y la potencia de entrada para una etapa o una serie de etapas. La ganancia de las componentes de RF de la etapa de potencia de un transmisor se puede expresar en decibelios (dB) como:

$$
G=10 \log \left(\frac{P_{\text {out }}}{P_{\text {in }}}\right)
$$

Ancho de banda de RF: $\Delta B$ es la banda de frecuencia cubierta por los puntos que están, sino se hace otra especificación, 10 dB por debajo de la potencia máxima. Una manera común de representar el ancho de banda de un amplificador es a través del ancho de banda fraccional $(F B)$, que se calcula a través del límite superior de frecuencia $\left(f_{h}\right)$ y el límite inferior $\left(f_{l}\right)$ como:

$$
F B=\frac{f h-f l}{f h+f l}
$$




\section{Rendimiento en un amplificador de potencia}

De las siguientes tres definiciones respecto a rendimiento, únicamente las dos primeras han sido usadas en este trabajo:

Rendimiento de drenador: $\eta_{d}$ es la relación entre la potencia de salida de RF y la potencia de la alimentación de drenador $\left(P_{d d}\right)$ :

$$
\eta_{d}=\frac{P_{\text {out }}}{P_{d d}}
$$

Cabe destacar que el rendimiento de drenador no tiene en cuenta la cantidad de potencia de entrada de RF consumida. Cuando la ganancia es baja (y la potencia de entrada es una parte importante de la potencia de salida) resulta conveniente emplear otra definición de rendimiento. Sin embargo, cuando la potencia de entrada es prácticamente insignificante respecto a la potencia de salida (para ganancias mayores de $10 \mathrm{~dB}$ ) esta definición es ampliamente utilizada.

Rendimiento de potencia añadida (PAE): tiene en cuenta la potencia de entrada consumida y se define como:

$$
\mathrm{PAE}=\frac{P_{\text {out }}-P_{\text {in }}}{P_{d d}}
$$

La PAE es siempre menor que el $\eta_{d}$, pero a medida que la ganancia aumenta el $\eta_{d}$ y la PAE tienden a converger hacia un mismo valor.

Rendimiento global o total: tiene en cuenta tanto la potencia de entrada como la potencia disipada en forma de calor, $P_{d i s}$, y se define como:

$$
\eta_{t}=\frac{P_{\text {out }}-P_{\text {in }}}{P_{d d}-P_{d i s}}
$$

\section{Elementos de un amplificador de potencia}

El diagrama de bloques de un PA elemental empleando un transistor de efecto de campo (FET) se puede ver en la Figura 1.2. Algunas definiciones son las mismas que para la etapa de potencia de la Figura 1.1. A continuación, se incluye terminología adicional: 


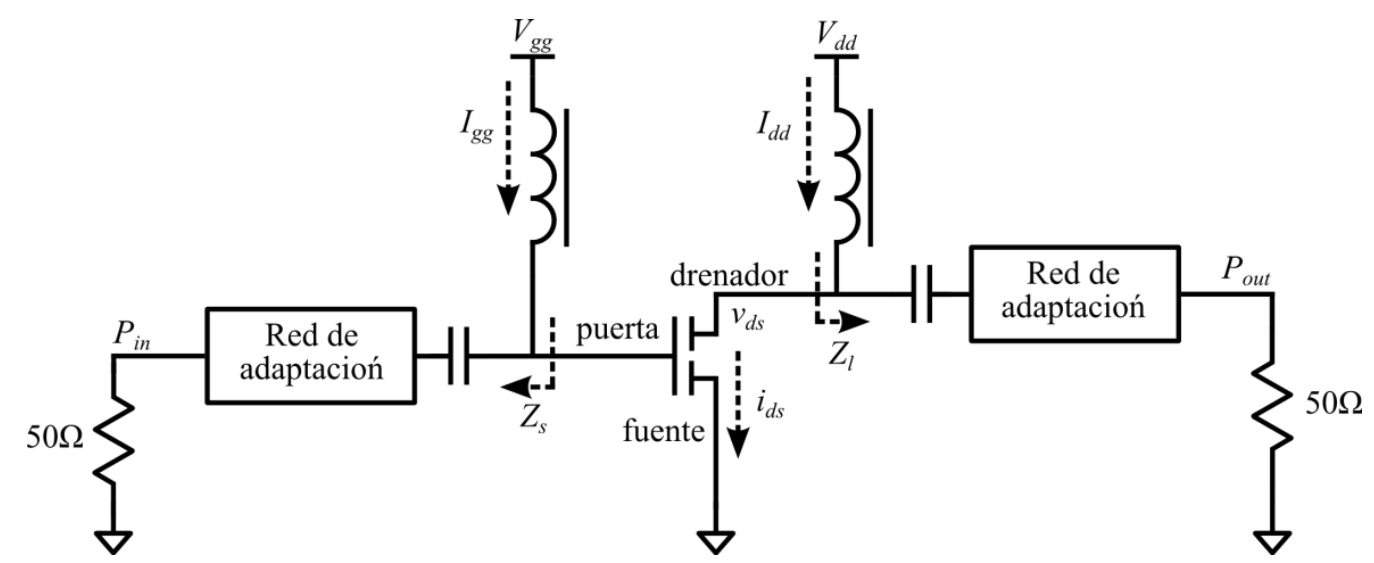

Figura 1.2. Diagrama de bloques de un PA

Transistor: es el dispositivo semiconductor que se encarga de producir una señal de salida en respuesta a otra señal de entrada.

Impedancia de fuente y de carga: $Z_{s}$ y $Z_{l}$ son las impedancias vistas por el transistor desde el terminal de fuente y de drenador respectivamente. Destacar que estas impedancias se definen no sólo a la frecuencia fundamental, sino también a los armónicos.

Tensión polarización de puerta: $V_{g g}$ es el valor de DC de la tensión de polarización de puerta.

Tensión y corriente de alimentación de drenador: $V_{d d}$ e $I_{d d}$ son los valores medios de las componentes de alimentación de drenador. Existen variaciones de este diagrama general en las que esta tensión de alimentación puede ser variable, como en el caso de amplificadores con modulación de envolvente.

Tensión y corriente de drenador: $v_{d s}$ e $i_{d s}$ son las componentes de tensión y de corriente instantánea a través de los terminales drenador y fuente. Si tensión y corriente coinciden al mismo tiempo en el drenador se produce disipación de potencia en el transistor.

\section{Modulaciones en amplificadores de potencia}

Las siguientes definiciones están relacionadas con las señales moduladas: 
Envolvente: una modulación de amplitud provoca variaciones lentas de la tensión de RF de pico de la portadora. Este nivel se corresponde con la envolvente de la señal modulada.

Representación en banda base: una modulación vectorial sobre una portadora de RF se puede expresar en términos de amplitud y fase o como la suma ortogonal de las componentes en fase y en cuadratura, de tal manera que:

$$
v(t)=A(t) \cos (\omega t+\phi(t))=\mathrm{I}(t) \cos (\omega t)-\mathrm{Q}(t) \sin (\omega t)
$$

Cuando se analizan señales moduladas es de gran utilidad la eliminación de la portadora de RF, dejando una representación en banda base mediante la representación IQ o la amplitud y la fase. Ambas formas son equivalentes.

Potencia de envolvente de pico (PEP): es el valor máximo instantáneo de la potencia de salida de RF.

Potencia media: $P_{\text {avg }}$ es la potencia de salida de RF media respecto al tiempo.

Factor de cresta: es la relación entre la amplitud de pico $\left(A_{\text {peak }}\right)$ y el valor cuadrático medio al cuadrado $\left(P_{R M S}\right)$.

$$
\xi=\frac{A_{\text {peak }}}{\sqrt{P_{R M S}}}
$$

Como regla general, la energía contenida en los armónicos superiores crece a medida que se incrementa el factor de cresta. A pesar de que las modulaciones espectralmente eficientes conllevan un factor de cresta alto, causa interferencia con los canales adyacentes y distorsión en banda, lo que explica el aumento de la tasa de bit erróneo. 
Relación de potencia pico-medio (PAPR): es el indicador del factor de cresta en unidades logarítmicas.

$$
P A P R=10 \cdot \log _{10}\left(\frac{A_{\text {peak }}}{\sqrt{P_{R M S}}}\right)^{2}=20 \cdot \log _{10}\left(\frac{A_{\text {peak }}}{\sqrt{P_{R M S}}}\right)
$$

Función de distribución de probabilidad (PDF): define la probabilidad de un nivel de potencia de salida modulada instantánea concreto a partir de un histograma normalizado de datos basados en el dominio del tiempo. 


\section{Capítulo 2}

Estado del arte 


\section{Capítulo 2}

\section{Estado del arte}

Características de las señales moduladas modernas, visión global de las tecnologías de semiconductores, amplificadores de potencia de alto rendimiento $y$ arquitecturas de linealización

\section{CONTENIDO}

2.1 Características de las señales moduladas modernas

2.2 Dispositivos para PA de alto rendimiento

2.3 Métodos de diseño de amplificadores de potencia

2.4 Arquitecturas de linealización

El rápido crecimiento y la demanda de mayores anchos de banda para soportar el alto tráfico de datos han impulsado el desarrollo de esquemas de modulación más complejos para permitir que haya más usuarios dentro del espectro limitado disponible. Sin embargo, estos esquemas de modulación eficientes desde el punto de vista espectral presentan un gran desafío de rendimiento energético en los amplificadores de potencia de las estaciones base debido a su envolvente variable en el tiempo y a su alta PAPR.

El amplificador de potencia de RF es el mayor responsable del consumo de potencia de los sistemas [9]. Mejorar el rendimiento del amplificador de potencia contribuirá significativamente a la reducción del coste de funcionamiento. Estos amplificadores en combinación con arquitecturas de alto rendimiento permiten trabajar con señales de alta PAPR.

En la primera parte de este capítulo se presentan los desafíos que presentan las señales con alta PAPR en el diseño de amplificadores de potencia. Posteriormente, se ofrece una descripción general de las tecnologías de semiconductores emergentes. Por último, se discute sobre el estado de arte de los amplificadores de potencia y de las diferentes arquitecturas de linealización más importantes en los últimos tiempos. 


\subsection{Características de las señales moduladas modernas}

La evolución de los sistemas de comunicación con el objetivo de incrementar la tasa de transmisión de datos dentro de un ancho de banda limitado conlleva esquemas de modulación más complejos. Este tipo de modulaciones normalmente se caracterizan por presentar envolventes no constantes con altas relaciones pico-medio provocando que los transmisores conviertan menor cantidad de potencia de DC en potencia de RF.

La amplificación de señales moduladas en amplitud debe mantener una linealidad que cumpla las especificaciones de los estándares de comunicaciones. Normalmente, está búsqueda de linealidad tiene su contrapartida en el rendimiento del amplificador de potencia. Por ello, es necesario buscar un equilibro entre rendimiento y linealidad cuando se trabaja con señales de alta PAPR.

De ahí que la PAPR de las señales sea un concepto muy importante a la hora de diseñar amplificadores de potencia para los sistemas de comunicaciones actuales [1]. Por ello, el primer paso es conocer las características de estas señales moduladas. En la Figura 2.1 se pueden ver un resumen de las características principales en la evolución de las señales de comunicaciones móviles inalámbricas y las típicas arquitecturas de comunicaciones utilizadas para amplificar cada una de esas señales, así como la tecnología de semiconductores empleada.

\subsubsection{Señales $2 G$}

La segunda generación (2G) de los sistemas de comunicaciones inalámbricas se conoce como Global System Mobile Communications (GSM). El primer sistema comercial GSM tuvo lugar en los años 90. Estaba basado en una modulación GMSK (Gaussian Minnimum Shift Keying) caracterizada por generar continuos cambios de fase manteniendo una envolvente constante, dando como resultado una relación PAPR nula. Al trabajar con envolventes constantes, los sistemas $2 \mathrm{G}$ se caracterizan por ser sistemas de banda estrecha cuyos amplificadores de potencia no necesitan grandes requisitos. 


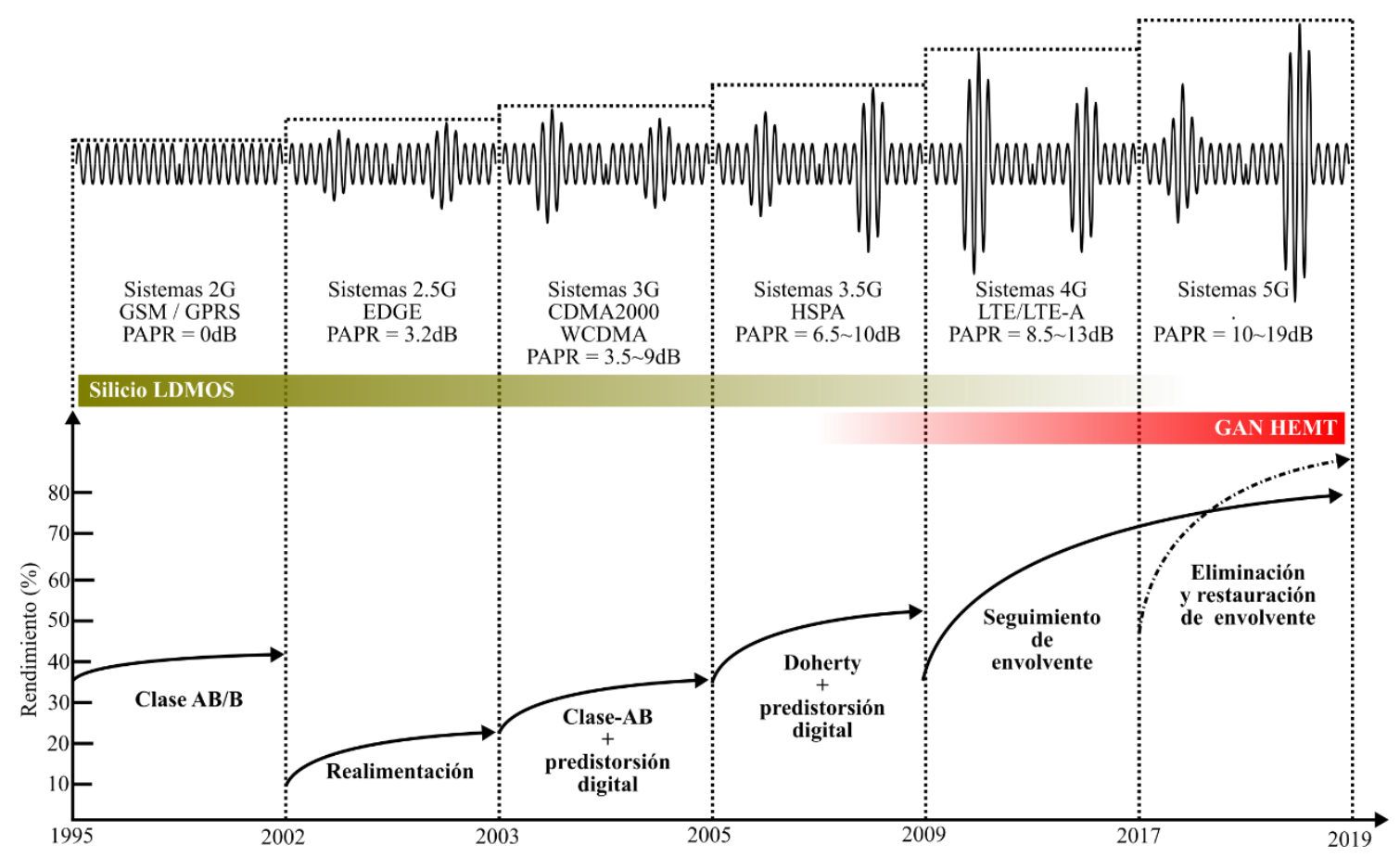

Figura 2.1. Evolución de la tecnología de amplificadores de RF en comunicaciones inalámbricas en función del incremento de la PAPR de las señales (basada en [9]).

Posteriormente surgió una evolución de 2G (2.5G) con el nombre de Enhanced Data Rates for Global Evolution (EDGE). Esta generación migró a una modulación más eficiente, 8-PSK (Phase Shift Keying). En ella, la trayectoria entre símbolos es más compleja presentando variaciones en la componente de amplitud lo que conlleva un incremento de la PAPR hasta alrededor de los $3.2 \mathrm{~dB}$. Esta situación se refleja en el amplificador de potencia de RF el cual requiere una mayor linealidad que para un sistema GSM. Por otro lado, un aumento de la PAPR se traduce en una reducción de la potencia media y, de ahí, una mejora en la PAE.

\subsubsection{Señales 3G}

A pesar de que a mediados de los años 90 surgió la tercera generación (3G) a través de Universal Mobile Telecomuminications System (UMTS), no fue hasta alrededor de 2003 cuando surgieron los primeros productos comerciales. En contraposición de tecnologías de acceso anteriores que emplean servicios de acceso por división en el tiempo o por división en frecuencia, 3G emplea un conjunto de técnicas de acceso múltiple por separación de código (WCDMA) cuyos datos son modulados en 
QPSK, la cual presenta una PAPR entre los 3.5 y 9 dB. A mayor relación entre la potencia de pico y la potencia media el amplificador de potencia genera mayores no linealidades y se produce una degradación en el rendimiento global.

La evolución de la tercera generación de telefonía móvil, conocida como 3.5G y concentrada en la tecnología HSPA, mejora significativamente la capacidad máxima de transferencia de información y la eficiencia espectral al incorporar nuevas técnicas de modulación; en un principio 16QAM en HSPA y posteriormente 64QAM en HSPA+. Dicha evolución es capaz de ofrecer una tasa de transmisión de datos en el enlace descendente de 14 y $42 \mathrm{Mbps}$, respectivamente. En cuando a la PAPR, las formas de onda de esta tecnología pueden alcanzar niveles por encima de los $10 \mathrm{~dB}$, degradando aún más el rendimiento total del sistema.

\subsubsection{Señales $4 G$}

La cuarta generación (4G) comenzó a forjarse en 2004 con el estándar Long Term Evolution (LTE), inicialmente enfocado en el desarrollo de Universial Terrestrial Radio Access (UTRA), más conocido como 3.9G. Esta especificación se comenzó a detallar en 2007 y terminó a finales de 2008. El principal objetivo de LTE es incrementar la tasa de transmisión de datos hasta los $100 \mathrm{Mbps}$ en el enlace descendente y hasta $50 \mathrm{Mbps}$ en el enlace ascendente, lo que significa 4 y 3 veces la tasa conseguida por HSPA, respectivamente. Esta última evolución, denominada LTE-Advanced (LTEA), se conoce mundialmente como 4G. El tipo de modulación elegida es OFDM y soporta los siguientes esquemas de modulación de datos: QPSK, 16QAM o 64QAM. La multiplexación OFDM permite obtener mayores tasas de transmisión de datos e incrementar la eficiencia espectral, pero estas señales multiplexadas presentan una PAPR más alta, normalmente comprendidas entre 8.5 y $13 \mathrm{~dB}$.

\subsubsection{Señales $5 G$}

La quinta generación (5G) lleva años en desarrollo y hoy en día ya es una realidad. Respecto a 4G, aumenta la velocidad de transferencia de datos del suscriptor y, además, amplia la cantidad de suscriptores atendidos por estación base. Este incremento 
de usuarios está más enfocado a IoT (Internet de las cosas) donde multitud de sensores estarán conectados a la red.

Técnicamente, el estándar 5G permite transferir datos a velocidades 10 veces mayor que 4G. Además, reduce significativamente la latencia lo que lo hace muy atractivo para aplicaciones de tiempo real. Esto permite no solo conectar personas sino conectar con otros sectores como la automoción, la medicina, los hogares... La demodulación sigue siendo OFDM con los mismos esquemas de modulación que LTE. Sin embargo, la PAPR se incrementa con niveles entre 10 y $19 \mathrm{~dB}$ y el ancho de banda espectral también aumenta hasta los $100 \mathrm{MHz}$.

En conclusión, a medida que han ido avanzando los sistemas de comunicaciones móviles inalámbricas los sistemas necesitan de amplificadores de potencia de RF más lineales.

\subsubsection{Señales RADAR}

En los apartados anteriores se han descrito las características de las señales moduladas modernas en el caso de comunicaciones móviles inalámbricas. Pero existen otras aplicaciones donde también se emplean amplificadores de potencia de RF, como por ejemplo en sistemas RADAR.

La forma de onda típica de un radar se puede escribir como:

$$
\bar{x}(t)=a(t) \sin [\Omega t+\theta(t)]
$$

donde $\Omega$ representa la frecuencia de portadora de la señal radar (en radianes por segundo), a(t) representa la modulación de amplitud de la portadora de RF y $\theta(\mathrm{t})$ representa la modulación de fase o de frecuencia de la portadora.

En un radar pulsado, la modulación de amplitud utilizada es una función rectangular que genera un pulso en la forma de onda de ON y OFF. La Figura 2.2 muestra varios ejemplos de formas de onda comunes en un radar pulsado.

Un único pulso es simplemente una ráfaga con amplitud constante a la frecuencia de RF. La frecuencia de un pulso modulado linealmente en frecuencia (LFM, Linear Frequency Modulated) se incrementa a un ritmo constante durante la duración 
del pulso en ON. Los pulsos LFM pueden también ser de decremento de frecuencia. El último caso es un pulso codificado en fase binario, donde la frecuencia es constante pero la fase puede cambiar $180^{\circ}$ en el tiempo.

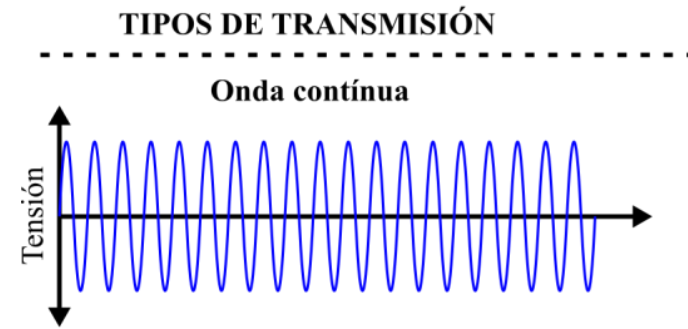

Tren de pulsos

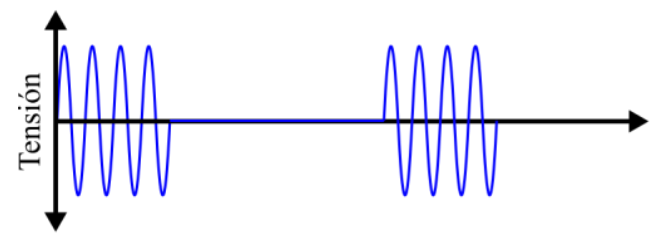

Pulso corto

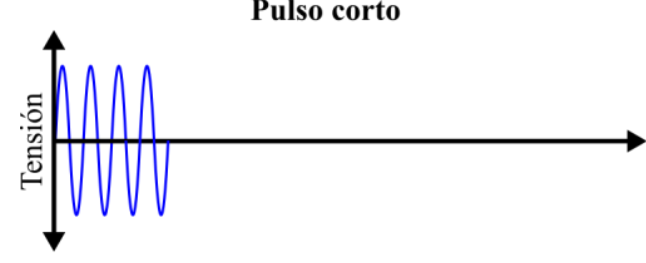

TIPOS DE MODULACIÓN

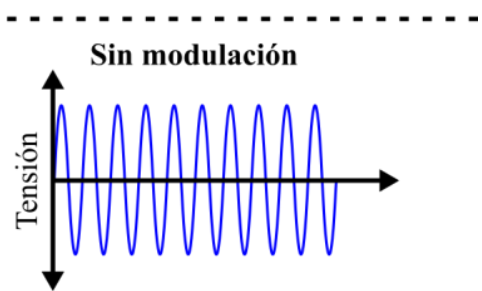

LFM (Modulación lineal en frecuencia)
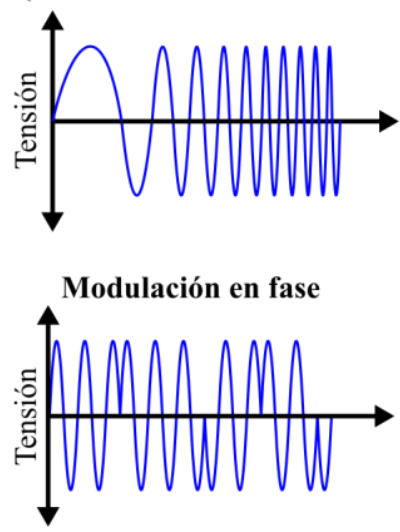

Figura 2.2. Tipos de transmisión y tipos de modulación de señales RADAR.

Por otro lado, las señales radar se pueden dividir en: radar de onda continúa (CW) o radar pulsado. Las formas de onda pulsadas se pueden definir basándose en un pulso continuo, en un tren de pulsos o en un único pulso. Tanto las formas de onda pulsadas como de onda continua pueden caracterizarse por la presencia o la ausencia de modulación de fase o de frecuencia. La modulación de fase puede ser bifase (con dos posibles estados) o polifase (con múltiples estados de fase); la modulación en frecuencia puede ser lineal o no lineal.

Este tipo de señales con envolvente constante pueden ser amplificadas con topologías de alto rendimiento como clase F o clase E. Sin embargo, es necesario tener en cuenta que la amplificación no produzca ningún decaimiento en la amplitud del pulso que implique la necesidad de incorporar un circuito de compensación de ganancia. Además, en caso de trabajar con señales moduladas en fase, será necesario caracterizar la distorsión de fase del amplificador y en caso de ser necesario corregirla con alguna técnica como por ejemplo predistorsión. 


\subsection{Dispositivos para PA de alto rendimiento}

A mediados del siglo XX, se descubrió que los materiales semiconductores podían ser utilizados para fabricar dispositivos electrónicos capaces de realizar las mismas funciones que las válvulas de vacío. Este descubrimiento hizo posible la aparición de los primeros circuitos integrados.

Desde entonces, se ha producido un amplio desarrollo de las tecnologías relacionadas con dichos materiales. Día a día van apareciendo una gran variedad de materiales semiconductores que pueden ser empleados para fabricar dispositivos electrónicos, cada uno con sus características y aplicaciones. La tendencia de los dispositivos basados en semiconductores de estado sólido es conseguir mayor densidad de potencia de salida, incrementar el rendimiento, trabajar a frecuencias más altas y mejorar la fiabilidad. Con el fin de conseguir estos requisitos, se han desarrollado novedosos diseños de dispositivos y tecnologías. En [10], [11] y [12] se puede profundizar en el diseño de este tipo de dispositivos.

Más de 35 años atrás, antes de la aparición del primer transistor de GaAs de efecto de campo, los diseñadores de amplificadores de estado sólido estaban limitados a los dispositivos activos disponibles: transistores bipolares de silicio ( $\mathrm{Si}$ ) y diodos GaAs Gunn. Por encima de $4 \mathrm{GHz}$, los diseñadores se vieron obligados a emplear diodos Gunn en las aplicaciones con las desventajas que ello acompañaba: ancho de banda limitado, alta cifra de ruido, bajo rendimiento y gran tamaño.

Todo esto cambió cuando los GaAs FETs aparecieron, ofreciendo ganancia suficiente para fabricar amplificadores hasta banda X. Desde entonces, el GaAs ha sido la tecnología dominante en el diseño e implementación de amplificadores de potencia. Sin embargo, hoy en día la tecnología GaAS está siendo superada por nuevas tecnologías cuyas prestaciones en aplicaciones de alta potencia, alta frecuencia y alta temperatura son más favorables; entre ellas se puede destacar la tecnología GaN.

Los semiconductores de GaN fueron inicialmente estudiados hace más 30 años y ya entonces eran considerados como los dispositivos ideales para el funcionamiento en alta potencia en microondas. Pero en esa época, la calidad del material de GaN era pobre para la producción de transistores para microondas. Todo esto comenzó a cambiar a principios de los 90 (ver Figura 2.3) cuando investigadores utilizaron GaN para 
fabricar los primeros diodos emisores de luz (LEDs) del mundo en verde, azul, violeta y blanco [13]. Este descubrimiento impulsó una rápida mejora de la calidad del material de GaN.

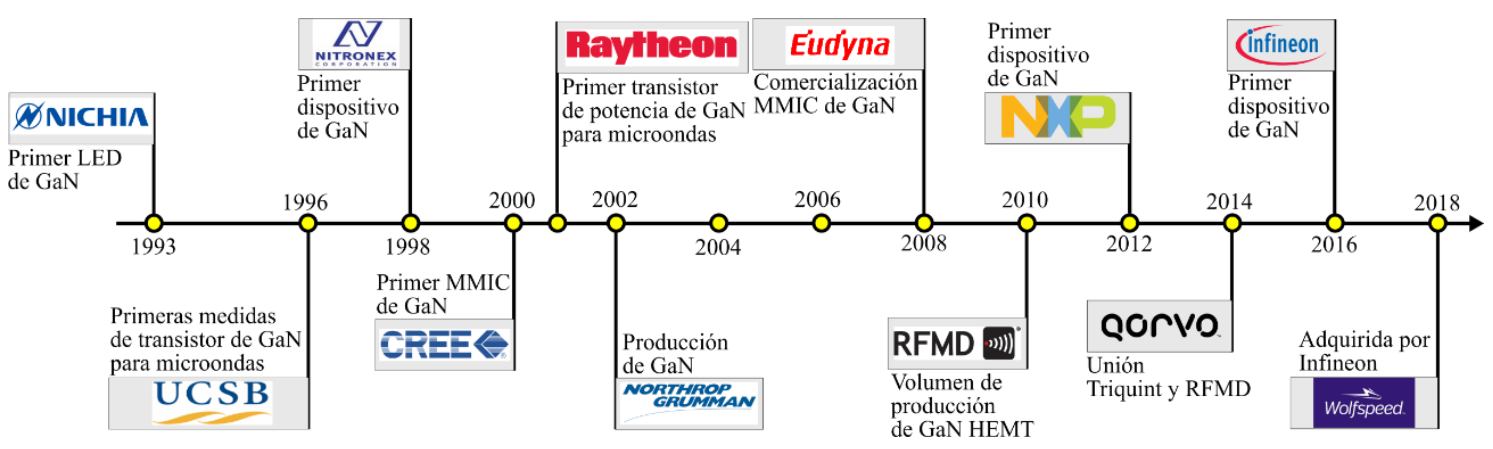

Figura 2.3. Cronología de la tecnología GaN

Otro obstáculo en el desarrollo de los transistores de GaN era la falta de sustrato de bajo coste. Tradicionalmente, el material empleado como sustrato en el transistor es el mismo material que el propio semiconductor, pero los investigadores no fueron capaces de producir obleas de gran tamaño de GaN con alta calidad. En 1996 se exhibió la primera demostración de funcionamiento de un transistor de potencia de $\mathrm{GaN}$ para microondas [14]. Los sustratos de zafiro se caracterizaban por su bajo coste y gran disponibilidad; sin embargo, su pobre conductividad térmica provocó que se volviera a dispositivos de GaN crecidos sobre sustratos semiconductores de SiC. El SiC tiene una buena estructura para $\mathrm{GaN}$ y es un excelente conductor térmico. El único inconveniente era que, a finales de los 90, los sustratos de $\mathrm{SiC}$ sólo estaban disponibles en pequeños tamaños (50mm de diámetro) y eran muy caros (100 veces el precio del GaAs). En los últimos 15 años, sin embargo, se ha visto una rápida mejora en el tamaño, calidad y coste de los sustratos de $\mathrm{SiC}$, lo que ha contribuido a la inserción de estos dispositivos de $\mathrm{GaN}$ en los sistemas comerciales [15].

$\mathrm{El} \mathrm{GaN} \mathrm{es} \mathrm{uno} \mathrm{de} \mathrm{los} \mathrm{materiales} \mathrm{semiconductores} \mathrm{con} \mathrm{mayor} \mathrm{potencial} \mathrm{de} \mathrm{futuro.}$ Las excepcionales propiedades físicas de este material hacen que sea especialmente adecuado para aplicaciones electrónicas de alta frecuencia y alta potencia, proporcionando además una elevada resistencia frente a altas temperaturas, radiación y ambientes químicos externos.

Los amplificadores de potencia de RF son partes esenciales tanto para las estaciones base (BTS) como para los equipos de usuario (UE) de los sistemas de 
comunicación inalámbricos. Son uno de los componentes más caros en los equipos de comunicaciones modernos y, tanto su funcionamiento como coste, son clave en las innovaciones de diseño radio. Rendimiento, tamaño, linealidad y fiabilidad son las principales preocupaciones.

La tecnología de semiconductores de potencia de RF para el diseño de amplificadores de potencia es el factor clave para la optimización de costes y capacidades. Con los servicios 4G emergentes, novedosas técnicas y materiales semiconductores están siendo usados para recudir el coste de los amplificadores de potencia mientras se mantiene su funcionamiento.

Aunque el principal interés de investigación es el desarrollo de dispositivos de alta densidad de potencia con materiales con banda prohibida ancha tales como carburo de silicio $(\mathrm{SiC})$ y nitruro de galio $(\mathrm{GaN})$, la producción de dispositivos de potencia de RF a larga escala, especialmente para propósitos comerciales, se suele realizar realmente usando silicio $(\mathrm{Si})$, arseniuro de galio ( $\mathrm{GaAs})$ y compuestos relacionados.

Elegir la tecnología de semiconductor para el transistor de potencia de RF es un punto esencial para aprovechar su funcionamiento en cuanto a parámetros de linealidad, ancho de banda, rendimiento, ganancia y potencia de salida. Entre todos los candidatos, los transistores bipolares de heterounión (HBT) de GaAs y los LDMOS de Si son todavía los triunfadores en los mercados de unidades móviles e infraestructuras de BTS, respectivamente. Sin embargo, nuevos rivales tales como las tecnologías CMOS y HEMT GaN están emergiendo y tomando más atención, aunque aún continua en marcha su investigación y comercialización.

Dispositivos tales como HBT de GaAs y HBT de SiGe se emplean para aplicaciones relativamente de baja potencia. También hay que mencionar los amplificadores de potencia que usan CMOS, el cual ha sido incorporado en años recientes debido a su atractivo coste. Existe una larga disputa de que el Si es superior al GaAs. Los argumentos afirman que el Si es más maduro y predecible y puede ser producido a un menor coste. Además, su integración en chip es más fácil que en el caso del GaAs.

Sin embargo, estos argumentos no serán verdaderos mucho más tiempo debido a las nuevas exigencias en las aplicaciones. Los inconvenientes de coste del GaAs han 
sido superados por el volumen, el cual recude enormemente el coste del sustrato. Además, el GaAs tiene características que no pueden siempre ser adaptadas al contrario que el Si. Sin embargo, existen serios inconvenientes al aumentar el tamaño del chip de GaAs debido a que la die (material semiconductor del circuito funcional) es más cara, y, aún más importante, tiene un impacto en el coste y en la dimensión del módulo.

Para aplicaciones de alta potencia y alta frecuencia tales como amplificadores de potencia para BTS, el LDMOS de Si domina el mercado actual. Sin embargo, la densidad de potencia de Si LDMOS es de 5 a 10 veces menor que su competidor GaN HEMT. Además, el GaN HEMT ha alcanzado la fase de comercialización en los últimos años, con el cual se pueden fabricar diseños de amplificadores de potencia de RF altamente eficientes, más compactos, fáciles de producir y más fiables.

\subsubsection{GaAs HBT}

Los HBT de GaAs han sido la tecnología de semiconductor más madura comercialmente durante décadas y presenta un mejor funcionamiento que los CMOS de SI o los BJT. GaAs trabaja a mayores frecuencias y potencia media que Si LDMOS y fácilmente superable en PAE y tamaño de la die. Sin embargo, GaAs puede competir con la tecnología GaN HEMT debido a su rápido desarrollo.

En contra a los convencionales BJT, la diferente banda prohibida o bandgap del HBT entre el emisor y la base mantiene una mayor ganancia. La resistencia de base es más baja que en los convencionales BJTs, dando lugar a una frecuencia de trabajo más alta. En comparación con Si BJT, HBT tiene una frecuencia de corte más alta y una tensión de ruptura mayor, manteniendo una resistencia de salida superior, una capacidad base-emisor más baja, una resistencia de base reducida y una reducción en la capacidad de acoplo con el substrato semi-aislante.

A diferencia de los dispositivos HEMT, la tecnología HBT necesita solo una tensión de alimentación única. Sin embargo, la fabricación de HBT de GaAs es todavía relativamente caras comparadas con la fabricación de CMOS. En el diseño de PAs de RF, la principal ventaja de los HBT de GaAs con respecto a los dispositivos HEMT es la mayor linealidad debida a la capacidad de unión base-emisor y su efecto positivo en la reducción de los productos de intermodulación. 


\subsubsection{CMOS}

Los transistores de efecto de campo (FET) de semiconductor óxido de metal complementario (CMOS) fueron inventados en 1963 por Fairchild Semiconductor. Son la tecnología más empleada durante los últimos 50 años y puede ser la tecnología de semiconductores más barata disponible hoy en día. El coste de la tecnología SiGe es aproximadamente de dos a tres veces el coste de la tecnología CMOS y el coste de GaAS es sobre 5 y 10 veces la del CMOS. Esta tecnología es la dominante en los circuitos digitales integrados y cada vez se emplea más en circuitos analógicos y de RF.

En años anteriores, los HBT de GaAs han sido una tecnología ampliamente empleada para la producción de amplificadores de RF. Sin embargo, cada vez más, la tecnología CMOS está siendo considerada como una alternativa para la implementación de circuitos de RF. Un funcionamiento a mayores frecuencias se puede alcanzar con tecnologías específicas tales como GaAs o SiGe. Sin embargo, la verdadera fuerza del CMOS está en el bajo coste y la posibilidad de integración con el procesado digital de la señal para mejorar el funcionamiento de interfaz de usuario de RF.

Un parámetro a menudo empleado cuando se comparan transistores para aplicaciones de RF es la frecuencia de corte. La frecuencia de corte se define como la frecuencia en la cual la ganancia de corriente del transistor es extrapolada para caer de la unidad cuando el dispositivo es cargado con un cortocircuito en pequeña señal. La frecuencia de corte depende de la corriente de polarización del dispositivo.

Gracias a los avances en los procesos de producción y en las tecnologías, se ha aumentado significativamente la frecuencia de corte de la tecnología basada en Si hasta el punto de rivalizar con los dispositivos de GaAs.

Sin embargo, uno de los desafíos de CMOS es la habilidad para sobrevivir bajo desadaptaciones de carga mientras se entrega la potencia de salida deseada. La tensión de ruptura del dispositivo es un parámetro muy importante en el diseño de amplificadores de potencia de RF. Con la reducción de tamaño de la tecnología CMOs, la tensión de ruptura también se reduce. Esto no solo limita la tensión de alimentación, sino también la fiabilidad. Por el contrario, la tensión de ruptura de los dispositivos de GaAs HBT es considerablemente más alta. 
Al contrario que ocurre en la tecnología GaAs HBT, el proceso de CMOs no permite la conexión a masa a través de vías, sino que se emplean hilos de bonding hacia el encapsulado. El inconveniente de los hilos de bonding es su inductancia, la cual es relativamente alta para aplicaciones de RF. Por ello, se suelen emplear varios hilos de bonding en paralelo para reducir la inductancia parásita asociada con las conexiones a masa. Esto es esencial para asegurar que se alcancen resultados de ganancia, rendimiento y estabilidad adecuados.

A pesar de los inconvenientes y dificultades, los dispositivos CMOS aportan una gran ventaja respecto a su uso en conmutadores. Por ello, son buenos candidatos para la implementación de amplificadores de potencia en modo de conmutación.

\subsubsection{Si LDMOS}

Los transistores de Si LDMOS son muy utilizados para amplificadores de alta potencia por debajo de los $3 \mathrm{GHz}$, pudiendo ser la tecnología dominante en aplicaciones inalámbricas. Los Si LDMOS mejora la estructura de los MOSFET lo que les hace especialmente adecuados para aplicaciones de alta potencia. Las principales razones son la maduración de la tecnología en términos de fabricación, el bajo coste y la fiabilidad del silicio.

La estructura de un Si LDMOS ayuda a incrementar la tensión de ruptura y a reducir la inductancia de fuente. Sin embargo, la resistencia de conducción es alta lo que incrementa las perdidas y degrada el rendimiento de RF [16]. Por ello, siempre existe un compromiso entre potencia de salida de RF y resistencia de conducción. Además, otra ventaja de su estructura es el incremento de la frecuencia de funcionamiento, siendo especialmente útiles desde UHF hasta por debajo de los $3 \mathrm{GHz}$, ya que la conexión directa a masa de su fuente elimina la inductancia de los hilos de bonding reduciendo la ganancia a altas frecuencias. 


\subsubsection{GaN HEMT}

Mientras que el GaN ha incrementado su interés en muchas aplicaciones de amplificadores de potencia de microondas tales como militares, los amplificadores de GaN para comunicaciones inalámbricas han sido la innovación durante algún tiempo. En la actualidad, existen investigaciones que abalan que los amplificadores de GaN remplazarán a los de Si-LDMOS. Aunque ha habido una buena penetración del mercado de los amplificadores de GaN para aplicaciones LTE, todavía encuentra retos para ser desplegados en la industria de las telecomunicaciones, en particular, para aplicaciones de BTS.

La principal razón de ello es que, para la misma potencia de salida, los HEMTs de GaN son más pequeños que los dispositivos convencionales de Si o de GaAs. La alta densidad de potencia de estos dispositivos de GaN permite la reducción de la anchura de puerta requerida o el número de celdas conectadas en paralelo. Además, la reducción del tamaño también implica que los dispositivos tengan impedancias de entrada y de salida más altas, lo que facilita el diseño de las redes de adaptación.

Las principales propiedades que debería tener un material semiconductor para el diseño de transistores de alta potencia y alta frecuencia incluyen un bandgap amplio, una alta tensión de ruptura, una alta velocidad y movilidad de electrones y una alta conductividad térmica.

Las capacidades del $\mathrm{GaN}$ en cuando a potencia y velocidad debidas a los parámetros del material se resumen a continuación. El amplio bandgap de energía y el campo eléctrico crítico permite el funcionamiento en alta tensión del transistor. Esto es esencial para la generación de alta potencia de RF [17]. Las propiedades de transporte de electrones tales como la movilidad de electrones y velocidad de saturación determinan sus características de alta frecuencia. La mayor conductividad térmica es muy importante en transistores de potencia para evitar la degradación de funcionamiento con el incremento de la temperatura del canal. Además, los dispositivos de GaN pueden trabajar a temperaturas ambiente mucho más grandes que su homólogo de Si. Experimentos han mostrado que los transistores de GaN continúan amplificando bien cuando la temperatura ambiente es de $300^{\circ} \mathrm{C}$ mientras que los transistores de $\mathrm{Si}$ paran de trabajar alrededor de $\operatorname{los} 140^{\circ} \mathrm{C}$ [18]. La constante dieléctrica relativamente baja de los semiconductores de bandgap ancho ayuda a generar potencias y corrientes 
de RF más altas, además de cargar al dispositivo con una baja capacidad [17], reduciendo los retardos parásitos en los HEMT de GaN.

Los avances actuales en el desarrollo de transistores de potencia de RF que usan material GaN de bandgap ancho muestran mayores potencias de salida, densidades de potencia, rendimiento y linealidad a altas frecuencias ya que las tecnologías actuales en el diseño de amplificadores de potencia de RF tales como Si LDMOS pueden haber alcanzado sus límites [19]. Los procesos de fabricación permiten realizar dispositivos de GaN HEMT con densidades de potencia hasta los 30W/mm sobre substratos de SiC [20]. Aunque la mayoría de los dispositivos son de pequeño tamaño con densidades de potencia que van desde los 2 a los $5 \mathrm{~W} / \mathrm{mm}$, se obtiene una mejora en un factor de 10 veces la densidad de potencia obtenida con GaAs o Si.

Las impedancias de entrada y de salida de los dispositivos de GaN HEMT son también superiores debido a sus densidades de potencia más altas, las cuales se trasladan en una simplificación del diseño de la red de adaptación de los amplificadores de potencia de RF para aplicaciones de banda ancha y de alta potencia. Sin embargo, la tecnología de tratamiento del GaN HEMT está madurando todavía. Algunos temas como la caída de la corriente de drenador, la fiabilidad y un encapsulado apropiado para la gestión térmica aún no han sido resueltos de manera coherente. El mayor obstáculo en la fabricación de HEMTs de GaN es la falta de un substrato adecuado, que sea un material compatible con GaN. Poco a poco, los substratos de GaN van estando comercialmente disponibles; mientras tanto se empleen otros substratos como carbono de silicio ( $\mathrm{SiC})$, zafiro (Al2O3) o silicio ( $\mathrm{Si})$.

\section{Propiedades del GaN}

Desde el punto de vista del diseño de amplificadores, los HEMTs basados en GaN presentan múltiples ventajas sobre otras tecnologías existentes. En la Figura 2.4 se ilustra una comparación de un módulo de amplificación con tecnología GaAs o GaN. 

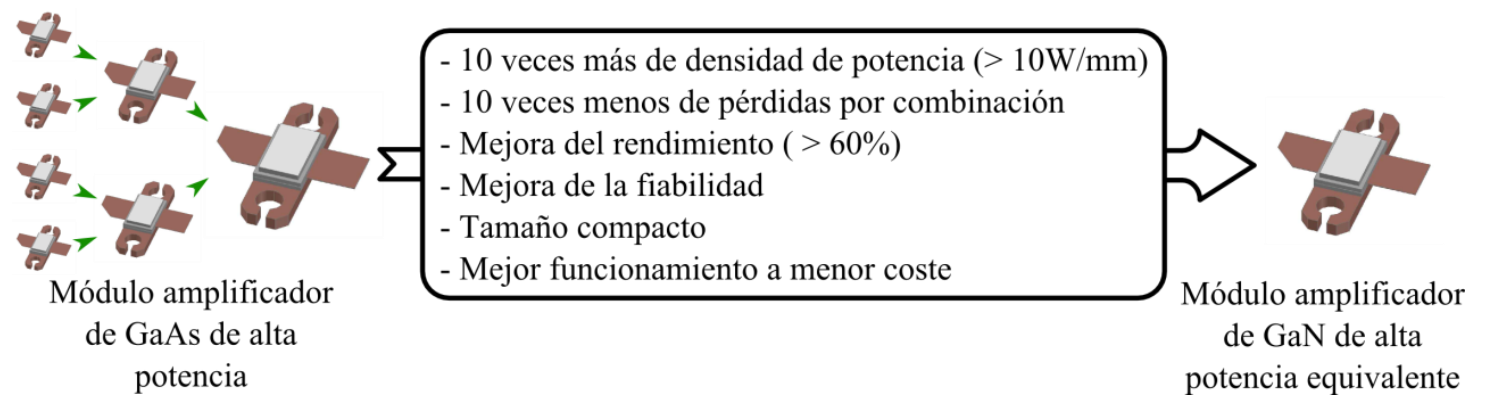

\section{Figura 2.4. Comparación ilustrativa de las ventajas del GaN respecto al GaAs}

La alta densidad de potencia, o potencia por unidad de superficie (típicamente medida en $\mathrm{W} / \mathrm{mm}$ ), permite la fabricación de dispositivos de menor tamaño (hasta 10 veces menor) [21] para la misma potencia de salida por lo que no sólo son más fáciles de construir, sino que también ofrecen impedancias más altas. Esto los hace mucho más fáciles para adaptarlos a los sistemas, lo que habitualmente es una tarea compleja como ocurre con los dispositivos de GaAs convencionales.

En la Figura 2.5 se resume los beneficios que se adquieren gracias a las propiedades de la tecnología GaN. Un campo eléctrico de ruptura alto permite el funcionamiento a tensiones altas, lo que significa que el mismo nivel de potencia se puede conseguir con impedancias de carga mucho más altas, derivando en menores pérdidas en el proceso de combinación de potencia en los sistemas y circuitos menos complejos.

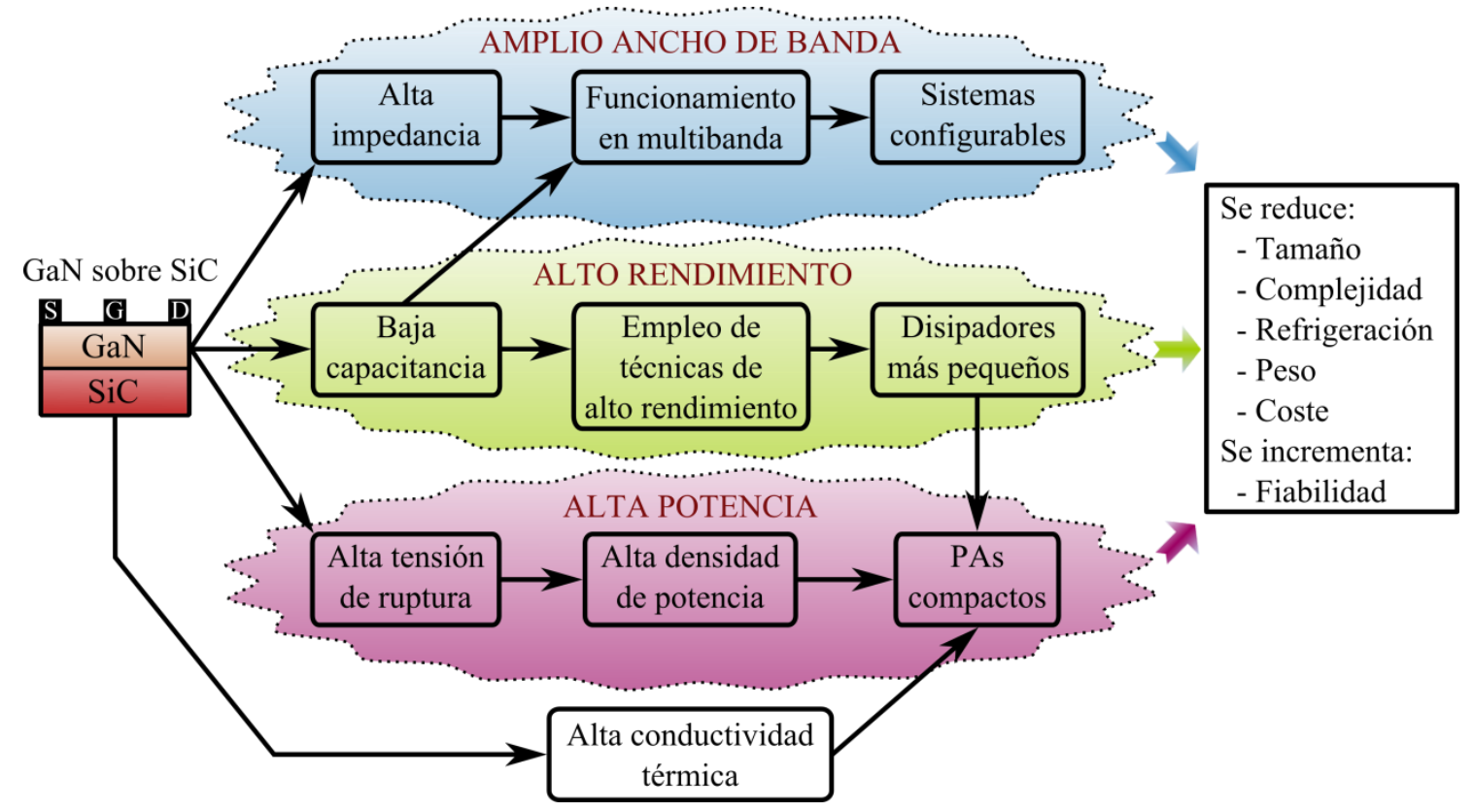

Figura 2.5. Propiedades y beneficios de la tecnología GAN 
El funcionamiento a altas tensiones, unido a la posibilidad del empleo de técnicas de alto rendimiento en el diseño de los amplificadores gracias a la baja capacidad por vatio que presentan estos dispositivos, también facilita la obtención de altos rendimientos, objetivo importante en los amplificadores de potencia. Un mayor rendimiento reduce el consumo de potencia y simplifica los sistemas de refrigeración, lo que es una ventaja importante ya que el coste y el tamaño de los sistemas de refrigeración son una parte significativa del coste global de los sistemas.

La alta estabilidad térmica intrínseca del material permite el funcionamiento a temperaturas más altas, haciéndolo menos estricto (o menos caro) a la gestión de potencia en amplificadores encapsulados. A nivel de producción, estas ventajosas características se traducen en una simplificación en el diseño del sistema, dando lugar a transistores encapsulados y chips más pequeños y permitiendo impedancias más altas con las que poder aumentar el ancho de banda.

La excelente conductividad térmica del $\mathrm{GaN}$ también le hace ideal para aplicaciones en ambientes extremos. Cuando se fabrica sobre sustratos de $\mathrm{SiC}$ la gestión térmica se mejora aún más debido a la alta conductividad térmica del $\mathrm{SiC}$, pese a su elevado coste comparado con otras alternativas como el zafiro y el silicio [22].

Las ventajas tecnológicas comentadas en los párrafos anteriores resultan de la combinación de un bandgap ancho y de la estructura del GaN, donde alta tensión, alta corriente y baja resistencia de conducción pueden ser proporcionadas al mismo tiempo dando como resultado un buen funcionamiento en alta potencia y alto rendimiento, a la vez que ofrece mayor robustez y fiabilidad.

La alta velocidad de electrones de la tecnología GaN permite el funcionamiento a altas frecuencias y con grandes anchos de banda. La combinación con la topología HEMT conlleva el diseño de dispositivos altamente lineales, incluso ofrecen mejor funcionamiento frente al ruido que los MESFETs [21].

Uno de los principales inconvenientes a lo largo del desarrollo de la tecnología $\mathrm{GaN}$ ha sido la fiabilidad. Mientras que el funcionamiento en potencia demostrado de los transistores de GaN ha sido siempre excelente, se ha dedicado una serie de años a la mejora de la fiabilidad y el rendimiento de los transistores [23]. 
En la última década, varios han sido los trabajos que han presentado resultados respecto a la fiabilidad de los transistores GaN [15] [24] [25]. En [26] han realizado estudios que confirman la no existencia de fallos ni degradación importante en la potencia de salida y en la ganancia del dispositivo. Estas pruebas proporcionaron un tiempo medio entre fallos (MTTF) de $10^{6}$ horas a una temperatura de $200^{\circ} \mathrm{C}$. Otros trabajos, como [27], han estudiado la degradación de la resistencia de conducción dinámica de los $\mathrm{GaN}$ en condiciones de conmutación de tensión continua hasta los $300 \mathrm{~V}$.

Diferentes proyectos a gran escala en Europa tales como KORRIGAN (Key Organisation for Research in Integrated Circuits in GaN Technology), con el apoyo de la Agencia de Defensa Europea (EDA) [28] [29] [30], y GREAT ${ }^{2}$ (GaN Reliability Enhancement and Technology Transfer Initiative), con el apoyo de la Agencia Espacial Europea (ESA) [31], centraron su objetivo en la fabricación de la tecnología y la mejora de la fiabilidad de los transistores. El objetivo final de estas actividades europeas fue desarrollar una cadena de suministro de transistores y MMICs de GaN que ofrezcan una alta fiabilidad [32].

Una vez afianzado el tema de la fiabilidad, el siguiente obstáculo es producir dispositivos y circuitos de RF a un coste asumible con respecto a sus oportunidades de inserción en el mercado.

\section{Aplicaciones del GaN}

El desarrollo de los semiconductores de GaN ha sido considerado como estratégico por los programas de Defensa de los países más avanzados del mundo. Numerosos sistemas militares futuros dependerán de la madurez tecnológica alcanzada en el desarrollo de dispositivos basados en estos materiales.

Las generaciones anteriores de semiconductores como el silicio, arseniuros, etc. presentan severas limitaciones para satisfacer los cada vez más exigentes requisitos de los sistemas electrónicos de defensa, fundamentalmente en lo que se refiere a generación y gestión de alta potencia, funcionamiento a altas frecuencias y con grandes anchos de banda y resistentes a altas temperaturas y ambientes muy severos. 
Entre los ámbitos de aplicación más importantes de la tecnología GaN se pueden destacar:

- Electrónica de radiofrecuencia: las aplicaciones más destacadas son las referentes a aviónica, el espacio y las comunicaciones. Amplificadores de alta potencia, de bajo ruido, de banda ancha, de alto rendimiento y chips integrados son algunos de los circuitos empleados en este tipo de aplicaciones.

- Electrónica de potencia: sistemas híbridos o completamente eléctricos de vehículos y plataformas de distribución de energía son las principales aplicaciones en este campo. La investigación y desarrollo de convertidores y conmutadores de potencia fijarán los avances futuros en este mercado.

Las aplicaciones militares de la tecnología de GaN tienen en la mayor parte de las ocasiones su contrapartida dual en el ámbito civil. En el campo de la electrónica de radiofrecuencia, los requisitos impuestos por las infraestructuras de telecomunicaciones actuales se traducen en un incremento de la frecuencia de trabajo, potencia de salida y tensión de funcionamiento para los sistemas de amplificación. Los dispositivos basados en semiconductores GaN HEMT ofrecen ventajas explotables desde el punto de vista de rendimiento, linealidad, ancho de banda, tensión y temperatura de funcionamiento.

Las comunicaciones inalámbricas son la principal aplicación de esta tecnología en el ámbito civil. Las áreas en las que puede suscitar mayor interés el uso de este tipo de semiconductores son los siguientes:

- Estaciones base para telefonía móvil.

- Estaciones base para WiMAX.

- Radiodifusión y comunicaciones por satélite, formando parte de los equipos de los futuros satélites de comunicaciones.

- Sistemas radar embarcados en automóviles para medir distancias, evitar colisiones y para facilitar el aparcamiento.

Con respecto al mercado de la electrónica de potencia, los circuitos de conversión de energía eléctrica conmutados forman parte de uno de los sistemas más importantes dentro este campo. La reducción de pérdidas de conversión, la mejora de la 
fiabilidad, el abaratamiento de los equipos y su reducción de tamaño son algunas de las características buscadas en este tipo de sistemas.

\section{Comparativa con tecnologías existentes}

En la actualidad, los sistemas de electrónica de RF de alta potencia están basados en dos tecnologías fundamentales: la tecnología de tubos de vacío y la tecnología de estado sólido.

La tecnología de tubos de vacío es una tecnología madura con más de 50 años de antigüedad. Los principales tipos de amplificadores de RF basados en esta tecnología son los magnetrones, klystrons y tubos de onda progresiva (TWT). Este tipo de tecnologías son capaces de ofrecer muy altas potencias a altas frecuencias, pero tienen el inconveniente de que son elementos muy voluminosos, frágiles, costosos y difíciles de fabricar.

Uno de los principales dispositivos de tubo de vacío utilizado en aplicaciones militares es el tubo de onda progresiva. Este dispositivo es capaz de suministrar elevados niveles de potencia en grandes anchos de banda, con rendimientos superiores al $60 \%$. Además, estos dispositivos han demostrado tener una alta fiabilidad y una larga vida de funcionamiento superior a los 20 años [33]. La alta potencia capaz de conseguir los transistores de GaN le convertirá en la primera tecnología de estado sólido capaz de competir en prestaciones con los tubos de vació. La sustitución de estos tubos de vacío por dispositivos basados en GaN permitirá reducir el peso, el volumen y el coste de fabricación de dichos equipos.

En lo que se refiere a la tecnología de estado sólido, los dispositivos más utilizados en electrónica de radiofrecuencia están basados en dos materiales semiconductores principalmente: GaAs y $\mathrm{Si}$. La gran ventaja de la tecnología de estado sólido frente a la de los tubos de vacío es la reducción en los costes de fabricación de los componentes y el menor peso y volumen de los mismos, aunque presenten unas prestaciones notablemente inferiores en potencia, ancho de banda y fiabilidad. En la Figura 2.6 se puede ver una comparativa de las propiedades físicas de varias tecnologías de semiconductores de estado sólido. 


$\left.\begin{array}{l|c|c|c|c|}\hline \multicolumn{1}{c|}{\text { Propiedad }} & \text { Si } & \text { GaAs } & \text { Sic } & \text { GaN } \\ \hline \begin{array}{l}\text { Banda prohibida } \\ (\mathrm{eV})\end{array} & 1.11 & 1.43 & 3.2 & 3.4 \\ \hline \begin{array}{l}\text { Campo eléctrico crítico } \\ (\mathrm{MV} / \mathrm{cm})\end{array} & 0.6 & 0.5 & 3.0 & 3.5\end{array}\right)$ Alta tensión

Figura 2.6. Comparativa de las propiedades físicas de diferentes semiconductores

Respecto a los dispositivos de GaAs, fueron desarrollados entre la década de los 80 y principios de los 90 . Hoy por hoy, es una tecnología madura y se encuentran principalmente en el sector de la telefonía móvil. Los dispositivos de GaAs se emplean en amplificadores pre-driver, driver y como etapa final en el rango de microondas y de ondas milimétricas. Sin embargo, las limitaciones inherentes al material impiden su utilización en aplicaciones de alta potencia, lo que supone que estos dispositivos tengan que ser combinados con dispositivos de tubos de vacío en aplicaciones donde se requiera alta potencia.

El mayor campo de ruptura del GaN con respecto al GaAs (aproximadamente 10 veces mayor), una banda prohibida más ancha (3.4 eV del GaN respecto a los $1.34 \mathrm{eV}$ del GaAs) y una velocidad de saturación de electrones superior conlleva a obtener dispositivos del mismo tamaño con 10 veces más de potencia y más fiables [20] [34]. Por otro lado, ya que el GaN ofrece 10 veces más de densidad de potencia para la misma potencia de salida, la relación de las impedancias de entrada y salida es significativamente favorable respecto al GaAs. Por ejemplo, en [21] la impedancia de entrada de un dispositivo de GaN fue de $1.5 \Omega$, mientras que para conseguir la misma potencia con un dispositivo de GaAs se requería $0.15 \Omega$, lo que implica una mayor complejidad ya que es 10 veces menor que la del GaN.

Aunque el coste de una oblea de $\mathrm{GaN}$ sobre $\mathrm{SiC}$ inicialmente es mayor que el de una oblea de GaAs, debido a la mayor densidad de potencia del GaN, el coste por W de 
potencia de salida para el GaN es significativamente inferior al del GaAs. Por ejemplo, incluso si se tiene en cuenta un caso extremo donde las obleas de GaN totalmente fabricadas cuestan 2 veces más que las obleas de GaAs, ya que el GaN produce en el caso más desfavorable 5 veces más de potencia que el GaAs, entonces en términos de coste por vatio, el GaN es un $40 \%$ más barato [15].

Por otro lado, a pesar del papel esencial del Si en el sector actual de la electrónica y de la microelectrónica, este material no ofrece buenas prestaciones a altas frecuencias y en elevados anchos de banda. Tanto es así que la velocidad de saturación de electrones del GaN es aproximadamente dos veces y media mayor que la del Si. Por ejemplo, los amplificadores basados en transistores LDMOS pueden trabajar a frecuencias máximas de unos $3.5 \mathrm{GHz}$. Por otro lado, los valores de potencia que manejan los dispositivos de Si están por debajo de los $200 \mathrm{~W}$, aunque es posible obtener módulos multietapa de hasta $1000 \mathrm{~W}$ a $1 \mathrm{GHz}$ [35].

La Figura 2.7 muestra una comparativa entre las prestaciones de distintos dispositivos de tubos de vacío y dispositivos de estado sólido. Aunque las previsiones son que, a medio plazo, los tubos vayan siendo sustituidos por dispositivos de estado sólido, todavía habrá aplicaciones cuyas restricciones de potencia exigirán la utilización de esta tecnología. Los dispositivos de $\mathrm{GaN}$ se encuentran en un proceso de maduración. La teoría dice de esta tecnología que será capaz de trabajar a altas potencias y a altas frecuencias. Por ello, se puede decir que engloba tanto las ventajas del Si (alta potencia) como las ventajas del GaAs (alta frecuencia).

\section{Perspectivas de la tecnología GaN}

Los avances tecnológicos comentados en los apartados anteriores sólo muestran una parte del interés que suscita el GaN. En realidad, existe un gran número de aplicaciones tanto militares como civiles en los que esta tecnología puede resultar un hecho en los próximos años.

El pronóstico era que los dispositivos de GaN de RF iniciarían la inserción en el mercado comercial en el campo de las infraestructuras inalámbricas. La idea era que el despliegue de las redes 4G, incluidos WiMAX y LTE, impulsara la demanda de pequeñas estaciones base sin comprometer los requisitos de potencia de salida, linealidad y rendimiento [36]. Sin embargo, un par de factores retrasaron la penetración 
en el mercado del GaN: los continuos avances de las tecnologías competentes, en particular los LDMOS de $\mathrm{Si}$, y la vacilación de los proveedores de servicios de desplegar una tecnología relativamente inmadura antes de que haya un historial de fiabilidad probado.

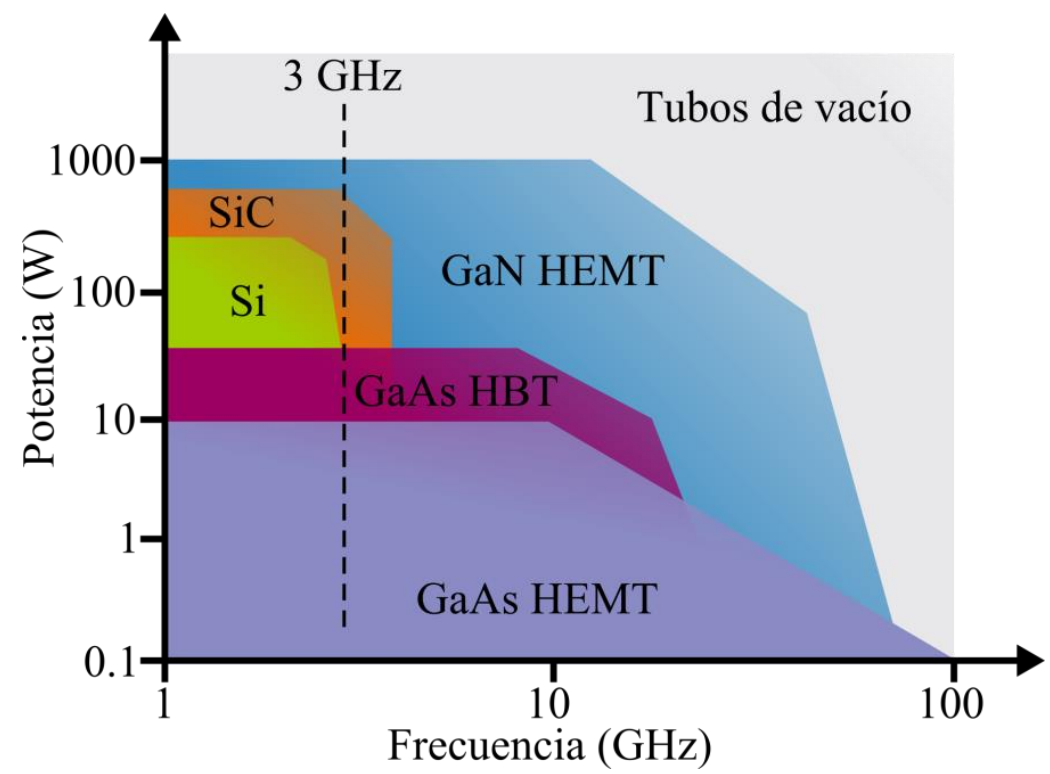

Figura 2.7. Limitaciones de los dispositivos de amplificación

La tecnología GaN presenta prestaciones ventajosas en las aplicaciones actuales de los dispositivos de estado sólido:

- Los amplificadores de alta potencia (HPA) de GaN son más pequeños, robustos y eficientes, presentando mayores márgenes de funcionamiento. Este tipo de dispositivos proporcionan una alta relación entre la corriente de pico y la capacidad de salida, así como una muy alta tensión de ruptura, además de una gran capacidad para gestionar gran densidad de potencia. Esa combinación es única y permite a los diseñadores abrir nuevas vías para alcanzar mejores prestaciones que las encontradas con otras tecnologías alternativas.

- Los LNA de GaN permiten diseñar el primer elemento del sistema receptor (también llamados front-ends) de RF más robustos, lo que se puede llegar a traducir en la desaparición del limitador (lo que aumentaría la sensibilidad del sistema, ya que al eliminar este elemento la cifra de ruido solo dependería del amplificador LNA) y permitiría 
disminuir el volumen y el peso del módulo de transmisión / recepción (TX/RX).

- La gestión térmica de los módulos de TX/RX de GaN será mucho más simple como consecuencia de la baja resistencia térmica entre la base de los MMIC y el canal, de los efectos de dispersión térmica debidos a la conductividad de los sustratos de $\mathrm{SiC}$ y de la mayor temperatura que soporta el GaN frente a los circuitos convencionales de GaAs. Estos módulos tendrán menor tamaño y peso, menor coste y serán más robustos.

- Diseños más avanzados de HPA (High Power Amplifier) basados en GaN permitirán obtener mayores rendimientos, con ahorros significativos en potencia y en gestión térmica. En este sentido, la baja capacidad parásita y la alta tensión de ruptura de los GaN HEMT convierte este tipo de dispositivos en ideales para fabricar amplificadores conmutados.

- El incremento de potencia de los dispositivos basados en GaN permitirá la utilización de ciclos de trabajo más cortos para la misma potencia media, dando como resultado, por ejemplo, más visibilidad de los objetos RADAR. Al permitir mayores densidades de potencia, el desarrollo de dispositivos de $\mathrm{GaN}$ va a permitir más flexibilidad a los diseñadores y de esta manera alcanzar mayores prestaciones en situaciones adversas.

- La adaptación de impedancias de entrada y de salida será más sencilla para dispositivos basados en GaN. De esta forma se generará más potencia en todo el ancho de banda. En este sentido, el GaN será una tecnología muy beneficiosa en el desarrollo de radares multibanda, los cuales presentan claras ventajas tales como mayor distancia de seguimiento, mejor funcionamiento en condiciones meteorológicas desfavorables (lluvia, etc.), mejor tolerancia contra perturbaciones, mayor libertad para escoger la forma de onda y mejores prestaciones para el reconocimiento de blancos.

El número de aplicaciones en las que la tecnología GaN puede ofrecer ventajas comparativas con respecto a otras soluciones va creciendo al avanzar la tecnología. Otras aplicaciones futuras podrían ser: 
- Células solares, en la fabricación de células solares multi-unión de alto rendimiento.

- Termoeléctricos basados en dispositivos que convierten el calor en energía eléctrica que es usada para refrigeración.

- Detectores de terahercios para aplicaciones de seguridad.

- Microelectromecanismos basados en tecnologías de GaN para operar en ambientes agresivos como sensores o actuadores.

- Hornos microondas, sustituyendo a los actuales magnetrones.

Como tendencia futura general, se prevé una paulatina implantación de sustratos de $\mathrm{GaN}$ con mejoras en el coste y en las prestaciones que pueden dar lugar a nuevas aplicaciones que hasta ahora han sido consideradas inviables. Se pretende que los futuros sistemas de RF continúen principalmente las siguientes tendencias:

- Sistemas multifunción, es decir, que realicen diferentes funciones empleando un hardware común de RF.

- Sistemas modulares y escalables, para reducir los costes de fabricación.

- Sistemas de reducido peso y volumen.

- Sistemas confortables a la superficie de la plataforma.

Los recientes progresos en el incremento de la vida útil y fiabilidad de los dispositivos de RF basados en $\mathrm{GaN}$ han sido significativos y con las tecnologías competentes continuamente mejorando, el coste parece ser el principal factor para generar un despliegue más amplio de los amplificadores de GaN.

\subsection{Métodos de diseño de amplificadores de potencia}

Muchas de las arquitecturas con las que mejorar el rendimiento de amplificadores de potencia de RF están actualmente en investigación. A pesar de ello, varias de ellas se están erigiendo como la corriente principal, particularmente en el caso de trabajar con señales de alta PAPR [37].

Entre las técnicas destacadas se pueden encontrar amplificadores en modo de conmutación (SMPA), Doherty y sus variantes, out-phasing/LINC, Eliminación y restauración de envolvente (EER) y seguimiento de envolvente (ET). Todas ellas 
presentan sus ventajas e inconvenientes como candidatas de amplificadores de potencia de alto rendimiento para modulaciones complejas. El rendimiento teórico en función de la potencia de salida se muestra en la Figura 2.8 [38].

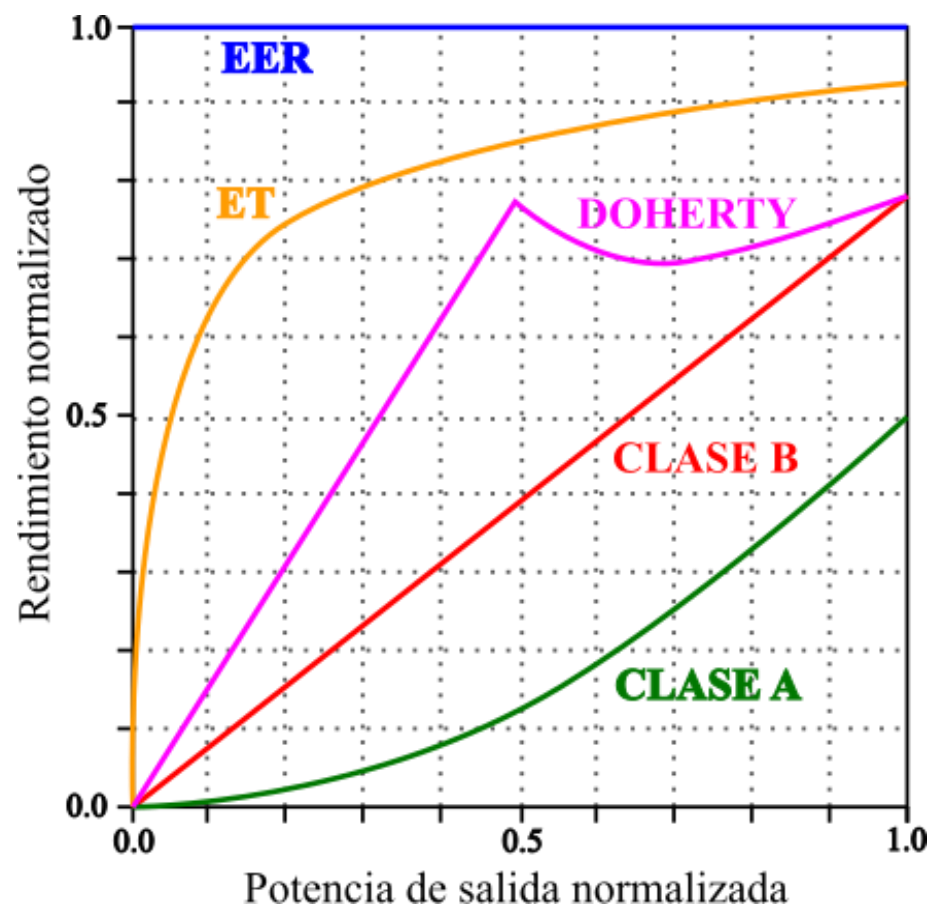

Figura 2.8. Rendimiento teórico de topologías y arquitecturas de amplificación respecto a la potencia de salida.

En la práctica, la amplificación en alto rendimiento de señales de RF de alta PAPR es un campo en continua investigación [37]. Inicialmente, la amplificación en alto rendimiento de señales de amplitud constante se podía conseguir haciendo trabajar al transistor entre saturación y corte siendo alimentado con una tensión de alimentación de DC fija. De esta manera, cuando el transistor trabaja en la zona de saturación, como se muestra en la Figura. 2.9, conduce corriente con una mínima tensión, de ahí que se minimiza la disipación de potencia del amplificador de RF. En la zona de corte, el transistor no conduce virtualmente corriente, aunque la tensión a través de sus terminales durante este tiempo puede ser bastante alta.

Idealmente, el transistor de RF actúa como un conmutador, por lo que este tipo de amplificadores, llamados SMPA, transfieren potencia de la alimentación a la carga con un rendimiento ideal del $100 \%$. Destacar, sin embargo, que este rendimiento ideal implica que los SMPAs deben ser fuertemente excitados con el fin de entregar siempre la máxima potencia de salida. Consecuentemente, los SMPAs se emplean generalmente solo con señales de envolvente constante. 


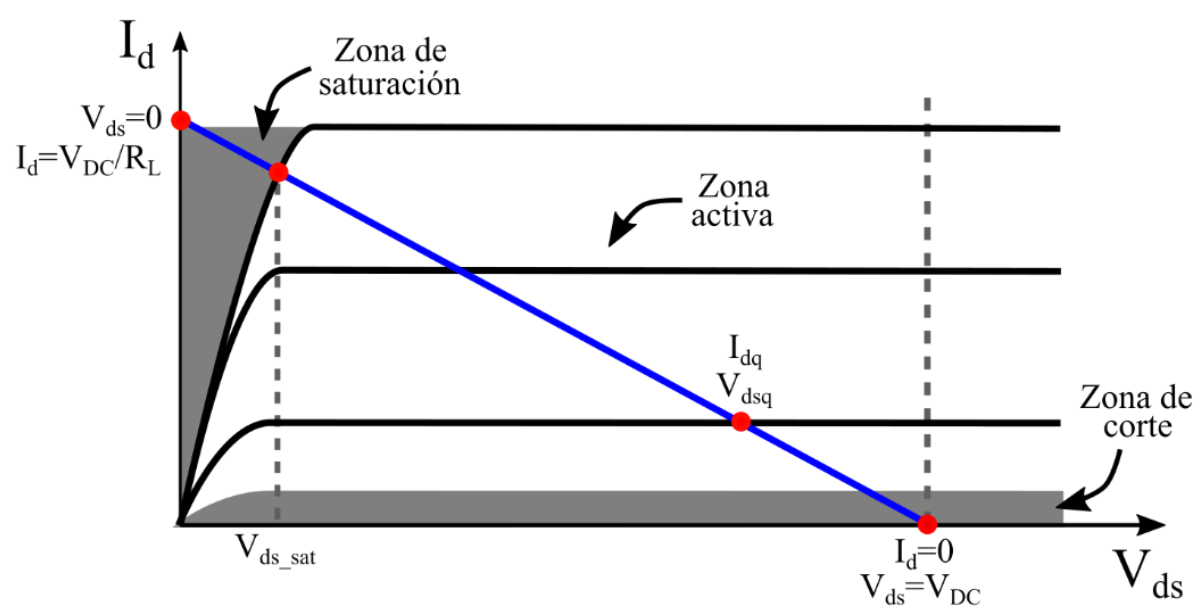

Fig. 2.9 Caracterización I-V del transistor y regiones de funcionamiento.

Los amplificadores de potencia lineales tales como clase A, AB y B manifiestan rendimientos máximos menores del 100\%. A diferencia de los SMPAs, los niveles de excitación de entrada de los amplificadores lineales se mantienen por debajo del punto en el cual el transistor se satura. Una excitación de entrada baja produce una potencia de salida baja, mientras que una excitación de entrada alta produce una potencia de salida alta. Esta correlación lineal ideal entre el nivel de excitación de entrada y la potencia de salida es característica de los amplificadores lineales, pero no es cierta en los SMPAs.

Sin embargo, la habilidad para linealizar señales moduladas en amplitud se lleva a cabo a consta del rendimiento. Un amplificador de RF lineal debe ser diseñado para ser capaz de entregar máxima potencia ante una demanda baja de niveles de señal, por lo que disipa mucha potencia ya que el transistor conserva una cantidad significativa de tensión y corriente simultánea. Es interesante mencionar que los modos tradicionales de amplificación clase $\mathrm{A}, \mathrm{AB}$ y $\mathrm{B}$ comparten la misma topología básica y difieren principalmente en términos de ángulo de conducción, el cual está relacionado con la corriente de polarización y la excursión.

De ahí que progresivas mejoras desde el $50 \%$ de rendimiento máximo de un amplificador clase A se pueden obtener reduciendo la corriente de polarización desde un ángulo de conducción de $\pi$ para clase $\mathrm{A}$, a un ángulo entre $\pi$ y $\pi / 2$ para clase $\mathrm{AB}$ y a un ángulo $\pi / 2$ para clase $B$, así como una polarización negativa virtual siendo el ángulo menor de $\pi / 2$ para clase $\mathrm{C}$. Las clases de amplificación $\mathrm{AB}$, B y C son amplificadores lineales en los que se mejora el rendimiento gracias a la reducción de la corriente de 
polarización; además, los amplificadores clase B y C tienen la ventaja añadida de tener corriente cero cuando no se aplica señal de entrada.

El diseño de amplificadores de potencia de alto rendimiento comienza con la consideración de un dispositivo activo, cuyo ejemplo de modelo simple puede ser el que se muestra en la Figura 2.10. Los FET son más comúnmente utilizados que los transistores de unión bipolar (Bipolar Junction Transistor, BJT), principalmente en aplicaciones de radiofrecuencia de alta potencia.

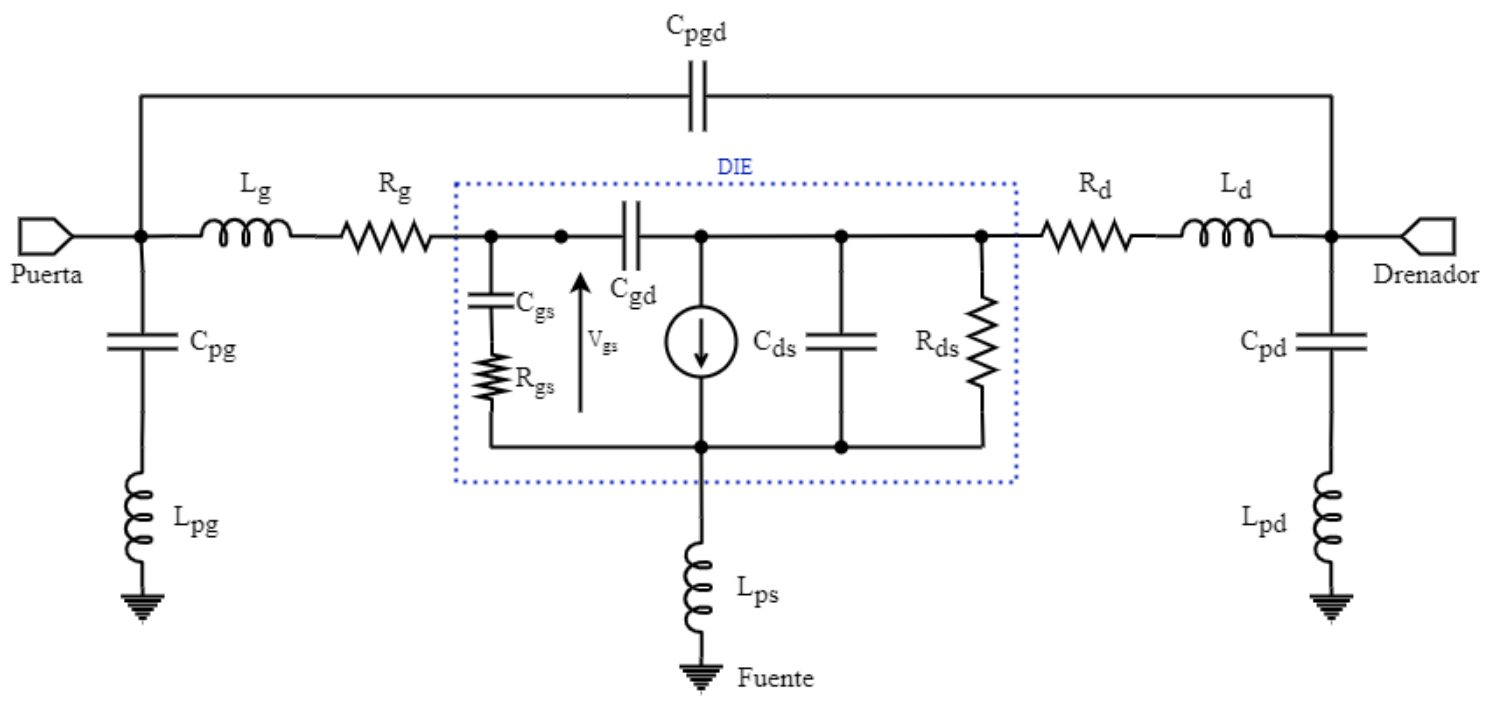

Figura 2.10. Modelo simplificado de un transistor FET.

El modelo más simple de un FET consta de una fuente de corriente controlada por tensión. En el caso de dispositivos de enriquecimiento, tales como los transistores LDMOS, se requiere una tensión de puerta vgs positiva creando un canal que permite que la corriente fluya del drenador a la fuente. Los dispositivos en modo de empobrecimiento, como es el caso de los HEMTs empleados en este trabajo, presentan un canal conductor en su estado de reposo por lo que requieren una tensión negativa que hace desaparecer ese canal impidiendo el flujo de corriente. Además, el canal puede formarse por un incremento en la concentración de electrones (dispositivos tipo N) o de huecos (dispositivos tipo P). Los primeros son los más utilizados en las aplicaciones de potencia en microondas.

Los análisis teóricos son herramientas muy útiles para comprender el funcionamiento de un amplificador de potencia. En la literatura, se simplifica el análisis teórico a base de despreciar o aproximar parámetros o condiciones de los transistores de 
alta potencia. Sin embargo, el diseño hardware real implica muchos efectos no ideales que son difíciles de analizar teóricamente.

Por ejemplo, un método de diseño de amplificadores se basa en las impedancias de carga que se presentan al dispositivo a las frecuencias armónicas (que será discutido con más detenimiento en este capítulo). Se puede simplificar un análisis forzando cortocircuitos o circuitos abiertos como carga a los armónicos para obligar a generar una forma de onda de tensión de drenador característica a la frecuencia fundamental; sin embargo, estas impedancias armónicas son complicadas de realizar en función de diversos parámetros del dispositivo, la frecuencia de funcionamiento y los efectos parásitos del encapsulado (aún más acentuados a altas frecuencias).

Muchos transistores incorporan redes de adaptación dentro del propio encapsulado con el fin de conseguir una adaptación con menores pérdidas y mayor ancho de banda, lo que se conoce como transistores preadaptados [39]. Estas redes de adaptación internas se pueden sintetizar fácilmente mediante hilos conductores (conocidos como hilos de Bonding) a modo de inductancia serie y con condensadores en chip actuando de capacidad en derivación. Con esta adaptación interna, además, se aumenta la impedancia en el plano de salida del dispositivo limitando el ancho de banda del dispositivo, así como la capacidad del diseñador para controlar las impedancias armónicas si se desea emplear para trabajar en modos de funcionamiento de alto rendimiento.

Otro inconveniente al trabajar con dispositivos de alta potencia es la dificultad de analizar y predecir el funcionamiento en gran señal, así como la estabilidad del dispositivo. Emplear los parámetros $\mathrm{S}$ en pequeña señal como método para diseñar amplificadores de potencia no es un método fiable debido a las no linealidades en el punto de trabajo.

Estas y muchas otras dificultades que aparecen cuando se trabaja con transistores de alta potencia en el rango de frecuencias de microondas no son reproducidas adecuadamente por muchos de los estudios teóricos y modelos no lineales, lo que lleva a buscar otros métodos de diseño, acentuando esta situación cuando se pretende un diseño de alto rendimiento, a altas potencias y/o a altas frecuencias. 


\subsubsection{Load Pull}

En muchas ocasiones, las nuevas tecnologías de transistores, como es el caso del $\mathrm{GaN}$ en este trabajo, carecen normalmente de modelos no lineales. En estos casos, la caracterización load pull representa el método de diseño experimental por excelencia [40] [41]. Consiste fundamentalmente en presentar un gran número de cargas al dispositivo activo y medir la variación de parámetros como la potencia de salida, el rendimiento o la ganancia. Muchos fabricantes de dispositivos de RF ofrecen en la hoja de características una versión reducida de la caracterización load pull en gran señal. Del mismo modo, en el caso de que las variaciones de carga se produzcan a la entrada del dispositivo se denomina source pull.

Este método de diseño se puede emplear tanto para dispositivos activos de bajo ruido como para los de alta potencia, o aquellos que trabajan a frecuencias de microondas o a unos pocos megahercios. Sin embargo, el equipamiento necesario para poder implementar un sistema load pull es muy costoso y especializado, generalmente en términos de frecuencia, de nivel de potencia o del parámetro específico a medir (ruido, potencia, rendimiento...). Además, el coste de los equipos aumenta considerablemente si se trata de elementos manuales o controlados por software [42] [43]. En la Figura 2.11 se muestran algunas fotografías de este tipo de equipamiento.
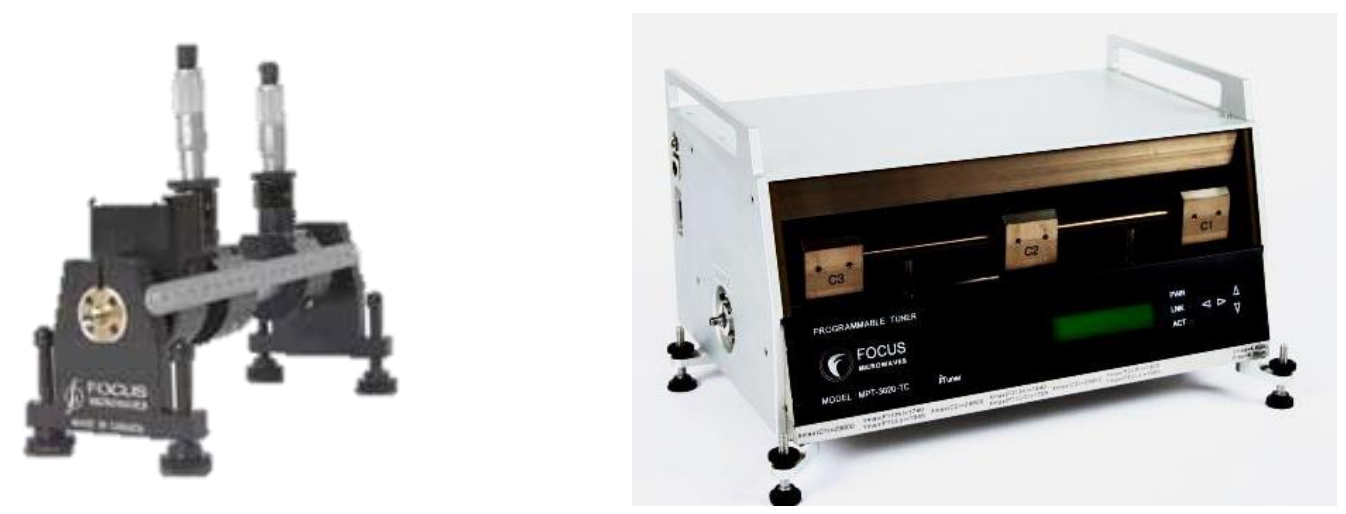

Figura 2.11. Sintonizadores de impedancia: manual (izq) y automático (drch).

Los sistemas de caracterización load pull ofrecen resultados realistas. Sin embargo, su principal defecto es que la información que aportan es exacta, pero para unas condiciones de operación muy puntuales y concretas: frecuencia de trabajo, tensión de alimentación, potencia de entrada, polarización, temperatura... 
Además, si no se disponen de medios sofisticados la caracterización load pull es compleja y está expuesta a una gran cantidad de posibles errores e imprecisiones, principalmente si se trata de sistemas manuales. Se necesita una gran experiencia tanto en la calibración como en la medición para su correcta implementación.

Este trabajo no pretende hacer una explicación exhaustiva del procedimiento sino describir los aspectos básicos del método utilizado. En [44] y [45] se puede encontrar una extensa biblioteca con notas de aplicación si el lector quiere profundizar sobre este tema. La creciente comercialización de sistemas load pull automáticos está facilitando la aplicación de estas técnicas con un mayor grado de precisión, pero a un coste muy elevado.

Por último, los sistemas load pull se pueden caracterizar en dos grupos: sistemas pasivos, donde se combinan elementos y componentes pasivos para buscar la funcionalidad deseada, y sistemas activos, donde el control de carga del sistema queda controlado completamente de forma electrónica. A continuación, se detalla cada uno de estos dos grupos y sus respectivas variantes.

\subsubsection{Sistemas load pull pasivos}

En general, un sistema load pull suele ser un sistema pasivo. Esto quiere decir que el sintonizador se combina con otros elementos y componentes pasivos como pueden ser un analizador de redes, generadores de señal, medidores de potencia, biastees, aisladores... con el fin de conseguir la funcionalidad buscada [46]. El principal desafío en la implementación de un sistema load pull es la calibración, que se lleva a cabo para eliminar los errores derivados de desadaptaciones, dispersiones e imperfecciones en los cables y/o componentes del sistema. En el Capítulo 3 se detallará este proceso de calibración.

En la Figura 2.12 se muestra el banco de medida load pull más simple. Consta de dos sintonizadores manuales que serán los encargados presentar las impedancias de carga necesarias a la frecuencia fundamental; el resto de elementos son instrumentos de medida con los que determinar la impedancia de carga presentada al dispositivo bajo prueba (DUT). 


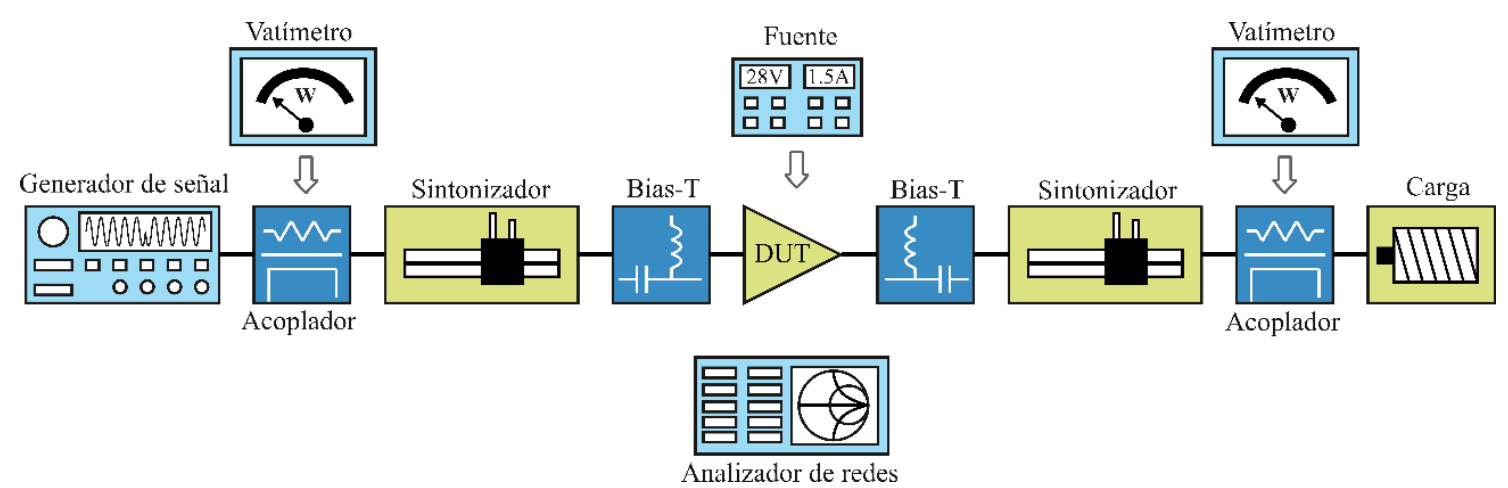

Fig. 2.12 Diagrama de bloques de un sistema load-pull manual.

Un sintonizador manual es un elemento electromecánico cuyo principio de funcionamiento se basa en transformar la impedancia estándar de $50 \mathrm{Ohm}$ a una nueva impedancia que será vista por el dispositivo a medir en un plano denominado plano de referencia. Al conjunto de impedancias que puede generar el sintonizador se denomina constelación. Como se puede ver en la Figura 2.13, estos sintonizadores normalmente constan de una línea de $50 \mathrm{Ohm}$ y un elemento metálico llamado slug que se posiciona con precisión a una cierta distancia del dispositivo a medir (movimiento horizontal) y a una cierta distancia del conductor central (movimiento vertical) [47].

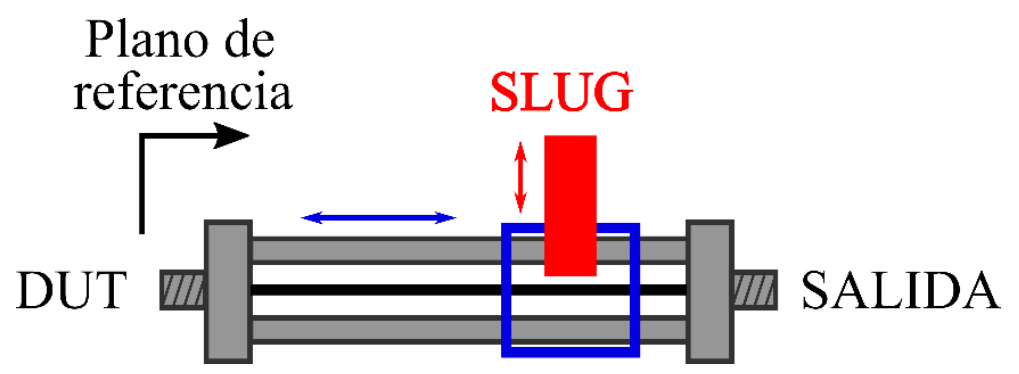

Figura 2.13 Esquema de un sintonizador de impedancia manual.

En la Figura 2.14a el slug está fuera del campo eléctrico que se propaga por el conductor central y no afecta a la impedancia del plano de referencia (normalmente 50 $\Omega$ ). Sin embargo, tan pronto como el slug se desplaza verticalmente acercándose al conductor central, el factor de reflexión en el plano de referencia aumenta (Figura 2.14b). Por otro lado, el movimiento horizontal del slug provoca una variación en la fase del coeficiente de reflexión (Figura 2.14c).

El procedimiento de medida en un sistema load pull manual es el siguiente: se fija el punto de polarización del DUT y se alimenta. A través de la configuración de los sintonizadores se presentan diferentes impedancias de entrada y de carga al dispositivo. 
Posteriormente, se mide la potencia de salida, R.O.E o cualquier otra magnitud que se desee analizar en función de la carga presentada. Finalmente, se desmonta el dispositivo activo y se mide con un analizador de redes la impedancia de carga y de generador que han sido presentadas al dispositivo. Este proceso se debe repetir cada vez que se varié la posición de uno de los sintonizadores o se cambie alguna de las condiciones de la medida.

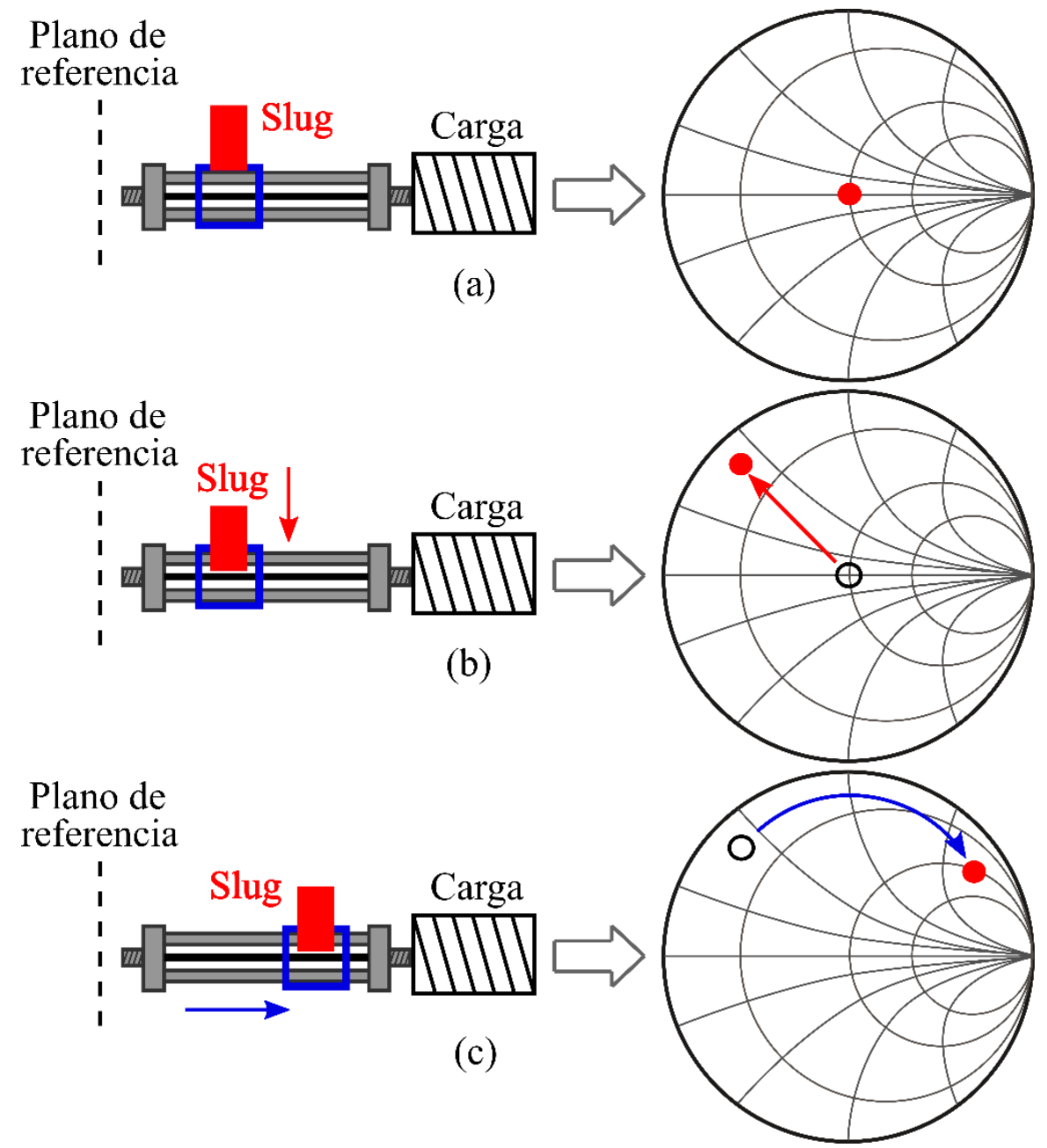

Fig. 2.14 Representación del funcionamiento de un sintonizador en la carta de Smicth.

Se trata de un procedimiento tedioso debido a la gran cantidad de medidas que deben ser realizadas para obtener una cierta calidad y precisión en el resultado. Típicamente, el método load pull permite generar unos contornos de carga para una determinada magnitud. En estos casos, el procedimiento debe ser realizado un gran número de veces, y si se modifican las condiciones de medida (por ejemplo, cambiar la 
frecuencia o la tensión de alimentación) el procedimiento debería volver a repetirse completamente para estas nuevas condiciones.

A mediados de los años 70 se comenzaron a utilizar los sistemas load pull automáticos. Estos sistemas se basan en la utilización de sintonizadores mecánicos actuados mediante motores y controlados por un sistema informático. Si el sistema está correctamente calibrado, presenta la ventaja de conocer la carga que se presenta al dispositivo sin necesidad de intercalar ningún elemento de medida entre el dispositivo activo y la carga.

Obviamente, la principal ventaja de un sistema automatizado es la de poder realizar muchas más medidas que en un sistema manual y con muchos menores errores. Sin embargo, el principal inconveniente de estos sintonizadores automáticos es su alto coste $\mathrm{y}$, además, dependiendo del modelo no se tiene control de la impedancia presentada a los armónicos. Igualmente, la sintonización de las cargas se ve limitada al trabajar a altas frecuencias.

\subsubsection{Sistemas load pull activos}

Con el fin de evitar los principales inconvenientes asociados a los sistemas loadpull basados en la utilización de sintonizadores (manuales o automáticos), existe la posibilidad de generar la carga de un sistema load pull de forma completamente electrónica. Cada carga dentro de una línea de transmisión está asociada con un cociente de fasores de onda incidente y reflejada.

Si fuera posible controlar la amplitud y la fase de la onda reflejada en función de la amplitud y fase de la onda incidente sería posible sintetizar cualquier carga. Conceptualmente, la idea es sencilla pero muy compleja en cuanto a la forma de realizarse.

\subsubsection{Sistemas load-pull multiarmónico}

Cualquier sistema load pull, ya sea pasivo o activo, presentará una utilidad limitada sino se permite controlar, o al menos conocer, la impedancia de carga 
presentada a los armónicos. Esta situación se acentúa aún más en el diseño de amplificadores de alto rendimiento, donde se demuestra la importancia del control de la carga armónica.

El control de impedancia a los armónicos se emplea para generar combinaciones de formas de onda de tensión y de corriente que eviten su solapamiento en el dominio del tiempo, reduciendo la disipación de potencia en el transistor en forma de calor al mismo tiempo que se puede alcanzar una potencia de salida más alta a la frecuencia fundamental.

De ahí que los sistemas load pull multiarmónicos presenten un gran beneficio en el diseño de amplificadores de alto rendimiento a través del control las impedancias armónicas. Sin embargo, no debe tratarse de una exploración de todas las impedancias armónicas posibles sino tener unos conocimientos básicos del efecto que puede generar el control de estos armónicos en la búsqueda del funcionamiento deseado.

$\mathrm{Al}$ igual que en los sistemas donde solo se controla la frecuencia fundamental, lo sistemas load pull multiarmónicos se puede dividir en sistemas pasivos o sistemas activos. El principio de funcionamiento de los primeros es prácticamente el mismo que para un sistema load pull pasivo donde solo se controle la carga a la frecuencia fundamental; la diferencia es que se repite el mismo esquema para cada una de las frecuencias armónicas que se quiere controlar, haciendo uso de mezcladores. En el caso de desear controlar únicamente los dos primeros armónicos, prácticamente es como tener tres sistemas pasivos (Figura 2.15) por lo que el número de medidas se triplicaría. En el caso de sintonizadores manuales, este sistema es prácticamente imposible de realizar debido a la gran cantidad de medidas, de tiempo y posibles errores, por lo que es más usual verlo con sintonizadores automáticos.

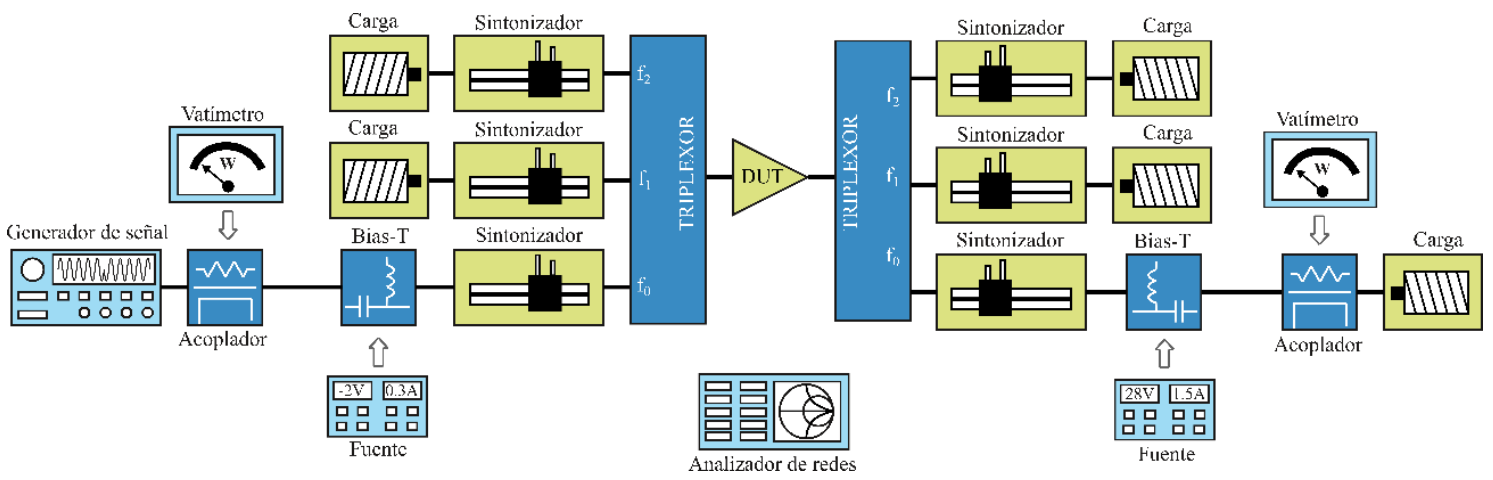

Figura 2.15. Diagrama de bloques de un sistema load pull multiarmónico pasivo. 
Los sistemas load pull multiarmónicos activos siguen una filosofía similar al caso en los que solo se controla la frecuencia fundamental, pero añadiendo varias ramas combinadas que trabajan a distintas frecuencias armónicas. Presentan las mismas ventajas e inconvenientes que el caso más simple, añadiendo la dificultad que supone el control de la carga a más de una frecuencia.

Por último, una alternativa limitada pero de mucho menor coste son los sistemas load-pull con redes de control de armónicos. Estos sistemas permiten conocer y, en cierta medida, controlar la carga presentada a los armónicos para posteriormente realizar el método load pull a la frecuencia fundamental. Generalmente, esta carga armónica requerida por los modos de amplificación de alto rendimiento está delimitada; cortocircuitos, circuitos abiertos, altas impedancias reactivas son las cargas armónicas más comunes requeridas para la amplificación de alto rendimiento.

En la Figura 2.16. se muestra un posible esquema para conseguir controlar las impedancias de carga al segundo y tercer armónico. El sistema se compone principalmente de un accesorio de prueba formado, generalmente, por una línea de $50 \Omega$ en la que se colocan unos stubs en circuito abierto, o cortocircuito, de longitud $\lambda / 4$ a la frecuencia del armónico correspondiente. Un circuito abierto a los armónicos presentará en su otro extremo un cortocircuito que podrá ser desplazado a lo largo de la línea fundamental. De esta manera, si se consideran las pérdidas despreciables, se puede controlar la impedancia reactiva a los armónicos. Este proceso se puede extender a tantos armónicos como se deseen controlar, con el inconveniente de que mayores pérdidas serán introducidas en el sistema.

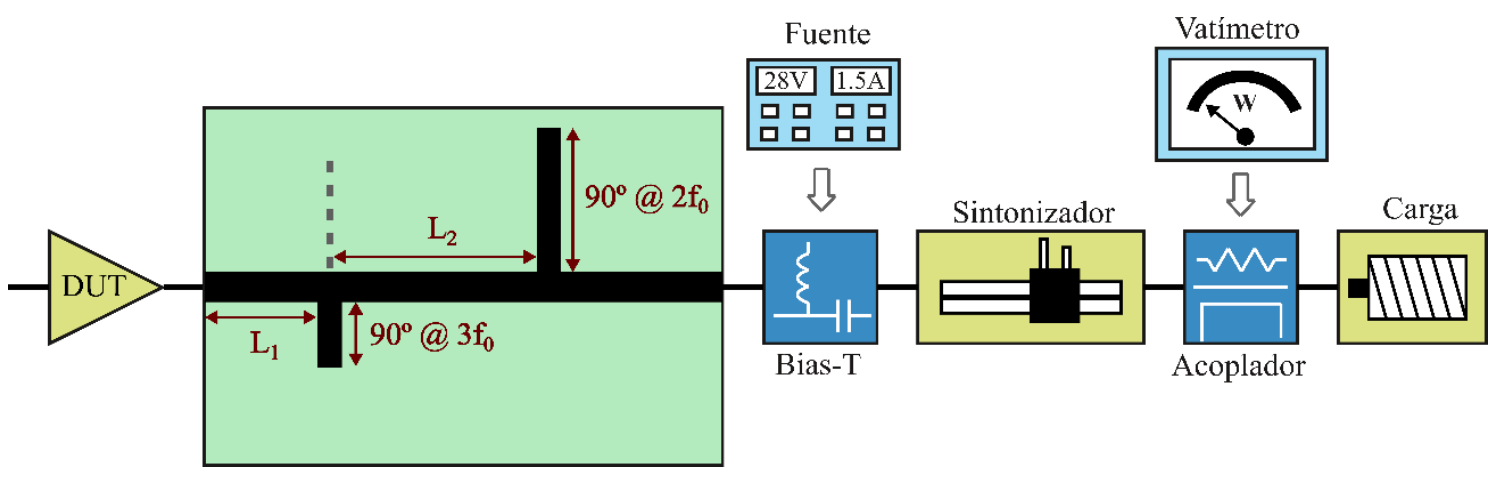

Figura 2.16. Diagrama de bloques de un sistema load pull con control de armónicos (etapa de salida). 
La principal ventaja de este sistema es su gran facilidad de implementación y su bajo coste tratándose de un sistema capaz de controlar también la carga a frecuencias armónicas.

\subsubsection{PA en modo de conmutación}

Alto rendimiento a altas potencias se puede conseguir a través de amplificadores de potencia en modo de conmutación (SMPA), a consta de inducir un comportamiento en potencia no lineal. Principalmente, las señales de RF moduladas en amplitud necesitan amplificación lineal y de ahí que los SMPAs no puedan ser utilizados solos con este tipo de señales. Algunas veces, la combinación de una SMPA junto con alguna técnica que permita mejorar la linealidad del amplificador, como puede ser predistorsión, puede considerarse una buena solución para obtener alto rendimiento. Sin embargo, en algunas otras soluciones, la no linealidad pero el alto rendimiento del amplificador de potencia puede ser útil como parte de la cadena del transmisor lineal, como ocurre en Doherty, LINC, EER y ET, que serán presentados más adelante.

Con el rápido desarrollo de los semiconductores, los SMPAs tienen ventajaa sobre las clases de amplificación lineales ya que teóricamente puden alcanzar un rendimiento del $100 \%$. Se denominan modo de conmutación porque el transistor de potencia de RF actúa como un conmutador en contraposición a una fuente de corriente en las tradicionales clases A, B, AB y C.

Bajo esta condición, los transistores de RF pueden ser modelados como un conmutador de dos maneras diferentes. Cada modelo tiene su efecto en las pérdidas. En la Figura 2.17a, conocido como modo zero voltage switching (ZVS), las perdidas ocurren durante la descarga del condensador tras la transición desde el estado de OFF a ON. Sin embargo, en la Figura $2.17 \mathrm{~b}$, conocido como modo zero current switching (ZCS), las pérdidas ocurren mientras se descarga la inductancia en el momento de la transición del estado ON a OFF. Ambos modos necesitan ya sea tensión cero a través del conmutador justo antes de conmutar a ON, modo ZVS, o corriente cero a través del conmutador justo antes de conmutar a OFF, modo ZCS. 


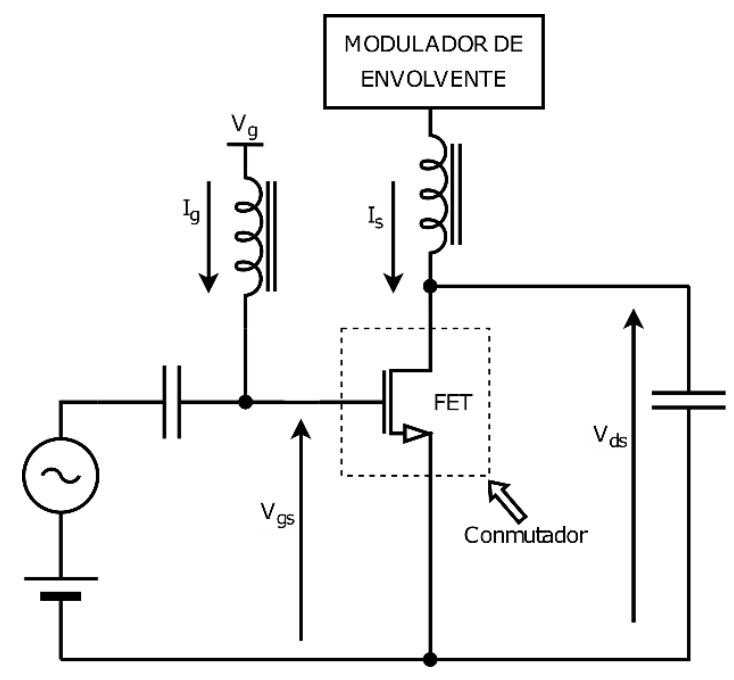

(a)

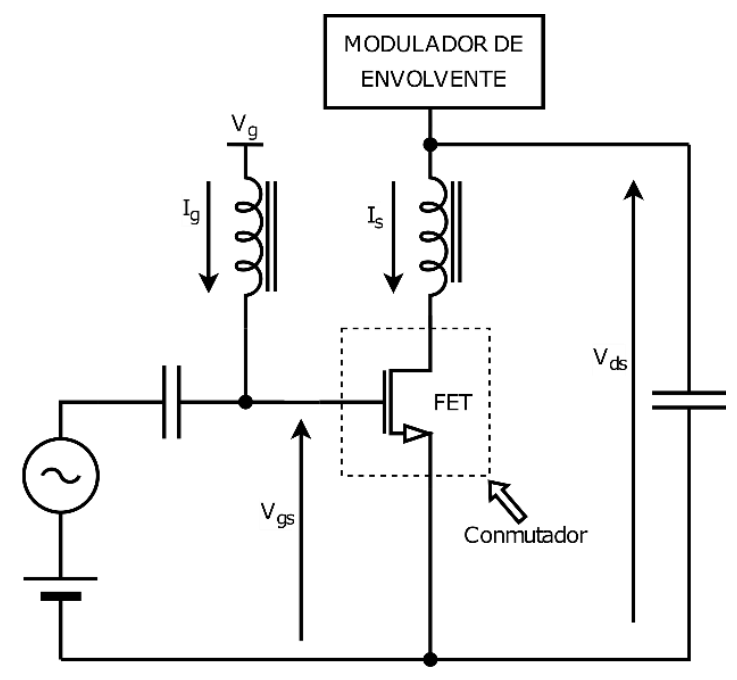

(b)

Figura 2.17. Esquema de un SMPA: ZVS o conmutación de tensión nula (a) y ZCS o conmutación de corriente nula (b).

En los siguientes apartados se van a definir algunas de las clases de amplificación de alto rendimiento más importantes.

\subsubsection{Amplificadores clase $\mathrm{D}$ y $\mathrm{D}^{-1}$}

La clase D fue propuesta por la compañía de semiconductores Harris, quien introdujo el primer driver para amplificadores clase D de audio en 1995, alcanzado rendimientos superiores al $90 \%$.

La topología clase D se emplea principalmente en aplicaciones de audio. En ella, el dispositivo activo actúa como un conmutador. El dispositivo se excita con una forma de onda cuadrada trabajando entre la zona de saturación y corte.

En un amplificador de potencia clase D, la anchura del pulso de la forma de onda de entrada es variable. A la salida del amplificador, un filtro paso bajo o un filtro paso banda atenúa la frecuencia de conmutación y sus armónicos, permitiendo el paso solamente de la versión amplificada de la forma de onda de entrada. Con el transmisor de potencia en clase D trabajando entre saturación y corte, las pérdidas son principalmente debidas a las caídas de tensión. Por ello, un amplificador de potencia clase D puede conseguir rendimientos prácticos mayores del 90\%, con niveles de distorsión cercanos a la de un amplificador en clase AB. Sin embargo, la debilidad de la 
topología clase D recae en la correcta supresión de la distorsión introducida en la conmutación.

Una topología de amplificador de potencia clase D es el modo tensión (VMCD) y se presenta en la Figura 2.18. Es la única clase que necesita dos transistores que trabajen con un desfase de $180^{\circ}$. La red de carga tiene un circuito resonante serie para pasar la corriente a la frecuencia fundamental a la carga y bloquear el resto de componentes de corriente.

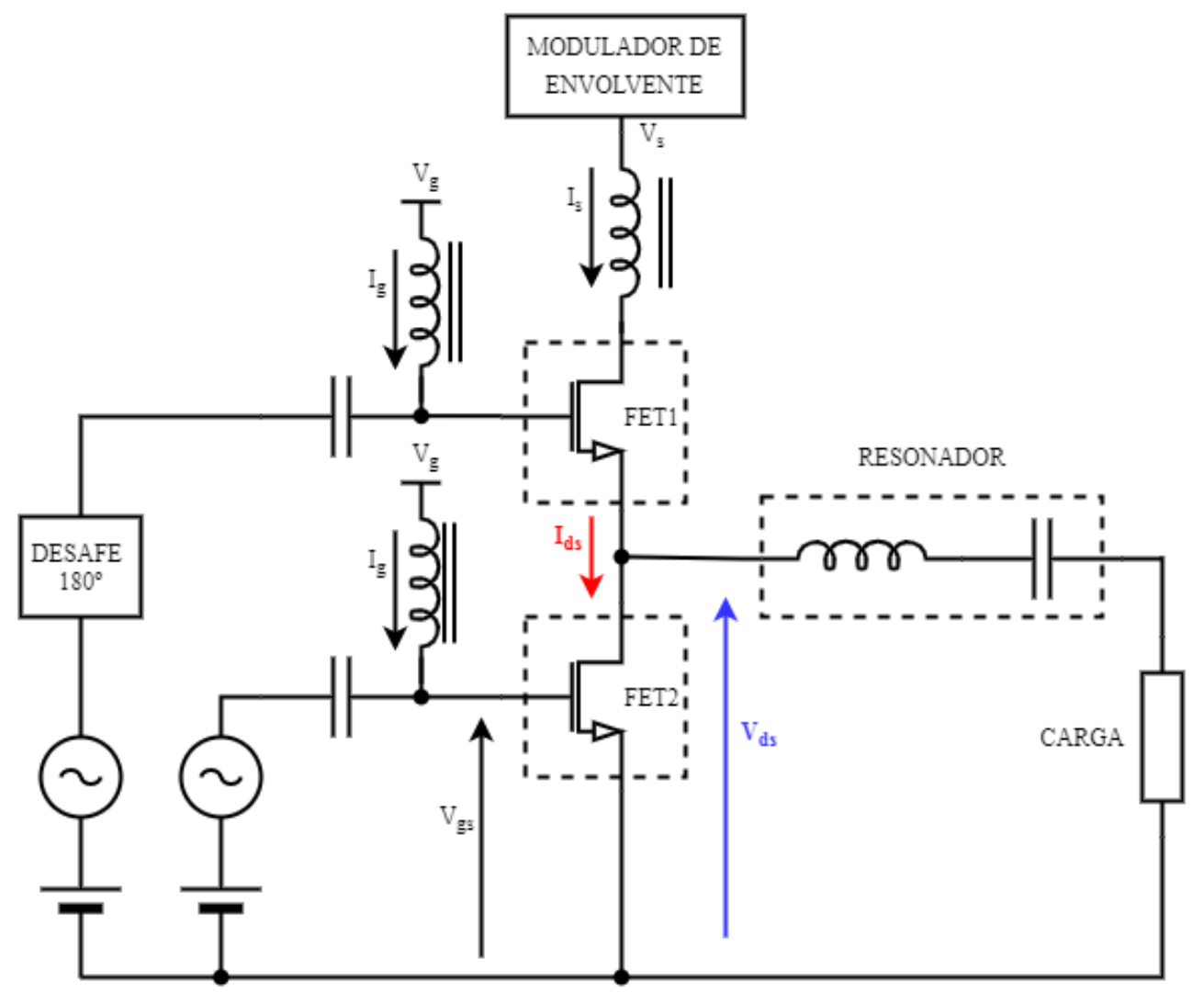

Figura 2.18. Esquema de un SMPA clase $D$ en modo tensión y formas de onda de tensión y de corriente.

Por ello, las formas de tensión y de corriente son una forma de onda cuadrada y sinusoidal, respectivamente, como se puede ver en la Figura 2.19. Para una mejor comprensión de este modo de funcionamiento, el transistor puede ser reemplazado por un conmutador, donde idealmente tensión y corriente no coinciden al mismo tiempo y por lo tanto no se disipa potencia obteniendo un rendimiento ideal del 100\%. En la práctica, el transistor no se comporta como un conmutador perfecto, por lo que se disipa potencia y se reduce el rendimiento. 

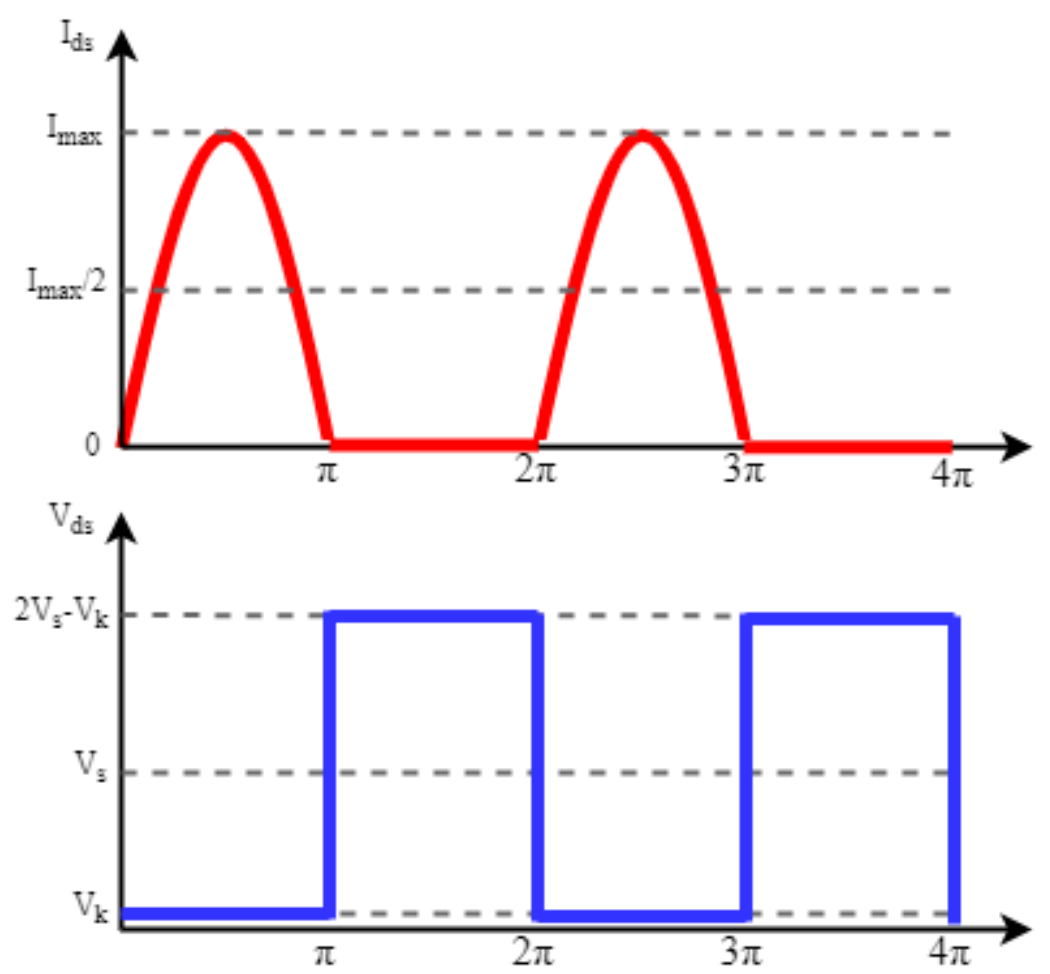

Figura 2.19. Formas de onda ideales de tensión y de corriente de un SMPA clase D.

En la Figura 2.20 se presenta otra variante conocida como clase D en modo de corriente $(\mathrm{CMCD})$ o $\mathrm{D}^{-1}$. Prácticamente es la topología inversa en cuando a formas de onda de tensión y corriente se refiere. La red de carga tiene un circuito resonante paralelo con una carga que pasa la frecuencia fundamental y corta todos los armónicos impares. Esto permite que la corriente sea una forma de onda cuadrada y la forma de onda de tensión sea sinusoidal, vistas en la Figura 2.21. Esta clase $\mathrm{D}^{-1}$ presenta una ventaja obvia sobre la tradicional clase D: poder incluir la capacidad de salida del transistor como parte del circuito resonante. Por ello, puede alcanzar un alto rendimiento, pero no del $100 \%$ debido a la región de tensión de codo, $V_{k}$.

Una de las razones de la degradación de rendimiento proviene del valor teórico de la capacidad de salida del transistor, que es muy significativa a altas frecuencias. La capacidad puede ser muy grande debido al nivel de potencia, especialmente para aplicaciones de alta potencia. Típicamente, la susceptancia de la capacidad de salida es muy alta a altas frecuencias y no puede ser despreciada. Correspondientemente, otra razón para no alcanzar el rendimiento teórico es que el transistor no opera como un conmutador ideal, permutando entre el estado de ON y de OFF instantáneamente. Con 
tiempos finitos de encendido y apagado, el solapamiento entre la corriente y la tensión puede causar pérdidas de conmutación en el transistor.

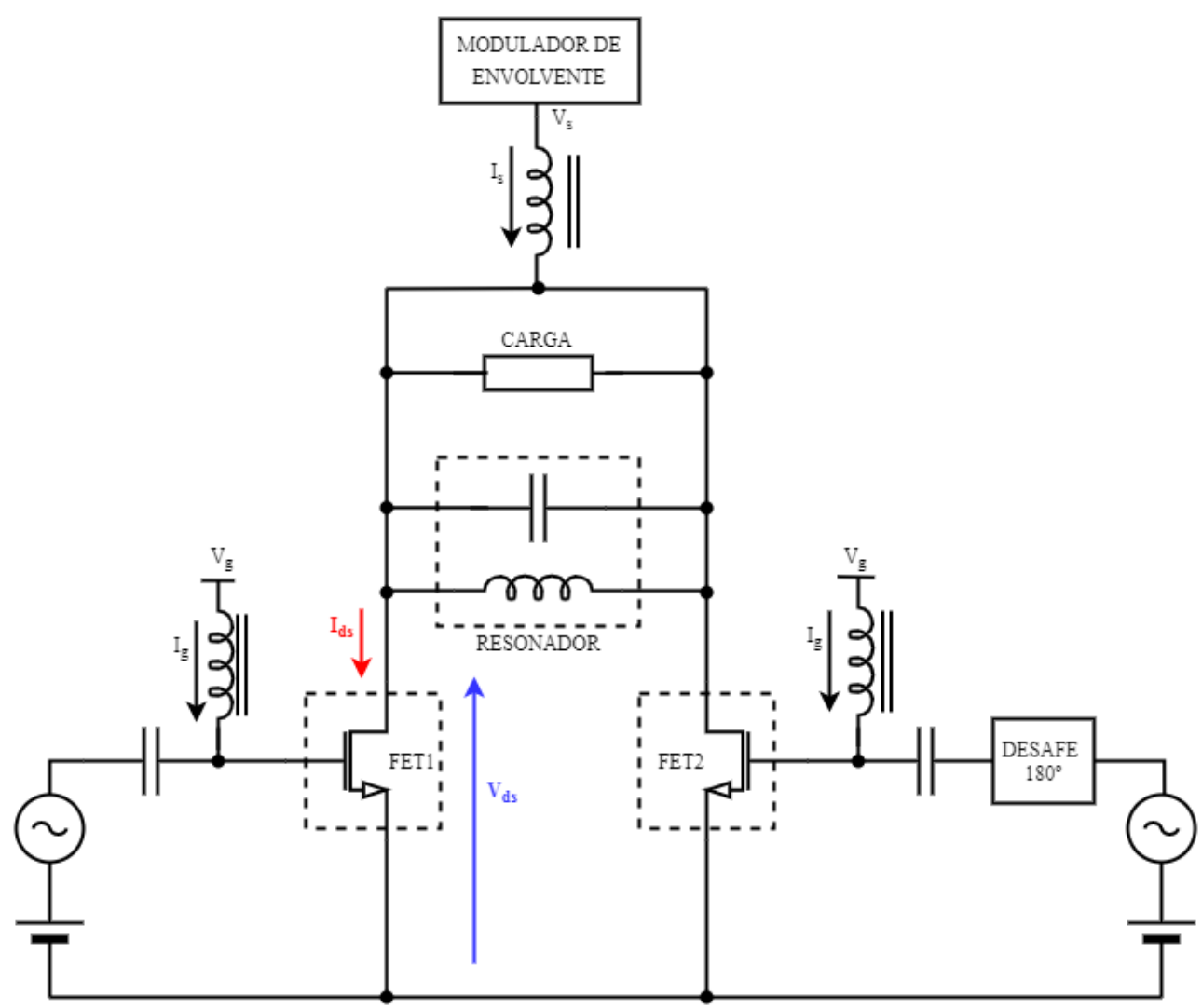

Figura 2.20. Esquema de un SMPA clase $D$ en modo corriente o clase $D^{-1}$.
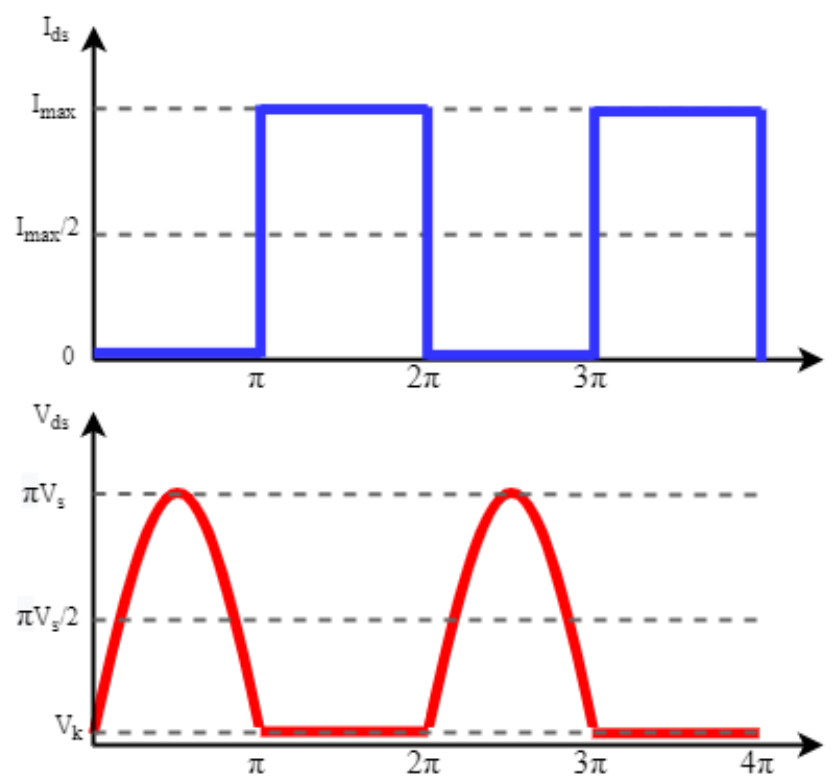

Figura 2.21. Formas de onda ideales de tensión y de corriente de un SMPA clase $D^{-1}$. 


\subsubsection{Amplificadores clase $\mathbf{E}$}

La amplificación clase E fue inventada por Nathan O. Sokal en 1972, pero no fue publicada hasta 1975 [48]. Sin embargo, hasta finales de los 90 no aparecieron los primeros prototipos a frecuencias de microondas [49]. Basado en las características de la topología, los amplificadores de potencia clase E utilizan un circuito tanque LC para adaptar, muy parecido a un amplificador clase C. Sin embargo, la topología clase C se aplican normalmente para trabajar a frecuencias por debajo de los $100 \mathrm{MHz}$, mientras que los amplificadores clase $\mathrm{E}$ se diseñan para frecuencias mucho más altas. La diferencia entre ambas topologías es que en clase $\mathrm{E}$ el dispositivo activo actúa como un conmutador.

La red de carga básica de un amplificador de potencia clase E, mostrada en la Figura 2.22, consta de un circuito LC serie con alto factor de calidad, sintonizado a la frecuencia de conmutación y que presenta circuitos abiertos a todas las frecuencias armónicas, y una capacidad en paralelo, que puede estar compuesta por la capacidad intrínseca del transistor más alguna capacidad adicional si fuera necesario. De esta manera, la capacidad del transistor ya no es una fuente de pérdidas de potencia, sino que se vuelve una parte fundamental en el diseño del circuito.

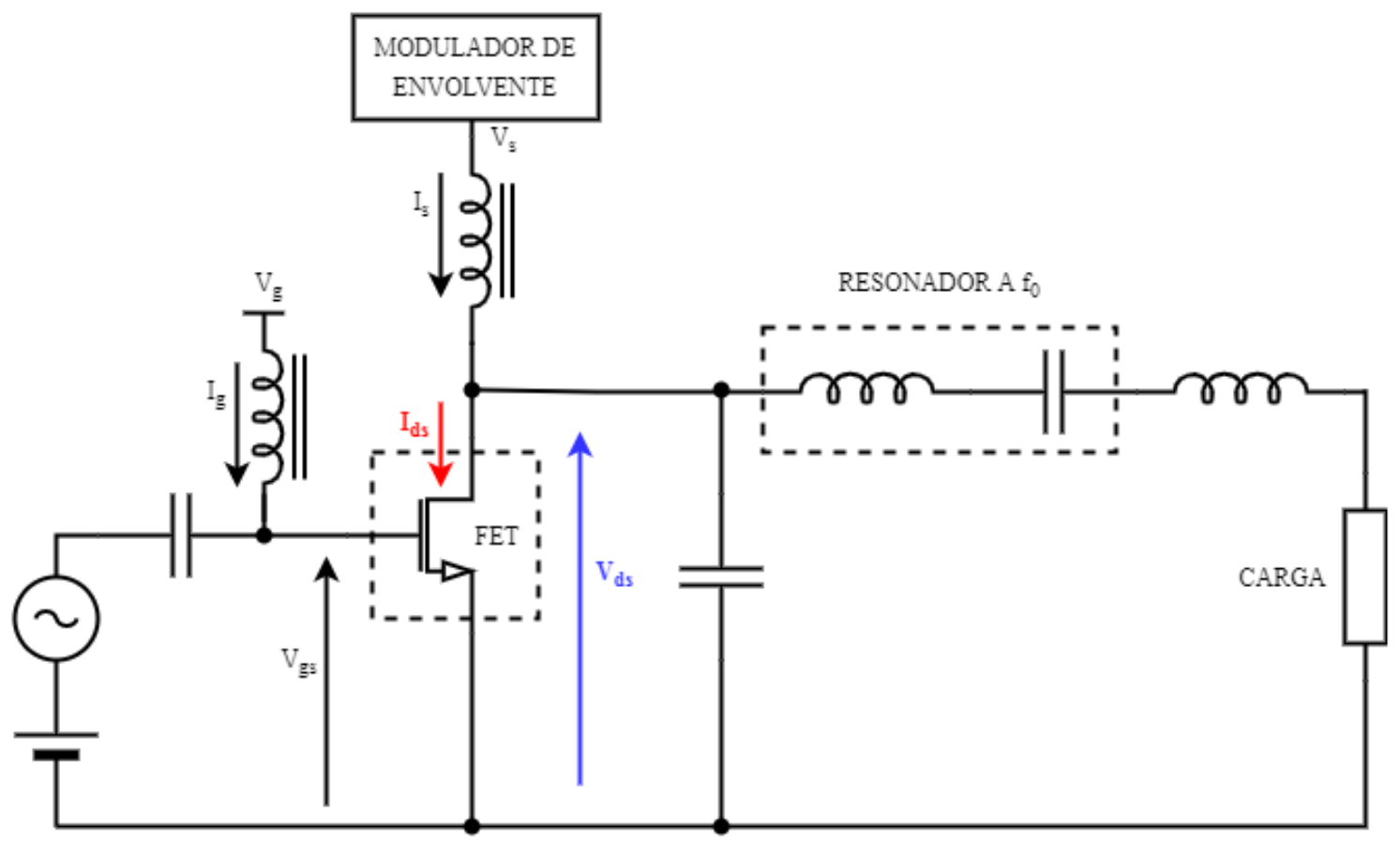

Figura 2.22. Esquema de un SMPA clase E básico. 
Para un modo de funcionamiento sin pérdidas, es necesario proporcionar las siguientes condiciones de tensión a través de conmutador en el instante cuando el transistor está saturado:

$$
\begin{aligned}
& \left.v(t)\right|_{\pi}=0 \\
& \left.\frac{d v(t)}{d t}\right|_{\pi}=0
\end{aligned}
$$

La segunda condición significa que la corriente de drenador no presenta saltos y es igual a cero en el momento que la capacidad de drenador está completamente descargada.

En el modo de funcionamiento óptimo, cuando las formas de onda de tensión y corriente de drenador mostradas en la Figura 2.23 no se solapan entre ellas, da como resultado la ausencia de pérdidas en el transistor. Las ecuaciones ya han sido publicadas en numerosos libros y artículos para describir el funcionamiento del amplificador clase $\mathrm{E}[50]$.

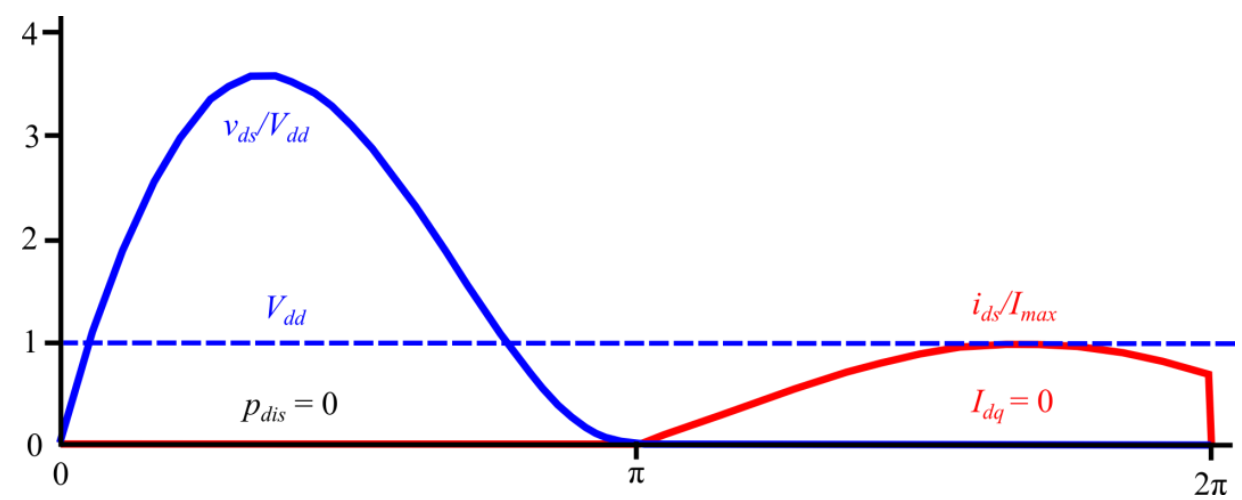

Figura 2.23. Formas de onda ideales de un SMPA en clase E.

Un amplificador de potencia clase E tiene dos ventajas principales: el suave efecto de conmutación para reducir las pérdidas y el sencillo circuito de adaptación comparado con otros modos de conmutación. Sin embargo, la topología clase E también tiene inconvenientes: la tensión de drenador tiene un valor de pico alto debido principalmente al alto valor de la capacidad de salida; también, es complicado incorporar dicha capacidad a la red de salida sintonizada, con el fin de conseguir la condición ZVS, de tal forma que toda la corriente debería ir a través de la combinación paralela del circuito LC, la capacidad drenador-fuente y la inductancia de salida. De ahí, que el amplificador clase E tiene una tolerancia límite para transistores con una alta 
capacidad de salida $C_{d s}$ que degrada el rendimiento en función de la frecuencia de operación máxima.

En un amplificador clase E convencional, se deben cumplir las condiciones ZVS y pendiente de conmutación de tensión nula con el fin de asegurar una conmutación suave. Sin embargo, la denominada topología clase $\mathrm{E}^{-1}$, cuyos primeros resultados fueron presentados en [51], utiliza condiciones ZCS y pendiente de conmutación de corriente nula para conseguir un funcionamiento óptimo [52]. El circuito básico, mostrado en la Figura 2.24, consta de una inductancia finita conectada a alimentación, una capacidad en derivación y un circuito LC sintonizado a la frecuencia fundamental. En este tipo de topología, las formas de onda de tensión y corriente se corresponden con las mismas formas de onda de corriente y de tensión de un amplificador clase $\mathrm{E}$, respectivamente.

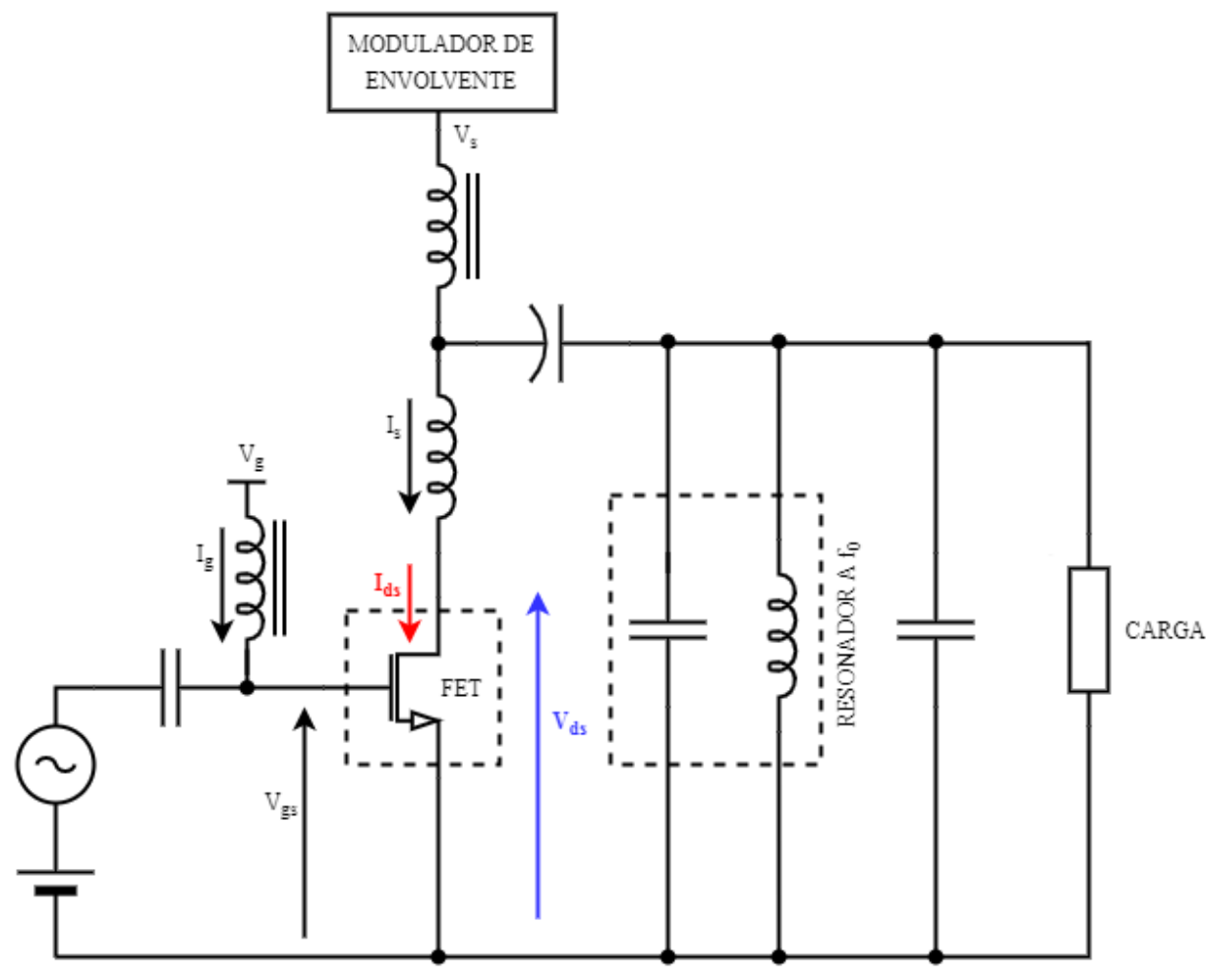

Figura 2.24. Esquema de un SMPA clase $\mathrm{E}^{-1}$ básico.

La ventaja de la topología clase $\mathrm{E}^{-1}$ es que trabaja aproximadamente con un $20 \%$ menos de tensión de conmutación máxima respecto al clase E tradicional. La tensión máxima de conmutación para un amplificador clase $\mathrm{E}$ nominal es de $3.562 \mathrm{~V}_{\mathrm{s}}$ mientras que para un amplificador clase $\mathrm{E}^{-1}$ el valor es de $2.862 \mathrm{~V}_{\mathrm{sm}}$. Esto relaja la tensión de ruptura requerida en el transistor de potencia. Segundo, un amplificador clase $\mathrm{E}^{-1}$ 
funciona con un valor de inductancia menor que en clase $\mathrm{E}$ tradicional. La inductancia parásita interna de los dispositivos encapsulados puede ser absorbida por la inductancia serie de esta topología [53]. Un valor de inductancia menor provoca que la resistencia serie del inductor se reduzca y que la frecuencia de resonancia propia sea mayor. Además, un amplificador clase $\mathrm{E}^{-1}$ es razonablemente más tolerante a las variaciones del valor de los componentes del circuito, así como a las variaciones en la frecuencia de conmutación y en el ciclo de trabajo.

\subsubsection{Amplificadores clase $\mathbf{F}$ y $\mathrm{F}^{-1}$}

Las clases $\mathrm{F} \mathrm{y} \mathrm{F}^{-1}$ trabajan sobre la manipulación de armónicos para conformar las ondas de tensión y de corriente. La clase F fue publicada por V. J. Tyler en 1958 [54], y posteriormente más investigado por Snider en 1967 [55] y por Raab en 1996 [56] como una forma de mejorar el rendimiento en gran señal de amplificadores de potencia. Estas clases de amplificación tiene mayor capacidad de potencia de salida que los amplificadores clase E [57].

Los amplificadores de potencia clase $\mathrm{F}$ requieren una red de adaptación más compleja respecto a otras clases debido a que necesita al menos 3 resonadores para cortocircuitar los armónicos pares y bloquear los armónicos impares. En la práctica, la red de adaptación típica controla hasta el quinto armónico. Por ello, un amplificador clase D se puede considerar como un caso ideal de amplificador clase F, en el cual la corriente es una forma de onda sinusoidal y la tensión es una onda cuadrada. La topología del circuito se muestra en la Figura 2.25.

La red de adaptación de un amplificador clase F, más compleja que la de un amplificador clase E, mejora la adaptación de impedancia entre la carga y el conmutador. Además, al ser diseñado para conformar una onda aproximadamente cuadrada mediante la eliminación de los armónicos pares, esto mejora el rendimiento ya que actúa más como una fuente de corriente que como un conmutador en clase $\mathrm{E}$, por lo que la intención es trabajar en saturación o corte durante el mayor tiempo posible dentro del ciclo de trabajo. 


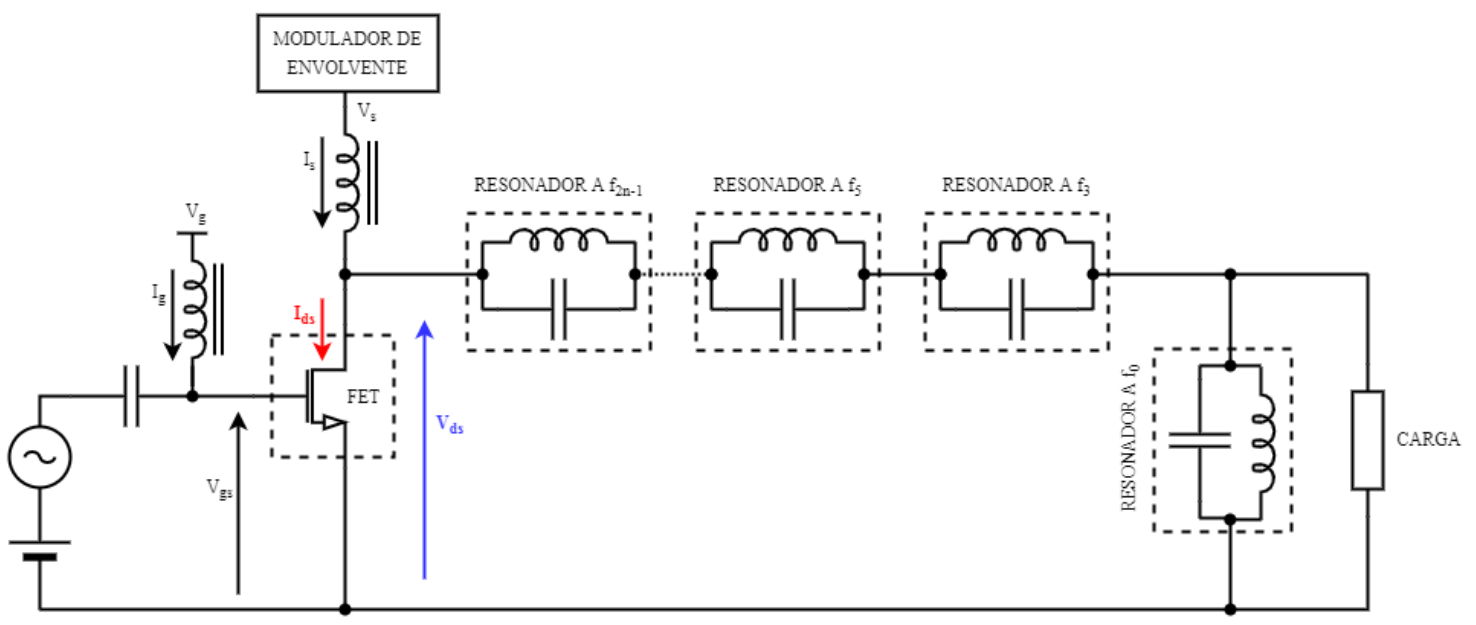

Figura 2.25. Esquema de un SMPA clase $F$ con infinitos resonadores.

En realidad, las formas de onda ideales (Figura 2.26) no se pueden conseguir debido a que se necesitaría un control de armónicos infinito. Una adecuada aproximación a una onda cuadrada se puede conseguir usando solo hasta la quinta componente de tensión armónica, donde la forma de onda resultante depende de la magnitud relativa a la componente del quinto armónico. Considerando el quinto armónico se alcanza un rendimiento teórico del $94.8 \%$ y un incremento de potencia de $0.82 \mathrm{~dB}$ con respecto a un amplificador clase B.
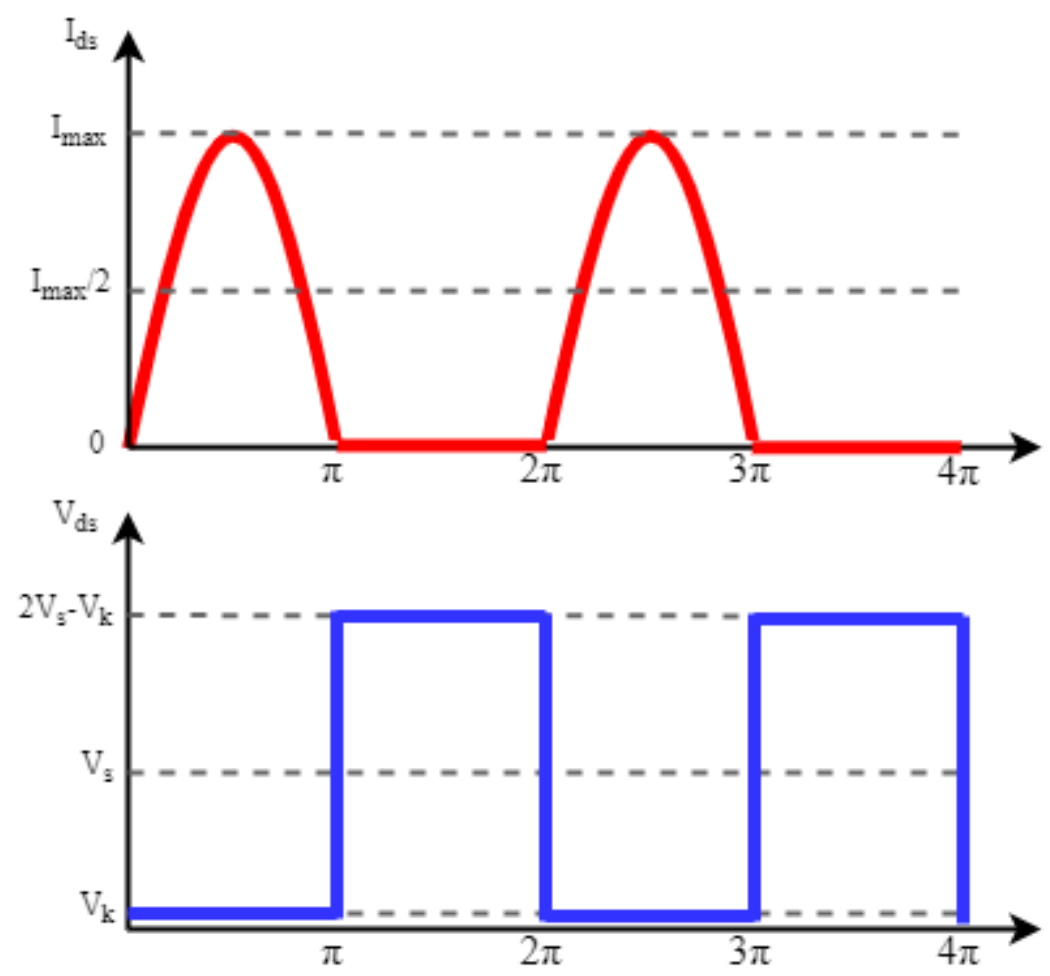

Figura 2.26. Formas de onda ideales de un SMPA en clase F. 
Al igual que con las topologías anteriores, también existe la clase complementaria denominada clase $\mathrm{F}^{-1} \mathrm{o}$ clase $\mathrm{F}$ inversa. Esta topología bloquea los armónicos pares y cortocircuita los impares. El esquema del circuito se muestra en la Figura 2.27. Se invierten las terminaciones armónicas pares e impares para conseguir una corriente cuadrada y una tensión sinusoidal.

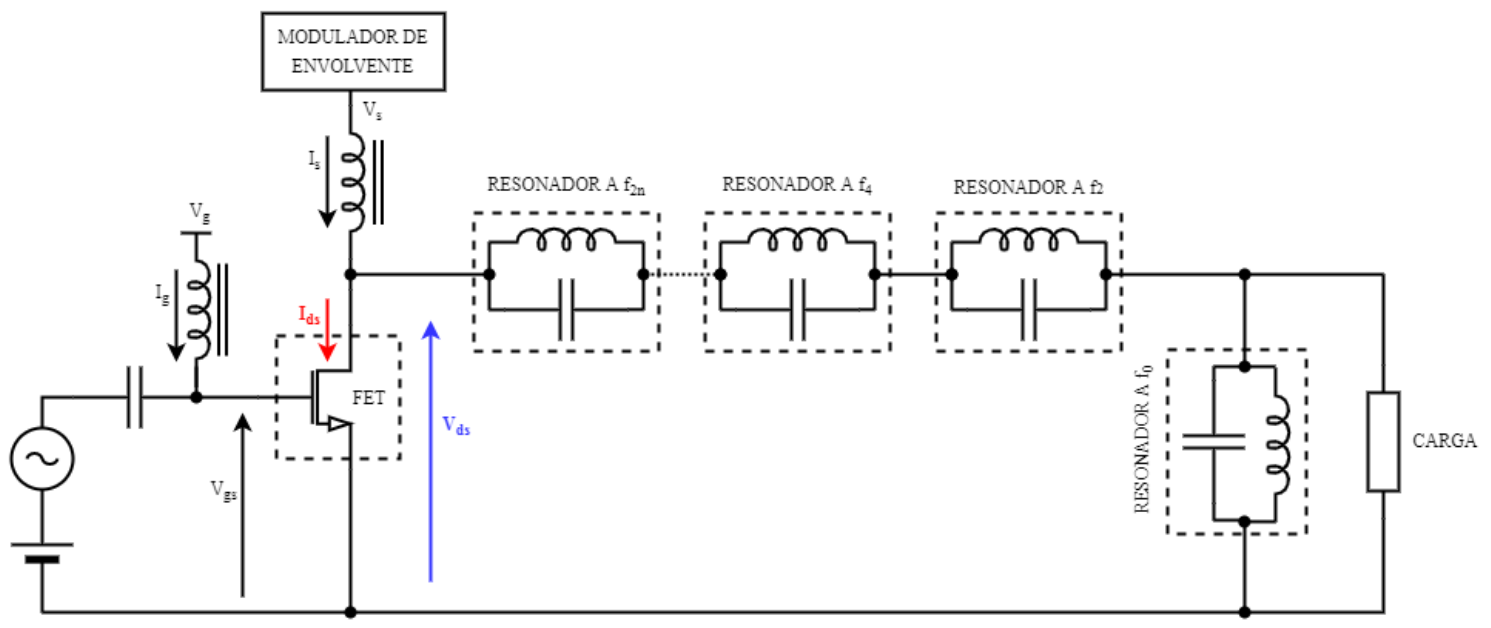

Figura 2.27 Esquema de un SMPA clase $\mathrm{F}^{-1}$.

La clase $\mathrm{F}^{-1}$ tiene unas formas de onda similares a la clase $\mathrm{D}^{-1}$, pero ofrece mayor rendimiento y menor tensión máxima que un amplificador clase E. Comparado con clase F, presenta mayores picos de tensión drenador-fuente y menores corrientes de pico. Alternativamente, la clase $\mathrm{F}^{-1}$ necesita menor tensión de alimentación de drenador que cualquier otra de las clases para la misma potencia de salida ya que la forma de onda de tensión tiene mayor amplitud máxima que la forma de onda cuadrada como la de un amplificador clase F. Por ello, es necesario prestar atención a la tensión de ruptura drenador-fuente del dispositivo activo. Menores picos de corriente reducen la degradación de rendimiento cuando se incrementa la resistencia de conducción del transistor. Debido a la baja corriente, la disipación es baja cuando la resistencia de conducción del transistor se incrementa basándose en $P=I^{2} \cdot R$, y teóricamente la impedancia de carga a fundamental es también mayor. Una mayor impedancia de carga a fundamental implica menores relaciones de transformación y reduce las pérdidas del circuito de adaptación.

Finalmente, los circuitos abiertos armónicos de alta frecuencia son más difíciles de realizar que las terminaciones armónicas de baja frecuencia. Por ello, cuando el diseño solo configura los dos primeros armónicos, en aplicaciones de alta frecuencia la 
topología clase $\mathrm{F}^{-1}$ es prácticamente más factible que la topología clase $\mathrm{F}$ ya que es menos complejo fijar una terminación en circuito abierto al segundo armónico que al tercer armónico. De ahí que por todas estas razones el diseño en clase $\mathrm{F}^{-1}$ es preferible para el funcionamiento a altas frecuencias.

Un diseño diferente reemplaza el circuito resonante armónico por una línea de transmisión $\lambda / 4$, que simula el equivalente a un número infinito de resonadores. El circuito de salida sintonizado paralelo es un cortocircuito para todas las frecuencias armónicas, sin embargo, la línea $\lambda / 4$ transforma esto en cortocircuitos a cada una de las frecuencias armónicas pares y circuitos abiertos a cada uno de los armónicos impares. El resultado final contiene el fundamental y los armónicos impares, los cuales conducen a una forma de tensión de drenador cuadrada y de ahí el funcionamiento del dispositivo como conmutador, consiguiendo mejorar el rendimiento del circuito.

\subsubsection{Amplificadores clase $S$}

El concepto clase S fue publicado por A. M. Sandman en 1982 [58] y se fue desarrollando junto con el concepto de modulación de pulso.

Hay una tendencia a investigar el funcionamiento del modo de conmutación clase S como una alternativa a los amplificadores de RF convencionales. Teóricamente, la clase $\mathrm{S}$ puede ofrecer hasta un $100 \%$ de rendimiento.

Un amplificador clase $S$ típico contiene una fuente de señal en banda base, un modulador de bit con modulación de pulso, un amplificador de potencia conmutado de alto rendimiento (típicamente una topología clase $\mathrm{D}$ o $\mathrm{D}^{-1}$ ) y un filtro de reconstrucción paso banda para restaurar la señal fundamental. En la Figura 2.28 se puede ver un diagrama de bloques de un amplificador clase $\mathrm{S}$. 


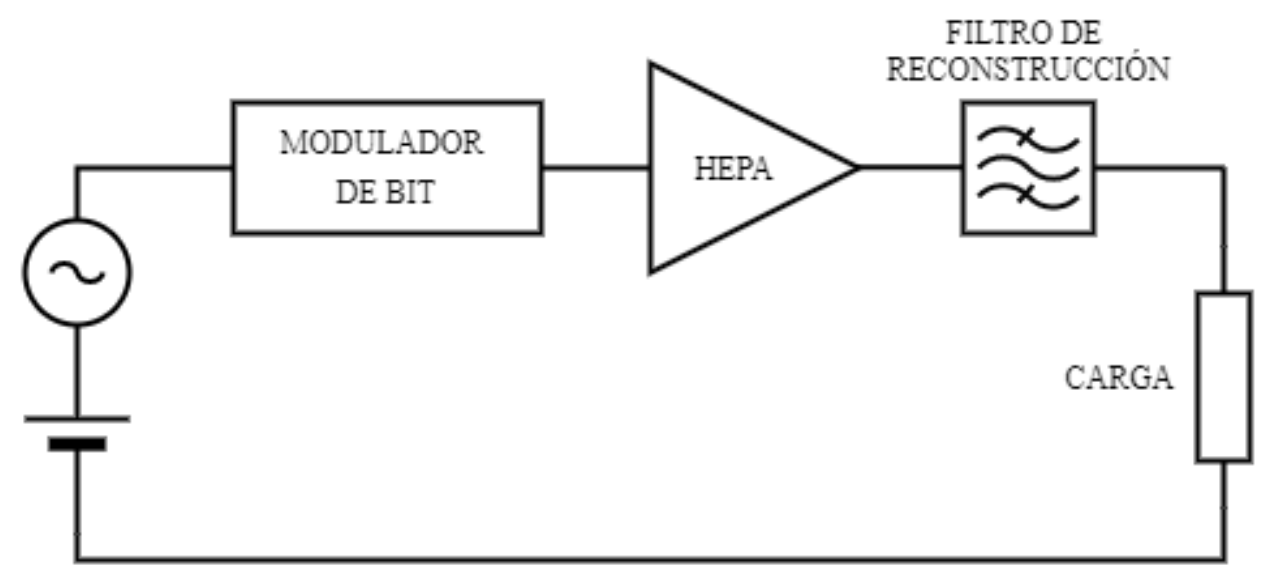

Figura 2.28. Diagrama de bloques de un SMPA clase S.

Para el diseño de un modulador de bit simple o múltiple, los esquemas de modulación por anchura de pulso (PWM) fueron utilizados en el desarrollo de esta topología [59]. Considerando la velocidad de conmutación no lineal, la modulación sigma delta (SDM) [60] fue propuesta para clase $\mathrm{S}$ debido a su perfil de ruido y su carácter inherentemente lineal. Mientras tanto, actualmente, la modulación por densidad de pulso (PDM) [61] y la modulación por posición del pulso (PPM) han sido también investigadas para paliar algunos inconvenientes [62].

El funcionamiento clase $\mathrm{S}$ se basa en una señal de conmutación de duración de pulso variable, que da como resultado de funcionamiento una condición de conmutación de tensión o corriente nula. Por lo tanto, una señal modulada digitalmente se considera como un tren de pulsos aperiódico y el empleo de un filtro de reconstrucción genera las formas de onda positivas y negativas. En otras palabras, el funcionamiento en clase $\mathrm{S}$ se caracteriza por unas transiciones de corriente-tensión no solo en el primer cuadrante sino también en el tercer cuadrante debido a las formas de onda de tensión y corriente negativas.

Existen dificultades a la hora de diseñar un amplificador clase S. La frecuencia a la cual deben conmutar los transistores debe ser mucho mayor que la frecuencia fundamental de la señal de salida y para ello se necesitan dispositivos con un gran ancho de banda de funcionamiento.

Al igual que otros amplificadores en modo de conmutación, la amplificación clase S también ve reducido su rendimiento teórico del $100 \%$ debido a los efectos de la 
tensión y resistencia de saturación, a las capacidades parásitas y a los tiempos de conmutación no nulos [63].

En la práctica, el empleo de moduladores de bit, como puede ser la modulación PWM, como entrada del amplificador conmutado provoca distorsión en la señal de salida. Esto es debido a los armónicos de la frecuencia de conmutación y a las bandas laterales espurias que presentan este tipo de modulaciones. Estos espurios pueden caer dentro de la banda de paso del filtro de salida e introducir una pequeña distorsión. De ahí que la frecuencia de conmutación deba ser mucho mayor que la frecuencia de trabajo.

\subsubsection{PA en ingeniería de onda}

La incorporación de energía armónica a las formas de onda de tensión y de corriente fundamentales puede ser una manera eficiente para conformar la onda en el dominio del tiempo y conseguir el funcionamiento en alto rendimiento. En principio se asume que la energía armónica de tensión y de corriente está siempre disponibles y puede tanto ser añadida como eliminada presentando las impedancias armónicas apropiadas en el drenador del transistor de potencia. Por lo tanto, las impedancias armónicas pueden ser usadas para generar combinaciones específicas de formas de onda de tensión y de corriente que eviten el solapamiento, pero mantengan una potencia de salida alta a la frecuencia fundamental.

Tradicionalmente, el diseño de amplificadores de RF se centra en las medidas de los parámetros de funcionamiento no lineales tales como potencia de salida, ganancia, rendimiento y linealidad como función de la impedancia de carga fundamental. Básicamente, el objetivo es encontrar, tanto por ensayo y error o vía una secuencia lógica de medidas, las impedancias de carga armónica con las que conseguir el funcionamiento óptimo. Teóricamente, el funcionamiento óptimo ocurrirá cuando se dé la forma apropiada a las ondas de tensión y corriente "ingenierizadas". Durante la investigación experimental, el diseñador del amplificador puede eficientemente modificar la forma de onda de corriente mediante la elección del punto de polarización de DC y el nivel de excitación de entrada y la forma de onda de tensión mediante la 
impedancia de carga fundamental. Por ello, muchos de los diseños de SMPA puede ser abordados como un proceso de ingeniería de forma de onda.

En todas estas clases de conmutación hay inconvenientes. Por ello, se debe encontrar un compromiso para conseguir un funcionamiento equilibrado entre rendimiento, linealidad y ancho de banda.

\subsubsection{Amplificador clase J}

Los amplificadores de potencia clase $\mathrm{J}$ fueron principalmente promovidos por Cripps [64]. Combina las ventajas de un amplificador clase $\mathrm{E}$ y de un amplificador clase $\mathrm{F}^{-1}$. Sin embargo, esta clase fue publicada con diferentes nombres, pero con el mismo patrón de funcionamiento: clase $\mathrm{E}$ de alto rendimiento [65] o clase E/F [66]. Cripss sugirió que los diseños clase B presentan terminaciones armónicas capacitivas en el plano de la capacidad de salida del propio transistor de potencia que se aproximan a un cortocircuito. De ahí se desarrolló la teoría para el modo clase J basándose en tales terminaciones armónicas y para una impedancia de carga reactiva. El esquema de un amplificador clase J se puede ver en la Figura 2.29.

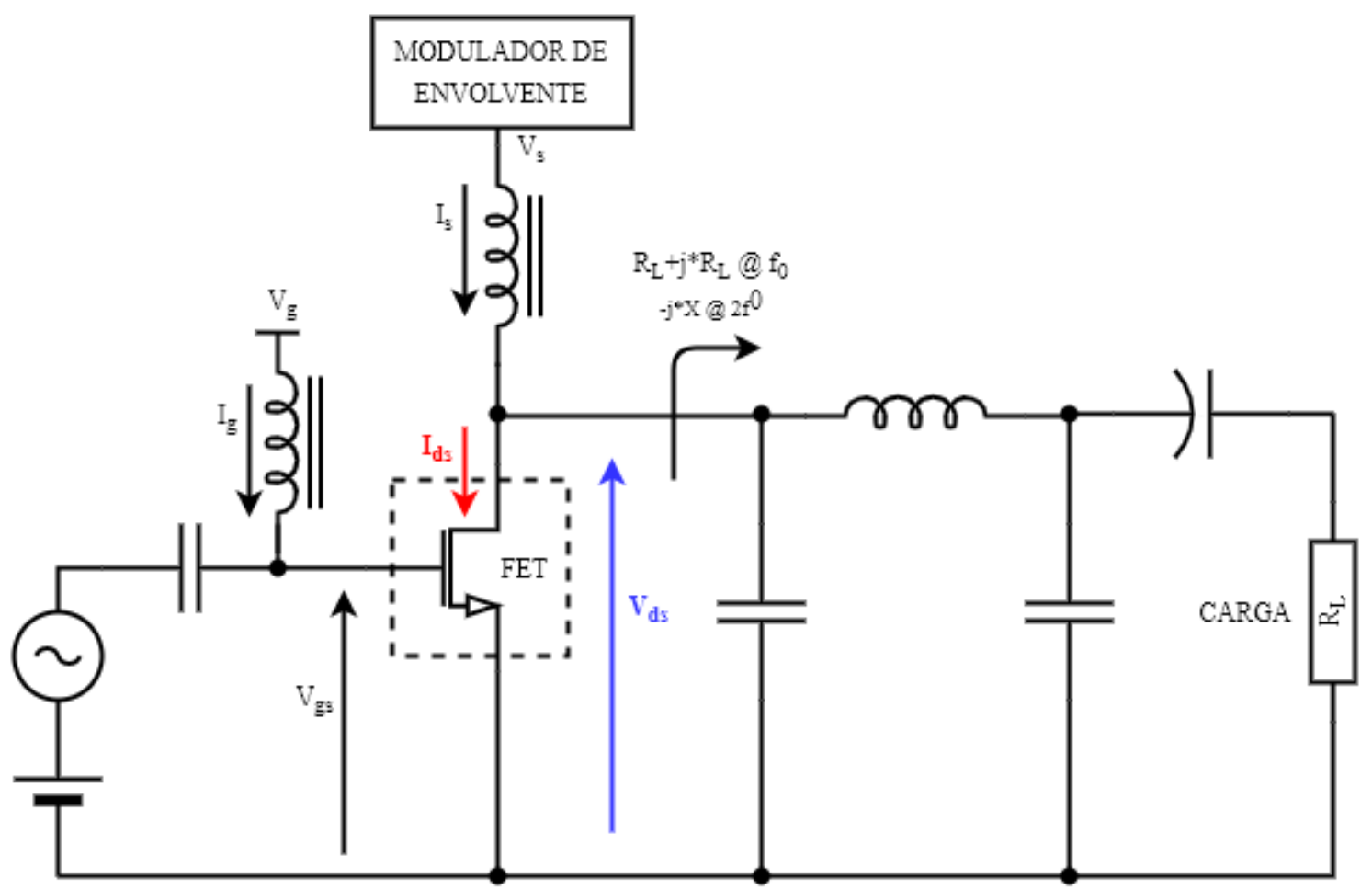

Figura 2.29. Esquema de un amplificador clase $\mathrm{J}$. 
Un amplificador de potencia clase $\mathrm{J}$ necesita una ligera carga inductiva a la frecuencia fundamental y solo una carga capacitiva al segundo armónico. El primer requisito es similar al de la clase E. Sin embargo, la clase J intenta hacer transiciones de conmutación más rápidas entre ON y OFF, lo que reduce las pérdidas e incrementa el rendimiento. Mientras, en un amplificador clase E las transiciones de conmutación a ON tienen menores pérdidas que las transiciones de conmutación a OFF para una señal de excitación sinusoidal. Por otro lado, la clase $\mathrm{F}^{-1}$ necesita un gran número de resonadores para conseguir alto rendimiento, aunque en la práctica la mayoría de los diseños controlan hasta el quinto armónico. En este punto, la topología clase J necesita solo un resonador para controlar el segundo armónico.

Varias ventajas se pueden reconocer en clase J. La primera y más importante, puede ser vista como un buen candidato para el funcionamiento en alto rendimiento y en banda ancha. Además, puede ser diseñada con cualquier clase de PA, tal como clase $\mathrm{A} / \mathrm{AB} / \mathrm{B}$.

Para eliminar la carga negativa al segundo armónico, la forma de onda de tensión debería ser desplazada al menos $45^{\circ}$ con la carga fundamental compleja y la del segundo armónico capacitiva, tal y como se muestra en Fig. 2.30. En este caso, hay algo de solapamiento entre las formas de onda de tensión y de corriente a fundamental, pero se pueden mantener las formas de onda de tensión y corriente medio-sinusoidal, lo que se convierte en un PA clase J. Debido al solapamiento entre las formas de onda, el clase $\mathrm{J}$ disipa potencia internamente $\mathrm{y}$ reduce la potencia y el rendimiento, dando como resultado el mismo rendimiento que un PA clase B, siendo del $78.5 \%$.

\subsubsection{Amplificador clase $P$}

Posteriormente al amplificador clase $\mathrm{J}$, investigadores de la Universidad de Ciencia y Tecnología de Pohang dieron a conocer la clase P [67], cuyo esquema se muestra en la Figura 2.31. En comparación con clase J, la gran ventaja es que se aprovecha del comportamiento no lineal de la capacidad de salida para realizar el proceso de ingeniería de forma de onda y fomentar el rendimiento máximo de potencia con una red de adaptación de salida sencilla a fundamental y armónicos. Sin embargo, el 
amplificador clase $\mathrm{P}$ contiene varias subcategorías de acuerdo a las diferentes técnicas de manipulación de armónicos.
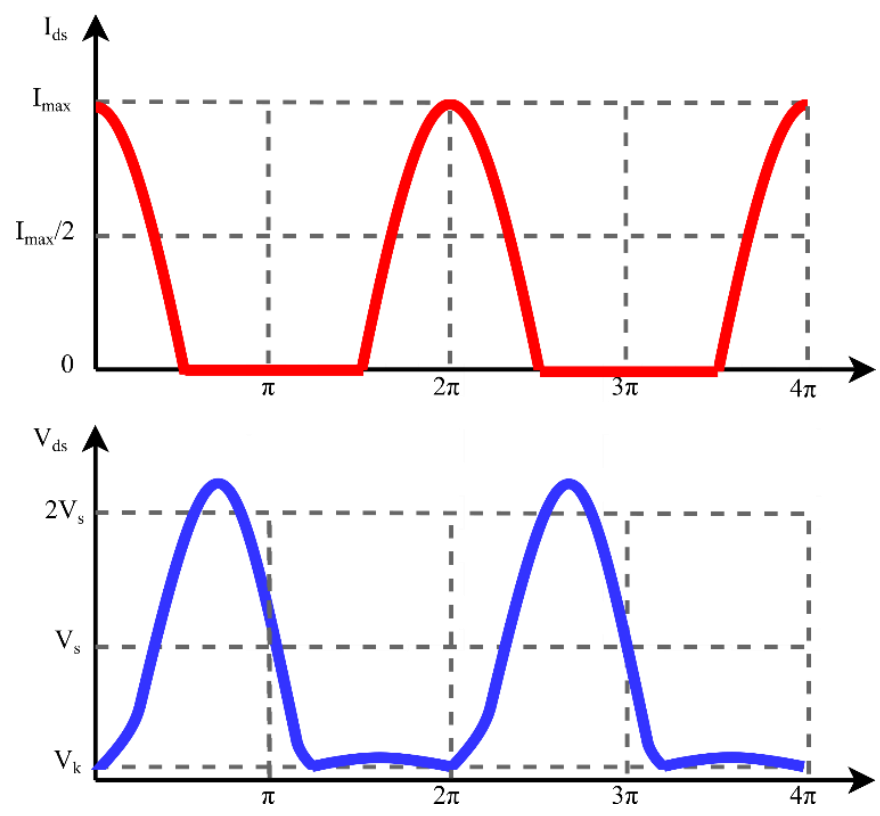

Fig. 2.30 Formas de onda ideales de un PA en clase J.

Los amplificadores clase $\mathrm{P}$ utilizan las tensiones armónicas generadas por el efecto no lineal de la capacidad de salida $C_{\text {out }}$ para dar forma a la onda de tensión. Esta capacidad no lineal está formada por las capacidades no lineales de la salida del transistor de RF, es decir, por la capacidad $C_{d s}$ y $C_{g d}$, que varían en función de la tensión $V_{d s}$ y $V_{g d}$ respectivamente. Las tensiones armónicas procedentes de $C_{\text {out }}$ contienen una gran componente al segundo armónico con magnitudes menores a armónicos de alto orden. Por ello, se puede generar de manera sencilla una tensión de media sinusoide a través de la capacidad usando una carga armónica grande.

Un amplificador clase $\mathrm{P}$ tiene una forma de onda de corriente quasirectangular/bifurcada y una forma de onda de tensión quasi medio-sinusoidal, que son similares a las formas de onda de un amplificador clase $\mathrm{F}^{-1}$. Las formas de onda de tensión y de corriente se les da forma usando las propias componentes armónicas generadas [Kim, highly efficient saturated power amplifier base don GaN - A class P amplifier, 2010]. Ya que la capacidad de salida en el transistor es altamente no lineal, se genera una gran cantidad de tensión armónica de segundo orden con unos bajos armónicos de orden superior. Por ello, la tensión armónica auto-generada ayuda a la formación de la tensión quasi medio-sinusoidal. Las tensiones armónicas pueden ser 
suficientemente grandes cuando el amplificador es excitado con una alta potencia de entrada. Bajo tal condición de excitación, la corriente se convierte en quasi-rectangular mediante la bifurcación de la corriente trabajando en saturación. Por ello, el rendimiento práctico de un amplificador clase $\mathrm{P}$ puede alcanzar el $90 \%$.

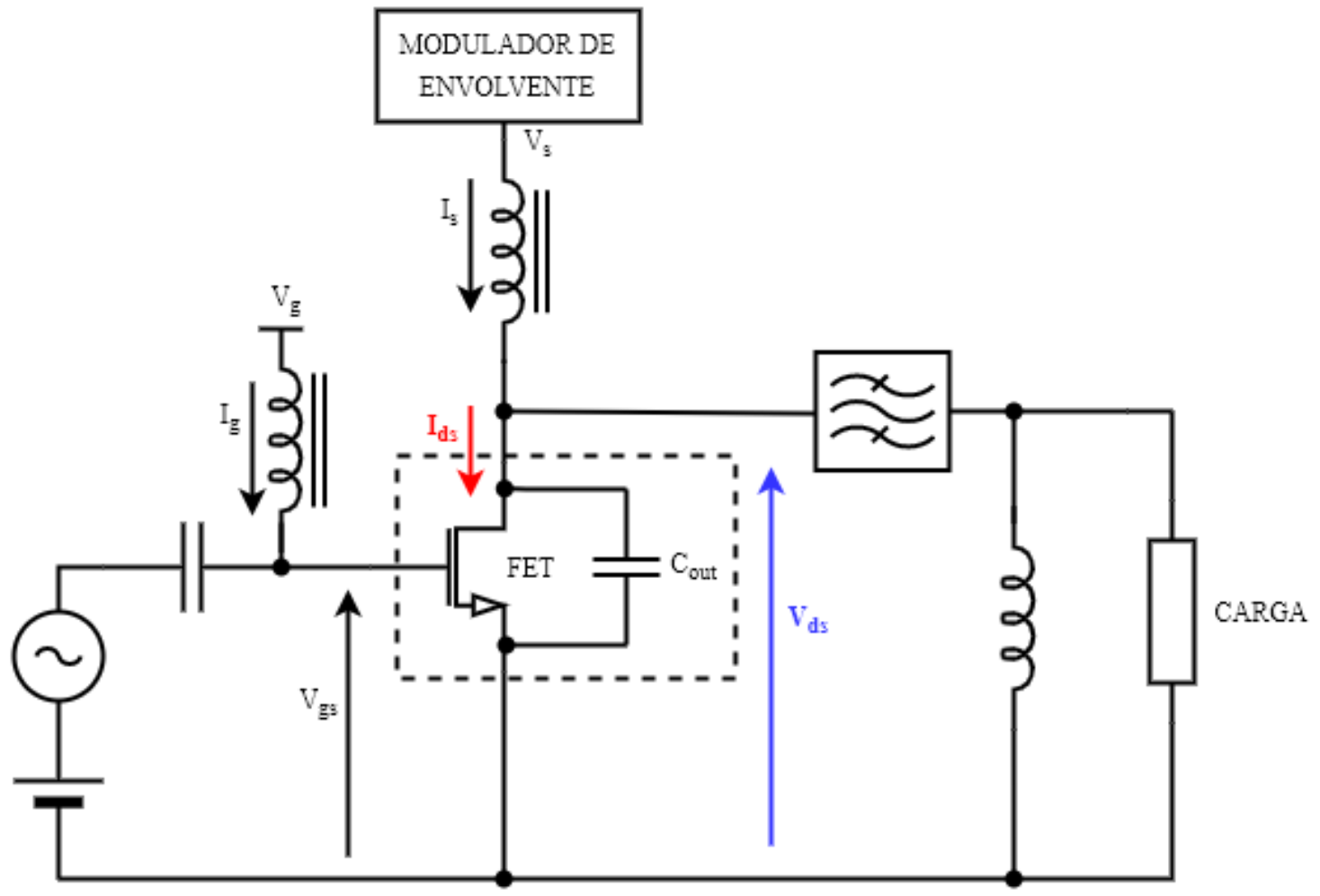

Figura 2.31. Diagrama de bloques de un PA en clase P.
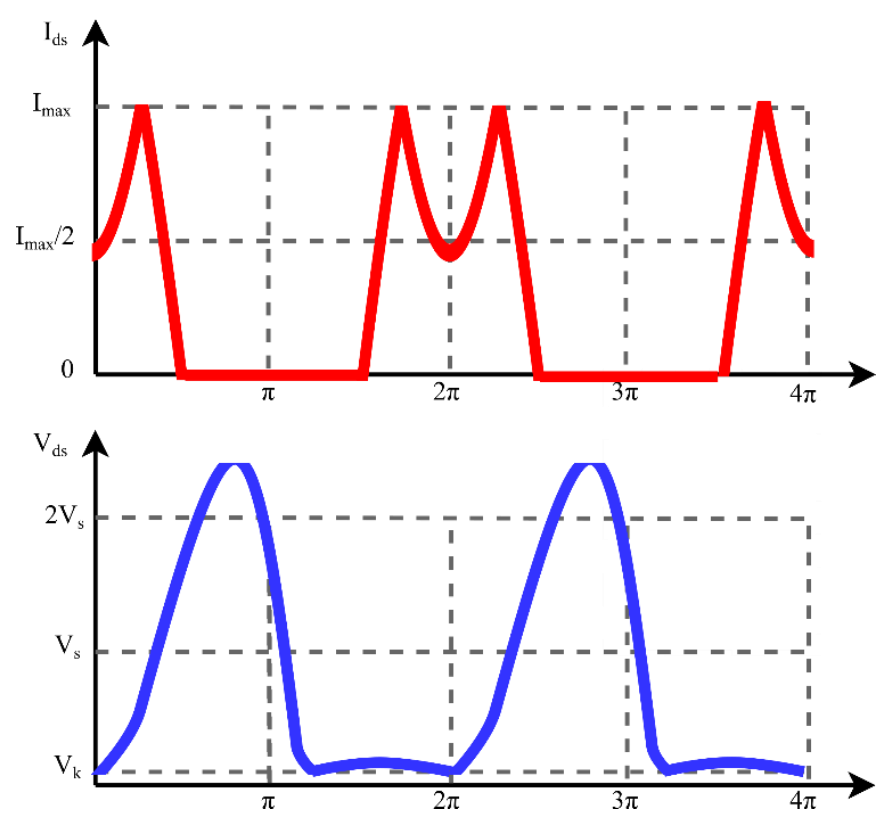

Fig. 2.32 Formas de onda ideales de un PA en clase P. 
La Tabla 2.1 resume las principales características de las clases de amplificación.

Tabla 2.1 Resumen de características de las clases de amplificación.

\begin{tabular}{ccccccc}
\hline \hline Clase & Complejidad & $\begin{array}{c}\text { Potencia } \\
\text { de salida }\end{array}$ & Rendimiento & Ganancia & Linealidad & $\begin{array}{c}\text { Ancho } \\
\text { de } \\
\text { banda }\end{array}$ \\
\hline A & Baja & Moderada & Bajo & Alta & Alta & Amplio \\
\hline B & Baja & Moderada & Medio & Moderada & Moderada & Amplio \\
\hline AB & Baja & Moderada & Medio & Moderada & Moderada & Amplio \\
\hline C & Baja & Baja & Alto & Baja & Baja & Amplio \\
\hline D & Moderada & Alta & Alto & Baja & Baja & Medio \\
\hline D-1 & Moderada & Alta & Alto & Baja & Baja & Medio \\
\hline E & Baja & Alta & Alto & Baja & Baja & Amplio \\
\hline E-1 & Baja & Alta & Alto & Baja & Baja & Amplio \\
\hline F & Alta & Alta & Alto & Baja & Baja & Estrecho \\
\hline F-1 & Alta & Alta & Alto & Baja & Baja & Estrecho \\
\hline S & Alta & Alta & Alto & Baja & Baja & Medio \\
\hline J & Moderada & Alta & Alto & Baja & Baja & Amplio \\
\hline P & Moderada & Alta & Alto & Baja & Baja & Medio \\
\hline \hline
\end{tabular}

\subsection{Arquitecturas de linealización}

Normalmente los amplificadores de alta potencia trabajan en la zona de compresión con el fin de conseguir alto rendimiento. Sin embargo, aparecen no linealialidades tales como distorsión de amplitud y de fase. Una opción para solucionar este efecto es reducir el rango dinámico de funcionamiento en una zona más lineal, donde el nivel de potencia de salida sería menor y también se reduciría el rendimiento. [68]. Otra opción se basa en emplear técnicas que pueden reducir las no linealidades en amplificadores, trabajando en la zona de alto rendimiento.

Aunque existen varias clases de técnicas de linealización, las más comunes son: feedback, feedforward y predistorsión. Algunas técnicas, como Doherty y Chireix 
todavía se emplean en algunas aplicaciones específicas. La técnica feedback se emplea normalmente en amplificadores de audio, ya que tiene fuertes limitaciones en cuanto a ancho de banda, retardos e inestabilidades a altas frecuencias. Feedforward es una técnica de linealización muy efectiva cuando se necesitan amplios anchos de banda y altas linealidades. No obstante, es una técnica muy compleja y, además, cuando se trabaja con alta potencia de salida, es la menos efectiva [69]. La técnica de predistorsión ha sido históricamente la favorita en implementaciones analógicas. Actualmente, es muy útil en aplicaciones digitales, ya que puede ser integrada en cualquier arquitectura digital, tal como DSP (Digital Signal Processor) o FPGA (Field Programmable Gate Array), que pueden llevar a cabo un funcionamiento aritmético más rápido [70].

Existen otras técnicas, como error-feedback, propuesta por Ying en [71], LINC (Linear Amplification using Non-linear Components), LIST (Linear Amplification by Sampling Techniques), ET (Envelope Tracking) o EER (Envelope Elimination and Restoration) que se utilizan en casos específicos.

\subsubsection{Doherty}

El principio básico del funcionamiento de un amplificador Doherty se basa en la modulación de carga, donde la impedancia de carga varía de acuerdo al nivel de potencia. La arquitectura Doherty es hoy en día una de las técnicas principales en el diseño de amplificadores de potencia de alto rendimiento para infraestructuras inalámbricas a pesar de que su primer desarrollo se produjo en 1936 por W. H. Doherty [72].

La mejora del rendimiento alcanzado mediante la técnica Doherty consigue que el rendimiento del amplificador sea dos o tres veces mayor que el de un amplificador convencional en clase B. Los amplificadores Doherty suelen basarse en amplificadores en clase B, por lo que la linealidad del sistema depende de la linealidad de ellos [73]. Sin embargo, otras técnicas adicionales podrían ser usadas para mejorar la linealidad del sistema.

La Figura 2.33 muestra la topología clásica de un sistema Doherty de dos etapas, llamado comúnmente Doherty uniforme o simétrico. Consta de dos amplificadores, comúnmente referenciados como amplificador principal y amplificador auxiliar, que 
actúan en función del nivel de entrada. En un amplificador Doherty con relación 1:1, la señal de RF de entrada se fragmenta mediante un divisor de $3 \mathrm{~dB}$ o un acoplador híbrido en dos caminos desfasados $90^{\circ}$. Cuando el nivel de potencia de entrada es bajo, el amplificador auxiliar deja de funcionar lo que da como resultado una impedancia de carga equivalente a $2 Z_{0}$ en el amplificador principal. A medida que se incrementa el nivel de potencia de entrada, el amplificador auxiliar entra en funcionamiento entrando en escena la red de adaptación y las líneas de desfase que modulan la carga del amplificador. Tras las amplificaciones producidas por los elementos principal y auxiliar, las señales de salida de RF son sumadas mediante un combinador Doherty.

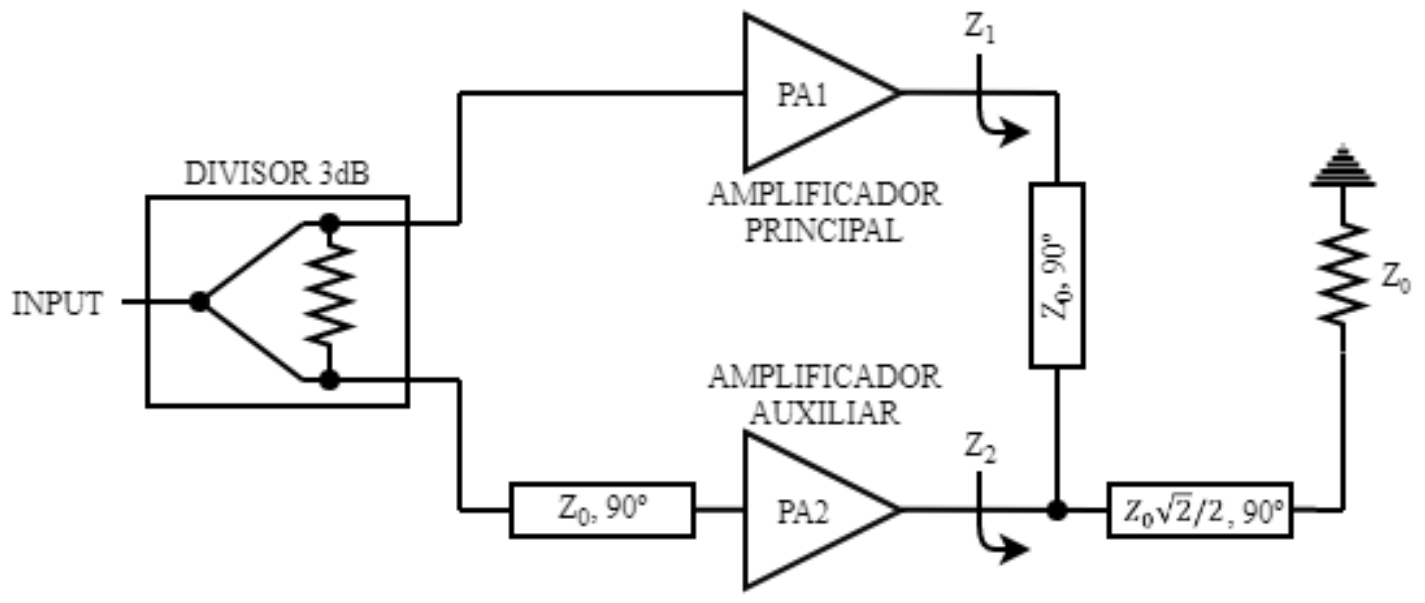

Figura 2.33. Diagrama de bloques de un sistema Doherty clásico.

Además del primer punto de rendimiento máximo que se produce con el elemento auxiliar, la curva de rendimiento se mantiene alta durante el modo de baja potencia mientras el amplificador auxiliar no actúa. Por ello, el elemento principal puede generar un segundo punto de rendimiento máximo a $6 \mathrm{~dB}$ por debajo de la potencia máxima de salida. Está característica se muestra en la Figura 2.34. Por esta razón, los amplificadores Doherty clásicos teóricamente presentan un rendimiento medio-alto al trabajar con señales moduladas en amplitud con una PAPR alrededor de $\operatorname{los} 6 \mathrm{~dB}$.

A pesar de este rendimiento medio-alto, el rápido desarrollo de los estándares en comunicaciones inalámbricas ha provocado que la PAPR de las señales se esté continuamente incrementando siendo los valores mayores a $6 \mathrm{~dB}$, lo cual da como resultado una degradación de rendimiento del sistema Doherty. Desde los años 70 se 
han desarrollado diferentes variantes para incrementar el rendimiento en señales con alta PAPR.

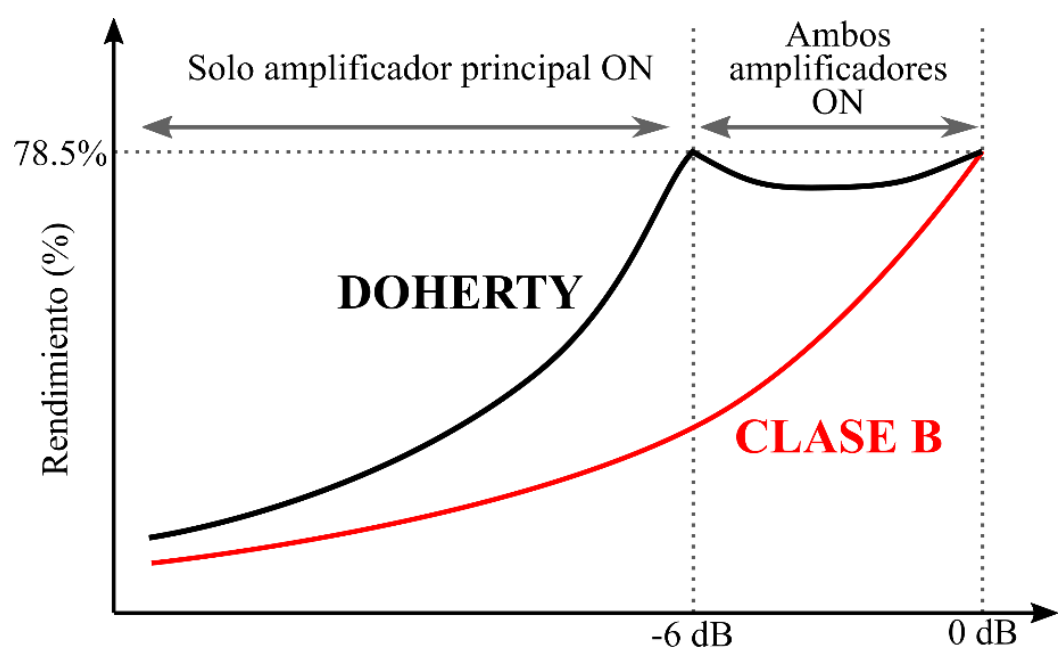

Potencia de back-off normalizada

Figura 2.34. Comparación del rendimiento de un sistema Doherty y un amplificador clase B.

De ahí, se propuso la arquitectura Doherty asimétrica con la intención de desplazar el segundo punto de rendimiento máximo hacia la zona de menor potencia para tolerar más de $6 \mathrm{~dB}$ de back-off.

Hay muchos métodos para implementar un Doherty asimétrico, tal como las configuraciones de tensión de drenador asimétrica y división asimétrica del amplificador. Una topología Doherty asimétrica típica, Figura 2.35, es una división y dimensionado asimétricos por $5 \mathrm{~dB}$ y relación 1:2, respectivamente.

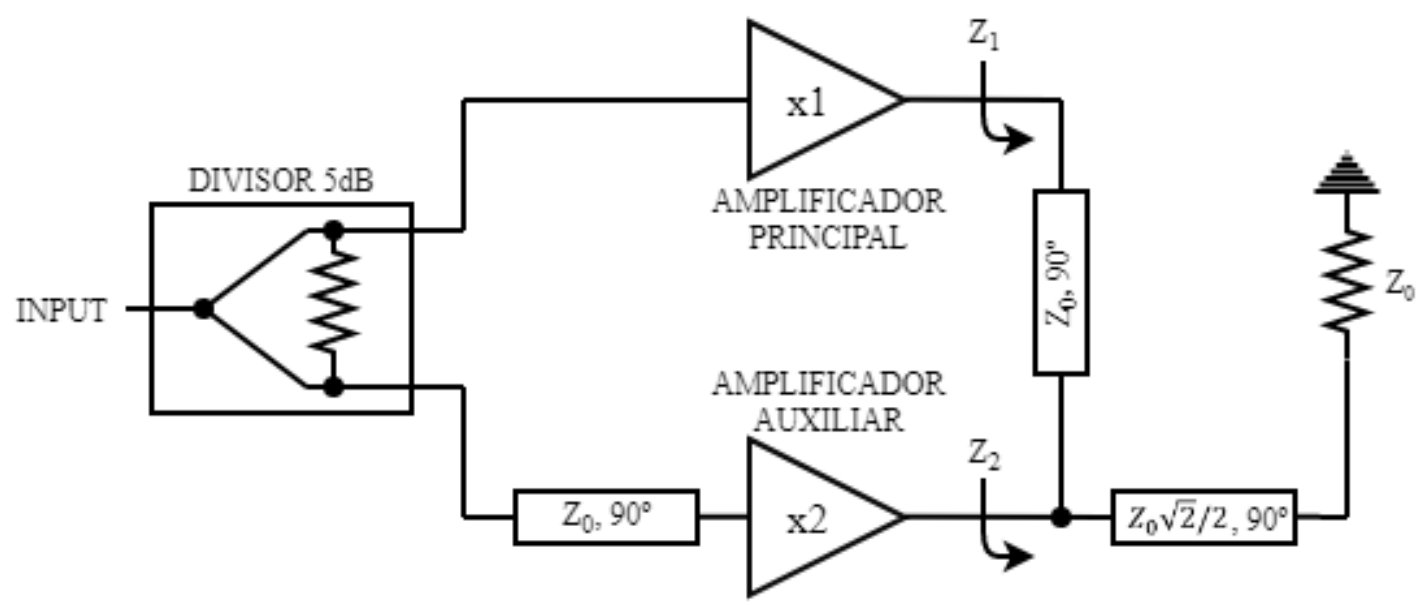

Figura 2.35. Diagrama de bloques de un sistema Doherty asimétrico. 
Con este tipo de estructura, el segundo pico de rendimiento se mueve a $9 \mathrm{~dB}$ de la posición de back-off para tolerar señales con alta PAPR (Figura 2.36). Sin embargo, la zona entre los dos picos de rendimiento sufrirá de una caída de rendimiento más severa. A medida que va disminuyendo la relación de potencia entre el elemento de principal y el auxiliar, es posible desplazar aún más el pico de rendimiento a costa de una mayor caída de rendimiento. Por ejemplo, para una relación de 1:3 el pico de rendimiento se desplaza a $12 \mathrm{~dB}$ de back-off. Además de la caida del rendimiento, estos esquemas asimétricos presentan importantes efectos memoria que dificutan la linealización empleando predistorsión.

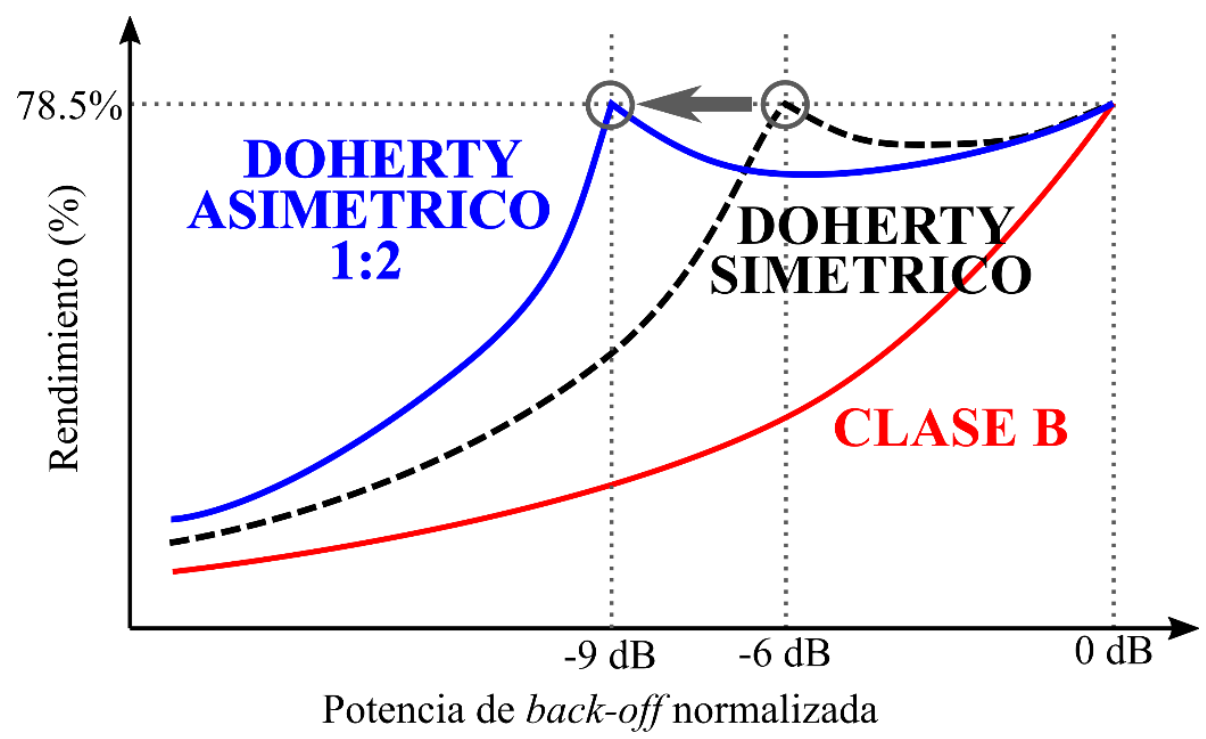

Figura 2.36. Comparación del rendimiento de un sistema Doherty y un amplificador clase B.

Otra variante del Doherty convencional es el Doherty invertido (Figura 2.37). En una arquitectura Doherty invertida, los elementos de la red de adaptación pueden ser sustituidos por líneas de transmisión $\lambda / 4$ cuyo valor de impedancia característica es arbitrario. Por medio de esto, se pueden conseguir importantes mejoras en linealidad y rendimiento con respecto a un Doherty simétrico tradicional. La principal ventaja es la sencilla implementación además de fomentar el incremento de ancho de banda de funcionamiento. 


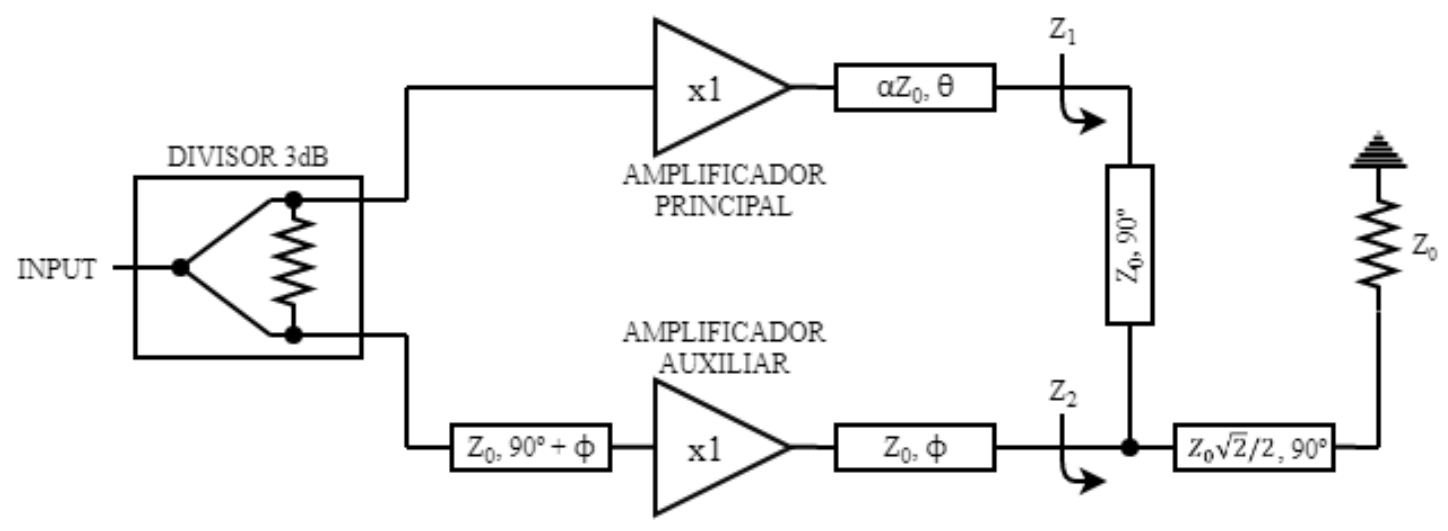

Figura 2.37. Diagrama de bloques de un sistema Doherty invertido.

Por último, con el incremento de la PAPR de las señales moduladas actuales, surgió el concepto de Doherty multietapa (Figura 2.38) capaz de proporcionar múltiples picos de rendimiento sobre un rango de back-off de potencia de salida para aliviar la caída de rendimiento y ajustarse a la función de densidad de potencia de las señales. Un Doherty multietapa se basa en una condición de encendidos secuenciales de varios elementos auxiliares con motivo de asumir múltiples regiones Doherty en una configuración en cascada, lo que reduce la degradación del valor medio debido al incremento del efecto de decaimiento en el rendimiento de Doherty, particularmente cuando se necesita un back-off de potencia de salida grande [74].

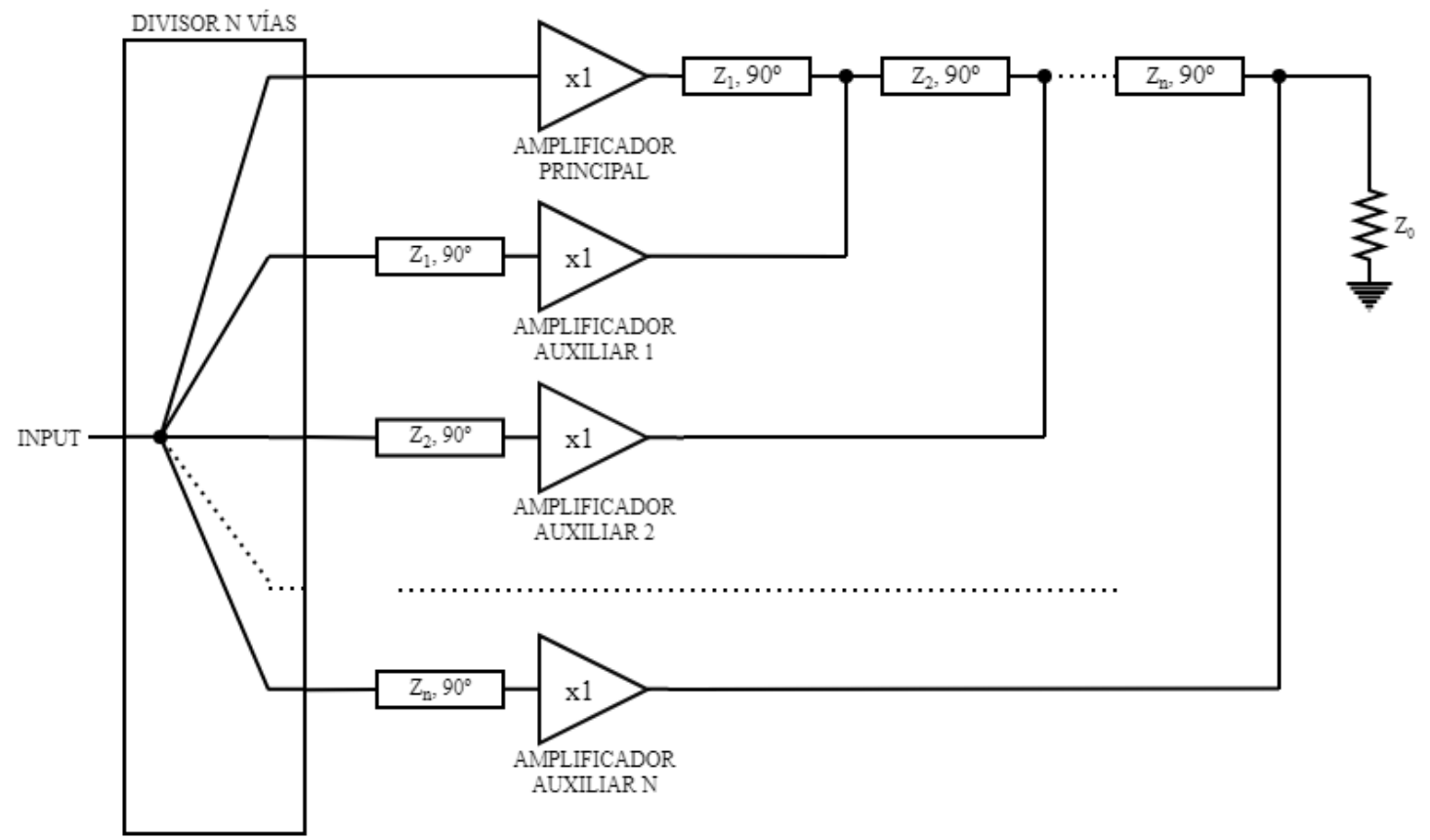

Figura 2.38. Diagrama de bloques de un sistema Doherty multietapa. 
El esquema de un amplificador Doherty multietapa general consta de un elemento principal y $\mathrm{N}$ elementos auxiliares. El elemento principal y el primer elemento auxiliar se diseñan para jugar los roles de amplificación principal y auxiliar como una arquitectura Doherty convencional. Además, cuando ambos elementos se saturan, el segundo elemento auxiliar entrará en funcionamiento para proporcionar otro pico de rendimiento. El resto de elementos auxiliares trabajan de la misma manera como la iteración inicial para proporcionar otros picos de rendimiento (Figura 2.39). Las redes de combinación de un amplificador Doherty multietapa es uno de los elementos de diseño más importantes. A pesar de ser una técnica muy estudiada, aún se encuentran dificultades prácticas para mantener el nivel de excitación adecuado para cada una de las etapas, lo que provoca no linealidades y efectos memoria importantes.

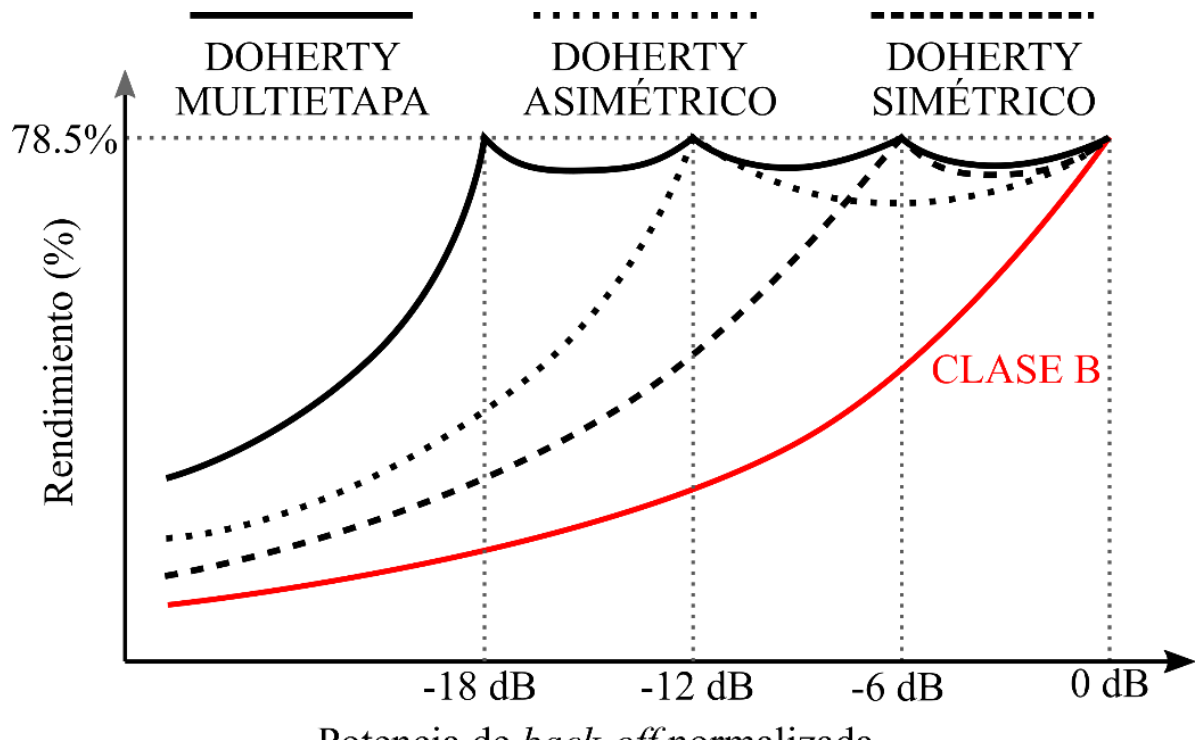

Potencia de back-off normalizada

Figura 2.39. Comportamiento teórico del rendimiento de un sistema Doherty multietapa.

\subsubsection{LINC y out-phasing}

La técnica de amplificación lineal usando componentes no lineales (LINC) fue propuesta por Cox en 1974 [75] como una arquitectura de transmisor con mejoras en rendimiento y linealidad. Como se puede ver en la Figura 2.40, la configuración del transmisor LINC utiliza dos caminos desfasados para amplificar la señal de envolvente constante mediante un SMPA con el fin de conseguir alto rendimiento, mientras la 
combinación de los dos caminos cancela las distorsiones y restablece una señal de salida lineal.

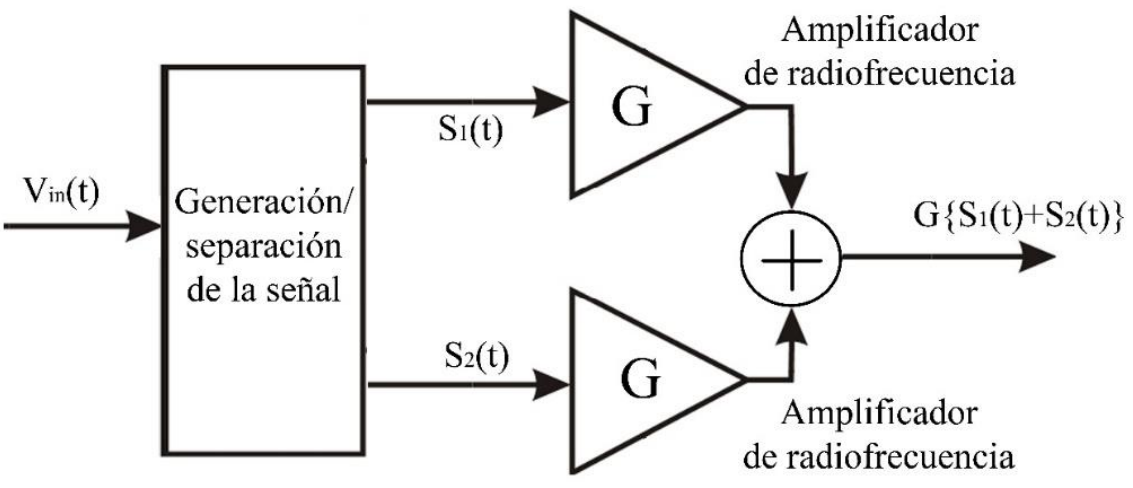

Figura 2.40. Diagrama de bloques de un transmisor LINC.

En un sistema transmisor LINC tradicional, hay un módulo separador de las componentes de señal para generar las señales en banda base con envolvente constante pero desfasadas usando un bloque de procesado digital de la señal (DSP).

Después del procesado, las señales complejas codificadas pasan a través de un convertidor digital analógico y se desplazan a frecuencia intermedia (IF). Posteriormente, se utiliza un modulador de frecuencia para subir las señales de IF a RF. Entonces cada una de estas dos señales con envolvente constante excita a su propio amplificador de potencia no lineal. Después de la amplificación individual, las señales se recombinan. La señal de salida resultante después de la suma es una versión amplificada de la señal de entrada sin ninguna distorsión añadida en un caso ideal. En otras palabras, el combinador LINC restablece la señal de RF en un amplificador modulado con las dos entradas de envolvente constante, en las que la distorsión de cada SMPA puede ser cancelada debido al desfase. El diseño del combinador es una de las partes más críticas en un transmisor LINC para mantener alta linealidad y alto rendimiento. La generación de las dos señales y la potencia combinada después de los amplificadores es también clave en este tipo de transmisores.

El rendimiento teórico del transmisor LINC es del $100 \%$ debido a que los amplificadores usados son amplificadores no lineales de alto rendimiento. Desde el punto de vista de la linealidad, el funcionamiento lineal está dominado por la ganancia de RF y la adaptación de fase entre las dos señales amplificadas. 
Aunque esta tecnología ha demostrado buenos rendimientos con una aceptable linealidad para aplicaciones en terminales móviles, es todavía un desafío aplicarla al diseño de alta potencia tales como estaciones base principalmente debido a la desadaptación entre los dos caminos, las pérdidas del combinador y la desadaptación del amplificador de potencia de RF.

\subsubsection{Chireix}

Esta técnica fue propuesta por H. Chireix en 1935 con el fin de satisfacer la necesidad de construir transmisores de radiodifusión que suministraran potencias por encima de las decenas de kilovatios. Fue una técnica utilizada en el diseño de estaciones base cuyos requisitos de consumo de potencia fueran mínimos y su rendimiento lo más alto posible. La fuente de alimentación y válvulas de alta potencia suponían la mayor parte de los costes de las estaciones [76].

El método Chireix se basa en una arquitectura de transmisores que genera una señal modulada mediante un alto nivel de amplitud. Se supone que la información está disponible en banda base (analógica o digitalmente). Una señal modulada en amplitud se genera a partir de un par de fuentes de onda continua, que tienen una compensación de fase variable entre sí. Este método se usa frecuentemente en sistemas AC y, por lo tanto, no es exclusivo del método Chireix. El componente adicional incluido por Chireix fue la capacidad de reducir el consumo de potencia por medio de los amplificadores de alto rendimiento, cuando se ajustan las fases entre las dos salidas para anularse entre sí. Este proceso se basa en el efecto llamado load-pulling de un dispositivo sobre el otro [39].

Una configuración básica se presenta en la Figura 2.41. Ambos elementos activos trabajan en modo común y tienen una carga conectada en modo diferencial. Presenta un desfasador variable en ambas entradas. Cada desfasador puede variarse entre 0 y $\pi / 2$ radianes. De esta manera, si se ajusta el tiempo transcurrido entre los dos caminos a $\pi$ radianes, se alcanzará el valor máximo de amplificación. En estas condiciones, la potencia de los elementos activos es totalmente absorbida por la carga de RF, mientras que el rendimiento global será el mismo en los dos amplificadores. Si el retardo fuera de 0 radianes, la salida de uno de los dispositivos anula la del otro 
(teóricamente), y no habrá potencia en la carga. En realidad, la cancelación no es perfecta. Por ello, se requieren algunas consideraciones para determinar la exactitud del control de fase con el fin de obtener un rango dinámico específico. La carga que es vista por los dos dispositivos está relacionada con el retardo de caminos.

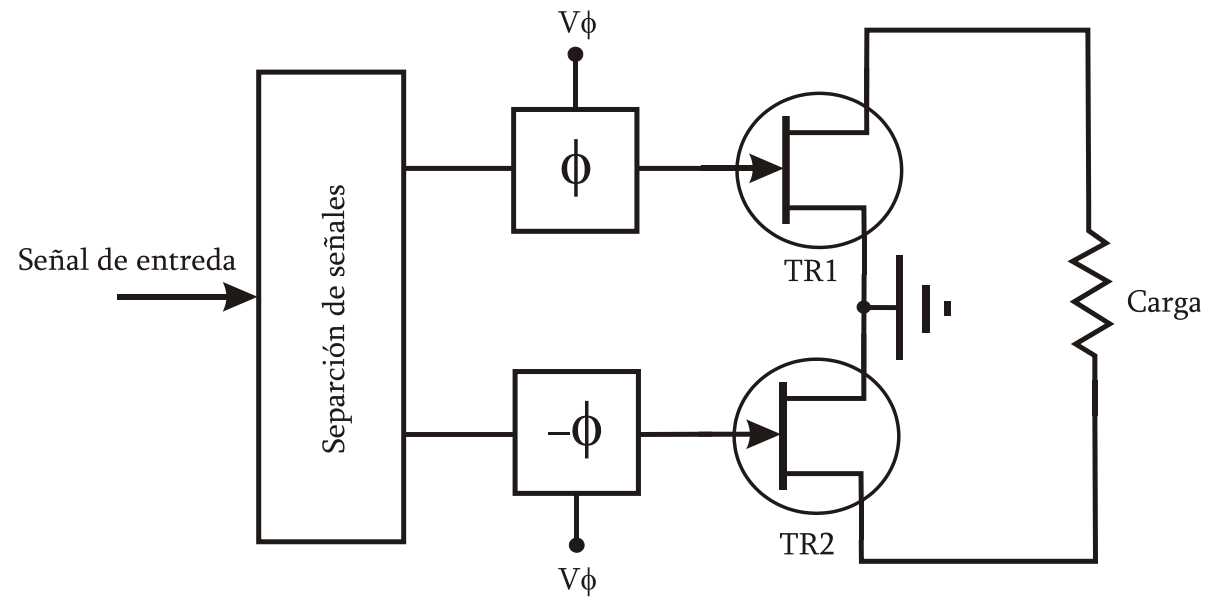

Figura 2.41. Diagrama de bloques de un transmisor Chireix.

El offset que existe entre las dos entradas aparecerá en la amplitud de salida. Se supone que ambos amplificadores trabajan en saturación, lo que genera una tensión sinusoidal de RF con una amplitud determinada $V$, que se relaciona con la polarización del amplificador. Por lo tanto, la tensión en la carga es:

$$
v_{o}=V \cos (\theta-\phi)-V \cos (\theta+\phi)=2 V \sin (\theta) \sin (\phi)
$$

De la Ecuación 2.4 se deduce que si es posible hacer un desfasador cuya respuesta en fase sea una función sinusoidal inversa a la tensión de control $\left(V_{\varphi}\right)$, como se muestra en la Ecuación 2.5, entonces, cada variación lineal de $V_{\varphi}$ producirá una variación lineal a la salida del amplificador. Hace algunos años, el diseño de la Ecuación 2.5 era un reto para implementarla analógicamente. Sin embargo, hoy en día es muy sencillo implementar cualquier función digitalmente. En este caso, una LUT en una arquitectura digital sería lo suficientemente buena para lograr una alta precisión.

$$
\phi=k \sin ^{-1}\left(V_{\phi}\right)
$$

A pesar de que las arquitecturas digitales han mejorado el diseño de la función $\phi$, no es fácil combinar los dos amplificadores. Se debe tener un especial cuidado a la hora del diseño e implementación. 


\subsubsection{Feedforward}

Esta teoría fue propuesta por H. S. Black en 1928 [77]. Sin embargo, no fue seriamente considerada hasta el final de los años sesenta, cuando se diseñaban amplificadores de alta potencia con una alta linealidad para señales de banda ancha. Algunas de las aplicaciones en las que se ha utilizado esta técnica son en comunicaciones militares de HF [78], en amplificadores de televisión por cable [79] y en amplificadores para satélites y sistemas celulares en aplicaciones móviles [80].

El principal efecto del lazo es suministrar la energía necesaria para incrementar gradualmente las características de compresión del amplificador de potencia. Esta potencia se suma a la salida del amplificador principal. La Figura 2.42 muestra el efecto de la mejora del lazo.

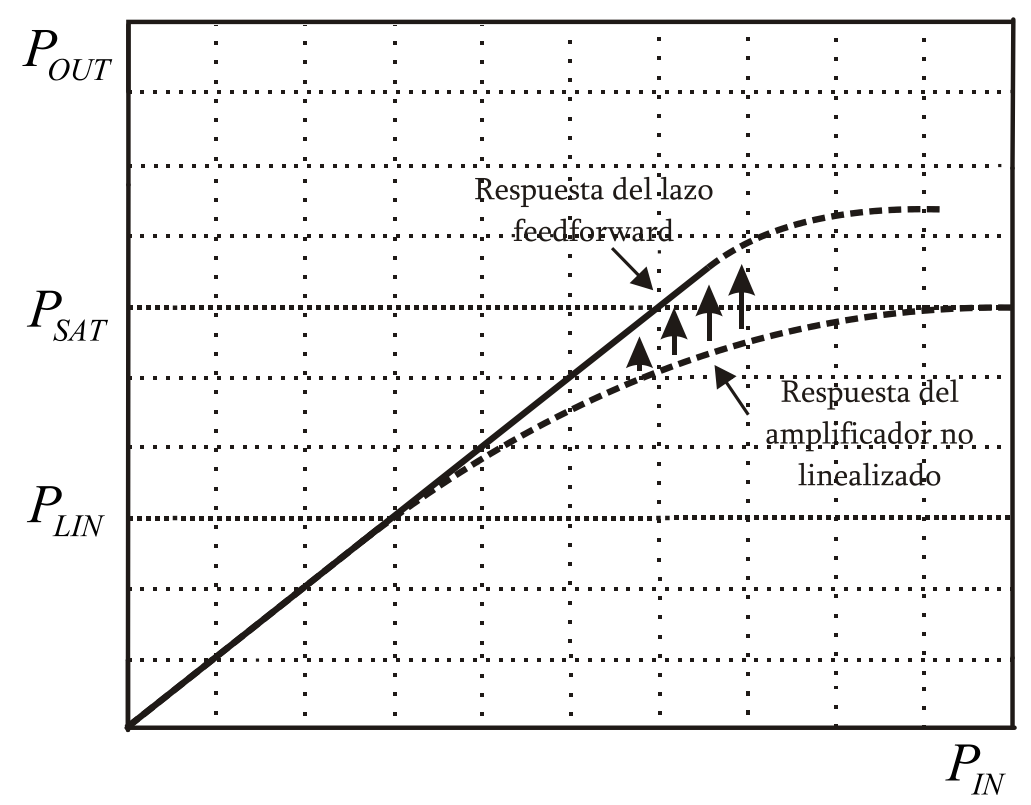

Figura 2.42. Efecto de la mejora del lazo feedforward.

La señal de corrección, que se suma a la generada por el amplificador, tiene una característica que anula cualquier distorsión AM-PM del amplificador principal.

Una configuración básica del lazo feedforward se presenta en la Fig. 2.43. Se compone de dos amplificadores (el principal y el de error), acopladores direccionales, líneas de transmisión y redes de control del lazo [81]. Los acopladores direccionales se utilizan para dividir o combinar la potencia. Las líneas de transmisión aseguran que el sistema pueda funcionar en un amplio ancho de banda. Además, las líneas de 
transmisión mantienen ambos caminos alineados en el tiempo. Los lazos de la red de control ajustan la amplitud y la fase a fin de mantener la señal de entrada y la de error en los niveles de corrección del lazo.

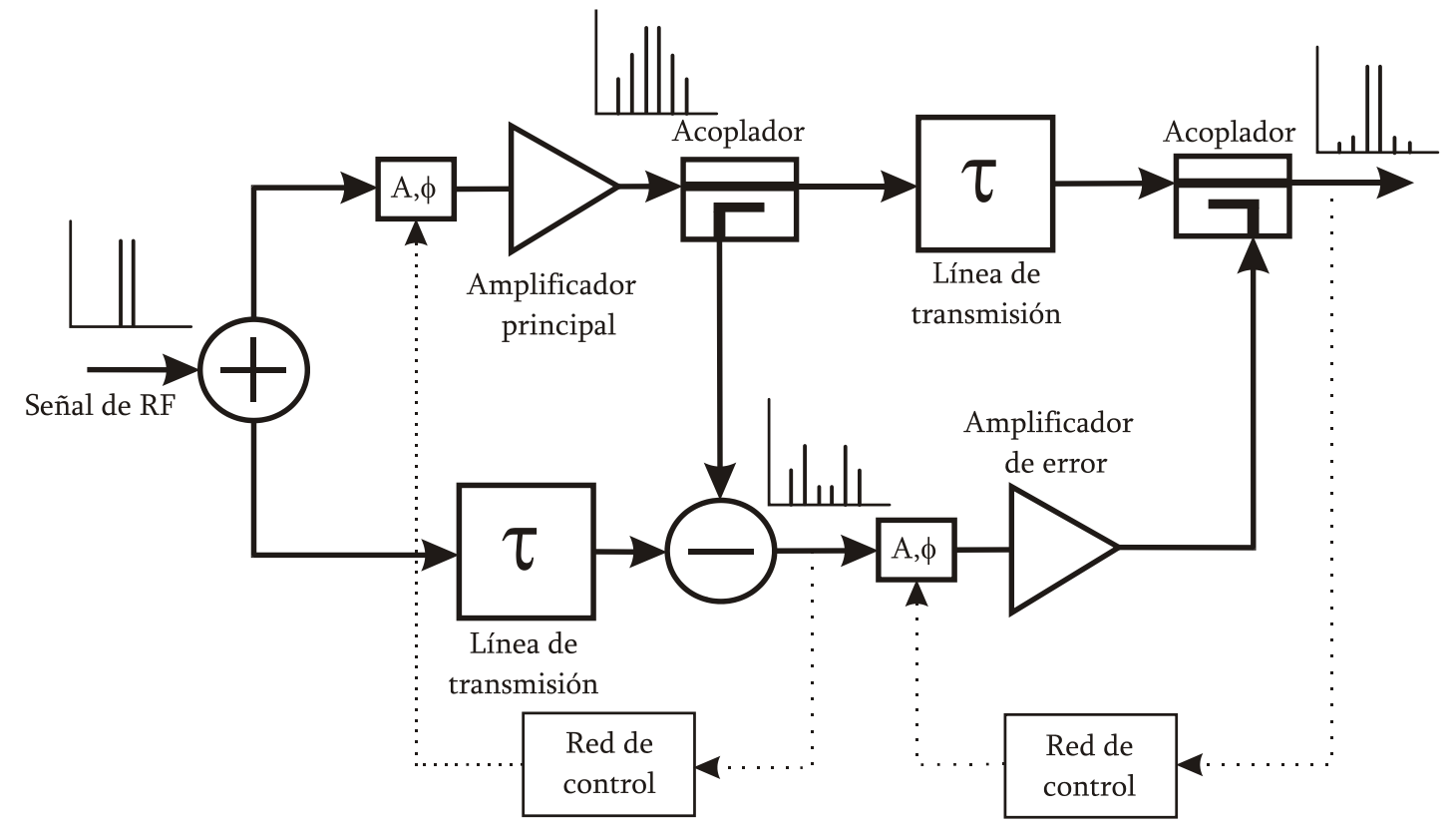

Figura 2.43 Diagrama de bloques de un transmisor basado en el lazo feedforward.

La tarea del amplificador de error es llevar a cabo el factor de escala, sin embargo, la ganancia del amplificador principal debe ser mayor que $1 / \alpha$ para compensar el factor de acoplamiento del acoplador de salida. Este último acoplador se utiliza para mezclar ambas señales, la salida del amplificador principal y la salida del amplificador de error.

El proceso de linealización se empeora debido a dos factores: en primer lugar, el amplificador de error no es totalmente lineal; y, en segundo lugar, es necesario mantener un control muy preciso de las amplitudes, retardos y ajustes de fase de los diferentes puntos del lazo.

De hecho, el amplificador de error generará productos de intermodulación que aparecerán a la salida del lazo. Por lo tanto, no pueden ser corregidos. No es un proceso iterativo, es decir, esta señal pasa una sola vez a través del lazo. De esta manera, no hay oportunidad de corregir el error en la salida del sistema. 


\subsubsection{Feedback}

La primera referencia de un sistema con lazo feedback o de realimentación empleada para la linealización de amplificadores de potencia pertenece a H.S. Black [77]. Las técnicas de realimentación han sido aplicadas a casi todos los sistemas de corrección desde muchos años atrás. Las técnicas de predistorsión y feedforward son complejas y caras, por lo que no son apropiadas para equipos móviles. La técnica feedback es más barata y más fácil de desarrollar. En la Figura 2.44 se representa un diagrama de bloques del lazo de realimentación.

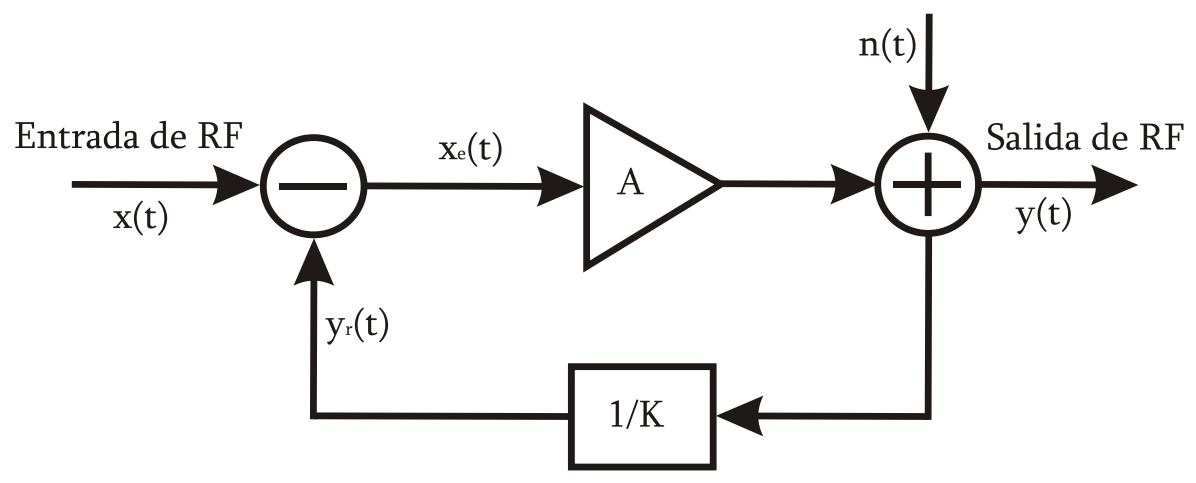

Figura 2.44. Esquema de un lazo de realimentación.

Existen diferentes técnicas de feedback:

- Lazo de realimentación de envolvente, donde la señal de error es una señal predistorsionada de la entrada. La amplificación de la señal de error genera la propia señal linealizada a la salida del transistor. El sistema puede incluir un modulador antes del amplificador, cuyo objetivo no es modular la señal, sino corregir la distorsión.

- Lazo de realimentación polar, basado en la combinación de un lazo de realimentación de envolvente y la técnica de Eliminación y Restauración de Envolvente.

- Lazo de realimentación cartesiano, similar a la realimentación polar pero la información en banda base se procesa en formato cartesiano. 


\subsubsection{Predistorsión}

El concepto de predistorsión en el campo de la linealización de amplificadores de potencia es muy simple. Básicamente, el objetivo es crear una función de transferencia complementaria a la distorsión generada en el amplificador. Después, esta se coloca antes del amplificador para compensar la función de transferencia distorsionada.

Las técnicas de predistorsión son en lazo abierto, por lo que, los niveles de linealidad alcanzados sólo se aproximan a los obtenidos mediante técnicas de lazo cerrado en un período de tiempo limitado y en un rango dinámico específico. Esta técnica suele complementarse con lazos feedforward o feedback. Por ejemplo, el diseño de un predistorsionador para un amplificador de potencia con un lazo feedforward puede reducir sustancialmente los requisitos de potencia del amplificador de error. Sin embargo, se logra un incremento significativo en el rendimiento global.

Una clasificación frecuente de las técnicas de predistorsión se basa en el rango de la frecuencia de funcionamiento:

- Predistorsión en RF: se basa en la predistorsión de la portadora.

- Predistorsión en IF: es posible que el bloque predistorsionador trabaje mejor a un rango de frecuencia específico. Por ello, la señal puede ser trasladada a IF (frecuencia intermedia) donde el funcionamiento del predistorsionador es más adecuado.

- Predistorsión en banda base: las arquitecturas digitales actuales han ayudado en el uso de este tipo de predistorsión. La función de predistorsión del amplificador de potencia se puede almacenar en la memoria o LUT digitalmente. De esta manera, la señal en banda base se predistorsiona antes de que sea trasladada mediante un up-converter a la frecuencia adecuada.

Un esquema básico de predistorsión se muestra en la Figura 2.45. La señal de entrada pasa a través del sistema cuya función de transferencia es la complementaria a la del amplificador de potencia. Por lo tanto, cuando la señal predistorsionada se aplica al amplificador de potencia, la distorsión es compensada e idealmente se obtiene una señal completamente lineal a la salida. 

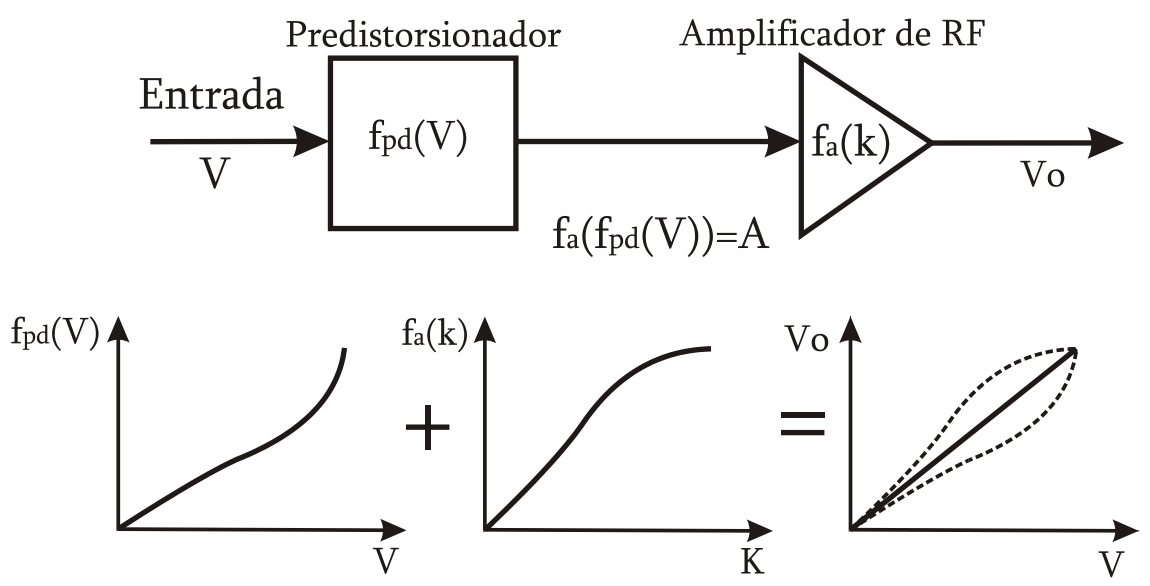

Figura 2.45. Concepto de predistorsión.

El punto clave de esta técnica es descubrir el dispositivo o mecanismo adecuado capaz de generar una función de predistorsión que sea complementaria a la del amplificador de potencia. Sin embargo, puede ser realmente difícil, ya que hay algunos casos en los que la predistorsión de los productos de intermodulación de tercer orden conlleva un incremento de los productos de orden superior.

Factores externos del sistema pueden afectar al comportamiento global de los transmisores, como pueden ser los cambios de temperatura, la ganancia y desequilibrios de fase entre los caminos del sistema, el envejecimiento y otros factores que no se hayan tenido en cuenta en el diseño del predistorsionador.

Existen otros métodos basados en algoritmos de adaptación, que se implementan en un DSP o una FPGA. Miden el error entre la entrada del amplificador y la salida, y con ello determinan la corrección adecuada a la curva de predistorsión.

Las principales ventajas de esta técnica son:

- Sencilla implementación.

- Es incondicionalmente estable. La técnica se realiza en lazo abierto, por lo que no hay problemas de estabilidad como en los de lazo cerrado.

- Se consiguen grandes anchos de banda de linealización.

Las principales desventajas son:

- La mejora en la linealidad no es muy alta.

- Los predistorsionadores de alto orden son difíciles de implementar. 


\subsubsection{EER}

La técnica EER fue inventada por L.R. Kahn en 1952, por ello también es conocida como técnica de Kahn, [82]. Esta técnica ha recibido mucha atención recientemente, especialmente en el campo de las telecomunicaciones. El diagrama de bloques de esta técnica se presenta en la Figura 2.46.

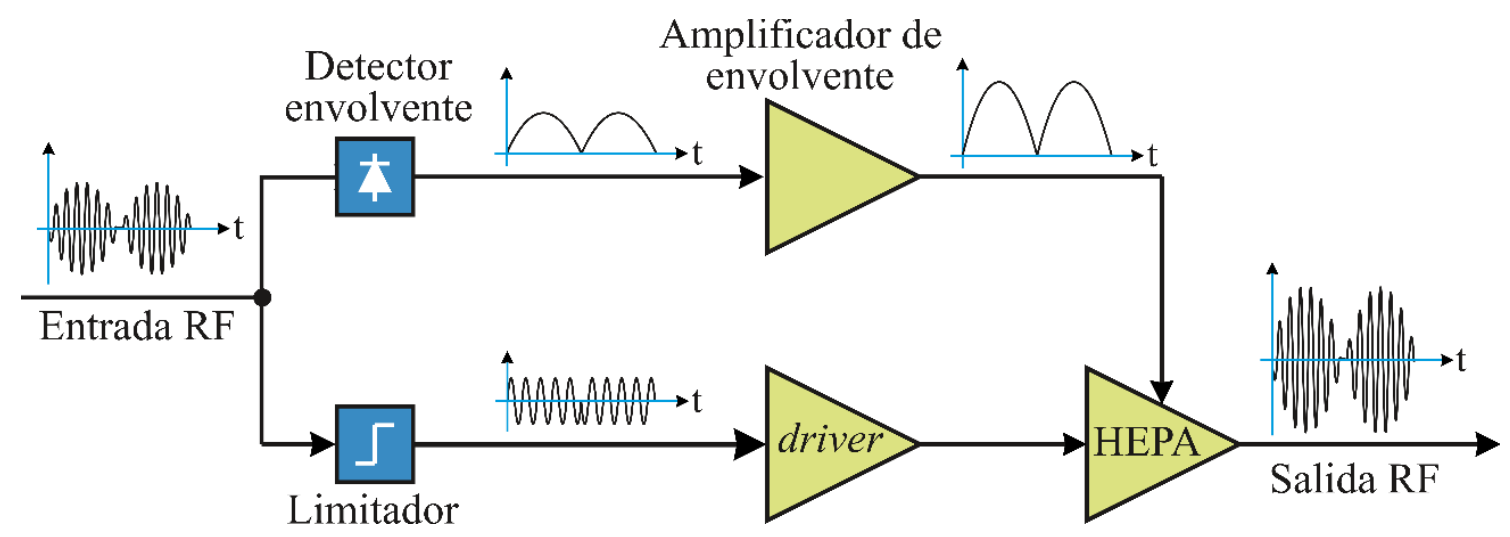

Figura 2.46. Diagrama de bloques de un transmisor EER.

Sin embargo, en aplicaciones de banda ancha aumenta la complejidad de diseño debido a los altos requisitos del modulador de envolvente. En banda estrecha, la técnica EER puede superar en rendimiento a las técnicas Doherty y LINC, siempre y cuando el amplificador de envolvente presente un alto rendimiento.

Esta técnica se basa en la separación de las señales de fase y de amplitud de la señal de entrada. De esta manera, pueden ser procesadas de forma independiente. La portadora de RF modulada en fase es amplificada por un amplificador de potencia de RF de alto rendimiento no lineal. La envolvente es amplificada por un modulador de amplitud de alto nivel, por ejemplo un amplificador clase $S$ o un convertidor DC-DC. La salida del modulador de amplitud será la fuente de alimentación del amplificador de potencia de RF. El transistor final mantiene un rendimiento medio muy alto, teóricamente del 100\%. [83].

Importantes avances en el campo de la lógica programable, como es el caso de los DSP y las FPGA, y en las técnicas de procesamiento de señal han hecho posible convertir las configuraciones básicas en otras más robustas y con mayor rendimiento. Algunos de los componentes que fueron necesarios en el pasado (detectores de envolvente, limitadores...) pueden ser sustituidos por elementos digitales, capaces de 
conseguir mejores resultados. Además, los algoritmos de procesado de la señal son más fáciles de implementar y de aplicar. Por ejemplo, es posible implementar algoritmos de adaptación que corrigen algunos de los errores presentados en los caminos de envolvente y de fase.

Hay varias fuentes de distorsión en un sistema EER: el ancho de banda del modulador de envolvente y el ajuste del retardo entre caminos son los factores que generan la mayor parte de la distorsión y se analizan profundamente en [84]. La linealidad en transmisores EER no depende del transistor de potencia de RF, sino de la exactitud al reproducir la información de fase y de envolvente de la señal modulada, así como de la sincronización entre ambas señales [84]. Otra fuente de distorsión es la variación de fase o de amplitud a la salida del sistema debidas principalmente a la variación de la capacidad el transistor en función de la tensión de alimentación.

El amplificador de potencia puede alcanzar una alta linealidad y, por lo tanto, niveles bajos de IMD, aunque funcione a máxima potencia y varios $\mathrm{dB}$ por encima del punto de compresión. Otros amplificadores tienen que trabajar a menor potencia y bajos rendimientos para conseguir el mismo nivel IMD. Además, si fuera necesario, la linealidad de un sistema EER puede ser mejorada mediante técnicas tales como realimentación de envolvente, realimentación cartesiana o esquemas avanzados de predistorsión.

En el método de Kahn original, la amplitud de la señal modulada en fase, que alimenta la entrada del driver, se mantiene lo suficientemente alta como para asegurar que el amplificador de potencia esté saturado con un nivel óptimo y, por lo tanto, trabaje con alto rendimiento al presentar un nivel de pico de envolvente fijo. Sin embargo, los bajos niveles de envolvente requerirían una señal de entrada más baja para saturar al amplificador y lograr un alto rendimiento. De esta manera, si la amplitud de entrada al driver se regula en función de la envolvente, el rendimiento se puede optimizar para cada valor de envolvente. Este método es conocido como modulación de driver. Incluye una topología EER y un control dinámico de potencia del driver.

La técnica de Kahn también ha tenido variaciones a lo largo de los años con el fin de mejorar las prestaciones del transmisor: 
- Método clásico de Kahn: mantiene la señal de entrada, que ataca al amplificador de potencia, con una amplitud constante, mientras que la envolvente se suministra por el drenador.

- Método de Kahn con Full-Drive Modulation (FDM): ajusta el nivel de la señal modulada en fase de amplitud constante, la cual alimenta al amplificador de potencia, en función del nivel de envolvente a la entrada del amplificador de envolvente.

- Método de Kahn con Parcial-Drive Modulation (PDM): es muy similar a la FDM, pero se estable un nivel de amplitud de entrada mínima, lo que aumenta el rendimiento a niveles de envolventes bajos.

Parece que sería conveniente realizar el control dinámico de potencia para mantener un alto rendimiento en el rango completo de amplitud de entrada. El método PDM, cuyo esquema se muestra en la Figura 2.47, logra mejores resultados respecto al rendimiento, pero aumenta la conversión AM-PM. Sin embargo, el método FDM tiene mejores prestaciones: obtiene mejor nivel de linealidad para el nivel de salida de pico y también mejora el rendimiento medio en más de 4.4 veces el obtenido por un amplificador no lineal con polarización fija [85].

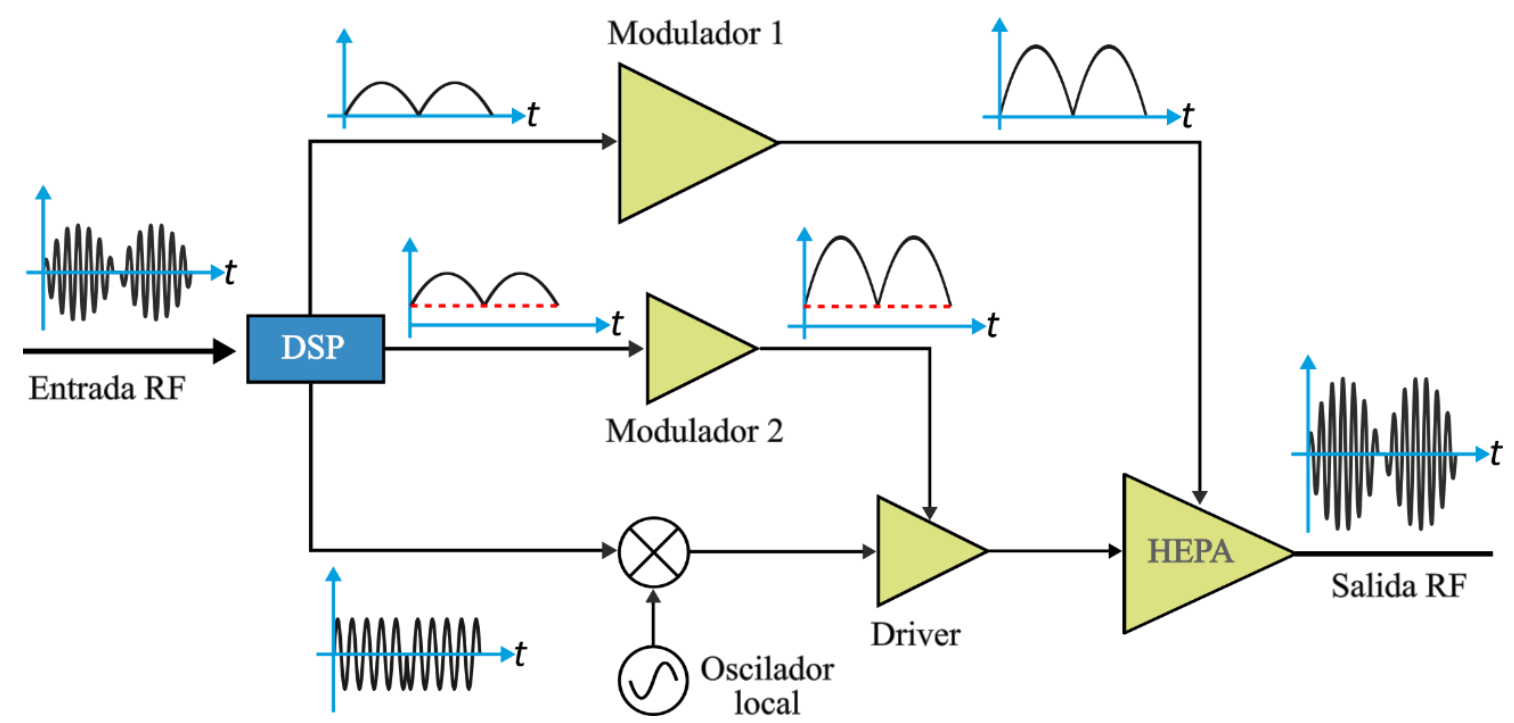

Figura 2.47. Arquitectura de un transmisor EER con modulación de driver parcial. 
Las principales ventajas de la técnica de EER son [84]:

- Su rendimiento es bastante lineal para una amplia gama de modulaciones cuya variación de envolvente es lenta, como $\pi / 4$-DQPSK. Cuando se utiliza en sistemas basados en modulaciones con variaciones de envolvente rápidas, la linealidad empeora.

- El sistema logra altos rendimientos. Básicamente, el rendimiento global del sistema es la multiplicación del rendimiento del amplificador de RF y del amplificador de envolvente. Típicamente, topologías clase D o E son utilizadas en el camino de fase y convertidores DC-DC de alto rendimiento en el camino de envolvente.

Los principales inconvenientes de la técnica de EER son:

- Las señales con alta PAPR son más difíciles de tratar, y reducen el rendimiento del amplificador de envolvente.

- La técnica EER original es de circuito abierto. Esto quiere decir que la entrada no tiene información de realimentación de la salida. Por ello, el sistema no puede corregir los efectos indeseables del hardware. Sin embargo, es posible añadir un lazo de realimentación a la versión original de sistema EER. Como resultado, los sistemas de EER pueden modificar sus señales de acuerdo a la salida con el fin de mejorar su funcionamiento.

\subsubsection{ET}

Existen dos variantes principales actuales de transmisores con alimentación variable. La primera es el transmisor EER que ejemplifica el uso de una SMPA con amplitud constante con una tensión de alimentación controlada dinámicamente. La otra es envelope tracking (ET) que utiliza amplificadores lineales cuya debilidad de bajo rendimiento durante los periodos de baja potencia se puede mitigar mediante el ajuste de la tensión de alimentación adecuadamente. Esta técnica fue propuesta en la década de 1930 [86] pero no fue implementada hasta hace un par de décadas. 
Normalmente, un transmisor ET se compone de un amplificador lineal de RF y un modulador de envolvente para proporcionar una tensión de alimentación modulada como alimentación dinámica del amplificador de potencia. La diferencia clave con respecto a EER es que el amplificador de RF trabaja con una señal modulada en fase y de envolvente no constante, o lo que es lo mismo, trabaja con una copia de la señal de entrada.

La idea básica de la técnica ET se presenta en la Figura 2.48. Consiste en conducir al amplificador de RF en su región de alto rendimiento independientemente de la magnitud de la señal de entrada. Esto se realiza mediante la detección de la envolvente de la señal de entrada y se emplea para modular la tensión de alimentación del amplificador lineal. En este sentido, es similar a la técnica EER pero no se emplea un limitador en el camino de excitación del amplificador de RF. Una de las ventajas de ET es que la alimentación de envolvente no tiene que ser una réplica tan exacta como en la técnica EER ya que tanto la señal de amplitud como de fase se amplifican en el camino de RF también.

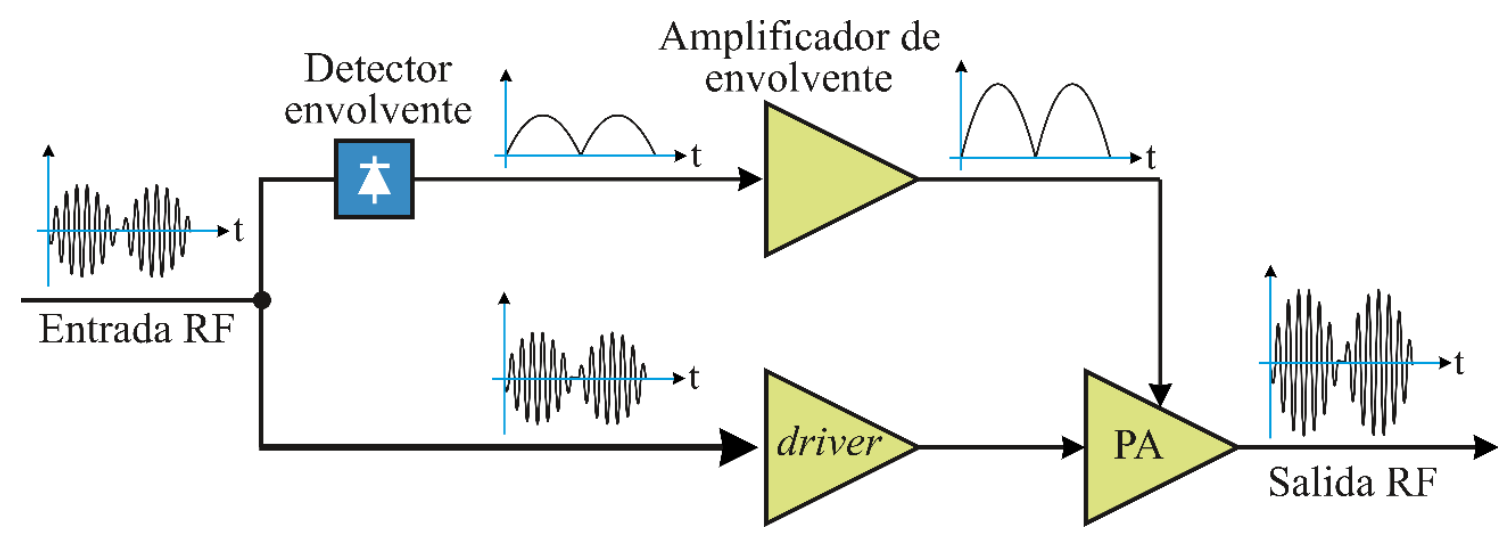

Figura 2.48 Diagrama de bloques de un transmisor ET.

En general, el rendimiento que se puede conseguir usando la técnica ET depende en gran medida del rendimiento del modulador de envolvente. El empleo de fuentes de alimentación conmutadas (SMPS) puede ser una solución para conseguir alto rendimiento, aunque podría tener algunas limitaciones en cuanto ancho de banda.

Una ventaja de los transmisores ET es que son relativamente sencillos de desplazar a otras frecuencias portadoras, ya que solo es necesario reemplazar el amplificador de RF manteniendo el modulador de envolvente. Otras ventajas frente a EER son: 
- ET necesita menor sensibilidad a las desadaptaciones temporales entre caminos que en el caso de EER.

- ET necesita menores requisitos de ancho de banda para el modulador de envolvente en el sentido de que el sistema global consigue la información de envolvente directamente a través del amplificador de RF y no del modulador de envolvente.

- ET relaja los requisitos de ancho de banda en el camino de RF. Esto le hace más adecuado para aplicaciones de banda ancha.

- ET tiene mayor ganancia a menores potencias de entrada ya que el modulador de envolvente mantiene al amplificador de potencia a trabajar en su zona lineal.

- ET tiene menos impacto en los cruces por cero de la señal de RF. En EER el llamado efecto feed-through es uno de sus prácticas limitaciones. En ET, este efecto se ve reducido debido a las formas de onda a bajos niveles de potencia.

Basado en estas razones, ET se va convirtiendo cada vez más atractivo para transmisores de alto rendimiento, de banda ancha y lineales para comunicaciones modernas tales como los sistemas $4 \mathrm{G}$ y posteriores:

- La técnica ET necesita usar un modulador de envolvente de alto rendimiento para incrementar el rendimiento total. Por ello, el problema de rendimiento se lleva a cabo en un nivel superior: el modulador de envolvente.

- El rendimiento de ET es menor a bajos niveles de entrada que a altos niveles. Esto implica que al trabajar con señales de alta PAPR, el rendimiento del sistema se vea drásticamente reducido.

- La distorsión desarrollada en el modulador de envolvente puede ser introducida al amplificador de RF y, por ello, requiere de métodos de linealización extra para corregirlos. Las necesidades de linealidad fuerzan el empleo de nuevos y más complejos algoritmos de 
predistorsión y un mayor ancho de banda de la señal requiere un grado mayor de precisión en la alineación temporal.

Finalmente, aunque linealidad y rendimiento son propiedades mutuamente exclusivas en los amplificadores de RF alimentados con tensión fija, los transmisores ET pueden amplificar linealmente las señales moduladas de fase y de amplitud con mucho mayor rendimiento. Tanto los amplificadores lineales como los SMPAs de alto rendimiento pueden ser beneficiosos en un sistema ET dado que la linealidad puede ser corregida con esquemas modernos de predistorsión.

Para eliminar la distorsión espectral al mínimo posible, varias consideraciones se tienen que tener en cuenta: el ancho de banda del modulador de envolvente, la desadaptación de tiempo, las distorsiones AM-AM y AM-PM y, finalmente, el desarrollo apropiado de la función de transferencia entre la tensión modulada media de salida y la envolvente de entrada.

La consecuencia de una desadaptación temporal aumenta considerablemente la distorsión por intermodulación espectral (IMD) y la magnitud de error vectorial (EVM), produciendo un degradamiento de la relación de potencia de canal adyacente (ACPR) y de la calidad de la modulación digital, respectivamente.

El ancho de banda de la señal de RF en un sistema ET es idéntico al ancho de banda de la señal en banda base, lo que lo hace mucho menos estricto en comparación con un sistema EER. Además, al preservar información de amplitud en la rama del amplificador de RF, permite unos requisitos de ancho de banda menores y también una menor precisión en el alineamiento temporal.

Muchos han sido los trabajos publicados enfocados en sistemas ET para amplificadores de alta potencia en las infraestructuras de las estaciones base de las comunicaciones móviles [87] [88]. Sin embargo, la empresa Nujira fue la primera en demostrar los beneficios del empleo de esta técnica para amplificadores de potencia en teléfonos móviles. Los resultados publicados por Nujira demuestran la implementación de un sistema ET en chip con un ancho de banda de hasta $20 \mathrm{MHz}$ que duplica el ahorro de energía de los amplificadores de potencia trabajando en LTE comparado con otras soluciones ET de menor ancho de banda. El sistema puede ofrecer un rendimiento del $80 \%$ ofreciendo un valor de potencia media de $1.2 \mathrm{~W}$ y un EVM del $3 \%$. 


\subsubsection{Comparación de las técnicas de linealización}

La Tabla 2.2 muestra una comparación entre las técnicas de linealización más importantes presentadas en este capítulo.

Tabla 2.2 Comparación de las técnicas de linealización.

\begin{tabular}{|c|c|c|c|c|c|}
\hline $\begin{array}{c}\text { Técnica de } \\
\text { linealización }\end{array}$ & Complejidad & Rendimiento & $\begin{array}{c}\text { Ancho } \\
\text { de } \\
\text { banda }\end{array}$ & $\begin{array}{l}\text { Cancelación } \\
\text { de espurios }\end{array}$ & $\begin{array}{l}\text { Principales } \\
\text { causas de } \\
\text { distorsión }\end{array}$ \\
\hline $\begin{array}{l}\text { Feedback } \\
\text { cartesiano }\end{array}$ & Medio & Alto & Estrecho & Alta & $\begin{array}{c}\text { Ancho de } \\
\text { banda del lazo }\end{array}$ \\
\hline Feedforward & Alta & Medio & Amplio & Alta & $\begin{array}{c}\text { Componentes } \\
\text { pasivos }\end{array}$ \\
\hline $\begin{array}{c}\text { Predistorsión } \\
\text { de RF }\end{array}$ & Baja & Alto & Amplio & Baja & $\begin{array}{l}\text { Amplificador } \\
\text { de potencia }\end{array}$ \\
\hline $\begin{array}{l}\text { Predistorsión } \\
\text { digital }\end{array}$ & Alta & Medio & Medio & Media & $\begin{array}{l}\text { Amplificador } \\
\text { de potencia y } \\
\text { filtros }\end{array}$ \\
\hline ET & Media & Medio & Medio & Media & $\begin{array}{l}\text { Convertidor } \\
\text { de envolvente }\end{array}$ \\
\hline EER & Media & Alto & Medio & Baja & $\begin{array}{l}\text { Amplificador } \\
\text { de potencia }\end{array}$ \\
\hline
\end{tabular}




\section{Capítulo 3}

Diseño de amplificadores de alto rendimiento 


\title{
Capítulo 3
}

\section{Diseño de amplificadores de alto rendimiento}

\author{
Caracterización de los transistores, métodos de diseño de amplificadores de \\ potencia de alto rendimiento y prototipos
}

\section{CONTENIDO}

3.1 Amplificador de potencia clase $\mathrm{F}$ en banda $\mathrm{L}$

3.2 Amplificador de potencia clase E sub-óptimo en banda $\mathrm{L}$

3.3 Amplificador de potencia clase E sub-óptimo en banda $\mathrm{S}$

En el Capítulo 2 se ha mostrado la teoría básica de funcionamiento de los amplificadores de potencia y sus métodos de diseño. Esta teoría está basada en suposiciones e idealismos para permitir la manejabilidad matemática y ofrecer una comprensión intuitiva. Sin embargo, no tiene en cuenta muchas de las no linealidades de los transistores de alta potencia, especialmente cuando se trabaja en modos de alto rendimiento.

Los modelos no lineales son muy utilizados en los diseños de amplificadores de potencia, pero su funcionamiento en la región de compresión para el diseño en alto rendimiento no es completamente fiable. Día a día, los fabricantes gastan esfuerzos en caracterizar y modelar los dispositivos para ofrecer a los usuarios una herramienta potente a la hora de diseñar amplificadores.

No obstante, las nuevas tecnologías usadas en la fabricación de transistores a menudo carecen de modelos no lineales. En estos casos, es necesario realizar una caracterización del dispositivo.

En este Capítulo se presentan varios diseños de amplificadores de potencia de alto rendimiento en diferentes bandas de frecuencia y diferentes características de potencia. Muchas de las conclusiones obtenidas durante el diseño de uno de ellos son de aplicación para el resto de los diseños. 


\subsection{Amplificador de potencia clase $F$ en banda $L$}

El funcionamiento en alto rendimiento de un amplificador de potencia se puede conseguir mediante el control de la carga a los armónicos y a la frecuencia fundamental [89]. En este apartado se mostrará el diseño de un amplificador clase F empleando un método simple y de bajo coste para el control y la variación sistemática de las impedancias armónicas.

El transistor utilizado en este amplificador será el RF3931 de RF Micro Devices (RFMD) [90]. RFMD fue absorbida por Qorvo tras la fusión con Triquint Semiconductor en 2015. Se trata de un transistor no adaptado de alta potencia diseñado para aplicaciones de infraestructura inalámbrica y de uso general en banda ancha. Este transistor de alta movilidad de electrones (HEMT) y alta densidad de potencia basado en la tecnología de nitruro de galio $(\mathrm{GaN})$ permite diseñar amplificadores con alto rendimiento y ganancia plana en un amplio rango de frecuencias, desde DC hasta 3 GHz. El RF3931 presenta un encapsulado cerámico y hermético que le proporciona una excelente estabilidad térmica a través del uso de avanzadas tecnologías de disipación de calor y de energía.

Según la hoja de datos del fabricante, este transistor es capaz de ofrecer picos de potencia de hasta $50 \mathrm{~W}$ con un rendimiento máximo del $65 \%$ y una ganancia lineal de $20 \mathrm{~dB}$ alimentado a $48 \mathrm{~V}$ y polarizado con una corriente de $130 \mathrm{~mA}$ y trabajando entre 880 y $920 \mathrm{MHz}$. Trabajando a $2140 \mathrm{MHz}$, permite obtener picos de potencia hasta 40W con un rendimiento máximo del $62 \%$ y una ganancia lineal de $15 \mathrm{~dB}$ alimentado a $48 \mathrm{~V}$ y polarizado con una corriente de $130 \mathrm{~mA}$ en un ancho de banda de $60 \mathrm{MHz}$ [91]. La Figura 3.1 presenta los módulos de evaluación proporcionados por RFMD para el transistor RF3931. 

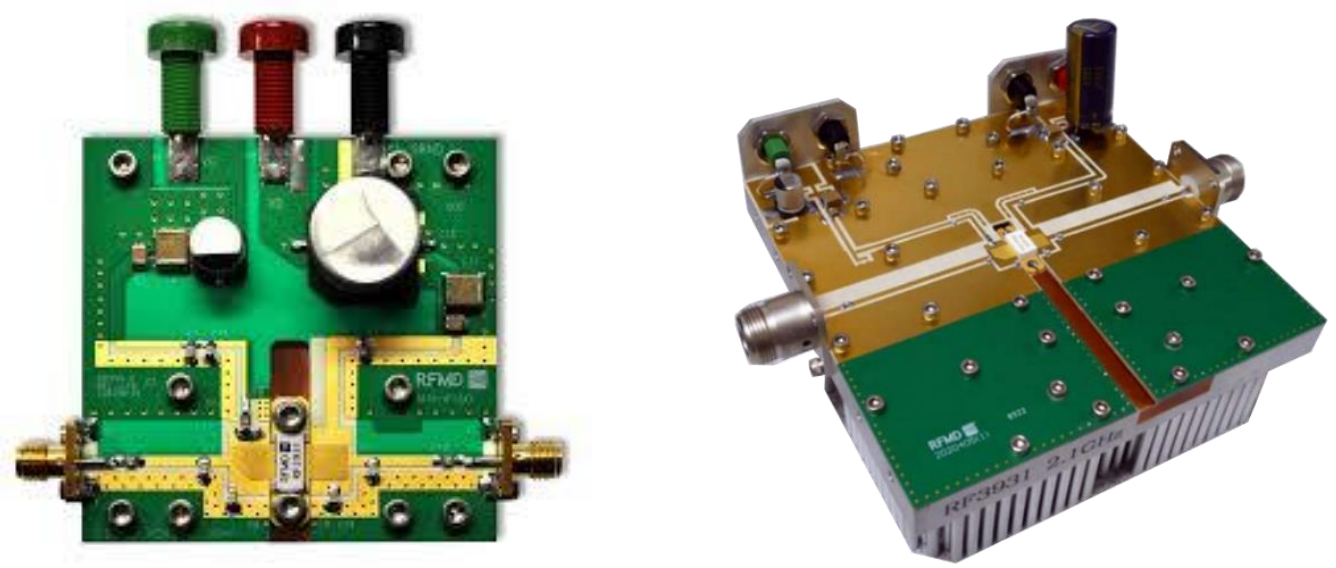

Fig. 3.2 Módulos de evaluación del transistor RF3931 proporcionados por RFMD cuya frecuencia central es $900 \mathrm{MHz}$ (izq) y es $2140 \mathrm{MHz}$ (drch).

Con el fin de facilitar el diseño de amplificadores de potencia con este dispositivo, en este caso, el fabricante ofrece tanto el modelo no lineal del transistor como el modelo de la die junto con los parámetros $\mathrm{S}$ del encapsulado para el software de simulación AWR Design Environment (Figura 3.2). A pesar de ello, otros parámetros necesarios para el diseño del amplificador no son ofrecidos por el fabricante por lo que habrá que hacer una caracterización del dispositivo de manera empírica.

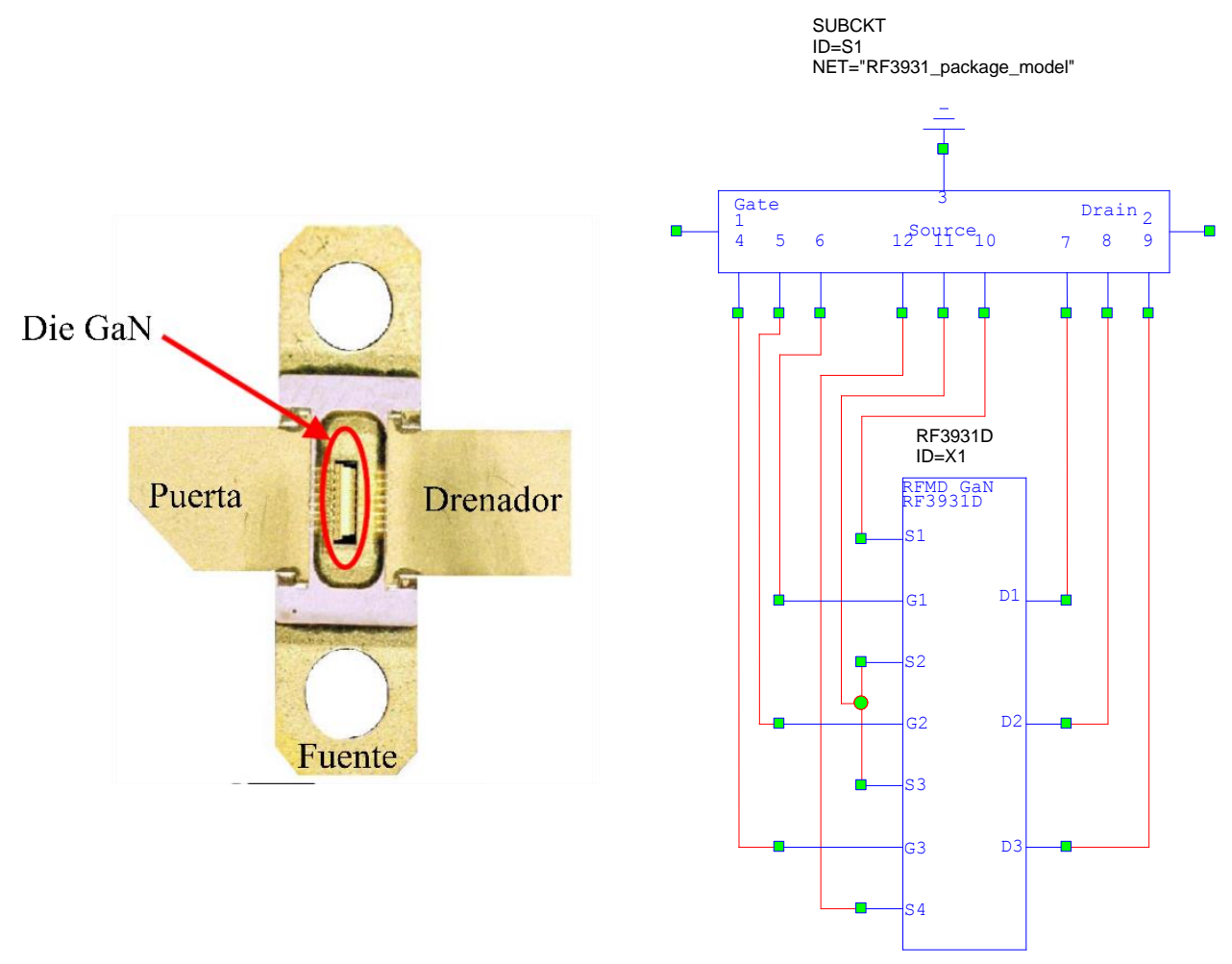

Fig. 3.2 Detalle de la die del transistor RF3931 (izq) y modelo para AWR de la die del dispositivo y de su encapsulado (drch). 


\subsubsection{Caracterización del transistor}

En el mercado se pueden encontrar diferentes tipos de dispositivos en función del tipo de aplicación para la que se utilicen. En aplicaciones de muy alta potencia, por encima de los cientos de vatios, se suelen emplear amplificadores ya adaptados internamente (MPT, Matched Power Transistors) y suelen ser empleados en amplificadores de onda continua, radares pulsados o amplificadores de banda ancha. Este tipo de transistores ofrecen un funcionamiento optimizado tanto en potencia como en rendimiento. Por otro lado, también están los transistores de banda ultra ancha, de baja potencia, pero de muy alta ganancia en un amplio rango de frecuencias. Suelen usarse en aplicaciones militares y normalmente se venden como circuitos integrados para integrar en los sistemas. Este tipo de dispositivos no permiten al diseñador plantear otro tipo de funcionamiento más que para el que fueron diseñados.

En el caso de esta tesis, con el fin de poder "jugar" con las impedancias armónicas del transistor y conseguir el funcionamiento en alta potencia y alto rendimiento deseado, se trabaja con transistores sin adaptación (UPT, Unmacthed Power Transistor). Estos transistores pueden alcanzar los cientos de vatios y permiten el diseño en banda ancha y con un alto rendimiento. Su uso es general, aunque también se pueden encontrar en aplicaciones militares, radares... Adicionalmente, debido a la dificultad de adaptación de los dispositivos actuales, ya que presentan unas impedancias muy bajas, los fabricantes están ofreciendo dispositivos pre-adaptados. Esta característica se puede dar tanto a la salida como a la entrada, pero tiene el inconveniente de que el diseñador no tiene tanto margen de maniobra para poder diseñar y configurar el amplificador de potencia.

Como ya se ha comentado, el RF3931 es un transistor de potencia no adaptado. Una de las características por las que fue elegido este transistor fue alta tensión de drenador de ruptura que presenta: hasta $150 \mathrm{~V}$. Este parámetro se debe tener muy en cuenta al trabajar con amplificadores conmutados, como es el caso de las clases F y E. En estas clases, la tensión de drenador puede llegar a ser varias veces la tensión de alimentación: para un amplificador clase F puede llegar a ser 2Vdd y en un amplificador clase E hasta 3.5Vdd. Además, en el caso particular del RF3931, se trabajará con una tensión de alimentación muy alta: $48 \mathrm{~V}$. 
Además, normalmente el fabricante también ofrece la impedancia de carga y de fuente óptimas a la frecuencia fundamental y para unas condiciones de polarización y tensión de alimentación concretas. En este caso, el fabricante ofrece los datos que se muestran en la Figura 3.3.

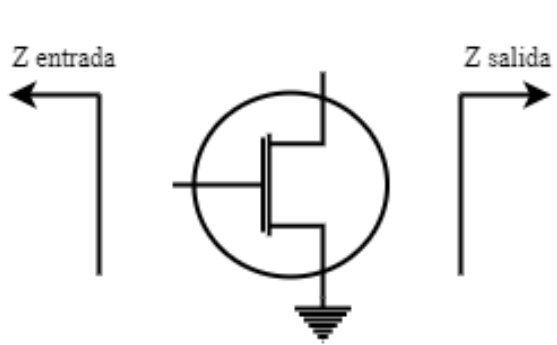

\begin{tabular}{|c|c|c|}
\hline Frecuencia $(\mathrm{MHz})$ & $\mathrm{Z}$ entrada $(\Omega)$ & $\mathrm{Z}$ salida $(\Omega)$ \\
\hline 450 & $5+10.5 \mathrm{j}$ & $20+8.2 \mathrm{j}$ \\
\hline 900 & $2.5+3.6 \mathrm{j}$ & $13.6+10.4 \mathrm{j}$ \\
\hline 1300 & $1.6+0.4 \mathrm{j}$ & $9.6+9.5 \mathrm{j}$ \\
\hline 1700 & $1.2-1.6 \mathrm{j}$ & $7.2+7.9 \mathrm{j}$ \\
\hline 2100 & $1.1-3.7 \mathrm{j}$ & $5.8+6.3 \mathrm{j}$ \\
\hline 2500 & $1.1-6.4 \mathrm{j}$ & $5.0+4.7 \mathrm{j}$ \\
\hline
\end{tabular}

Figura 3.3. Impedancias óptimas vistas por el dispositivo polarizado a $V_{D S}=48 \mathrm{~V}$ y $I_{D Q}=$ 130mA [90].

Con estos datos el diseñador solo debería diseñar la red de adaptación de entrada y de salida capaz de sintonizar dichas impedancias de fuente y de carga para reproducir los resultados mostrados en la hoja de datos. Sin embargo, en esta tesis se va a ser más ambicioso y se van a explorar los límites que se pueden obtener con el transistor. El objetivo es diseñar un amplificador capaz de obtener la máxima potencia posible y con un rendimiento muy superior al ofrecido por el fabricante sobre un ancho de banda amplio. Para ello, y trabajando a altas frecuencias, los efectos no lineales del dispositivo y las cargas a frecuencias armónicas influyen considerablemente en el diseño del amplificador.

El diagrama de bloques de la etapa de salida de un amplificador clase $\mathrm{F}$ se muestra en la Figura 3.4 [92]. La salida del transistor se puede simular como una fuente de corriente en paralelo con la capacidad no lineal de salida del transistor. A continuación, se definiría el encapsulado del transistor seguido de las redes de preadaptación a las frecuentas armónicas y por último la adaptación a la frecuencia fundamental a la carga de salida. 


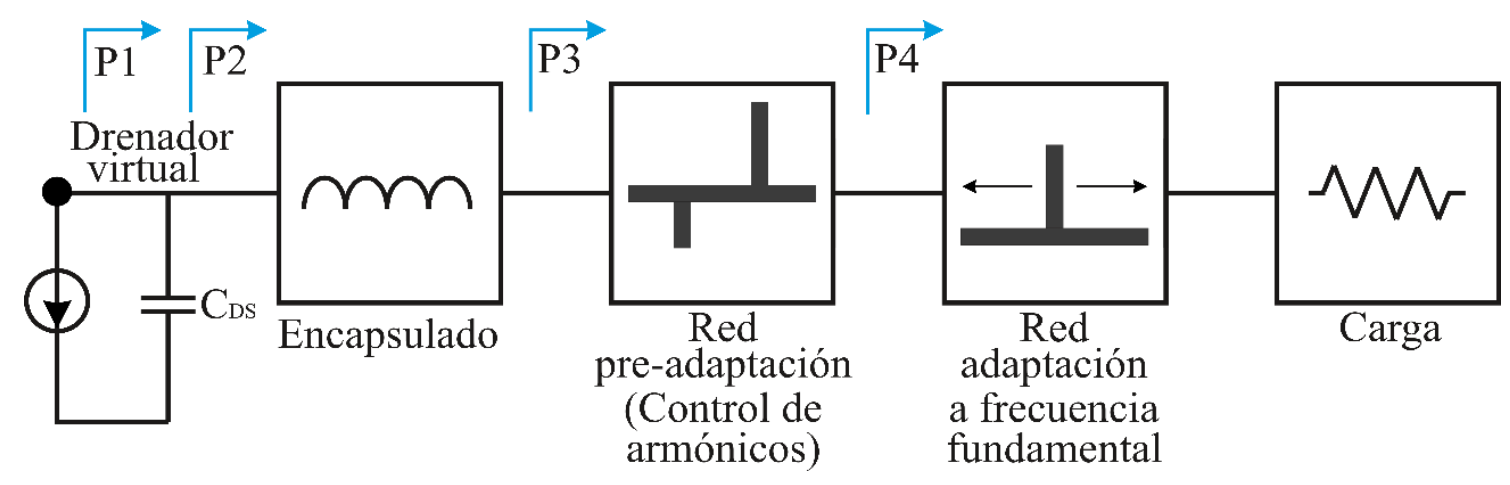

Figura 3.4. Diagrama de bloques de la red de salida de un amplificador clase $F$ especificando los diferentes planos de referencia de carga.

Convencionalmente, la impedancia de salida a la frecuencia fundamental queda referenciada al plano P3 y las impedancias a las frecuencias armónicas son referidas al plano P1. Este plano de referencia es intrínseco y se conoce como drenador virtual. Simétricamente se define el plano de referencia de la puerta como puerta virtual. Las terminaciones armónicas deben quedar definidas en estos planos para conseguir el funcionamiento óptimo del amplificador. Gracias al modelo del encapsulado, algunas veces ofrecido por el fabricante, y a la medida de la capacidad de salida, se puede estimar la impedancia en el plano P1 basándose en la impedancia medida en el plano P3. Pero para ello es necesario estimar la capacidad no lineal de salida del transistor $\left(C_{d s}\right)$ y, homólogamente, la de entrada $\left(C_{g s}\right)$.

\subsubsection{Capacidades no lineales del transistor}

La tecnología GaN HEMT ha sido en los últimos años una tecnología emergente muy empleada en el diseño de dispositivos de alta potencia para RF debido a su máxima frecuencia de operación, su alta tensión de ruptura, su alta densidad de potencia y su alta temperatura de funcionamiento [23].

Como ya se ha comentado, disponer de un modelo preciso es crucial para el diseño de los circuitos. Un modelo de circuito equivalente en gran señal empírico es más simple y sencillo de implementar en los programas de simulación, y ya han sido validados en diseños basados en otras tecnologías de semiconductores [93]. Sin embargo, encontrar un modelo fiel para los dispositivos de GaN es un tema de discusión 
muy extendido debido a la existencia de efectos térmicos y de dispersión en los dispositivos [94].

En esta tesis no se pretende generar un modelo en gran señal del dispositivo por dos razones: la primera es porque el fabricante ya ofrece un modelo en gran señal que habrá que comprobar su fiabilidad comparando las simulaciones y las medidas realizadas; y en segundo lugar porque para el diseño del amplificador principalmente interesan solamente una serie de parámetros, como pueden ser las capacidades no lineales del transistor.

Dos de las claves para conseguir un alto rendimiento en el amplificador son la capacidad de drenador-fuente del transistor $C_{d s}$ y la resistencia de conducción $R_{o n}$ o $R_{d s}$ [95]. La capacidad $C_{d s}$ dificulta la posibilidad de controlar los armónicos de la red de carga. Un dispositivo cuya frecuencia de funcionamiento máxima sea muy alta comparada con la frecuencia de trabajo ayuda a generar armónicos de alto orden necesarios para conformar las ondas. A este respecto, los transistores de GaN HEMT son buenos candidatos para ser empleados en el diseño de amplificadores clase F. La alta densidad de potencia de esta tecnología da lugar a capacidades de entrada y de salida más bajas para la misma potencia de salida. Además, al igual que los LDMOS, los transistores de $\mathrm{GaN}$ tienen tensiones de ruptura muy altas y consecuentemente impedancias de drenador más grandes a la frecuencia de funcionamiento.

Un modelo de circuito equivalente en pequeña señal ayudará a caracterizar el dispositivo de RF. En la literatura existen múltiples estudios sobre modelos de caracterización de estos dispositivos de RF [96] [97]. Con el fin de facilitar la compresión y el diseño del amplificador, en esta tesis se va a utilizar un modelo simple y de pocos elementos basado en el modelo de encapsulado que ofrece el fabricante para este dispositivo y en un modelo simplificado equivalente de dispositivo interno [98] tal y como se puede ver en la Figura 3.5. 


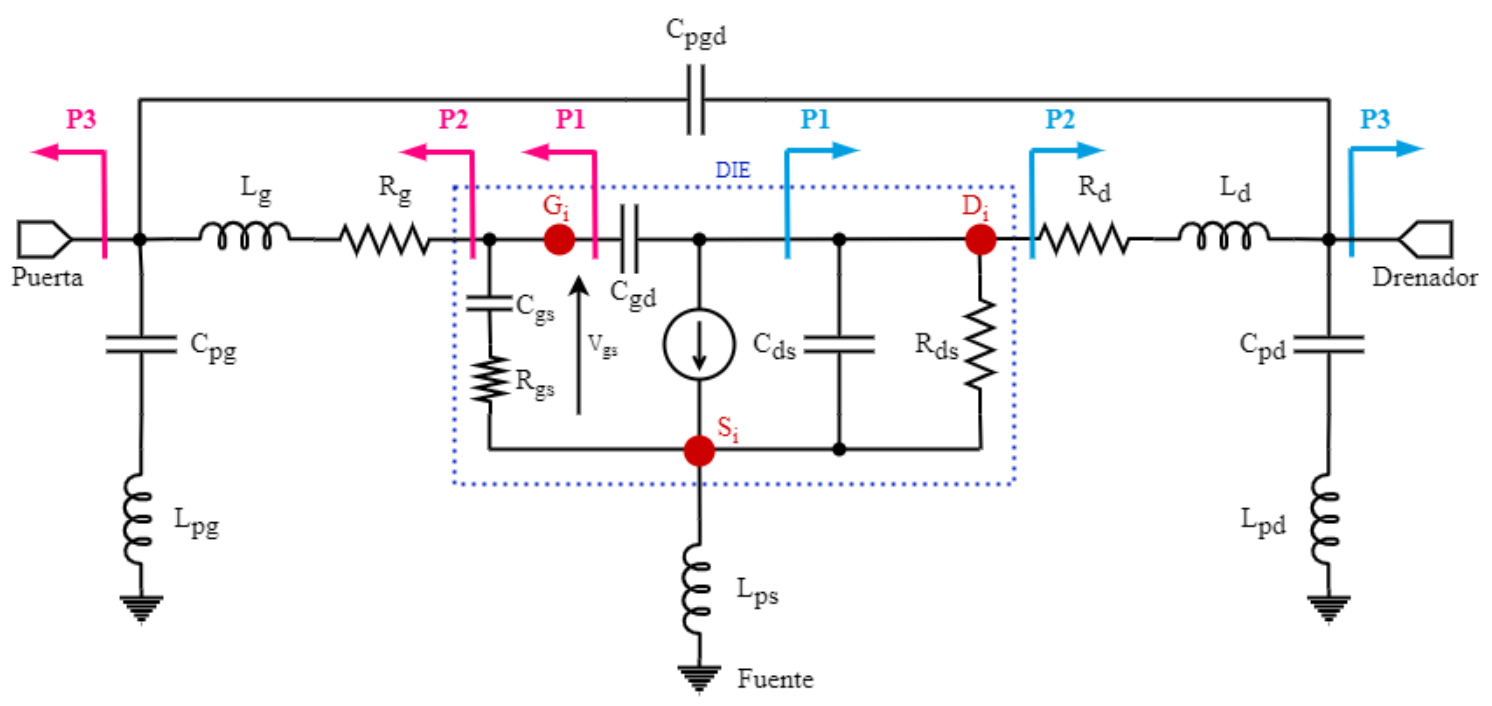

Figura 3.5. Modelo de transistor FET básico (die y encapsulado) y planos de referencia de carga.

En la Figura 3.5 existen parámetros conocidos, como los del encapsulado ofrecidos por el fabricante, y otros no conocidos, como las capacidades y resistencias internas. El modelo de encapsulado presenta, por un lado, unas inductancias $\left(L_{g}\right.$ y $\left.L_{d}\right)$ y resistencias $\left(R_{g} \mathrm{y} R_{d}\right)$ en serie que simulan la conexión con hilos de Bonding entre la die y el encapsulado y, por otro lado, capacidades parásitas que representan el efecto de los pads del encapsulado. Los efectos de la capacidad parásita del encapsulado entre puerta y drenador $\left(C_{g d}\right)$ son muy pequeños y son despreciables si la frecuencia de funcionamiento está por debajo de los 10GHz [93].

El modelo equivalente del dispositivo presenta a su salida una fuente de corriente dependiente de la tensión puerta-fuente $\left(V_{g s}\right)$. Otros parámetros del modelo simplificado equivalente de la die incluyen las capacidades intrínsecas no lineales entre puerta y fuente $\left(C_{g s}\right)$ y entre drenador y fuente $\left(C_{d s}\right)$, así como sus respectivas resistencias intrínsecas $\left(R_{g s}\right.$ y $\left.R_{d s}\right)$. Estos parámetros son los que deben ser determinados.

La dificultad de determinar los valores de los componentes del modelo equivalente puede ser ambigua. Esta ambigüedad hace referencia a que para un mismo conjunto de parámetros de dispersión (parámetros S) podrían existir diferentes circuitos equivalentes o diferentes conjuntos de valores de los componentes de un circuito equivalente. La clave para eliminar está ambigüedad y obtener una solución única es poder realizar diferentes experimentos que permitan aislar diferentes partes del modelo 
equivalente para poder caracterizar el dispositivo. Esto se puede conseguir aplicando una determinada polarización al dispositivo que haga que se comporte de una manera predecible.

Fuera del régimen de funcionamiento normal del dispositivo, un HEMT puede ser forzado para comportarse prácticamente como un cortocircuito o un circuito abierto. De esta manera, para la extracción de las capacidades intrínsecas, el dispositivo debe ser polarizado de tal manera que se simplifiquen el número de elementos a extraer.

Desde este punto de vista, se puede forzar la polarización inversa en la puerta del dispositivo ( $V_{g s} \leq-6 \mathrm{~V}$ para el caso del RF3931) de tal manera que el transistor entre completamente en la región de corte para cualquier tensión aplicada en el drenador. En la literatura se suele encontrar este método con el nombre de pinch-off. Cuando el dispositivo tiene polarización inversa, el comportamiento en pequeña señal y a baja frecuencia (menor de $500 \mathrm{MHz}$ ) del dispositivo intrínseco se puede modelar como un circuito abierto [93]. Esta polarización simplifica el modelo de la Figura 3.5, siendo el resultado el mostrado en la Figura 3.6.

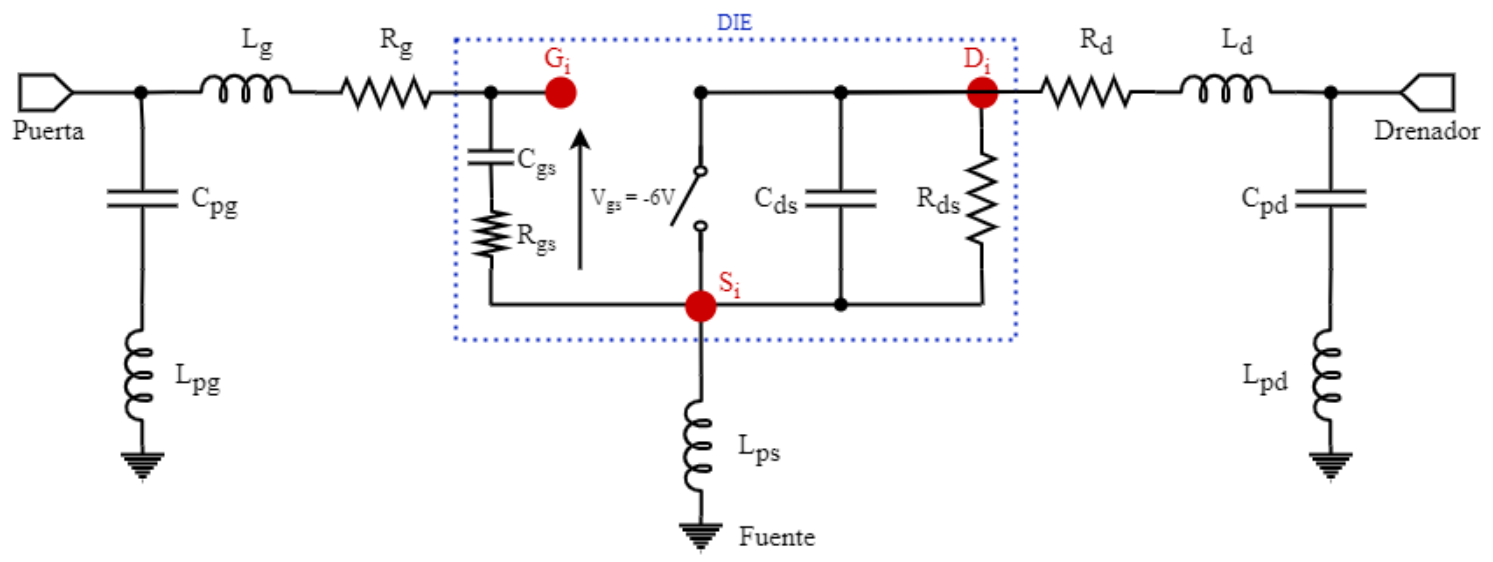

Figura 3.6. Modelo de transistor bajo polarización inversa o pinch-off.

Fijada la polarización de entrada, el objetivo es averiguar el valor de la capacidad de salida $\left(C_{\text {out }}\right)$ no lineal, la cual está formada tanto por la capacidad intrínseca entre drenador y fuente como por la capacidad parásita del encapsulado en el puerto de salida. Para ello, se procede a variar la tensión entre los puertos de drenador y fuente dentro de los límites del transistor y se realiza la medida de los parámetros S a la salida del dispositivo en un rango de frecuencias hasta $500 \mathrm{MHz}$. El diagrama de 
bloques empleado en la realización de esta medida se muestra en la Figura 3.7. Se emplearon dos bias-T para polarizar el transistor y variar la tensión en el drenador:

- ZNBT-60-1W de Mini-Circuits [99].

- ZFBT-4R2GW-FT de Mini-Circuits [100].

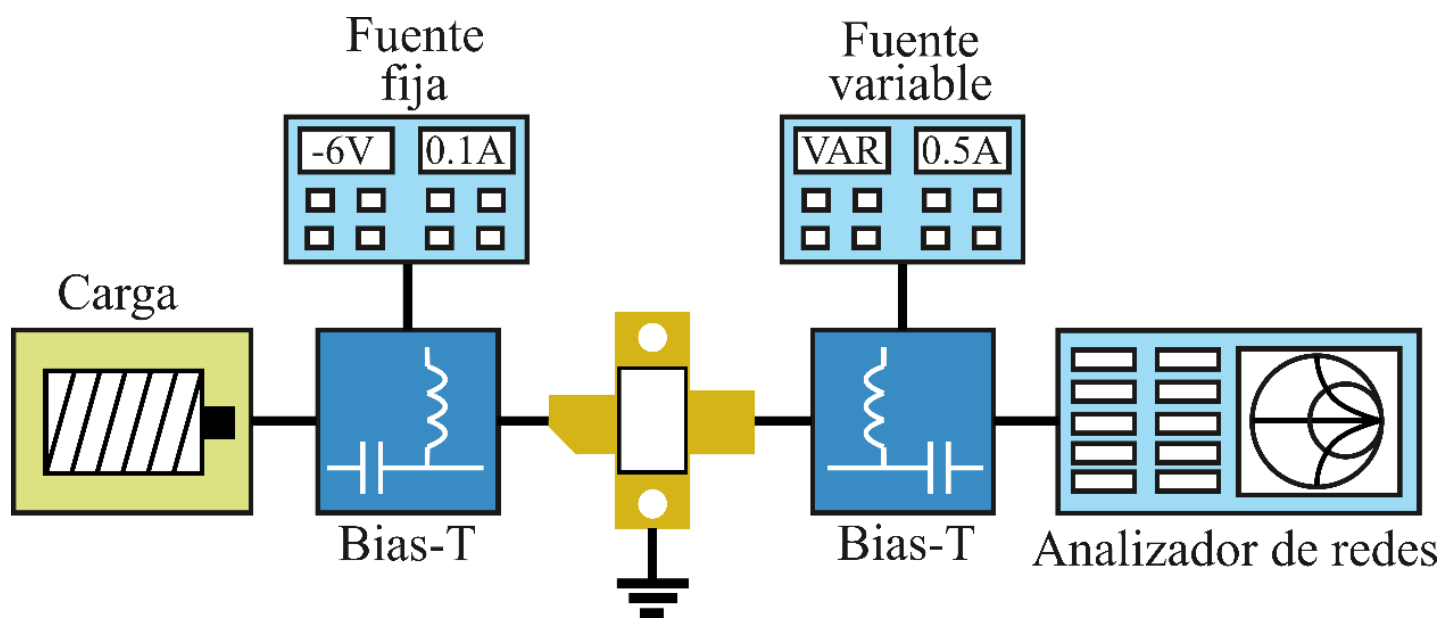

Figura 3.7. Diagrama de bloques del procedimiento de medida de la capacidad de salida equivalente del dispositivo.

Como se puede comprobar en la hoja de características [99] [100], ambos bias-T tienen como tensión de DC límite 30 voltios. Para obtener la capacidad no lineal en todo el rango de tensiones de drenador del dispositivo habría que hacer un barrido desde $0 \mathrm{~V}$ hasta la tensión de ruptura del transistor, que para el RF3931 es de 175 V [90]. Sin embargo, como se verá más adelante, la capacidad de salida tiende hacia un valor constante al ir incrementando la tensión de drenador. Por ello, en este caso concreto, es suficiente con caracterizar la capacidad de salida hasta un límite de $30 \mathrm{~V}$. En la Figura 3.8 se muestra una fotografía real del procedimiento de medida. 


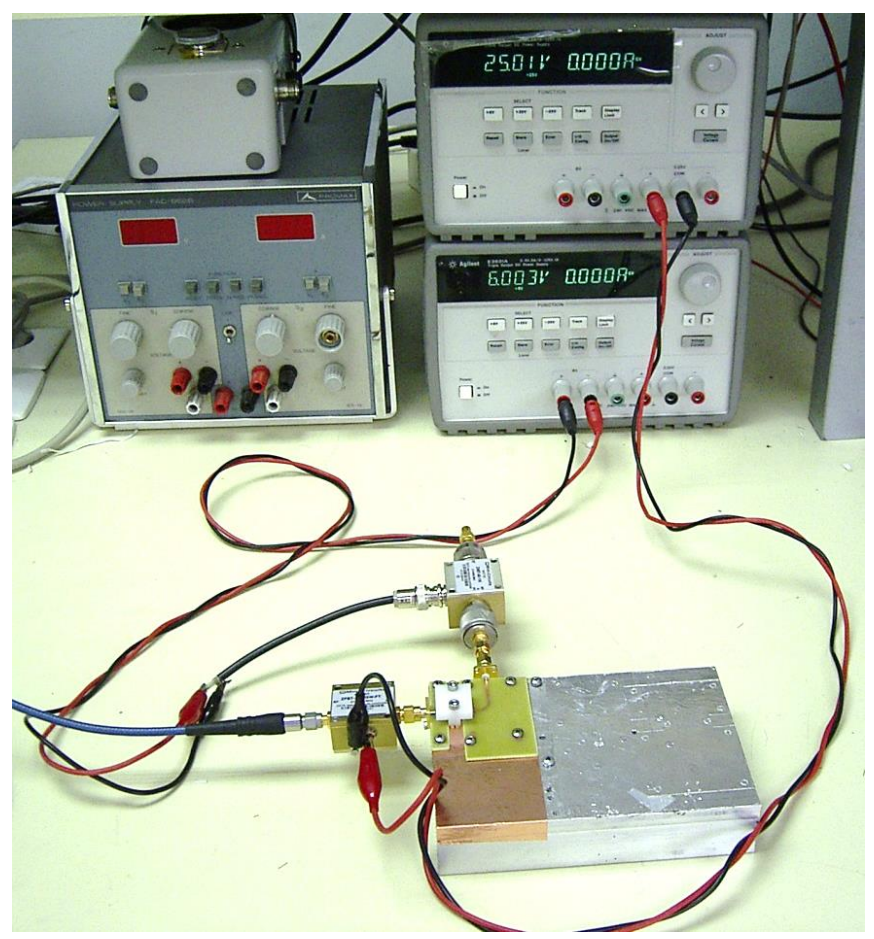

Figura 3.8. Fotografía del proceso de medida de la capacidad de salida del dispositivo.

De manera semejante se puede obtener el valor de la capacidad de entrada $\left(C_{i s s}\right)$ no lineal, que consta de la capacidad intrínseca entre puerta y fuente y de la capacidad parásita del encapsulado. En este caso habría que forzar que el dispositivo actúe como un cortocircuito. Para ello, se puede fijar la tensión entre drenador y fuente a $0 \mathrm{~V}$, siendo el circuito equivalente del dispositivo en pequeña señal y a baja frecuencia el mostrado en la Figura 3.9.

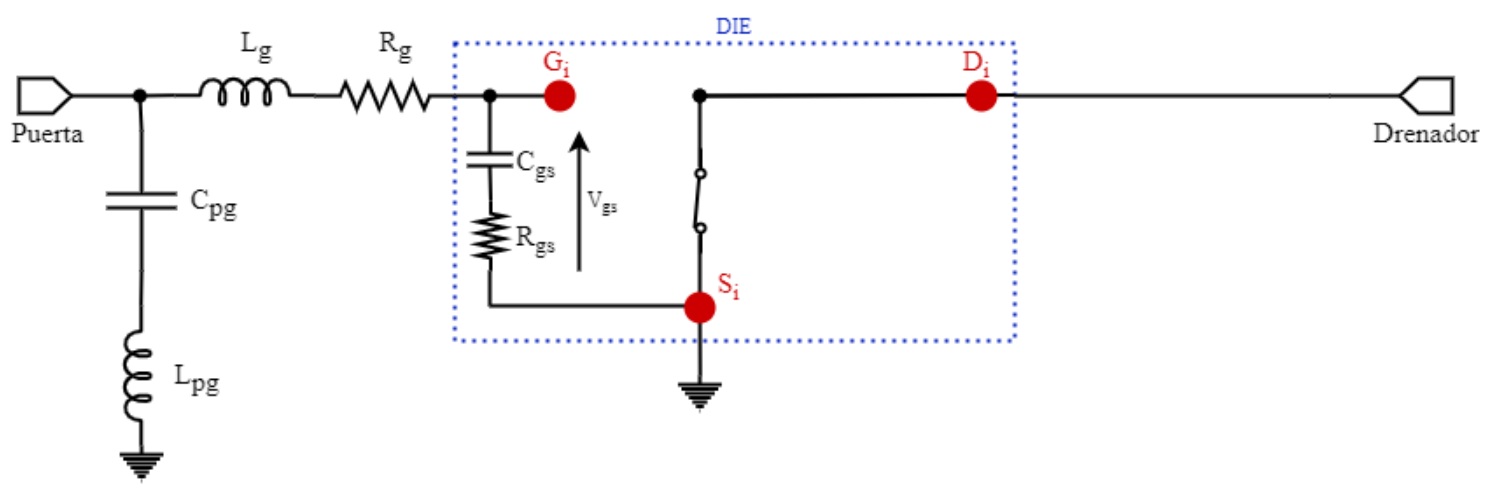

Figura 3.9. Modelo de transistor cortocircuitando drenador y fuente.

Fijada esta condición en el puerto de salida del dispositivo, se procedería a variar la tensión entre los puertos de puerta y fuente dentro de los límites del transistor (desde $-8 \mathrm{~V}$ a $+2 \mathrm{~V}$ ) y se realizarían las medidas de los parámetros $\mathrm{S}$ a la entrada del dispositivo 
en un rango de frecuencias hasta $500 \mathrm{MHz}$. El diagrama de bloques empleado en la realización de esta medida se muestra en la Figura 3.10.

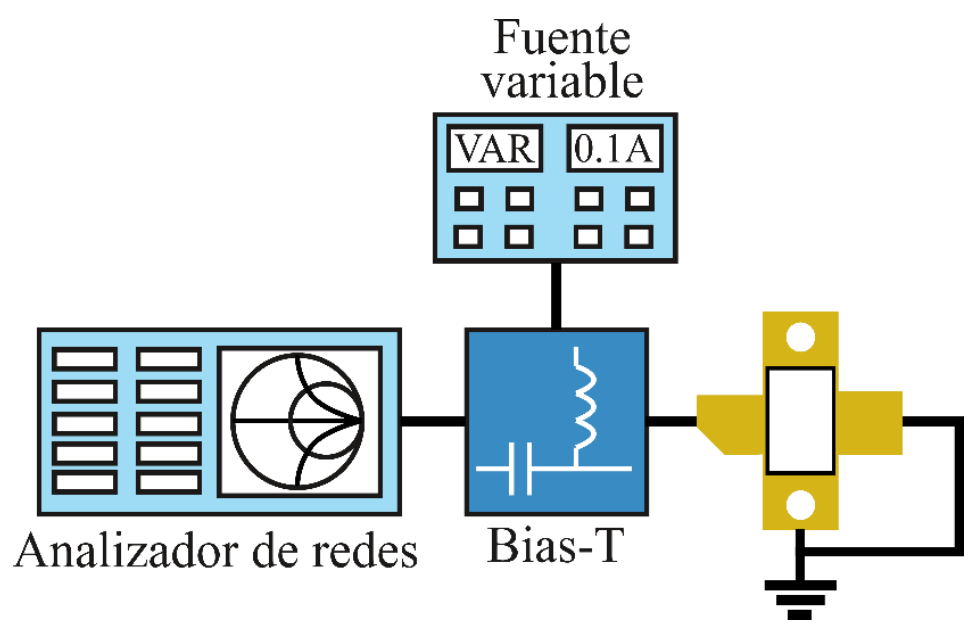

Figura 3.10. Diagrama de bloques del procedimiento de medida de la capacidad de entrada equivalente del dispositivo.

En la Figura 3.11 se muestra una fotografía real del procedimiento de medida de la $C_{i s s}$. En ella se observa que el drenador del dispositivo se ha cortocircuitado con la fuente.

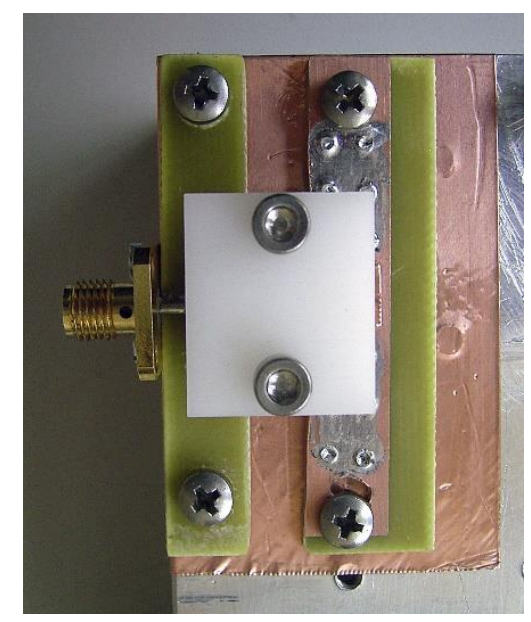

Figura 3.11. Detalle del procedimiento de medida de la capacidad de entrada del dispositivo.

A partir de las medidas obtenidas con el analizador de redes y conociendo el circuito equivalente del transistor y las condiciones de alimentación de entrada y de salida, se puede averiguar el valor de los elementos del circuito usando un programa de simulación, en este caso AWR. 
Los resultados obtenidos de las medidas del transistor RF3931 encapsulado son los siguientes (Figura 3.12):

a) Como era de esperar, la capacidad de entrada se incrementa sustancialmente una vez que es superior a la tensión de codo, pasando de unos $13 \mathrm{pF}$ a $30 \mathrm{pF}$ aproximadamente.

b) En cuanto a la capacidad de salida, a muy bajas tensiones se aprecia su no linealidad, pero a medida que la tensión de drenador va aumentando se estabiliza en un valor cercano a los $7 \mathrm{pF}$.
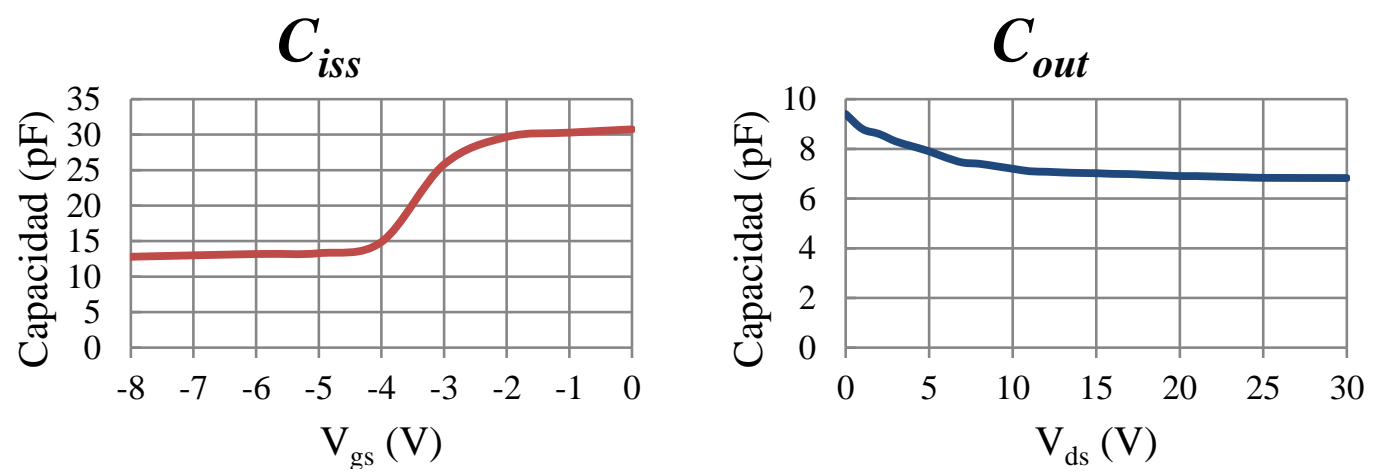

Figura 3.12. Capacidades equivalentes de entrada $\left(C_{\text {iss }}\right)$ y de salida $\left(C_{\text {out }}\right)$ del transistor RF3931.

A partir de estas medidas de capacidad internas se ha obtenido uno de los parámetros más influyentes en el diseño de amplificadores de alta potencia y alto rendimiento que trabajen en modo de conmutación, ya que habitualmente el dispositivo trabajará en corte o en saturación. Normalmente, el fabricante ofrece estos parámetros para un punto de polarización concreto, pero estos valores cambian en función de la tensión aplicada al dispositivo, como se ha podido comprobar.

\subsubsection{Planos de transformación}

Una vez se dispone de los valores aproximados de las capacidades internas de entrada y de salida y gracias a los parámetros $\mathrm{S}$ del encapsulado del dispositivo ofrecidos por el fabricante, el siguiente paso es ver la influencia de dichos parámetros a la hora de sintonizar las impedancias de carga. De esta manera se puede estimar la impedancia en el plano P1 basándose en la impedancia fijada en el plano P3. 
Por lo tanto, el siguiente objetivo es determinar que impedancia debería ser presentada por la red de carga en el plano P3 para conseguir las impedancias armónicas necesarias en el drenador virtual (plano P1). En el caso de un amplificador clase F, como ya se ha comentado en el Capítulo 2, estas impedancias de carga son cortocircuitos para los armónicos pares y circuitos abiertos para los armónicos impares.

$$
\begin{array}{cc}
Z_{n e t}\left(2 n \omega_{0}\right)=0 & \text { para armónicos pares } \\
Z_{n e t}\left[(2 n+1) \omega_{0}\right]=\infty & \text { para armónicos impares }
\end{array}
$$

En la Figura 3.13 y Figura 3.14 se muestra la transformación de impedancias simulada mediante el software AWR provocadas por el encapsulado, ofrecido por el fabricante, y la capacidad interna previamente calculada (en la región de saturación), para los dos primeros armónicos y a dos frecuencias fundamentales distintas: $2.14 \mathrm{GHz}$ y $1.64 \mathrm{GHz}$.

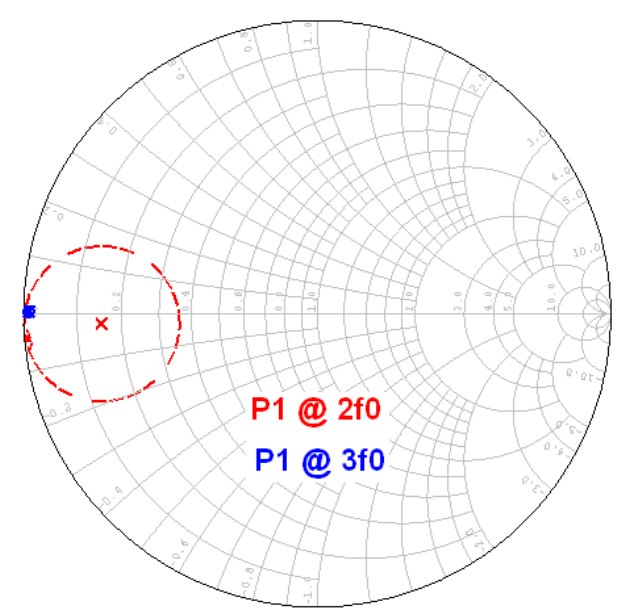

(a)

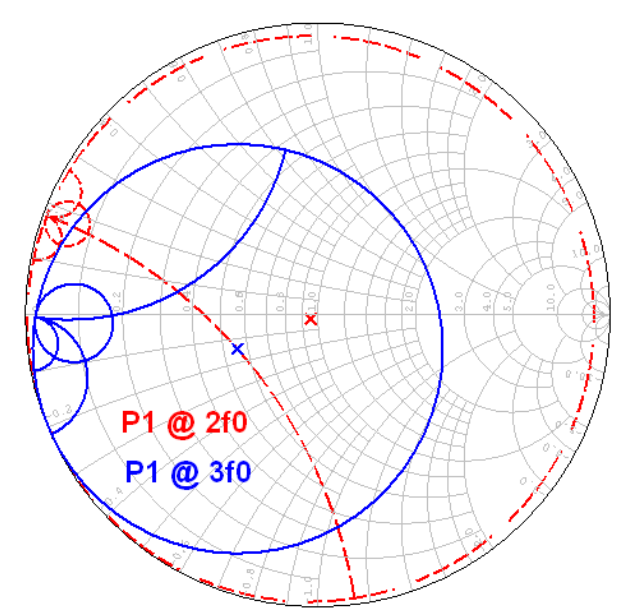

(b)

Figura 3.13. Simulación de la transformación de impedancias del P3 al P1 a $2.14 \mathrm{GHz}$ provocada por el encapsulado: entrada (a) y salida (b).

De las simulaciones realizadas se pueden obtener las siguientes conclusiones:

- A la entrada, prácticamente con cualquier impedancia que se cargue el dispositivo presentará un cortocircuito al segundo armónico para ambas frecuencias.

- A la salida, a mayor frecuencia se dificulta la sintonización del dispositivo para generar cortocircuitos a 2 fo y circuitos abiertos a 3 fo. 


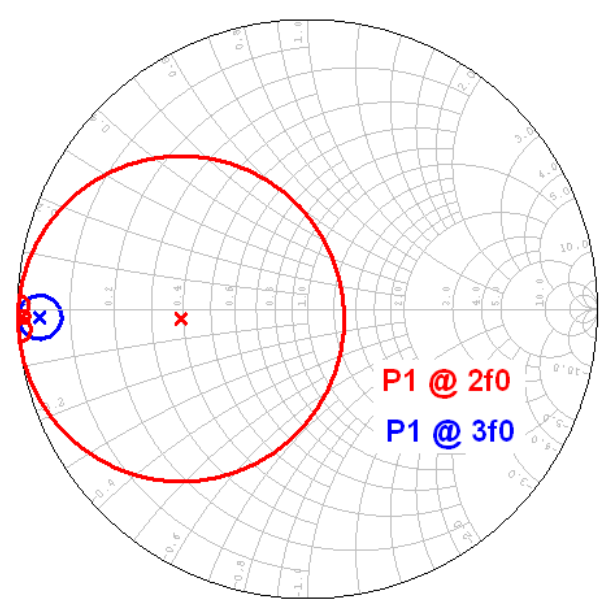

(a)

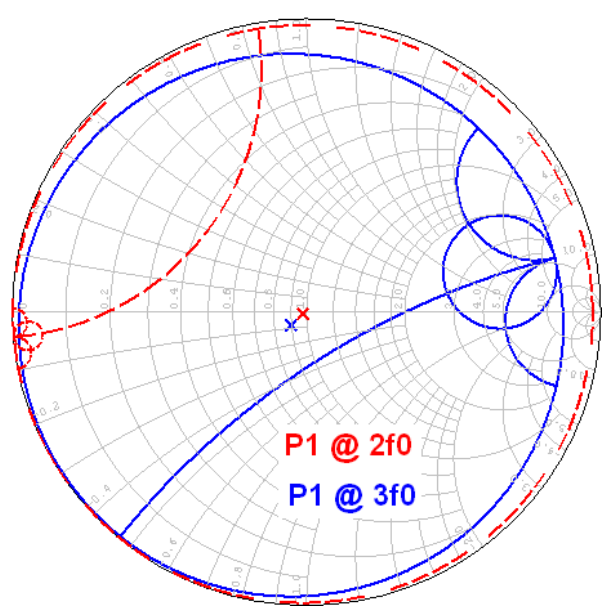

(b)

Figura 3.14. Simulación de la transformación de impedancias del P3 al P1 a $1.64 \mathrm{GHz}$ provocada por el encapsulado: entrada (a) y salida (b).

Con el fin optimizar la forma de onda de excitación de entrada para mejorar la conmutación del transistor, el encapsulado junto con la capacidad interna de entrada provoca que sea prácticamente imposible fijar un circuito abierto al tercer armónico a ambas frecuencias. La idea es poder excitar la puerta del transistor con una onda cuadrada que facilite una rápida conmutación del dispositivo y para ello, los armónicos pares deben ser una impedancia nula y los impares una impedancia infinita. En este caso, al no ser posible generar armónicos impares de alta impedancia, sino que solo se pueden generar armónicos pares e impares nulos, la forma de onda de excitación será sinusoidal. Ejercitar esta onda sinusoidal en todo el rango dinámico de la puerta ayuda a una correcta excitación y facilita la rápida conmutación.

Por consiguiente, el trabajar a una frecuencia mayor (cercana a la frecuencia de trabajo del transistor) provoca que la dupla del encapsulado y capacidad interna del dispositivo dificulten el control de las impedancias armónicas en la puerta y drenador virtuales. Basándose en estos resultados obtenidos, cabe destacar que la implementación de un amplificador clase $\mathrm{F}$ a la frecuencia de $2.14 \mathrm{GHz}$ no va a ser posible debido a que no sería viable establecer las impedancias armónicas necesarias. Esto conlleva reducir la frecuencia de trabajo; en este caso se ha fijado a $1.64 \mathrm{GHz}$ para el diseño del amplificador usando este dispositivo. 


\subsubsection{Caracterización load pull}

La impedancia y ganancia de dispositivos activos para funcionar en pequeña señal es normalmente medida con un analizador de redes con puertos de $50 \Omega$. Por linealidad, matemáticamente se podría post-procesar el comportamiento del dispositivo con puertos distintos de $50 \Omega$.

El comportamiento del transistor en gran señal, en contra, cambia de manera no lineal con la impedancia del puerto, necesitando que las medidas sean realizadas con la impedancia de puerto apropiada. El método load pull implica medidas de dispositivos activos bajo la variación de las impedancias de carga. A su vez, matizar que source pull denota la variación de la impedancia de entrada.

Es necesario tener una gran experiencia tanto en calibración como en medidas para implementar correctamente el método load pull en la caracterización de dispositivos no lineales. Esta sección describe los aspectos más destacados de los métodos utilizados en este trabajo; de ninguna manera es una explicación exhaustiva del procedimiento y no cubre todos los pasos intermedios necesarios para obtener los resultados presentados. Focus Microwaves dispone de una biblioteca con notas de aplicación útiles [44].

La mayoría de los modelos de transistores no lineales no están diseñados para reproducir su comportamiento a frecuencias armónicas y bajo una fuerte compresión. En otros casos, los modelos no lineales no están disponibles. Por ello, el método load pull se emplea normalmente junto con métodos analíticos en el diseño de amplificadores de alta potencia.

\subsubsection{Sintonizadores mecánicos y circuitos de preadaptación}

El método source pull y load pull tradicional hace uso de sintonizadores mecánicos con el que se varían la impedancia de puerta y de drenador, respectivamente. A raíz de ello, se miden los parámetros deseados (rendimiento, potencia...) sobre una constelación de impedancias a la frecuencia fundamental mientras que las impedancias armónicas se permiten variar arbitrariamente. 
El método load pull armónico tiene en cuenta, además de la impedancia a la frecuencia fundamental, las impedancias a los armónicos. Como se ha comentado en el Capítulo 2, existen sintonizadores mecánicos capaces de controlar la impedancia de carga tanto a la frecuencia fundamental como a los armónicos. Sin embargo, una solución más común y de menor coste es el control de los armónicos a través de un circuito resonante.

En microondas, el control de la impedancia a los armónicos se puede realizar mediante stubs de longitud $\lambda / 4$ a la frecuencia armónica situados a una distancia concreta con respecto al plano de referencia, como se muestra en la Figura 3.15.

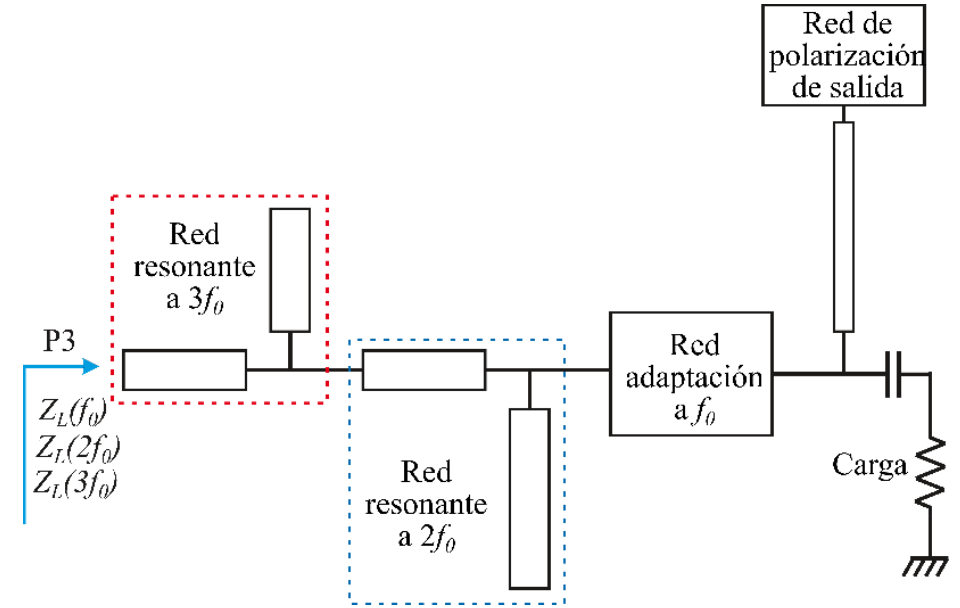

Figura 3.15. Ejemplo de red de salida con control de armónicos usando líneas de transmisión.

El objetivo perseguido en el diseño del amplificador clase $\mathrm{F}$ es maximizar el rendimiento sin comprometer demasiado la ganancia del transistor, minimizando el coste y la complejidad. De ahí que una buena alternativa es emplear un sistema load pull con redes de control de armónicos, vista en el Capítulo 2 y desarrollada a continuación. El empleo de este método permite conocer y controlar de una manera bastante precisa la carga presentada a los armónicos para posteriormente aplicar el método load pull tradicional a la frecuencia fundamental.

El procedimiento para la implementación de este método es encontrar la red resonante necesaria al tercer y segundo armónico con el fin de posteriormente variar la impedancia a la frecuencia fundamental a partir de un sintonizador manual. Sin embargo, generar diferentes prototipos con diferentes redes resonantes para encontrar cuál de ellas es la que consigue mejores resultados de rendimiento, potencia... no es ni 
práctico ni económico. Por ello, una posible solución es el diseño de una placa de circuito impreso que permita diferentes configuraciones de impedancia tanto al segundo como al tercer armónico.

Este útil consiste en una red de entrada y/o salida compuesta por una serie de stubs de longitud $90^{\circ}(\lambda / 4)$ a la frecuencia armónica colocados en una determinada posición. Esto debería provocar que la impedancia interna del transistor a la frecuencia armónica sea modificada con la finalidad de buscar las impedancias de carga necesarias, que en el caso de un amplificador clase F pueden ser o cortocircuitos o circuito abiertos. Destacar que el resonador de frecuencia más alta debe estar lo más próximo a la puerta o drenador del dispositivo, quedando los resonadores de menor frecuencia a continuación y así sucesivamente, de tal manera que los resonadores de frecuencia más baja no influyan en los de frecuencia más alta. Otro punto a tener en cuenta son las redes de polarización; conviene que la red de polarización se coloque lo más cerca posible de la puerta y drenador, debido a que ayuda a la estabilización de dispositivo.

En los diversos prototipos que a continuación se muestran se podrá ver el diseño de control de armónicos realizado; se muestra como se han implementado múltiples stubs de longitud $\lambda / 4$ a la frecuencia del tercer armónico separados una cierta longitud entre ellos (en este caso 30 grados eléctricos). De la misma manera, también se han implementado múltiples stubs de longitud $\lambda / 4$ a la frecuencia del segundo armónico separados una cierta longitud entre ellos (también 30 grados eléctricos). Esto permite generar una constelación de impedancia con alta relación de onda estacionaria (ROE) desplazadas 30 grados eléctricos entre cada una de ellas. En la Figura 3.16 se puede ver un ejemplo de la constelación de impedancias en función de la longitud eléctrica a la cual se coloca el resonador armónico.

Manualmente, estos stubs se irán conectando a la línea principal usando tintura de plata. Cada configuración cargará al dispositivo con unas impedancias armónicas determinadas que, junto con el encapsulado del transistor y los elementos intrínsecos, condicionaran el funcionamiento del dispositivo. 

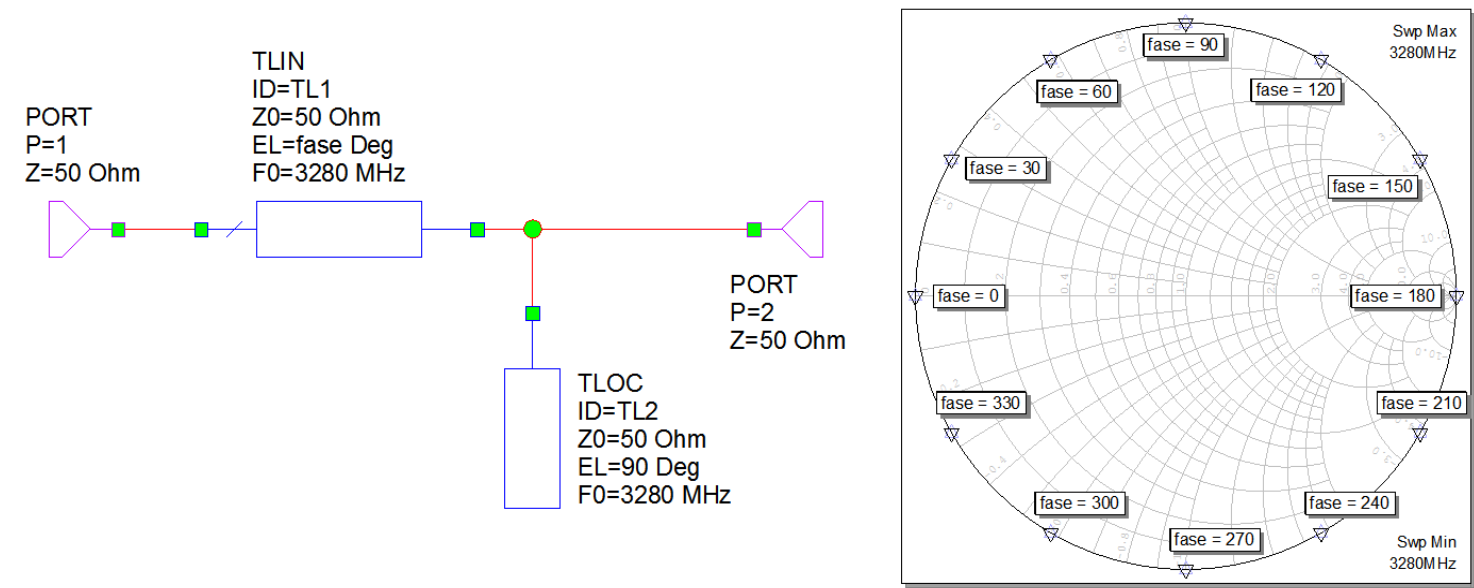

Figura 3.16. Constelación de la impedancia vista en el puerto 1 a la frecuencia armónica en función de la longitud eléctrica a la cual se posiciona el resonador armónico.

Fijadas las impedancias armónicas, el próximo paso es encontrar la impedancia a la frecuencia fundamental que maximiza el parámetro buscado (rendimiento, potencia...). Para ello, se ha usado un sintonizador manual conectado a la salida del útil. De manera análoga, se ha realizado en mismo procedimiento para la entrada. La Figura 3.17 muestra un diagrama de bloques del banco de pruebas utilizado.

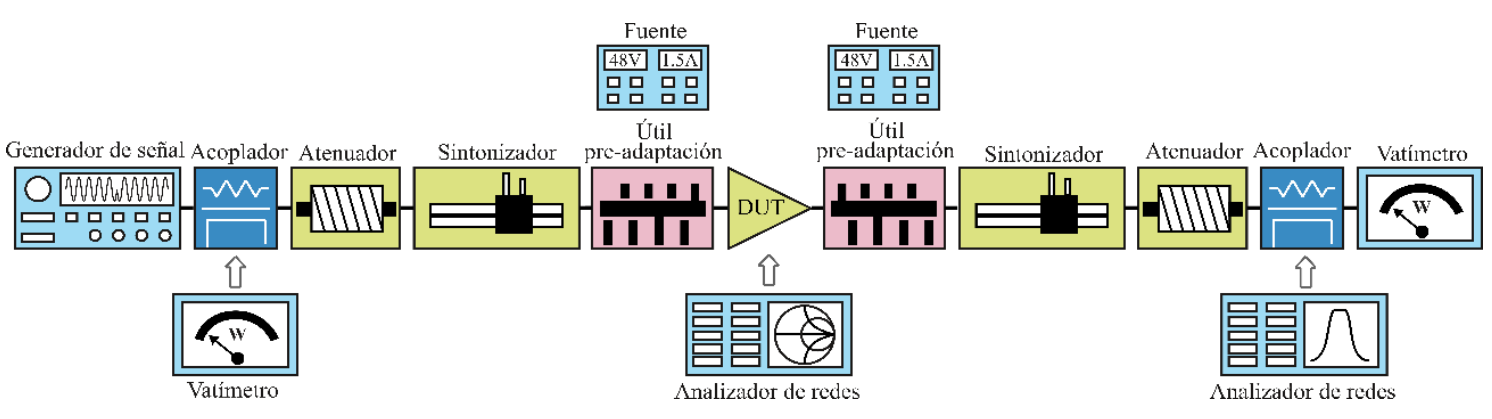

Figura 3.17. Diagrama de bloques del bando de prueba utilizado en la caracterización load pull.

Encontrada la combinación de resonadores armónicos y sintonizada la impedancia a la frecuencia fundamental que maximiza el parámetro buscado, el siguiente paso es averiguar el valor de las impedancias que generan ese funcionamiento. Para ello, será necesario medir las impedancias a la frecuencia fundamental y a los armónicos vistas por el dispositivo. Esta medida se realiza a través de un analizador de redes correctamente calibrado y perfectamente adaptado, con el fin de no cometer errores en la medida. El próximo apartado muestra el complejo proceso de calibración y adaptación de impedancias del analizador de redes para conseguir este fin. 


\subsubsection{Calibración}

Con el fin de averiguar qué impedancias son las que consiguen un cierto punto de funcionamiento es necesario recurrir a un método de calibración preciso. Antes de realizar cualquier medida load pull, es necesario caracterizar correctamente los circuitos de entrada de salida y de pre-adaptación.

A frecuencias de microondas, y especialmente en el caso de trabajar con MMIC (Monolithic Microwave Integrated Circuits), la calibración SOLT (Short-Open-Load and Thru) tradicional con extensión de puertos no es recomendable. En este caso se emplea la calibración TRL (Thru-Reflect-Line) que es un conjunto de técnicas de calibración que miden al menos dos estándares de transmisión y un estándar de reflexión para determinar el plano de referencia calibrado.

La calibración TRL es extremadamente precisa (aunque depende de la calidad y de la repetitividad de los estándares), pero tiene una limitación a frecuencias bajas dependiendo del estándar Line utilizado. Al contrario que con la calibración SOLT, muy pocos kits de calibración contienen estándares TRL. La calibración TRL se usa con mayor frecuencia cuando se requiere un alto nivel de precisión y no se dispone de estándares de calibración con el mismo tipo de conector que la DUT [101]. Por ello, en algunos casos como ocurre en este trabajo, se debe construir y caracterizar estándares en el mismo tipo de substrato que la DUT. Es más sencillo fabricar y caracterizar los tres estándares TRL que los cuatro estándares SOLT. Sin embargo, las discontinuidades físicas degradarán la calibración TRL.

Pequeños errores en las medidas de parámetros $\mathrm{S}$ debida a imprecisiones en la calibración presenta dos inconvenientes: el primero es obvio, una impedancia ligeramente diferente de entrada y de salida que será presentada a la DUT; el segundo, un error en los parámetros $\mathrm{S}$ influye en el cálculo del coeficiente de reflexión, y de ahí un error en la medida de la potencia de salida. El error será diferente para cada impedancia en la constelación, dando como resultado una variación del error en la medición de la potencia sobre el conjunto de impedancias.

Varias medidas de verificación pueden ser realizadas para determinar la exactitud de la calibración para cada puerto del sistema. Una de ellas puede ser la medida de los contornos de ganancia cuando no hay DUT, y la fuente está adaptada 
muy cerca de los $50 \Omega$. Si el sistema load pull está bien calibrado, esta medida debería presentar $0 \mathrm{~dB}$ de ganancia a la impedancia de carga conjugada y contornos de ganancia decreciente uniformes a medida que se incrementa el coeficiente de reflexión debido a las pérdidas por desadaptación, como se puede ver en la Figura 3.18.

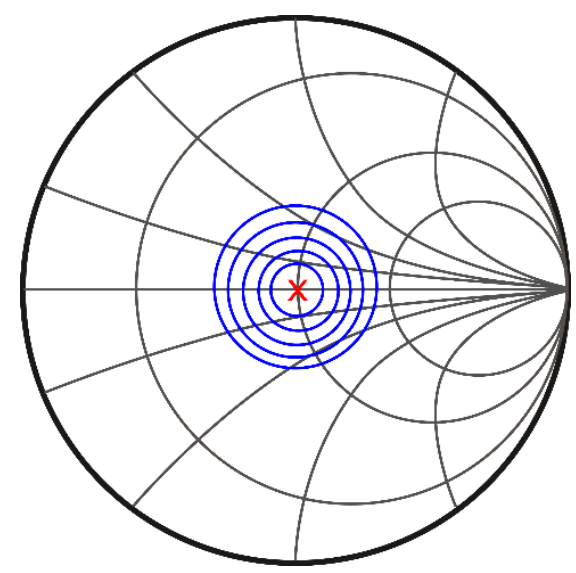

Figura 3.18. Contornos de ganancia de verificación de la correcta calibración de un sistema load pull.

En general, prácticamente se dedica más tiempo a la calibración del sistema load pull que a las medidas de la DUT. Además, es crítico trabajar a altas potencias y altos rendimientos, ya que pequeñas variaciones de potencia, tensión o corrientes medidas pueden tener un gran impacto en los resultados. Pequeños errores en las medidas load pull son tolerables, pero errores que varían significativamente sobre la constelación de impedancias no son aceptables y falsean los resultados.

En este proyecto se han diseñado los estándares TRL con el fin de calibrar el plano de referencia en la DUT. Las guías de diseño de los estándares TRL se pueden consultar en [102]. El estándar Thru puede ser de longitud cero o no cero. Cuanto menor sea la longitud del Thru más preciso será el estándar debido a que presentará menos pérdidas y, en el caso de longitud cero, no exhibirá reflexiones. El único requisito del estándar Thru es que no puede tener la misma longitud eléctrica que el estándar Line. La impedancia característica en el plano de referencia de los estándares Thru y Line define la impedancia de referencia de la calibración. A veces, con el fin de adaptar la conexión del estándar a la DUT, esta impedancia característica no es la misma que la del equipo de medida de RF (normalmente $50 \Omega$ ). En este caso, se suelen emplear transformadores de impedancia que ofrecen mayor precisión sobre los parámetros de la DUT. 
El estándar Reflect puede ser cualquier elemento que presente una alta reflexión, siempre que sea el mismo que se conecte a ambos puertos del PNA (Power Network Analyzer). Mientras que la magnitud real de la reflexión no necesita ser conocida, la fase del estándar debe conocerse y ser menor o igual a 1/4 de la longitud de onda.

El estándar Line tiene una serie de restricciones:

- Debe ser de la misma impedancia y constante de propagación que el estándar Thru.

- No puede tener la misma longitud que el estándar Thru.

- Debe ser una longitud eléctrica adecuada para el rango de frecuencia: en cada frecuencia, la diferencia de fase entre el Thru y la Line debe ser mayor de 20 y menor de 160 grados eléctricos.

- Si se desea ampliar el ancho de banda de frecuencia que puede medir el estándar, es posible utilizar múltiples estándares Line [103]:

○ Un estándar puede cubrir un ancho de banda de 8:1.

○ Dos estándares pueden cubrir un ancho de banda de 64:1.

○ Tres estándares pueden cubrir un ancho de banda de 512:1.

○ Cuatro estándares pueden cubrir un ancho de banda de 4096:1.

- A bajas frecuencias, el estándar Line puede llegar a ser demasiado largo para un uso práctico. La longitud óptima del estándar Line es de 1/4 de la longitud de onda dentro de la media geométrica del intervalo de frecuencia (raíz cuadrada de $f_{l} \times f_{2}$ ).
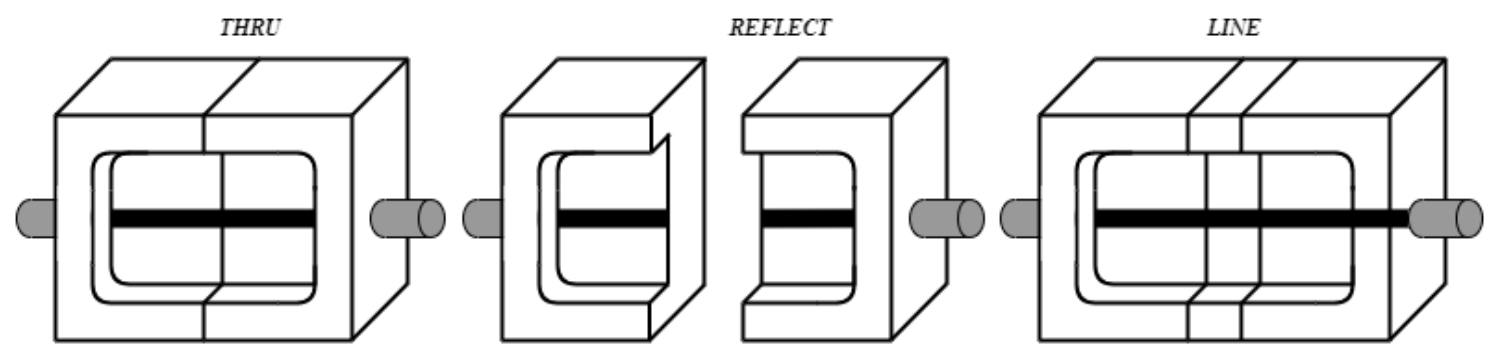

Figura 3.19. Estándares de calibración TRL. 
La Figura 3.19 muestra un ejemplo de cada uno de los estándares de la calibración TRL.

Conocidos los requisitos que deben cumplir los estándares de la calibración TRL, se procedió a realizar un primer kit de calibración capaz de elaborar medidas a la frecuencia fundamental de $1640 \mathrm{MHz}$ y varios armónicos empleando los estándares diseñados. Un punto importante es configurar correctamente el analizador. Para ello, se realizaron 3 estándares:

- Un Thru con una longitud eléctrica de $90^{\circ}\left(45^{\circ}\right.$ cada lado).

- Un Reflect con una longitud eléctrica igual que el Thru $\left(45^{\circ}\right)$.

- Un Line con una longitud eléctrica de $30^{\circ}$.

Adicionalmente se realizaron otros 2 estándares Line más por si fuera necesario aumentar el ancho de banda de la calibración: uno de ellos con una longitud eléctrica de $75^{\circ}$ y otro con una longitud eléctrica de $150^{\circ}$. Todos los estándares fueron diseñados a $1300 \mathrm{MHz}$ y con una impedancia característica de $50 \mathrm{Ohm}$ y sobre el substrato TACONIC RF35. En la Figura 3.20 se pueden ver los estándares realizados. Además, se diseñaron unos bloques de aluminio anodizado con el fin de ensamblar mecánicamente unos con otros.
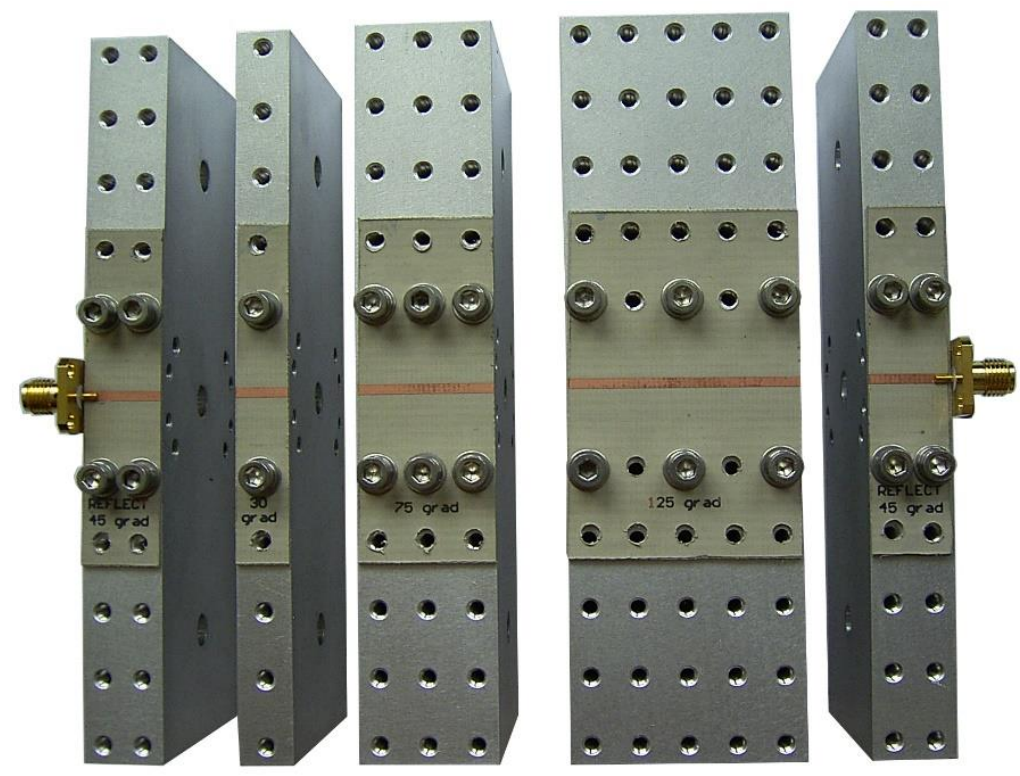

Figura 3.20. Fotografía real de los estándares de calibración TRL diseñados. 
Desde la Figura 3.21 a la Figura 3.26 se muestra una comparación real entre la calibración coaxial con extensión de puertos y la calibración TRL usando los estándares descritos. Si nos fijamos en las pérdidas de retorno $\left(\mathrm{S}_{11}\right)$ y en las pérdidas de inserción ( $\mathrm{S}_{21}$ ), se comprueba que la calibración empleando el método TRL es mucho más precisa en todo el rango de frecuencias.

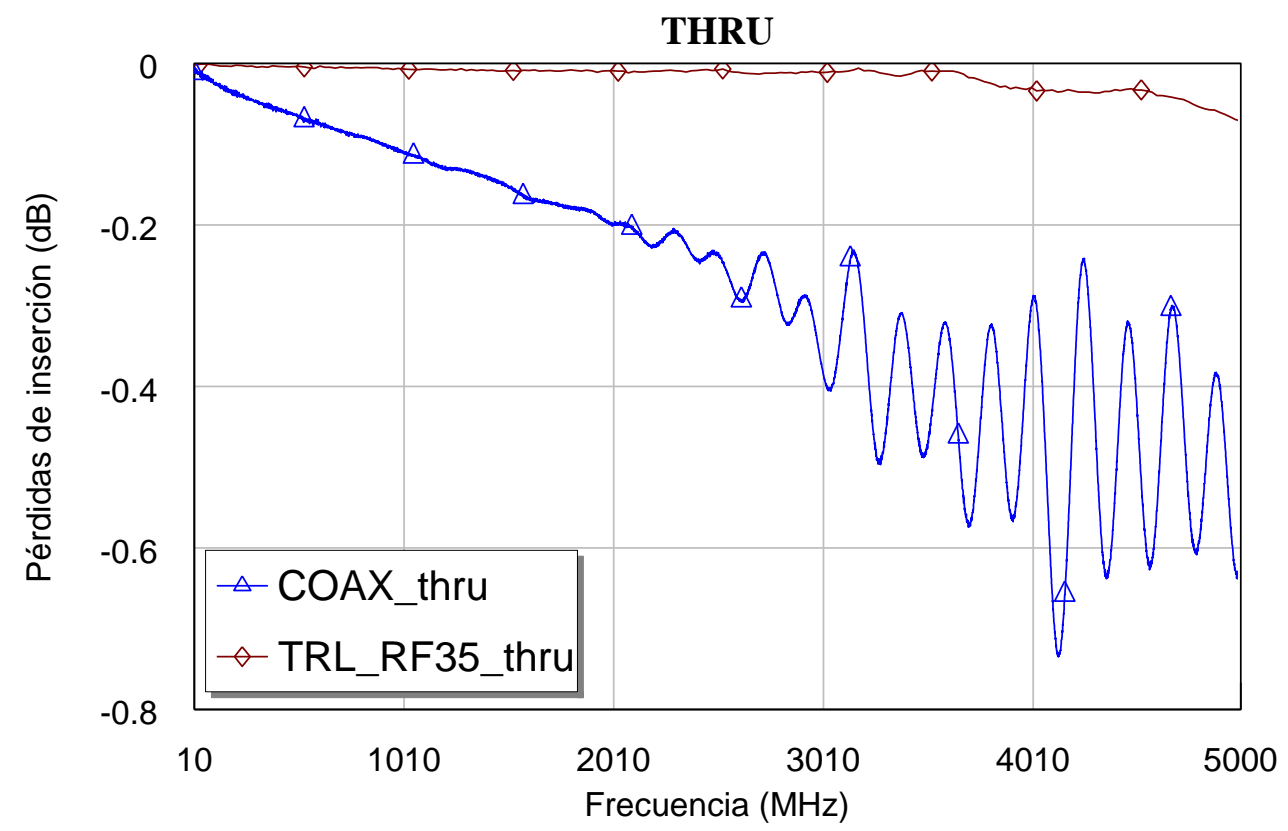

Figura 3.21. Comparación de las pérdidas de inserción de un cortocircuito usando calibración TRL y calibración coaxial con extensión de puertos.

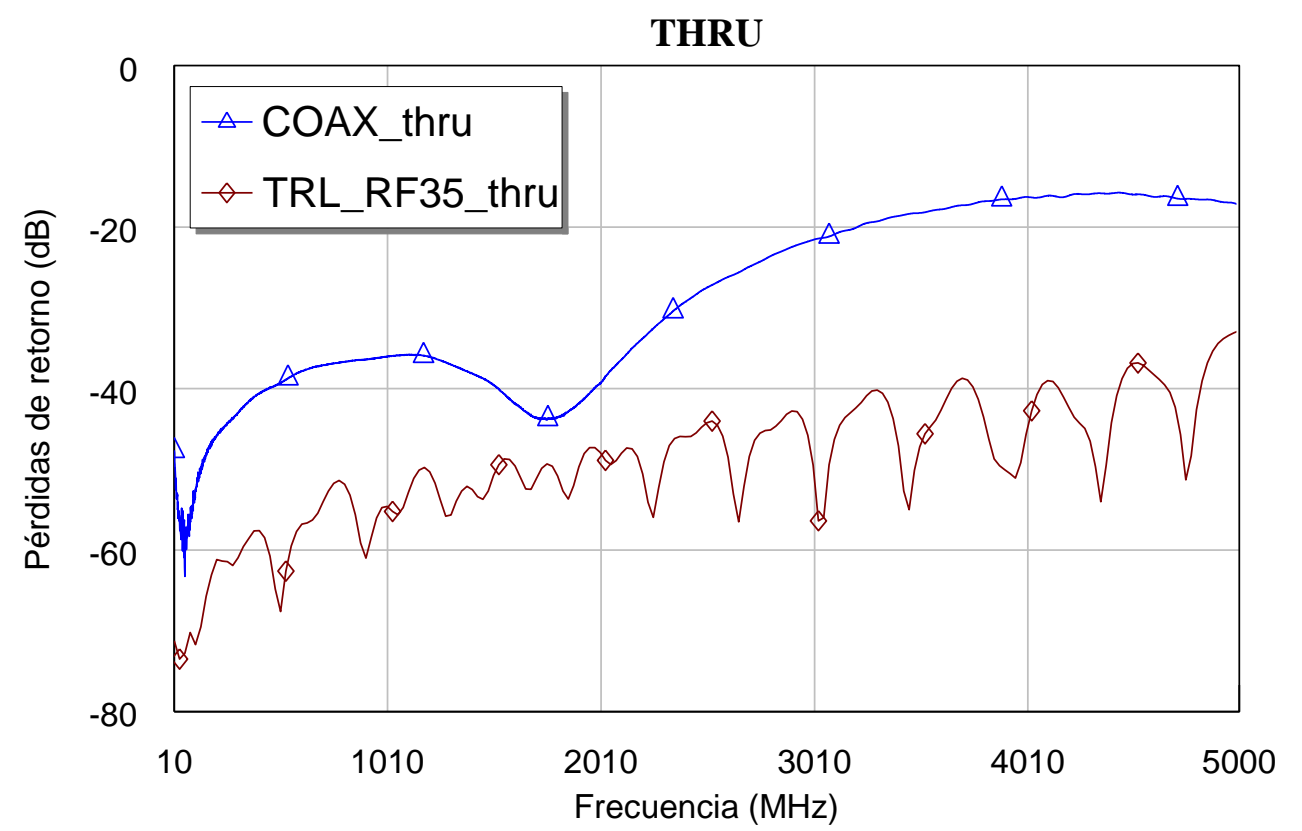

Figura 3.22. Comparación de las pérdidas de retorno de un cortocircuito usando calibración TRL y calibración coaxial con extensión de puertos. 


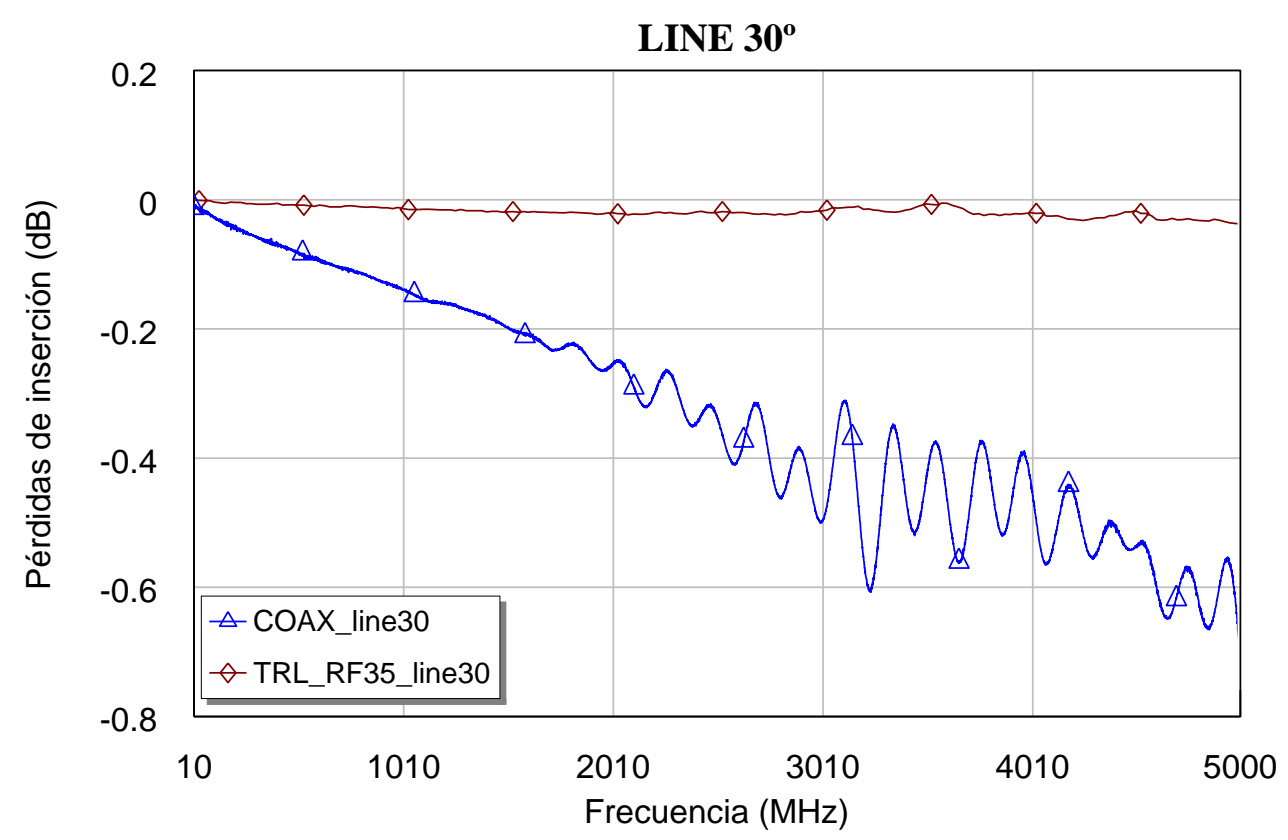

Figura 3.23. Comparación de las pérdidas de inserción de una línea de $30^{\circ}$ eléctricos usando calibración TRL y calibración coaxial con extensión de puertos.

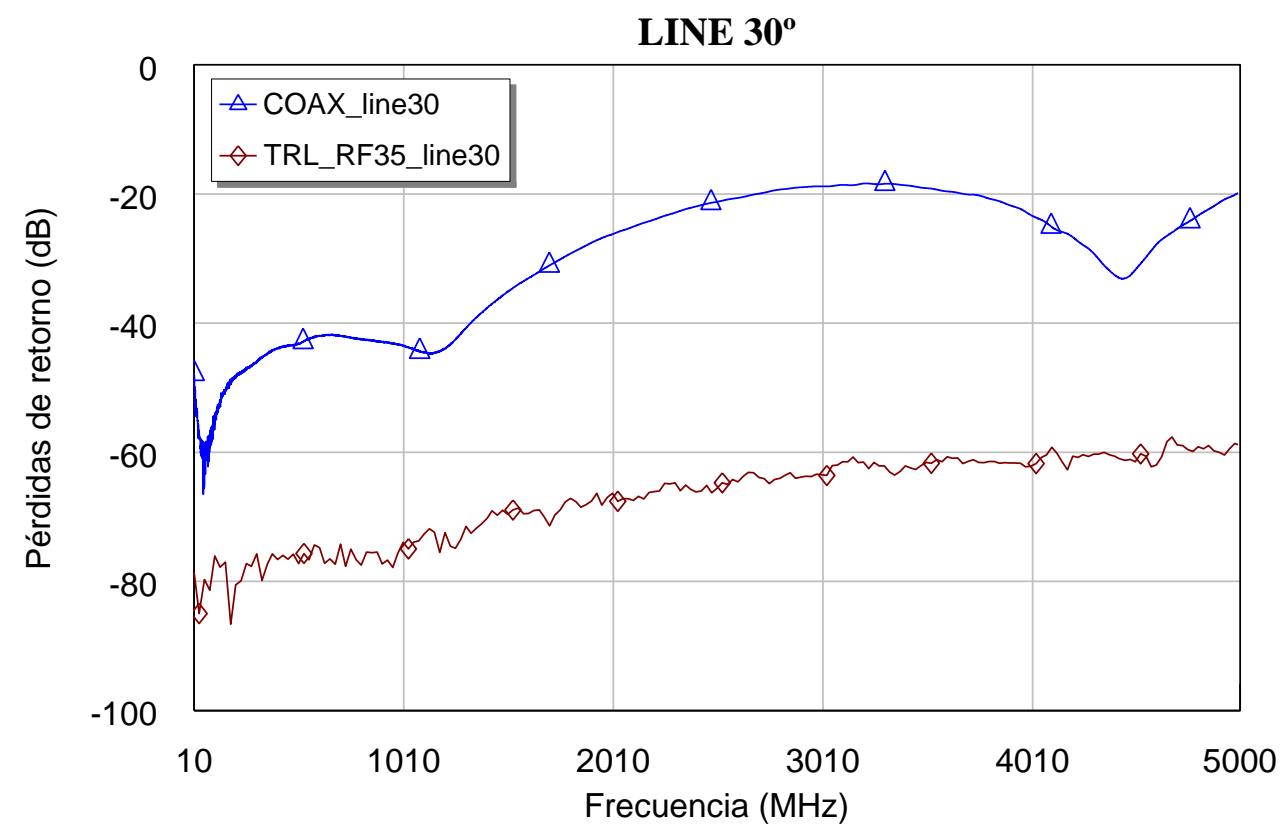

Figura 3.24. Comparación de las pérdidas de retorno de una línea de $30^{\circ}$ eléctricos usando calibración TRL y calibración coaxial con extensión de puertos. 


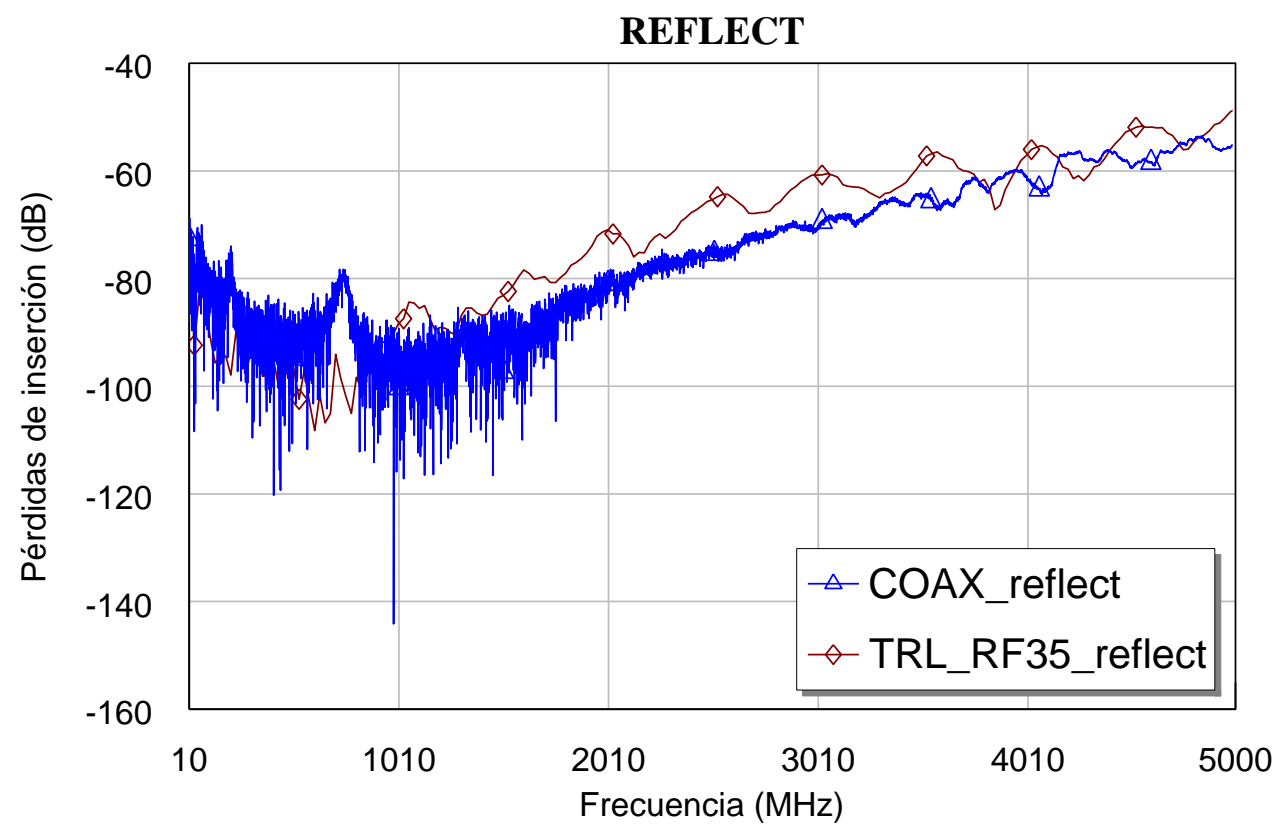

Figura 3.25. Comparación de las pérdidas de inserción de un circuito abierto usando calibración TRL y calibración coaxial con extensión de puertos.

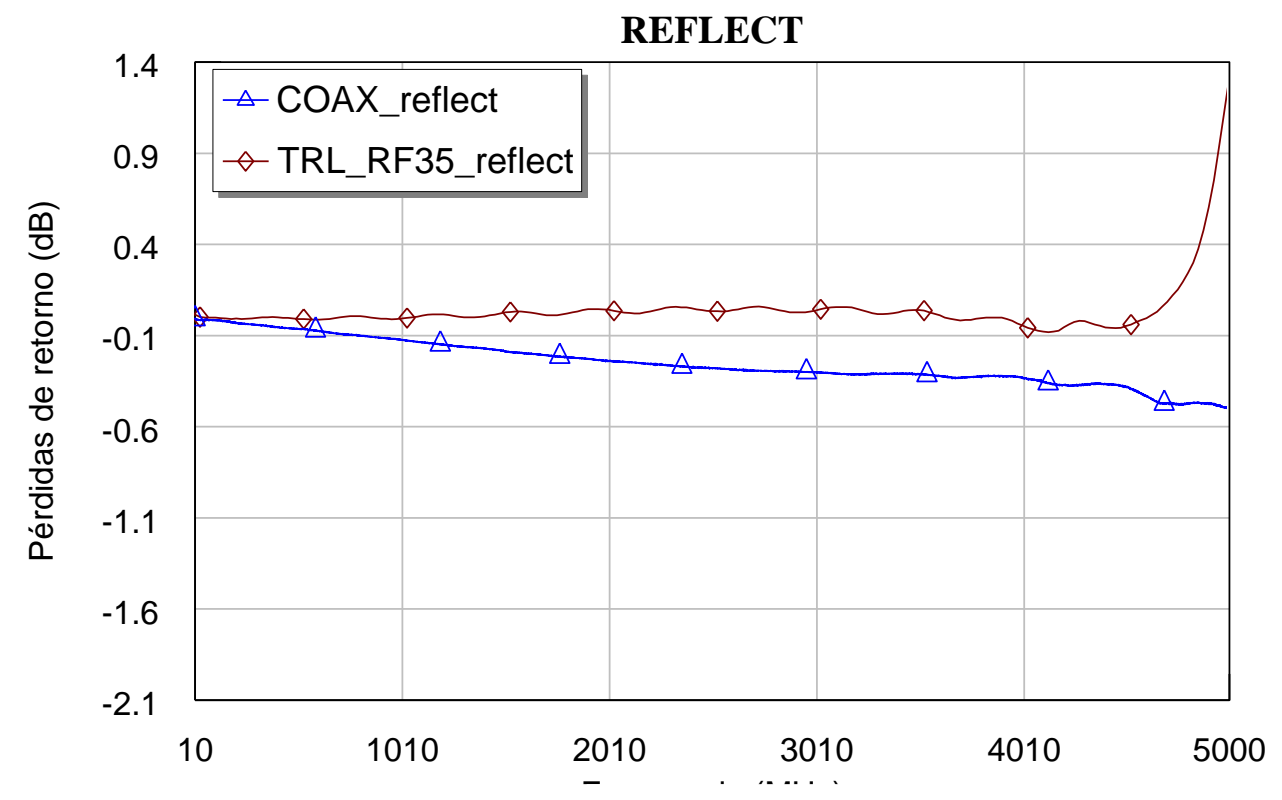

Figura 3.26 Comparación de las pérdidas de retorno de un circuito abierto usando calibración TRL y calibración coaxial con extensión de puertos.

Siguiendo este proceso de calibración TRL se definirá el plano de referencia de medida. A partir de ahí, entran en juego los sintonizadores mecánicos y los módulos de pre-adaptación. 
En un método load pull tradicional, donde únicamente exista un circuito de preadaptación y un sintonizador mecánico, el primer paso sería medir los parámetros $\mathrm{S}$ del circuito de pre-adaptación sobre una constelación de impedancias generadas con el sintonizador mecánico. Los parámetros $\mathrm{S}$ de cada punto de la constelación están conectados en cascada con los parámetros $\mathrm{S}$ del circuito de pre-adaptación, dando lugar a una nueva constelación de impedancias en el plano de referencia de medida, así como los factores de calibración correspondientes. Dependiendo de la resolución y precisión que se desee, el número de medidas a realizar es muy elevado, y se incrementa en función de diferentes puntos de polarización o si son varios los parámetros que se desean medir. Posteriormente, se conecta el circuito de pre-adaptación al DUT y se le carga con esa misma constelación de impedancias y se miden los parámetros deseados. De esta manera, se podrá identificar cada punto de la constelación con los resultados obtenidos.

En este trabajo, con el fin de reducir considerablemente el número de medidas, el método load pull es ligeramente diferente: únicamente se buscará el punto máximo de funcionamiento del parámetro deseado, ya sea potencia, rendimiento o ganancia. Hay que tener en cuenta que, en este caso, el circuito de pre-adaptación es un útil que consta de múltiples configuraciones lo que incrementaría el número de medidas ya que es como si tuviéramos múltiples circuitos de pre-adaptación.

El proceso load pull seguido en este trabajo es el siguiente: se realizarán las medidas de potencia, rendimiento y ganancia para cada una de las configuraciones del útil buscando con el sintonizador mecánico la impedancia que consigue maximizar cada uno de esos parámetros. Posteriormente, y para cada uno de esos parámetros, se medirá la impedancia vista por el dispositivo a la frecuencia fundamental y a los armónicos usando los bloques TRL (Figura 3.27). Por último, utilizando el software de simulación AWR se desempotrarán las impedancias armónicas vistas en el P3 (drenador exterior) hasta el P1 (drenador intrínseco). 


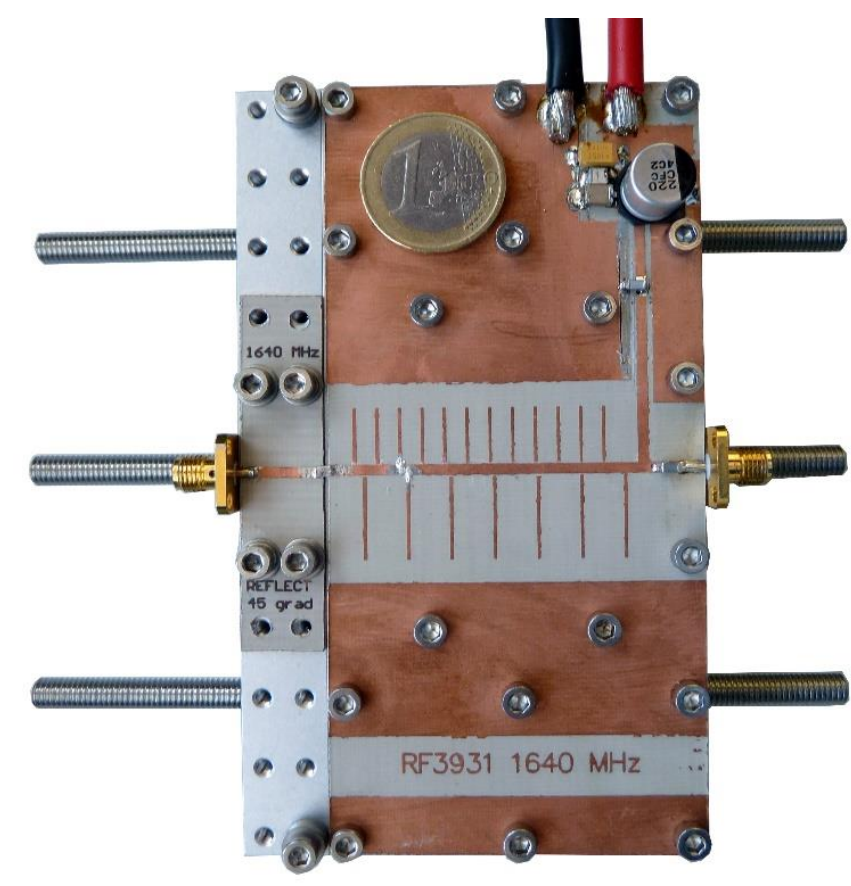

Figura 3.27 Proceso de medida de la impedancia vista por el dispositivo utilizando los bloques TRL con los que se ha calibrado.

\subsubsection{Banco de medidas}

Las medidas load pull se han realizado a partir de un banco de medida cuyo diagrama de bloques ya se mostró en la Figura 3.17. El banco de medidas real que se presenta en la Figura 3.28 está formado por:

- Generador de funciones: E4438C de Keysight o SM300 de Rohde \& Schwarz.

- Driver: ZHL-10W-2G+ de Mini Circuits.

- Sintonizadores mecánicos: modelo $1643 \mathrm{~N}$ de Maury Microwave

- Atenuador $3 \mathrm{~dB}$ : BW-N3W20+ de Mini Circuits.

- Circuitos de pre-adaptación y DUT

- Carga 50 S: BW-40N100W de Mini Circuits.

- Analizador de espectros: N9320B de Keysight. 
- Analizador de redes: N5242A de Keysight.

- Bloques TRL.

- Fuentes de alimentación: E3631, E3634 y N5767A de Keysight.

- Acopladores direccionales: ZABDC20-252H-N+ y ZGDC20-33HP+ de Mini Circuits.

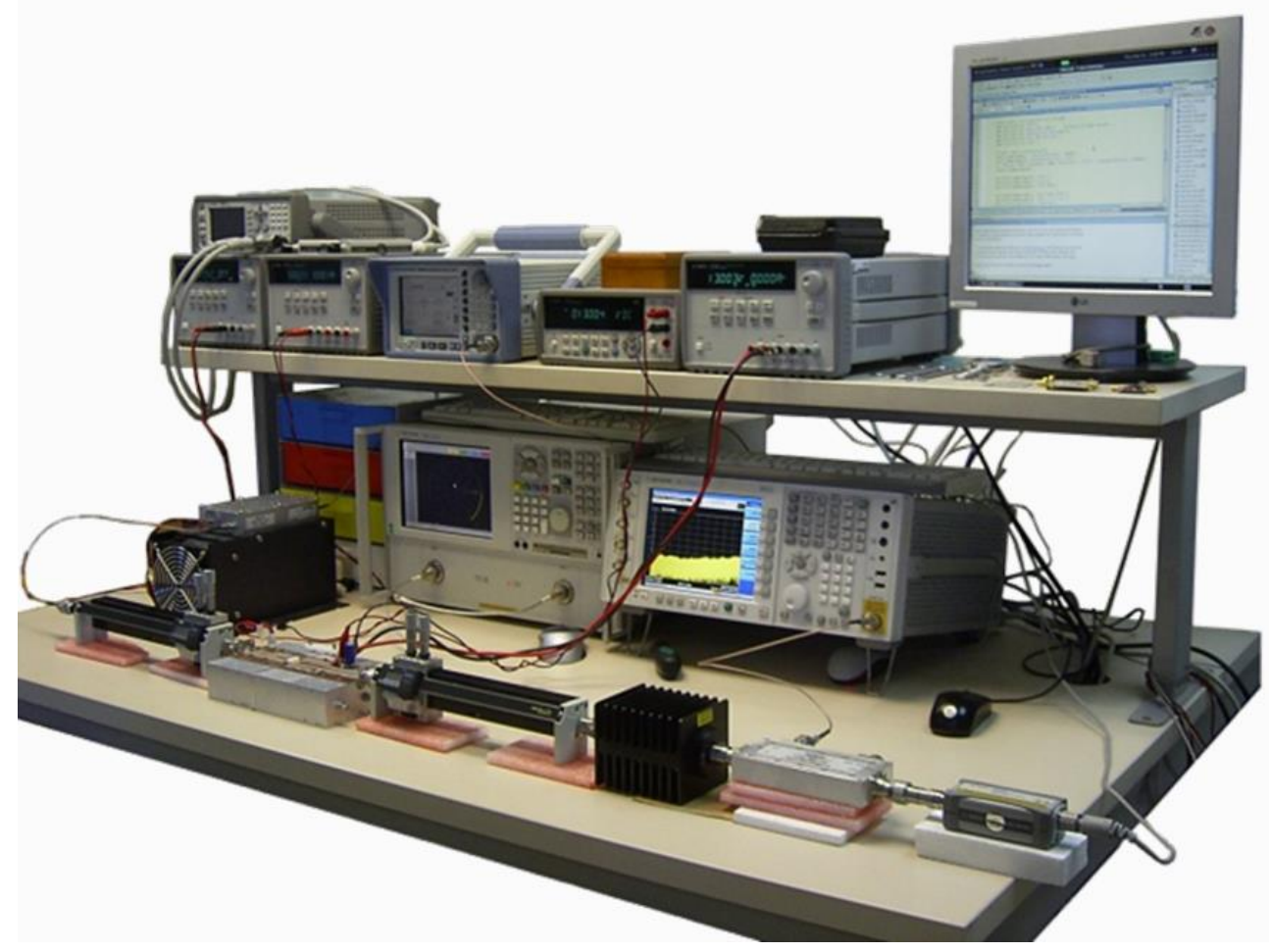

Figura 3.28. Banco de medida implementado para realizar el proceso load pull.

Después del proceso de calibración, el primer paso es polarizar el transistor. A la hora de realizar este paso es muy importante realizarlo lentamente observando posibles oscilaciones que puedan romper el transistor. En algunos casos se puede ajustar la impedancia de salida o de entrada, o mejorar la red de polarización, para evitar inestabilidades en el transistor.

Sin embargo, un transistor no es unilateral, es decir, que su funcionamiento depende tanto de la impedancia de entrada como de la impedancia de salida. Por ello, el primer paso es realizar el método source pull en pequeña señal en búsqueda de la impedancia de entrada que consigue la máxima ganancia. Durante este proceso, la 
impedancia de carga debe ser controlada, típicamente con el valor teórico de la impedancia calculada por el método de la línea de carga [104].

En muchas ocasiones, es posible que durante el proceso de load pull se deba desadaptar intencionalmente la impedancia de entrada de su punto de máxima ganancia. Esto se realiza para llegar a un compromiso entre ganancia y estabilidad. Afortunadamente, normalmente la impedancia de entrada no suele tener un gran impacto en la potencia de salida y en el rendimiento por lo que con una pequeña desadaptación se puede obtener prácticamente el mismo nivel de ganancia.

Las medidas de funcionamiento del transistor buscando que impedancia maximizan la potencia de salida, el rendimiento o la ganancia se realizan bajo unas condiciones de potencia de entrada que ofrezcan una ganancia de compresión constante.

\subsubsection{Prototipos}

En esta sección se presentan varios prototipos de amplificador de potencia clase F diseñados a partir del método load pull. El DUT, como ya se ha comentado anteriormente, es un transistor GaN HEMT (RF3931 de RFMD) no adaptado internamente capaz de producir 40W a 2140MHz.

Un amplificador clase $\mathrm{F}$ puede conseguir un rendimiento de drenador teórico del $100 \%$ dando forma a las onda de tensión y corriente en el drenador virtual [38]. Como ya se comentó en el Capítulo 2, en un amplificador clase F la forma de onda de tensión se asemeja a una onda cuadrada, y la forma de onda de corriente puede ser representada como una onda sinusoidal rectificada. La forma de onda de tensión cuadrada contiene solo frecuencias armónicas impares, lo que significa un circuito abierto en el drenador virtual del transistor (P1). La forma de onda de corriente solo contiene armónico pares, lo que requiere que todos los armónicos pares presenten un cortocircuito en el drenador virtual.

En un amplificador de RF no es práctico cargar en un número infinito de armónicos. Raab en [57] y [105] ha demostrado que la mayor parte del aumento del rendimiento debido a la conformación de onda puede ser realizada con solo la presencia de unos poco armónicos correctamente terminados. Las impedancias de la red de carga 
a la frecuencia fundamental (Ecuación 3.3) y a lo armónicos (Ecuación 3.1 y 3.2) en clase F para controlar las formas de onda de tensión y de corriente del dispositivo con el objetivo de obtener el máximo rendimiento son iguales a:

$$
Z_{\text {net }}\left(\omega_{0}\right)=R=\frac{8}{\pi^{2}} \frac{V_{d d}}{I_{0}}
$$

donde $R$ es la impedancia de la red de carga a la frecuencia fundamental (o resistencia de la línea de carga óptima), $V_{d d}$ es la tensión de alimentación e $I_{0}$ es la componente de corriente continua [50].

El funcionamiento en clase $\mathrm{F}$ se ha empleado para conseguir más de un $80 \%$ de PAE a $2 \mathrm{GHz}$ con una potencia de salida de $16.5 \mathrm{~W}$ en [106] mediante el control de las impedancias a $2^{\circ}, 3^{\circ}$ y 4 armónico. En [107] se presenta un diseño de amplificador de potencia clase $\mathrm{F}$ inversa con terminación multiarmónica que emplea un transistor de $\mathrm{GaN}$ que opera a $2.5 \mathrm{GHz}$ y ofrece una potencia de salida de $12.7 \mathrm{~W}$ con $0.5 \mathrm{~W}$ de potencia de entrada y un $73.5 \%$ de PAE. Esta clase de funcionamiento proporciona como beneficio que la impedancia de carga a la frecuencia fundamental es alta y reduce la sensibilidad a la resistencia de conducción del transistor [108].

\subsubsection{Prototipo 1}

El primer diseño de amplificador clase F se implementó empleando el útil de pre-adaptación de impedancias a los armónicos. En este caso, se diseñó una red de adaptación de entrada y de salida que contenía una serie de stubs a diferentes distancias de la DUT y todos conectados a una línea principal de $50 \mathrm{Ohm}$. El útil fue diseñado para controlar los dos primeros armónicos. En la Figura 3.29 se puede ver el prototipo implementado. 


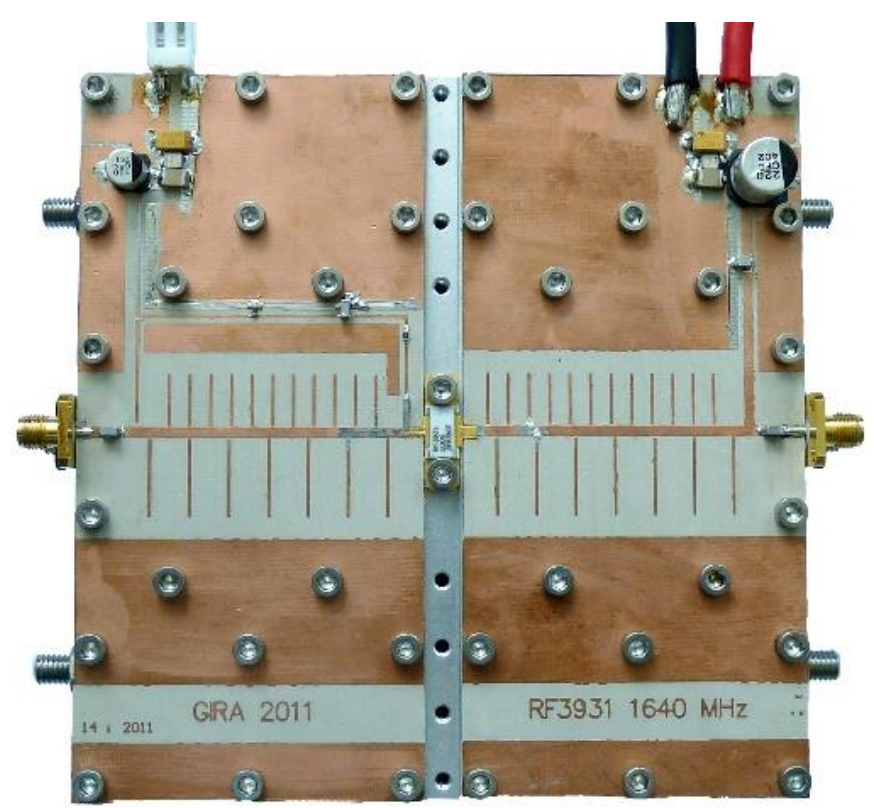

Figura 3.29. Prototipo de amplificador clase $F$ con control de armónicos a través de un útil de pre-adaptación de impedancias a los armónicos (prototipo 1).

Con la ayuda de un programa de simulación y gracias al modelo del encapsulado ofrecido por el fabricante y a las capacidades internas del dispositivo calculadas en este capítulo, se va a determinar que configuraciones de stubs son las que más se acercan al objetivo buscado, es decir, que configuración hace que se pueda presentar cortocircuitos a $2 f_{0}$ y $3 f_{0}$ en la red de entrada y un cortocircuito a $2 f_{0}$ y un circuito abierto a $3 f_{0}$ en la red de salida, todo ello en el plano P1 (drenador intrínseco).

Tras analizar cada una de las configuraciones, teniendo en cuenta que el resonador más cercano a la DUT debe ser el de frecuencia más alta, la combinación de stubs que permite implementar esas impedancias a los armónicos es:

- Para la red de entrada, el primer $s t u b$ a $3 f o$ y el segundo stub a $2 f o$.

- Para la red de salida, el tercer stub a $3 f_{0}$ y el segundo stub a $2 f_{0}$.

Simulando estás combinaciones anteriores en AWR, se obtienen la siguiente transformación de impedancias del plano de referencia P4 al P1. De la Figura 3.30 se pueden obtener las siguientes conclusiones: a $2 f o$, prácticamente cualquier impedancia de carga tenderá a fijar un cortocircuito, tanto a la entrada como a la salida; a $3 f o$, para cualquier impedancia de carga se fijará un cortocircuito a la entrada y un circuito abierto a la salida. 

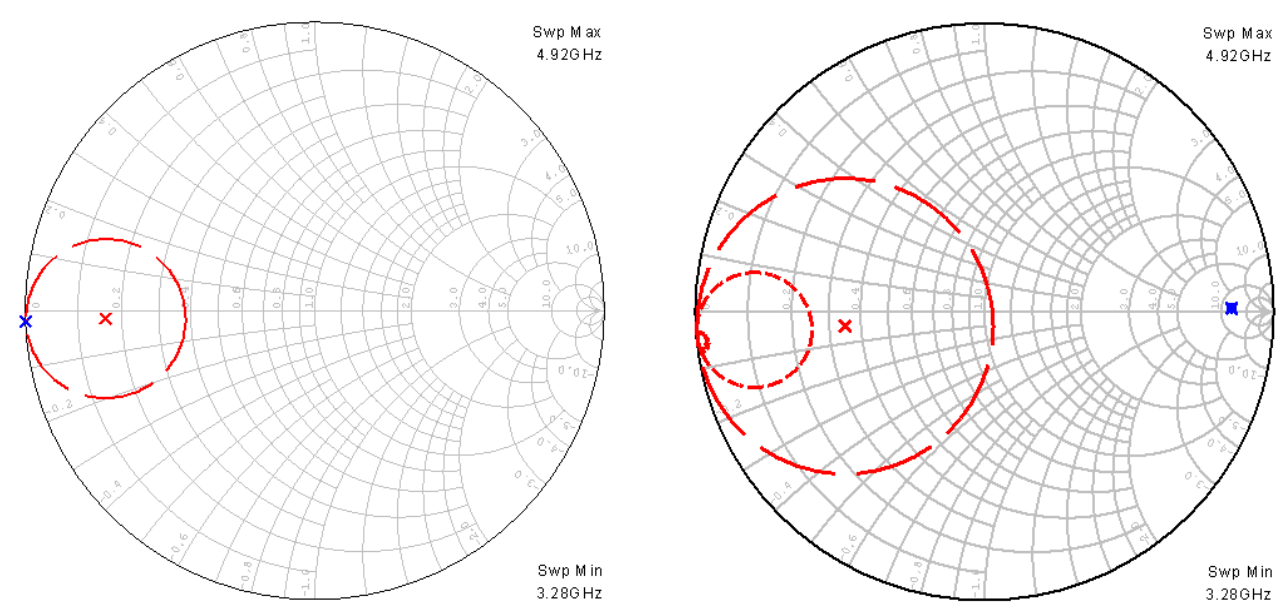

Figura 3.30. Transformación de impedancias del P1 al P4 a la entrada (izq) y a la salida (drch) del prototipo 1. En rojo $2 f_{0}$ y en azul $3 f_{0}$, siendo la $f_{0}=1640 \mathrm{MHz}$.

El siguiente paso es aplicar el método source pull para fijar la máxima ganancia del dispositivo. Tras aplicar la correcta polarización al dispositivo se sintoniza el tunner de entrada en busca de la impedancia que genera máxima ganancia. Fijada dicha impedancia se procede con el método load pull. De la misma manera que el método source pull, se manipula el sintonizador manual a la salida de tal manera que se fije aquella impedancia de carga que consiga alguno de estos 2 objetivos:

- Máxima potencia de salida a la frecuencia fundamental.

- Máximo rendimiento a la frecuencia fundamental.

Normalmente estas dos impedancias no suelen coincidir, es más, muchas veces provocan una reducción de las prestaciones con respecto al otro parámetro. Esto quiere decir que sintonizando la impedancia de carga para conseguir máximo rendimiento puede que reduzca considerablemente la potencia de salida que puede entregar el dispositivo, y al contrario. Por ello, en muchas ocasiones a la hora de diseñar el amplificador de potencia se busca una impedancia de compromiso para conseguir una alta potencia y un alto rendimiento.

En este trabajo, tras sintonizar ambas impedancias y analizar los resultados obtenidos empíricamente, se decidió sintonizar una impedancia de compromiso. Una vez fueron fijadas las impedancias de entrada y de salida en los sintonizadores mecánicos se procedió a realizar las medidas de las impedancias vistas por el DUT en el plano P3 usando los bloques TRL con los que se calibró el analizador de redes. 
A continuación, haciendo uso del programa de simulación AWR, se procedió a desempotrar el encapsulado del dispositivo y las capacidades internas con el fin de representar las impedancias vistas por el dispositivo intrínseco. En la Figura 3.31 se pueden ver las impedancias vistas por el dispositivo en el plano P1. A la entrada se puede verificar, como se preveía de la Figura 3.30, que las impedancias a los armónicos son cortocircuitos en un ancho de banda de $120 \mathrm{MHz}$; a la salida, en cambio, a $2 f_{0}$ sí se fija un cortocircuito dentro de ese ancho de banda, pero a $3 f_{0}$ se fija un circuito abierto a la frecuencia central y a medida que se desvía de esa frecuencia se mantiene el coeficiente de reflexión pero cambia la fase. Esta situación provocará que se vayan perdiendo las características de funcionamiento en clase F.
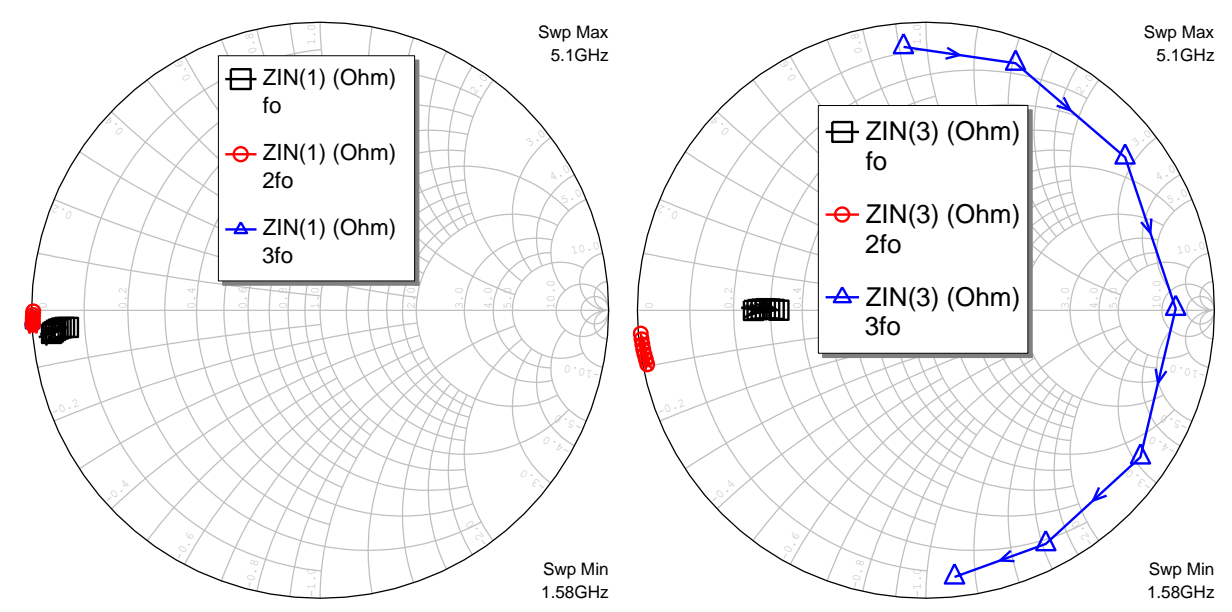

Figura 3.31. Medida de las impedancias intrínsecas a partir de la transformación de impedancias del P3 al P1 a la entrada (izq) y a la salida (drch) del prototipo 1. En rojo $2 f_{0}$ y en azul $3 f_{0}$, siendo la $f_{0}=1640 \mathrm{MHz}$.

Conocidas las impedancias de entrada y de salida que generan alta potencia y alto rendimiento en un funcionamiento en clase $\mathrm{F}$, se puede simular el comportamiento del amplificador. En este caso, se va a emplear un diseño simplificado del amplificador real por lo que es posible que existan pequeñas diferencias respecto a las medidas reales. En la Figura 3.32 se puede ver el esquema del amplificador de potencia: consta de los sintonizadores de entrada y de salida, de las redes de pre-adaptación simplificadas, del bloque de parámetros $\mathrm{S}$ del encapsulado y del modelo no lineal de la die del dispositivo (estos dos últimos ofrecidos por el fabricante). Las condiciones de trabajo que se emplean en la simulación son las mismas que las utilizadas en las medidas empíricas con el método load pull: $V_{g s}=-3.5 \mathrm{~V}$ y $V_{d d}=48 \mathrm{~V}$. 


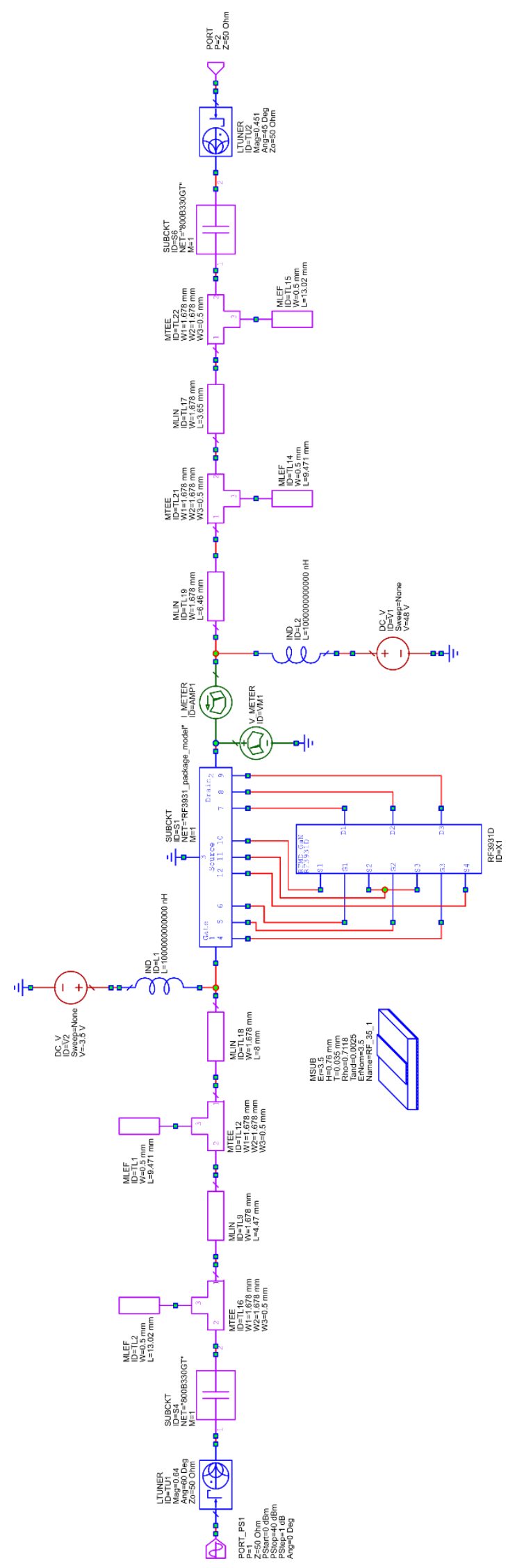

Figura 3.32. Esquema del amplificador de potencia clase $F$ diseñado con el método load pull a partir del útil de pre-adaptación de impedancias (prototipo 1). 
A continuación, se pueden ver los resultados de la simulación del amplificador clase F diseñado. La Figura 3.33 muestra las formas de onda en el plano P2 del amplificador en la zona de saturación: se puede apreciar que la onda de tensión se aproxima a una onda cuadra y la onda de corriente a una señal sinusoidal rectificada, características de un amplificador clase F. Recordar que las formas de onda de un amplificador clase F deberían ser tomadas en el plano P1, es decir, en el drenador intrínseco del dispositivo. Sin embargo, este punto no está accesible en la simulación, pero la simulación en el plano P2 es una buena manera de comprobar el funcionamiento. Otro punto que nos indica que el amplificador está trabajando en clase F es la tensión de drenador: como ya se comentó en el Capítulo 2, la tensión de drenador en un amplificador clase $\mathrm{F}$ es aproximadamente 2 veces la tensión de alimentación. Además, la impedancia a la frecuencia fundamental de un amplificador clase F es resistiva [89], como se ha podido comprobar en la Figura 3.31.

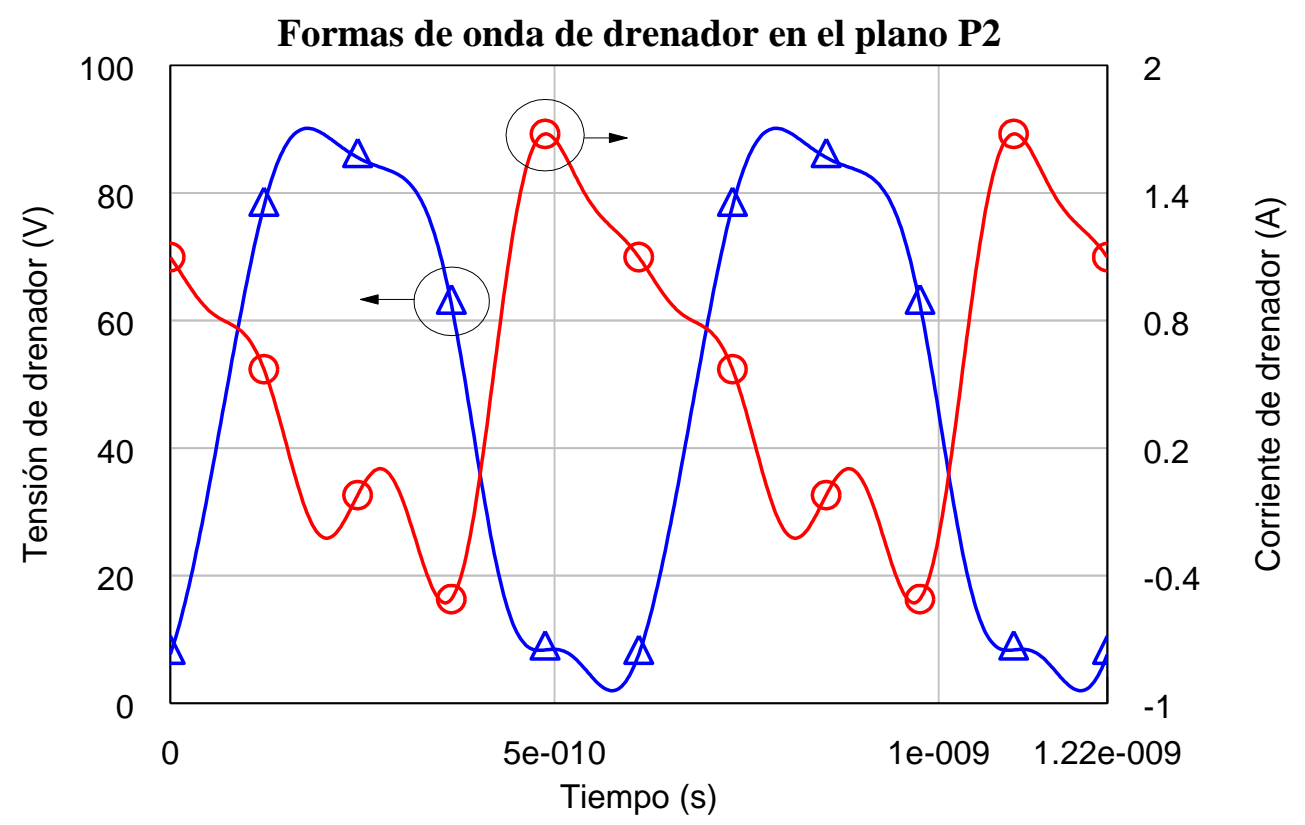

Figura 3.33. Simulación de las formas de onda de drenador en el plano P2 del amplificador de potencia clase $F$ diseñado (prototipo 1).

En la Figura 3.34 y la Figura 3.35 se muestra la simulación de funcionamiento del amplificador en función de la frecuencia y de la potencia de entrada, respectivamente. A la frecuencia central y excitado con $32 \mathrm{dBm}$, el amplificador es capaz de ofrecer una potencia de salida de $46.83 \mathrm{dBm}(48.2 \mathrm{~W})$ con un rendimiento de drenador del 66\% y una ganancia de $14.83 \mathrm{~dB}$. En la zona de saturación, el amplificador 
puede alcanzar un rendimiento de drenador del $75 \%$ y una potencia máxima de 47.78 $\mathrm{dBm}(\approx 60 \mathrm{~W})$. Un funcionamiento por encima del $60 \%$ de rendimiento de drenador se puede conseguir dentro de un ancho de banda de aproximadamente $120 \mathrm{MHz}$, siendo la potencia superior a $46.17 \mathrm{dBm}(41.4 \mathrm{~W})$. Estos resultados simulados mejoran las prestaciones indicadas por el fabricante del dispositivo en su hoja de características [90]

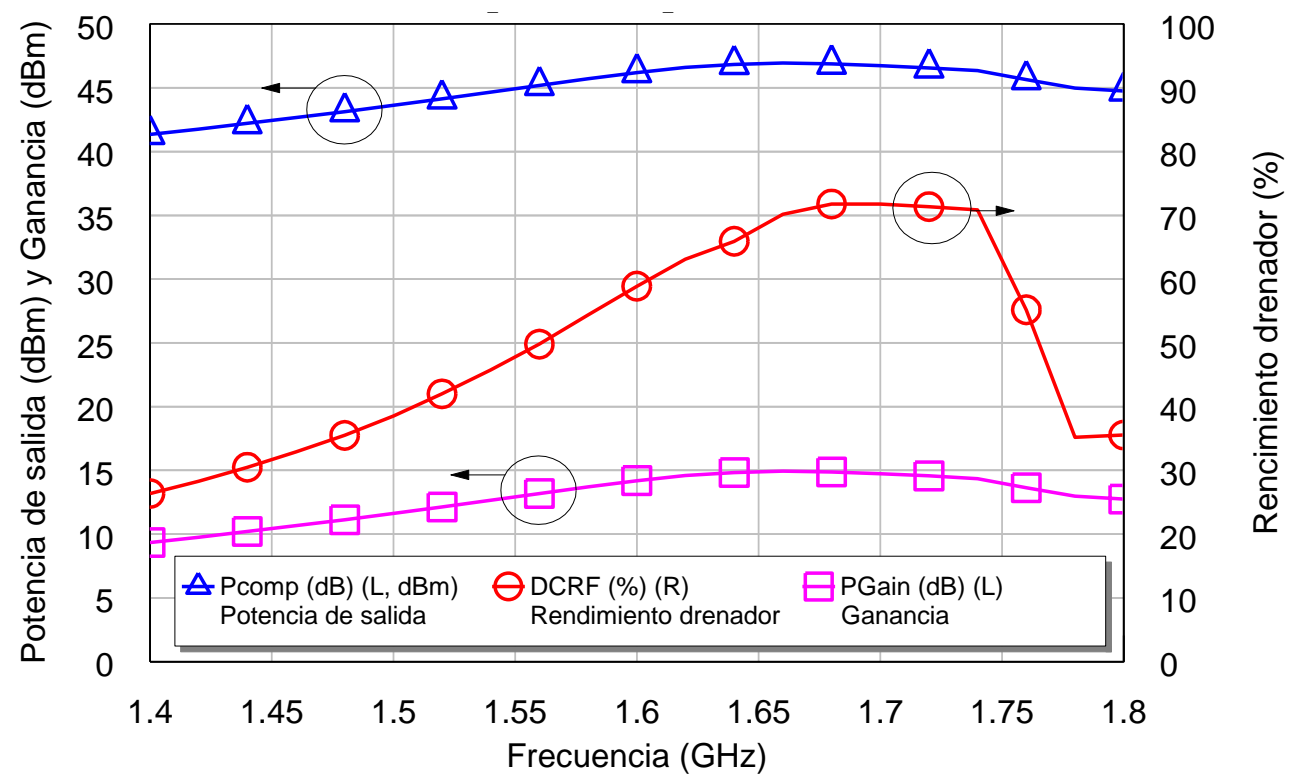

Figura 3.34. Simulación del funcionamiento del amplificador clase $F$ (prototipo 2 ) en función de la frecuencia.

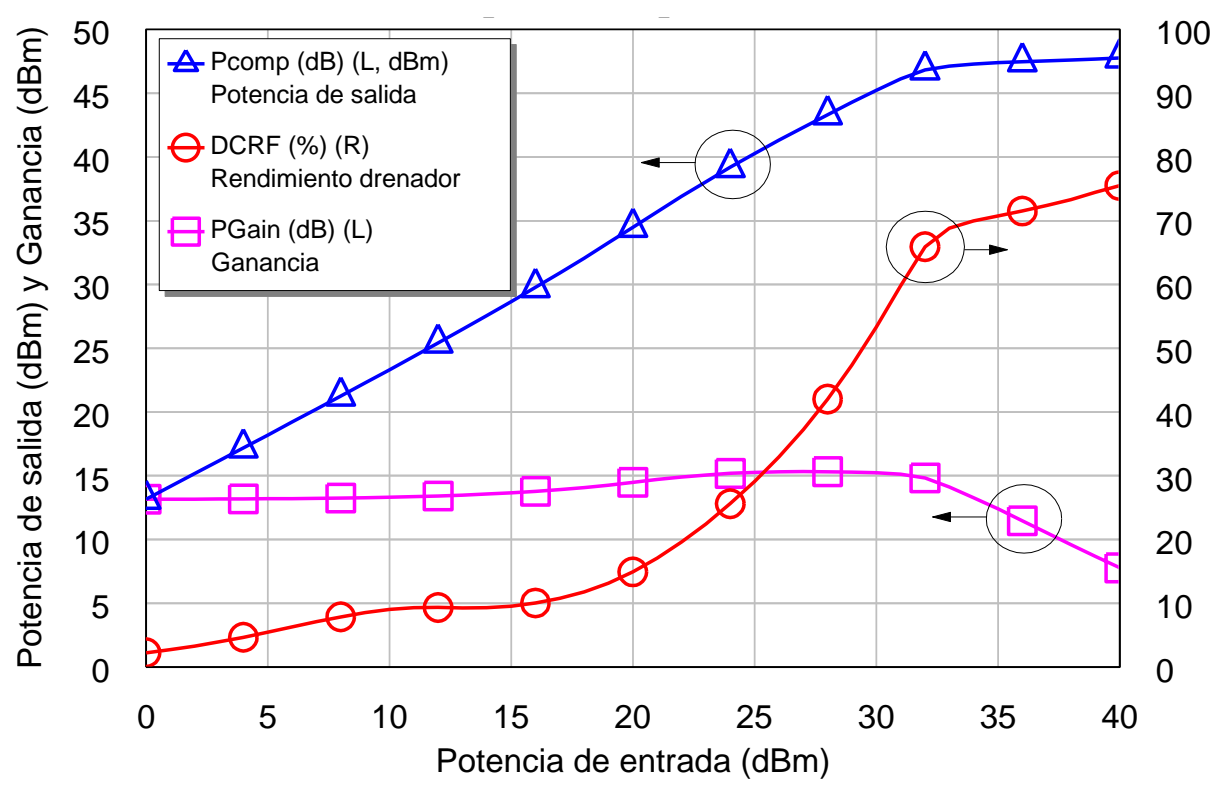

Figura 3.35. Simulación del funcionamiento del amplificador clase $F$ (prototipo 1) en función de la potencia de entrada. 


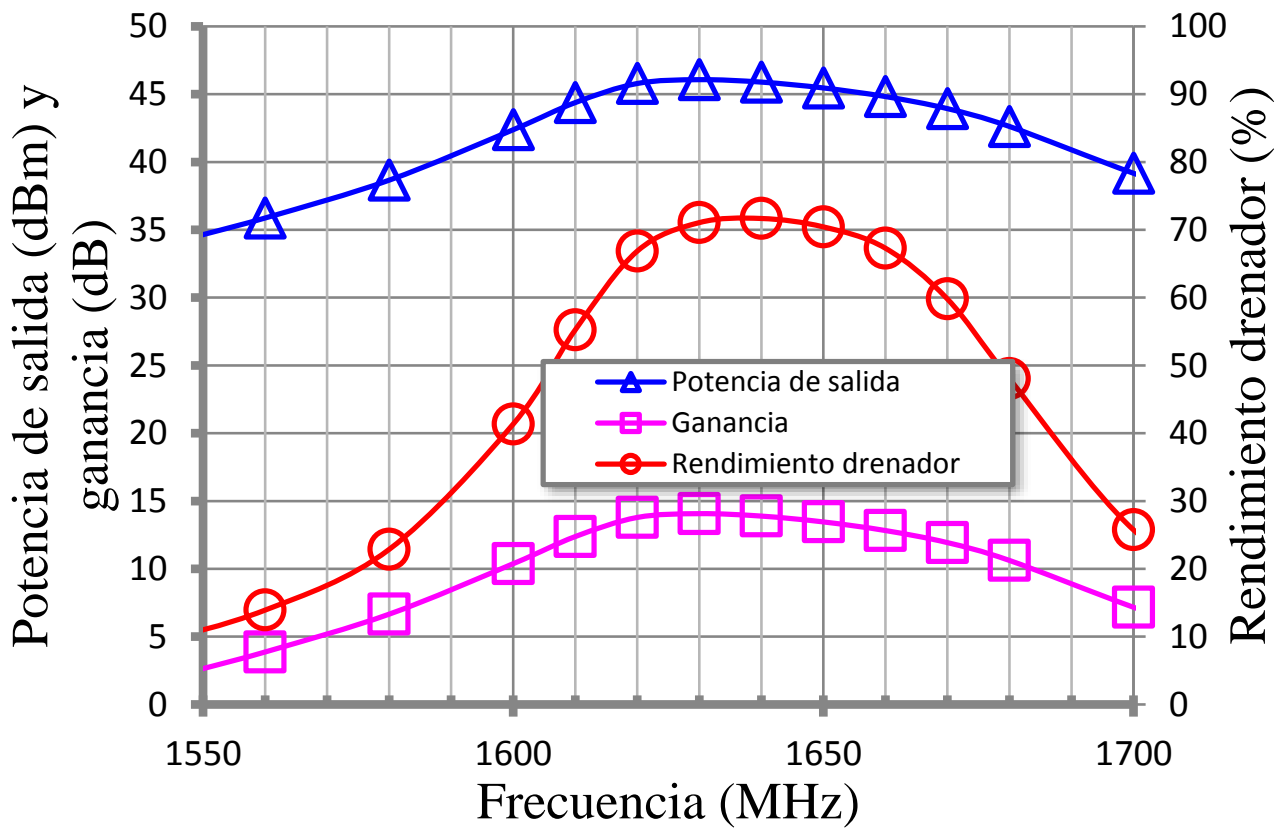

Figura 3.36. Medidas del funcionamiento del amplificador clase F (prototipo 1) en función de la frecuencia.

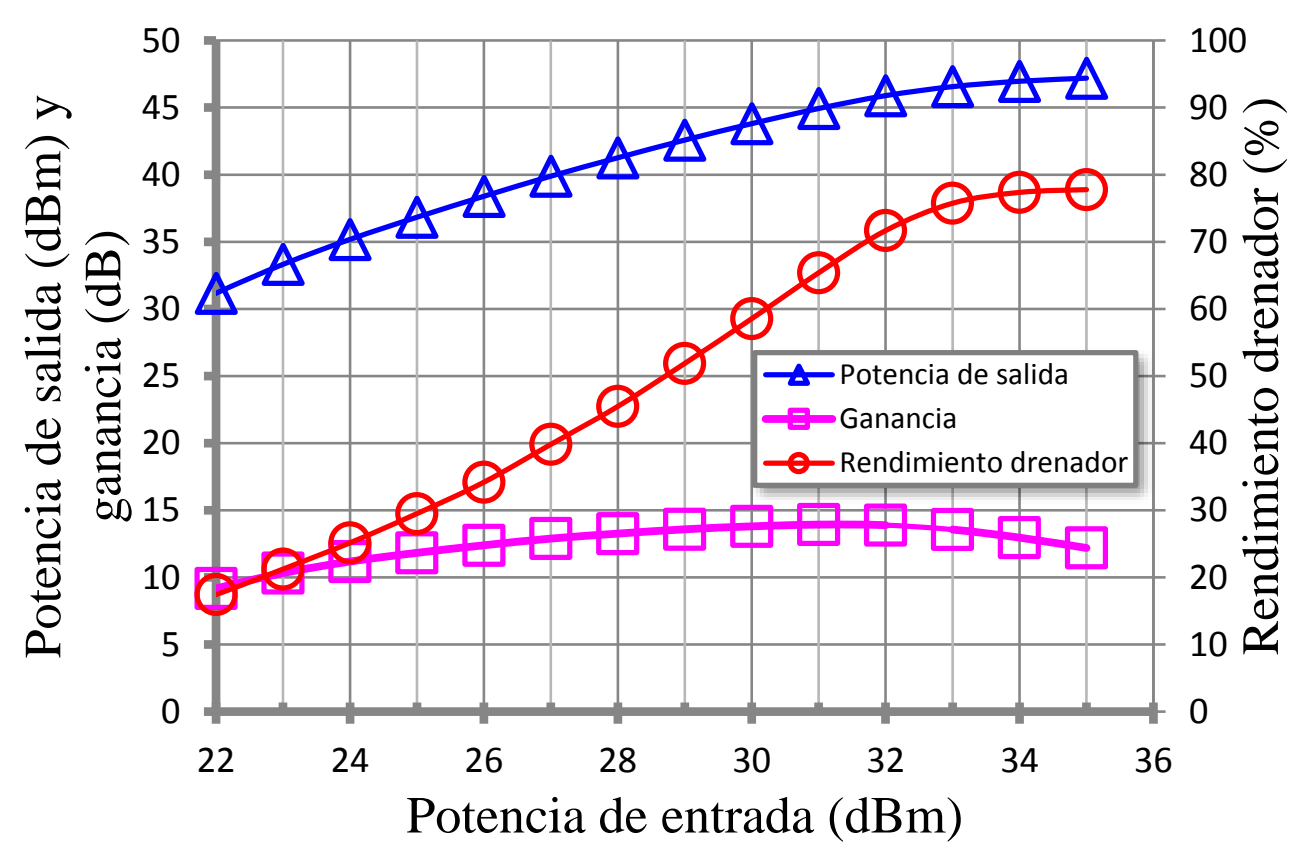

Figura 3.37. Medidas del funcionamiento del amplificador clase $F$ (prototipo 1) en función de la potencia de entrada.

En la Figura 3.36 y la Figura 3.37 se muestra los resultados obtenidos de las medidas empíricas realizadas con el método load pull. A la frecuencia central y excitado con $32 \mathrm{dBm}$, el amplificador es capaz de ofrecer una potencia de salida de $45.89 \mathrm{dBm}$ $(38.81 \mathrm{~W})$ con un rendimiento de drenador del $72 \%$ y una ganancia de $13.89 \mathrm{~dB}$. En la zona de saturación, el amplificador puede alcanzar un rendimiento de drenador del $78 \%$ 
y una potencia máxima de $47.19 \mathrm{dBm}(\approx 52.36 \mathrm{~W})$. Un funcionamiento por encima del $60 \%$ de rendimiento de drenador se puede conseguir dentro de un ancho de banda de aproximadamente $60 \mathrm{MHz}$, siendo la potencia superior a $44 \mathrm{dBm}(25.11 \mathrm{~W})$.

Comparando las simulaciones con las medidas se puede comprobar que hay grandes diferencias en cuanto a potencia y ancho de banda. Esto puede ser debido a:

- En el diseño simulado no se han tenido en cuenta las pérdidas debidas a la línea de $50 \Omega$ que conecta los stubs con los sintonizadores y también las pérdidas de los sintonizadores. Esto puede provocar una gran diferencia en cuanto a potencia obtenida.

- En el diseño simulado, las redes de alimentación se han simulado como ideales. En el circuito real, estas redes también pueden influir en los resultados, aunque si estuvieran correctamente diseñadas no deberían modificar significativamente el comportamiento.

- Los stubs generan el comportamiento deseado (cortocircuito o circuito abierto) en banda estrecha, lo que explica que el funcionamiento en clase F no se mantenga si se desplaza de la frecuencia central, reduciendo el ancho de banda.

- El transistor RF3931 presenta un encapsulado con unos pads de conexión mucho más anchos que una línea de $50 \Omega$. Con el fin de evitar una mayor complejidad, se recortaron esos pads de conexión de entrada y salida para hacerlos coincidir con una anchura igual a la línea de $50 \Omega$. Esto provoca que el encapsulado quede modificado y por lo tanto ya no sea comparable con el ofrecido por el fabricante, añadiendo un nuevo error de medida. En la Figura 3.38 se puede ver en detalle esta modificación.

El siguiente prototipo intentará corregir alguna de estas peculiaridades intentando mantener los buenos resultados obtenidos con el método load-pull. 


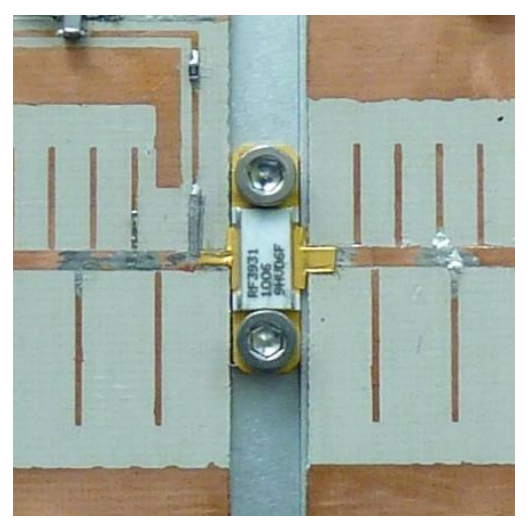

Figura 3.38. Detalle de la adaptación/recorte de los pads del dispositivo en la realización de las medidas load pull.

\subsubsection{Prototipo 2}

El segundo diseño de amplificador clase $\mathrm{F}$ también fue diseñado utilizando el método source pull y load pull con la diferencia respecto al anterior prototipo de que se tuvieron en cuenta algunas de las imprecisiones que se cometieron conscientemente. En la Figura 3.39 se presenta el prototipo implementado.

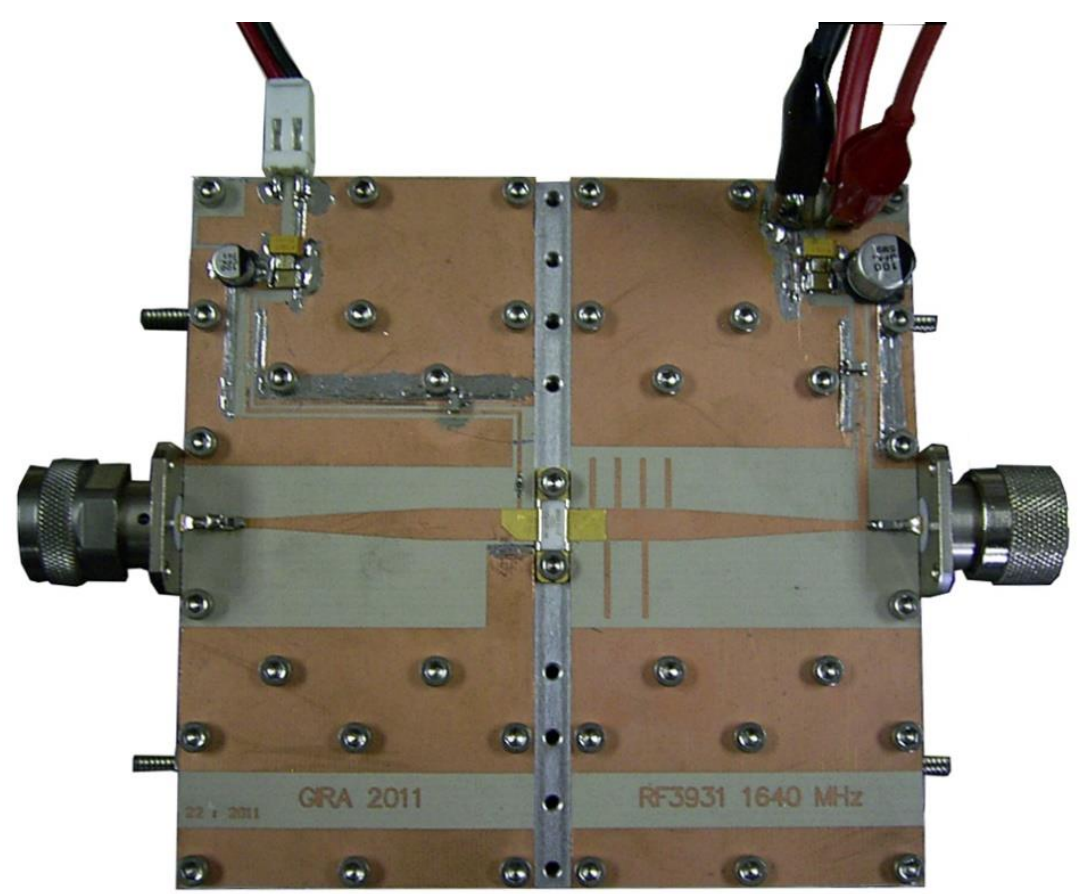

Figura 3.39. Prototipo de amplificador clase $F$ con control de armónicos a través de un útil de pre-adaptación de impedancias a los armónicos (prototipo 2). 
En primer lugar, se ha tenido en cuenta la anchura de los pads del transistor. Incrementar la anchura de la pista hace que la impedancia característica de la línea se reduzca. Ello puede ayudar a adaptar el dispositivo ya que normalmente presenta impedancias muy pequeñas debida a las capacidades internas. Por ello, en este prototipo se optó por la opción de incluir un transformador de impedancia.

Existen múltiples opciones a la hora de diseñar un transformador de impedancia con línea de transmisión. El más práctico y sencillo, especialmente para adaptar impedancias reales, es un transformador $\lambda / 4$. También es posible adaptar una impedancia compleja usando este tipo de transformador, pero necesita una longitud adicional de línea de transmisión entre la carga y el transformador que transforma la impedancia de carga compleja a una impedancia real. Este transformador consiste en una línea de transmisión de esa longitud (90 grados eléctricos) a la frecuencia fundamental y cuya impedancia característica es la raíz cuadrada del producto de las impedancias características de ambos extremos. El principal inconveniente de este elemento es que adapta en un ancho de banda limitado.

Con la intención de extender el funcionamiento del transformador en un rango mayor de frecuencia, se puede usar transformadores con múltiples secciones donde cada una presenta una impedancia característica diferente. Cada una de las secciones debe tener la misma longitud y la respuesta del coeficiente de reflexión deseado en función de la frecuencia se puede conseguir seleccionando correctamente la impedancia característica de cada sección. En el caso de tener un número infinito de secciones, el transformador tiene forma cónica. Sin embargo, otras formas pueden ser consideradas en función de las características de funcionamiento: lineal, triangular, exponencial...

Para una longitud de transformador concreta, el transformador de Klopfenstein se considera el más óptimo desde el punto de vista de minimizar el coeficiente de reflexión dentro de la banda de paso [109].

En el caso de este prototipo, y por consideraciones prácticas de implementación, se utilizará un transformador multisección lineal con una longitud de $\lambda / 4$, como se puede ver en la Figura 3.40. 


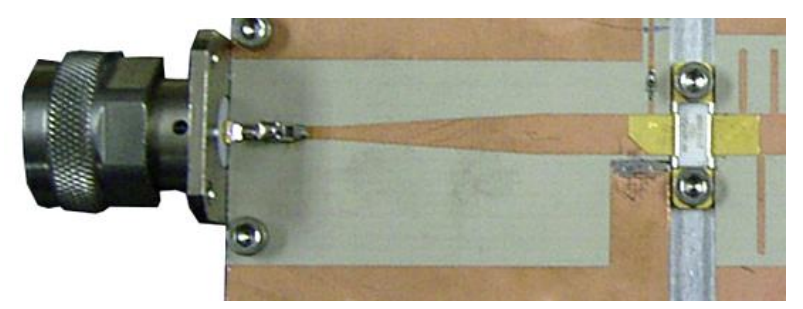

Figura 3.40. Detalle de la red de entrada del amplificador clase $F$ (prototipo 2) donde se ve el transformador multisección lineal con una longitud de $\lambda / 4$.

Debido a la utilización del transformador, hay que tener en cuenta que la impedancia característica en el plano P3 cambia (ya no es $50 \Omega$ como en el prototipo 1 ). Esto implica que el kit de calibración también debe ser rediseñado. En la Figura 3.41 se muestra el kit de calibración empleado para medir las impedancias vistas por el dispositivo. Como se puede observar, se han implementado los tres estándares (Thru, Reflect y Line) con la peculiaridad de que presentan un transformador de impedancia multisección lineal. La longitud de este transformador es independiente para la calibración y las medidas, ya que se fija en el plano de referencia la misma impedancia característica que ve el dispositivo.

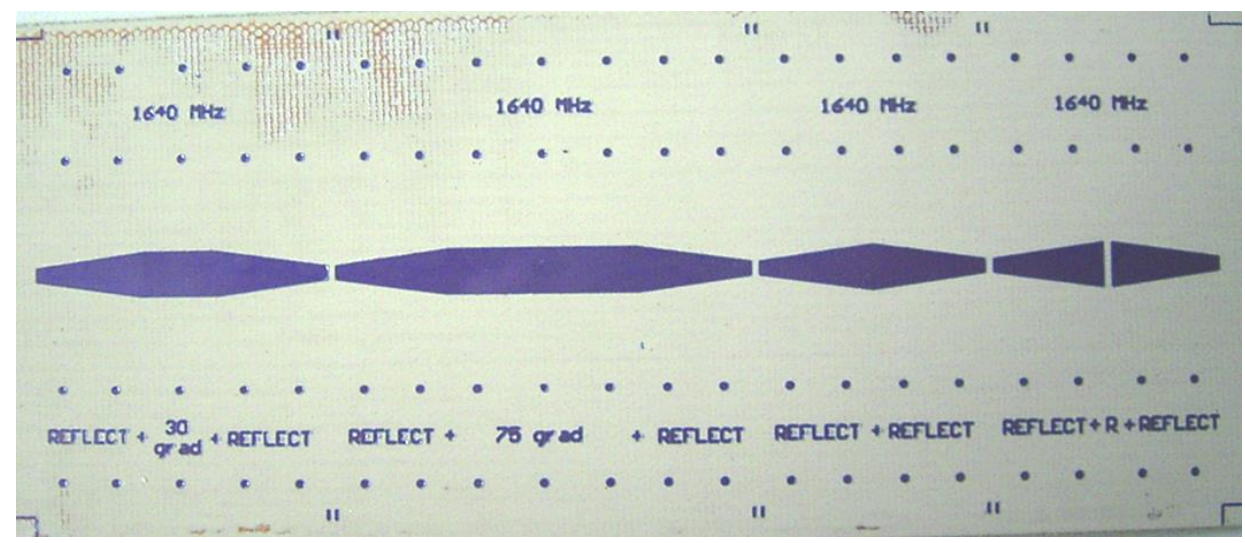

Figura 3.41. Fotografía real de los estándares de calibración TRL diseñados con el transformador multisección lineal.

Otro punto a tener en cuenta en el diseño de este prototipo es que se va a prescindir de controlar los armónicos en la red de entrada. Esto se debe a que simplemente con el encapsulado del dispositivo y sus elementos internos prácticamente se fijan cortocircuitos a los dos primeros armónicos, generando una onda de excitación adecuada para conmutar el dispositivo eficientemente. A la salida si se han incluido los stubs para controlar la impedancia a los dos primeros armónicos. 
Al igual que en el prototipo 1, con la ayuda de un programa de simulación y el modelo de encapsulado y las capacidades internas del dispositivo previamente calculadas, se determinó que configuraciones de stubs eran las que más se acercaban al objetivo de fijar un cortocircuito a $2 f_{0}$ y un circuito abierto a $3 f_{0}$ en la red de salida en el plano P1 (drenador intrínseco).

Tras analizar cada una de las configuraciones, la combinación de stubs que permite implementar esas impedancias a los armónicos en la red de salida son: el segundo stub a $3 f o$ y el segundo stub a $2 f_{0}$.

En la Figura 3.42 se muestra la transformación de impedancias del plano de referencia P4 al P1 resultante de la simulación de esa combinación de stubs. De la simulación se pueden obtener las siguientes conclusiones: a $2 f_{0}$, prácticamente cualquier impedancia de carga tenderá a fijar un cortocircuito; a $3 f_{0}$, se tenderá a fijar un circuito abierto, pero hay un gran abanico de impedancias de carga que no cumplirían esa premisa.
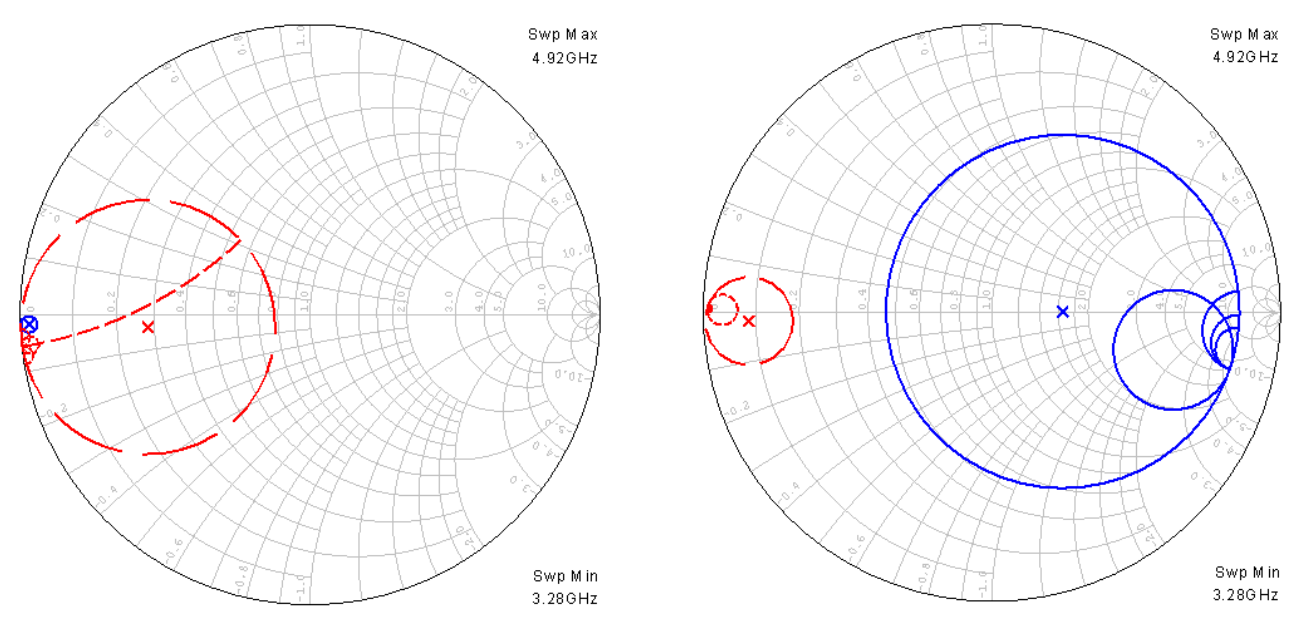

Figura 3.42. Transformación de impedancias del P1 al P4 a la entrada (izq) y a la salida (drch) del prototipo 2. En rojo $2 f_{0}$ y en azul $3 f_{0}$, siendo la $f_{0}=1640 \mathrm{MHz}$.

Posteriormente, se polarizó el dispositivo y se procedió a aplicar el método source pull en búsqueda de la impedancia a la entrada que genera la máxima ganancia del dispositivo. Tras ello, se implementó el método load pull variando la impedancia del sintonizador de salida hasta conseguir encontrar aquella impedancia que consigue una alta potencia y un alto rendimiento, solución de compromiso entre la impedancia que consigue la máxima potencia de salida y aquella que consigue el máximo rendimiento 
de drenador. Y, posteriormente, se procedió a realizar las medidas de las impedancias vistas por la DUT en el plano P3 a partir de los nuevos bloques TRL.

Conocidas las impedancias de entrada y de salida que fijaban el funcionamiento del amplificador, se procedió a desempotrar el encapsulado del dispositivo y las capacidades internas para representar las impedancias vistas por el dispositivo intrínseco (Figura 3.43). Tal y como se preveía, a la entrada se puede verificar que las impedancias a los dos primeros armónicos son prácticamente cortocircuitos en un ancho de banda de más de $280 \mathrm{MHz}$; a la salida, es más complicado mantener la impedancia deseada dentro de ese ancho de banda, por lo que, como en el caso anterior, a medida que nos vayamos alejando de la frecuencia central se irán perdiendo las características de funcionamiento en clase $\mathrm{F}$.
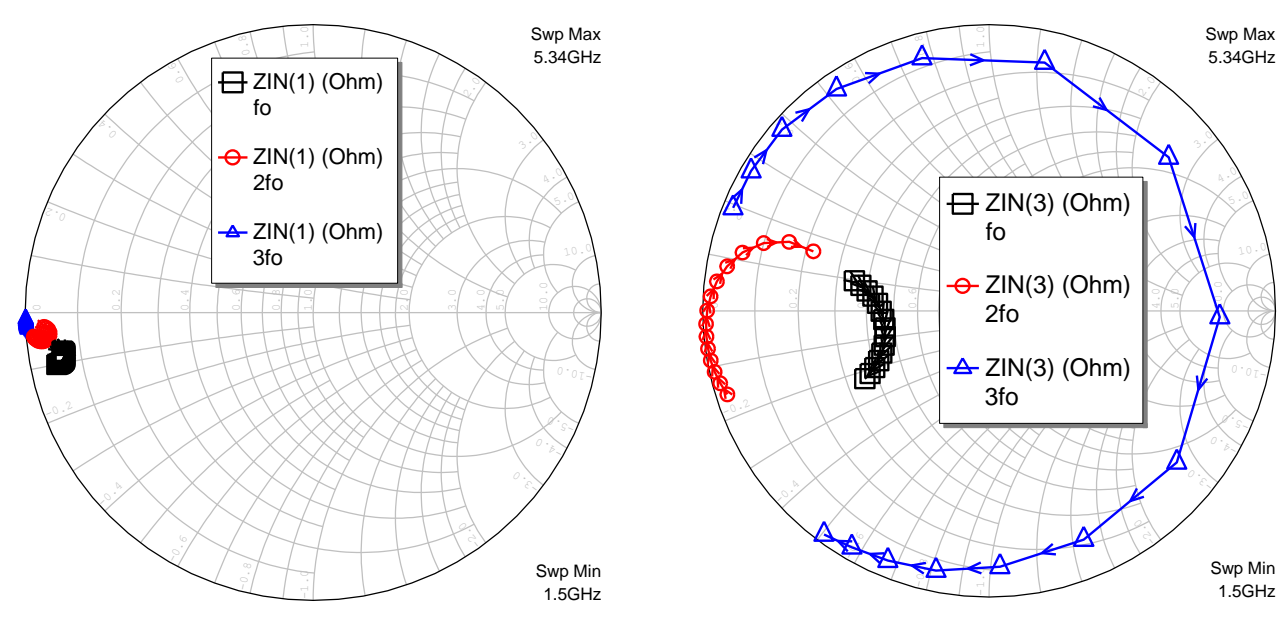

Figura 3.43. Medida de las impedancias intrínsecas a partir de la transformación de impedancias del P3 al P1 a la entrada (izq) y a la salida (drch) del prototipo 2. En rojo $2 f_{0}$ y en azul $3 f_{0}$, siendo la $f_{0}=1640 \mathrm{MHz}$.

En este punto, se puede simular el comportamiento del amplificador clase F. En la Figura 3.44 se puede ver el esquema del amplificador de potencia. Las condiciones de trabajo que se emplean en la simulación son las mismas que las utilizadas en las medidas empíricas con el método load pull:

- Tensión puerta-fuente (Vgs): $-3.5 \mathrm{~V}$

- Tensión de alimentación (Vdd): 48V 


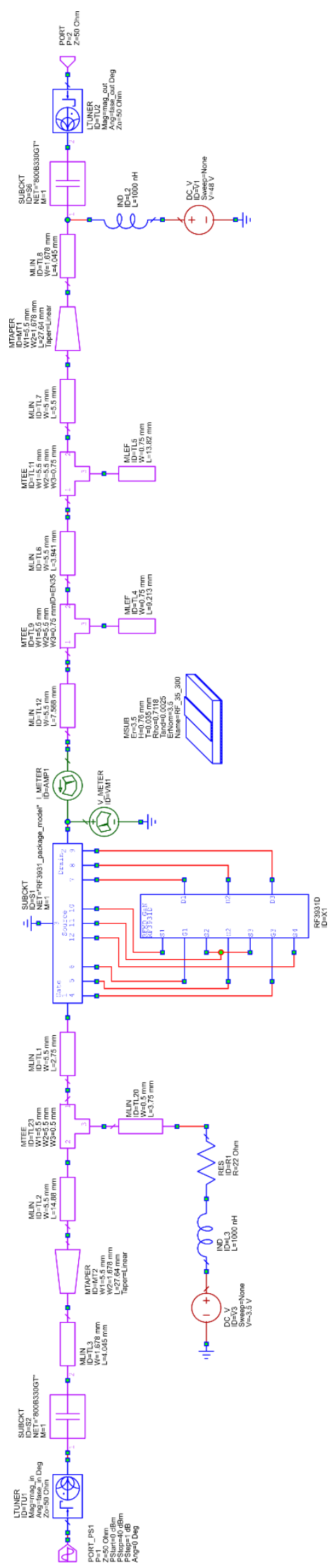

Figura 3.44. Esquema del amplificador de potencia clase $F$ diseñado con el método load pull a partir del útil de pre-adaptación de impedancias (prototipo 2). 
A continuación, se pueden ver los resultados de la simulación del amplificador clase F diseñado. La Figura 3.45 muestra las formas de onda en el plano P2 del amplificador en la zona de saturación: se puede apreciar que la onda de tensión se aproxima a una onda cuadra y la onda de corriente a una señal sinusoidal rectificada, características de un amplificador clase F.

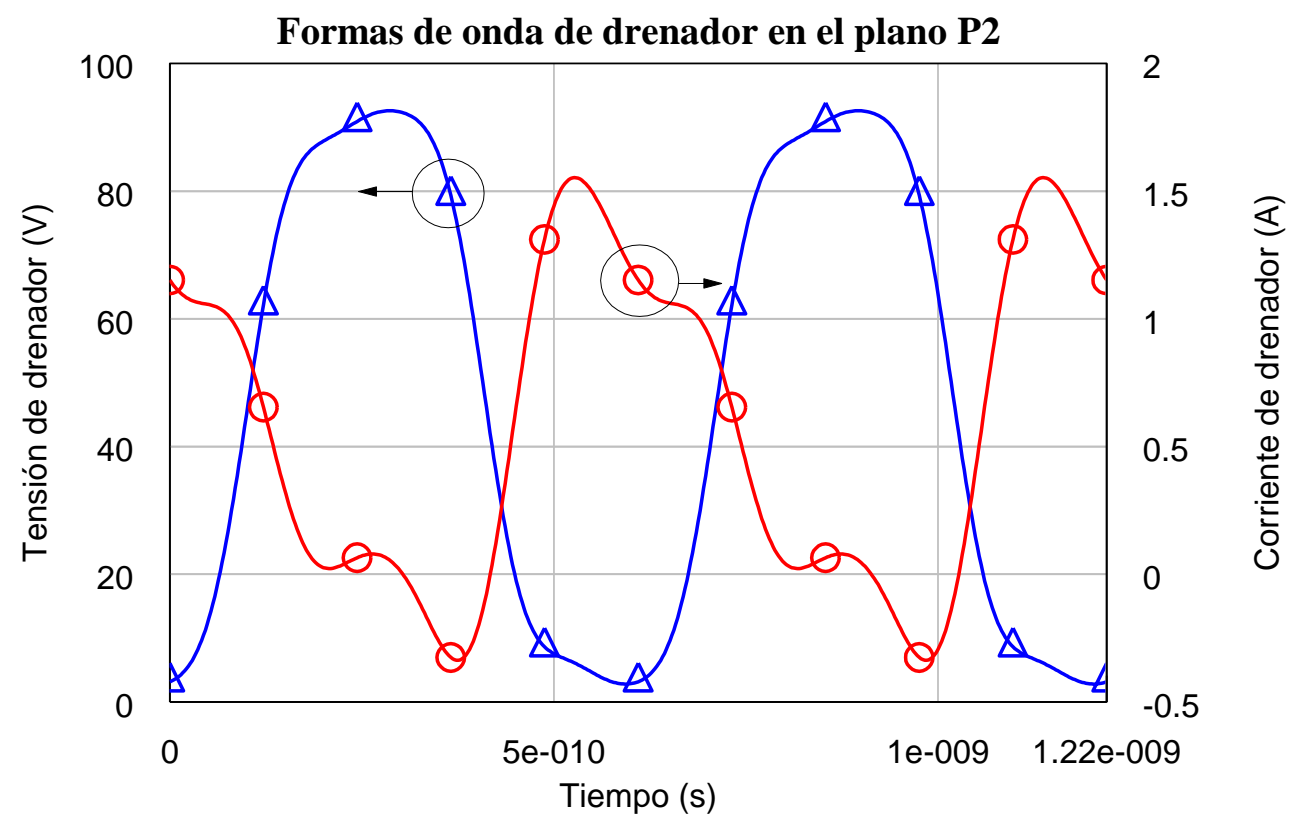

Figura 3.45. Simulación de las formas de onda de drenador en el plano P2 del amplificador de potencia clase $\mathrm{F}$ diseñado (prototipo 2 ).

En la Figura 3.46 y la Figura 3.47 se muestra la simulación del amplificador en función de la frecuencia y de la potencia de entrada, respectivamente. A la frecuencia central y excitado con $36 \mathrm{dBm}$, el amplificador es capaz de ofrecer una potencia de salida de $47.45 \mathrm{dBm}(55.59 \mathrm{~W})$ con un rendimiento de drenador del $69 \%$ y una ganancia de $11.45 \mathrm{~dB}$. En la zona de saturación, el amplificador puede alcanzar un rendimiento de drenador del $77 \%$ y una potencia máxima de $47.61 \mathrm{dBm}(\approx 57 \mathrm{~W})$. Un funcionamiento por encima del $60 \%$ de rendimiento de drenador se puede conseguir dentro de un ancho de banda de aproximadamente $300 \mathrm{MHz}$, siendo la potencia superior a $46.65 \mathrm{dBm}(46.3 \mathrm{~W})$. Estos resultados simulados mejoran las prestaciones indicadas por el fabricante del dispositivo en su hoja de datos [90], sobre todo en cuanto a potencia de salida se refiere. 


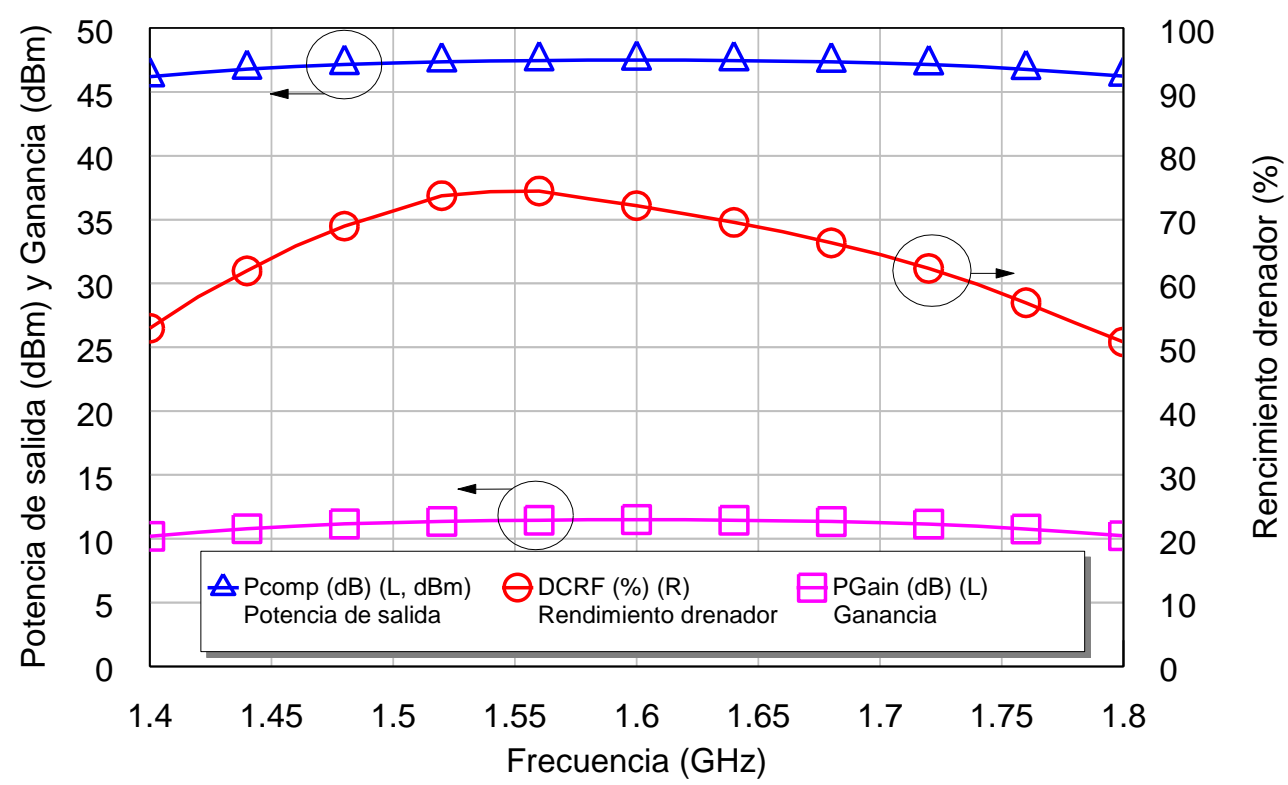

Figura 3.46. Simulación del funcionamiento del amplificador clase $F$ (prototipo 2) en función de la frecuencia.

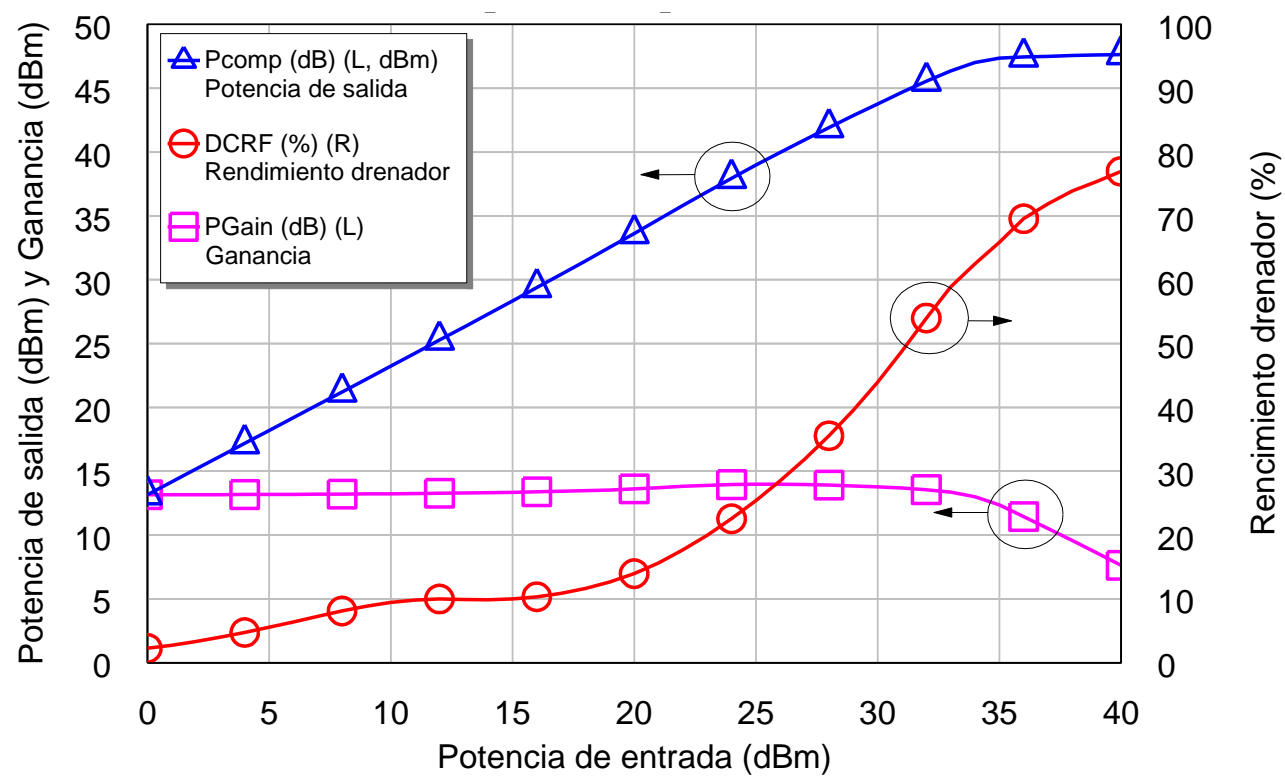

Figura 3.47. Simulación del funcionamiento del amplificador clase $F$ (prototipo 2 ) en función de la potencia de entrada.

En la Figura 3.48 y la Figura 3.49 se muestran los resultados obtenidos de las medidas empíricas realizadas con el método load pull. A la frecuencia central y excitado con $36 \mathrm{dBm}$, el amplificador es capaz de ofrecer una potencia de salida de $44.9 \mathrm{dBm}$ (30.90W) con un rendimiento de drenador del $74.5 \%$ y una ganancia de $8.9 \mathrm{~dB}$. En la zona de saturación, el amplificador puede alcanzar un rendimiento de drenador del $75.5 \%$ y una potencia máxima de $45 \mathrm{dBm}(31.62 \mathrm{~W})$. Un funcionamiento por encima 
del $60 \%$ de rendimiento de drenador se puede conseguir dentro de un ancho de banda de aproximadamente $80 \mathrm{MHz}$, siendo la potencia superior a $43.61 \mathrm{dBm}(23 \mathrm{~W})$.

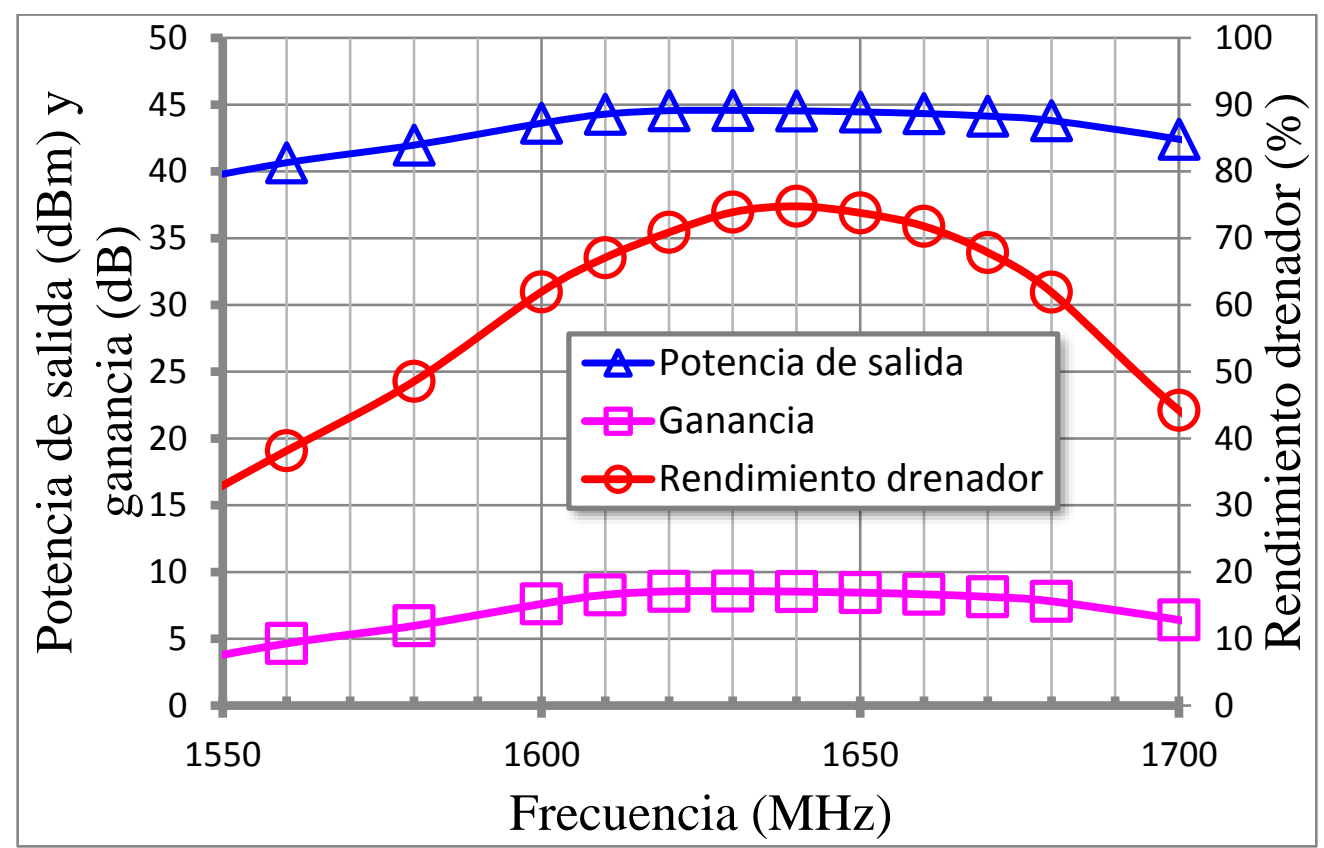

Figura 3.36. Medidas del funcionamiento del amplificador clase $F$ (prototipo 2) en función de la frecuencia.

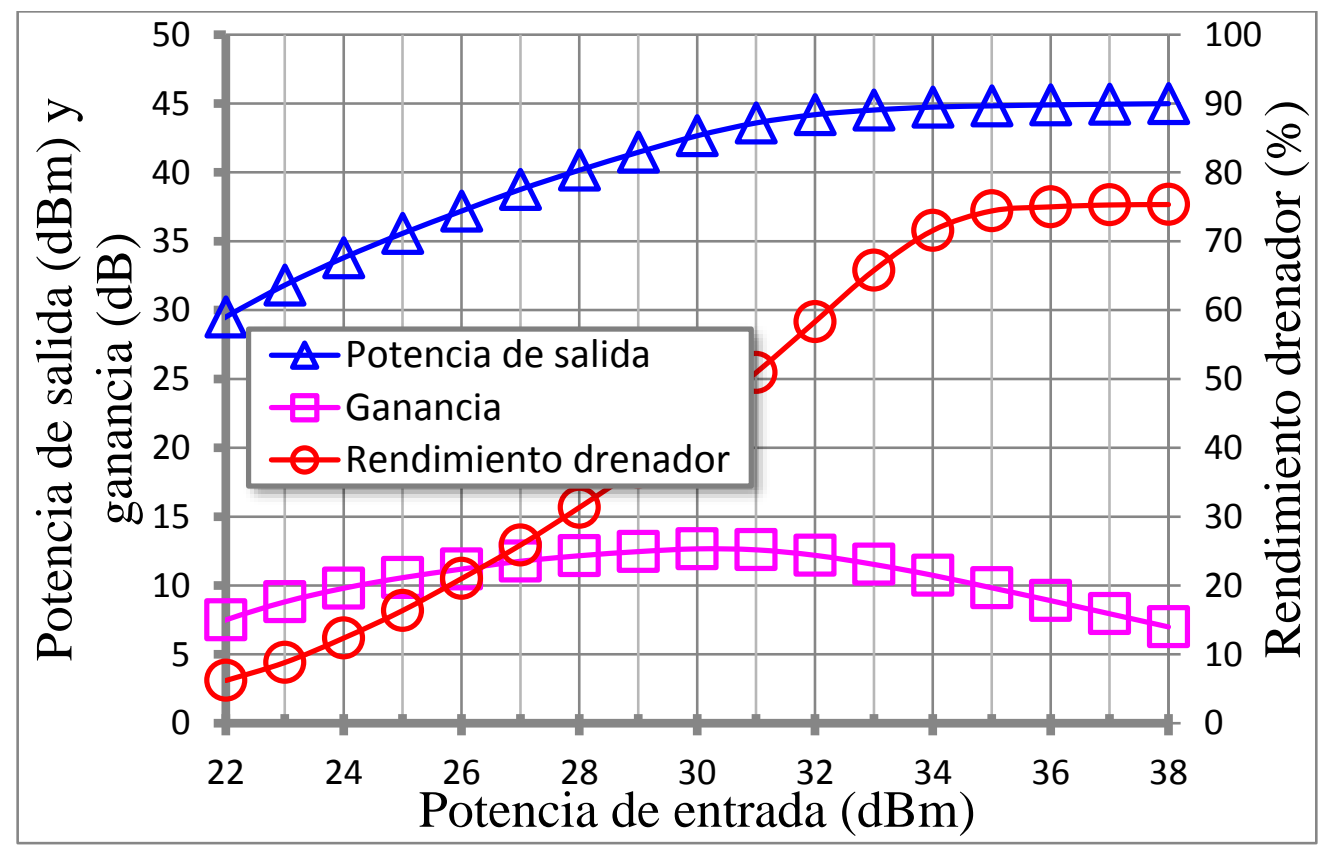

Figura 3.49. Medidas del funcionamiento del amplificador clase $F$ (prototipo 2 ) en función de la potencia de entrada. 
Comparando las simulaciones con las medidas se puede comprobar que hay grandes diferencias en cuanto a la potencia y ancho de banda, tal y como ocurría en el prototipo anterior. En este caso, estas diferencias son debidas a las altas pérdidas del sintonizador y por las imprecisiones a la hora de realizar las medidas de las impedancias vistas por el dispositivo. A pesar de ello, estos dos prototipos han servido para verificar que el modelo de transistor y encapsulado ofrecido por el fabricante puede ser de gran ayuda a la hora de diseñar el amplificador, como se verá en el prototipo 3.

\subsubsection{Prototipo 3}

El tercer y último prototipo de amplificador clase $\mathrm{F}$ que fue diseñado parte de la simulación de las redes de entrada y de salida una vez verificado que el modelo del transistor y del encapsulado que ofrece el fabricante obtiene resultados muy parecidos a los obtenidos empíricamente a través del método load pull. En la Figura 3.50 se puede ver el prototipo implementado.

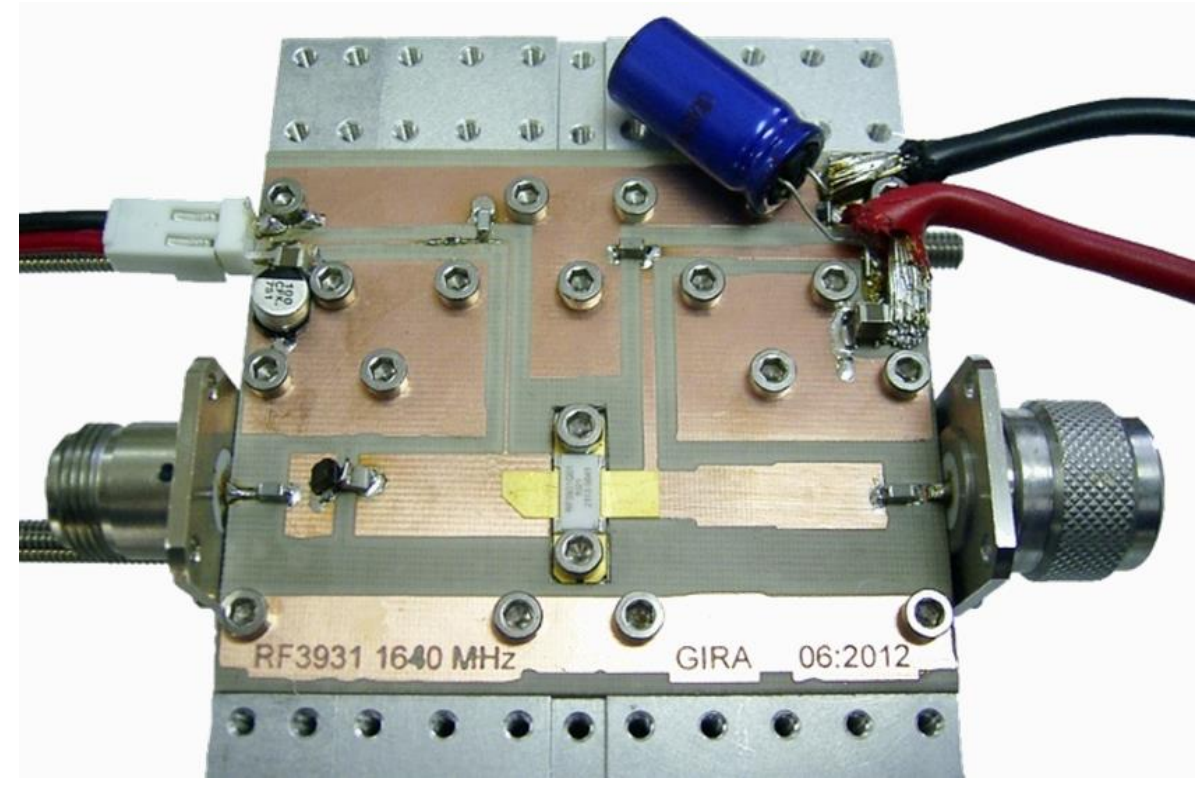

Figura 3.50. Prototipo de amplificador clase F con control de armónicos (prototipo 3).

En este caso, el procedimiento de diseño es totalmente diferente. El simulador AWR será la principal herramienta en el diseño de este nuevo prototipo de amplificador clase F. Para ello, se tendrán en cuenta las siguientes condiciones: 
- Reducción de tamaño como principal objetivo. El útil empleado en los prototipos 1 y 2 implicaba un gran tamaño del amplificador. Al reducir el tamaño se acortarán las líneas de transmisión y también se reducirán las pérdidas.

- Diseño bi-armónico, es decir, como en los prototipos 1 y 2, únicamente se controlarán los dos primeros armónicos.

- Impedancias en el conector de entrada y de salida de $50 \Omega$. En este caso no se emplearán sintonizadores manuales, lo que reducirá las perdidas.

El diseño de las redes de entrada y de salida están basadas en [110]. Este artículo emplea el mismo transistor obteniendo una potencia de salida de pico de $46.17 \mathrm{dBm}$ $(41.4 \mathrm{~W})$ a $1.5 \mathrm{GHz}$ y con un rendimiento del $67.43 \%$. El amplificador mantiene un rendimiento por encima del $60 \%$ y una ganancia superior a $12 \mathrm{~dB}$ en un ancho de banda de $20 \mathrm{MHz}$. Sin embargo, dicho artículo no investiga la razón por la que esas redes de entrada y de salida consiguen el funcionamiento en alto rendimiento y en alta potencia del dispositivo. A continuación, se explicará este funcionamiento y, además, varios cambios serán realizados con respecto a ese diseño con el fin de hacer las redes de entrada y de salida más sencillas y reproducibles.
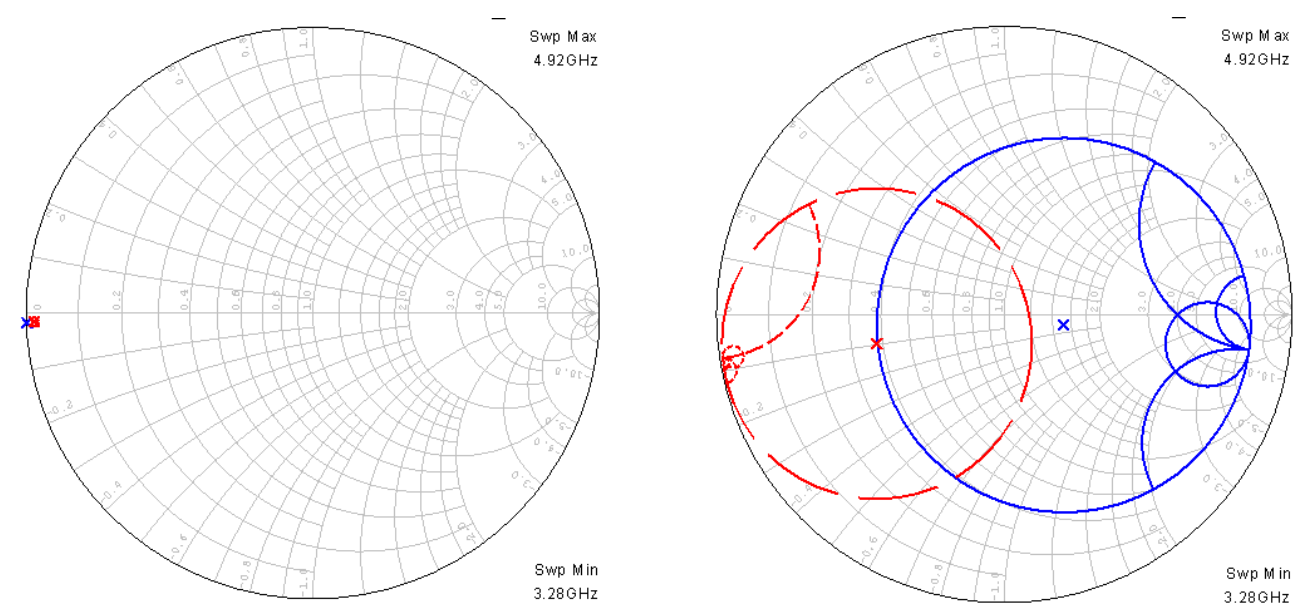

Figura 3.51. Transformación de impedancias del P1 al P4 a la entrada (izq) y a la salida (drch) del prototipo 3 . En rojo $2 f_{0}$ y en azul $3 f_{0}$, siendo la $f_{0}=1640 \mathrm{MHz}$.

De la misma manera que en los prototipos 1 y 2, en la Figura 3.51 se muestran el mapeo de impedancia vistas por el dispositivo en el plano P1 debidas a las redes de entrada y de salida. Se pueden obtener las siguientes conclusiones: a la entrada, 
cualquier impedancia de carga tenderá a fijar un cortocircuito a los dos primeros armónicos; a la salida, será sencillo sintonizar un cortocircuito a $2 f_{0}$ pero sin embargo será complicado sintonizar un circuito abierto a $3 f 0$. Estas impedancias a los armónicos tienden a prever que con las redes de salida se puede diseñar un amplificador en clase F.

En la Figura 3.52 se muestra las impedancias vistas por el dispositivo intrínseco una vez tenido en cuenta su encapsulado y las capacidades internas. Esta imagen muestra la respuesta en frecuencia de las redes diseñadas. Se puede verificar que a la entrada se mantienen las impedancias a los dos primeros armónicos en un gran ancho de banda (280 MHz); a la salida, esta situación no ocurre, lo que conlleva a imaginar que el funcionamiento en clase F de la red de salida será en una banda limitada.
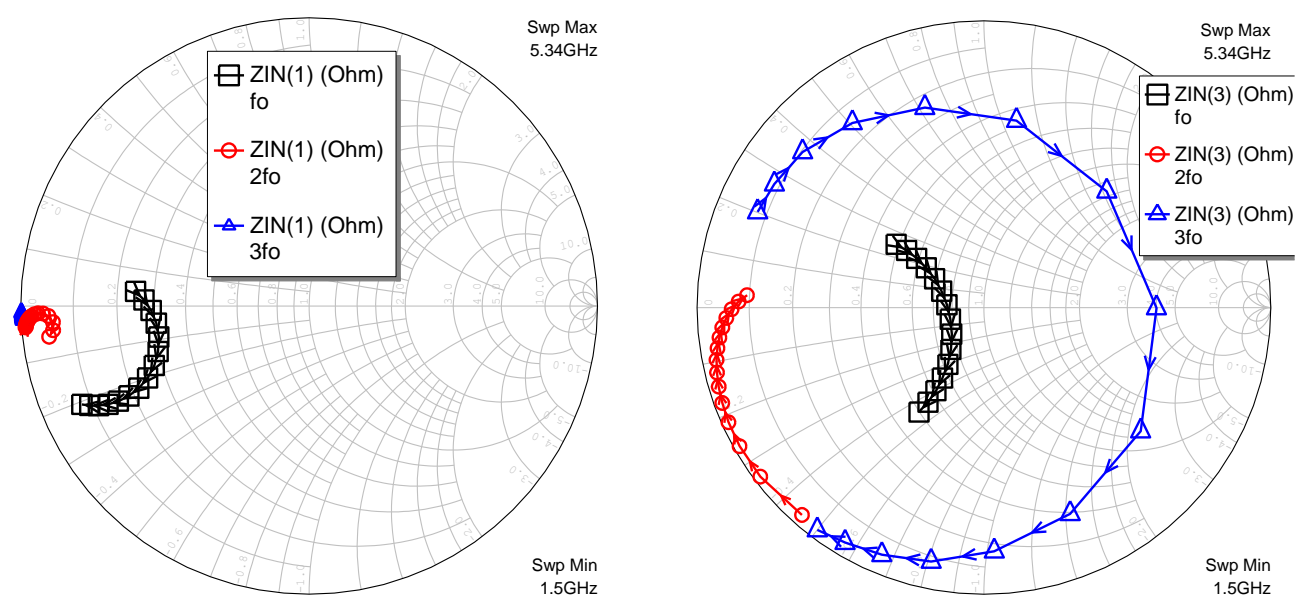

Figura 3.52. Medida de las impedancias intrínsecas a partir de la transformación de impedancias del P3 al P1 a la entrada (izq) y a la salida (drch) del prototipo 3 . En rojo $2 f_{o}$ y en azul $3 f_{0}$, siendo la $f_{0}=1640 \mathrm{MHz}$.

En este punto, se puede simular el comportamiento del amplificador clase F. En la Figura 3.53 se puede ver el esquema del amplificador de potencia. Las condiciones de trabajo que se emplean en la simulación:

- Tensión puerta-fuente (Vgs): $-3.35 \mathrm{~V}$

- Tensión de alimentación (Vdd): 48V 


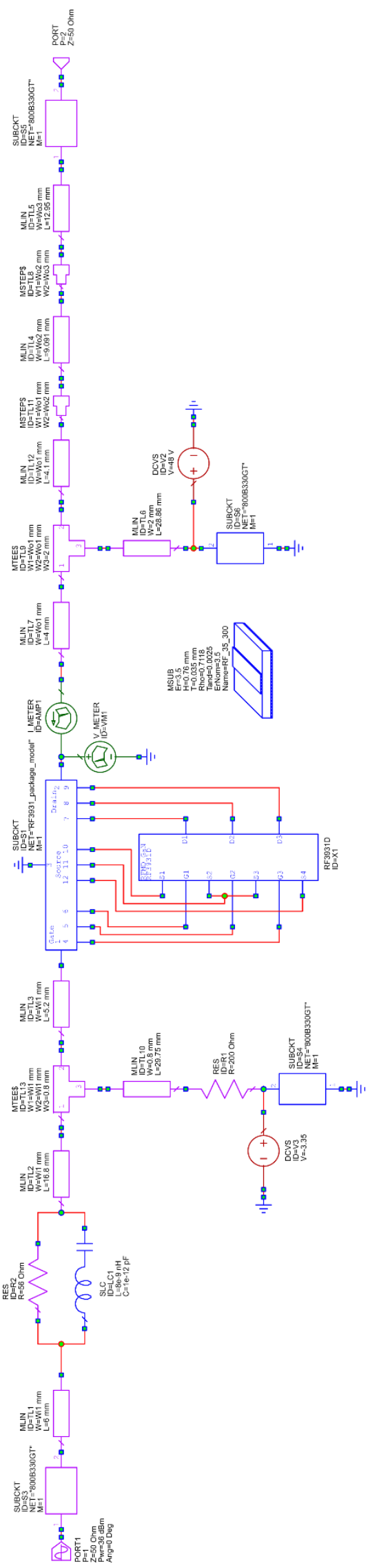

Figura 3.53. Esquema del amplificador de potencia clase $\mathrm{F}$ diseñado por simulación con control de armónicos (prototipo 3). 
A continuación, se pueden ver los resultados de la simulación del amplificador diseñado. La Figura 3.54 muestra las formas de onda en el plano P2 del amplificador en la zona de saturación. No poder fijar un correcto circuito abierto a la salida en $3 \mathrm{f0}$ implica que las formas de onda del amplificador clase $\mathrm{F}$ difieran de las ideales reduciendo las prestaciones en cuanto a potencia y rendimiento se refiere.

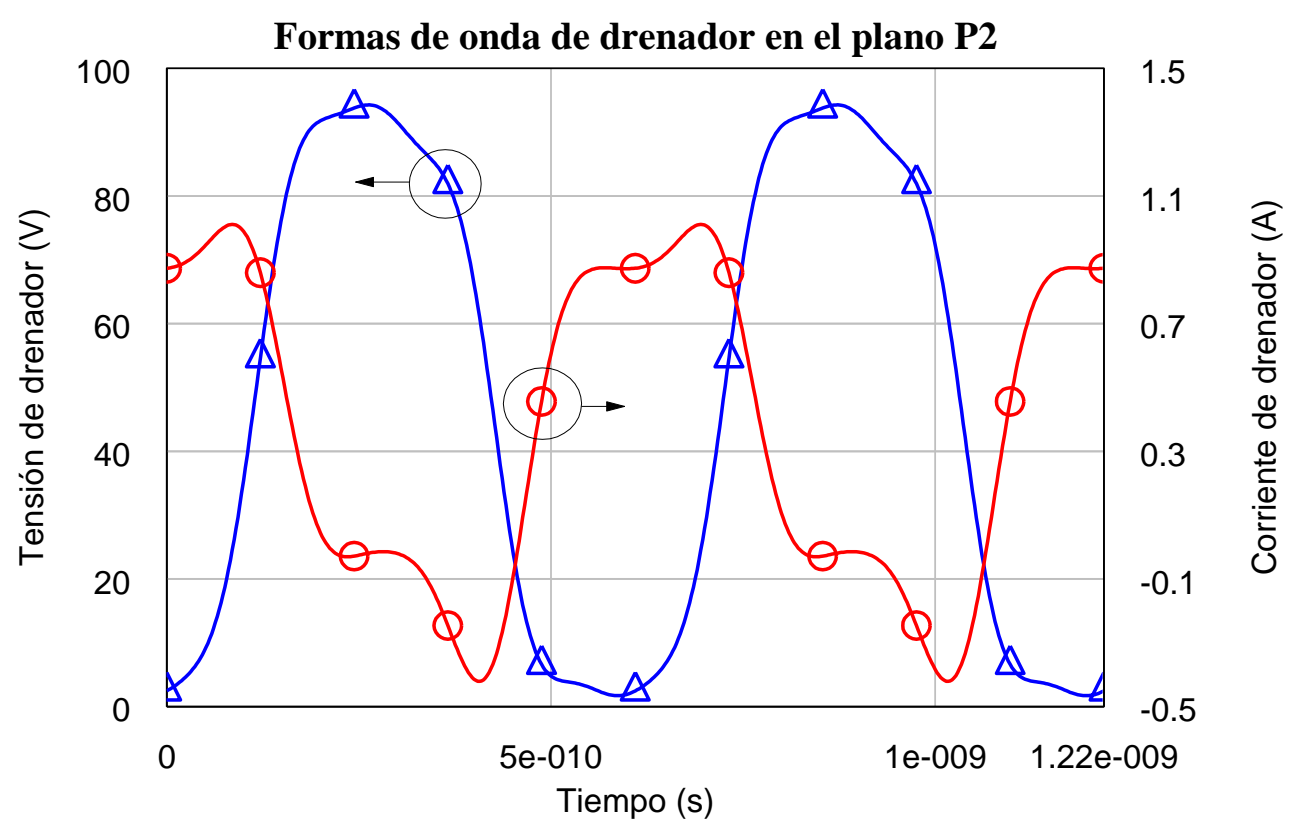

Figura 3.54. Simulación de las formas de onda de drenador en el plano P2 del amplificador de potencia clase $\mathrm{F}$ diseñado (prototipo 3 ).

En la Figura 3.55 y la Figura 3.56 se muestra la simulación del amplificador en función de la frecuencia y de la potencia de entrada, respectivamente. A la frecuencia central y excitado con $37 \mathrm{dBm}$, el amplificador es capaz de ofrecer una potencia de salida de $45.3 \mathrm{dBm}(33.88 \mathrm{~W})$ con un rendimiento de drenador del $69.6 \%$ y una ganancia de $8.3 \mathrm{~dB}$. En la zona de saturación, el amplificador puede alcanzar un rendimiento de drenador del $77.6 \%$ y una potencia máxima de $46.5 \mathrm{dBm}(44.7 \mathrm{~W})$. Un funcionamiento por encima del $60 \%$ de rendimiento de drenador se puede conseguir dentro de un ancho de banda de aproximadamente $220 \mathrm{MHz}$, siendo la potencia superior a $44.62 \mathrm{dBm}$ (29 $\mathrm{W})$. 


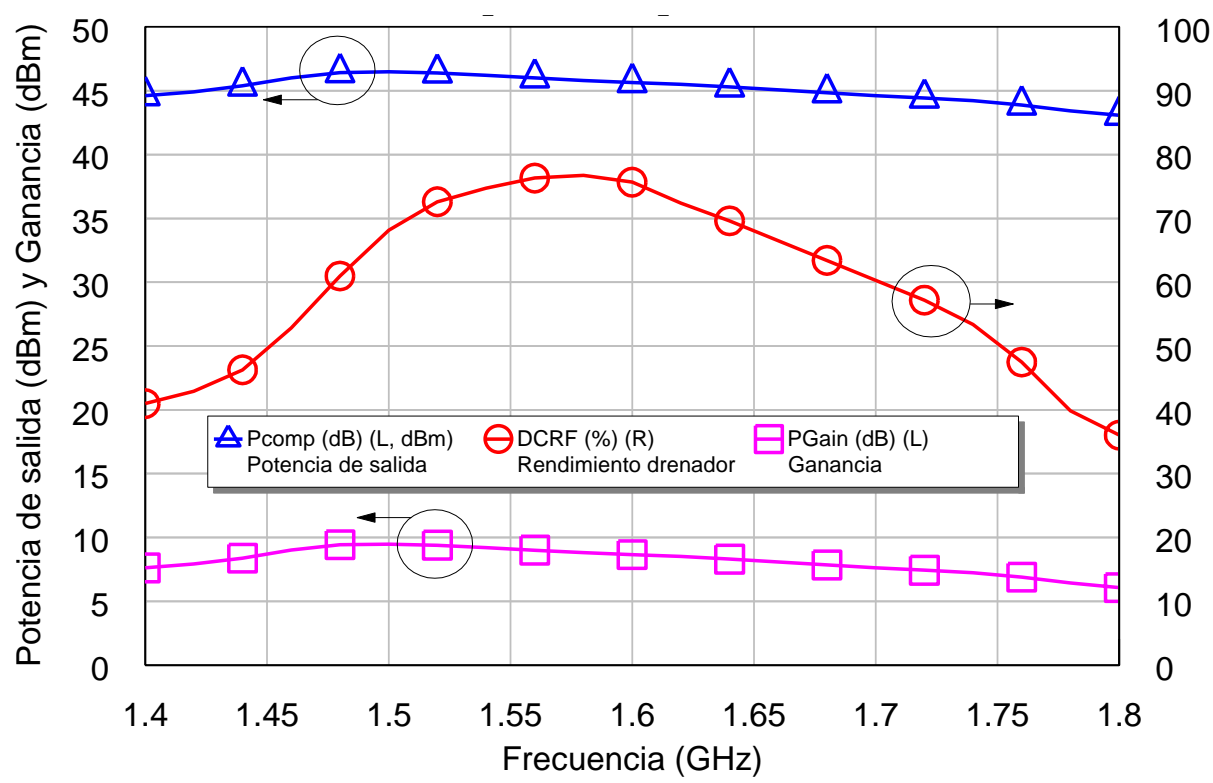

Figura 3.56. Simulación del funcionamiento del amplificador clase $F$ (prototipo 3) en función de la frecuencia.

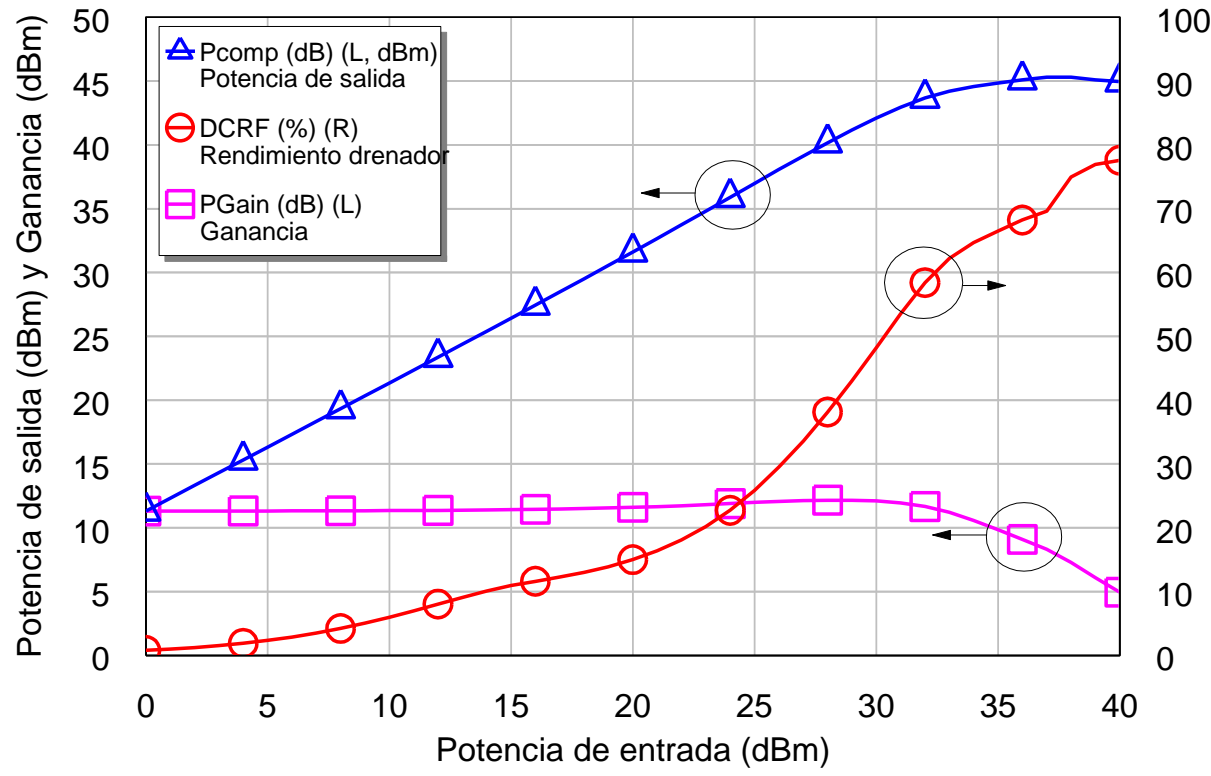

Figura 3.56. Simulación del funcionamiento del amplificador clase $F$ (prototipo 3 ) en función de la potencia de entrada.

En la Figura 3.57 y la Figura 3.58 se muestra los resultados obtenidos de las medidas reales realizadas al amplificador con las mismas condiciones de polarización que las utilizadas en la simulación. A la frecuencia central y excitado con $37 \mathrm{dBm}$, el amplificador es capaz de ofrecer una potencia de salida de $46.3 \mathrm{dBm}(42.65 \mathrm{~W})$ con un rendimiento de drenador del $65.71 \%$ y una ganancia de $9.3 \mathrm{~dB}$. En la zona de 
saturación, el amplificador puede alcanzar un rendimiento de drenador del $70 \%$ y una potencia máxima de $46.55 \mathrm{dBm}(45.18 \mathrm{~W})$. El amplificador presenta un comportamiento plano en rendimiento en un gran ancho de banda.

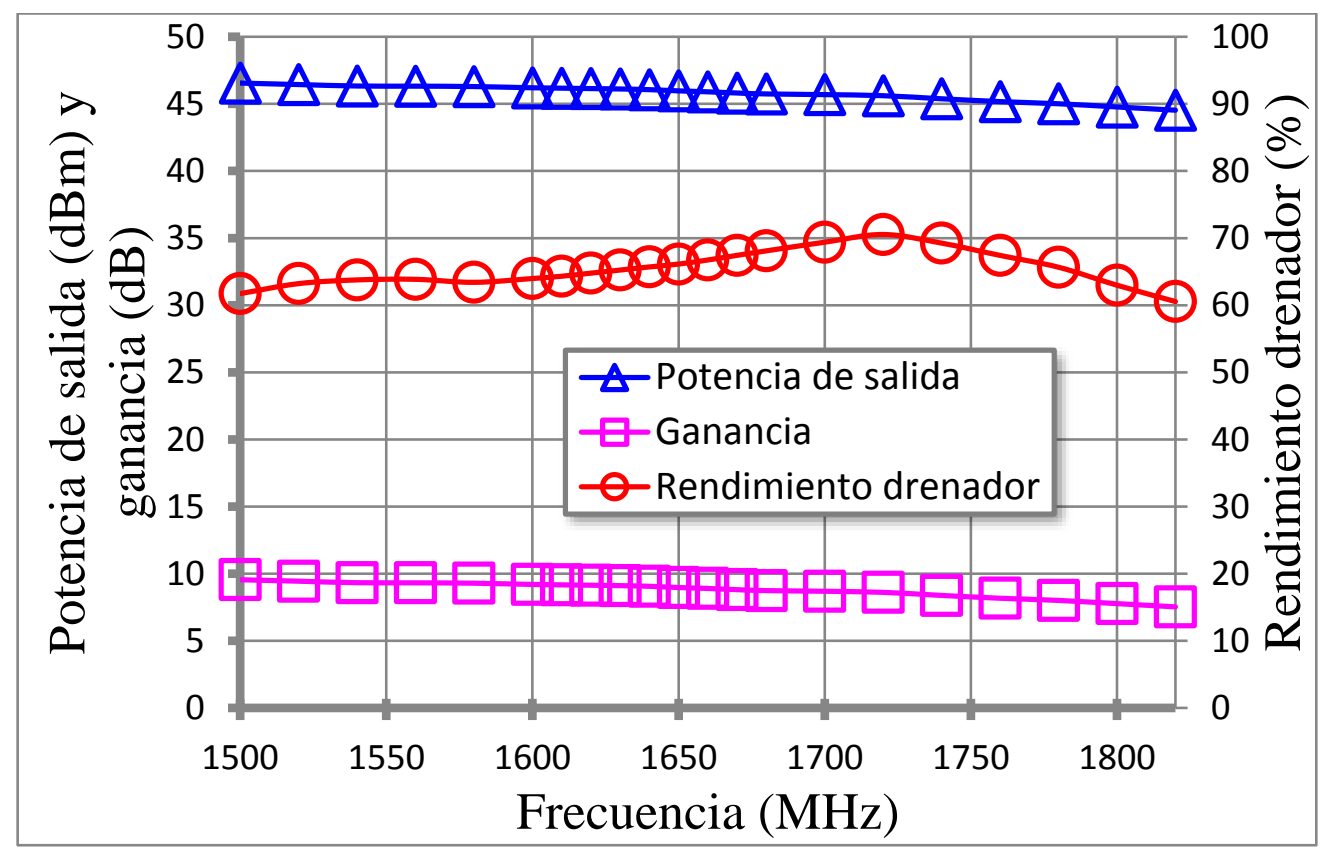

Figura 3.57. Medidas del funcionamiento del amplificador clase $F$ (prototipo 3 ) en función de la frecuencia.

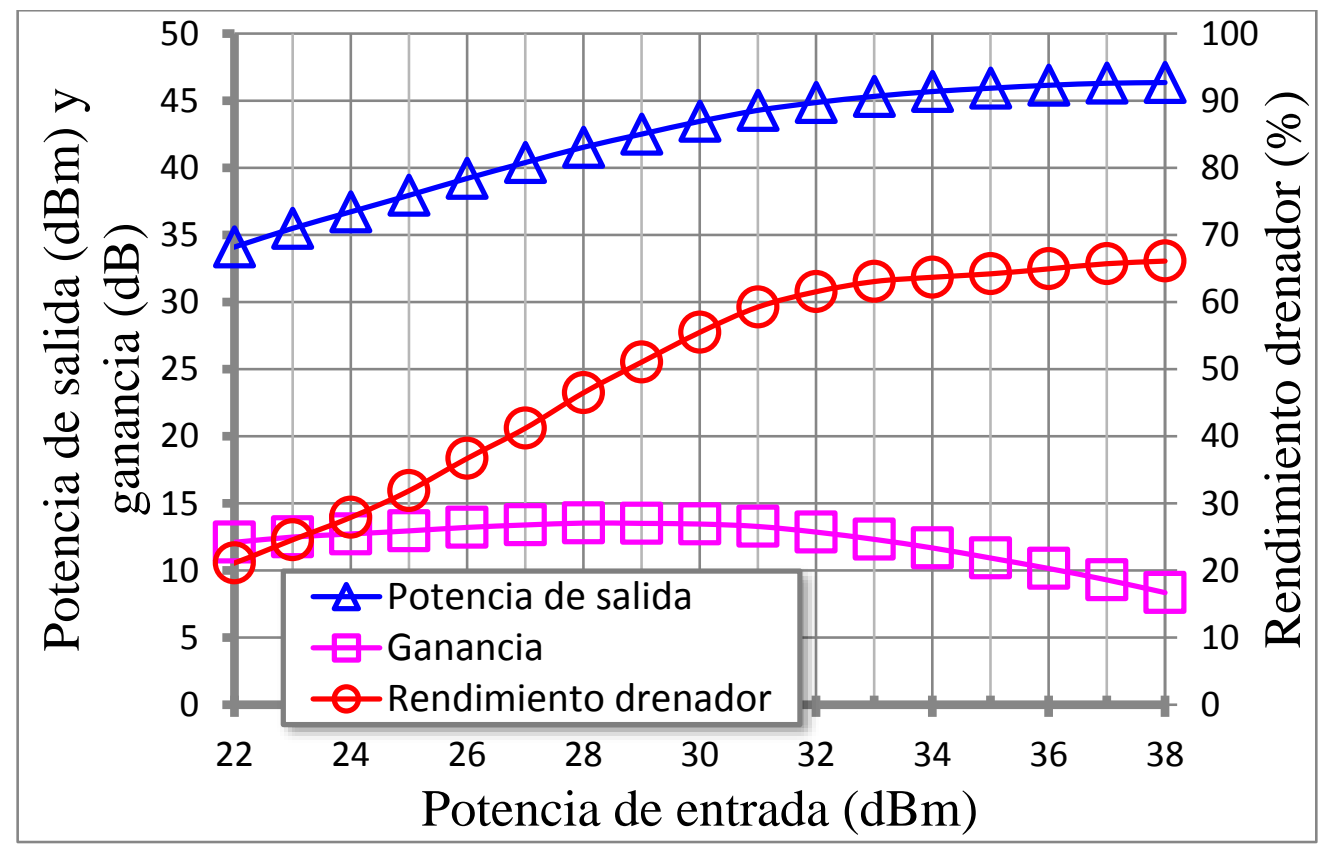

Figura 3.58. Medidas del funcionamiento del amplificador clase $F$ (prototipo 3) en función de la potencia de entrada. 
Estas prestaciones del amplificador superan las especificadas en la hoja del fabricante [90]. El diseño de las redes implementado permite su fácil reproducción en un reducido tamaño, cumpliendo los objetivos de diseño que se había fijado.

\subsection{Amplificador de potencia clase $\mathbf{E}$ subóptimo en banda $L$}

Las técnicas de amplificación de potencia con alto rendimiento proporcionan importantes ventajas en los sistemas de RF y microondas, especialmente en aplicaciones de alta potencia o cuya alimentación proceda de una batería. Hoy en día es muy conocido el uso de amplificadores de potencia en modo conmutación con el fin de minimizar el consumo de potencia, la temperatura, el tamaño y el peso de los transmisores; sin embargo, en la práctica, es difícil la integración de este tipo de amplificadores en escenarios con altos niveles de potencia o altas frecuencias debido a las capacidades intrínsecas y a los tiempos de conmutación de los dispositivos, a los efectos del encapsulado, incluso, a en función de las aplicaciones donde vayan a ser usados.

Entre la multitud de clases de amplificación conmutadas que ofrecen alto rendimiento, la amplificación clase E suele ser muy empleada debido a la tolerancia en las imperfecciones del transistor. Además, las redes de carga pueden ser fácilmente sintonizadas para trabajar tanto en banda estrecha como en banda ancha [111].

La capacidad intrínseca equivalente de salida del transistor $\left(C_{\text {out }}\right)$ impone un límite de frecuencia de funcionamiento máximo de un amplificador clase E nominal $\left(f_{\max }\right)$ [112] [113]. En la práctica, $f_{\max }$ es insuficiente para cubrir algunas de las más importantes aplicaciones de potencia de RF y microondas. Esto quiere decir que los dispositivos comerciales disponibles hoy en día no permiten diseñar amplificadores clase E nominales.

Una solución para estos casos es la amplificación clase E subóptima. Este modo de funcionamiento mantiene la mayoría de los beneficios de un funcionamiento óptimo por encima de $f_{\max }$, además de tolerar mayores valores de $C_{\text {out }}$ en detrimento del rendimiento de drenador [114]. Asimismo, los requisitos de carga para un amplificador 
clase E subóptimo por encima de $f_{\max }$, tanto a la frecuencia fundamental como a los armónicos, son muy simulares a los de un amplificador clase E nominal.

Por otro lado, los transistores de GaN muestran importantes ventajas para su uso en amplificadores conmutados en la banda de microondas en comparación con otras tecnologías de semiconductores. Su alta tensión de ruptura los hace más adecuados que otras tecnologías como GaAs para la amplificación en modo de conmutación. Además, típicamente los transistores de $\mathrm{GaN}$ de potencia con alta tensión de ruptura presentan impedancias de carga más altas, lo que contribuye a simplificar las redes de carga, presentando menores perdidas y, por lo tanto, incrementando el rendimiento global del amplificador.

En este apartado se presenta el diseño de un amplificador clase E subóptimo de alta potencia y banda ancha en banda L [115]. Puede trabajar tanto en modo continuo (CW) como en modo pulsado proporcionando una potencia de salida de $180 \mathrm{~W}$ en el rango de 900 a $1500 \mathrm{MHz}$. Exhibe un rendimiento de drenador hasta el 85\%, una ganancia de potencia máxima de $14.7 \mathrm{~dB}$ y un rendimiento de potencia añadida (PAE) máximo de $81 \%$. Este amplificador cubre las subbandas RADAR más importantes en banda L en todos los modos de funcionamiento sin necesidad de ningún ajuste. Además, no requiere ningún circuito de compensación de ganancia cuando se usa en aplicaciones de RADAR pulsado.

\subsubsection{Transistor de GaN}

El amplificador diseñado empleará el transistor CGH40180PP de Cree Inc mostrado en la Figura 3.59. Se trata de un transistor de alta movilidad de electrones (HEMT) basado en tecnología GaN. Es un transistor no adaptado, característica que es crucial en el diseño del amplificador, ya que, como se mostrará en las próximas secciones, los elementos intrínsecos del transistor, así como su encapsulado serán partes relevantes en las redes de entrada y de salida. Presenta un encapsulado cerámico y hermético que le proporciona una excelente estabilidad térmica, dentro del cual se alojan dos células de amplificación. 


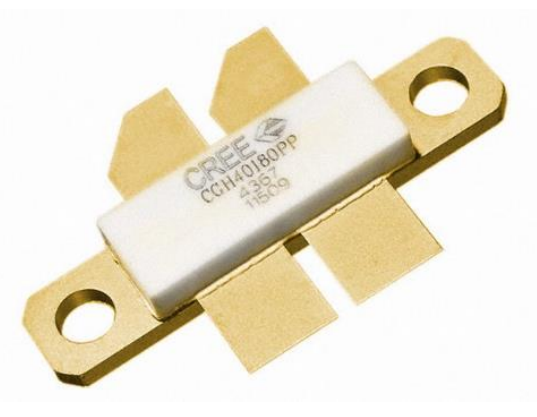

Figura 3.59. Transistor CGH40180PP empleado en el amplificador clase E subóptimo.

Este transistor presenta una tensión de ruptura de $120 \mathrm{~V}$, lo que supone un funcionamiento seguro del dispositivo en el caso de diseñar un amplificador clase E nominal a una tensión de alimentación de $28 \mathrm{~V}$. Sin embargo, antes de cualquier consideración, es necesario determinar si la frecuencia de funcionamiento del amplificador está por encima o por debajo de la $f_{\max }$ permitida por el transistor.

$f_{\max }$, denominada también frecuencia crítica, se define como la frecuencia a la cual la capacidad de drenador del transistor es suficiente por sí misma para proporcionar la susceptancia necesaria para el funcionamiento óptimo en clase E. En [116] se realiza un análisis de la amplificación clase E y presenta la Ecuación 3.4 que cuantifica la frecuencia crítica $f_{\max }$ de funcionamiento para una topología clase E con capacidad en derivación y filtro en serie:

$$
f_{\max }=\frac{I_{\max }}{56.5 V_{D D} C_{\text {out }}}
$$

donde $I_{\max }$ es la corriente máxima de drenador, $V_{D D}$ es la tensión de alimentación y $C_{\text {out }}$ es la capacidad equivalente de salida del transistor. Esta ecuación también puede ser reescrita en función de la potencia de salida tal y como se ve en la Ecuación 3.5 [117]:

$$
f_{\max }=\frac{0.057 P_{o}}{V_{D D}^{2} C_{o u t}}
$$

donde $P_{o}$ es la potencia de salida, $V_{D D}$ es la tensión de alimentación y $C_{o u t}$ es la capacidad equivalente de salida del transistor.

De acuerdo con el fabricante [118], la capacidad drenador-fuente $\left(C_{d s}\right)$ es de $9.6 \mathrm{pF}$ a $28 \mathrm{~V}$ medida a $1 \mathrm{MHz}$ y la capacidad puerta-drenador $\left(C_{g d}\right)$ es de $1.6 \mathrm{pF}$ a $28 \mathrm{~V}$ medida a $1 \mathrm{MHz}$ por cada célula del transistor. La capacidad de salida del transistor será la suma de las capacidades anteriores. A partir de la Ecuación 3.5, se puede obtener la 
Figura 3.60 donde se representa la $f_{\max }$ del transistor en función de la potencia de salida y para diferentes tensiones de alimentación, siendo la capacidad de salida del transistor será la suma de las capacidades $C_{d s}$ y $C_{g d}$ (Ecuación 3.6).

$$
C_{o u t}=C_{d s}+C_{g d}
$$

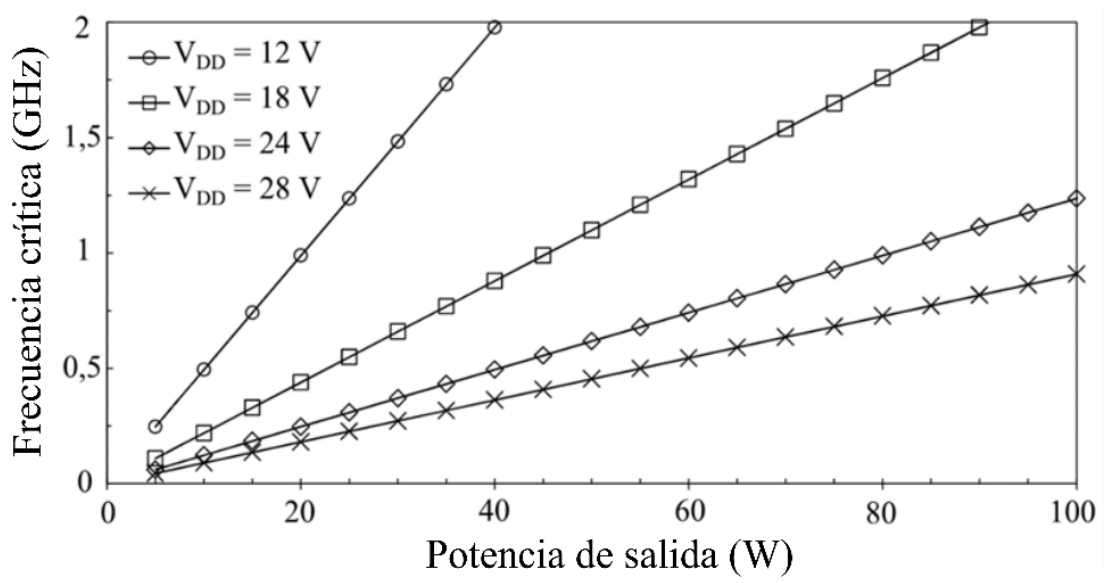

Figura 3.60. Frecuencia crítica de un amplificador clase E en función de la potencia de salida para el transistor CGH40180pp.

Considerando una potencia de salida de $90 \mathrm{~W}$ por célula, la frecuencia crítica $f_{\max }$ es aproximadamente de $818 \mathrm{MHz}$ a $28 \mathrm{~V}$. Esta frecuencia es claramente menor que la frecuencia inferior del objetivo buscado dentro del ancho de banda para el cual se pretende diseñar el amplificador. De la Figura 3.60 se puede deducir que debe existir un compromiso entre potencia de salida y tensión de alimentación, pero esta solución incrementaría la corriente de pico de drenador, y ello conllevaría el diseñar una red de salida para una impedancia de carga más baja.

Al igual que con el amplificador clase F, el fabricante proporciona un modelo compilado del transistor que incluye los efectos del encapsulado, pero no muestra los valores de los componentes del circuito equivalente del transistor necesarios para el diseño del amplificador. Por lo tanto, el modelo propuesto por Sokal y Redl para transistores en modo de conmutación [119] fue empleado para averiguar los parámetros del circuito equivalente del encapsulado. En la Figura 3.61 se puede ser el modelo de encapsulado y en la Tabla 3.1 se muestran los valores de los elementos. 

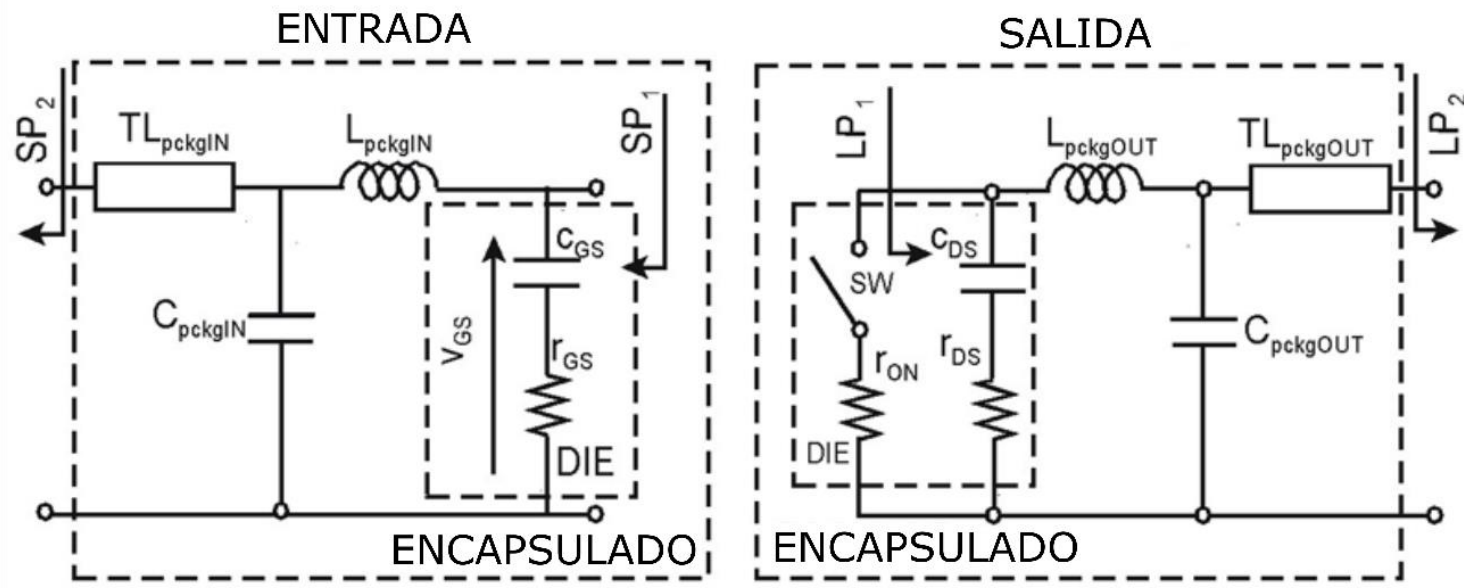

Figura 3.61. Modelo de la entrada y la salida para un transistor encapsulado.

Tabla 3.1. Valores del modelo simplificado para el transistor CGH40180pp.

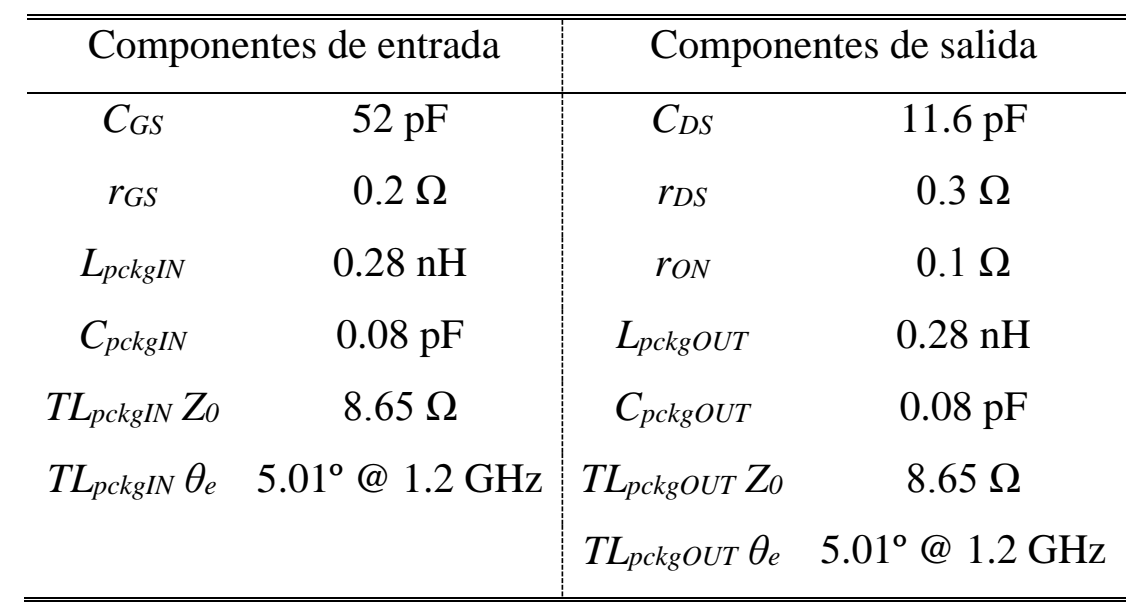

\subsubsection{Funcionamiento subóptimo en clase $E$ en banda $L$}

En el Capítulo 2 se mostró la teoría básica de una amplificador clase E. A modo de recordatorio, este amplificador consiste idealmente en un conmutador de tensión controlado con una capacidad en derivación a la salida, un choque de RF y un circuito resonante R-L-C serie como salida. El choque de RF permite a la corriente constante de DC fluir a través del conmutador cuando está en $\mathrm{ON}$, mientras que actúa como un circuito abierto para las señales de alterna cuando el conmutador está en OFF. La capacidad en derivación se carga y descarga durante el ciclo ON-OFF. El tanque resonante serie permite que una onda sinusoidal fundamental aparezca a la salida. 
El funcionamiento en clase E óptimo se consigue si no hay carga almacenada en la capacidad y tampoco fluye corriente a través de él en el momento de conmutar a ON. Raab analiza en [120] que, si las formas de onda de tensión y de corriente en un amplificador clase E óptimo cumplen dos condiciones, Ecuación 2.2 y Ecuación 2.3 explicadas en el Capítulo 2, asegura que no se disipa energía incluso durante la transición en la conmutación. Únicamente bajo estas condiciones se puede obtener un rendimiento del $100 \%$ y se puede entregar la máxima energía a la carga, aunque en la práctica es difícil de conseguir debido a diferentes variables como son: la capacidad parásita y la resistencia de conducción del transistor, el factor de calidad del circuito resonante serie $\mathrm{u}$ otras no idealidades asumidas.

Si la tensión de la señal de tensión desaparece antes del tiempo óptimo o el condensador de drenador no está descargado en el momento de la conmutación, como se puede ver en la Figura 3.62, se denomina funcionamiento sub-óptimo. En [121] [122] se puede ver el análisis teórico de un amplificador clase E tanto en su funcionamiento óptimo como en subóptimo.

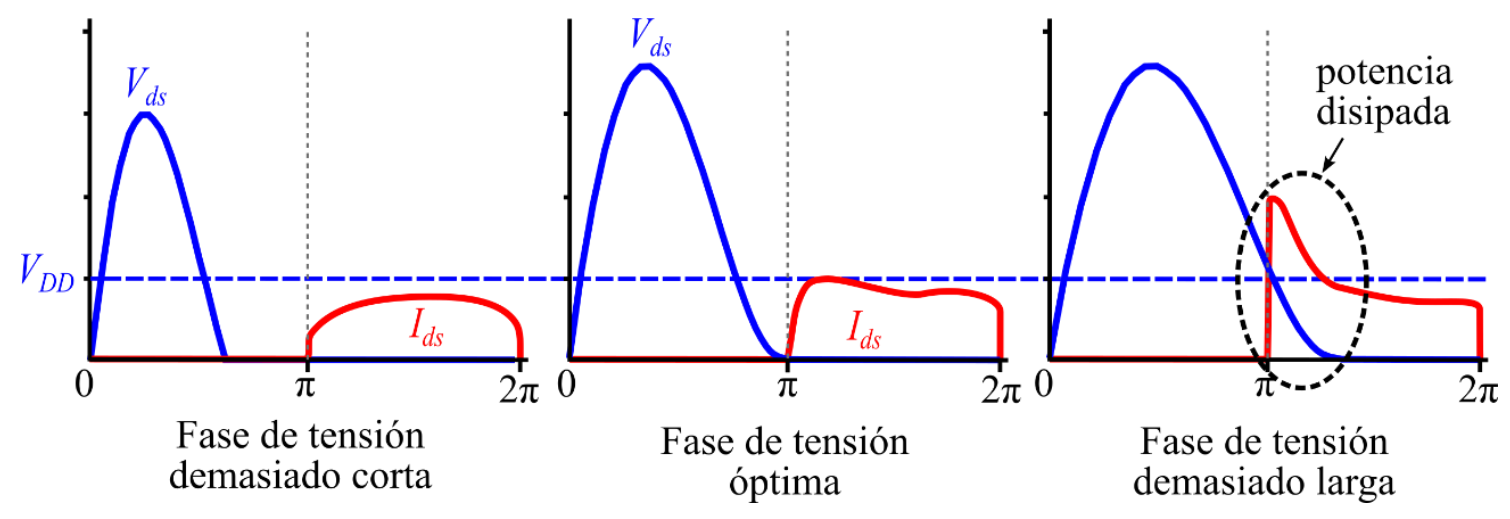

Figura 3.62. Formas de onda de un amplificador clase E en modo subóptimo y óptimo.

A una determinada frecuencia $f_{\max }$, la capacidad en derivación necesaria para un funcionamiento óptimo es proporcionada únicamente por la capacidad interna del transistor. Por debajo de esta $f_{\max }$, es posible ajustar la reactancia serie de la carga para conseguir el funcionamiento óptimo. Sin embargo, por encima de $f_{\max }$, es imposible conseguir un rendimiento del $100 \%$ variando la reactancia serie de la carga [122]. Consecuentemente, el rendimiento máximo que se puede conseguir disminuye a medida que se incrementa la frecuencia por encima de $f_{\max }$. De la misma manera, a medida que 
la frecuencia aumenta por encima de $f_{\max }$, la capacidad de potencia de salida es ligeramente más alta [122]. Otro aspecto importante respecto al modo subóptimo por encima de $f_{\max }$ es que la relación entre la tensión de pico de drenador y la de alimentación ( $V_{d s p e a k} / V_{d d}$ ) es menor que la de una clase E nominal, lo que suavizará los requisitos de los transistores de RF y microondas en cuanto a tensión de ruptura se refiere.

Aunque el amplificador clase E subóptimo no pueda proporcionar $100 \%$ de rendimiento de drenador por encima de $f_{\max }$, puede igualar el rendimiento de otras clases de amplificación de alto rendimiento como la clase $\mathrm{F}$ o clase $\mathrm{F}^{-1}$, con la ventaja de implementar redes de carga más simples incluso para el funcionamiento en banda ancha.

En [122], [114] y [123] muestran que el máximo rendimiento de drenador factible para un amplificador subóptimo clase $\mathrm{E}$ es del $97 \%$ a $1.56 f_{\max }$ y del $92 \%$ a 2 $f_{\max }$. Además, la relación $V_{D S p e a k} / V_{d d}$ disminuye hasta $\operatorname{los} 3.077$ a $1.56 f_{\max }$ y 2.677 a $2 f_{\max }$, siendo está relación de 3.56 para un amplificador clase E nominal. Estas cifras son similares a las obtenidas con otras clases de amplificación tales como la amplificación clase F [124], [125] y [126]. La Figura 3.63 representa gráficamente el efecto sobre el rendimiento, sobre la potencia, sobre la tensión y sobre la corriente en función de la frecuencia con respecto a $f_{\max }$.

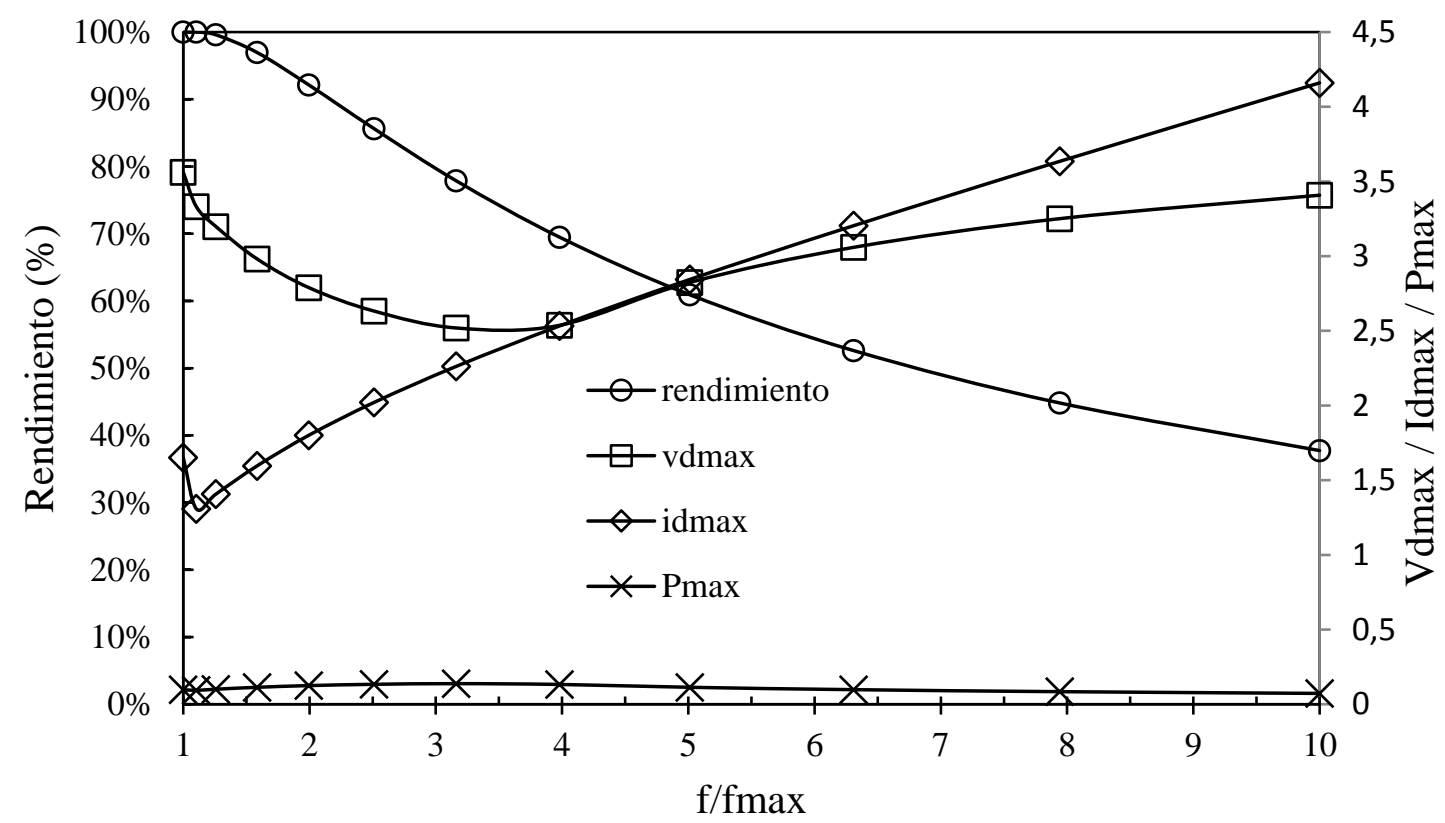

Figura 3.63. Variación del rendimiento, potencia, tensión y corriente máximas en función de la frecuencia de trabajo normalizada por $f_{\max }$. 
Similar a un amplificador clase $\mathrm{E}$ nominal, el funcionamiento en clase $\mathrm{E}$ subóptimo por encima de $f_{\max }$ necesita una impedancia compleja a la frecuencia fundamental y una carga capacitiva pura a los armónicos, siendo el ángulo de la fase de la admitancia de carga a la frecuencia fundamental la principal diferencia entre ambos funcionamientos. En [122] se analiza el valor de carga ideal que maximiza el rendimiento de drenador de un amplificador que trabaje en clase E subóptima por encima de $f_{\max }$. En la Figura 3.64 se presenta el ángulo de la impedancia óptima necesaria para el funcionamiento en clase E subóptimo considerando un transistor ideal ( $\sin$ pérdidas) con tiempos de conmutación nulos. $-35.945^{\circ}$ es el ángulo óptimo de la fase de la admitancia de carga para un amplificador clase E nominal. En el caso de un amplificador clase E subóptimo, este ángulo es de $-23.7^{\circ}$ a $1.56 f_{\max }$ y de $-12.4^{\circ}$ a $2 f_{\max }$.

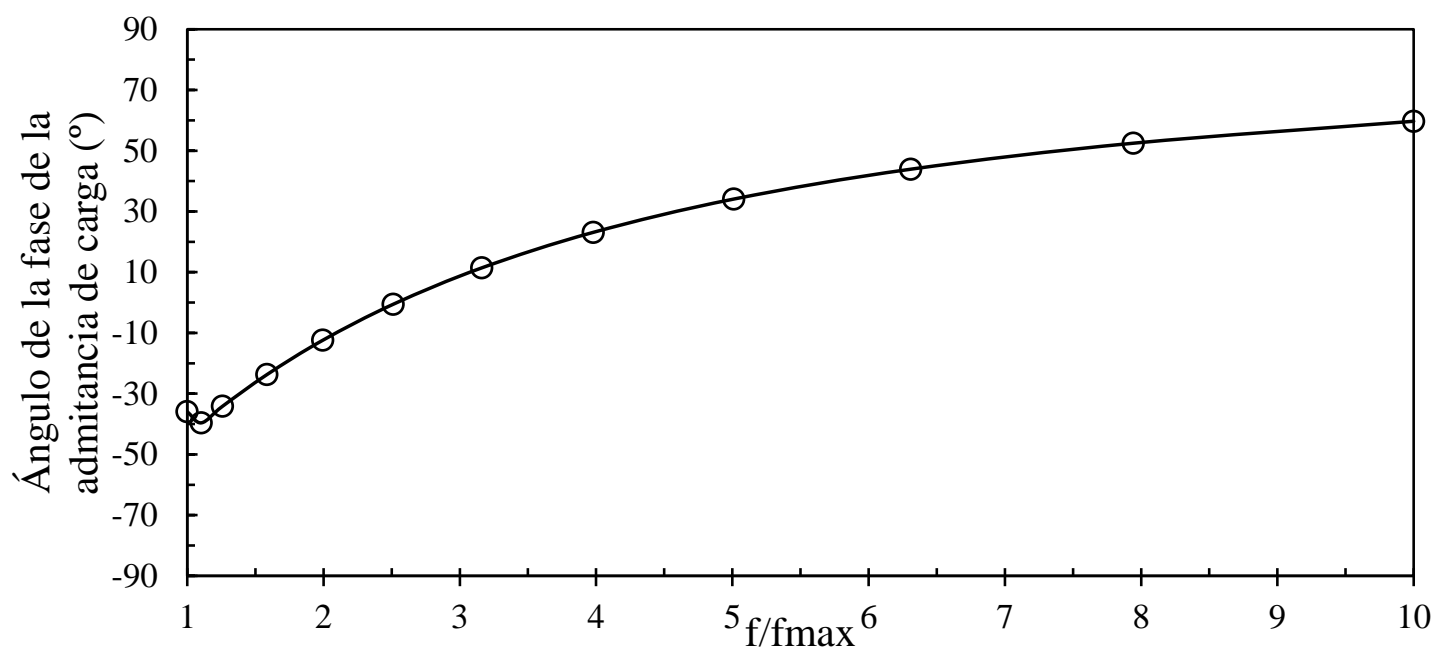

Figura 3.64. Ángulo óptimo de la admitancia para un amplificador clase E subóptimo trabajando por encima de $f_{\max }$.

Las siguientes conclusiones se pueden obtener de la Figura 3.64:

- Existe un ángulo de carga óptima a la frecuencia fundamental que maximiza el rendimiento de drenador que depende de la relación con respecto a $f_{\max }$.

- La admitancia de carga necesaria en función de la frecuencia es muy simular a la de un amplificador clase E nominal, por lo que las redes de carga empleadas en el diseño de un amplificador clase E nominal pueden ser fácilmente adaptadas para el funcionamiento subóptimo. 


\subsubsection{Diseño del amplificador clase E subóptimo}

El amplificador clase E más sencillo fue descrito por Sokal en [48] y patentado en 1975 [127]. Años después se publicaron nuevos análisis más elaborados y exactos [128] [129]. El funcionamiento básico y las redes de carga ya han sido descritas en el Capítulo 2.

El diseño del amplificador de este trabajo ha sido llevado a cabo a través de la técnica de síntesis de la admitancia de carga [130]. Esta técnica consiste en implementar una red de salida cuya admitancia de carga a fundamental y armónicos vista desde el interior del transistor sea la requerida para trabajar en clase E. De la misma manera, esta técnica es extensible al funcionamiento subóptimo. Según [57], en la práctica solamente es necesario calcular las admitancias de carga a la frecuencia fundamental y a los dos primeros armónicos.

La principal ventaja de esta técnica de diseño, con respecto al método clásico basado en diseñar una red de carga específica, es que las condiciones de carga calculadas son universales y están calculadas sobre un determinado ancho de banda, de tal forma que es posible elegir cualquier topología adecuada de red de carga para diseñar el amplificador: elementos concentrados, líneas de transmisión o incluso cargas activas. Además, para determinar la impedancia de carga a través de esta técnica se tienen en cuenta la capacidad intrínseca del transistor y los parámetros del encapsulado. Desafortunadamente, a pesar de que esta técnica facilita la implementación de un amplificador clase E en la banda de microondas, donde el encapsulado del transistor tiene que ser tenido en cuenta, tiene una flexibilidad limitada y dificulta su implementación en banda ancha.

Existen infinitos circuitos y soluciones para proporcionar la impedancia de carga que necesita el amplificador clase E. Se pueden sintetizar redes de carga de alto orden con una gran precisión sobre un gran ancho de banda a expensa de altas pérdidas y falta de repetitividad. Por otro lado, emplear redes de carga de bajo orden proporcionan menores pérdidas a costa de reducir el perfil de frecuencia de la potencia de salida y el rendimiento. Este problema se puede resolver asemejando las especificaciones a un filtro paso banda con carga asimétrica e impedancia fuente [130]. 
La admitancia de carga necesaria para el diseño del amplificador clase E subóptimo depende de la reactancia de la capacidad en derivación $\left(X_{C o u t}\right)$ a la frecuencia más alta de funcionamiento, de la tensión de alimentación $\left(V_{d d}\right)$ y del nivel de potencia de salida del amplificador $\left(P_{\text {out }}\right)$. En el caso de este trabajo, la capacidad en derivación $C_{\text {out }}$ tiene un valor de $11.6 \mathrm{pF}$, la frecuencia más alta de funcionamiento será de 1500 $\mathrm{MHz}$, la tensión de alimentación será de $28 \mathrm{~V}$ y se pretende generar una potencia de salida por célula de 90W. Considerando todos estos factores, la admitancia de carga se puede calcular de [122] y [131] siendo, a la frecuencia fundamental, el módulo de la admitancia $0.1 \mathrm{~S}$ y la fase de $-25^{\circ}$ en el centro de la banda. Está fase varía desde los $-30^{\circ}$ a los $-23^{\circ}$ en el ancho de banda del amplificador. En cuanto a los armónicos, la fase de la admitancia de carga debe ser $90^{\circ}$.

Estos valores de admitancia de carga fueron ajustados usando un optimizador por balance armónico en todo el ancho de banda del amplificador con el fin de conseguir los objetivos de potencia de salida y rendimiento. Esta admitancia de carga fue presentada usando una red de carga que transforma la impedancia de $50 \Omega$ del puerto de salida al valor requerido por el amplificador. La red diseñada para este amplificador se muestra en la Figura 3.65.

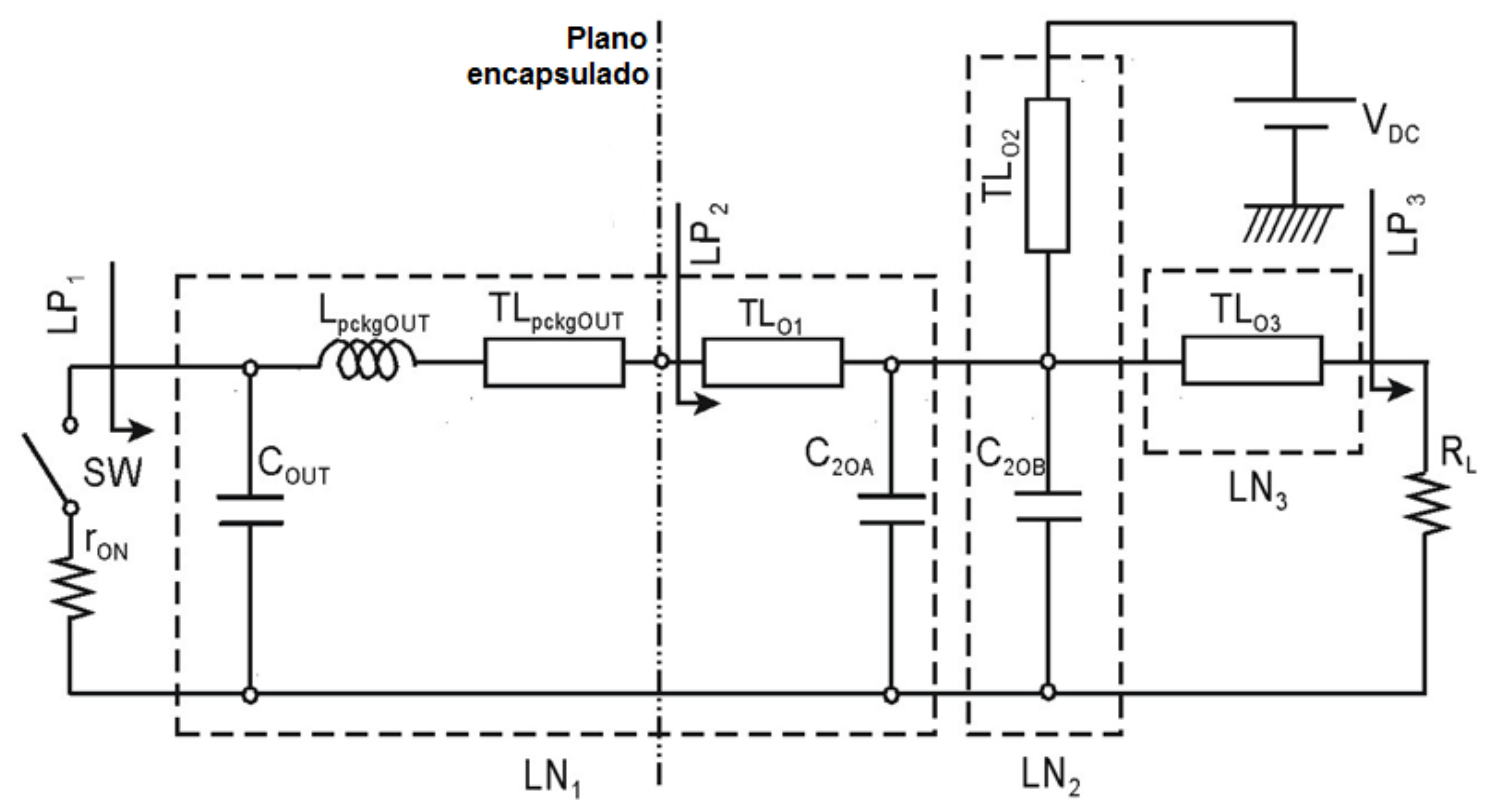

Figura 3.65. Esquema de la red de salida del amplificador clase E subóptimo en banda L.

Se trata de un circuito de compensación de doble-reactancia que además hace la función de transformación de impedancias e incluye un puerto para la inyección de la 
alimentación [116]. Este circuito es muy usado para mejorar el ancho de banda de los amplificadores en la banda de microondas. La Tabla 3.2 muestra los valores de los elementos de la red de carga.

Tabla 3.2. Valores los componentes de la red de salida.

\begin{tabular}{cc|cc}
\hline \hline CoUt & $11.6 \mathrm{pF}$ & $C_{2 O A}$ & $11.6 \mathrm{pF}$ \\
$L_{p c k g O U T}$ & $0.28 \mathrm{nH}$ & $C_{2 O B}$ & $6.95 \mathrm{pF}$ \\
$T L_{p c k g O U T} Z_{0}$ & $8.65 \Omega$ & $T L_{O 2} Z_{0}$ & $19.8 \Omega$ \\
$T L_{p c k g O U T} \theta_{0}$ & $5.01^{\circ} @ 1.2 \mathrm{GHz}$ & $T L_{O 2} \theta_{e}$ & $10.55^{\circ} @ 1.2 \mathrm{GHz}$ \\
$T L_{O 1} Z_{0}$ & $14.9 \Omega$ & $T L_{O 3} Z_{0}$ & $23.83 \Omega$ \\
$T L_{O 1} \theta e$ & $10.56^{\circ} @ 1.2 \mathrm{GHz}$ & $T L_{O 3} \theta e$ & $91.62^{\circ} @ 1.2 \mathrm{GHz}$ \\
\hline \hline
\end{tabular}

En la Figura 3.65 se han marcado tres planos de carga. LP1 es el plano de carga virtual localizado justamente en el conmutador SW equivalente que modela el transistor. Este plano es donde se debe proporcionar la admitancia de carga necesaria para el correcto funcionamiento del amplificador. LP2 es el plano de carga drenador-fuente a la salida del encapsulado del transistor, donde las medidas de impedancia pueden ser llevadas a cabo en la práctica. LP3 es el plano de carga de $50 \Omega$ a la salida del amplificador.

Por otro lado, se pueden identificar tres subredes dentro de la red de salida del amplificador:

- LN1 está compuesta por la capacidad en derivación $C_{\text {out, }}$ los elementos parásitos del encapsulado en el puerto de salida del transistor, Lpckg_out y $T L_{p c k g \_o u t,}$ y dos componentes externos: una corta línea de trasmisión, $T L_{o 1}$, y una capacidad cerámica multicapa, $C_{2 o a}$. Estos componentes forman una red paso bajo en $\pi$ y es equivalente a un transformador $\lambda / 4$ con línea de transmisión (en condiciones de banda estrecha).

- LN2 consta de una línea microstrip en cortocircuito que actúa como una inductancia, $T L_{o 2}$, y una capacidad cerámica multicapa, $C_{2 o b}$. Estos componentes actúan como un circuito resonante paralelo. Además, la terminación en cortocircuito de la línea $T L_{o 2}$ se usa como puerto de alimentación de DC. 
- LN3 es un transformador $\lambda / 4$ con línea de transmisión. Lleva a cabo la mayor transformación de impedancia de la red de salida y también contribuye a conseguir el efecto de compensación de doble reactancia manteniendo el ángulo de la admitancia de carga aproximadamente constante en todo el ancho de banda del amplificador.

El perfil de admitancia de carga $Y_{L}(f)$ en el plano virtual LP1 conseguido por la red de carga descrita se muestra en la Figura 3.66. Los dos picos vistos en el perfil de admitancia de carga será responsable de un perfil de potencia de salida con dos picos, como se verá más adelante. Como se puede ver en la Figura 3.66, los perfiles de magnitud y fase de la admitancia de carga a la frecuencia fundamental no pueden mantenerse constantes en todo el ancho de banda del amplificador; de ahí que no sea posible conseguir potencia de salida y rendimiento planos simultáneamente. En la práctica, normalmente las aplicaciones demandan perseguir un funcionamiento con potencia de salida plana más que un rendimiento plano dentro del ancho de banda. Por ello, este amplificador fue optimizado para proporcionar un perfil de potencia de salida plana a costa de reducir el rendimiento en la parte alta de la banda de frecuencia.

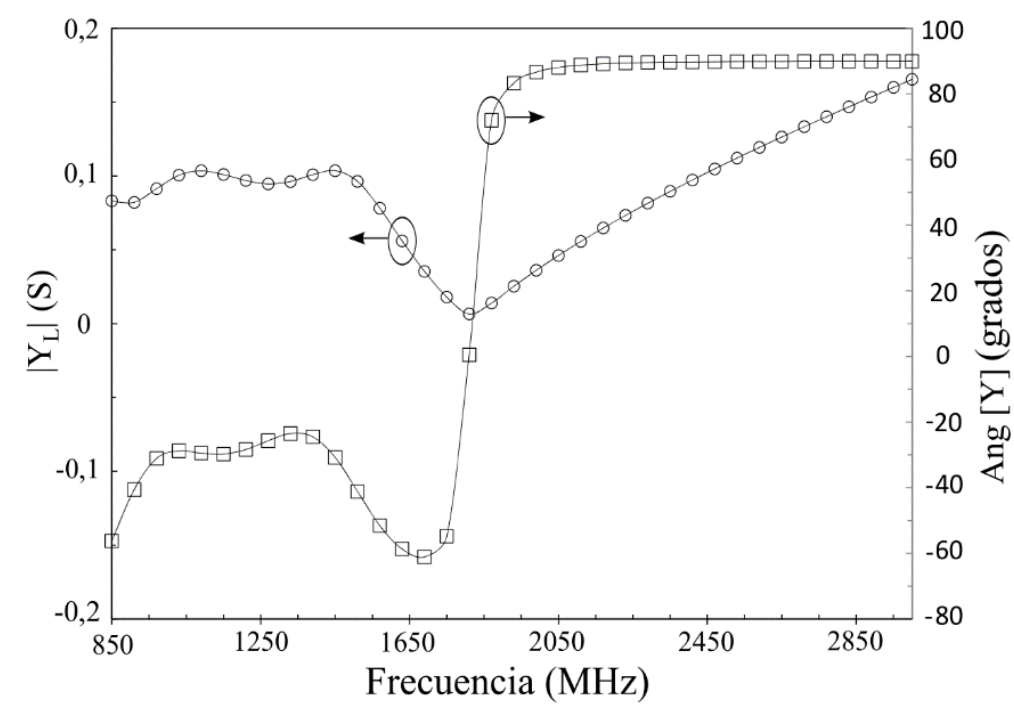

Figura 3.66. Perfil de admitancia proporcionado en el plano LP1 por la red de salida del amplificador.

Respecto a la red de entrada, no existe mucha documentación técnica que cubra el diseño de métodos de excitación óptima y redes de entrada para amplificadores de potencia conmutador en RF y microondas. Una excitación ineficiente del amplificador 
se traduce en unas pérdidas de ganancia de potencia importantes lo que reduce el rendimiento global del amplificador [132].

Los amplificadores en modo de conmutación que emplean dispositivos semiconductores por transconductancia, como es el caso del GaN en este trabajo, necesitan una forma de onda de tensión en el puerto de entrada capaz de conducir al dispositivo de su estado de ON a OFF, y viceversa, con los tiempos de subida y de bajada más cortos posible. En la práctica, tiempos inferiores al 30\% del periodo de conmutación se considera suficiente para un correcto funcionamiento [115]. Tiempos de transición más pequeños es difícil de conseguir debido al efecto de los parámetros intrínsecos y del encapsulado del puerto de entrada del transistor.

En [115] considera que la forma de onda más eficiente a la hora de trabajar con amplificadores de potencia conmutados es una onda de tensión trapezoidal. Sin embargo, debido a los parásitos del dispositivo y del encapsulado esta forma de onda es muy difícil de implementar en la práctica. En el amplificador descrito en este trabajo, la capacidad de entrada equivalente y los parásitos del encapsulado del transistor impiden la implementación de esa forma de onda trapezoidal, por lo que se utilizará una excitación por onda de tensión sinusoidal con un ciclo de trabajo del 50\% a expensas de reducir la ganancia en potencia.

Otro punto a tener en cuenta en la excitación de amplificadores conmutados es mantener un nivel de potencia de entrada constante en todo el ancho de banda. Esto se garantiza fijando un perfil de ganancia de potencia plano.

La red de entrada de amplificador propuesto en este trabajo se diseñó cumpliendo las dos consideraciones anteriores, además de tener un especial cuidado en conseguir unas pérdidas de potencia mínimas. En la Figura 3.67 se muestra la red de entrada implementada y en la Tabla 3.3 se presentan los valores de cada uno de los elementos. 


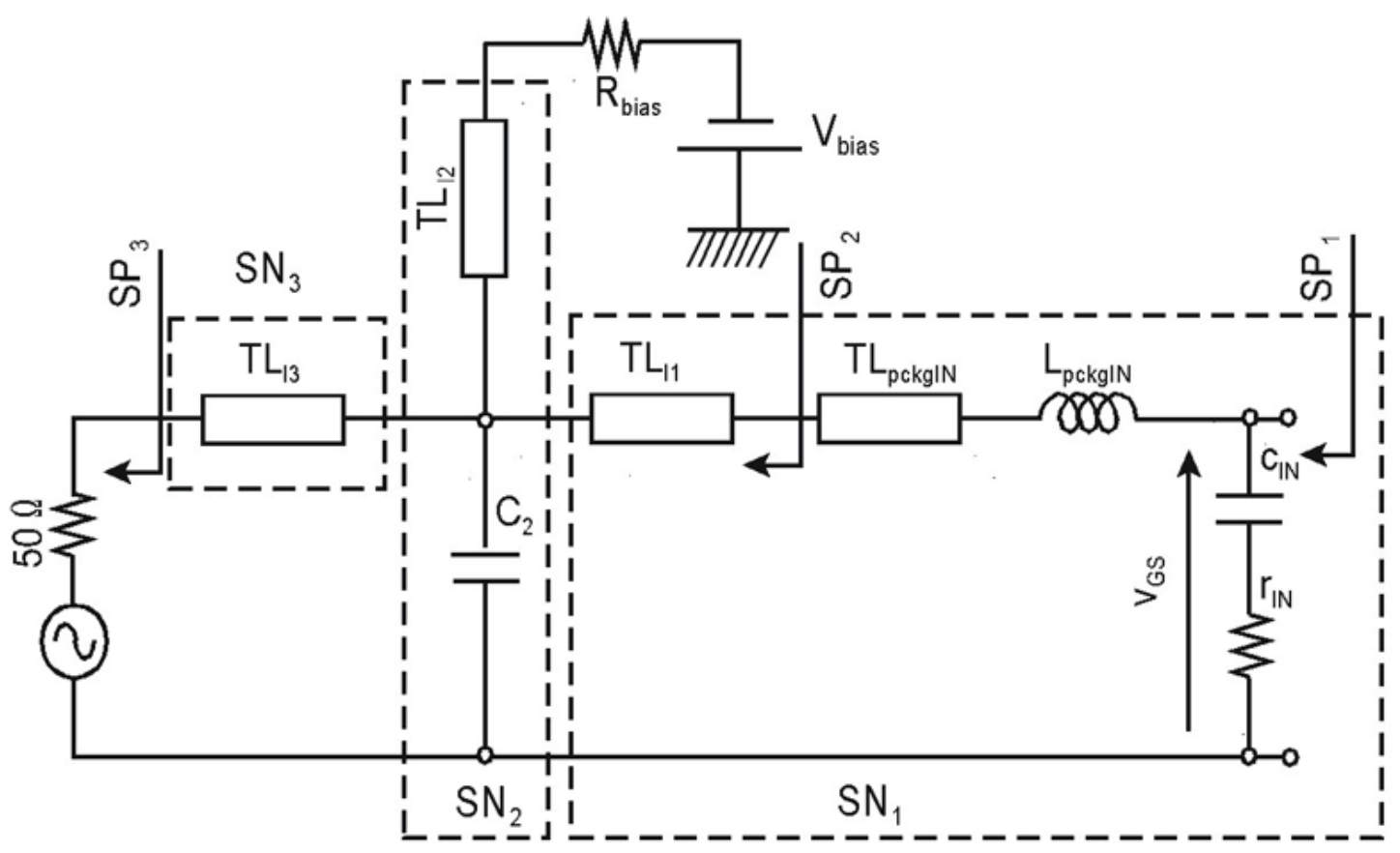

Figura 3.67. Esquema de la red de entrada del amplificador clase E subóptimo en banda $L$.

Tabla 3.3. Valores los componentes de la red de entrada.

\begin{tabular}{|c|c|c|c|}
\hline $\mathrm{C}_{\text {IN }}$ & $52 \mathrm{pF}$ & $\mathrm{C}_{2}$ & $16.69 \mathrm{pF}$ \\
\hline $\mathrm{L}_{\text {pckgIN }}$ & $0.28 \mathrm{nH}$ & RBIAS & $3.9 \Omega$ \\
\hline $\mathrm{TL}_{\text {pckgIN }} \mathrm{Z}_{0}$ & $8.65 \Omega$ & $\mathrm{TL}_{12} \mathrm{Z}_{0}$ & $13.73 \Omega$ \\
\hline $\mathrm{TL}_{\mathrm{pckgIN}} \theta_{0}$ & $5.01^{\circ} @ 1.2 \mathrm{GHz}$ & $\mathrm{TL}_{\mathrm{I} 2} \theta_{\mathrm{e}}$ & $51.89^{\circ} @ 1.2 \mathrm{GHz}$ \\
\hline $\mathrm{TL}_{11} \mathrm{Z}_{0}$ & $19.58 \Omega$ & $\mathrm{TL}_{13} \mathrm{Z}_{0}$ & $24.57 \Omega$ \\
\hline TLI1 $\theta e$ & $15.72^{\circ} @ 1.2 \mathrm{GHz}$ & TLi3 $\theta_{\mathrm{e}}$ & $91.23^{\circ} @ 1.2 \mathrm{GHz}$ \\
\hline
\end{tabular}

Al igual la red de carga, se define tres planos de entrada. El plano SP1 es un plano virtual que se localiza hacia la izquierda de la capacidad de entrada equivalente del transistor $\left(C_{i n}\right)$ y de su resistencia de entrada equivalente $\left(R_{i n}\right)$. El plano SP2 se sitúa a la entrada del encapsulado del transistor, donde se pueden medir las impedancias de entrada de una manera práctica). Por último, el plano SP3 se localiza en el puerto de entrada de $50 \Omega$ del amplificador.

La red de entrada del amplificador se puede dividir en tres subredes. SN1 incluye la capacidad de entrada equivalente $\left(C_{i n}\right)$ y la resistencia de entrada equivalente del transistor $\left(R_{i n}\right)$, los parásitos del encapsulado del transistor $\left(L_{p c k g \_i n}\right.$ y $\left.T L_{p c k g \_i n}\right)$ y una 
línea de transmisión externa $T_{L i 1}$. SN2 se compone de una línea microstrip en cortocircuito $T_{L i 2}$, que será usada para inyectar la tensión de polarización puerta-fuente negativa ( $V_{\text {bias }}$ ) necesaria por el transistor, y de un condensador cerámico $C_{2}$. Finalmente, SN3 es un transformado $\lambda / 4$ con línea de transmisión que lleva a cabo la transformación de la impedancia de la red de entrada.

Esta red de entrada diseñada proporciona ganancia de tensión plana desde el puerto de entrada del amplificador (plano SP1) hasta la capacidad de entrada equivalente del transistor (plano SP3). Además, aporta un adecuado funcionamiento con respecto a las pérdidas de retorno (IRLs), ya que para conseguir ganancia en tensión plana en todo el rango de frecuencias es necesario reflejar parte de la potencia de excitación de entrada. Esta situación se puede ver en la Figura 3.68, donde el amplificador presenta unas cifras de IRL muy buenas en la parte alta de frecuencia, pero sin embargo debe reflejar una gran cantidad de potencia en la parte baja.

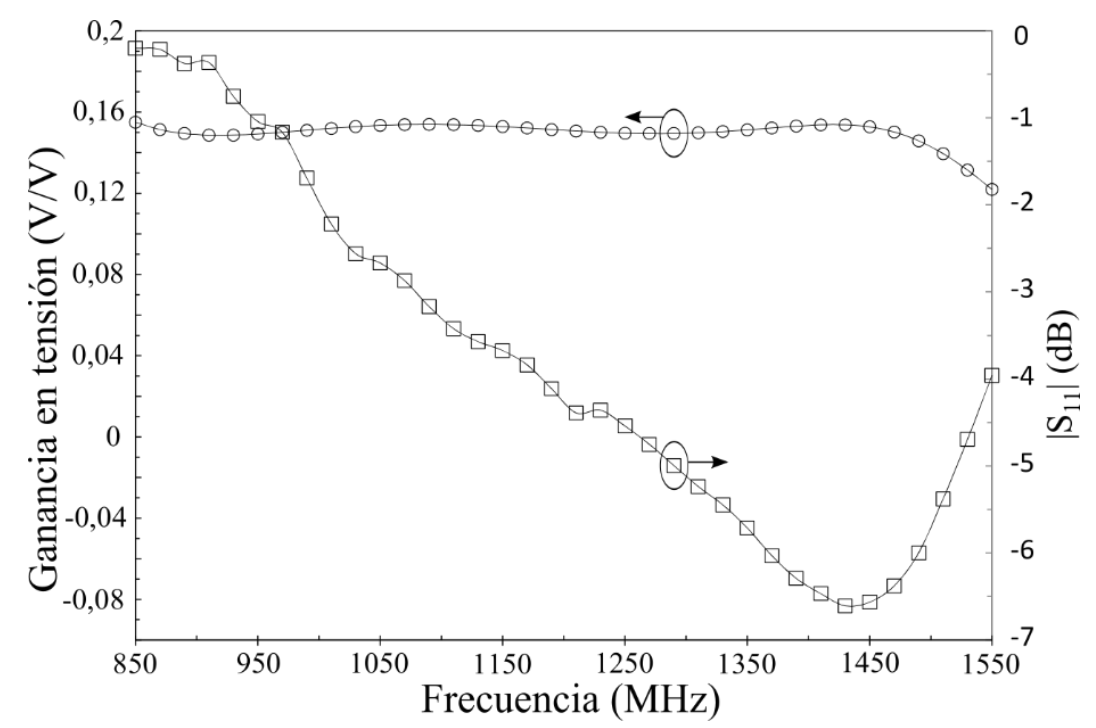

Figura 3.68. Perfil de ganancia en tensión del plano SP3 al SP1 y pérdidas de retorno en el plano SP3 en función de la frecuencia.

Con el fin de obtener unas pérdidas de retorno adecuadas y duplicar la potencia de salida del amplificador, dos células del transistor fueron combinadas, construyendo un amplificador balanceado a través de un combinador híbrido en cuadratura. En la Figura 3.69 se puede ver el esquema completo del amplificador clase E subóptimo balanceado. 


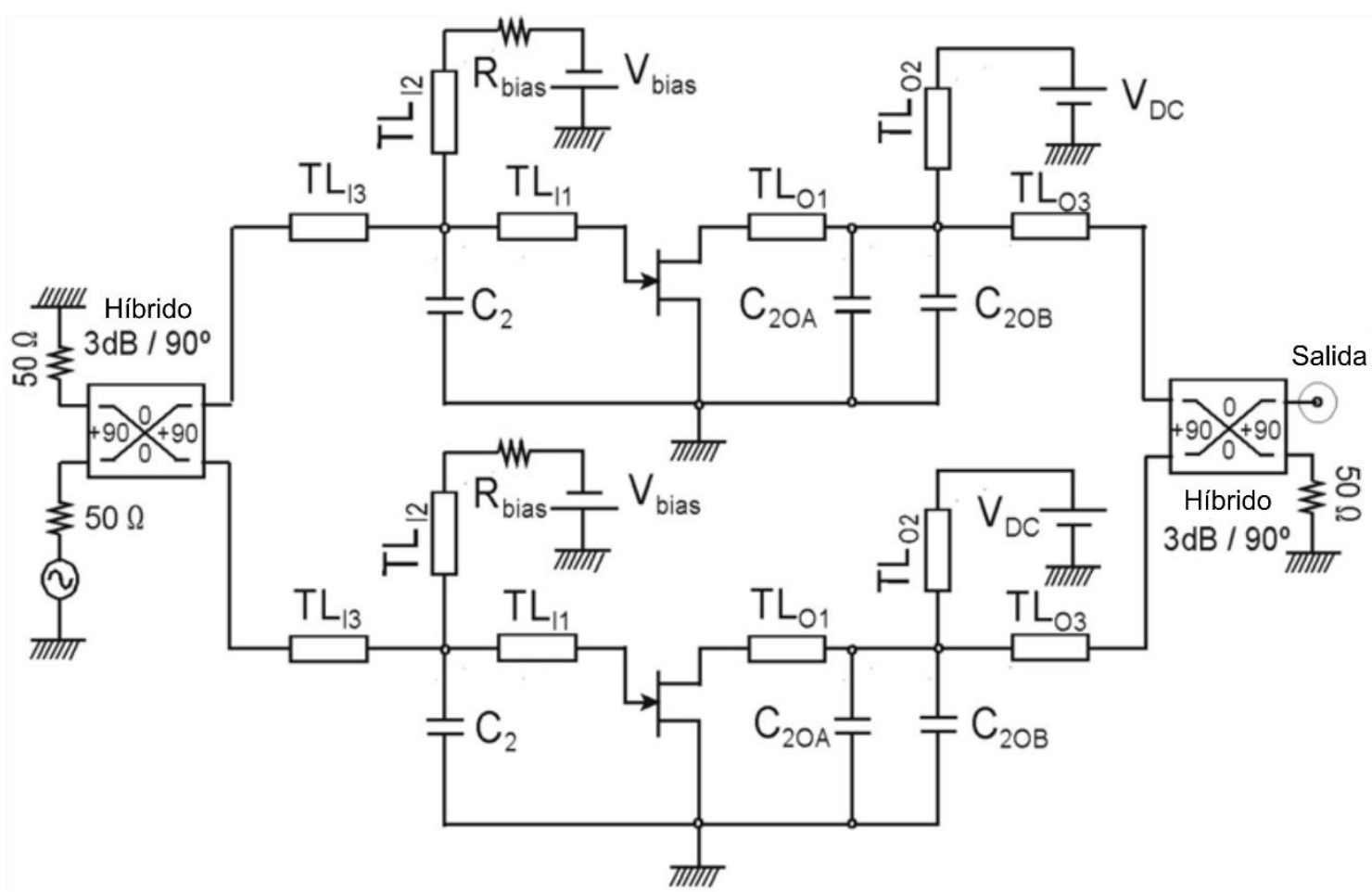

Figura 3.69. Esquema completo del amplificador clase E subóptimo balanceado.

\subsubsection{Simulación del amplificador clase E subóptimo en banda $\mathbf{L}$}

La simulación y optimización del amplificador se realizó usando el software AWR Microwave Office Design y el modelo no lineal del transistor proporcionado por el fabricante. En el momento temporal en el que se realizó esta simulación, los modelos no lineales de los transistores de GaN ya estaban mucho más maduros que los modelos utilizados en el amplificador clase F presentado en este Capítulo 3. Esto se pudo comprobar a la hora de prácticamente no tener problemas de convergencia durante el proceso de simulación.

La Figura 3.70 muestra la simulación de la potencia de salida del amplificador (para una única célula del transistor) en función de la frecuencia para diferentes valores de tensión de alimentación $V_{d d}$. Se puede observar que la potencia de salida se mantiene aproximadamente plana desde los 900 a los $1500 \mathrm{MHz}$. Tal y como se comentó durante el diseño de la admitancia de carga, se observan dos suaves picos debidos al perfil de admitancia visto en la Figura 3.66. 


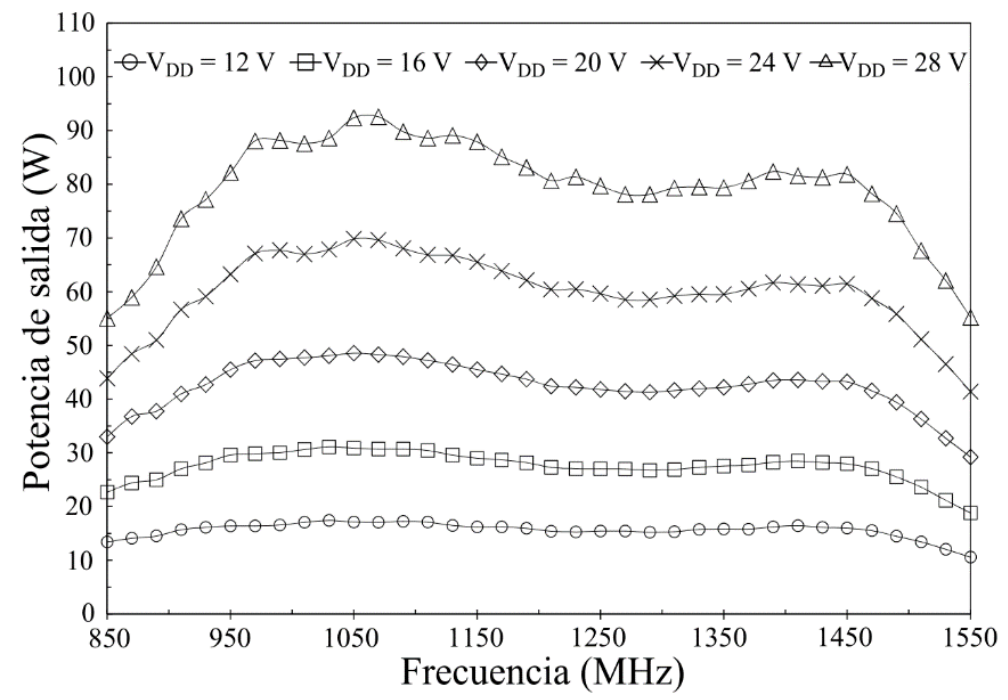

Figura 3.70. Simulación de la potencia de salida en función de la frecuencia en CW (para una célula del amplificador).

La Figura 3.71 presenta el rendimiento de drenador simulado (para una única célula del transistor) bajo las mismas condiciones anteriores. Una ligera reducción de rendimiento se produce a media que aumenta la frecuencia, aunque se mantiene próximo al $80 \%$ en la parte alta de la banda. La dependencia del rendimiento de drenador con respecto a la tensión de alimentación $V_{d d}$ es moderada.

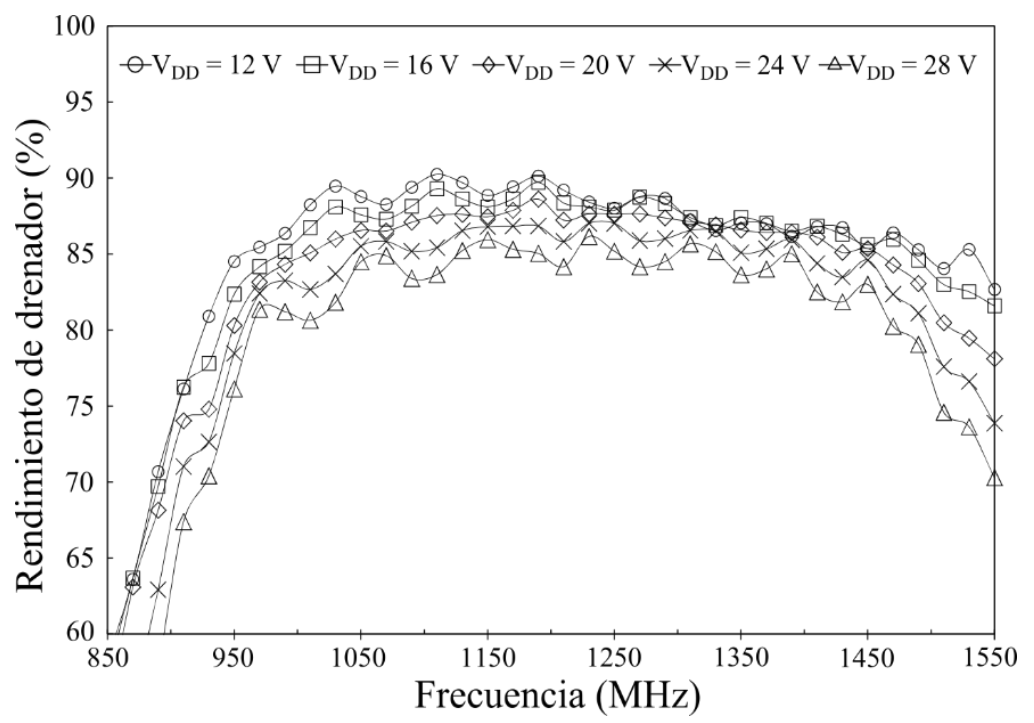

Figura 3.71. Simulación del rendimiento de drenador en función de la frecuencia en CW (para una célula del amplificador). 


\subsubsection{Implementación del amplificador clase $\mathbf{E}$ subóptimo en banda $\mathbf{L}$}

Tras optimizar la simulación del amplificador, se procedió a construir un prototipo sobre un substrato RO4350B de Rogers Corporation con un espesor de 0.508 mm. El layout del amplificador (Figura 3.72) fue cuidadosamente diseñado en Autodesk Autocad con el fin de minimizar cualquier elemento parásito adicional.

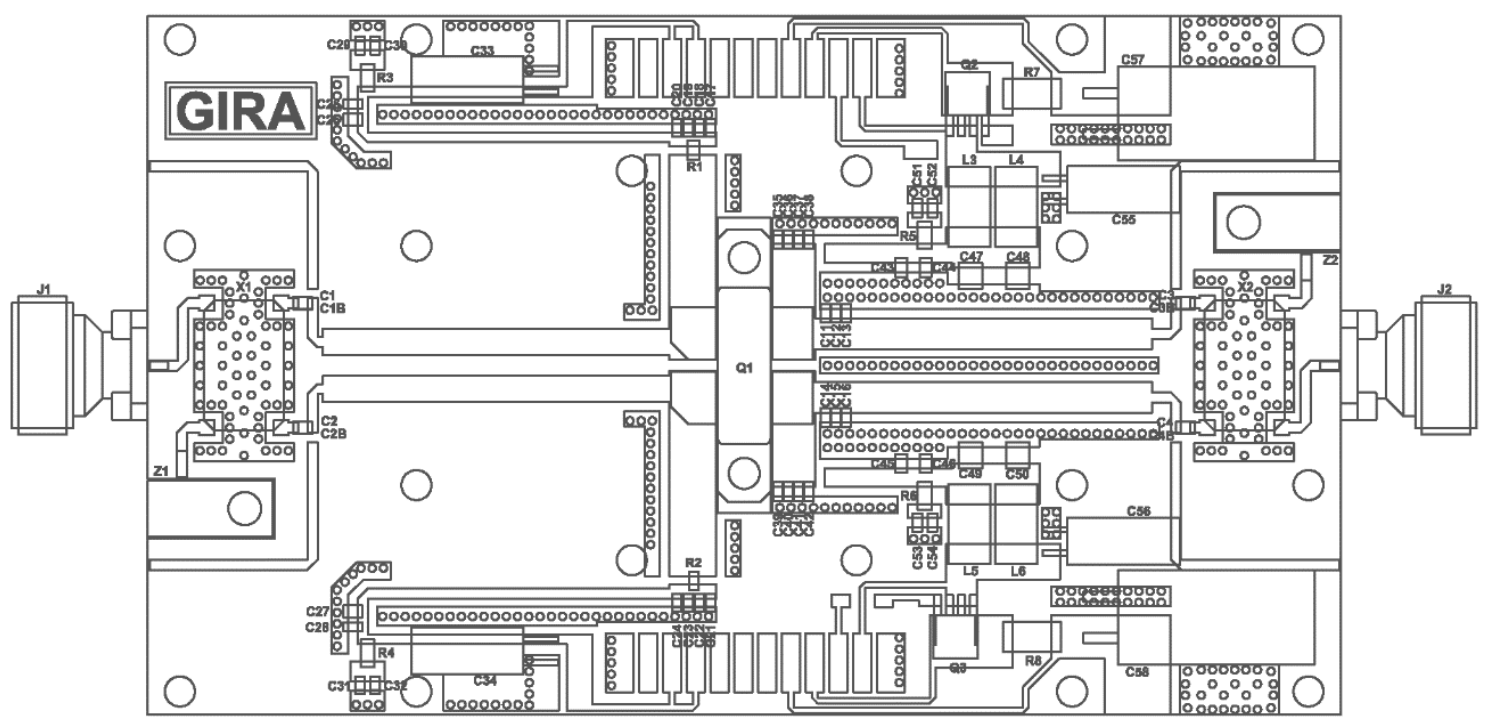

Figura 3.72. Layout diseñado en Autocad del amplificador balanceado.

Como era de esperar, se certificó que el posicionamiento de los componentes es crucial para conseguir el funcionamiento previsto. En la Figura 3.73 se muestra una fotografía del amplificador.

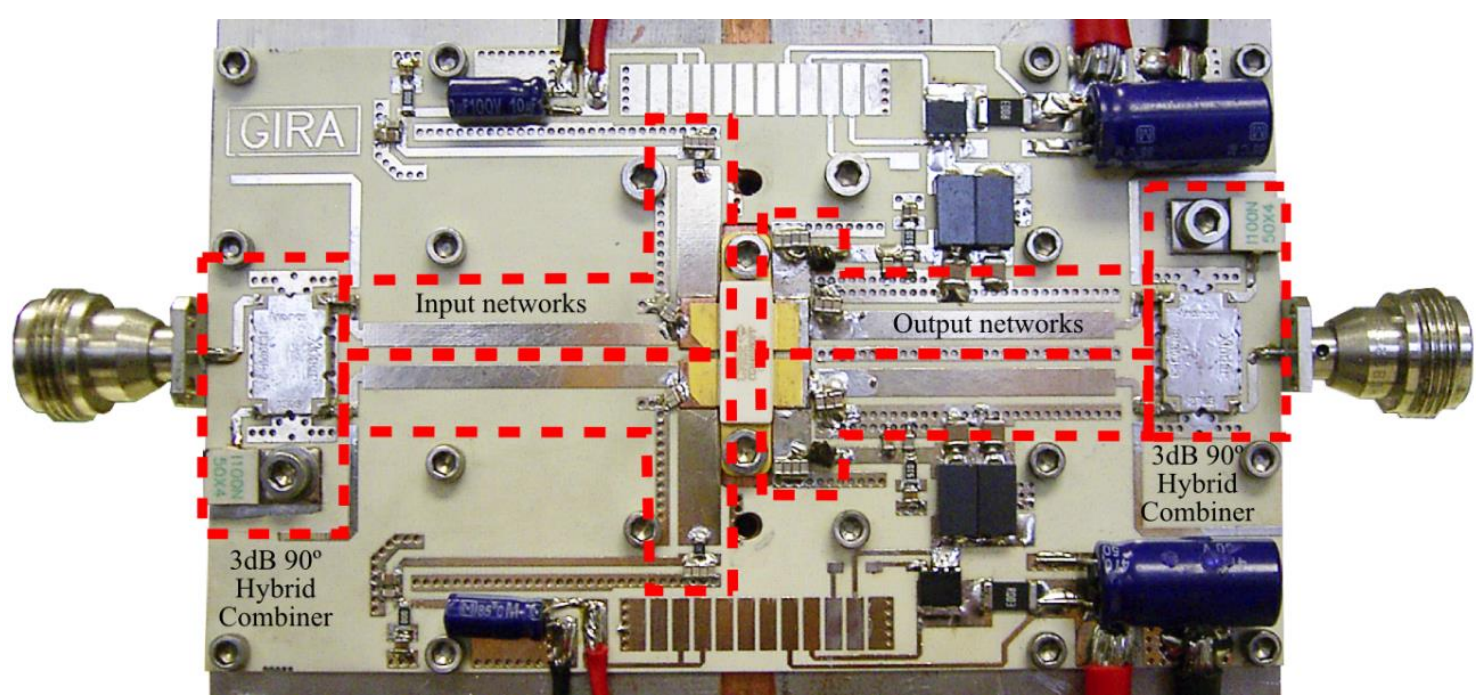

Figura 3.73. Fotografía del amplificador balanceado clase E subóptimo implementado. 
Tanto la potencia de salida como el rendimiento de drenador fueron medidas en condiciones de onda continua CW. Para ello, se confeccionó un banco de pruebas controlado por ordenador a través de Matlab, que se muestra en la Figura 3.74. Este banco de medida consta de:

- Un generador de señal: E4438C de Keysight.

- Un driver: ZHL-10W-2G+ de Mini Circuits.

- Acopladores direccionales: ZABDC20-252H-N+ y ZGDC20-33HP+ de Mini Circuits.

- Un atenuador 3dB: BW-N3W20+ de Mini Circuits.

- Fuentes de alimentación lineales: E3631, E3634 y N5767A de Keysight.

- Multímetro digital: 34410A de Keysight.

- Medidor de potencia: N1912A de Keysight.

- Analizador de espectros: N9320B de Keysight

- Ordenador para el control de equipos a través de Matlab.
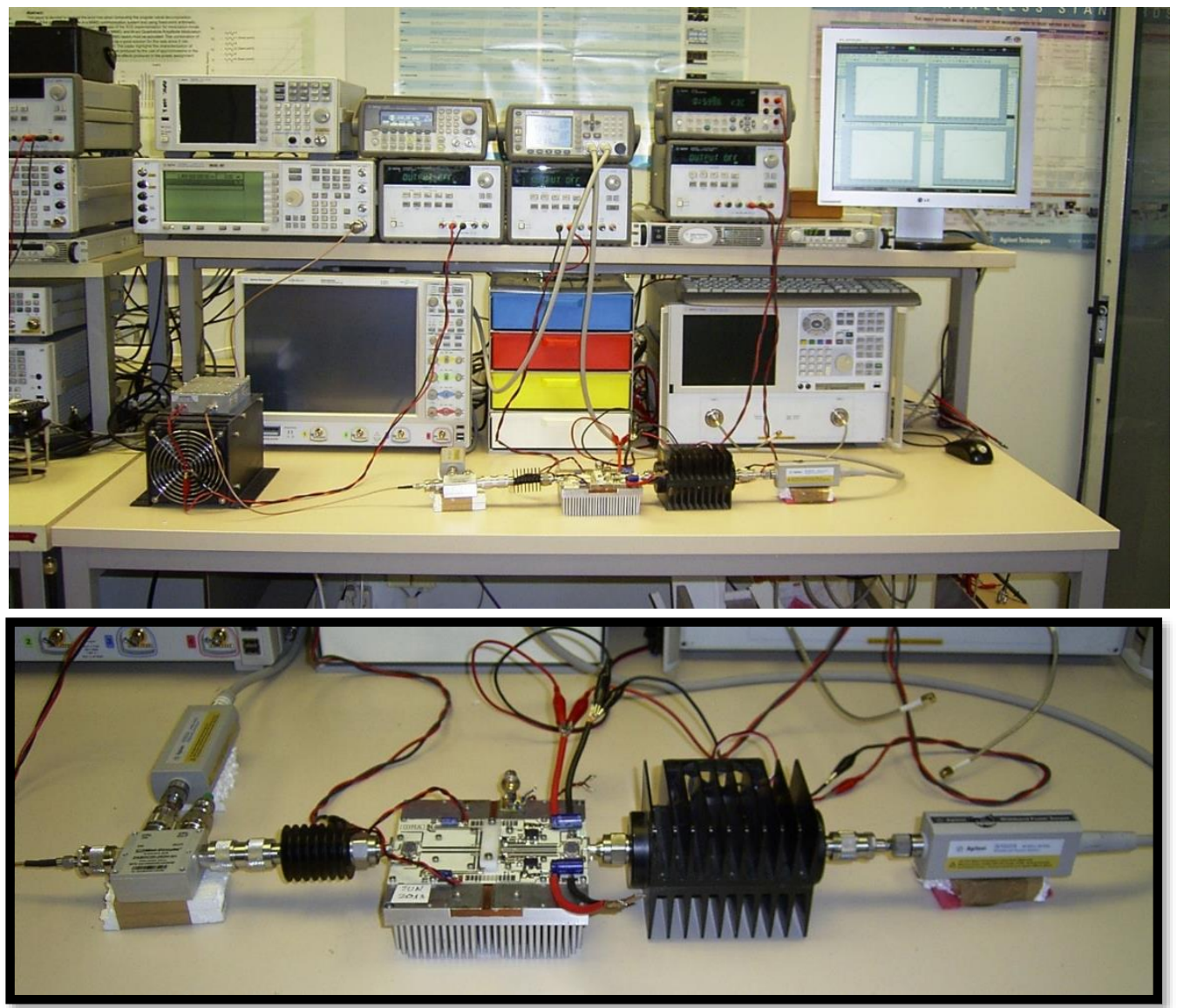

Figura 3.74. Banco de medida controlado por ordenador para la realización de las medidas del amplificador clase E subóptimo balanceado. 
Los resultados de las medidas reales de potencia de salida y rendimiento de drenador para una única célula del transistor mostrados en la Figura 3.75 y la Figura 3.76, respectivamente, fueron obtenidos con una excitación constate de $36 \mathrm{dBm}$ en todo el ancho de banda. Comparando estas medidas con las simulaciones vistas en la Figura 3.70 y la Figura 3.71, existe una gran concordancia.

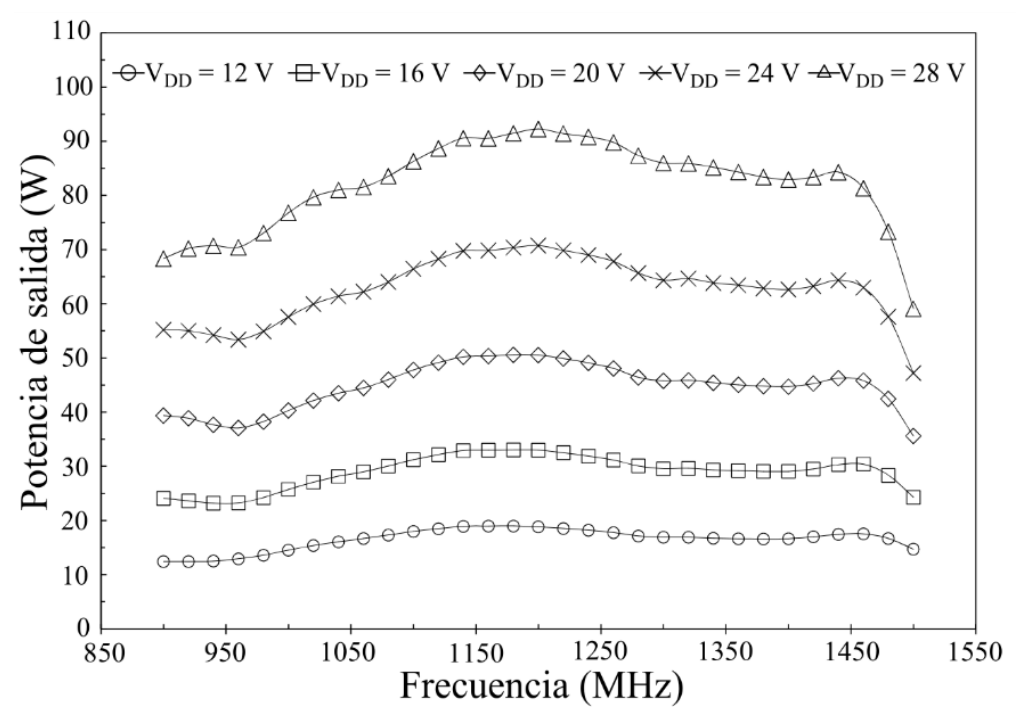

Figura 3.75. Medida de la potencia de salida en función de la frecuencia en CW (para una célula del amplificador).

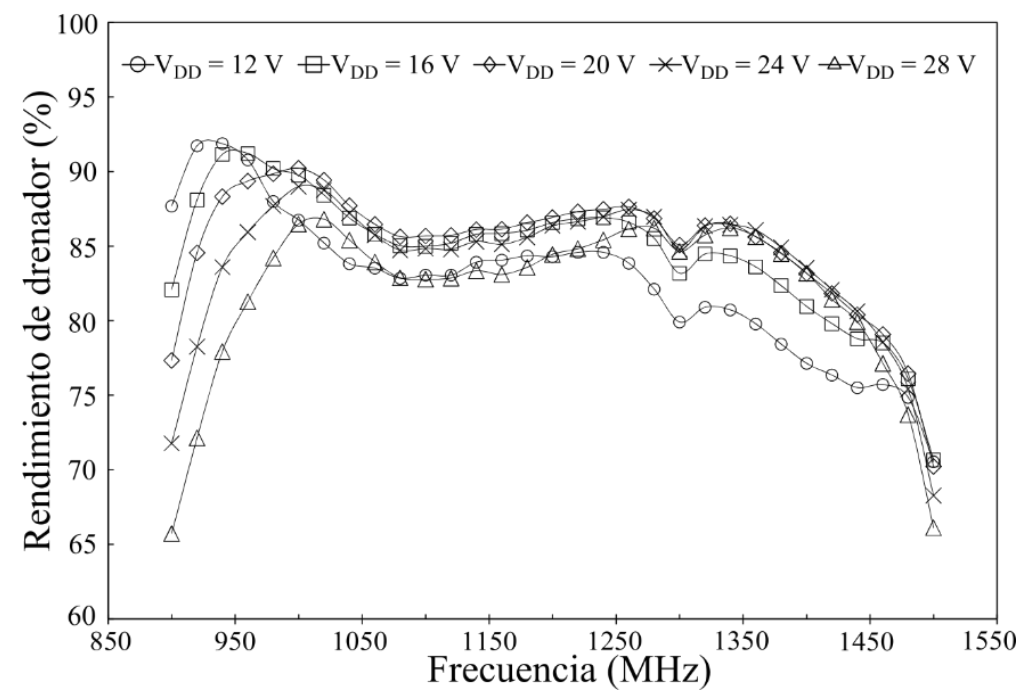

Figura 3.76. Medida del rendimiento de drenador en función de la frecuencia en CW (para una célula del amplificador).

La Figura 3.77 muestra la medida de rendimiento de potencia añadida (PAE) para diferentes valores de alimentación $V_{d d}$. Se puede observar una fuerte dependencia 
de la PAE respecto a $V_{d d}$ debido a la dependencia de la ganancia en potencia dependiente de la tensión de alimentación $V_{d d}$. Este efecto es característico de los amplificadores en modo de conmutación. Por ejemplo, en el caso de este amplificador, la ganancia en potencia a $28 \mathrm{~V}$ es de $15 \mathrm{~dB}$ mientras que a $12 \mathrm{~V}$ es de únicamente $8 \mathrm{~dB}$. El rendimiento de potencia añadido máximo (PAEmax) se obtiene para este caso particular a 28V. En [133] se presenta un amplificador optimizado para trabajar de manera más efectiva alimentado por debajo de la tensión de alimentación máxima. Esta situación se suele dar en aplicaciones donde se optimiza el amplificador para trabajar con señales en función de la densidad de probabilidad.

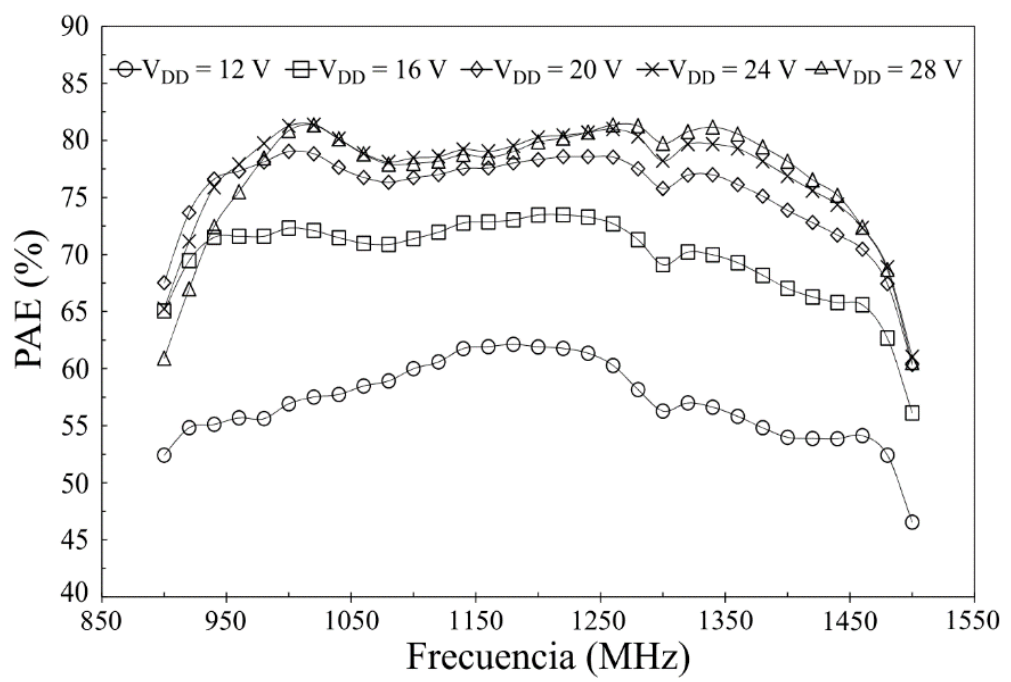

Figura 3.77. Medida de la PAE en función de la frecuencia en CW (para una célula del amplificador).

La Figura 3.78 presenta la potencia de salida en función de la potencia de entrada para un funcionamiento en onda continua y una única célula a diferentes frecuencias. Además del funcionamiento saturado del amplificador, éste también puede ser utilizado en su zona lineal proporcionando una ganancia de unos $20 \mathrm{~dB}$ a cambio de reducir el rendimiento de drenador. 


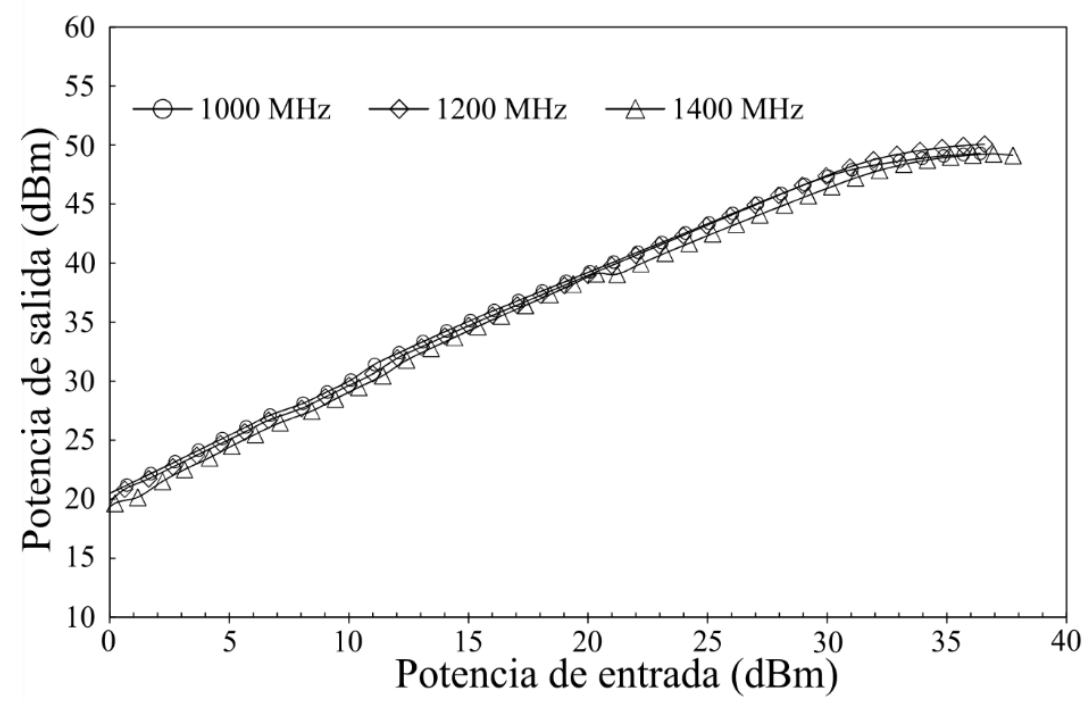

Figura 3.78. Medida de la potencia de salida en función de la potencia de entrada en CW (para una célula del amplificador).

El transistor CGH40180PP dispone de dos células que permiten construir dos amplificadores idénticos que pueden ser combinados en un amplificador balanceado mediante el uso de un combinador híbrido $3 \mathrm{~dB} 90^{\circ}$ para duplicar la potencia de salida y reducir la relación de onda estacionaria de entrada a niveles aceptables. En este caso se utilizó el combinador 1E1305 de Anaren.

Ya que la potencia de salida del amplificador balanceado excede de la potencia máxima del combinador en onda continua $\mathrm{CW}$, solo podrá trabajar a máxima potencia en modo pulsado y con un ciclo de trabajo del 50\%. Esta situación ocurre solo a tensiones de alimentación por encima de los $20 \mathrm{~V}$, donde la potencia de salida es superior a los 100W. Esta limitación es debida únicamente al combinador, lo que no quiere decir que el amplificador balanceado no pueda trabajar en modo continuo si se emplea un combinador de alta potencia adecuado.

La potencia de salida y el rendimiento de drenador fueron medidos también en modo pulsado, siendo la anchura del pulso de $1 \mathrm{~ms}$ y la frecuencia de repetición del pulso (PRF) de $100 \mathrm{~Hz}$. Para la realización de estas medidas en pulsado se diseñó un sintetizador muy simple capaz de generar pulsos de alimentación en función de las características del modo pulsado. En la Figura 3.79 se puede ver el esquema de este sintetizador de pulsos. 


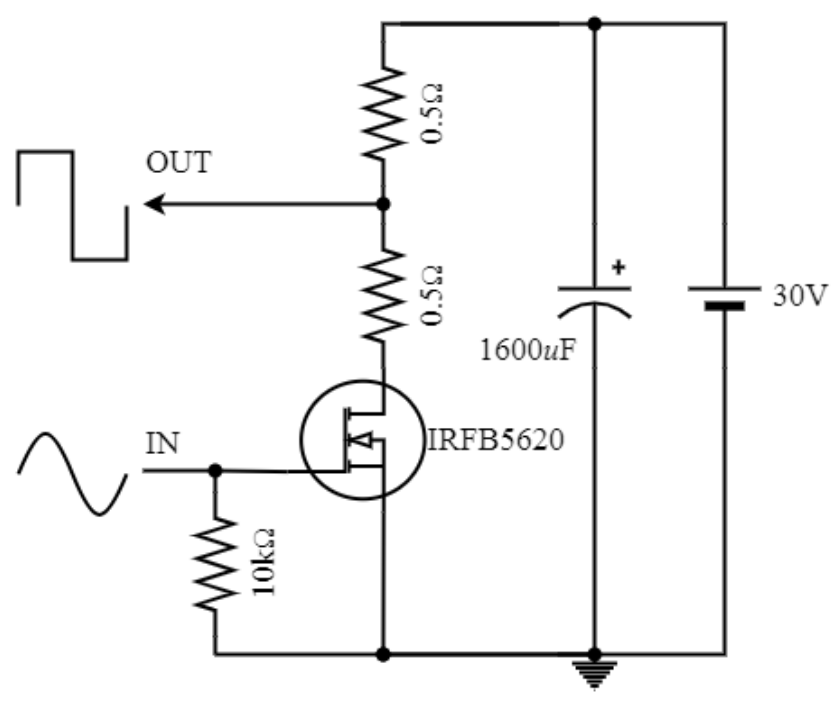

Figura 3.79. Sintetizador de pulsos para las medidas del amplificador en modo pulsado.

En modo pulsado, el amplificador puede proporcionar hasta 94W (por célula) en comparación con los $90 \mathrm{~W}$ bajo condiciones de onda continua. Picos de rendimiento de drenador de hasta el $90 \%$ a $28 \mathrm{~V}$ fueron medidos en modo pulsado respecto al $85 \%$ a 28V medido en CW.

Las medidas de potencia de salida y del rendimiento de drenador del amplificador balanceado funcionando en modo pulsado (la anchura del pulso es de $1 \mathrm{~ms}$ y la frecuencia de repetición del pulso es de $100 \mathrm{~Hz}$ ) se muestran en la Figura 3.80 y la Figura 3.81, respectivamente.

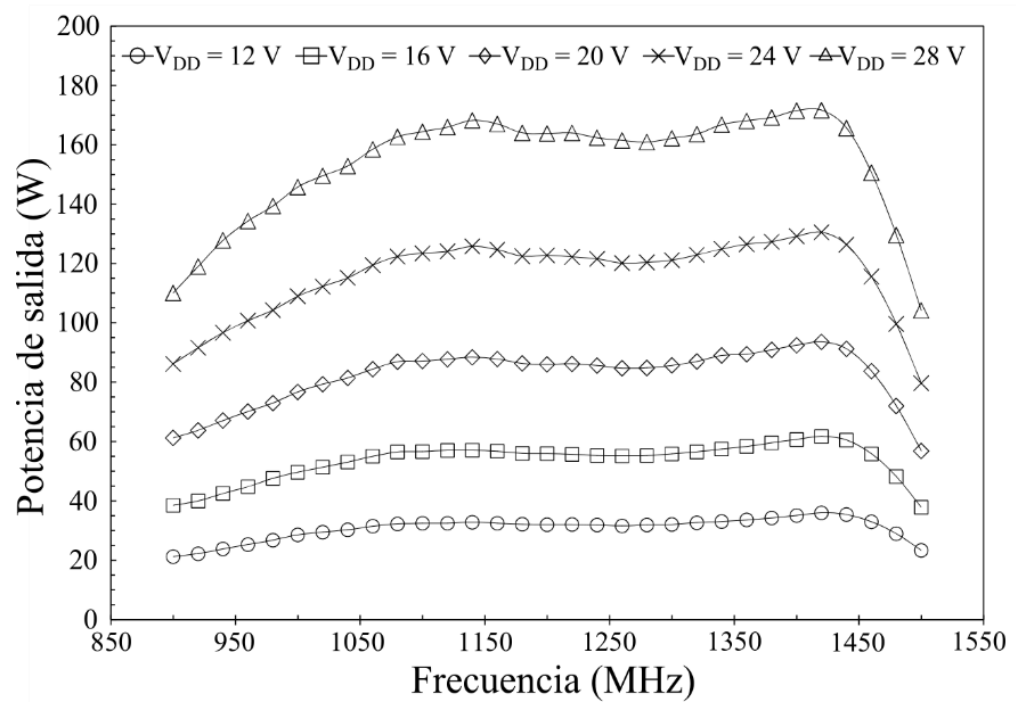

Figura 3.80. Medida de la potencia de salida en función de la frecuencia en modo pulsado (para dos células del amplificador). 


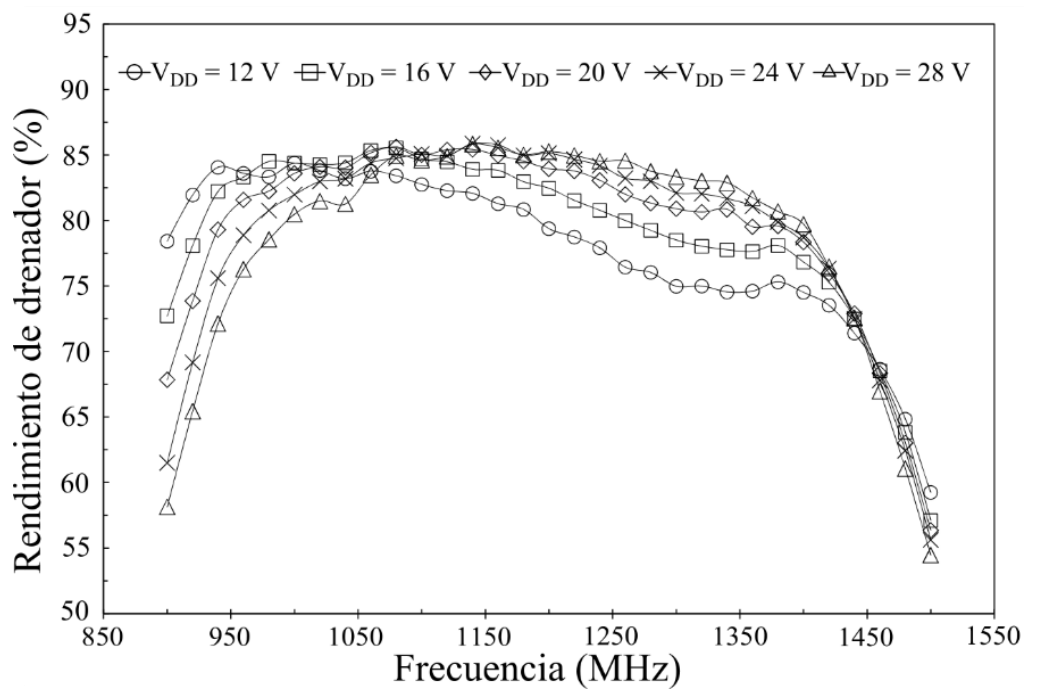

Figura 3.81. Medida del rendimiento de drenador en función de la frecuencia en modo pulsado (para dos células del amplificador).

Como se ha mencionado anteriormente, uno de los beneficios al implementar el amplificador balanceado es la importante mejora de las pérdidas de retorno y de la relación de onda estacionaria de tensión (VSWR). La Figura 3.82 muestra dichas medidas.

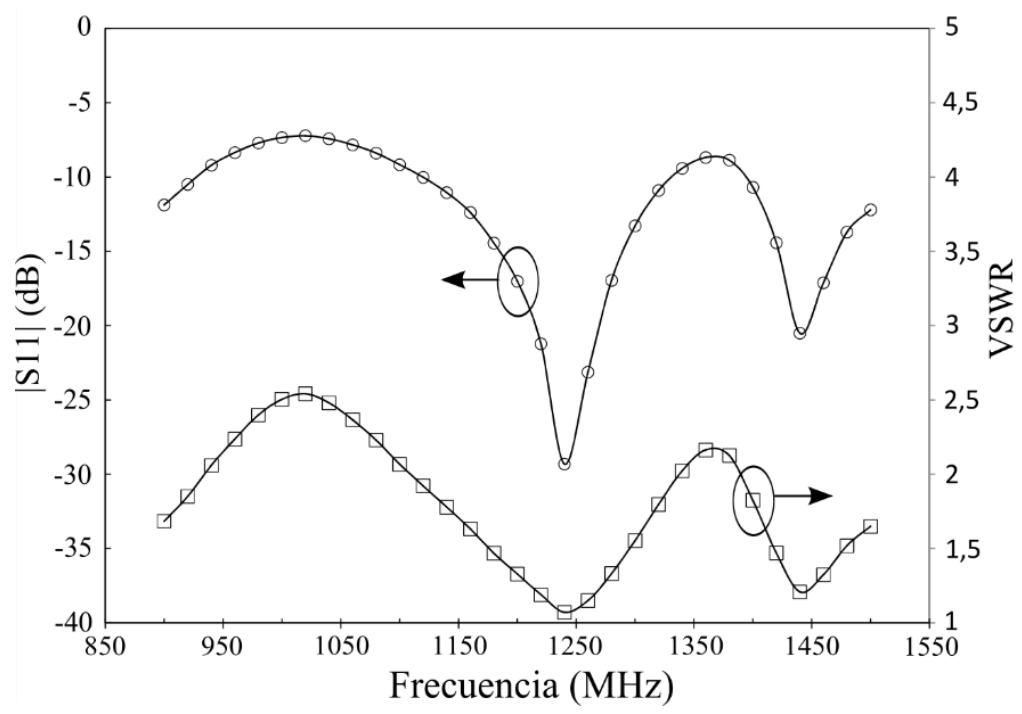

Figura 3.82. Medida de las pérdidas de retorno y de la relación de onda estacionaria de tensión en función de la frecuencia (para dos células del amplificador).

Una de las más importantes y prometedora aplicaciones para este amplificador es el funcionamiento como etapa de RF en transmisores linealizados a través de la técnica eliminación y restauración de envolvente (EER) o seguimiento de envolvente 
(ET), por ejemplo, en servicios de transmisión de radio digital (DAB, Digital Audio Broadcasting) o en comunicaciones móviles por satélite. Por ello, la respuesta $\mathrm{V}_{\mathrm{DD}} \mathrm{AM}$ y $V_{D D}-P M$ necesitan ser caracterizadas para estas aplicaciones [134].

El método seguido para realizar esta caracterización se muestra en la Figura 3.83. Consiste en modular la alimentación del amplificador trabajando en saturación con una señal triangular de baja frecuencia que cubra todo el rango dinámico, en este caso desde $0 \mathrm{~V}$ a $28 \mathrm{~V}$. Esto se puede conseguir controlando por ordenador una fuente de alimentación lineal.

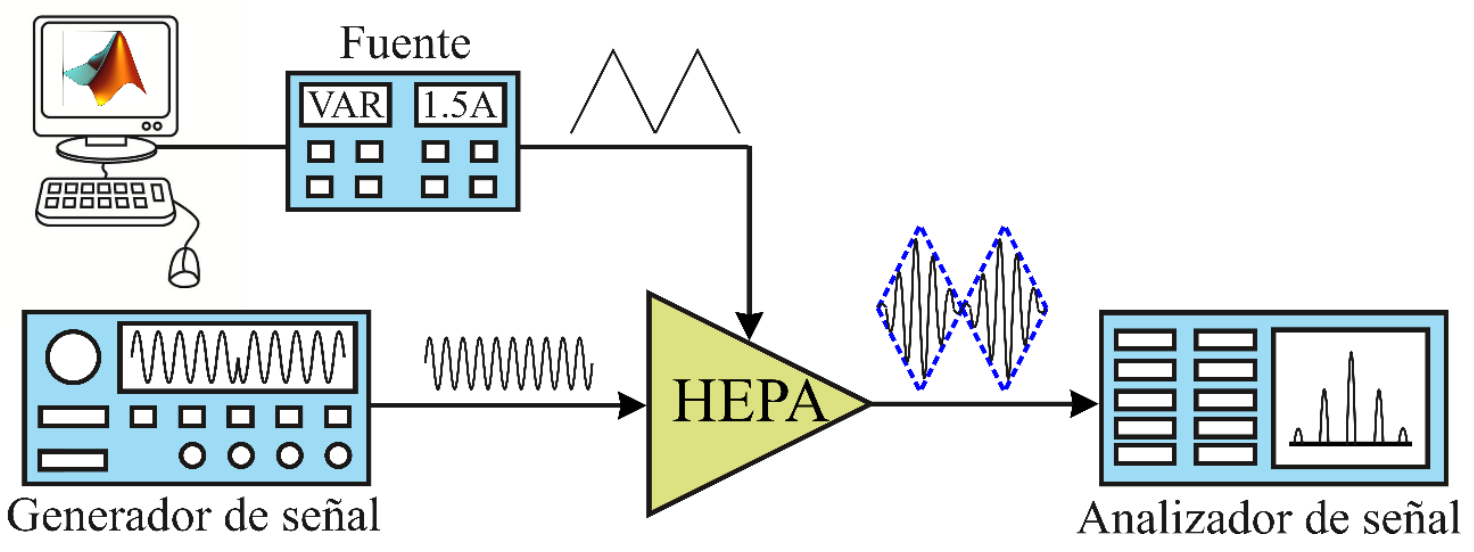

Figura 3.83. Diagrama de bloques del método utilizado para caracterizar la distorsión $V_{D D}-A M$ y $V_{D D}-P M$ del amplificador balanceado.

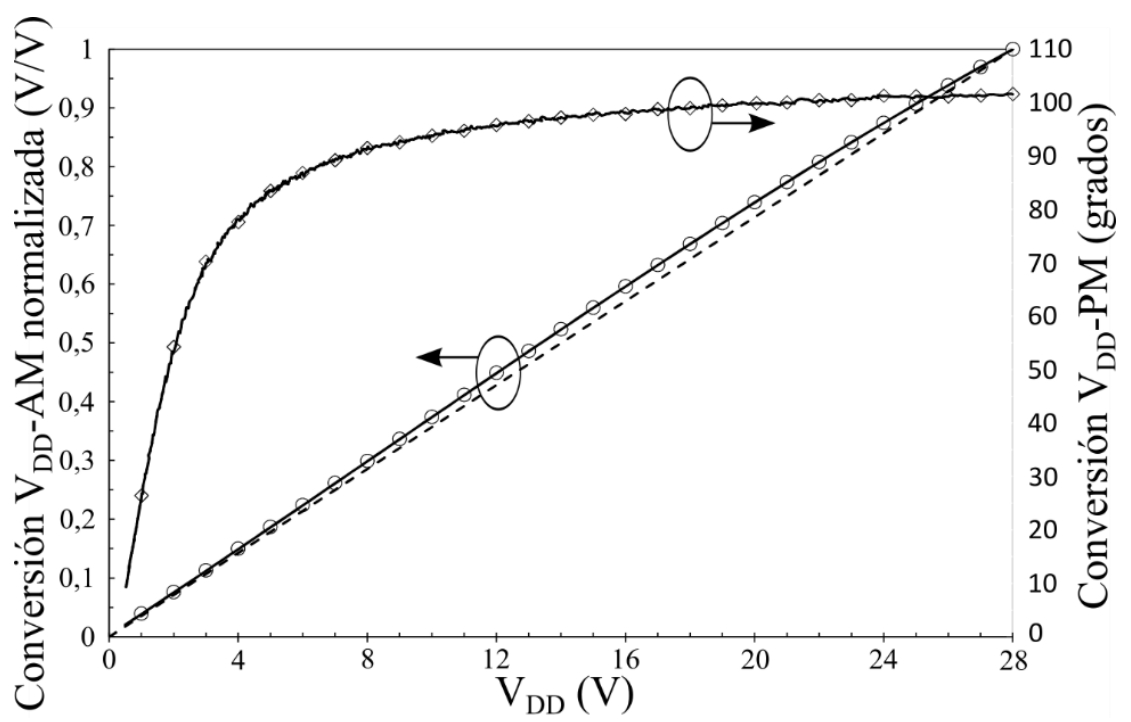

Figura 3.84. Caracterización de la conversión $\mathrm{V}_{\mathrm{DD}}-\mathrm{AM}$ y $\mathrm{V}_{\mathrm{DD}}-\mathrm{PM}$ del amplificador balanceado. 
La Figura 3.84 muestra la respuesta en amplitud y en fase respecto a la tensión de alimentación a $1300 \mathrm{MHz}$. De la figura se puede atestiguar que la conversión $\mathrm{V}_{\mathrm{DD}^{-}}$ AM es prácticamente insignificante; sin embargo, la conversión $\mathrm{V}_{\mathrm{DD}}-\mathrm{PM}$ (causada por la naturaleza no lineal de la capacidad equivalente del transistor $C_{o u t}$ ) debería ser corregida mediante predistorsión u otro mecanismo para lograr el funcionamiento lineal razonable en aplicaciones EER/ET [133] [135].

Otra de las aplicaciones donde este amplificador balanceado puede ofrecer muy buenas prestaciones es en RADAR pulsado en banda L. La Figura 3.85 muestra la señal demodulada de un pulso RADAR de anchura $1 \mathrm{~ms}$ y frecuencia de repetición de $100 \mathrm{~Hz}$ a $1300 \mathrm{MHz}$, entregando una potencia de 180W. El análisis del pulso demodulado no muestra decaimientos $\mathrm{y}$, consecuentemente, no es necesario ningún circuito de compensación de ganancia.

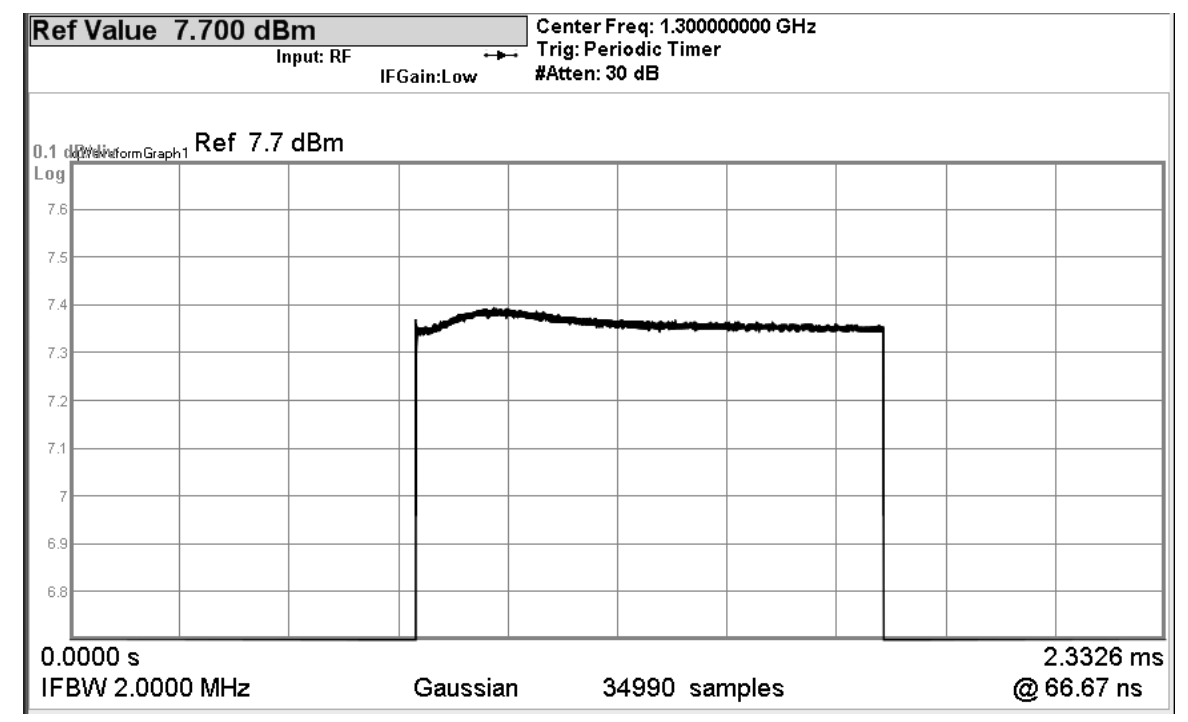

Figura 3.85. Medida de un pulso RADAR de anchura $1 \mathrm{~ms}$ y frecuencia de repetición 100 $\mathrm{Hz}$ a $1300 \mathrm{MHz}$ empleando el amplificador balanceado.

Los radares comerciales en la banda de frecuencia de $1250 \mathrm{MHz}$ a los 1350 MHz, como el RADAR Lanza, son sistemas de muy alta potencia, típicamente de varios kW. Múltiples unidades del amplificador mostrado en este trabajo podrían ser combinadas con el fin de obtener una potencia de salida muy alta manteniendo unos parámetros de funcionamiento muy prometedores. En la Figura 3.86 se muestra un esquema con esta propuesta empleando combinadores Wilkinson. Otros tipos de combinadores pueden ser estudiados de acuerdo al número de amplificadores que se deseen combinar, presentando diferentes ventajas e inconvenientes [136]. 


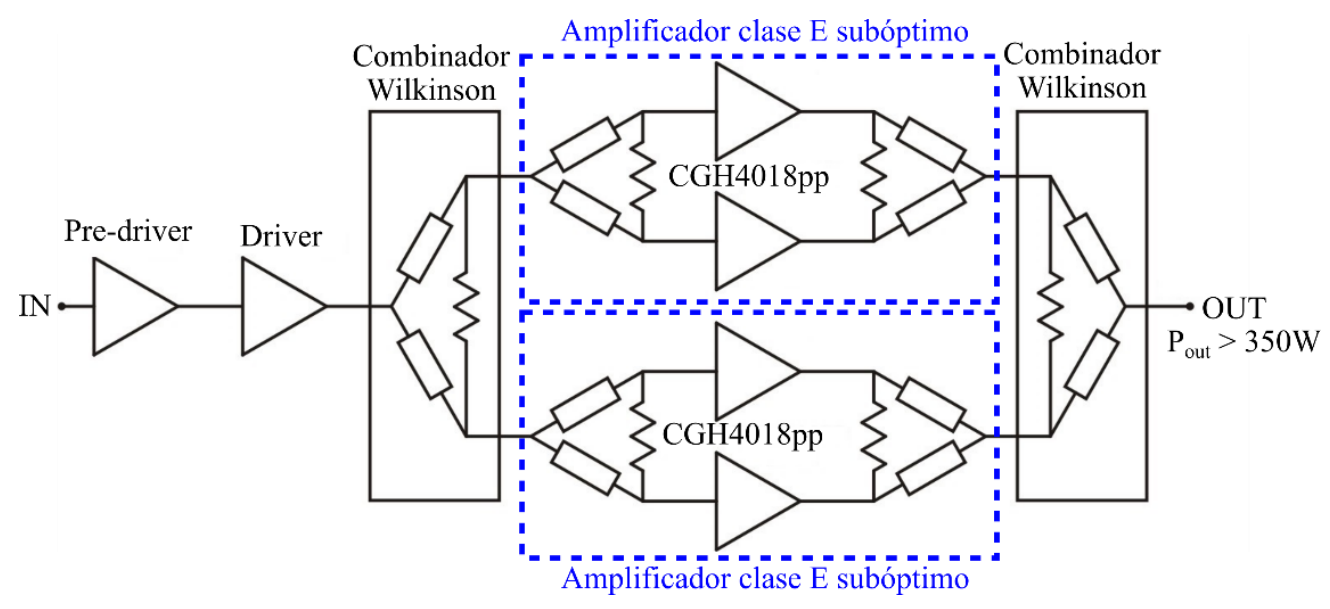

Figura 3.86. Propuesta de combinación de varios amplificadores balanceados mediante combinadores Wilkinson para duplicar la potencia de salida.

La Figura 3.87 muestra los niveles de armónicos medidos del amplificador balanceado, donde se puede comprobar que los más significativos se encuentran a más de $30 \mathrm{dBc}$.

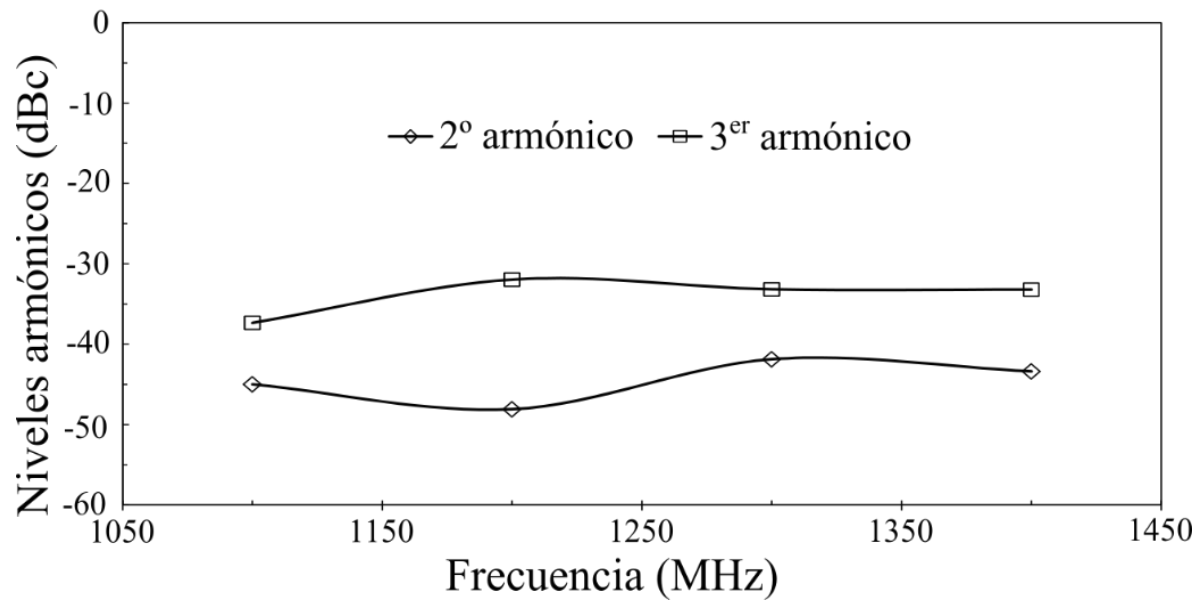

Figura 3.87. Nivel de los armónicos en función de la frecuencia del tono empleado en la medida.

Según el conocimiento de los autores, el funcionamiento de este amplificador supera a otros amplificadores en la misma banda de frecuencia y nivel de potencia de salida, especialmente los de categoría comercial, como se muestra en la Tabla 3.4. 
Tabla 3.4. Comparación del amplificador diseñado en este trabajo con respecto al estado del arte de amplificadores de potencia en banda $L$.

\begin{tabular}{lccccc}
\hline \hline Referencia & $\begin{array}{c}\text { Frecuencia } \\
(\mathbf{G H z})\end{array}$ & $\begin{array}{c}\mathbf{P}_{\text {out }} \\
(\mathbf{W})\end{array}$ & $\begin{array}{c}\text { Gain } \\
(\mathbf{d B})\end{array}$ & $\begin{array}{c}\boldsymbol{\eta}_{d} / \text { PAE } \\
(\boldsymbol{\%})\end{array}$ & $\begin{array}{c}\text { Clase de } \\
\text { amplificación }\end{array}$ \\
\hline $2006[137]$ & 1.5 & 500 & 17.8 & $50 /-$ & Class-B \\
$2009[138]$ & $0.6-1$ & 49 & 16.3 & $87.8 / 80.6$ & Class-E \\
$2011[139]$ & $1-2$ & 90 & 11 & $-/ 50$ & Class-AB \\
$2011[140]$ & 1.19 & 15 & 18.5 & $-/ 74$ & Class-AB \\
$2011[141]$ & 1.275 & 10 & 10 & $81 / 73$ & Class-E \\
$2011[142]$ & $1.2-2$ & 20 & 13 & $84 / 79$ & Class-E \\
$2012[143]$ & $1.2-1.3$ & 140 & 12.5 & $60 /-$ & Class-AB \\
Este trabajo & $0.9-1.5$ & 180 & 14 & $85 / 81$ & SO Class-E \\
\hline \hline
\end{tabular}

Como conclusión, a partir de las medidas obtenidas y de la comparación con otros trabajos para la misma banda de frecuencia y potencia, el diseño de amplificadores basado en la topología clase E subóptima es una solución no extensamente usada. Esta clase de funcionamiento permite al transistor proporcionar alto rendimiento a frecuencia por encima de su frecuencia de trabajo en clase E nominal compitiendo con otras topologías como clase $\mathrm{F}$ o clase $\mathrm{F}^{-1}$ para la misma banda de frecuencia y potencia, además de disponer de una red de carga más simple.

\subsection{Amplificador de potencia clase $\mathbf{E}$ subóptimo en banda $S$}

Hoy en día, las técnicas de amplificación conmutadas en la banda de microondas están ganando en popularidad debido al desarrollo comercial de dispositivos de GaN que presentan una mayor fiabilidad y cuyos fabricantes han madurado los modelos y herramientas para reducir y facilitar el diseño de estos amplificadores. Además, también se han incrementado los estudios que combinan estas técnicas de amplificación con técnicas de linealización basadas en el procesado digital de la señal.

Estos avances han facilitado el diseño de amplificadores de GaN a más altas frecuencias, como es el caso del amplificador que se va a mostrar a continuación. El objetivo es diseñar un amplificador de alto rendimiento y en banda ancha basado en tecnología GaN capaz de entregar una potencia de salida por encima de $10 \mathrm{~W}$. 
Trabajando en banda S, puede cubrir multitud de aplicaciones como por ejemplo ser parte de un transmisor en la etapa de RF de un sistema de comunicaciones por satélite, o para aplicaciones de transferencia inalámbrica de energía (Wireless Power Transfer, WPT), o incluso en aplicaciones industriales o de servicios médicos.

\subsubsection{Transistor de GaN}

El amplificador diseñado empleará el transistor CGH40010F de Cree Inc (Figura 3.88). Se trata de un HEMT basado en tecnología GaN. Como en el caso anterior, es un transistor no adaptado, lo que beneficia a la hora de diseñar las redes de adaptación. Se presenta en un encapsulado cerámico y hermético que le proporciona una excelente estabilidad térmica.

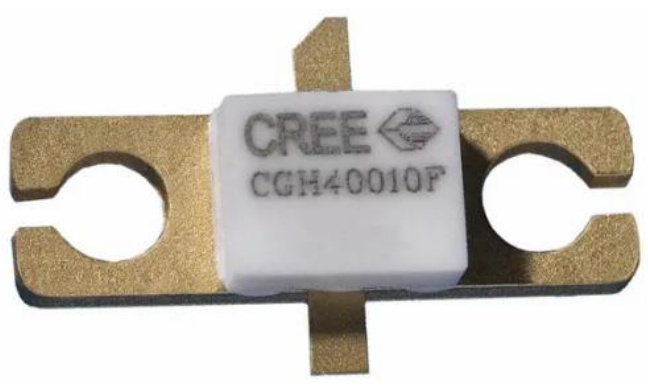

\section{Figura 3.88. Transistor CGH40010P empleado diseño del amplificador clase $E$ en banda $S$.}

Este transistor presenta una tensión de ruptura de $120 \mathrm{~V}$, lo que le convierte en un buen candidato para ser integrado en un amplificador clase E alimentado a $28 \mathrm{~V}$. Sin embargo, antes hay que analizar la frecuencia máxima a la que este transistor puede trabajar en clase E nominal. En este caso, la red de carga utilizada, como se verá más adelante, es una red con capacidad en derivación y filtro en derivación [144]. La frecuencia máxima de funcionamiento de una clase $\mathrm{E}$ nominal con capacidad en derivación y filtro en derivación está definida por la capacidad de salida del dispositivo y de puede calcular usando Ecuacion 3.7.

$$
f_{\max }=\frac{0.097 P_{o}}{V_{D D}^{2} C_{o u t}}
$$

donde $P_{o}$ es la potencia de salida, $V_{D D}$ es la tensión de alimentación y $C_{o u t}$ es la capacidad equivalente de salida del transistor. Esta ecuación puede ser reescrita para 
hacerla dependiente de la corriente máxima de drenador, como se puede ver en la ecuación 3.8 .

$$
f_{\max }=\frac{0.035 I_{\max }}{V_{D D} C_{\text {out }}}
$$

donde $I_{\max }$ es la corriente máxima de drenador, $V_{D D}$ es la tensión de alimentación y $C_{o u t}$ es la capacidad equivalente de salida del transistor.

De la hoja de características del fabricante [145] se pueden obtener los valores de los elementos necesarios para calcular esta frecuencia máxima a partir de la Ecuación 3.7 y 3.8. Siendo la capacidad equivalente de salida la suma de la capacidad drenadorfuente $\left(C_{d s}\right)$ cuyo valor es $1.3 \mathrm{pF}$ a $28 \mathrm{~V}$ medido a $1 \mathrm{MHz}$ y la capacidad puerta-drenador $\left(C_{g d}\right)$ siendo $0.2 \mathrm{pF}$ a $28 \mathrm{~V}$ medida $1 \mathrm{MHz}$, para una potencia de salida de $14 \mathrm{~W}$ y alimentado a $28 \mathrm{~V}$ la frecuencia máxima en la que este transistor puede trabajar en clase E nominal es de aproximadamente $1155 \mathrm{MHz}$. Además, a media que baja la potencia también disminuye la frecuencia crítica. En la Figura 3.89 se pude ver la relación que existe entre la frecuencia máxima y la potencia de salida o la corriente máxima de drenador.

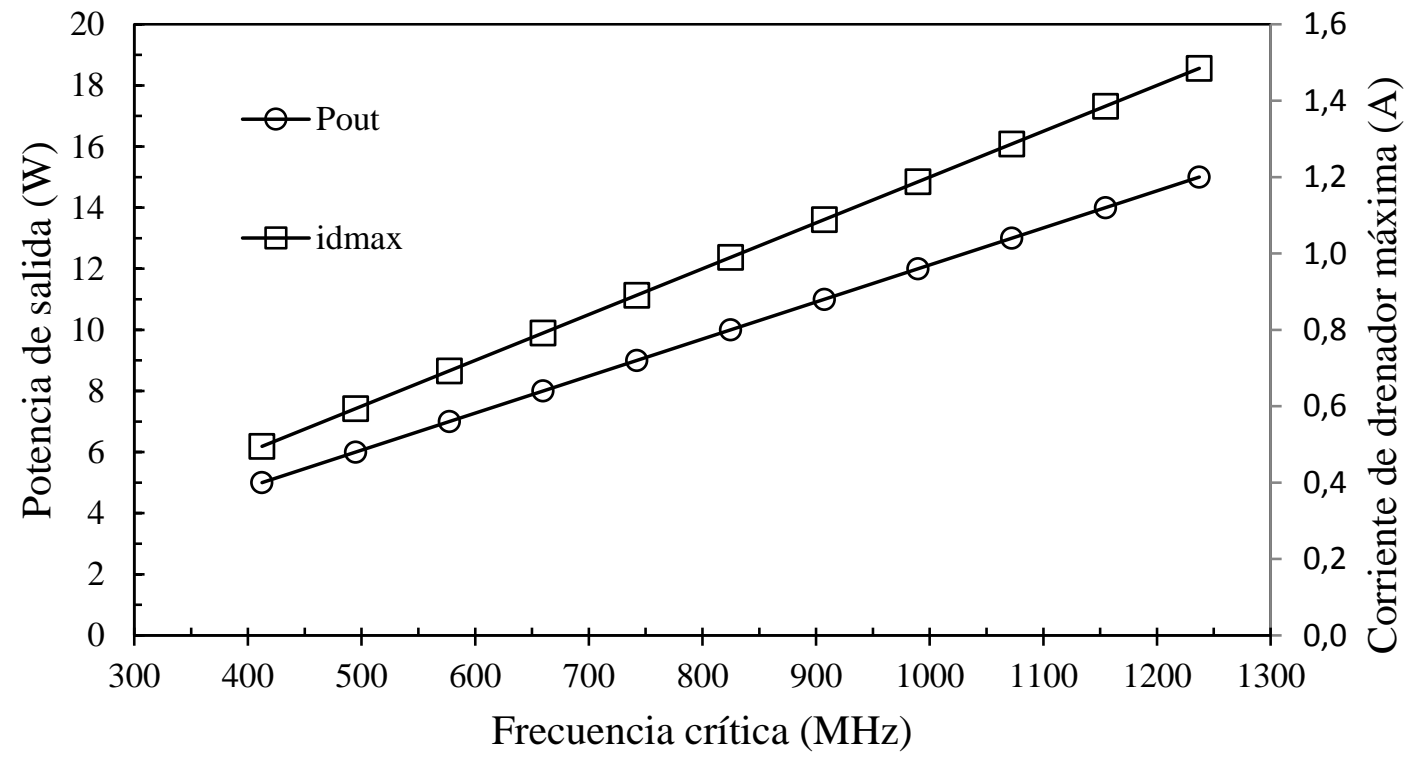

Figura 3.89. Frecuencia crítica de un amplificador clase $E$ en función de la potencia de salida para el transistor CGH4001P.

El ángulo óptimo a $f_{\max }$ para esta red de carga es de $-32.945^{\circ}$. Además, la tensión máxima de drenador es de 3.677 veces la tensión de alimentación, es decir, 
aproximadamente $103 \mathrm{~V}$ y la corriente máxima de drenador es de 2.768 veces la corriente de alimentación, lo que se traduce en unos 2.11A. Estos valores cumplen con los valores máximos permitidos por el transistor según el fabricante, lo que permite su uso en este amplificador. Cabe recordar que al trabajar a una frecuencia por encima de esta frecuencia máxima, que sería el peor caso posible en cuanto a requisitos del transistor, estos valores se vuelven menos restrictivos por no estar trabajando de manera óptima.

La frecuencia central de diseño de este amplificador es poco más de dos veces la frecuencia máxima a la cual puede funcionar en modo clase E nominal. Esto implica trabajar en modo subóptimo lo que conlleva que el rendimiento máximo ideal que se puede conseguir disminuya hasta el 90\%. Este funcionamiento en clase E subóptimo ya ha sido extensamente explicado en el Apartado 3.2 de este capítulo.

\subsubsection{Diseño del amplificador clase $\mathbf{E}$ subóptimo en banda $S$}

Al igual que el amplificador clase E subóptimo en banda L presentado en el Apartado 3.2, el diseño también ha sido llevado a cabo mediante la técnica de síntesis de la admitancia de carga.

Como ya se comentó, la admitancia de carga necesaria para el diseño del amplificador clase E subóptimo con capacidad en derivación y filtro en derivación depende de la reactancia de la capacidad en derivación $\left(X_{\text {cout }}\right)$ a la frecuencia más alta de funcionamiento, de la tensión de alimentación $\left(V_{d d}\right)$ y del nivel de potencia de salida del amplificador $\left(P_{\text {out }}\right)$. En el caso de este amplificador, la capacidad en derivación $C_{\text {out }}$ tiene un valor de $1.5 \mathrm{pF}$, la frecuencia más alta de funcionamiento será de $2600 \mathrm{MHz}$, la tensión de alimentación será de $28 \mathrm{~V}$ y se pretende generar una potencia de salida de 14W. Considerando todos estos factores, la admitancia de carga se puede calcular como en [122] y [145] siendo, a la frecuencia fundamental, el módulo de la admitancia 0.023 $\mathrm{S}$ y la fase de $-20^{\circ}$ en el centro de la banda. Esta fase varía desde los $-28^{\circ}$ a los $-18^{\circ}$ dentro del ancho de banda del amplificador. En cuanto a los armónicos, la fase de la admitancia de carga debe ser $90^{\circ}$.

Estos valores de admitancia de carga fueron ajustados usando un optimizador por balance armónico en todo el ancho de banda del amplificador con el fin de 
conseguir los objetivos de potencia de salida y rendimiento. Esta admitancia de carga fue presentada usando una red de carga que transforma la impedancia de $50 \Omega$ del puerto de salida al valor requerido por el amplificador. La red diseñada para este amplificador se muestra en la Figura 3.90.

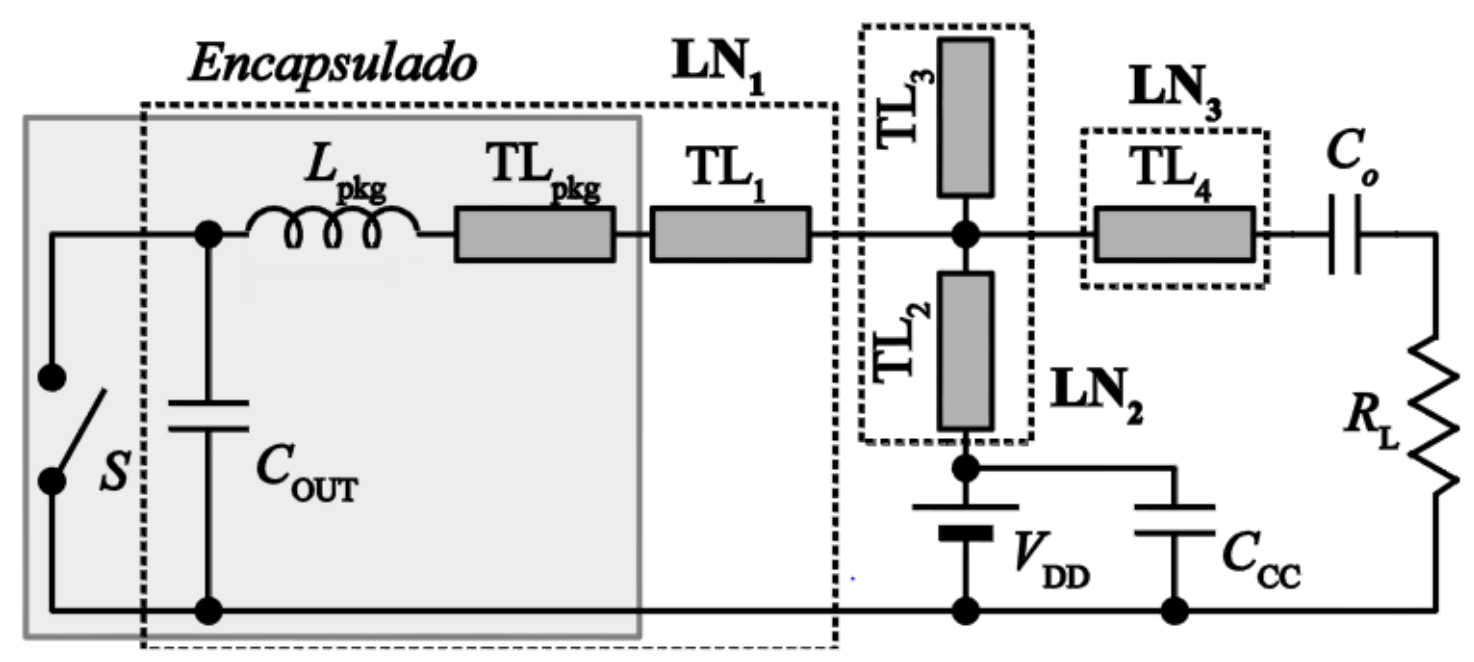

Figura 3.90. Esquema de la red de salida del amplificador clase E subóptimo en banda S.

El circuito implementa una red de compensación de doble-reactancia para conseguir un funcionamiento en banda ancha [116]. La Tabla 3.5 muestra los valores de los elementos de la red de carga.

Tabla 3.5. Valores de los componentes de la red de salida.

\begin{tabular}{cc|cc}
\hline \hline CoUT & $1.5 \mathrm{pF}$ & $T L_{O 2} Z_{0}$ & $15.1 \Omega$ \\
$L_{p c k g O U T}$ & $0.55 \mathrm{nH}$ & $T L_{O 2} \theta_{e}$ & $59.5^{\circ} @ 2.4 \mathrm{GHz}$ \\
$T L_{p c k g O U T} Z_{0}$ & $17.7 \Omega$ & $T L_{O 3} Z_{0}$ & $15.1 \Omega$ \\
$T L_{p c k g O U T} \theta e$ & $6.08^{\circ} @ 2.4 \mathrm{GHz}$ & $T L_{O 3} \theta_{e}$ & $30.2^{\circ} @ 2.4 \mathrm{GHz}$ \\
$T L_{O 1} Z_{0}$ & $37.57 \Omega$ & $T L_{O 4} Z_{0}$ & $25.6 \Omega$ \\
$T L_{O 1} \theta e$ & $13.8^{\circ} @ 2.4 \mathrm{GHz}$ & $T L_{O 4} \theta e$ & $91.8^{\circ} @ 2.4 \mathrm{GHz}$ \\
\hline \hline
\end{tabular}

La red de carga se puede subdividir en tres redes tal y como se describe en [115]:

- LN1 está compuesta por la capacidad equivalente de salida del transistor $C_{\text {out }}$, los elementos parásitos del encapsulado en el puerto de salida,

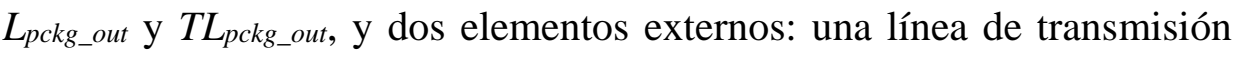
de corta longitud, $T L_{o 1}$ y una parte del efecto capacitivo que genera la 
línea $T L_{o 3}$. Estos elementos forman una red paso bajo en $\pi$ y es equivalente a un transformador $\lambda / 4$ con línea de transmisión (en condiciones de banda estrecha).

- LN2 consta de una línea microstrip en cortocircuito, $T L_{o 2}$, y la parte restante de la línea de transmisión en circuito abierto, TLo3. Esta red genera las condiciones necesarias de esta clase de amplificación a los armónicos pares. Además, $T L_{o 2}$ se emplea como puerto de alimentación de DC.

- LN3 es un transformador $\lambda / 4$ con línea de transmisión, $T L_{o 4}$. Además de fijar las condiciones de la clase de amplificación al tercer armónico, esta red lleva a cabo la mayor transformación de impedancias de la red de salida. Este elemento equivale, en banda estrecha, a un filtro en derivación.

El perfil de admitancia de carga $\mathrm{Y}_{\mathrm{L}}(\mathrm{f})$ a la derecha del conmutador equivalente conseguido por la red de carga descrita se muestra en la Figura 3.91. Las mismas conclusiones obtenidas de la Figura 3.66 son aplicables a este perfil de admitancia.

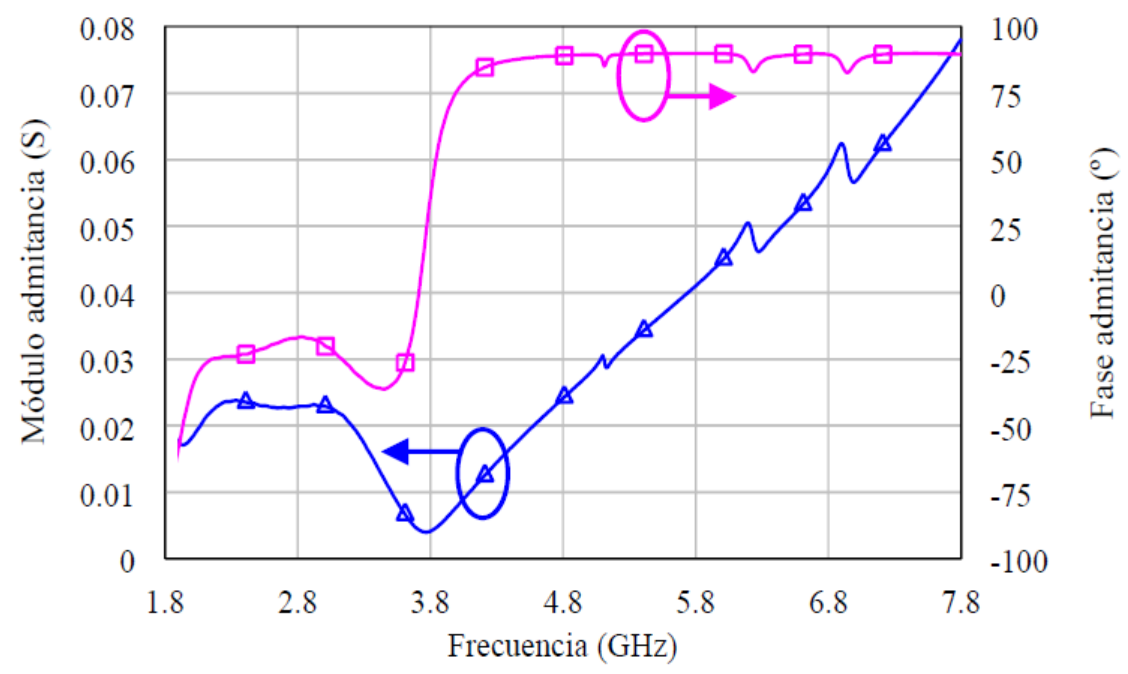

Figura 3.91. Perfil de admitancia proporcionado por la red de salida del amplificador a la derecha del conmutador equivalente.

El siguiente paso es realizar el diseño de la red de entrada, no menos importante que la red de salida. La principal premisa de esta red es excitar al amplificador de una manera eficiente de tal forma que no se produzcan excesivas pérdidas que afecten a una reducción en la ganancia del amplificador y por lo tanto en su rendimiento global. 
La red de entrada diseñada sigue las mismas consideraciones del Apartado 3.2.3. Debido a los efectos de la capacidad de entrada equivalente del transistor y de los parásitos de su encapsulado, se empleará una forma de onda de tensión sinusoidal con un ciclo de trabajo del 50\%. Además, esta red de entrada mantendrá un nivel de potencia de excitación constante en todo el ancho de banda, a costa de reflejar parte de la potencia a determinadas frecuencias. Basado en todo lo anterior, la red de entrada propuesta en este trabajo se muestra en la Figura 3.92 y los valores de los elementos se presentan en la Tabla 3.6.

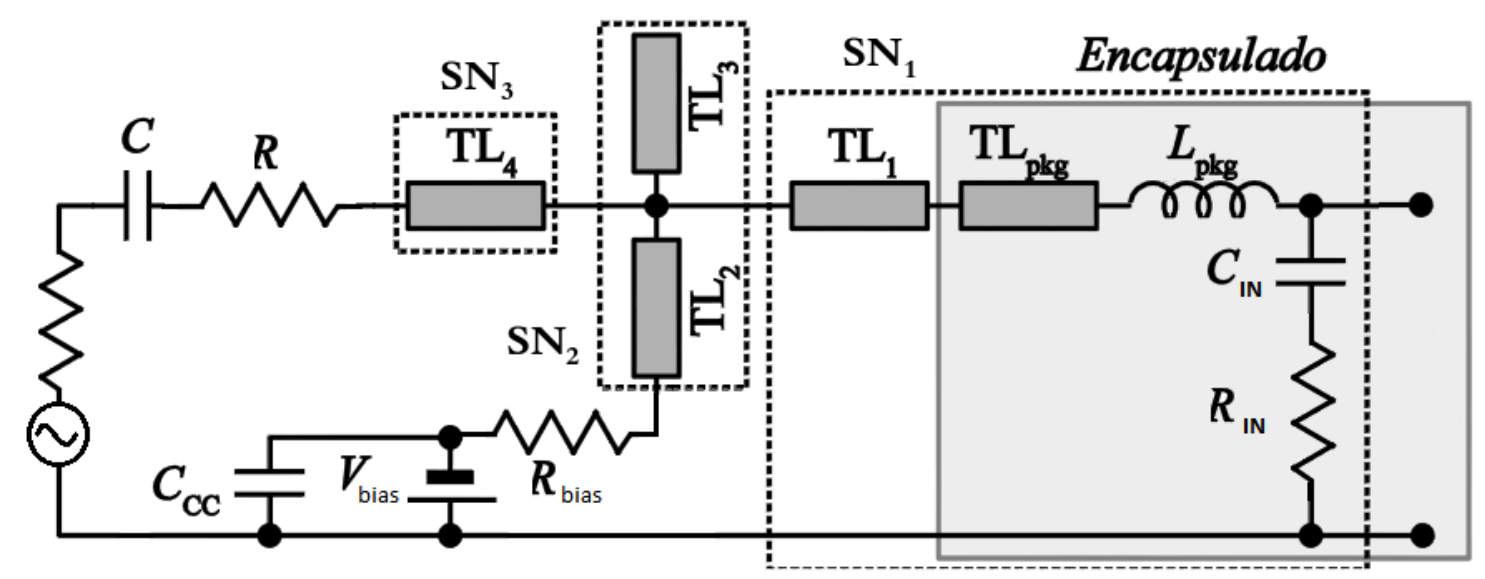

Figura 3.92. Esquema de la red de entrada del amplificador clase E subóptimo en band S.

Tabla 3.6. Valores de los componentes de la red de entrada.

\begin{tabular}{cc:cc}
\hline \hline$C_{I N}$ & $4.7 \mathrm{pF}$ & $T L_{I 2} Z_{0}$ & $39.15 \Omega$ \\
$L_{p c k g I N}$ & $0.55 \mathrm{nH}$ & $T L_{I 2} \theta_{e}$ & $56.33^{\circ} @ 2.4 \mathrm{GHz}$ \\
$T L_{p c k g I N} Z_{0}$ & $17.7 \Omega$ & $T L_{I 3} Z_{0}$ & $39.15 \Omega$ \\
$T L_{p c k g I N} \theta e$ & $6.08^{\circ} @ 2.4 \mathrm{GHz}$ & $T L_{I 3} \theta_{e}$ & $48.49^{\circ} @ 2.4 \mathrm{GHz}$ \\
$T L_{I 1} Z_{0}$ & $21.95 \Omega$ & $T L_{I 4} Z_{0}$ & $18.95 \Omega$ \\
$T L_{I 1} \theta e$ & $8.12^{\circ} @ 2.4 \mathrm{GHz}$ & $T L_{I 4} \theta e$ & $30.70^{\circ} @ 2.4 \mathrm{GHz}$ \\
$R$ & $7.5 \Omega$ & $R_{\text {bias }}$ & $20 \Omega$ \\
\hline \hline
\end{tabular}

La red de entrada también se subdivide en tres subredes. SN1 incluye la capacidad de entrada equivalente $C_{\text {in }}$ y la resistencia de entrada equivalente Rin, los parásitos del encapsulado del transistor, $L_{p c k g} i n$ y $T L_{p c k g} i n$, y dos líneas de transmisión externas, $T L_{i 1}$ y $T L_{i 3 a}$. SN2 se compone de una línea microstrip en cortocircuito $T L_{i 2}$, por donde será inyectada la tensión de polarización puerta-fuente $\left(V_{\text {bias }}\right)$, y de la parte restante de la línea de transmisión en circuito abierto $T L_{i 3 b}$. Además, una resistencia de 
polarización $R_{\text {bias }}$ ayuda a mantener la estabilidad del punto de funcionamiento del transistor. SN3 es una línea de transmisión que actúa como transformador de impedancia. En serie con la línea de excitación de RF se ha situado una resistencia cuyo objetivo es mantener la estabilidad del amplificador para cualquier impedancia de carga.

La red de entrada diseñada proporciona ganancia plana en todo el ancho de banda de funcionamiento. Además, muestra unos valores adecuados de pérdidas de retorno. Esta situación se puede comprobar en la Figura 3.93.

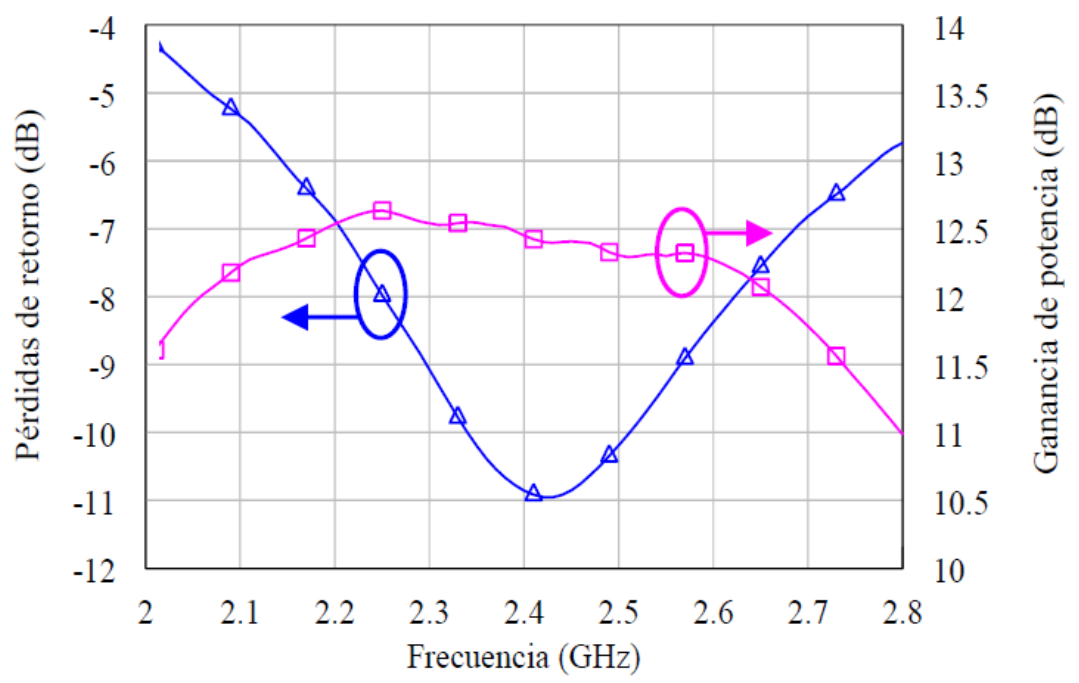

Figura 3.93. Perfil de ganancia en potencia y pérdidas de retorno del amplificador en función de la frecuencia.

\subsubsection{Simulación del amplificador clase $\mathbf{E}$ sub-óptimo en banda $S$}

La simulación y optimización del amplificador se realizó usando el software AWR Microwave Office Design y el modelo no lineal del transistor proporcionado por el fabricante. Durante este proceso, diversos problemas de convergencia a frecuencias y puntos de polarización y excitación puntuales fueron obtenidos hasta implementar el diseño final. Esto quiere decir que aún no están suficientemente maduros y depurados los modelos no lineales de transistores de GaN. A pesar de ello, una de las ventajas que incorporaba el modelo no lineal ofrecido por el fabricante es que presentaba un acceso al drenador intrínseco del transistor como se puede ver en la Figura 3.94. 


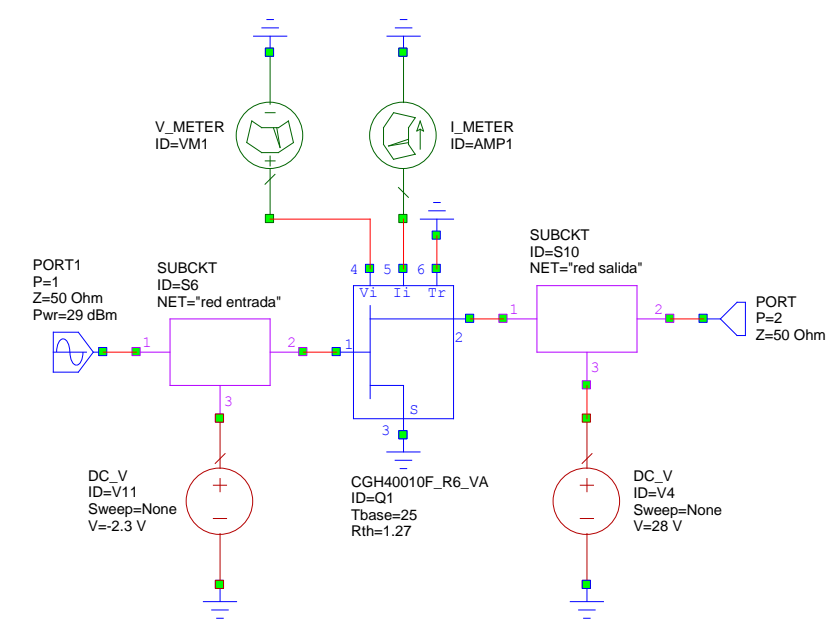

Figura 3.94. Modelo del transistor CGH40010p ofrecido por el fabricante donde se aprecia un acceso al drenador intrínseco.

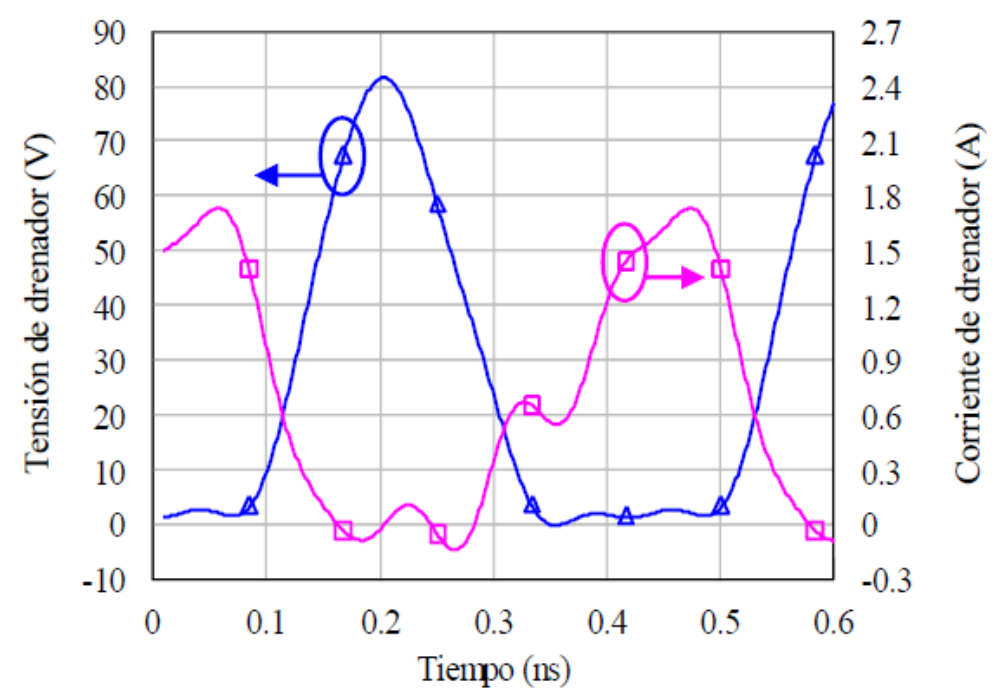

Figura 3.95. Formas de onda simuladas del amplificador clase E subóptimo en banda $S$.

Gracias a estos puertos se pueden comprobar por simulación las formas de onda del amplificador clase E subóptimo. En la Figura 3.95 se muestra la forma de onda de tensión y de corriente en el drenador intrínseco a la frecuencia central $(2.4 \mathrm{GHz})$ y para las siguientes condiciones de polarización: $I_{d q}=200 \mathrm{~mA}$ y $V_{d d}=28 \mathrm{~V}$. Las formas de onda simuladas confirman el correcto diseño del amplificador clase E subóptimo diseñado. Este funcionamiento se puede verificar debido a que la forma de onda de tensión se superpone con la forma de onda de corriente puesto que en el momento de la conmutación el condensador del transistor aún tiene carga, no cumpliendo una de las condiciones para el funcionamiento en clase E nominal. Ello provoca que se disipe 
potencia en el transistor y por lo tanto se reduzca el rendimiento. Otros parámetros que inducen a confirmar el modo de operación son los valores máximos de las formas de onda: la tensión máxima de drenador es de 82 voltios (2.93 veces $\left.V_{d d}\right)$ y la corriente máxima de drenador es de 1.7 A (2.23 veces $\left.I_{o}\right)$.

La Figura 3.96 muestra la simulación de la potencia de salida y del rendimiento de drenador del amplificador en función de la frecuencia para unas condiciones de polarización fijas: $\mathrm{Idq}=200 \mathrm{~mA}$ y $\mathrm{Vdd}=28 \mathrm{~V}$. Cabe destacar que tanto el rendimiento como la potencia se mantienen bastante planos en todo el margen de trabajo del amplificador. El rendimiento de drenador se mantiene sobre el $80 \%$ dentro de la banda comprendida entre los 2.1 y $2.6 \mathrm{GHz}$. La potencia de salida entregada supera los 41 $\mathrm{dBm}$ en todo el ancho de banda.

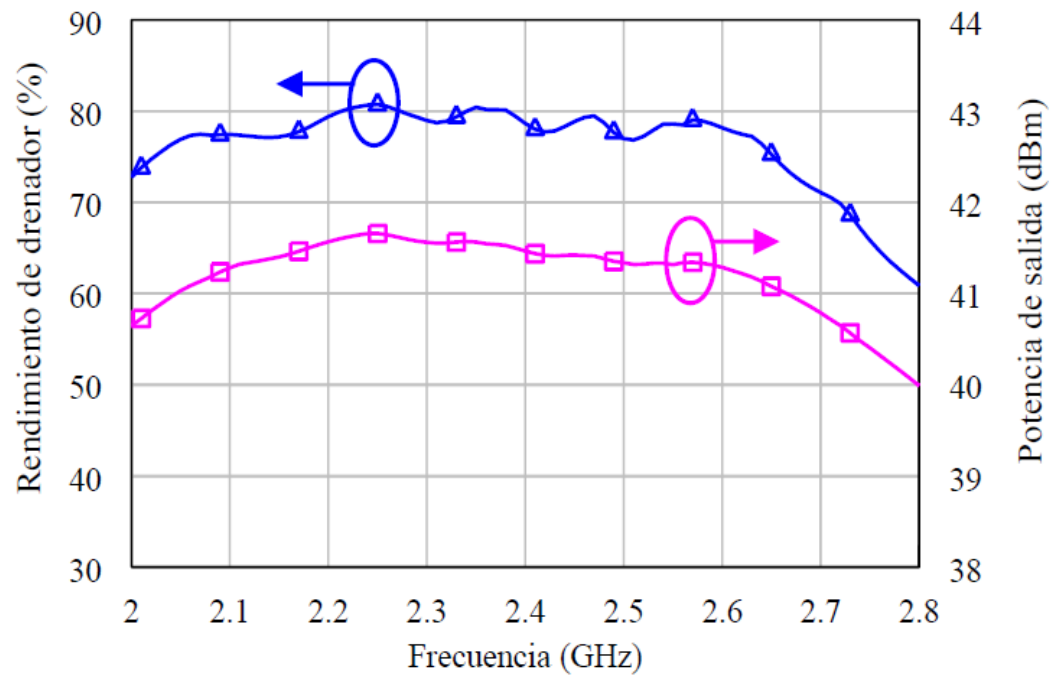

Figura 3.96. Simulación de la potencia de salida y del rendimiento de drenador en función de la frecuencia para el amplificador clase E subóptimo en banda S.

\subsubsection{Implementación del amplificador clase $\mathrm{E}$ subóptimo en banda $\mathrm{S}$}

La fabricación del prototipo de amplificador clase E subóptimo se ha realizado en base a las simulaciones realizadas anteriormente. El circuito se construyó sobre un substrato RO4350B de Rogers Corporation con un espesor de $0.508 \mathrm{~mm}$. El layout del amplificador fue cuidadosamente diseñado en Autodesk Autocad con el fin de minimizar cualquier imprecisión, ya que a medida que se aumenta la frecuencia la precisión en la fabricación ha de ser una prioridad. 
La ventaja de este diseño, con respecto al amplificador clase E subóptimo en banda L visto en el Apartado 3.2, es que no depende de elementos concentrados cuyo posicionamiento es crítico para conseguir el funcionamiento previsto. En este caso, los elementos críticos de las redes de entrada y de salida han sido implementados con líneas de transmisión. Esto ofrece una ventaja en cuanto a repetitividad y facilidad de montaje. Además, las redes de polarización y de alimentación han sido integradas dentro del diseño, eliminando ese elemento de la fase de diseño. La Figura 3.97 muestra una fotografía del amplificador.

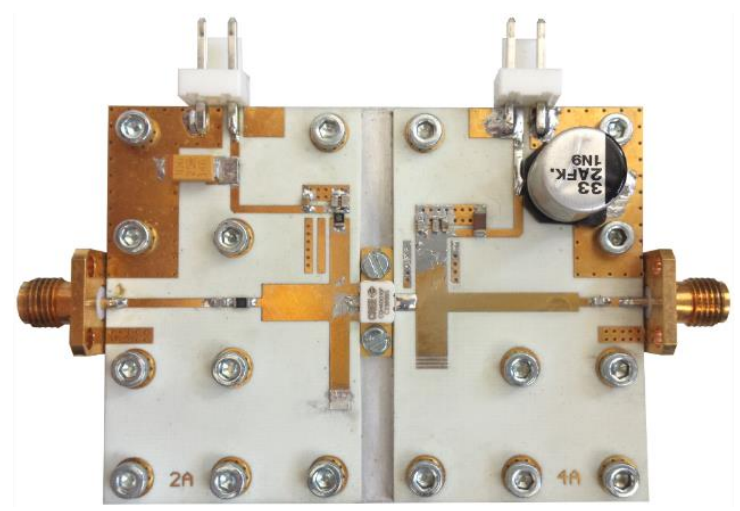

Figura 3.97. Fotografía del amplificador clase E subóptimo en banda $\mathrm{S}$ implementado.

Un disipador a medida fue diseñado y construido en aluminio anodizado para este amplificador. Este material y su acabado presentan buenas características de conducción y de disipación térmicas que, junto con su bajo precio con respecto a otros materiales como el cobre, lo hace adecuado para esta aplicación.

Las medidas en onda continua fueron realizadas utilizando el mismo banco de medida controlado por ordenador mostrado en la Figura 3.74. Los resultados de las medidas reales de potencia de salida y de rendimiento de drenador mostrados en la Figura 3.98 fueron obtenidos con una excitación constante de $29 \mathrm{dBm}$ en todo el ancho de banda. Pequeñas diferencias se han encontrado en los valores obtenidos en las medidas reales con respecto a los valores obtenidos en la simulación que fueron mostrados en la Figura 3.96. 


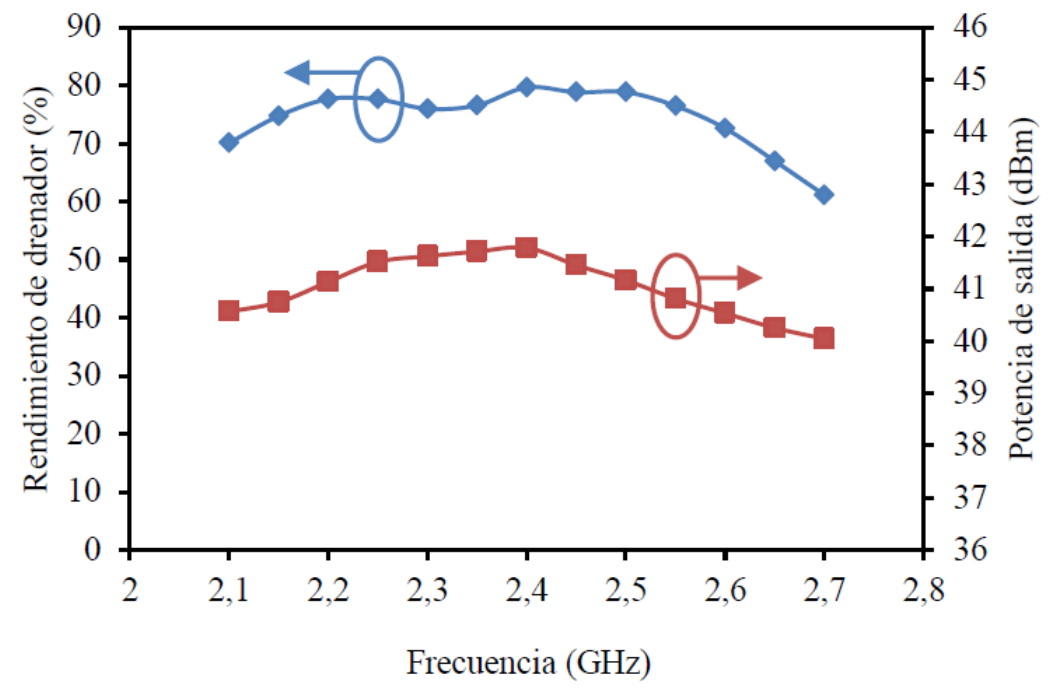

Figura 3.98. Medida de la potencia de salida y del rendimiento de drenador del amplificador clase E subóptimo en banda $\mathrm{S}$.

En resumen, el rendimiento de drenador medido se mantiene aproximadamente entre el 70 y $80 \%$ dentro del ancho de banda comprendido entre 2.1 y $2.6 \mathrm{GHz}$. De la misma forma, la potencia de salida se mantiene por encima de los $40 \mathrm{dBm}$ en ese ancho de banda.

Como se ha mencionado anteriormente, la red de entrada está diseñada para mantener una excitación constante en toda la banda. Esto hace que el amplificador refleje gran cantidad de potencia a frecuencia bajas para mantener esa excitación, como se puede ver en la Figura 3.99. Además, se puede observar que la ganancia de este amplificador se mantiene por encima de los $11 \mathrm{~dB}$ en todo el ancho de banda, llegando a alcanzar los $13.6 \mathrm{~dB}$ en la frecuencia central.

Los resultados obtenidos con este amplificador, sobre todo en un gran ancho de banda, lo hacen adecuado para su uso en aplicaciones espaciales donde el ahorro energético es muy importante. 


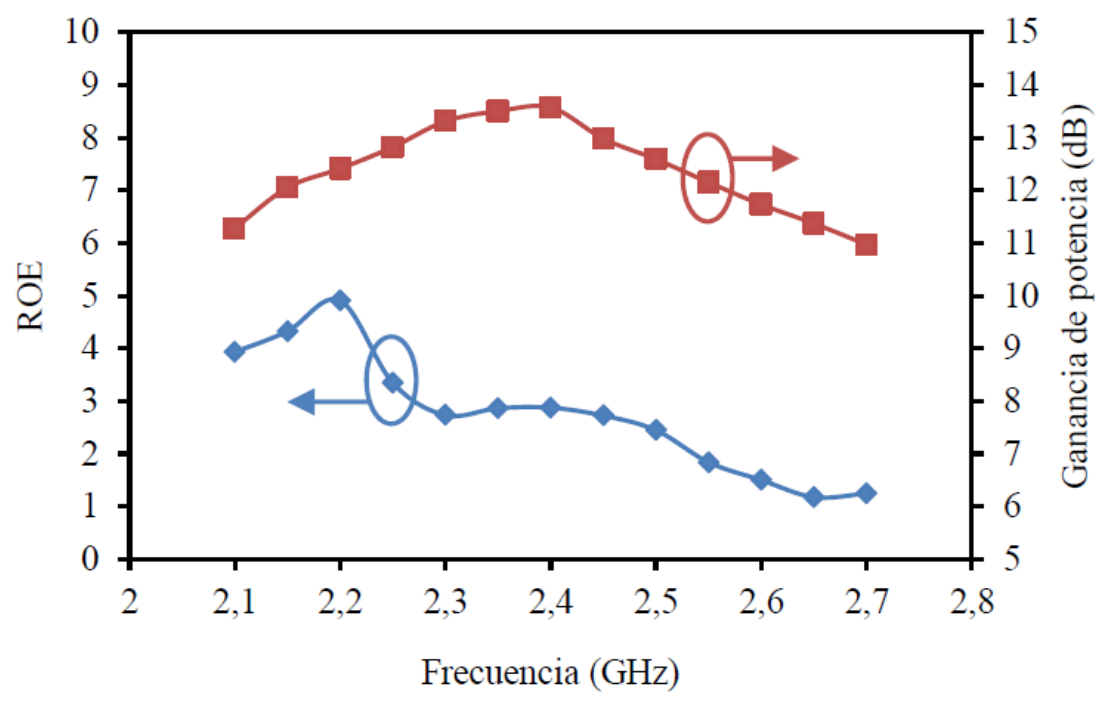

Figura 3.99. Medida de la relación de onda estacionaria y de la ganancia en potencia del amplificador clase E subóptimo en banda S.

Observando los resultados obtenidos y el estado del arte mostrado en la Tabla 3.7 se puede determinar que los amplificadores clase E trabajando por encima de su frecuencia nominal son una clara alternativa en el diseño de amplificadores de potencia como clase F o F-1. Además, esta topología permite el funcionamiento en banda ancha con una red de carga muy simple.

Tabla 3.4. Comparación del amplificador diseñado en este trabajo con respecto al estado del arte de amplificadores de potencia en banda $\mathrm{S}$.

\begin{tabular}{cccccc}
\hline \hline Referencia & $\begin{array}{c}\text { Frecuencia } \\
(\mathbf{G H z})\end{array}$ & $\begin{array}{c}\mathbf{P}_{\text {out }} \\
(\mathbf{W})\end{array}$ & $\begin{array}{c}\text { Gain } \\
(\mathbf{d B})\end{array}$ & $\begin{array}{c}\boldsymbol{\eta}_{\boldsymbol{d}} / \text { PAE } \\
(\boldsymbol{\%})\end{array}$ & $\begin{array}{c}\text { Clase de } \\
\text { amplificación }\end{array}$ \\
\hline $2010[146]$ & 2.5 & 12 & 15.8 & $-/ 75.8$ & Class F \\
$2011[147]$ & $2.5-2.6$ & 13.8 & 15.4 & $75.2 / 73$ & Class F $^{-1}$ \\
$2011[148]$ & 1.95 & 10 & 13 & $-/ 67$ & Class E \\
$2014[149]$ & $1.6-2.5$ & 10 & 15 & $70 /-$ & Class J \\
$2016[150]$ & $1.4-2.7$ & 12.6 & 9 & $72 /-$ & Class E \\
Este trabajo & $2.1-2.6$ & 14 & 13.6 & $79 / 76$ & SO Class-E \\
\hline \hline
\end{tabular}




\section{Capítulo 4}

\section{Linealización de amplificadores}

Moduladores de envolvente, arquitecturas de linealización y prototipos 


\section{Capítulo 4}

\section{Linealización de amplificadores}

Moduladores de envolvente, arquitecturas de linealización y prototipos

\section{CONTENIDO}

\subsection{Introducción}

4.2 Transmisor lineal basado en amplificador clase $\mathrm{F}$

4.3 Transmisor lineal basado en amplificador clase $\mathrm{E}$

A medida que las señales de comunicaciones se hacen más complejas para aumentar velocidad y ajustarse a un espectro limitado, el diseño de los moduladores de envolvente se hace más complejo. Por ejemplo, $5 \mathrm{G}$ tiene un ancho de banda de $20 \mathrm{MHz}$ lo que implica que el ancho de banda de RF necesario en el modulador debe ser entre 3 y 10 veces más, dependiendo de la aplicación.

En este capítulo se presentan varias soluciones a esta problemática. En primer lugar, se muestra una propuesta donde el amplificador de potencia clase F presentado en el Capítulo 2 se combina con un convertidor buck multifase para aplicaciones ET y EER. En segundo lugar, otra propuesta de transmisor basada en el amplificador clase E en banda L del Capítulo 2 será investigada junto con otro convertidor buck multifase.

Previamente a ello, se resumirá las principales dificultades que los diseñadores se encuentran a la hora de implementar este tipo de soluciones aplicando técnicas ET y EER y una pequeña introducción de las principales tendencias en el diseño de amplificadores de envolvente. 


\subsection{Introducción}

En la Figura 4.1 se muestra un diagrama de bloques simplificado de un transmisor de potencia que emplea un modulador de envolvente. Básicamente está formado por un amplificador de envolvente que se emplea para modular la tensión de alimentación del amplificador de RF.

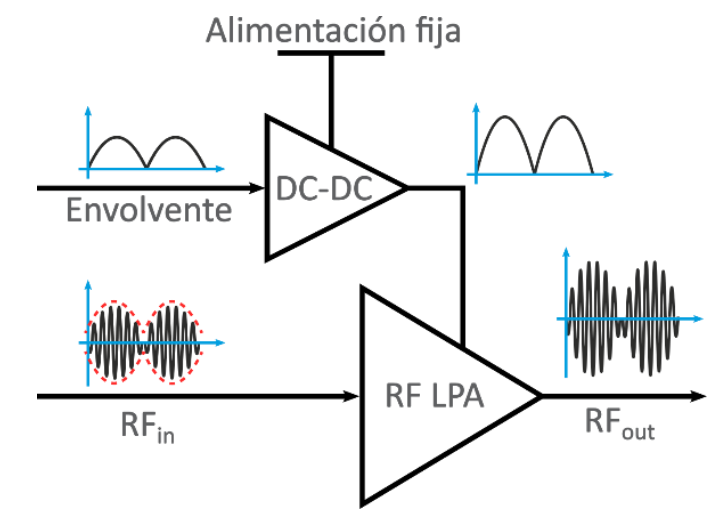

Figura 4.1. Diagrama de bloques simplificado de un transmisor de RF formado por un modulador de envolvente y amplificador de RF lineal.

El amplificador de envolvente más simple es un regulador lineal alimentado a una tensión fija. En este caso, la tensión de alimentación necesaria deberá ser ligeramente superior a la tensión de salida máxima, para no saturar y provocar distorsión. Esta sencilla solución, sin embargo, presenta un muy bajo rendimiento especialmente al trabajar con señales de alta PAPR. En la Figura 4.2 se muestra el perfil de funcionamiento donde se representa en rojo la cantidad de energía disipada en calor.

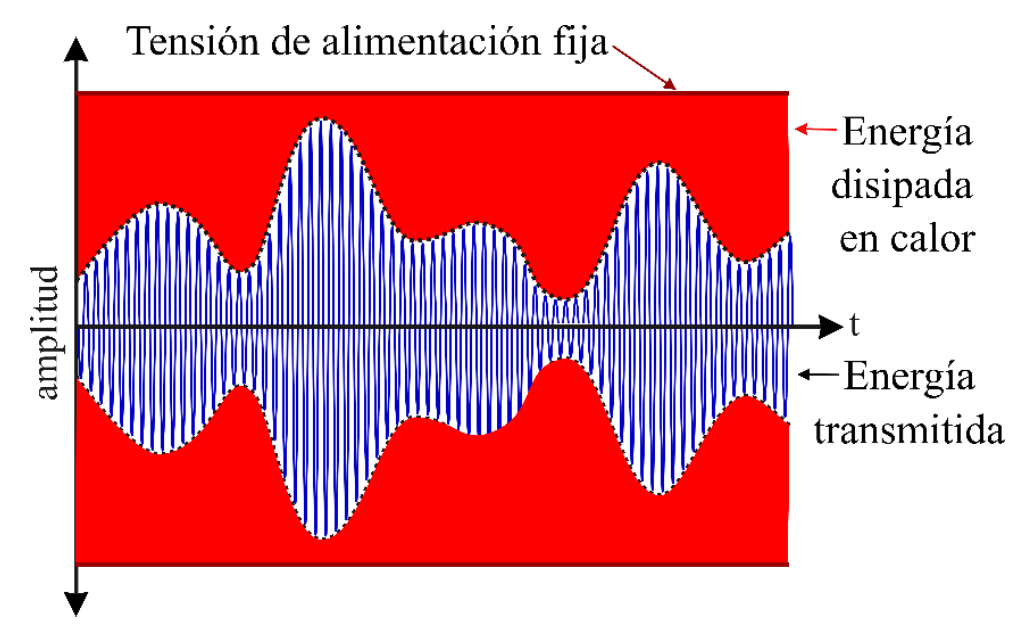

Figura 4.2. Funcionamiento de un amplificador de envolvente con tensión de alimentación fija. 
Con el fin de solventar este problema, se puede emplear una estrategia de control de la tensión de alimentación de tal manera que se acerque al valor de la envolvente de la señal en cada instante, como se puede ver en la Figura 4.3. De esta manera se disipa menos energía en forma de calor y, por lo tanto, se incrementa el rendimiento global del transmisor. Ello implica un diseño más complejo del amplificador de envolvente.

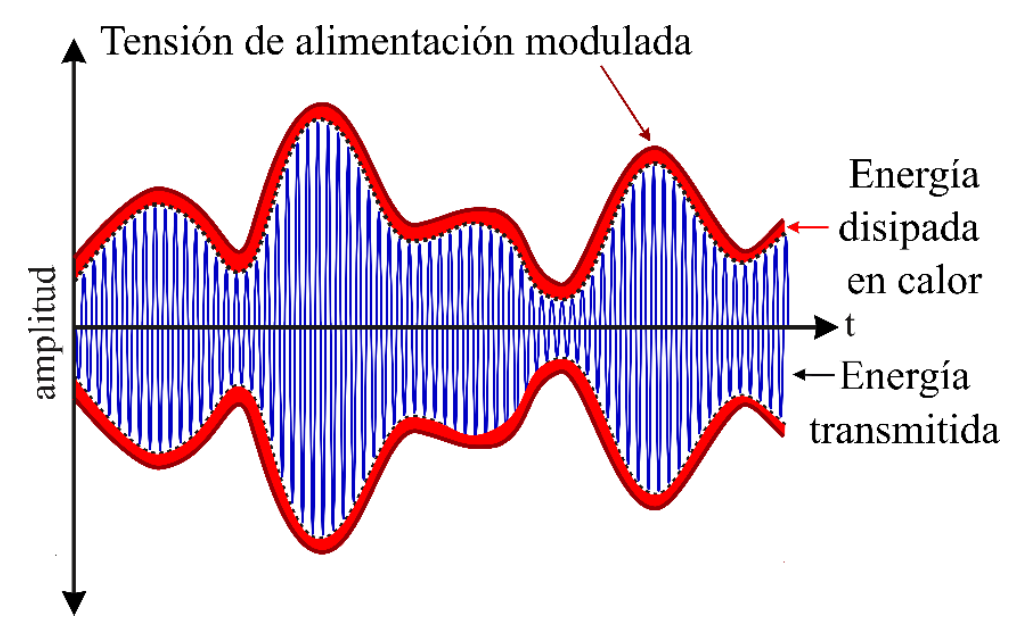

Figura 4.3. Funcionamiento de un amplificador de envolvente con tensión de alimentación modulada en función de la envolvente de la señal.

En esta tesis se trabajará con dos sistemas que implementan la modulación de la tensión de alimentación: EER y ET. En el caso de la aplicación EER, es necesario proporcionar la envolvente exacta al amplificador de RF, incrementando la complejidad del amplificador de envolvente. Aquí, el amplificador de RF solo se encarga de amplificar la información de fase de la señal de entrada y posteriormente esta información amplificada se modula con la envolvente amplificada por el amplificador de envolvente. En este caso, si el amplificador de envolvente tiene un alto rendimiento y el amplificador de RF también, el rendimiento global del transmisor será muy alto.

Por otro lado, en una aplicación ET no es necesario reproducir exactamente la envolvente de la señal, ya que el amplificador de RF también transporta la información de envolvente junto con la información de fase, a costa de un rendimiento menor. De ahí que los requisitos a la hora de diseñar el amplificador de envolvente sean menores que para una aplicación EER. Además, un amplificador de envolvente para una aplicación ET no necesita un proceso de integración muy complejo como en la técnica EER, donde la sincronización temporal tiene una relación directa con la linealidad del sistema. 
En este punto es importante entender que la elección de la técnica de linealización, EER o ET, tiene un gran impacto en el diseño del amplificador de envolvente.

\subsubsection{Moduladores de envolvente}

Además de una deseable linealidad, incrementar el rendimiento global del transmisor de potencia es el principal objetivo buscado cuando se emplean amplificadores de envolvente para modular la tensión de alimentación del amplificador de potencia de RF. Sin embargo, se incrementa la complejidad del sistema y además muchos otros parámetros de diseño han de ser tenidos en cuenta como pueden ser el ancho de banda, la distorsión...

Las nuevas señales de comunicaciones inalámbricas generan grandes restricciones en el diseño de los moduladores de envolvente. La principal razón es el gran ancho de banda necesario en las aplicaciones, lo que implica altas frecuencias de conmutación.

A continuación, se muestra un breve resumen del estado del arte de moduladores de envolvente construidos a partir de convertidores DC/DC. Existen dos fuertes tendencias: la primera consiste en utilizar un único convertidor conmutado, siendo la solución más óptima desde el punto de vista energético; la segunda combina un convertidor conmutado con un amplificador lineal, de tal manera que se mejora la reproducción de la envolvente de las señales de comunicaciones actuales a costa de reducir el rendimiento global.

\subsubsection{Convertidores conmutados}

El convertidor más usado como modulador de envolvente es el convertidor reductor, también conocido como buck, cuyo esquema se presenta en la Figura 4.4. Este convertidor destaca por su sencillez y facilidad de control. 


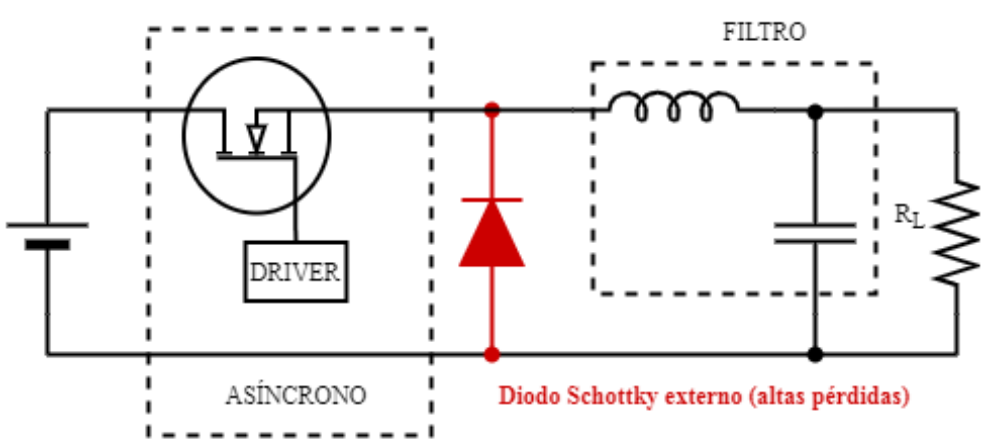

(a)

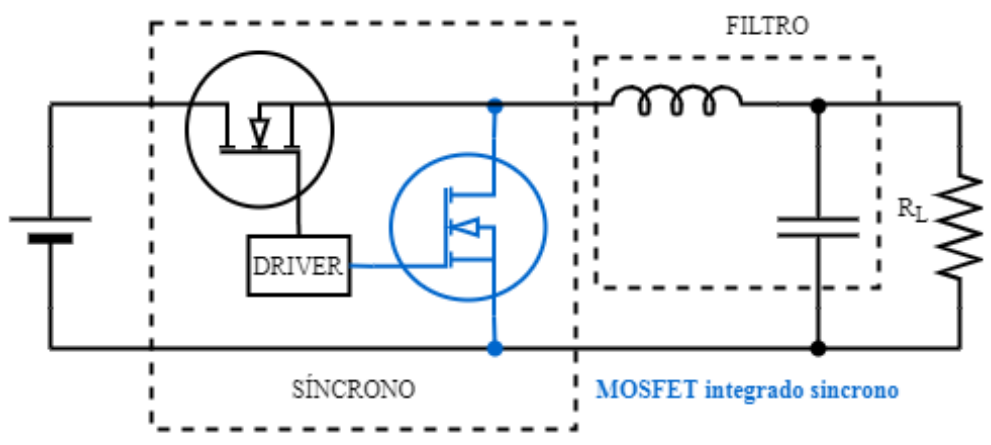

(b)

Figura 4.4. Esquema de un convertidor reductor: asíncrono (a) y síncrono (b).

Está formado por una red de conmutación formada por transistor y un diodo en su versión asíncrona (o dos transistores en su versión síncrona) y un filtro LC paso bajo de salida. El funcionamiento es muy sencillo: la red de conmutación genera un pulso, de amplitud $V_{i n}$, que es filtrado por el circuito LC, aplicándose a la carga una tensión igual al valor medio del pulso. El driver controla la puerta de los transistores. Comúnmente se emplea una modulación por anchura de pulso (PWM), de tal forma que la tensión de salida es directamente proporcional al ciclo de trabajo [151], de forma que:

$$
V_{\text {out }}=D \cdot V_{\text {in }}
$$

donde $D$ es el ciclo de trabajo.

Esta característica lineal entre la tensión de salida y el control de entrada hace de este tipo de convertidor interesante como modulador de envolvente. Además, su sencillez facilita su empleo a frecuencias de conmutación más altas, lo que permite aumentar el ancho de banda del filtro. Además, hay que tener en cuenta que cuanto se trabaja con técnicas de linealización junto con este tipo de convertidores, la frecuencia de conmutación debería ser al menos 3 veces, en el caso de ET [9], y más de 5 veces, en el caso de EER [152], mayor que el ancho de banda de envolvente necesario. No 
obstante, a mayores frecuencias de conmutación mayores pérdidas por conmutación en los semiconductores. Esta situación ha conllevado la investigación de variaciones basadas en esta topología.

Una primera variación consiste en incrementar el orden del filtro [153] de tal manera que la frecuencia de conmutación puede ser más baja incrementando el rendimiento del convertidor.

Otra variante se basa en repartir la corriente de entrada en múltiples redes de conmutación, lo que se conoce como la variante multifase [154]. En ella, se mejoran los problemas de disipación y rendimiento que aparecen en los convertidores de una sola fase al trabajar con corrientes superiores a los 25A. Además, permite reducir la frecuencia de conmutación de tal manera que para conseguir aproximadamente el mismo ancho de banda de funcionamiento que un monofase, la frecuencia de conmutación de un convertidor multifase sería la frecuencia de conmutación del monofase dividida entre el número de fase. El principal inconveniente de esta variante es el control de cada una de las fases.

Una variante diferente es el convertidor multinivel, que consiste en el uso de conmutadores adicionales con el fin de reducir la tensión de entrada dinámicamente de acuerdo a la forma de onda de referencia de envolvente. [155]. Esta topología reduce las pérdidas de conmutación, tiene una respuesta dinámica más rápida y permite incrementar el rendimiento global del transmisor.

Por último, otra idea es la variante multientrada [156]. Está técnica tiene varias tensiones de entrada de tal manera que en cada momento se aplica una de esas tensiones al filtro LC. Está opción es muy parecida a la anterior, pero es este caso se suele trabajar a frecuencias más altas, disipando más potencia y penalizando en rendimiento.

En conclusión, la topología reductora es muy utilizada para su uso en aplicaciones EER y ET debido a su alto rendimiento. Sin embargo, cuando grandes anchos de banda son necesarios, la frecuencia de conmutación debería ser alta provocando pérdidas y limitando la potencia del convertidor. En estos casos, otras alternativas pueden ser usadas como la variante multifase o multinivel que, a pesar de ser más complejas, mejoran el rendimiento. De ahí que las variantes comentadas puedan 
ser combinadas entre sí de tal manera que cada una aporte sus beneficios y contrarresten sus inconvenientes.

\subsubsection{Convertidores conmutados asistidos linealmente}

El incremento del ancho de banda necesario en las señales de comunicaciones ha ido incrementándose a lo largo de los años, haciendo que los convertidores conmutados no sean capaces por si solos de ser integrados en aplicaciones EER y ET. Por ello, una solución es combinarlos con etapas de amplificación lineal. De esta manera, el convertidor conmutado, limitado en ancho de banda pero con un rendimiento elevado, se encarga de proporcionar la potencia contenida a frecuencias bajas de envolvente; mientras tanto, el amplificador lineal, con gran ancho de banda y un rendimiento bajo, garantiza el suministro de potencia a frecuencias elevadas. Esta combinación puede hacerse de dos formas diferentes: en serie o en paralelo con el convertidor conmutado.

En la combinación en serie [157], tanto la tensión como la corriente demandada por el amplificador RF es suministrada por el amplificador lineal. En este caso, el amplificador lineal está alimentado con el convertidor conmutado de tal manera que aproxima la tensión de alimentación de la etapa lineal a la tensión de salida, reduciendo las pérdidas. En la Figura 4.5 se muestra esta técnica.

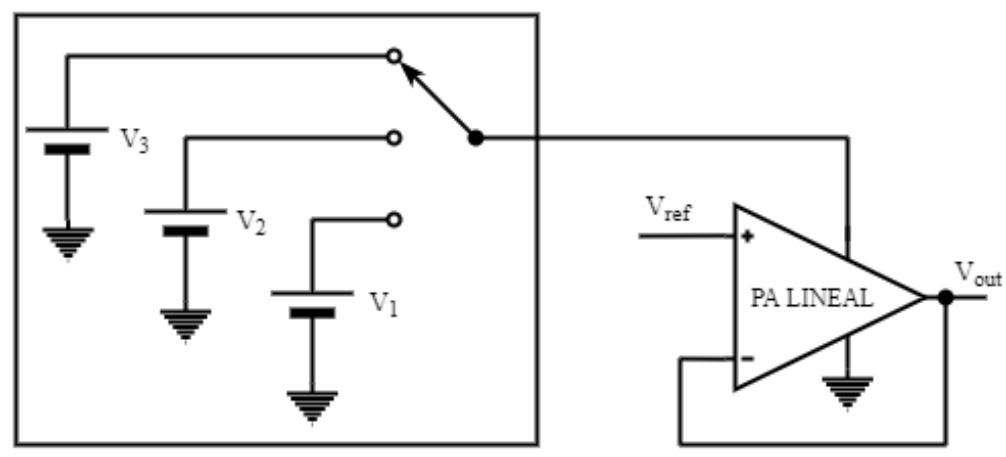

Fuente de tensión

Figura 4.5 Esquema de un convertidor reductor asistido en serie por un amplificador lineal.

En la combinación paralelo [158], la corriente necesaria por el amplificador de RF es suministrada únicamente por el convertidor conmutado. En este caso, la etapa lineal únicamente suministra corriente al amplificador de RF cuando la dinámica de la 
etapa conmutada no permita seguir la envolvente con precisión. Un ejemplo de este funcionamiento se puede ver en la Figura 4.6.

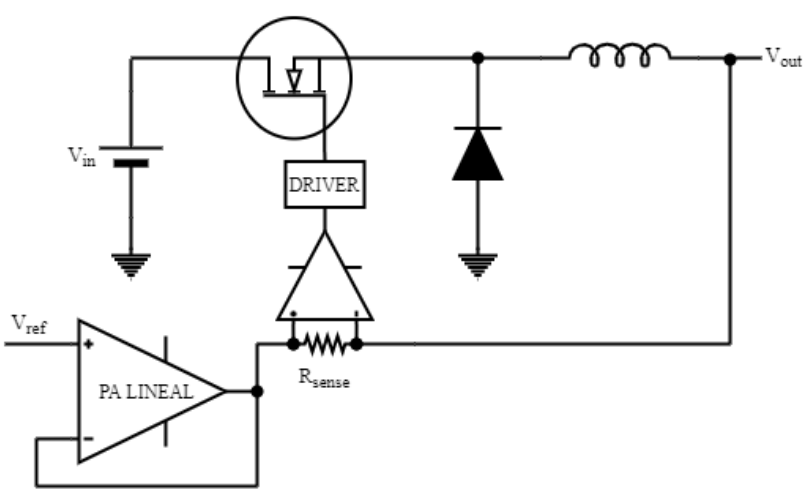

Figura 4.6 Esquema de un convertidor reductor asistido en paralelo por un amplificador lineal.

La razón que explica porque resulta eficiente la combinación de etapas conmutadas y lineales se debe a que la mayor parte de la potencia de la señal de envolvente se encuentra en la parte baja del espectro. Por ello, si la mayor parte de la potencia es suministrada por la etapa conmutada de manera eficiente, el rendimiento global del sistema será elevado, ya que la etapa lineal aporta muy poca potencia al sistema.

\subsubsection{Generación de señales}

Este apartado hace referencia a la generación o extracción de la información de envolvente y de fase de las señales que van a ser empleadas en las pruebas de los transmisores diseñados. Esta acción se puede realizar de manera analógica, empleando un detector de envolvente y un limitador en el camino de fase, o de manera digital procesando matemáticamente la señal en banda base.

Hoy en día, no es posible la extracción de la envolvente mediante medios analógicos debido a las características de las señales de las aplicaciones de RF [9]. Por ello, actualmente es más común generar las componentes de la señal digitalmente.

En este trabajo, las señales de prueba que se emplearán para probar los transmisores diseñados han sido generadas por ordenador usando Matlab. Posteriormente, un periodo de estas señales digitales ha sido cargado en un generador de 
señal arbitrario 81150A de Agilent Technologies (actualmente Keysight Technologies): por un lado, la envolvente de la señal en banda base y, por otro, las magnitudes en fase (I) y en cuadratura (Q) de la señal. De esta manera, el canal de envolvente del generador arbitrario será la referencia de entrada del amplificador de envolvente y los canales I y Q del generador arbitrario serán las entradas del modulador IQ del generador de señal (en este caso, E4438C de Agilent Technologies) que generará la señal de RF para ser amplificadas por el amplificador de RF. En la Figura 4.7 se puede ver el diagrama de bloques utilizado para generar las señales de prueba. La resolución de los equipos y la frecuencia de trabajo permiten realizar la generación de las señales con una precisión suficiente.

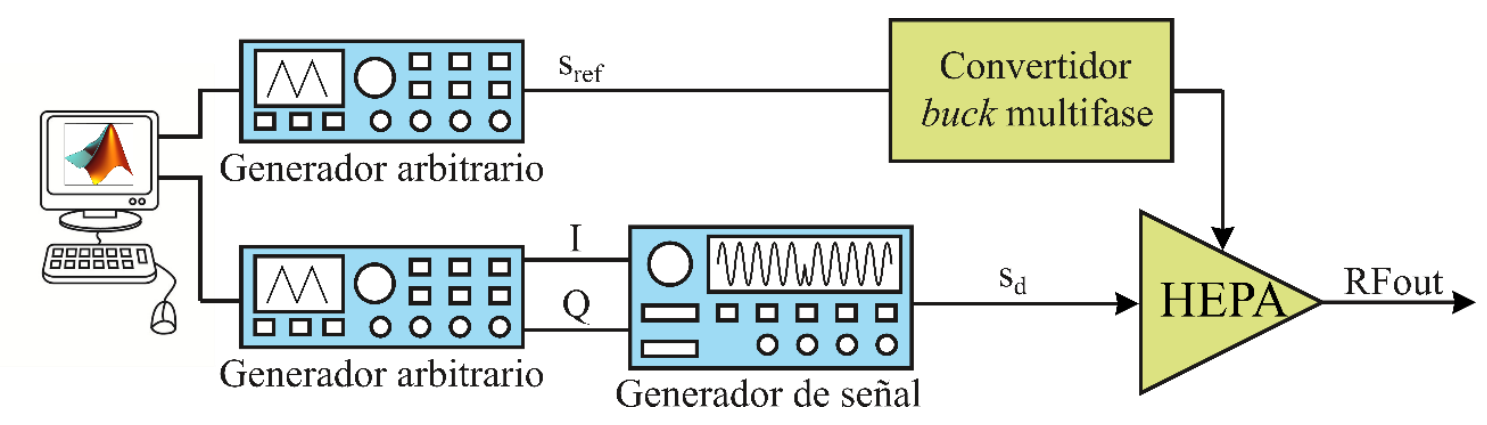

Figura 4.7 Diagrama de bloques del esquema empleado en la generación de señales.

\subsubsection{Alineamiento temporal}

Aunque el empleo de un modulador de envolvente en aplicaciones ET y EER puede incrementar el rendimiento del amplificador, otros factores también influyen en el rendimiento global del sistema. La precisión de la compensación en el retardo entre la señal de RF y la señal de envolvente en un sistema ET o EER es crucial en la linealidad del sistema. Tal y como se puede ver en la Figura 4.8, en un sistema EER un desalinealimiento temporal es más perjudicial que en un sistema ET. Esta alinealidad temporal puede provocar degradaciones en el EVM (Error Vector Magnitude) de señales moduladas digitalmente, reducciones de rendimiento y de potencia o incremento de emisiones no deseadas fuera de banda [84]. Además, esta desadaptación temporal también puede dar como resultado efectos memoria que pueden ser difíciles de corregir. 


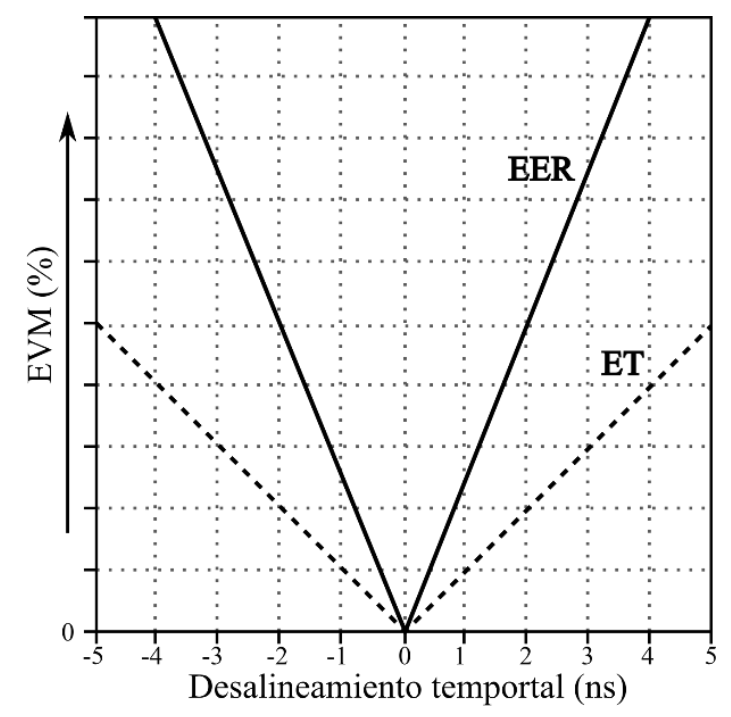

Figura 4.8. Ilustración gráfica de como un desalineamiento temporal afecta en el EVM de un sistema EER y de un sistema ET.

En esta tesis, el alineamiento temporal de las señales se ha realizado de dos maneras diferentes: de manera manual variando el retardo de uno de los caminos hasta obtener el mejor ACPR (Adjacent Channel Power Ratio) o mediante un banco automático de medida que calcula el retardo, en muestras, a partir de la correlación cruzada entre la señal de salida y la de entrada. El primer caso es algo más inexacto y laborioso, pero gracias a los instrumentos de generación de señal es sencillo realizar una buena aproximación. El segundo caso requiere de una calibración y sincronización de los equipos de medida y, a pesar de ello, no se asegura una exacta alineación temporal.

Un punto a tener en cuenta en el caso de emplear predistorsión digital junto con el transmisor de RF es que la alineación temporal debe ser realizada antes de la predistorsión de tal forma que sus algoritmos proporcionen una corrección adecuada en cada instante.

\subsection{Transmisor lineal basado en amplificador clase $\mathbf{F}$}

Los sistemas de comunicaciones modernos permiten la transmisión de datos a altas velocidades mediante el uso de complejas modulaciones que conllevan amplios anchos de banda y alta PAPR. Con el fin de garantizar la linealidad del transmisor, típicamente se han utilizado amplificadores trabajando entre 4 y 10 dB de su tensión de 
back-off, reduciendo considerablemente el rendimiento del sistema. En el Capítulo 2 se mostraron diversas técnicas para superar este problema de rendimiento.

Entre ellas, ET y EER son dos prometedoras técnicas que se adaptan perfectamente a este tipo de señales y con las que se puede mejorar el rendimiento y la linealidad de los sistemas. En ambas, el transmisor está compuesto por un amplificador de RF y un amplificador de envolvente. Este último alimenta al amplificador de RF en función de la envolvente de la señal transmitida. Con el fin de conseguir alto rendimiento y alta linealidad, el diseño del amplificador de envolvente es un elemento crucial del sistema. Además, en los sistemas EER el alto rendimiento del amplificador de RF también es transcendental en el rendimiento global del sistema.

En este apartado se presenta la combinación del amplificador clase F presentado en el Capítulo 3 junto con un amplificador de envolvente con el fin de implementar un transmisor basado en técnicas ET y EER. En primer lugar, se mostrará el amplificador de envolvente que va a ser utilizado y posteriormente se exhiben las medidas realizadas.

\subsubsection{Amplificador de envolvente}

El amplificador de envolvente utilizado en este apartado es un convertidor buck multifase ( 8 fases) con control de tiempo mínimo para el seguimiento de tensión multinivel asistido por un amplificador lineal en serie [159]. Ha sido diseñado por el Centro de Electrónica Industrial de la Universidad Politécnica de Madrid. El esquema básico del convertidor multinivel se muestra en la Figura 4.9.

El amplificador de envolvente está formado por un convertidor buck multifase capaz de generar ocho niveles de tensión mediante la selección adecuada del ciclo de trabajo. Además, con el fin de que los cambios entre niveles de tensión de salida sean los más rápidos posibles, se emplea una técnica de control del tiempo de transición entre niveles mínima y no lineal. Para más información sobre su funcionamiento se puede consultar [160]. 


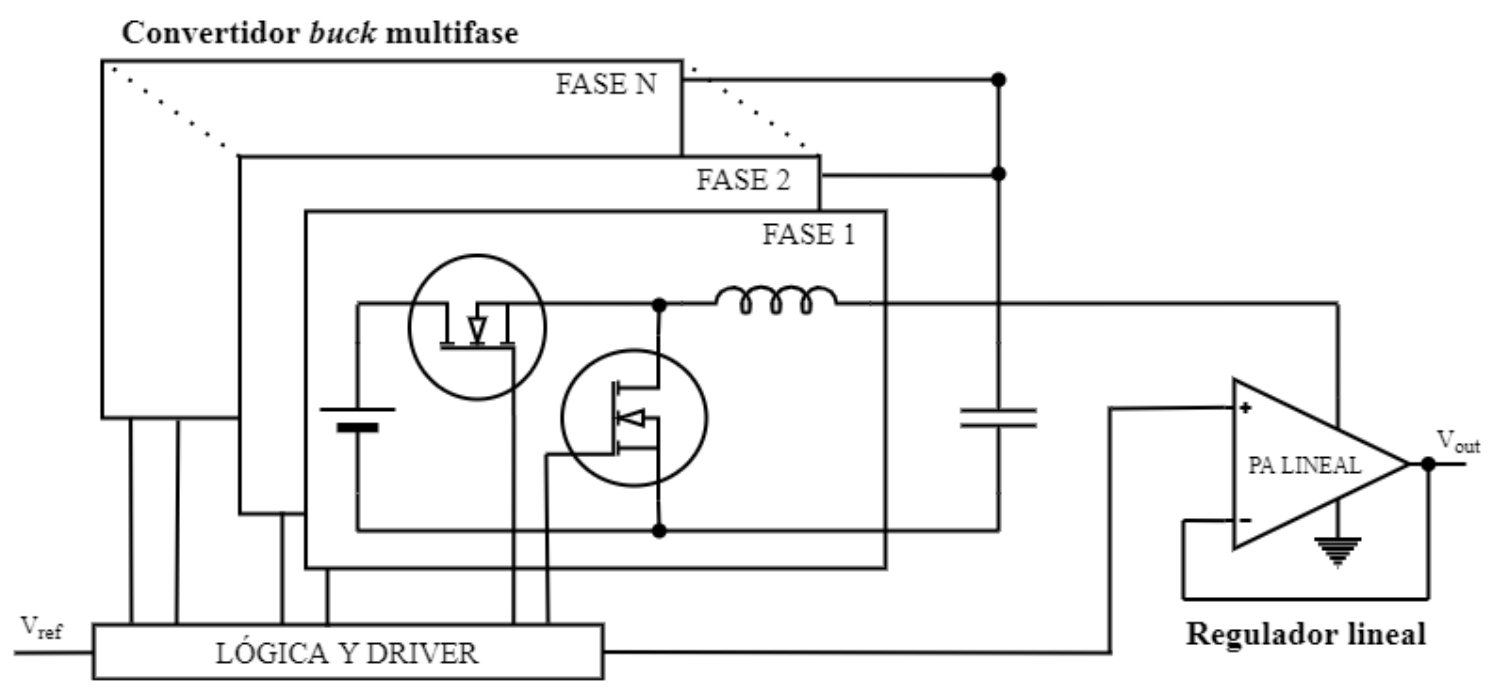

Figura 4.9. Esquema simplificado del convertidor multifase y multinivel en serie con un regulador lineal.

Aplicando estas nuevas técnicas de control, el convertidor multifase es capaz de seguir señales hasta los $10 \mathrm{MHz}$ de ancho de banda [159]. El control de cada una de las fases se realiza a través de una FPAG Virtex-5 de Xilinx con una frecuencia de reloj de $200 \mathrm{MHz}$.

Las características principales de este convertidor son:

- Tensión de entrada: $48 \mathrm{~V}$

- Rango de tensión de salida: de $0 \mathrm{~V}$ a $42 \mathrm{~V}$

- Potencia de salida de pico: $170 \mathrm{~W}$

- Ancho de banda de la señal de RF: $5 \mathrm{MHz}-10 \mathrm{MHz}$

El regulador lineal a la salida de este convertidor será empleado para probar su funcionamiento en una aplicación EER. En este caso, se utilizará el PA107 de APEX Microtechnology como regulador lineal de alta velocidad. Por otro lado, debido a las características de la técnica ET, se realizarán medidas del convertidor buck sin el regulador lineal, donde la forma de envolvente no es tan crítica como en EER.

Antes de integrar el amplificador de envolvente con el amplificador de RF se va a mostrar las medidas experimentales del convertidor buck de 8 fases. En la Figura 4.10 se puede ver el rendimiento estático de este convertidor. De las medidas obtenidas se puede comprobar que el convertidor está optimizado para trabajar a altas corrientes 
(entre $\operatorname{los} 2$ y 4A) siendo el rendimiento superior al 85\%. El rendimiento cae significativamente a cargas bajas debido a las pérdidas por consumo de potencia de los drivers y aisladores.

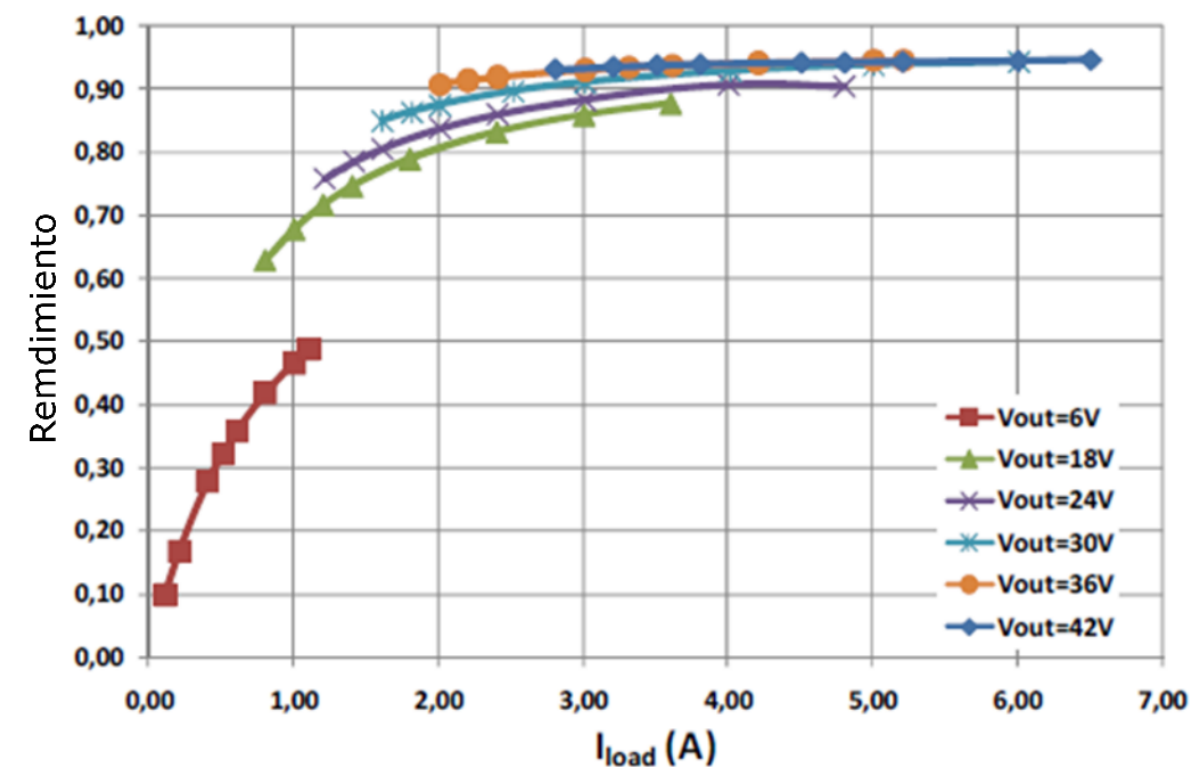

Figura 4.10. Medida del rendimiento estático de convertidor buck multifase [159].

El convertidor asistido por el regulador lineal fue probado con una señal WCDMA cuyo ancho de banda es de 5MHz y presenta un PAPR de $6.5 \mathrm{~dB}$, aplicado a una carga de $10 \Omega$. El convertidor genera los niveles correctos en función de la envolvente de referencia y como el regulador lineal es capaz de reproducir la tensión de referencia, aunque no de manera idéntica (esta situación influirá en la linealidad del transmisor EER como se verá posteriormente). El rendimiento global del amplificador de envolvente para esta señal fue de $61.3 \%$.

\subsubsection{Transmisor ET y EER}

Con el fin de validar el concepto de ahorro de energía, se procedió a implementar un prototipo compuesto por el convertidor buck anteriormente presentado y el amplificador clase F mostrado en el capítulo anterior. Este prototipo sirvió para verificar el funcionamiento del transmisor en aplicaciones ET y EER.

El esquema del sistema montado se muestra en la Figura 4.11. Básicamente, a través de Matlab se generan las señales de referencia necesarias: por un lado, se genera 
un periodo de la señal de envolvente que se cargará en el generador arbitrario 81150A de Agilent y que servirá como referencia para el amplificador lineal; por otro, se proporcionaran las señales de control del convertidor multifase a través de la FPGA Virtex-5 basándose en la señal de envolvente de entrada; y por último, se generaran los canales IQ de la señal que será entrada del amplificador clase F (en el caso de ET sería una señal con información de fase y de envolvente, mientras que en el caso de EER será una señal únicamente con información de fase con envolvente constante) como entradas del generador de señal de RF E4438C de Agilent.

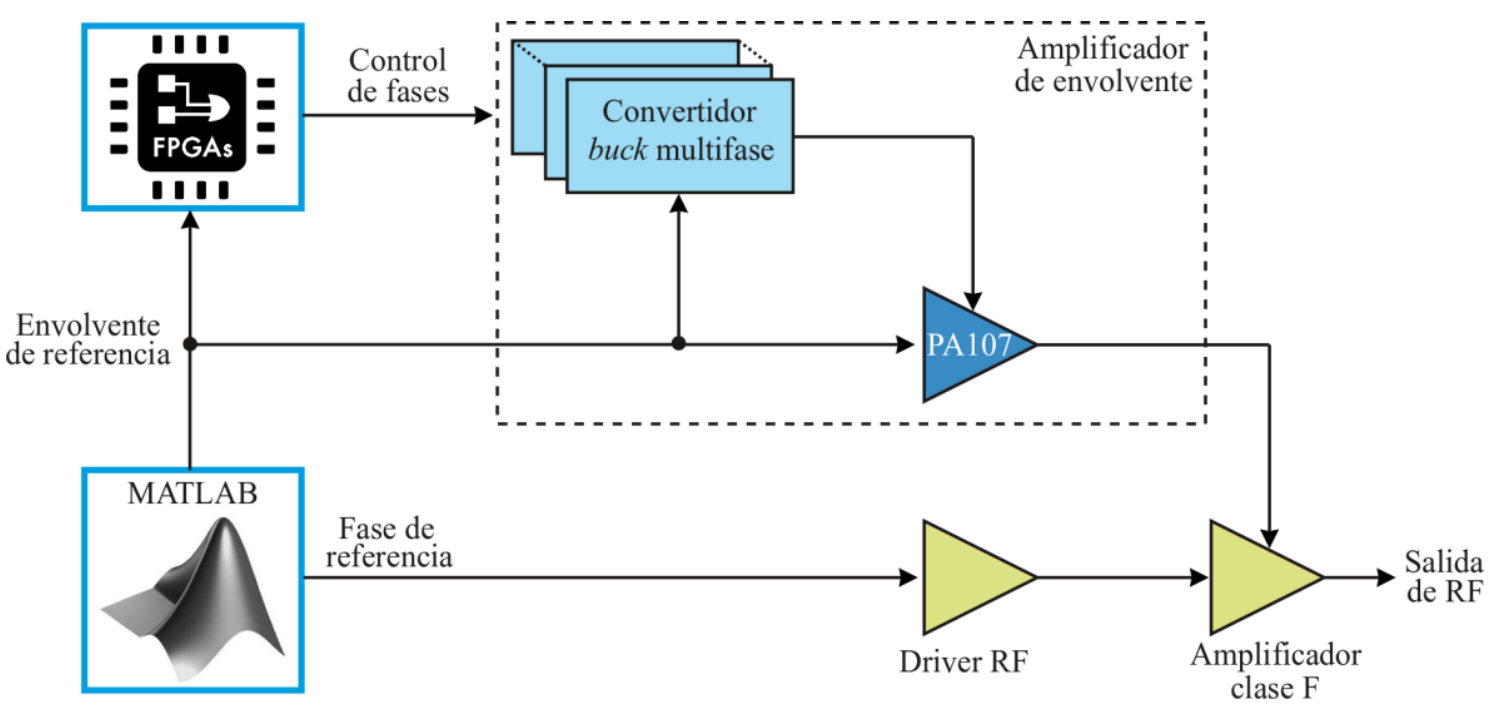

Figura 4.11. Esquema del sistema para aplicaciones ET y EER.

En el diseño de este transmisor existen dos retos previos a resolver: la sincronización entre la FPGA y los equipos de generación y medida, y la alineación entre la información de fase y la información de amplitud. La primera puede resolverse a través de un reloj de referencia estable, para lo cual se utilizó la referencia del equipo 53131A de Agilent. La segunda difícilmente puede ser solucionada visualmente. La mejor opción es mantener fija una de las dos referencias, la del amplificador de RF o la del amplificador de envolvente, y retardar la otra hasta minimizar la distorsión. En este caso, la señal de referencia del amplificador de RF se mantuvo fija y se retardó la señal de referencia de la envolvente.

La Figura 4.12 presenta una fotografía del prototipo montado. En ella se puede distinguir claramente el convertidor buck multifase seguido del regulador lineal PA107 que alimenta al amplificador de potencia de RF clase F cuya salida es aplicada a una carga de $50 \Omega$. 


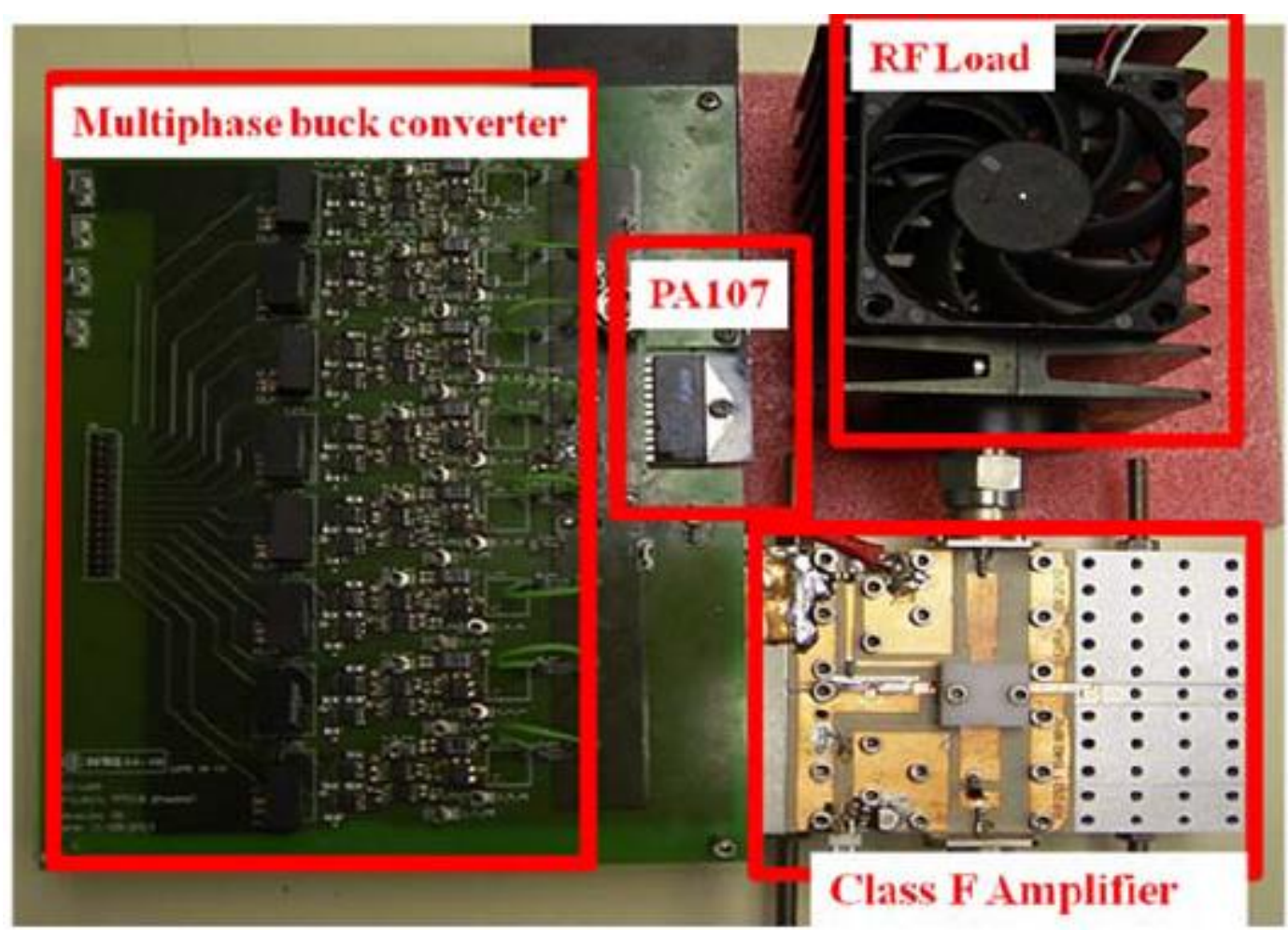

Figura 4.12 Fotografía del prototipo implementado para aplicaciones ET y EER [159].

En la Figura 4.13 se puede ver el banco de medida empleado en la caracterización EER y ET de este sistema. Este banco de medida es prácticamente el mismo que se presentó en el Apartado 3.2 del Capítulo 3 añadiendo alguna fuente de alimentación extra y los generadores arbitrarios de señal para generar las señales de referencia.

\subsubsection{Aplicación EER}

En este apartado se caracteriza el prototipo de transmisor para aplicaciones EER. Tal y como ya se ha comentado anteriormente, en esta aplicación la información de envolvente será amplificada exclusivamente por el amplificador de envolvente por lo que debe corresponderse de la manera más exacta posible para reducir la distorsión. Debido a ello, el amplificador de envolvente constará del convertidor buck seguido por el regulador lineal para cumplir este cometido. Respecto a la información de fase, el amplificador de RF será el encargado de amplificar eficientemente dicha señal de 
envolvente constante trabajando en la zona de saturación. En la Figura 4.14 se presenta un diagrama de bloques con las formas de onda obtenidas en cada punto.

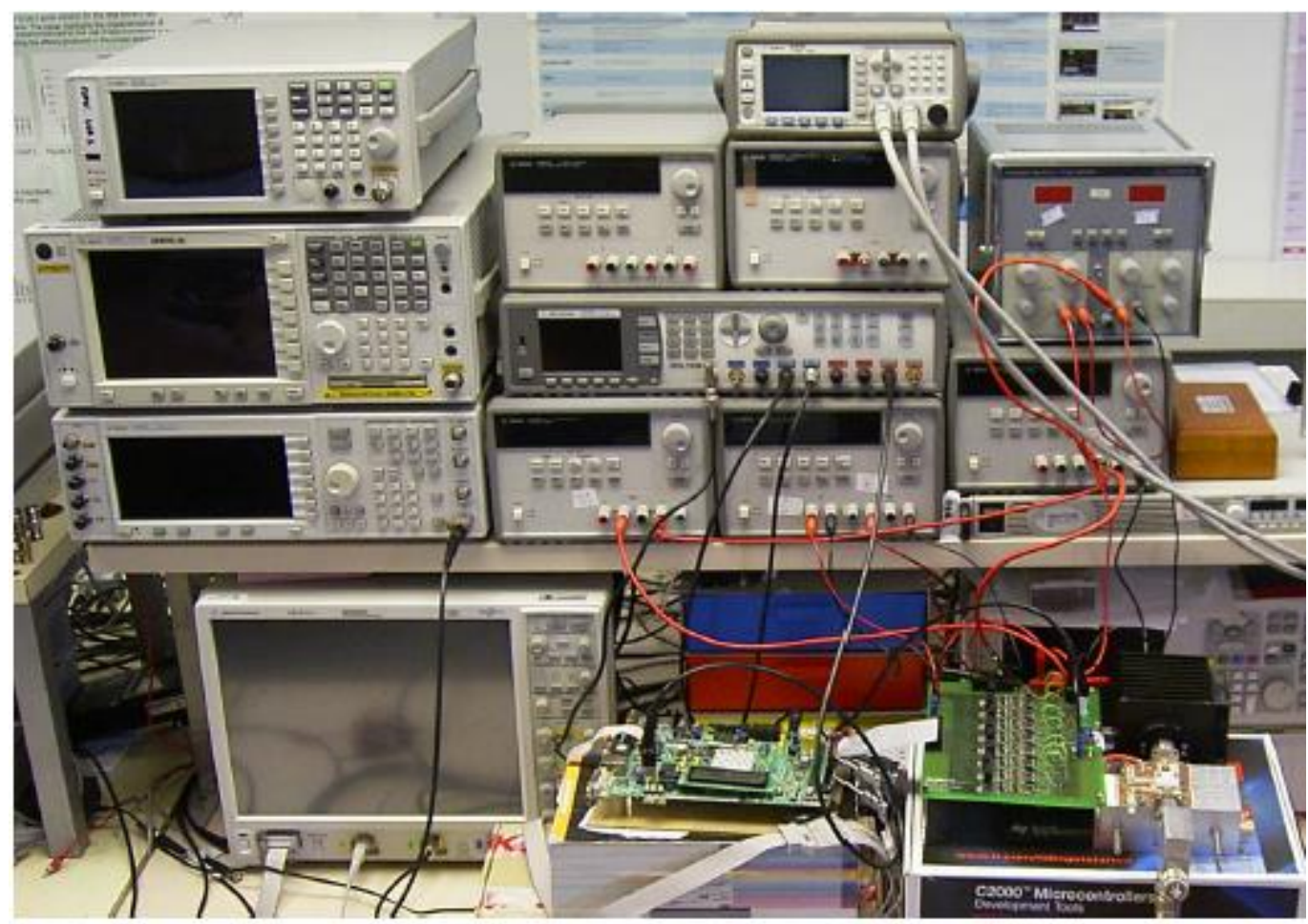

Figura 4.13. Banco de medida del transmisor para aplicaciones ET y EER.

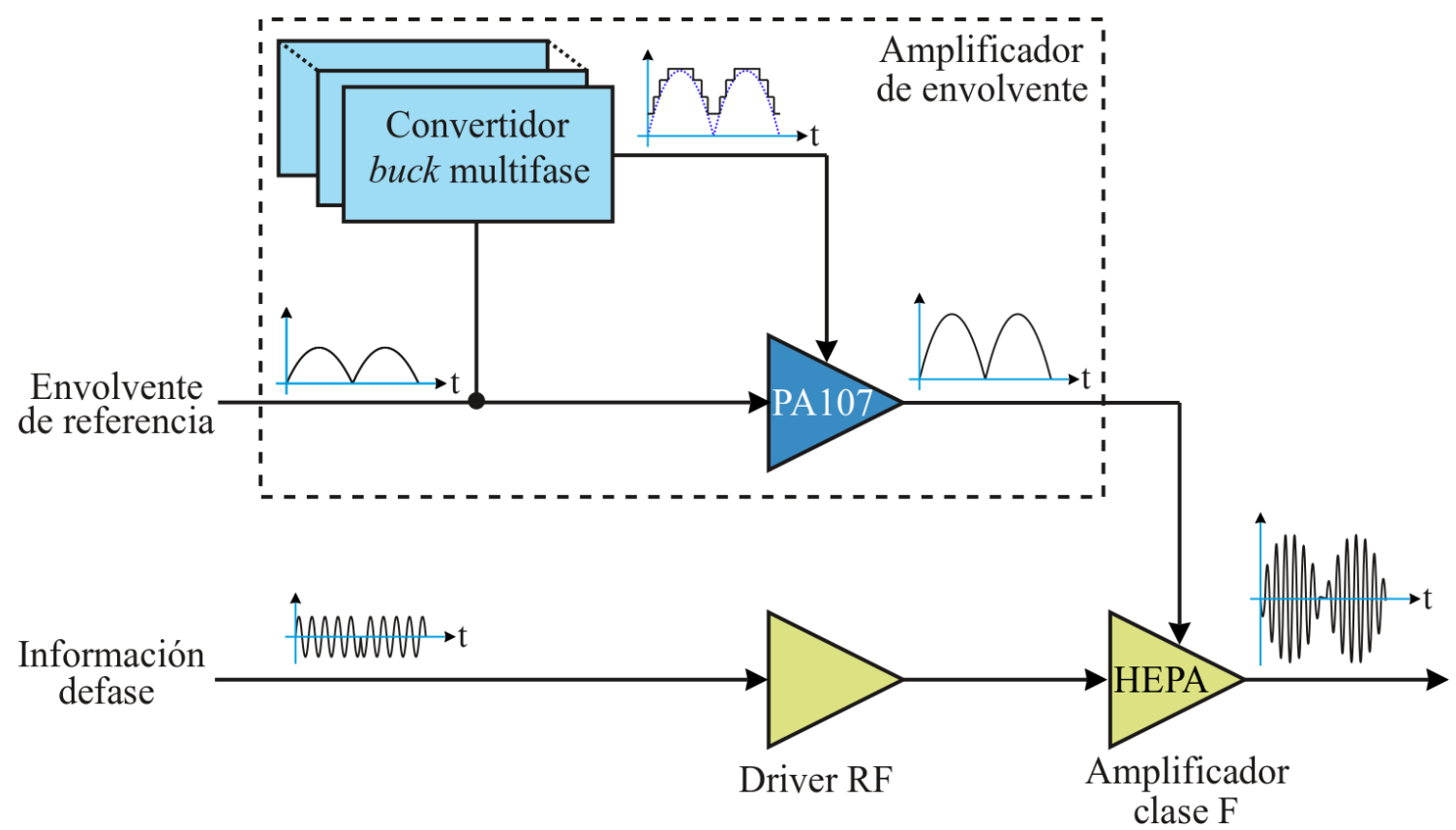

Figura 4.14 Diagrama de bloques transmisor EER con convertidor multinivel asistido por amplificador lineal. 
Con el fin de caracterizar el transmisor, se realizaron medidas de linealidad y de rendimiento. Para ello, se empleó como señal de prueba una modulación digital 64QAM con diferentes anchos de banda.

Desde la Figura 4.15 hasta la Figura 4.19 se muestra un segmento de la señal de prueba amplificada por el transmisor EER. Tal y como fue diseñado, la salida del convertidor buck multifase cambia instantáneamente con el nivel siguiendo la envolvente y además siempre ofrece un nivel mayor que ella con el fin de evitar distorsión de amplitud. En el caso de bajos anchos de banda (Figura 4.15), la tensión del multinivel es capaz de seguir de cerca la envolvente debido a su lenta variación en el tiempo. Sin embargo, a medida que aumenta el ancho de banda de la señal la envolvente presenta variaciones temporales más rápidas haciendo que el convertidor multifase no sea capaz de fijar rápidamente los niveles. En la Figura 4.19, donde el ancho de banda de la señal es grande, se observa claramente como el convertidor multifase no es capaz de responder a las rápidas variaciones de envolvente. En este caso, el convertidor se salta algunas transiciones entre niveles, pero mantiene una tensión mayor que el nivel de envolvente necesario.

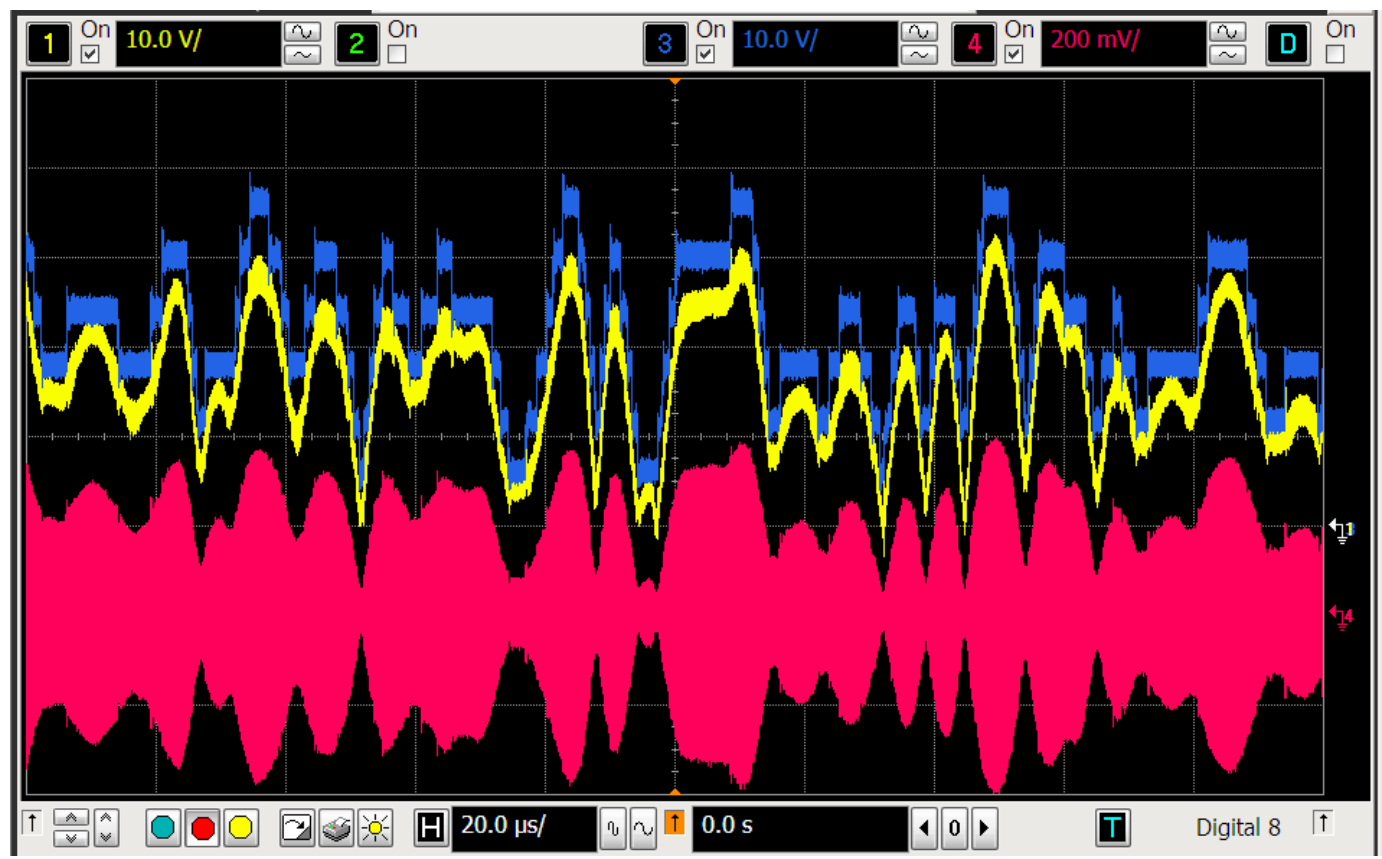

Figura 4.15 Formas de onda de la salida del convertidor buck multifase (canal 1), de la envolvente de salida del convertidor (canal 3) y de la salida de RF (canal 4) de una señal 64QAM con un ancho de banda de RF de $300 \mathrm{KHz}$. 


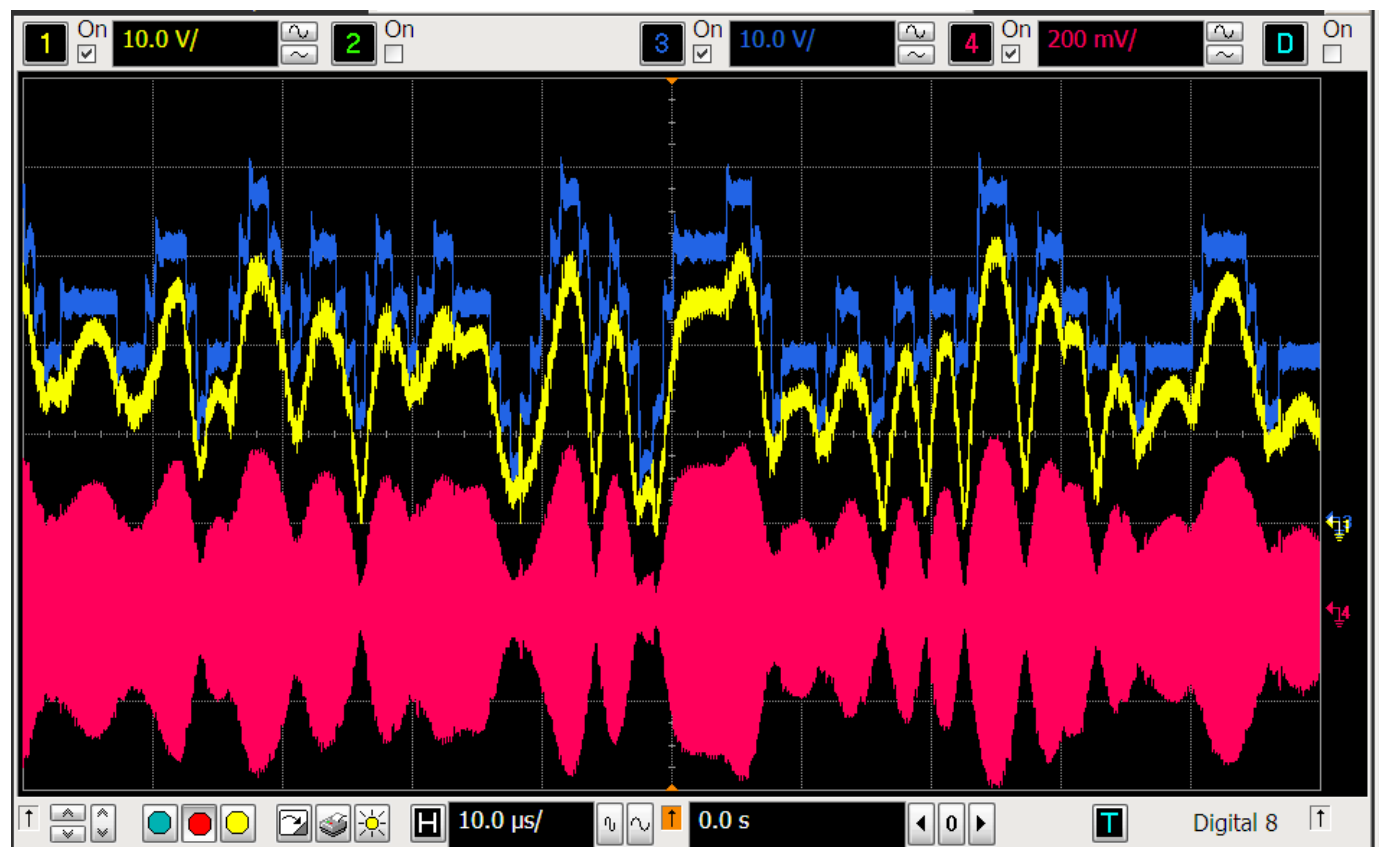

Figura 4.16 Formas de onda de la salida del convertidor buck multifase (canal 1), de la envolvente de salida del convertidor (canal 3) y de la salida de RF (canal 4) de una señal 64QAM con un ancho de banda de RF de $600 \mathrm{KHz}$.

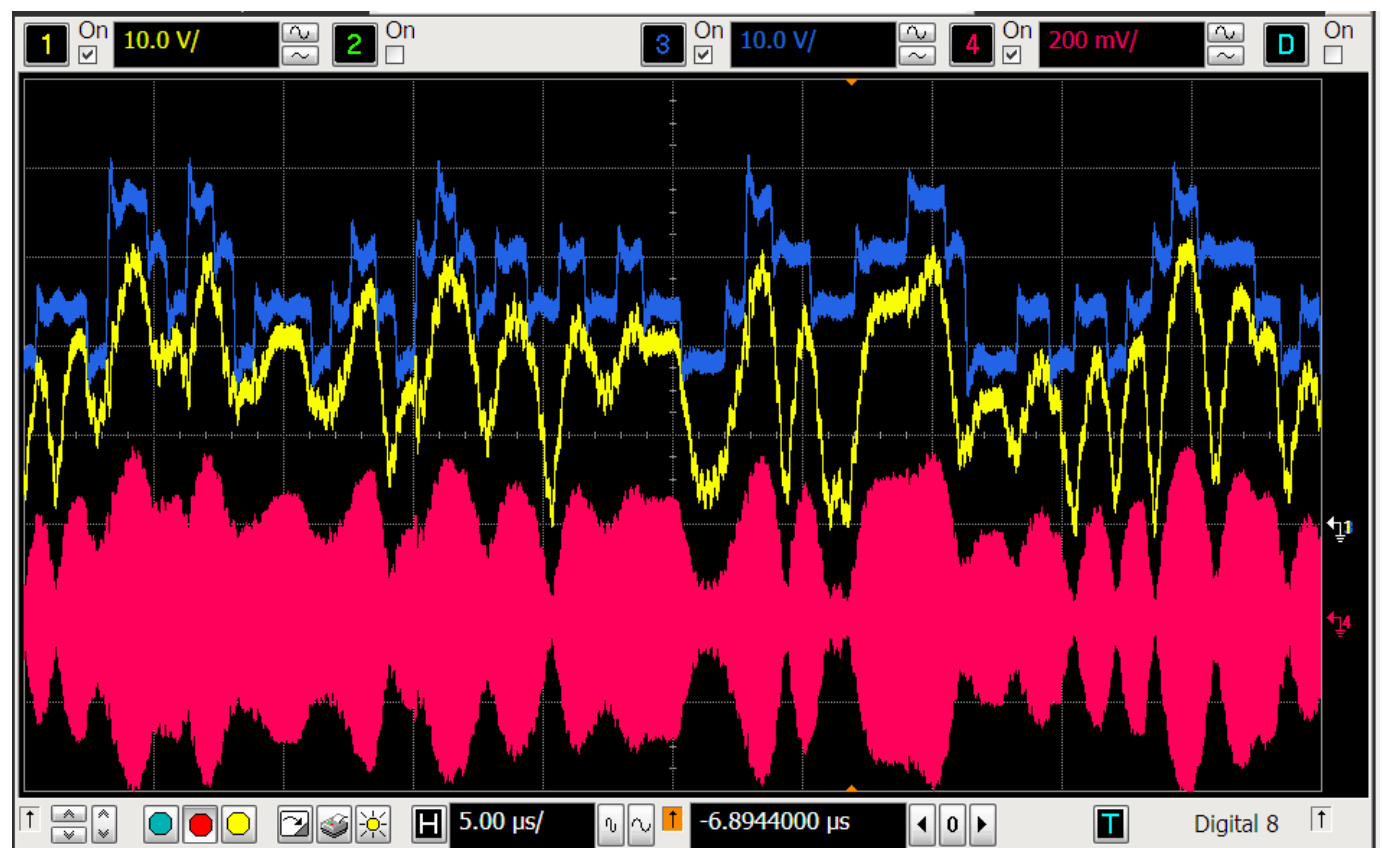

Figura 4.17 Formas de onda de la salida del convertidor buck multifase (canal 1), de la envolvente de salida del convertidor (canal 3) y de la salida de RF (canal 4) de una señal 64QAM con un ancho de banda de RF de $1200 \mathrm{KHz}$. 


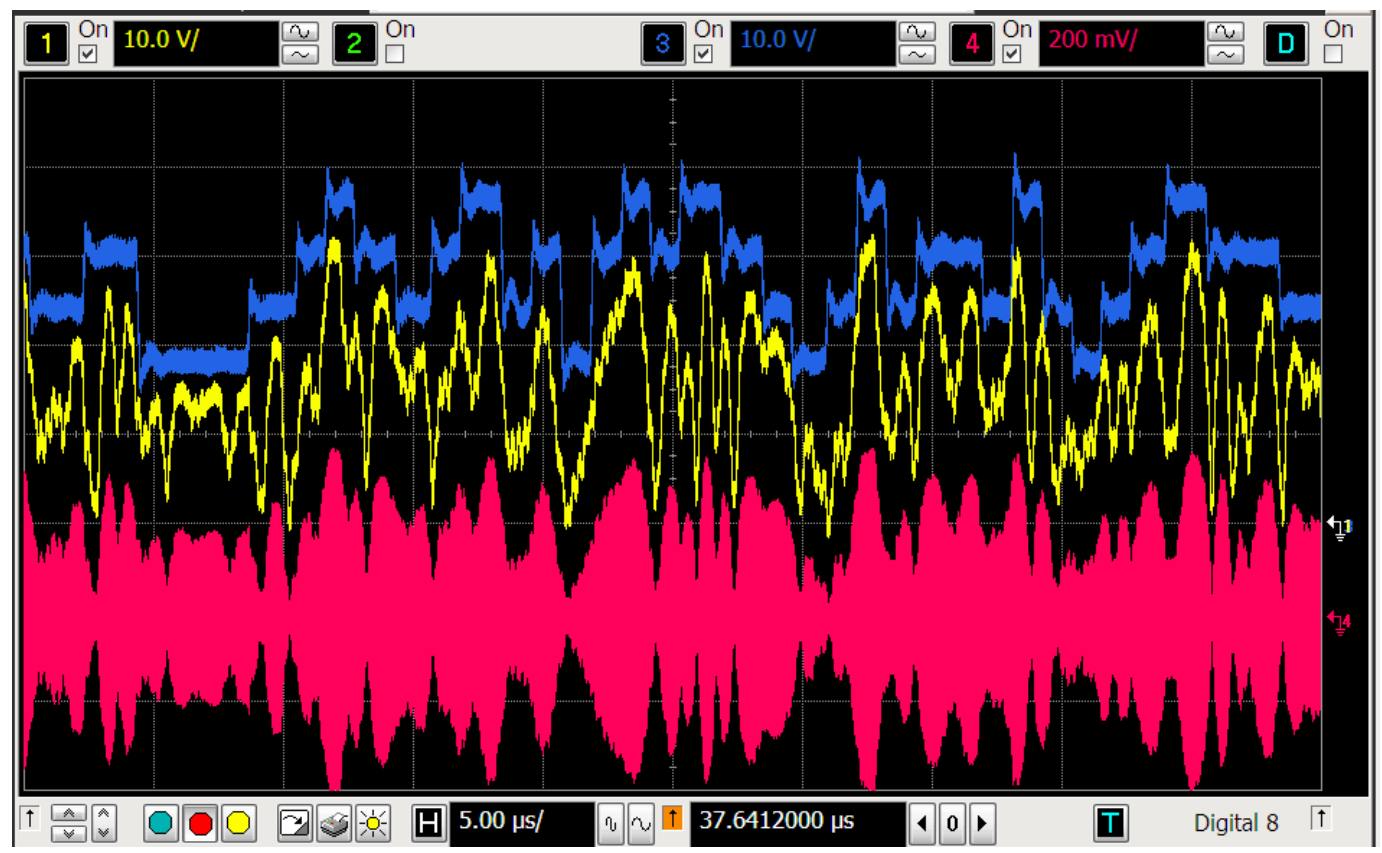

Figura 4.18 Formas de onda de la salida del convertidor buck multifase (canal 1), de la envolvente de salida del convertidor (canal 3) y de la salida de RF (canal 4) de una señal 64QAM con un ancho de banda de RF de $2400 \mathrm{KHz}$.

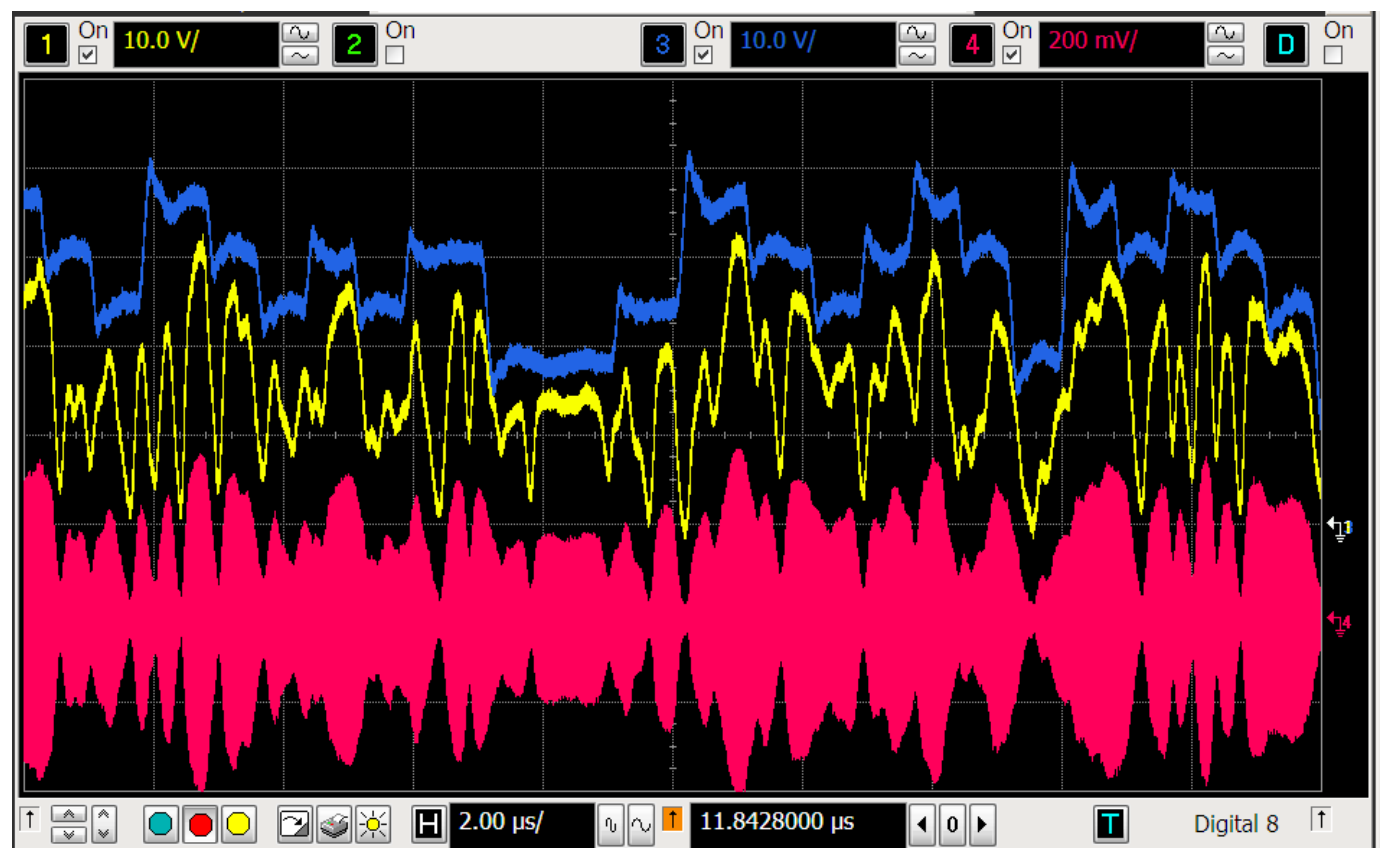

Figura 4.19 Formas de onda de la salida del convertidor buck multifase (canal 1), de la envolvente de salida del convertidor (canal 3) y de la salida de RF (canal 4) de una señal 64QAM con un ancho de banda de RF de $4800 \mathrm{KHz}$. 
Las mismas medidas han sido realizadas haciendo que el convertidor buck fije una tensión continua máxima con la que alimentar al regulador lineal. De esta forma, se puede ver cuál es la mejora que introduce el convertidor buck multinivel frente a un nivel fijo de alimentación. Las formas de onda del transmisor para las mismas señales de prueba se muestran desde la Figura 4.20 hasta la Figura 4.24. De ellas, se puede concluir que el convertidor multinivel introduce algo de distorsión debido a sus transiciones y a medida que aumenta el ancho de banda de la envolvente el regulador lineal no es capaz de seguir fielmente la envolvente de referencia, generando distorsión, aunque menor que en el caso del funcionamiento multinivel.

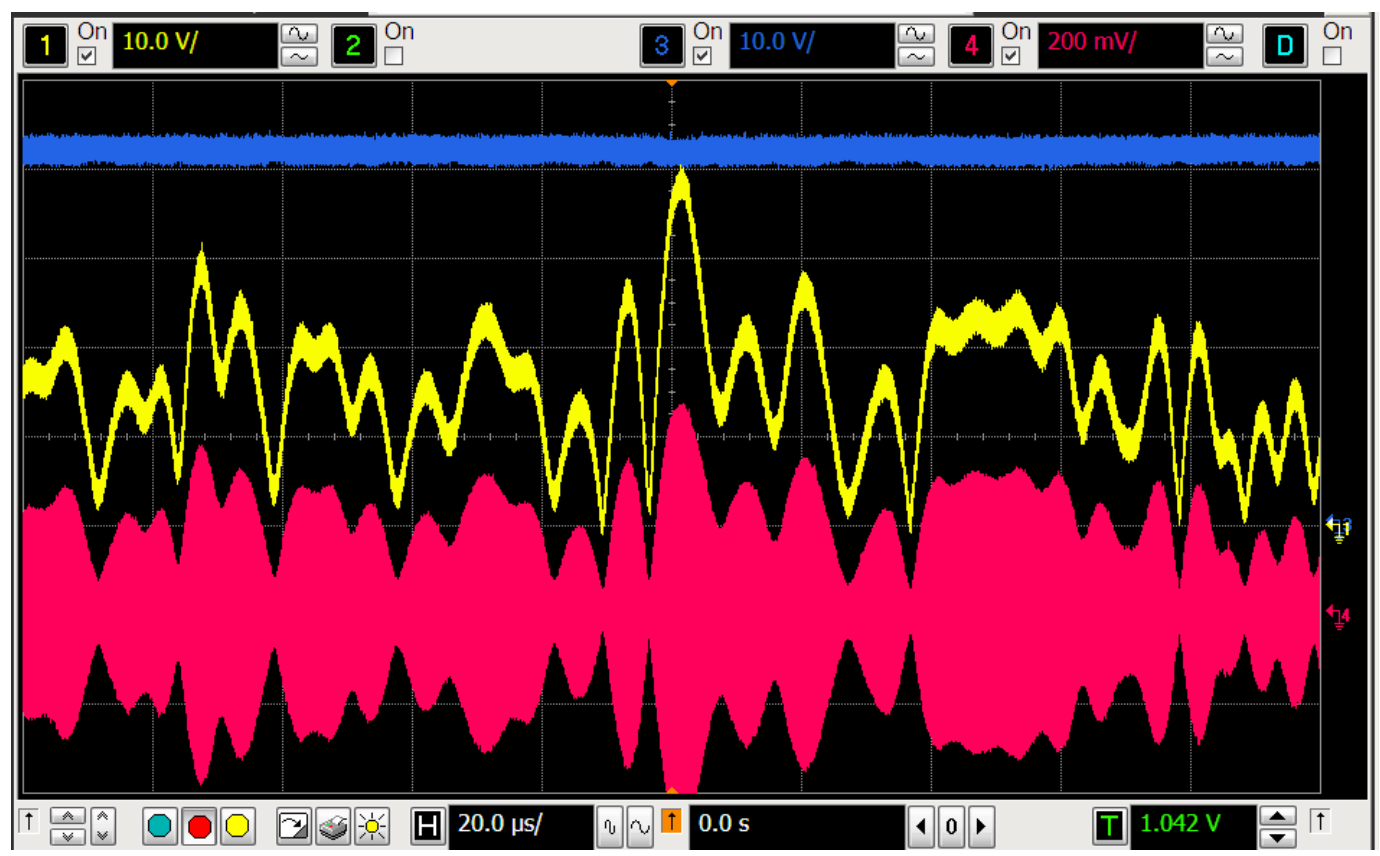

Figura 4.20 Formas de onda del nivel fijo del convertidor buck multifase (canal 1), de la envolvente de salida del convertidor (canal 3 ) y de la salida de $R F$ (canal 4) de una señal 64QAM con un ancho de banda de RF de $300 \mathrm{KHz}$. 


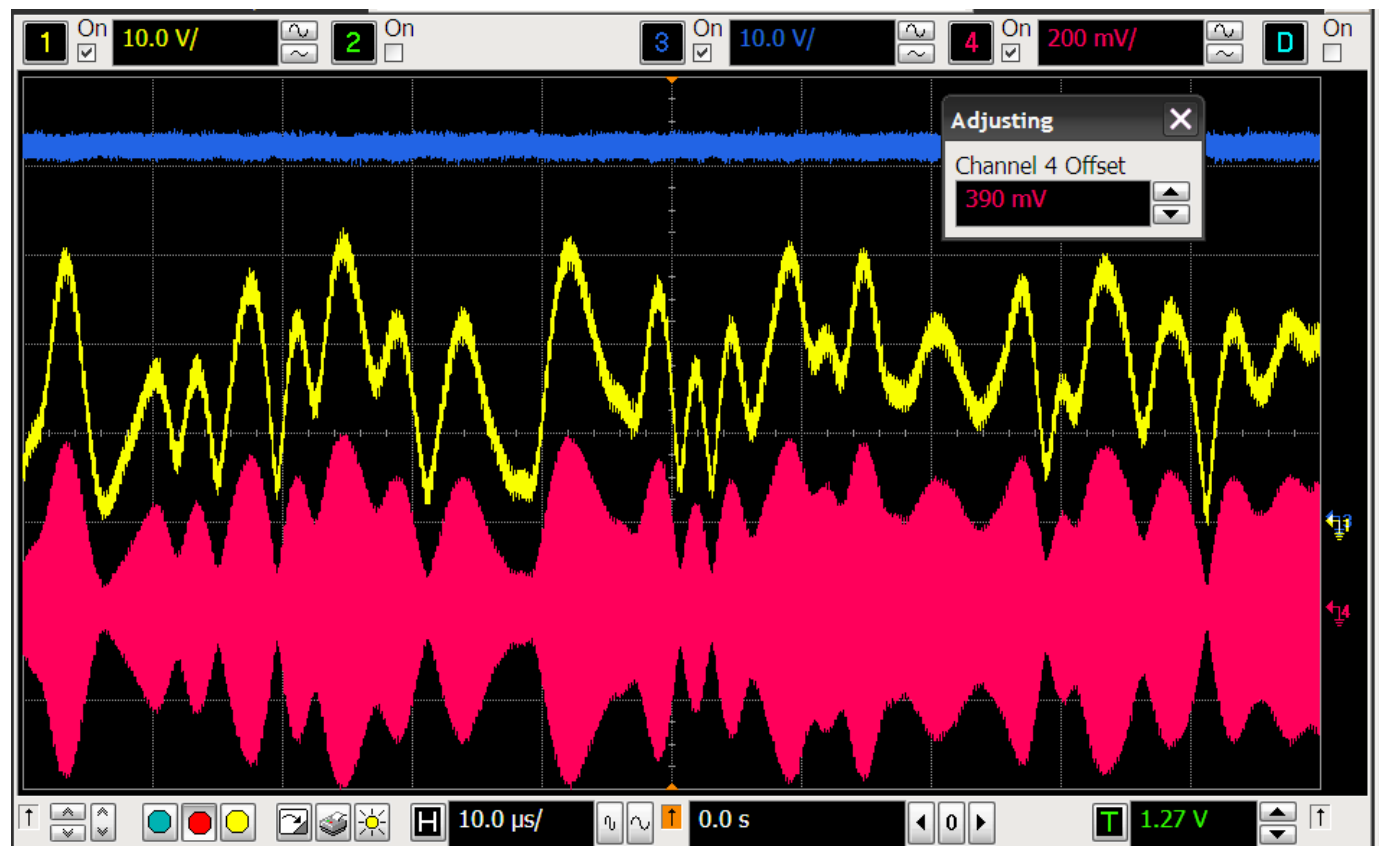

Figura 4.21 Formas de onda del nivel fijo del convertidor buck multifase (canal 1), de la envolvente de salida del convertidor (canal 3) y de la salida de RF (canal 4) de una señal 64QAM con un ancho de banda de RF de $600 \mathrm{KHz}$.

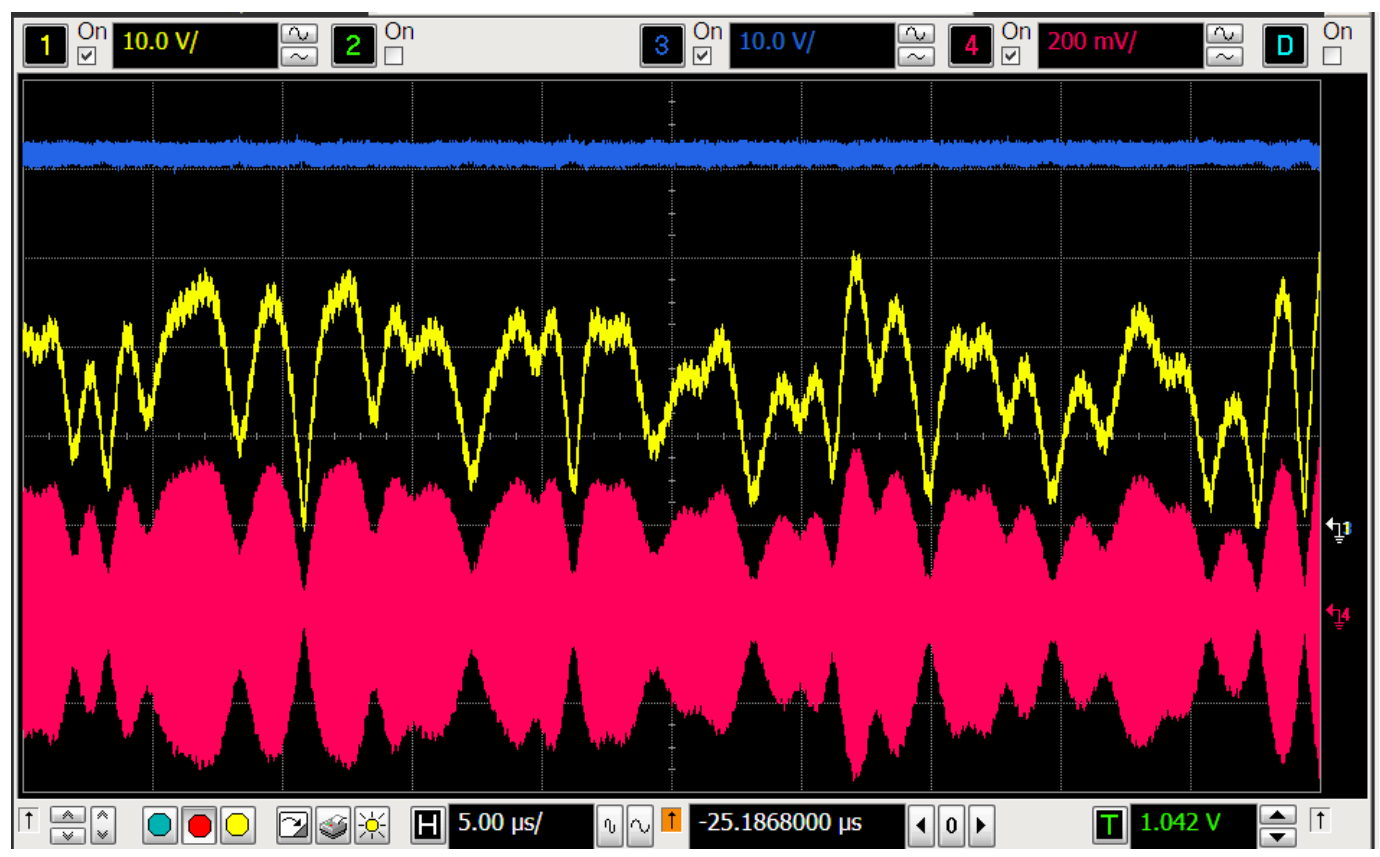

Figura 4.22 Formas de onda del nivel fijo del convertidor buck multifase (canal 1), de la envolvente de salida del convertidor (canal 3) y de la salida de $\mathbf{R F}$ (canal 4) de una señal 64QAM con un ancho de banda de RF de $1200 \mathrm{KHz}$. 


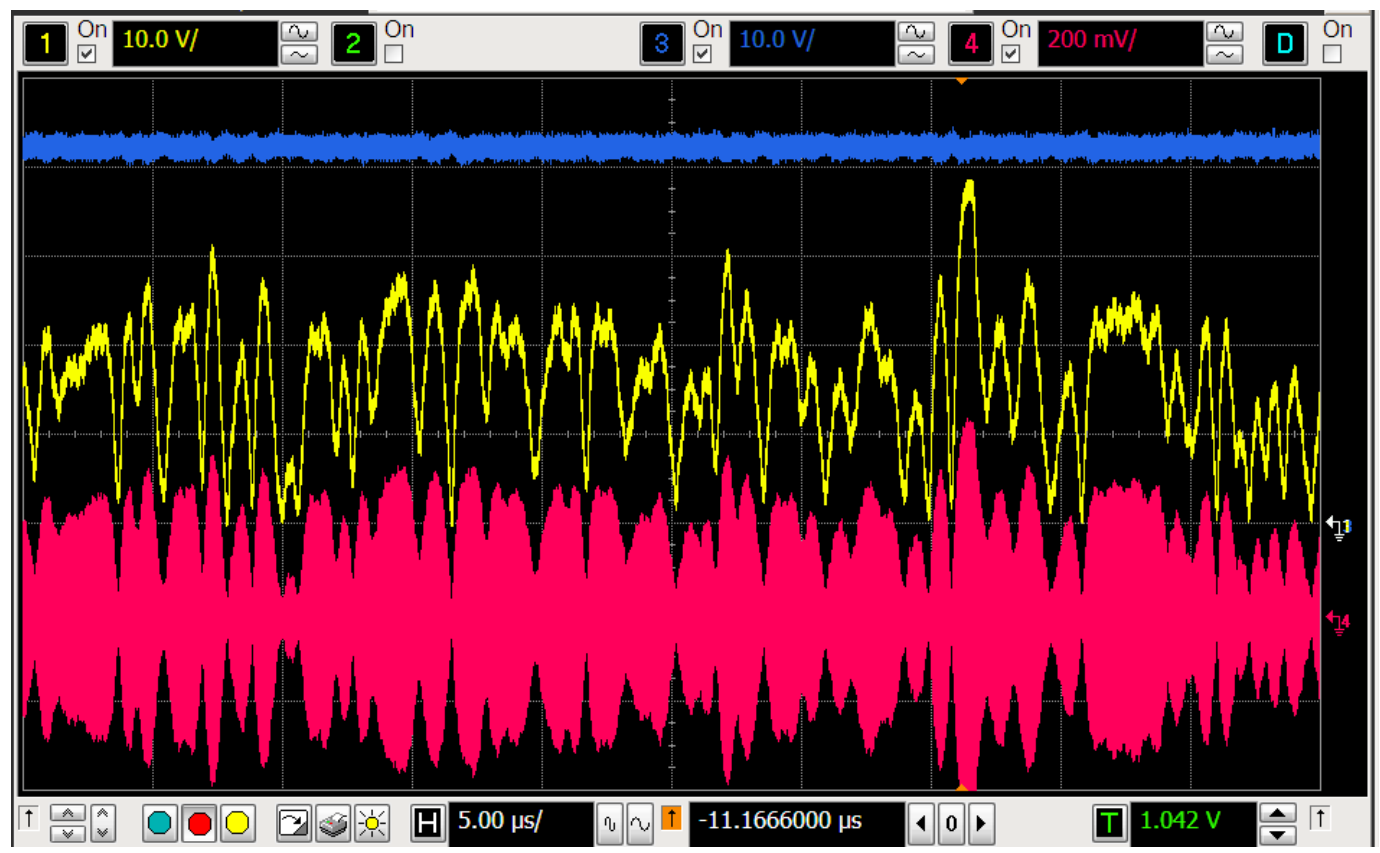

Figura 4.23 Formas de onda del nivel fijo del convertidor buck multifase (canal 1), de la envolvente de salida del convertidor (canal 3) y de la salida de RF (canal 4) de una señal 64QAM con un ancho de banda de RF de $2400 \mathrm{KHz}$.

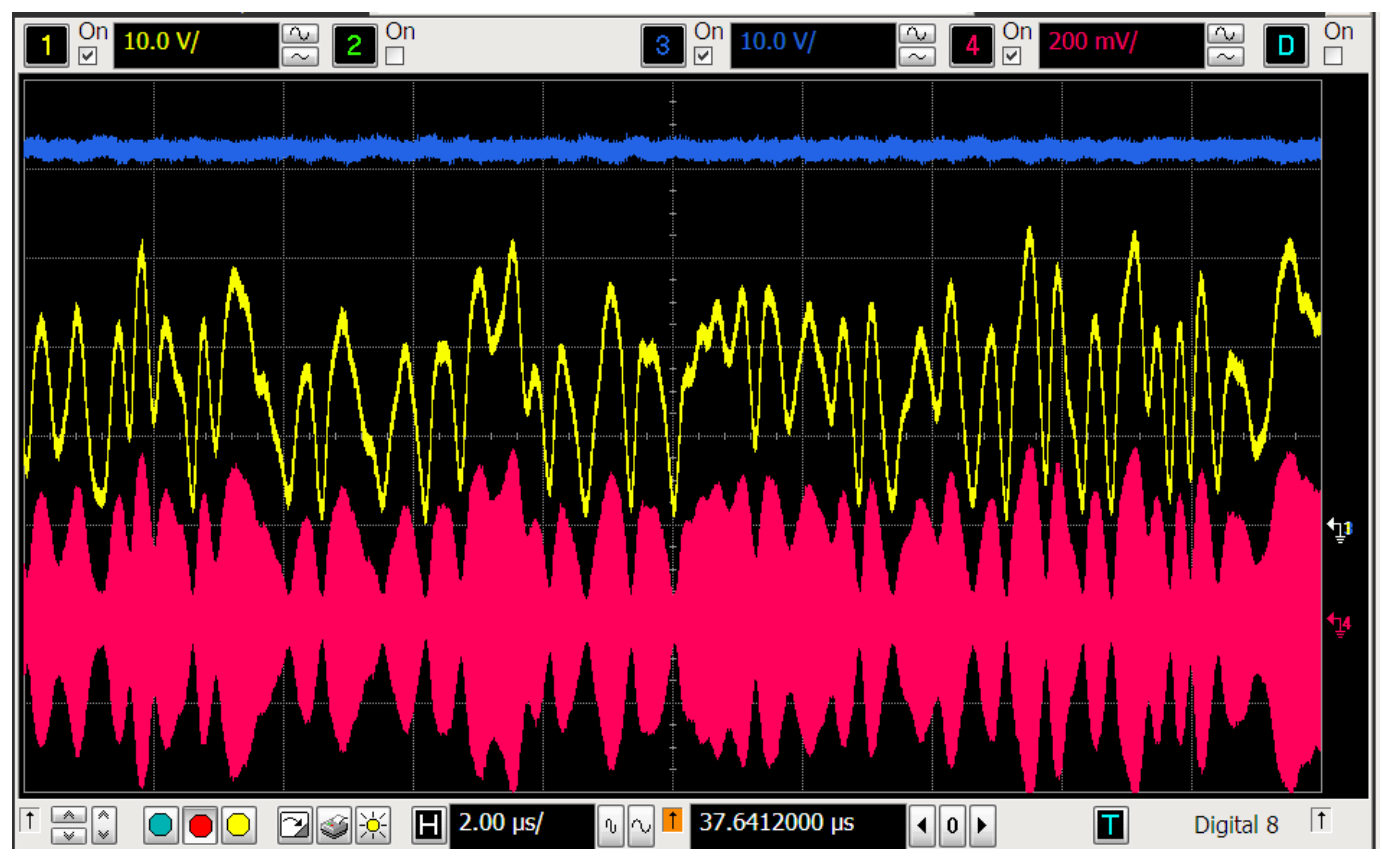

Figura 4.24 Formas de onda del nivel fijo del convertidor buck multifase (canal 1), de la envolvente de salida del convertidor (canal 3) y de la salida de $\mathbf{R F}$ (canal 4) de una señal 64QAM con un ancho de banda de RF de $4800 \mathrm{KHz}$. 
Esta situación se puede ver más claramente en las medidas espectrales. Desde la Figura 4.25 hasta la Figura 4.29 se muestra la comparación del espectro de salida de RF para los diferentes anchos de banda de la señal de prueba aplicada al sistema EER donde el convertidor presenta un comportamiento multinivel o presenta un nivel fijo de tensión. Las medidas de ACPR muestran la ligera influencia del convertidor multifase en la linealidad del amplificador EER, principalmente motivada por los tiempos de transición. Además, también se observa que a medida que aumenta el ancho de banda de la señal el error cometido en la alineación temporal entre el camino de fase y el de envolvente es mayor y, por ello, los niveles de ACPR a la derecha y a la izquierda de la frecuencia central son diferentes.

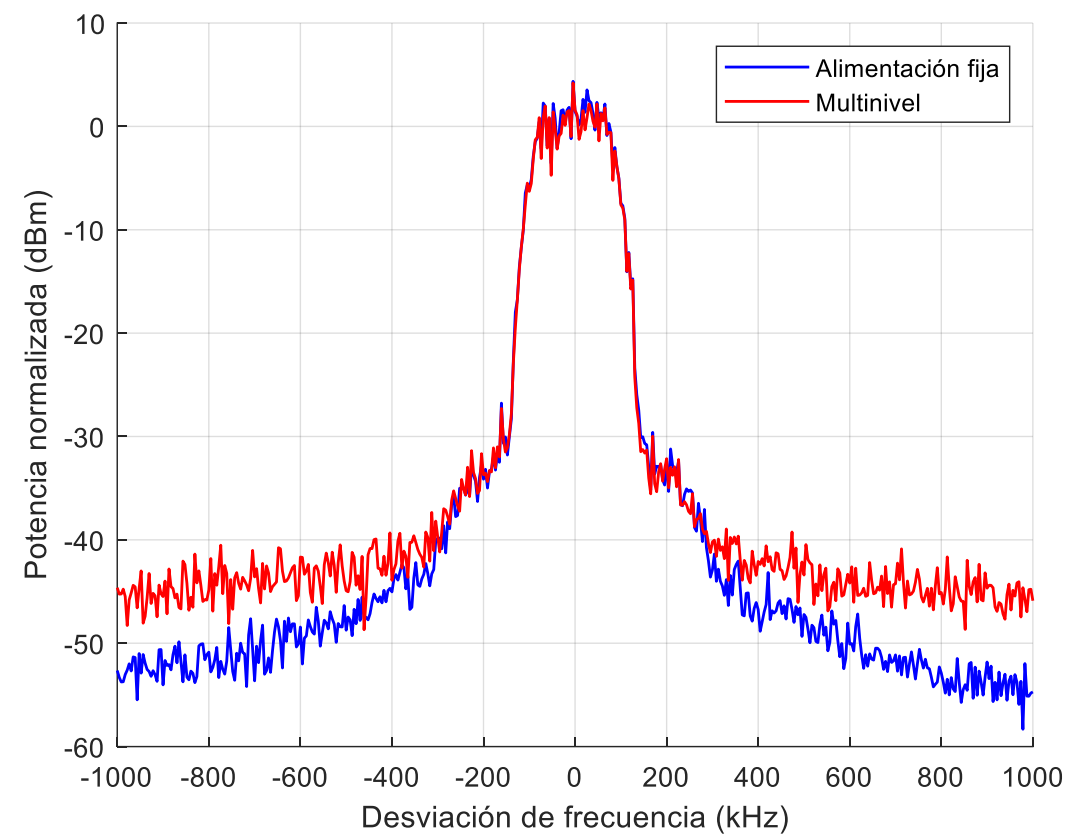

Figura 4.25 Espectro de salida normalizado del amplificador EER para una señal 64QAM con un ancho de banda de RF de $300 \mathrm{KHz}$. 


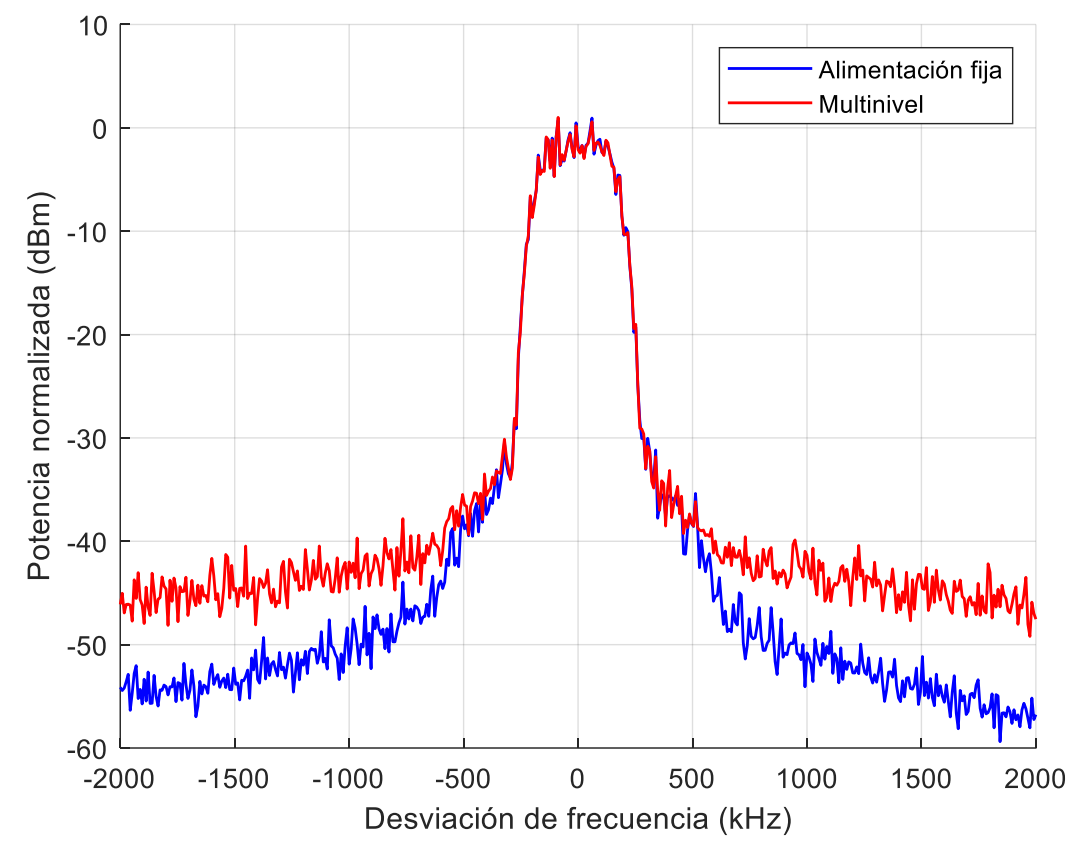

Figura 4.26 Espectro de salida normalizado del amplificador EER para una señal 64QAM con un ancho de banda de RF de $600 \mathrm{KHz}$.

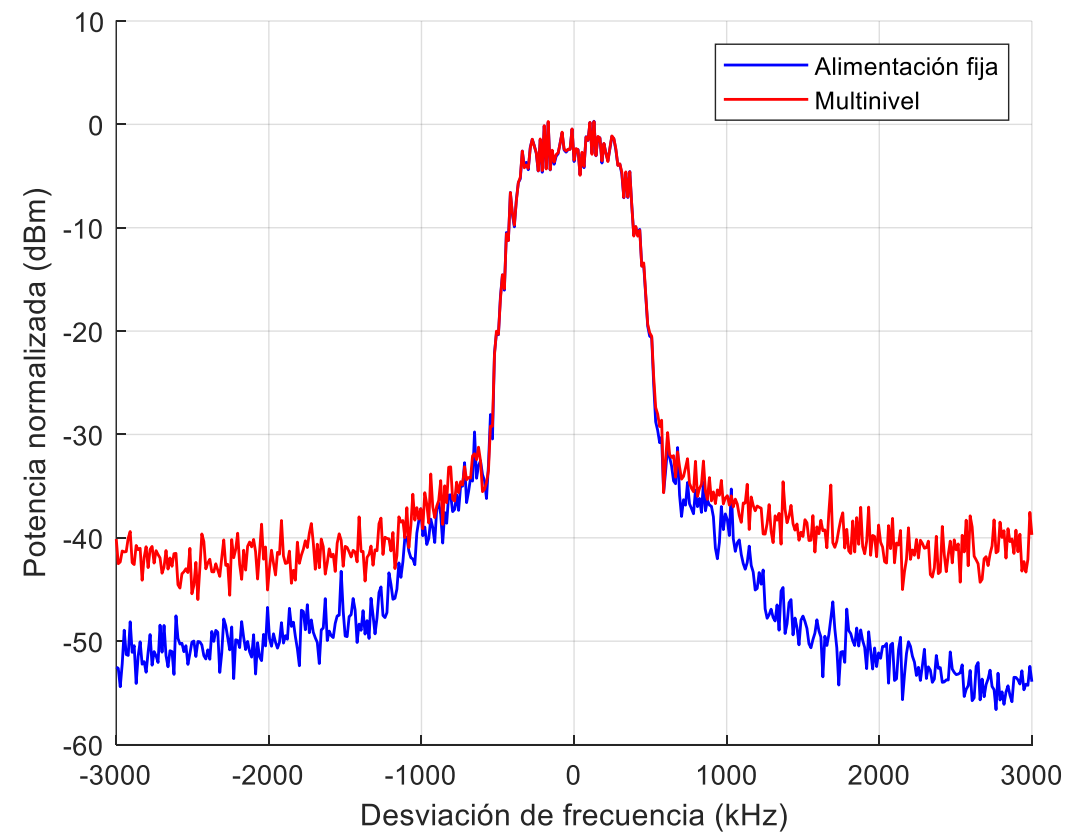

Figura 4.27 Espectro de salida normalizado del amplificador EER para una señal 64QAM con un ancho de banda de RF de $1200 \mathrm{KHz}$. 


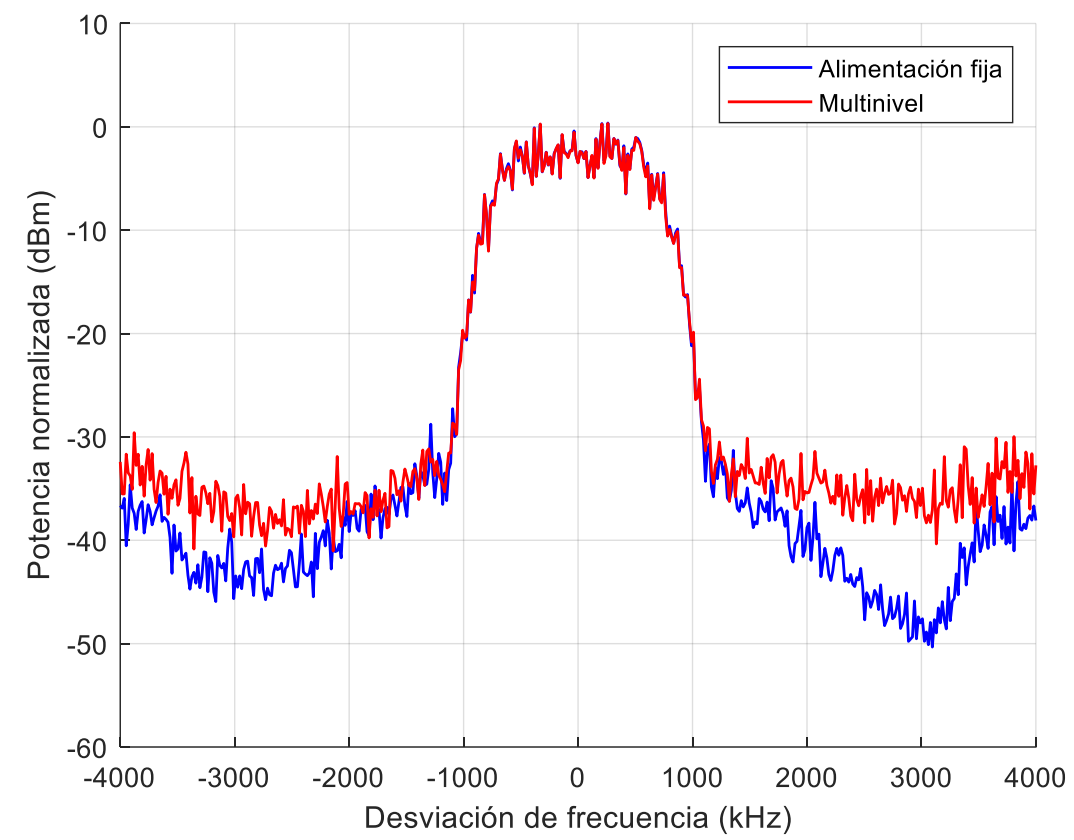

Figura 4.28 Espectro de salida normalizado del amplificador EER para una señal 64QAM con un ancho de banda de RF de $2400 \mathrm{KHz}$.

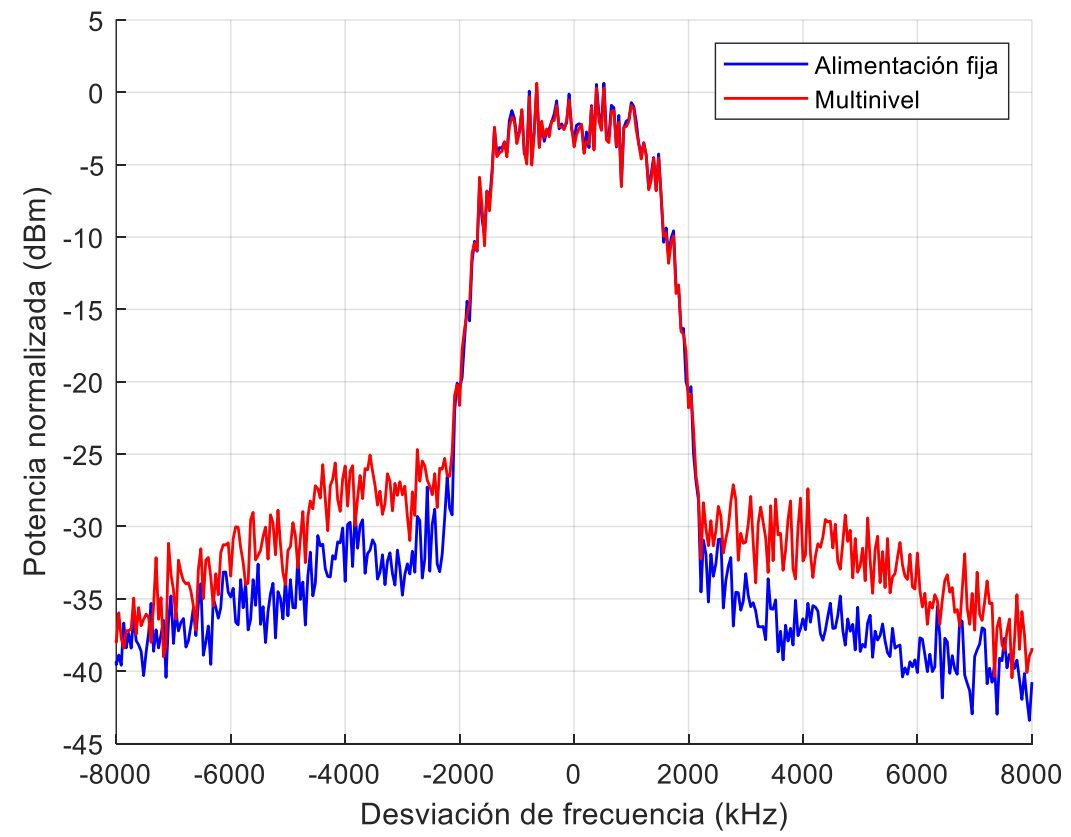

Figura 4.29 Espectro de salida normalizado del amplificador EER para una señal 64QAM con un ancho de banda de RF de $4800 \mathrm{KHz}$. 
La Figura 4.30 resume las medidas de linealidad para los diferentes anchos de banda de la señal de prueba. Para ello, se han hallado los valores de ACPR a la izquierda y a la derecha del canal. Como se puede observar en la Figura 4.30, la linealidad se degrada a medida que aumenta el ancho de banda de la señal de prueba. Principalmente ello se debe a la limitación en frecuencia del regulador lineal. Por otro lado, las diferencias entre el ACPR a la derecha o a la izquierda del canal pueden ser debida a dos causas: a efectos memoria, los cuales no fueron tenidos en cuenta, o a un error en la exactitud de la alineación temporal entre la señal de fase y la señal de envolvente, característica muy importante en un sistema EER. Estas medidas de linealidad podrían mejorar en el caso de aplicar predistorsión digital, tarea que se plantea como trabajo futuro.

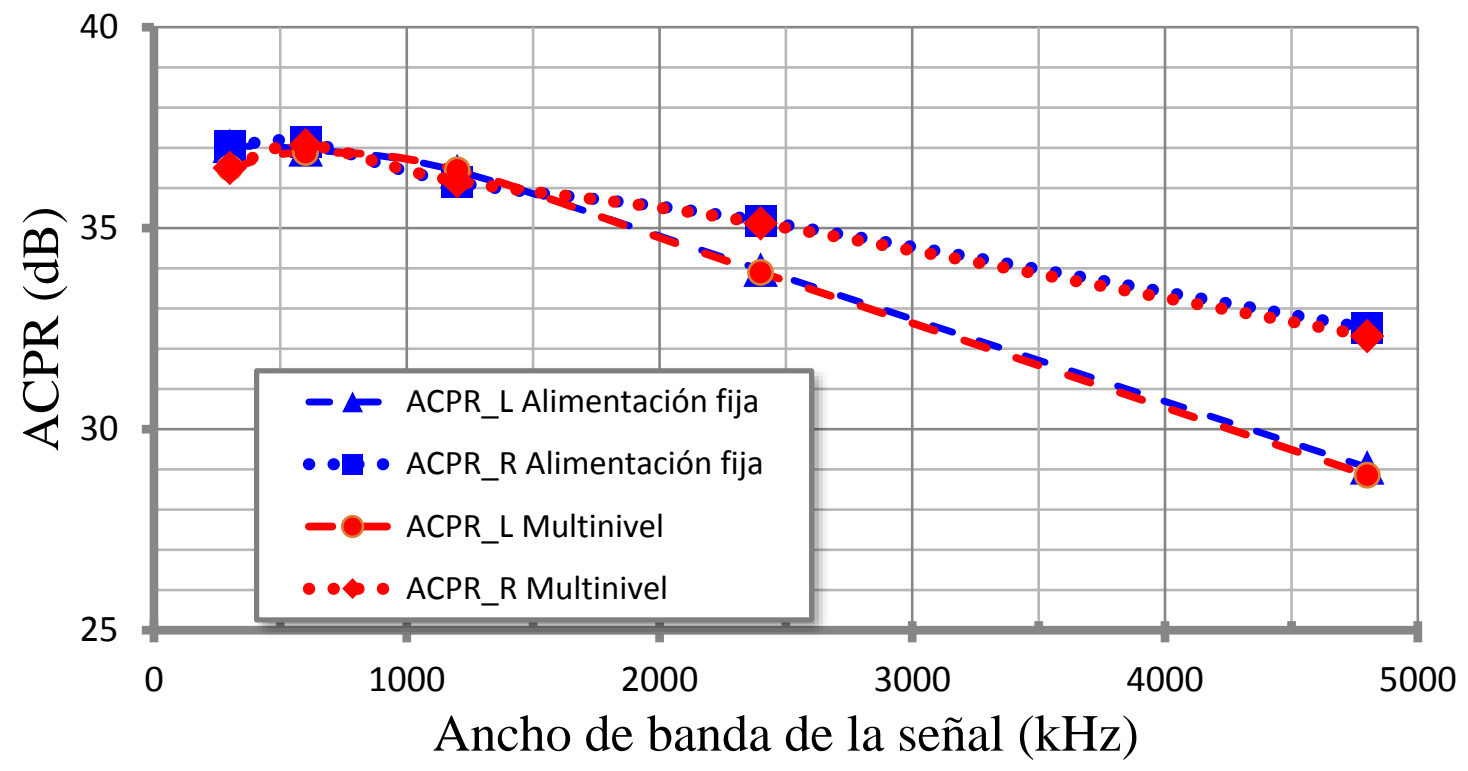

\section{Figura 4.30 Resumen de la linealidad del sistema EER en función del ancho de banda de la señal.}

De la misma manera, también se realizaron medidas de rendimiento. Las medidas se realizaron con el objetivo de cuantificar la mejora que introduce el convertidor multinivel en el sistema EER. Para ello, al igual que con las medidas de linealidad, se analizaron dos métodos para la misma señal de prueba con diferentes anchos de banda: por un lado, el convertidor trabaja en modo multinivel y, por otro, el convertidor trabaja a una tensión fija.

En la Figura 4.31 se presentan las medidas de rendimiento asociadas al transmisor EER. A bajos anchos de banda, se ve la influencia del convertidor buck 
obteniendo mejoras en el rendimiento de más del 9\%. Sin embargo, a medida que va aumentando el ancho de banda de la señal, el convertidor no es capaz de seguir los cambios en la envolvente y esto provoca mayores pérdidas en el regulador lineal.

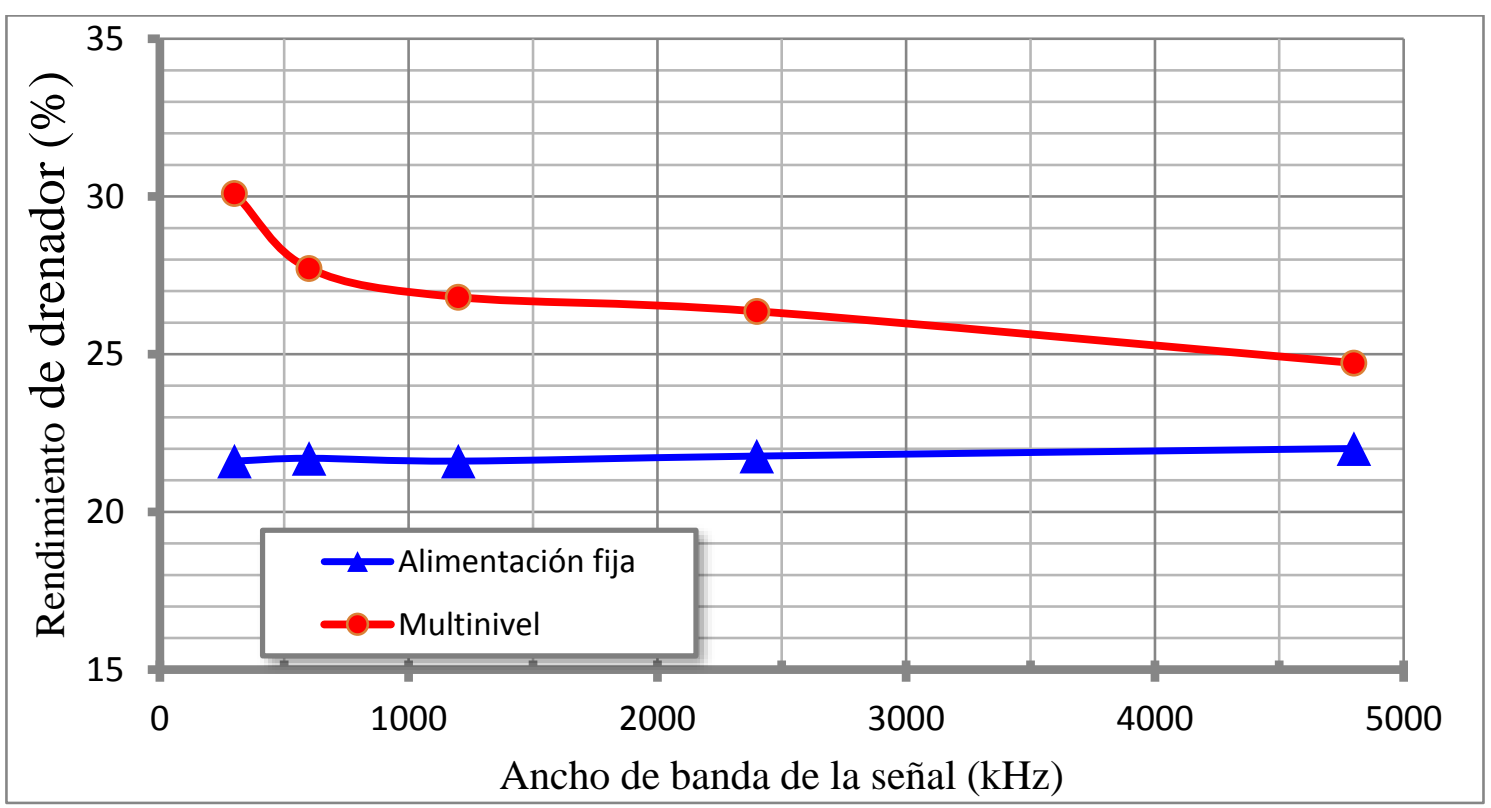

Figura 4.31 Rendimiento de drenador del transmisor EER en función del ancho de banda de la señal de prueba.

En conclusión, la integración del amplificador clase F con el amplificador de envolvente para implementar un sistema EER ha mostrado las siguientes conclusiones:

- El sistema EER fue probado con una señal 64QAM para diferentes anchos de banda hasta los $4.8 \mathrm{MHz}$.

- Los niveles de linealidad empeoran a medida que aumenta el ancho de banda de la señal debido a que el convertidor buck no es capaz de seguir la envolvente y a la limitación en ancho de banda del regulador lineal.

- La aplicación de predistorsión digital en el sistema EER ayudaría a mejorar estos niveles de linealidad con el fin de hacer cumplir las especificaciones de una aplicación concreta.

- La incorporación del convertidor multifase mejora el rendimiento de drenador del sistema, lo que ofrece una ligera ventaja respecto al caso de alimentación constante. 


\subsubsection{Aplicación ET}

En este apartado se caracteriza el prototipo de transmisor para aplicaciones ET. Esta caracterización difiere un poco de la anterior aplicación. Aquí el amplificador de envolvente trabajará únicamente con el convertidor buck (sin el regulador lineal) alimentando al amplificador de RF con un nivel superior al de la tensión de salida. De esta manera, la información de envolvente y de fase es tratada exclusivamente por el amplificador de RF. Con el fin de no provocar distorsión, es necesario que el amplificador de RF trabaje en su zona lineal por lo que se deberá reducir el nivel de excitación del amplificador clase F para que trabaje en su zona lineal. Esto implica una reducción en la potencia de salida y en el rendimiento que puede ofrecer este amplificador de RF. En la Figura 4.32 se presenta un diagrama de bloques de este transmisor ET.

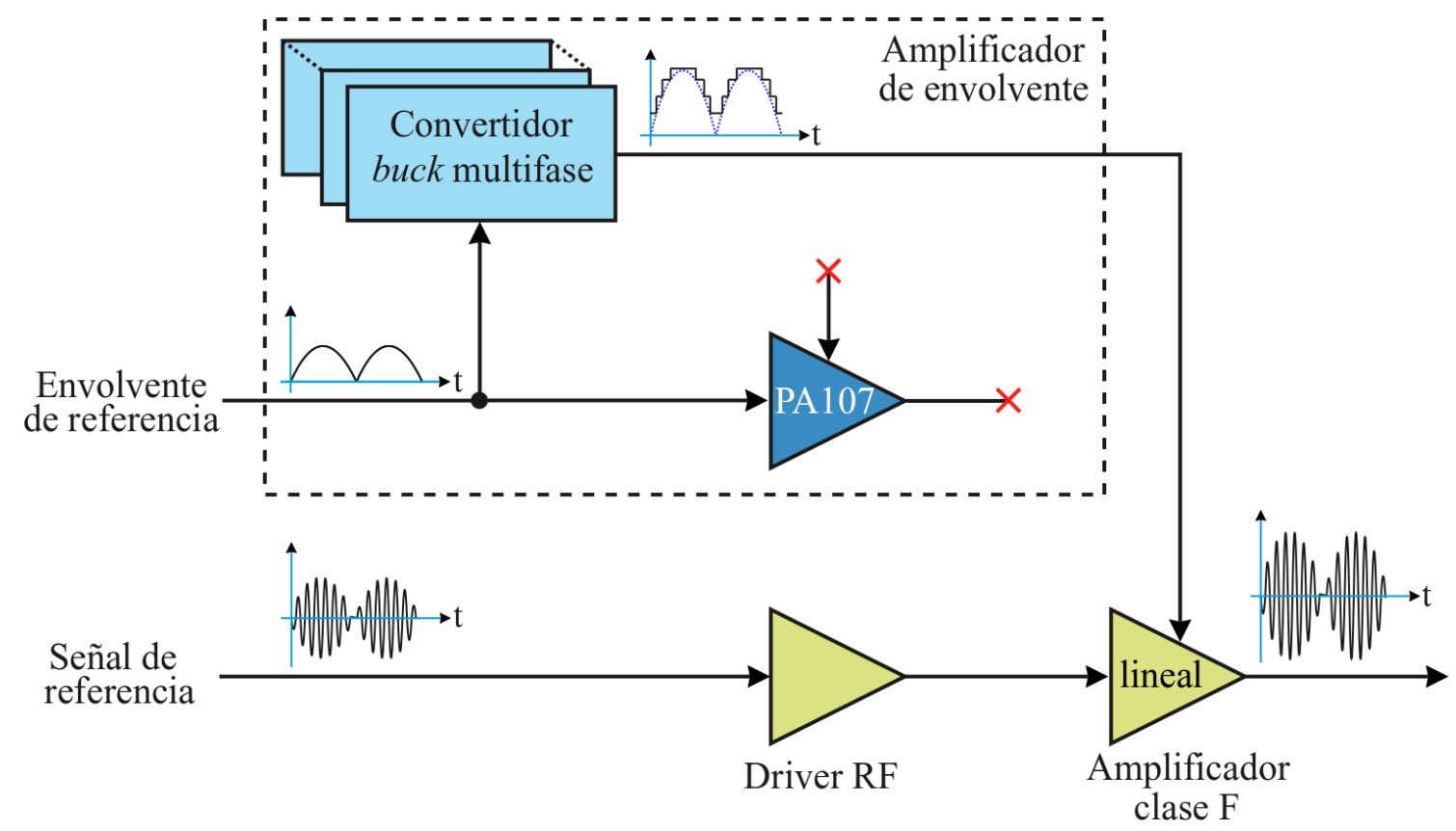

Figura 4.32 Diagrama de bloques transmisor ET con convertidor multinivel.

$\mathrm{Al}$ igual que en el caso del transmisor EER del apartado anterior, se realizaron medidas de linealidad y rendimiento al transmisor ET implementado. Como señal de prueba también será empleada una modulación digital 64QAM con diferentes anchos de banda.

Desde la Figura 4.33 hasta la Figura 4.37 se muestra un segmento de la señal de prueba amplificada por el transmisor ET. En este caso, el convertidor buck multifase 
alimenta al amplificador de RF con un nivel de tensión superior al de la envolvente de salida en cada instante. A bajos anchos de banda de la señal (Figura 4.33), el convertidor buck multifase es capaz de seguir de cerca la envolvente de la señal de salida debido a su lenta variación en el tiempo. Sin embargo, a medida que aumenta el ancho de banda de la señal de envolvente (Figura 4.37) presenta variaciones temporales más rápidas haciendo que el convertidor multifase no sea capaz de adaptar los niveles. En este caso, el convertidor no realiza transiciones entre niveles de tensión lo que reducirá el rendimiento.

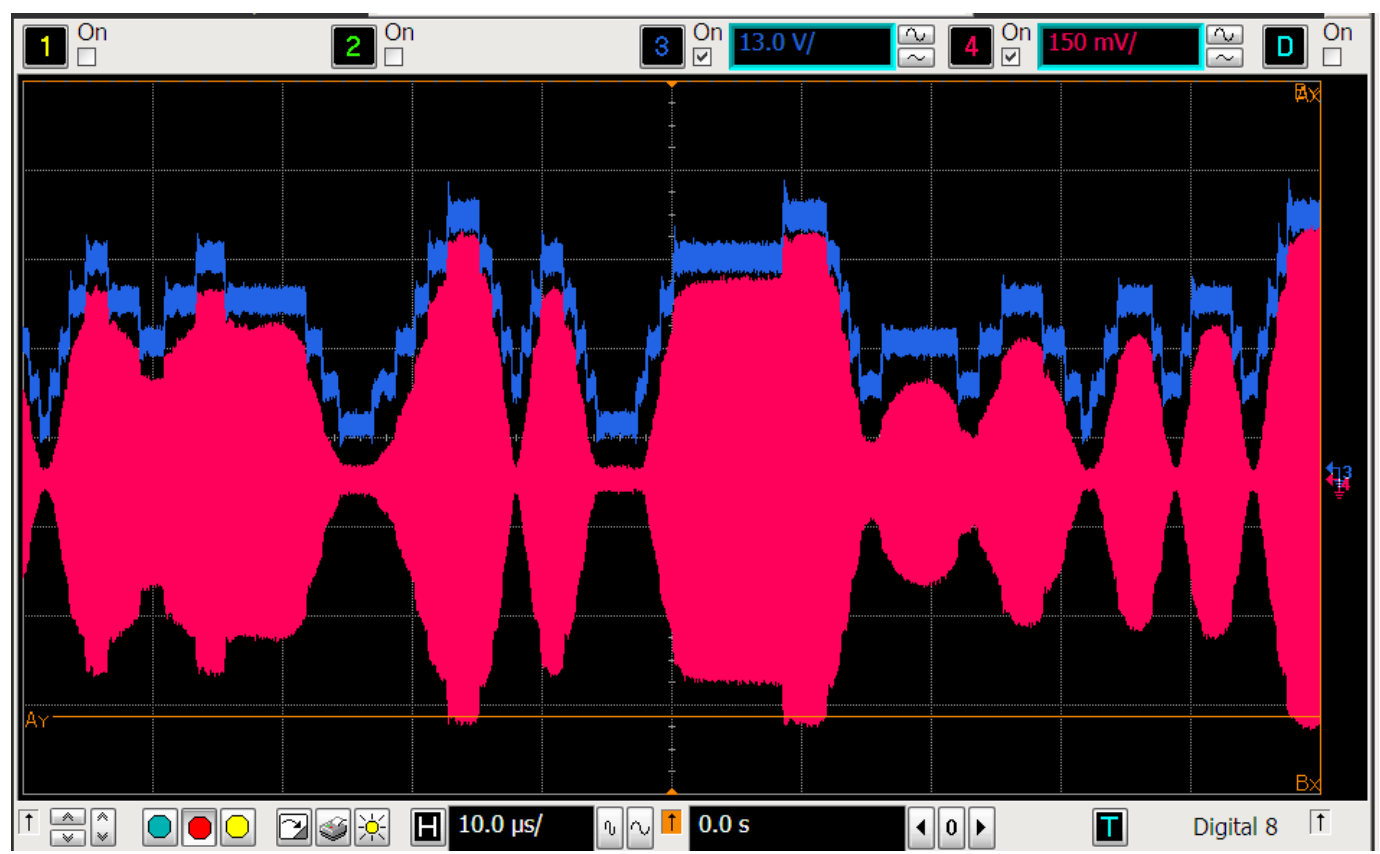

Figura 4.33 Formas de onda del transmisor ET a la salida del convertidor buck multifase (canal 1) y a la salida de RF (canal 4) para una señal 64QAM con un ancho de banda de RF de $300 \mathrm{KHz}$. 


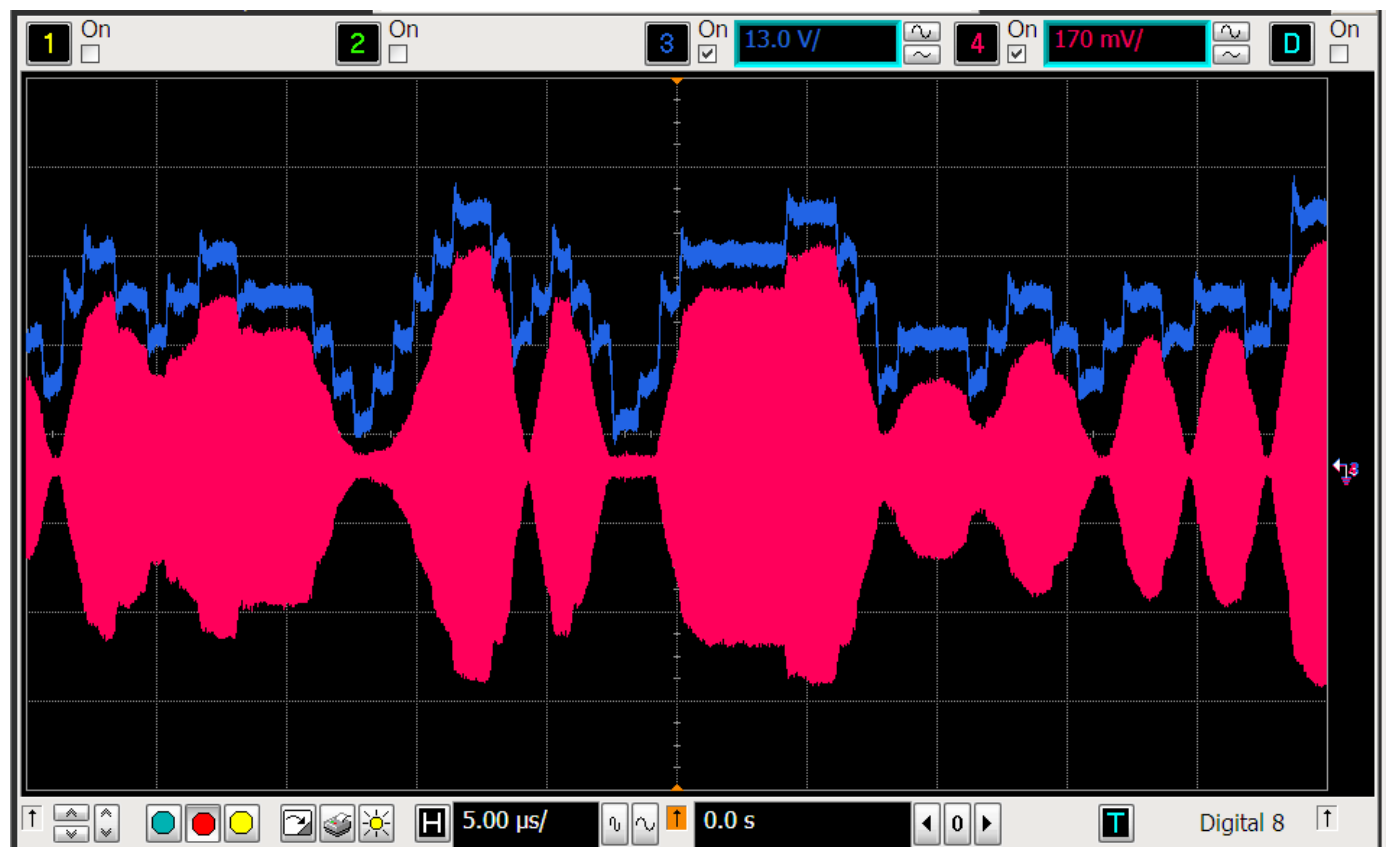

Figura 4.34 Formas de onda del transmisor ET a la salida del convertidor buck multifase (canal 1) y a la salida de RF (canal 4) para una señal 64QAM con un ancho de banda de RF de $600 \mathrm{KHz}$.

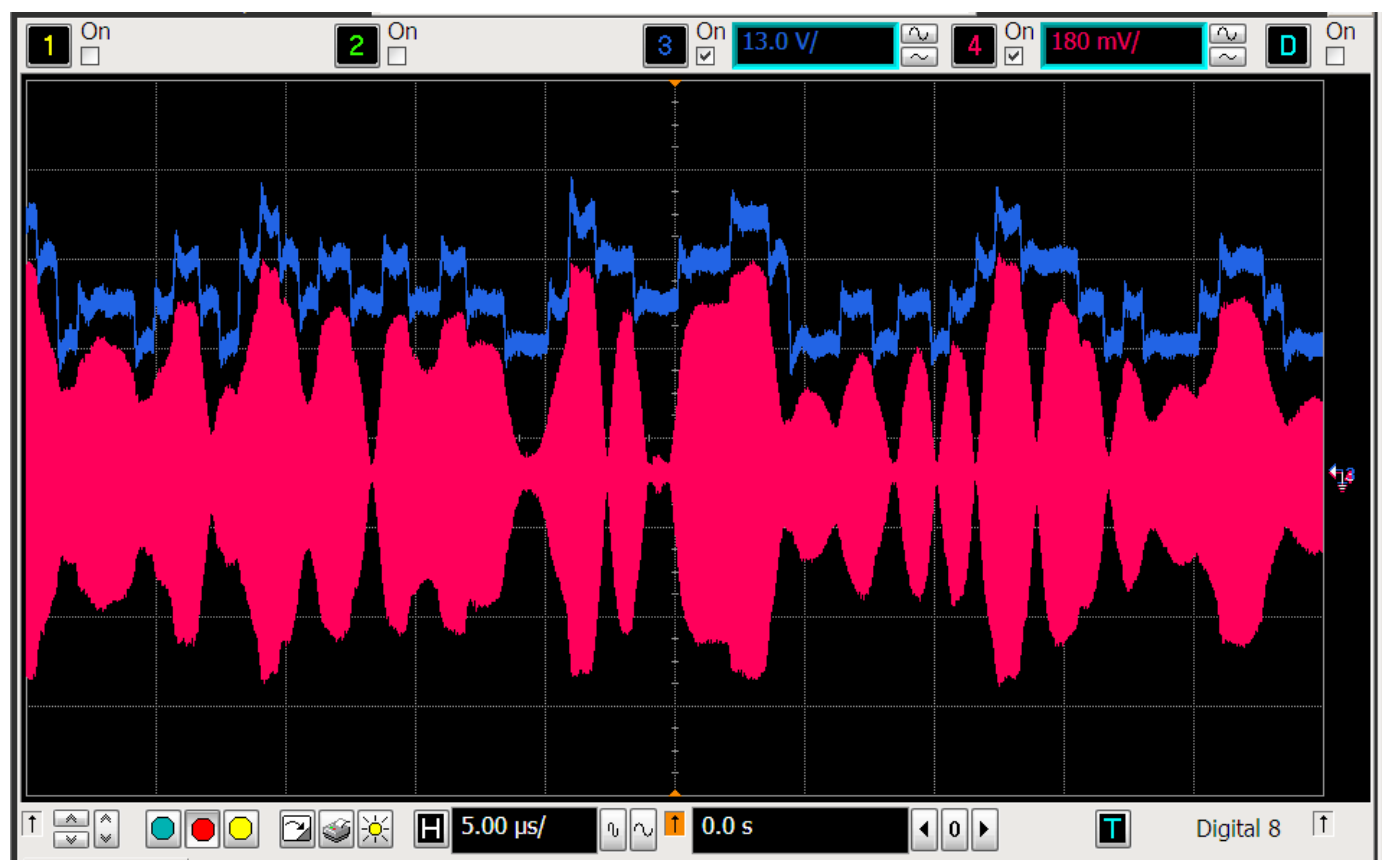

Figura 4.35 Formas de onda del transmisor ET a la salida del convertidor buck multifase (canal 1) y a la salida de RF (canal 4) para una señal 64QAM con un ancho de banda de RF de $1200 \mathrm{KHz}$. 


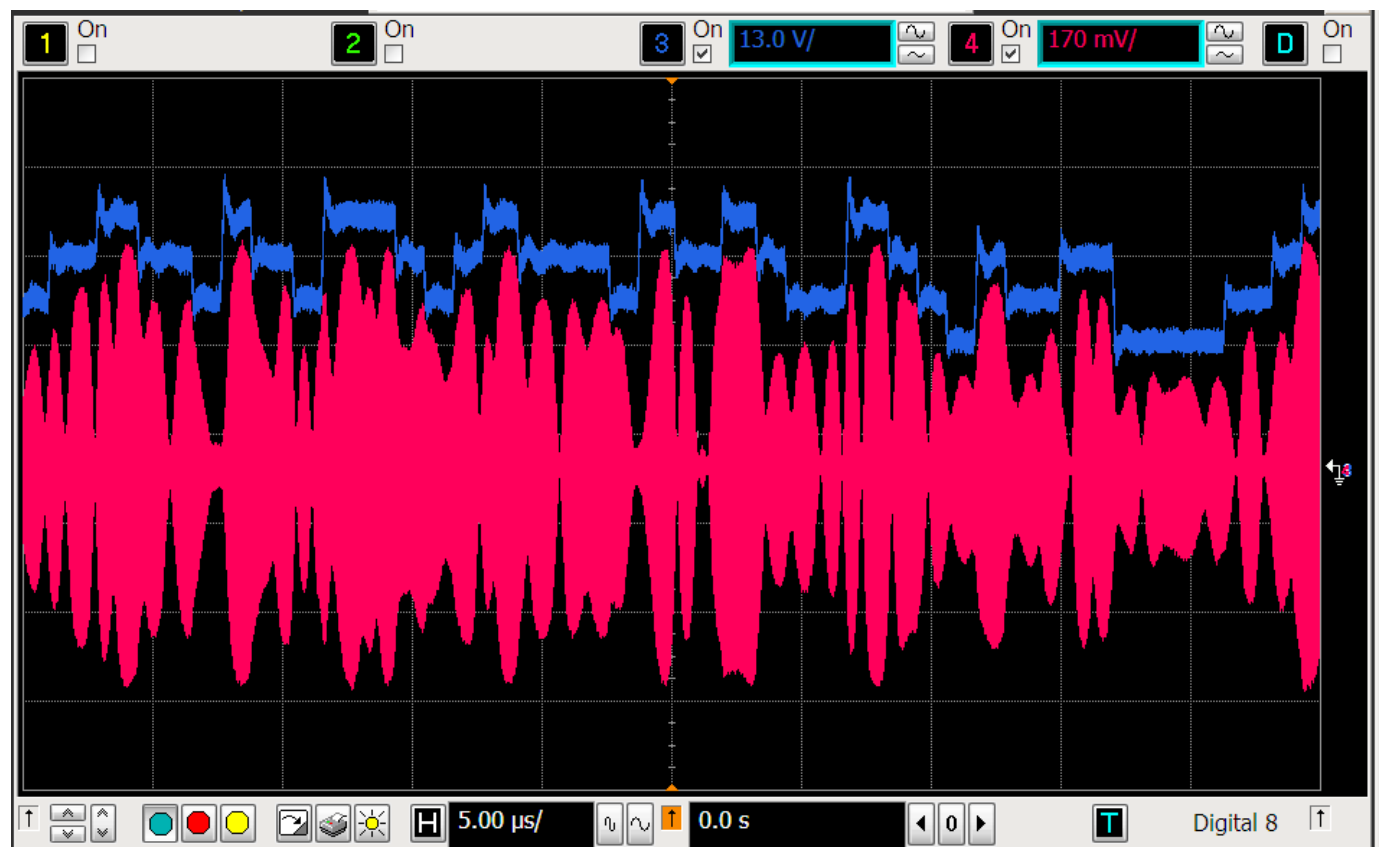

Figura 4.36 Formas de onda del transmisor ET a la salida del convertidor buck multifase (canal 1) y a la salida de RF (canal 4) para una señal 64QAM con un ancho de banda de RF de $2400 \mathrm{KHz}$.

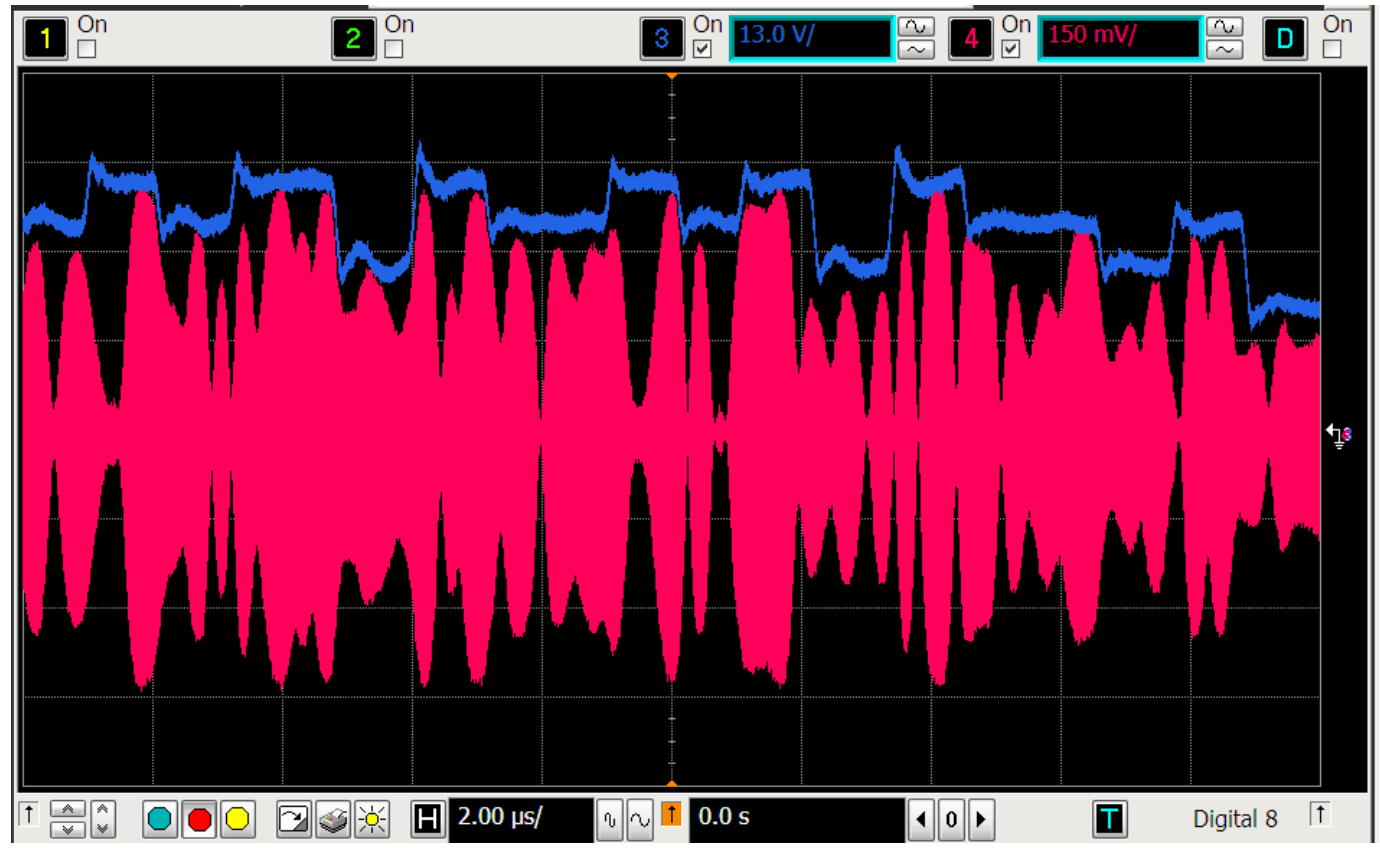

Figura 4.37 Formas de onda del transmisor ET a la salida del convertidor buck multifase (canal 1) y a la salida de RF (canal 4) para una señal 64QAM con un ancho de banda de RF de $4800 \mathrm{KHz}$. 
La linealidad del transmisor ET se puede ver más claramente en las medidas espectrales. Desde la Figura 4.38 hasta la Figura 4.42 se muestra el espectro de salida de RF del transmisor ET para diferentes anchos de banda de la señal de prueba. Las medidas de ACPR muestran niveles de linealidad

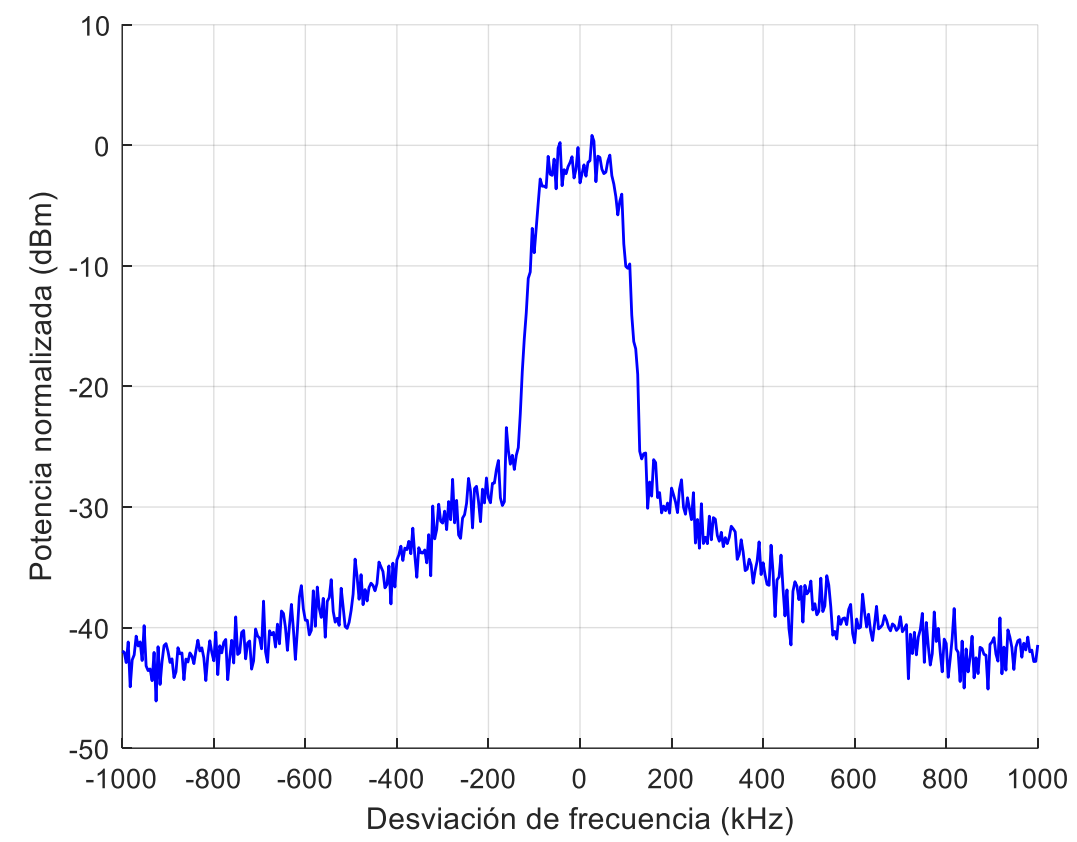

Figura 4.38 Espectro de salida normalizado del amplificador ET para una señal 64QAM con un ancho de banda de RF de $300 \mathrm{KHz}$.

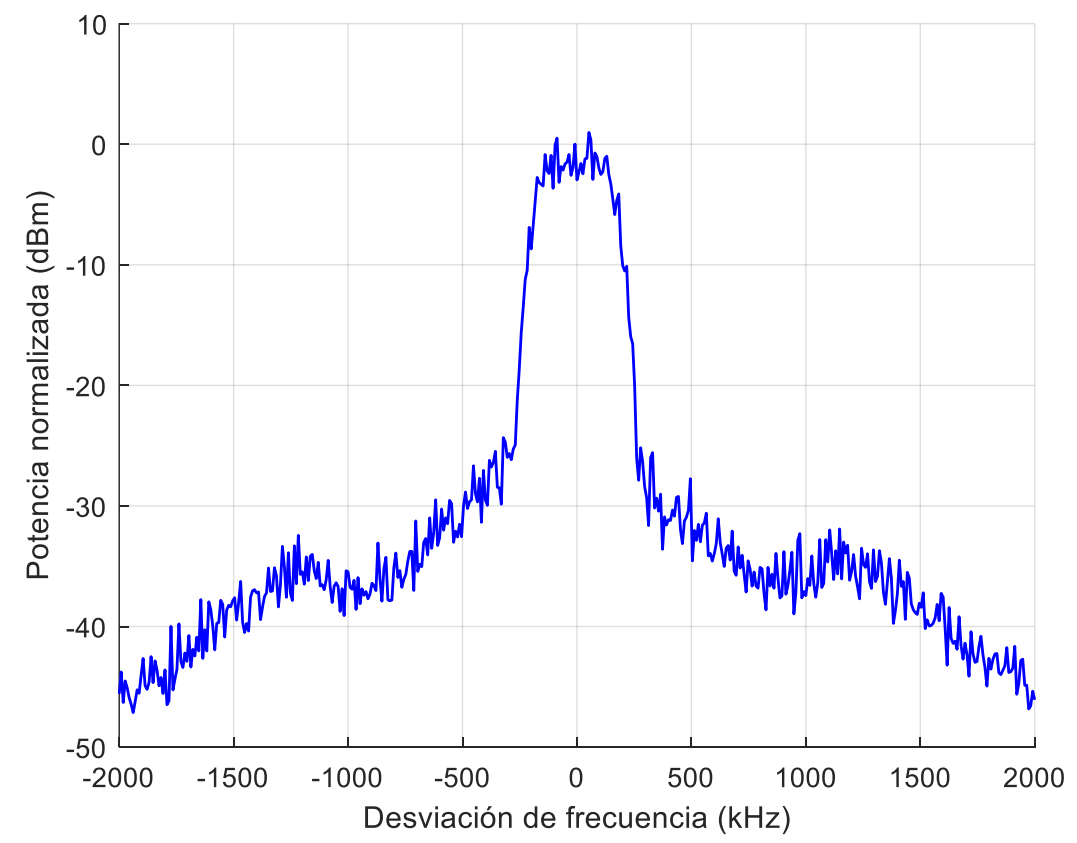

Figura 4.39 Espectro de salida normalizado del amplificador ET para una señal 64QAM con un ancho de banda de RF de $600 \mathrm{KHz}$. 


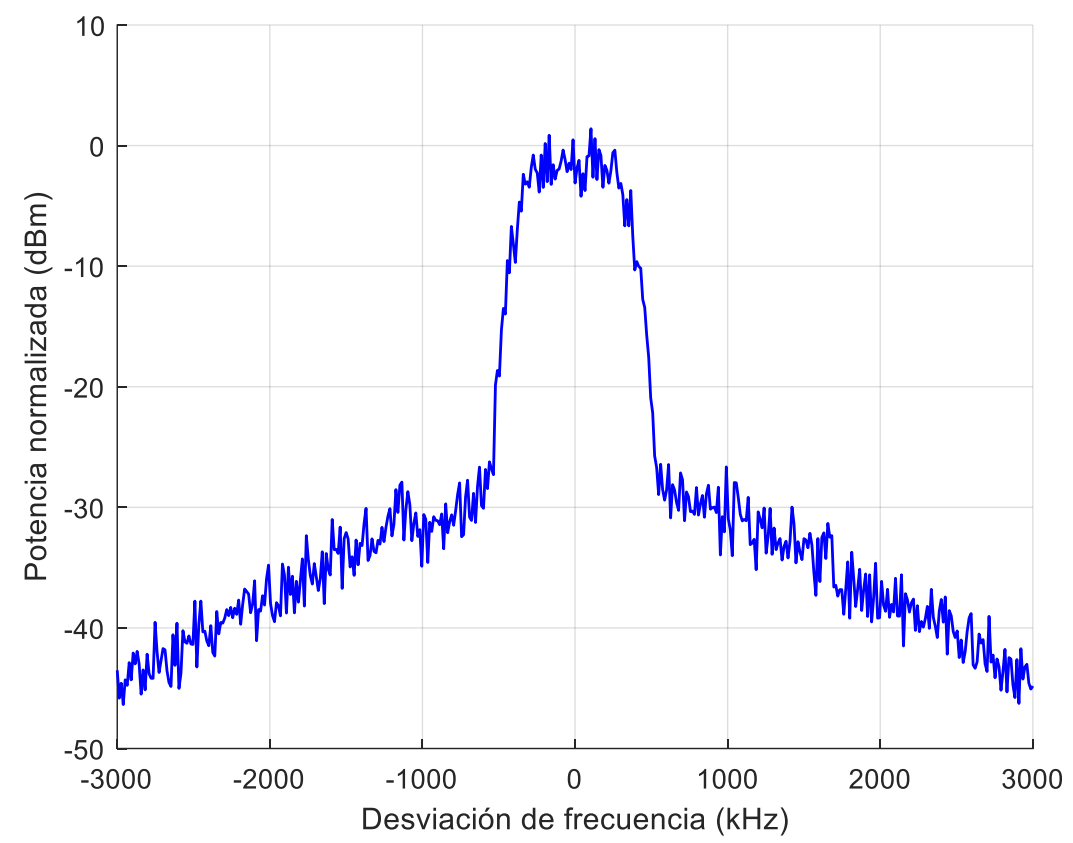

Figura 4.40 Espectro de salida normalizado del amplificador ET para una señal 64QAM con un ancho de banda de RF de $1200 \mathrm{KHz}$.

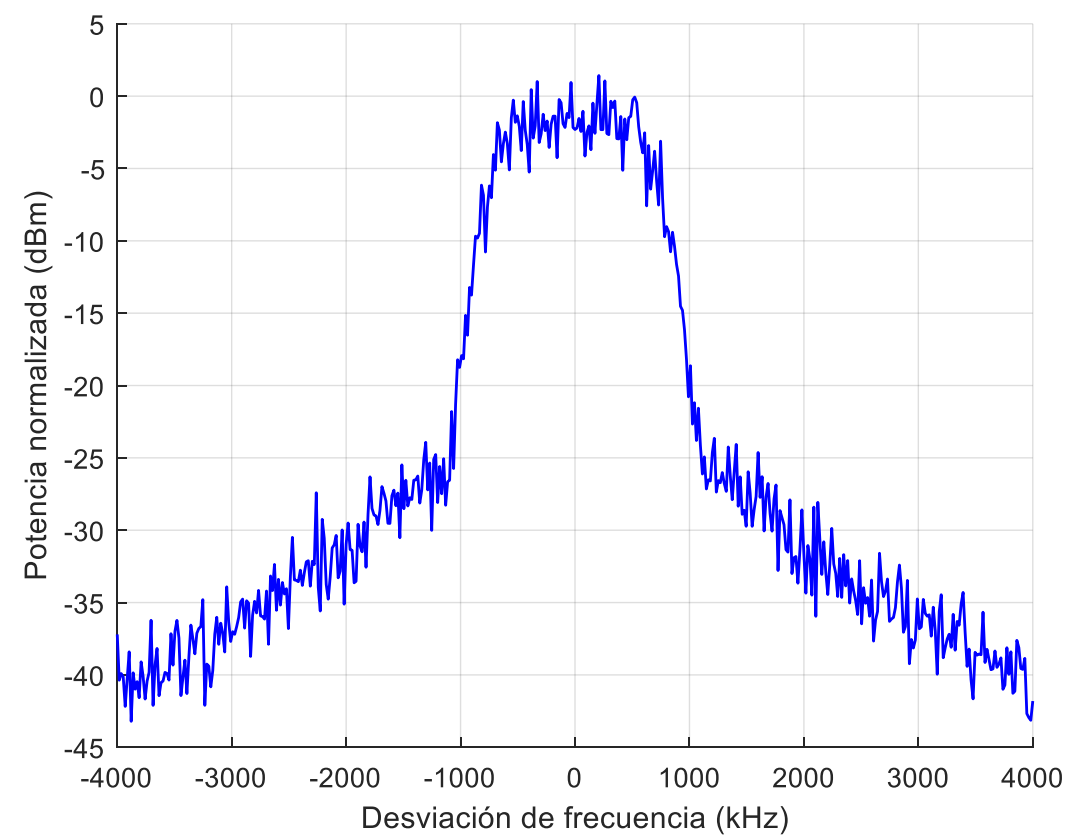

Figura 4.41 Espectro de salida normalizado del amplificador ET para una señal 64QAM con un ancho de banda de RF de $2400 \mathrm{KHz}$. 


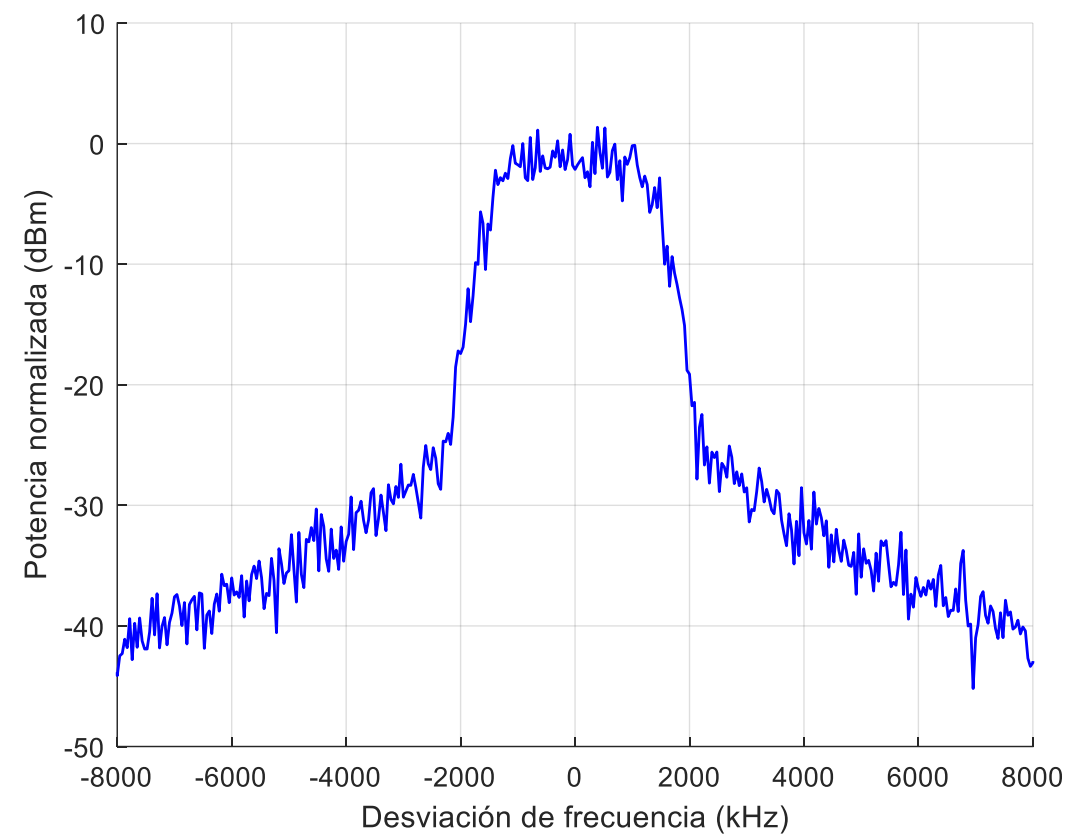

Figura 4.42 Espectro de salida normalizado del amplificador ET para una señal 64QAM con un ancho de banda de RF de $4800 \mathrm{KHz}$.

La Figura 4.43 resume las medidas de linealidad para los diferentes anchos de banda de la señal de prueba. Por ello, se han hallado los valores de ACPR a la derecha y la izquierda del canal. Como era de esperar, las medidas del ACPR se mantienen prácticamente constante para cada uno de los anchos de banda de la señal de prueba. En este caso, el convertidor multifase no afecta a la linealidad que puede conseguir el amplificador de RF trabajando en su zona lineal.

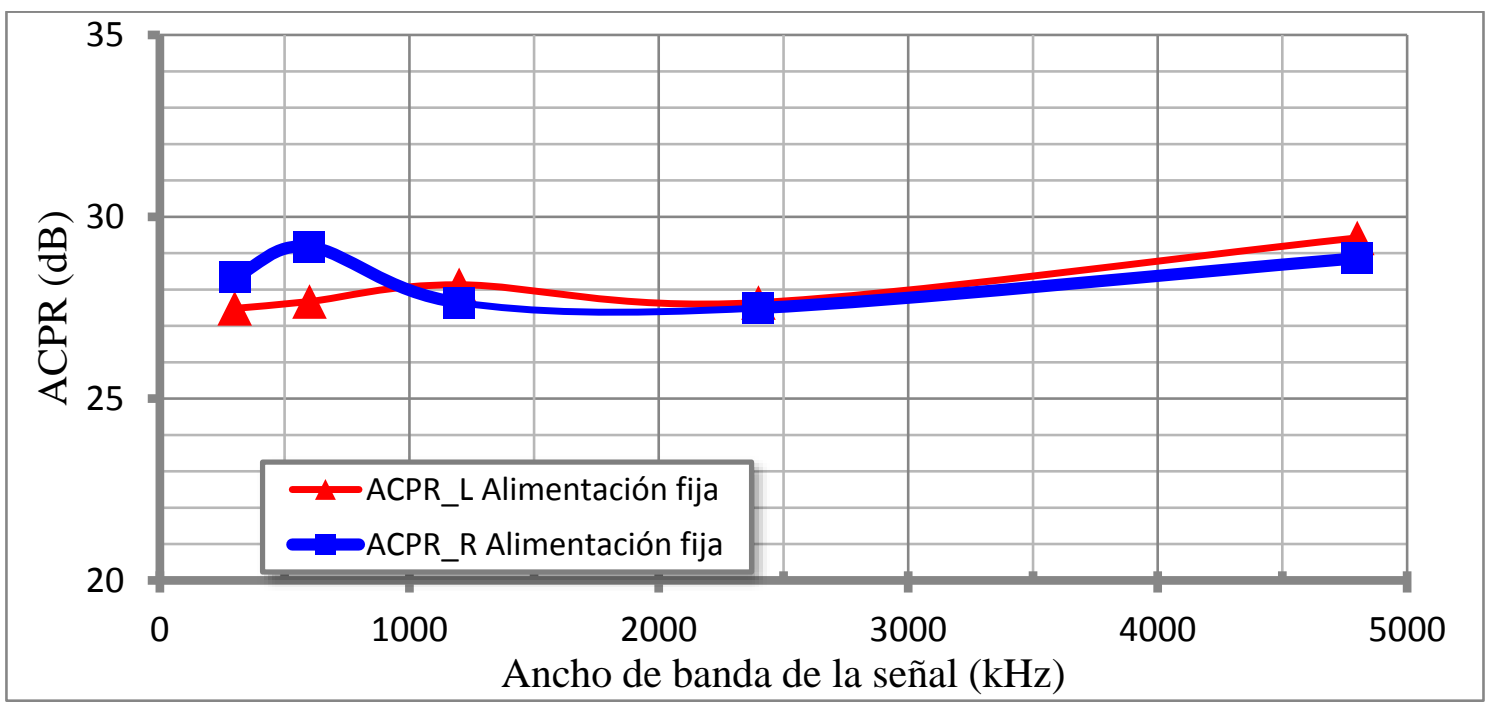

Figura 4.43 Resumen de la linealidad del sistema ET en función del ancho de banda de la señal. 
En cuando a las medidas de rendimiento, el convertidor multifase si juega un papel importante. En la Figura 4.44 se muestra el rendimiento del transmisor ET en función del ancho de banda. Se puede observar que a medida que aumenta el ancho de banda de la señal el rendimiento se va estabilizando. Esto se debe a que hay menos variaciones de nivel en el convertidor debido a las rápidas variaciones temporales de la envolvente. Si el ancho de banda de la señal es pequeño, el convertidor multifase es capaz de seguir fielmente la envolvente generando más transiciones entre niveles. Esto provoca que se reduzca el rendimiento que es capaz de ofrecer el convertidor y por lo tanto se degrada el rendimiento del transmisor ET.

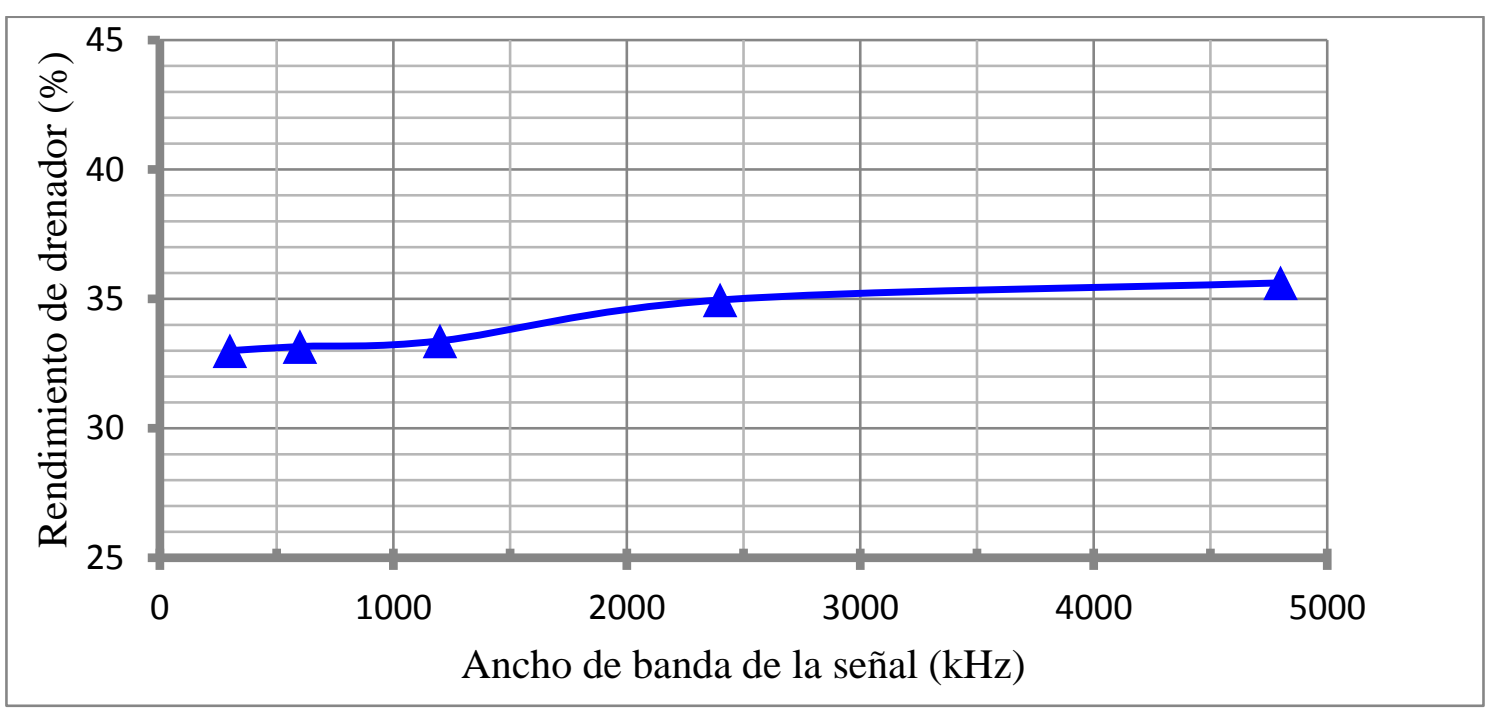

\section{Figura 4.44 Rendimiento de drenador del transmisor ET en función del ancho de banda de la señal de prueba.}

En conclusión, la integración del amplificador clase F trabajando en su zona lineal con el convertidor multifase para implementar un sistema ET ha mostrado las siguientes conclusiones:

- El sistema ET fue probado con una señal 64QAM para diferentes anchos de banda hasta los $4.8 \mathrm{MHz}$.

- Los niveles de linealidad permanecen prácticamente constantes para cada ancho de banda de la señal, lo que indica que el convertidor multifase no influye significativamente sobre esta característica.

- La aplicación de predistorsión digital en el sistema ET ayudaría a mejorar los niveles de linealidad. 
- Las transiciones entre niveles del convertidor multifase degradan el rendimiento del transmisor ET, sobre todo en anchos de banda estrechos donde los niveles de tensión siguen más de cerca la envolvente de la señal.

\subsubsection{Comparación EER y ET}

En los apartados anteriores se ha mostrado la implementación de dos arquitecturas de linealización empleando el mismo hardware. Esta situación es perfecta para poder comparar ambas arquitecturas y obtener las respectivas conclusiones.

En este apartado se va a realizar la comparación del transmisor EER donde el amplificador de envolvente consta del convertidor multifase asistido por un regulador lineal y del transmisor ET donde el amplificador de envolvente solo consta del convertidor multifase.

En general, la señal de envolvente con gran ancho de banda y alta PAPR degrada tanto el rendimiento como la linealidad del amplificador de envolvente. De ahí que puede introducir una importante distorsión en la salida de RF.

En cuanto a linealidad, se han obtenido características de linealidad ligeramente mejores con el transmisor EER. Esto se debe a que la combinación del convertidor multifase con el regulador lineal ofrece una envolvente más fiel a la original que en el caso del transmisor ET. En ambos sistemas, la linealidad podría ser mejorada usando predistorsión digital.

Respecto al rendimiento, es significativamente superior el rendimiento obtenido por el sistema ET para la misma señal de prueba. Esto indica que el regulador lineal que asiste al convertidor multifase introduce importantes pérdidas en el transmisor EER. Además, hay que tener en cuenta que el rendimiento obtenido por el transmisor ET es gracias al empleo del amplificador de RF en su zona lineal lo que reduce su rendimiento considerablemente.

Por todo ello, se puede concluir que la mejor integración del sistema se puede conseguir con una arquitectura ET a pesar de tener una menor linealidad, situación que podría ser corregida con predistorsión digital. Al contrario que la técnica EER, en la 
cual la envolvente de la salida de RF solo es controlada por el amplificador de envolvente, una arquitectura ET permite una mayor libertad en la elección de la forma de onda de alimentación.

\subsection{Transmisor lineal basado en amplificador clase $\mathbf{E}$}

Uno de los retos perseguidos en todo sistema de comunicación inalámbrico es reducir el consumo de las fuentes de alimentación de los sistemas. El empleo de amplificadores de potencia de alto rendimiento en modo de conmutación puede ayudar a cumplir este objetivo. Además, permiten mejorar la gestión del calor generado lo que puede ayudar a prescindir o reducir equipos de refrigeración y, de esta manera, reducir el tamaño de los transmisores de radiofrecuencia [116] [59]. Sin embargo, su naturaleza no lineal dificulta su incorporación en los sistemas de comunicaciones inalámbricas modernos.

Para salvar estas limitaciones de los amplificadores de potencia en modo de conmutación se han propuesto diversas técnicas de linealización capaces de mantener el comportamiento en alto rendimiento. Entre ellas, la técnica EER, también conocida como la técnica de Kahn [82], es una candidata excelente para sistemas que requieren un rendimiento en potencia alto en combinación con unas características de linealidad moderada [38].

El funcionamiento de la técnica original EER ya ha sido presentado en el Capítulo 2. Sin embargo, esta técnica presenta limitaciones debidas a la generación de productos de distorsión adicionales basados en una serie de fuentes de distorsión que causan crecimiento espectral fuera de banda. Entre estas fuentes de distorsión se pueden destacar [84]:

- La alineación temporal entre el camino de envolvente y de fase.

- Limitación en el ancho de banda en el proceso de separación de la envolvente y de la fase de la señal.

- Conversiones no lineales en las variaciones de envolvente del amplificador de RF provocadas por el amplificador de envolvente.

- Efecto feed-through [161]. 
En un sistema EER, la alineación temporal entre el camino de envolvente y de fase es una de las mayores fuentes de distorsión. El desalineamiento temporal genera reducciones de potencia y rendimiento, incremento del nivel de emisiones no deseadas fuera de banda y, en el caso de señales digitales una degradación en el EVM.

Otro punto de distorsión se produce en la separación de la información de fase y de envolvente. En el caso de que esta separación se realice de manera hardware a través de detectores de envolvente y limitadores, la limitación en ancho de banda de estos módulos de detección introducirá distorsión en el sistema.

Por otro lado, otro elemento importante en una arquitectura EER es el amplificador de envolvente. Una solución típica es usar un convertidor buck de alta velocidad. Algunos problemas asociados a esta solución son la alta frecuencia de conmutación necesaria y el alto orden del filtro de salida paso bajo necesario para permitir grandes anchos de banda de envolvente, lo que se traduce en distorsión a niveles de envolvente bajos. Además, incrementar el orden y la complejidad del filtro de salida con el fin de maximizar el ancho de banda [162] [163] implica que la forma de onda de salida sea más sensible a las variaciones de carga. Esta situación es especialmente importante en los cruces por cero (cuando la tensión de alimentación del amplificador de RF es cero) o a bajos niveles de envolvente, incrementando la distorsión en esta zona.

Un amplificador lineal puede ser combinado con un amplificador de envolvente para solventar estos problemas [164], pero la implementación se hace más compleja y el rendimiento del convertidor se degrada.

Y, por último, otro elemento de distorsión es el efecto feed-through. Este efecto fue descubierto en 1996 por Funk y Johnston [165] mientras implementaban la linealización de un amplificador clase E. Este efecto limita la distorsión por intermodulación (IMD) y contribuyó a un cambio de fase en el amplificador de RF causando un espectro asimétrico. El efecto feed-through resulta de la capacidad parásita del dispositivo activo entre puerta y drenador; además, el valor de esta capacidad es dependiente de la tensión. Cuando la tensión de drenador está por debajo de un determinado nivel, la señal de excitación de RF en la puerta comienza a filtrarse al drenador a través de la capacidad puerta-drenador $(\mathrm{Cgd})$ provocando distorsión de fase y de amplitud. 
Estas fuentes de distorsión pueden ser ligeramente corregidas combinando la técnica EER con otras técnicas como pueden ser predistorsión digital (Digital Predistortion, DPD) o la modulación de señal de excitación (Drive modulation, DM) y de esta manera ayudar a corregir estos elementos de distorsión con el fin de que la linealidad del sistema cumpla con las especificaciones requeridas por la aplicación del sistema.

En este apartado se muestra una técnica capaz de reducir estos productos de distorsión explicados anteriormente manteniendo los beneficios de la arquitectura EER. Esta técnica EER híbrida mantiene el comportamiento de un amplificador EER típico a altos niveles de envolvente y funciona como un amplificador lineal a niveles bajos de envolvente, presentando una impedancia de entrada constante en el puerto de alimentación lo que permite al modulador de envolvente trabajar linealmente. Además, con el fin de analizar la mejora del sistema híbrido, se realizará una comparación con una arquitectura EER convencional. El amplificador de RF empleado será el amplificador clase E subóptimo en banda L presentado en el capítulo 3.

\subsubsection{Amplificador de envolvente}

El amplificador de envolvente (Figura 4.45) utilizado en este apartado es un convertidor buck de dos fases de alta velocidad de conmutación [162]. Está fabricado con transistores LDMOS de RF de silicio controlado por un driver aislado de alta velocidad. Es capaz de entregar una potencia de salida máxima de $100 \mathrm{~W}$ sobre una carga de $5 \Omega$ alimentado a $28 \mathrm{~V}$, siendo el rendimiento medio típico de hasta el $88 \%$. El convertidor trabaja en lazo abierto, lo que requiere de una impedancia de carga relativamente constante para su correcto funcionamiento, situación donde la carga del amplificador de RF en clase E puede ser beneficioso. 


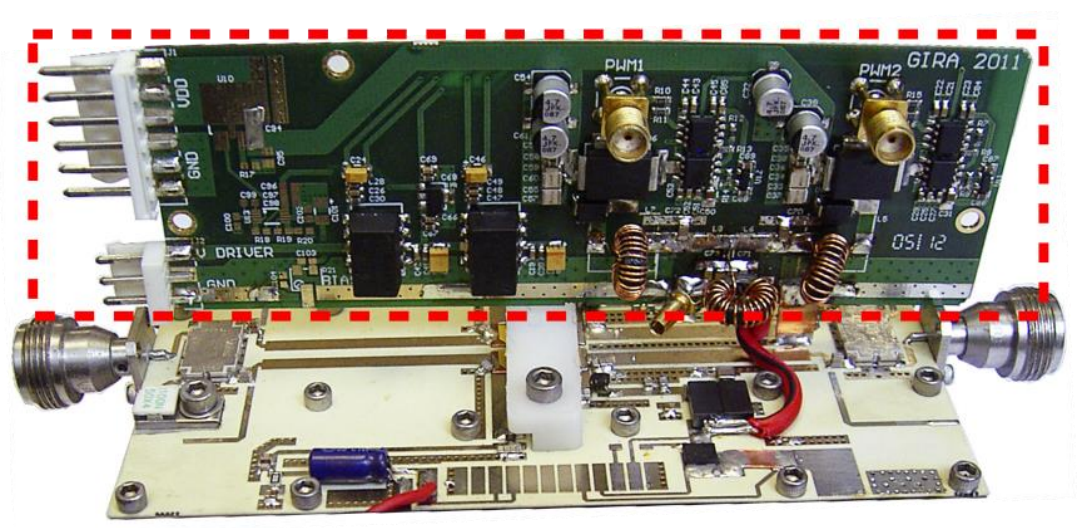

Figura 4.45 Prototipo amplificador de envolvente.

Entre otras ventajas, la topología multifase favorece la eliminación de la frecuencia de conmutación del convertidor; el resto de las componentes no deseadas a la salida del modulador son atenuadas mediante la incorporación de un filtro paso bajo de cuarto orden tipo Legendre-Papoulis diseñado específicamente para permitir el mayor ancho de banda de la señal procedente del amplificador de envolvente mientras atenúa simultáneamente las componentes no deseadas en su banda atenuada [166].

El amplificador de envolvente es capaz de trabajar con frecuencias de conmutación de hasta $10 \mathrm{MHz}$. Altas frecuencias de conmutación causan una reducción de rendimiento significativa debido a las perdidas por conmutación. De ahí que elegir el semiconductor adecuado sea una tarea importante. En ese caso, se han empleado dispositivos de silicio como transistores de potencia. Estos dispositivos pueden ser usados hasta altas frecuencias de RF debido a su baja capacidad parásita y a sus rápidos tiempos de conmutación.

\subsubsection{Transmisor EER}

A continuación, el prototipo de un transmisor EER ha sido implementado con el amplificador de envolvente presentado anteriormente y el amplificador clase E en banda L mostrado en el capítulo anterior. Este prototipo sirvió para verificar las limitaciones de esta arquitectura e investigar posibles soluciones.

La técnica EER propuesta inicialmente por Kahn [82] emplea un nivel fijo de excitación asegurando la saturación del amplificador de RF siempre y cuando no se trabaje a niveles bajos de envolvente. Esto proporciona una buena linealidad, pero 
presenta altos niveles medios de potencia de excitación y el efecto feed-through, sobre todo bajos niveles de señal, lo que reduce el rendimiento.

En 1999, Raab propuso una técnica de modulación de driver para amplificadores EER que solventaba estos inconvenientes a niveles bajos de señal (Figura 4.46) [83]. Consiste en modular completamente la excitación de tal manera que se reduce la potencia media de excitación, pero en cambio se genera distorsión no lineal en el amplificador de RF y además modifica el modo de funcionamiento del amplificador de envolvente a niveles bajos de envolvente, es decir, se modifica la carga vista por el amplificador de envolvente. La modulación completa de la excitación es efectiva en la reducción del feed-through. Sin embargo, la ganancia de los dispositivos de RF se reduce a niveles de alimentación bajos, debido a que los dispositivos entran en la zona de corte cuando las tensiones de drenador y puerta son bajas.

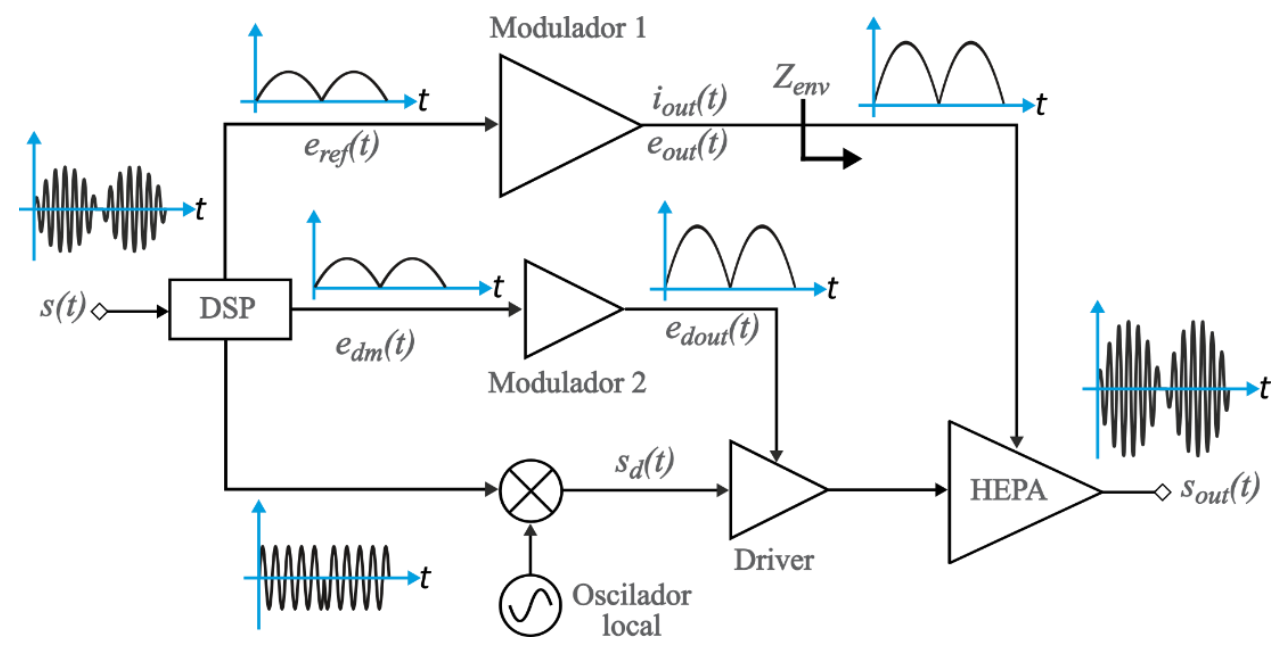

Figura 4.46. Esquema de un sistema EER con modulación de excitación completa.

Una solución para reducir la potencia media de excitación son degradar la linealidad fue propuesta por Raab [167] y se muestra en la Figura 4.47. Consiste en modular parcialmente la excitación del amplificador de RF añadiendo a la señal de envolvente un nivel mínimo de excitación E0. Este nivel mínimo de excitación E0 se determina empíricamente para asegurar la saturación del amplificador de RF a bajos niveles de envolvente. Esta técnica presenta como ventaja que maximiza el rendimiento del driver y, además, elimina los desvanecimientos de la señal de RF de salida a bajos niveles de señal. 


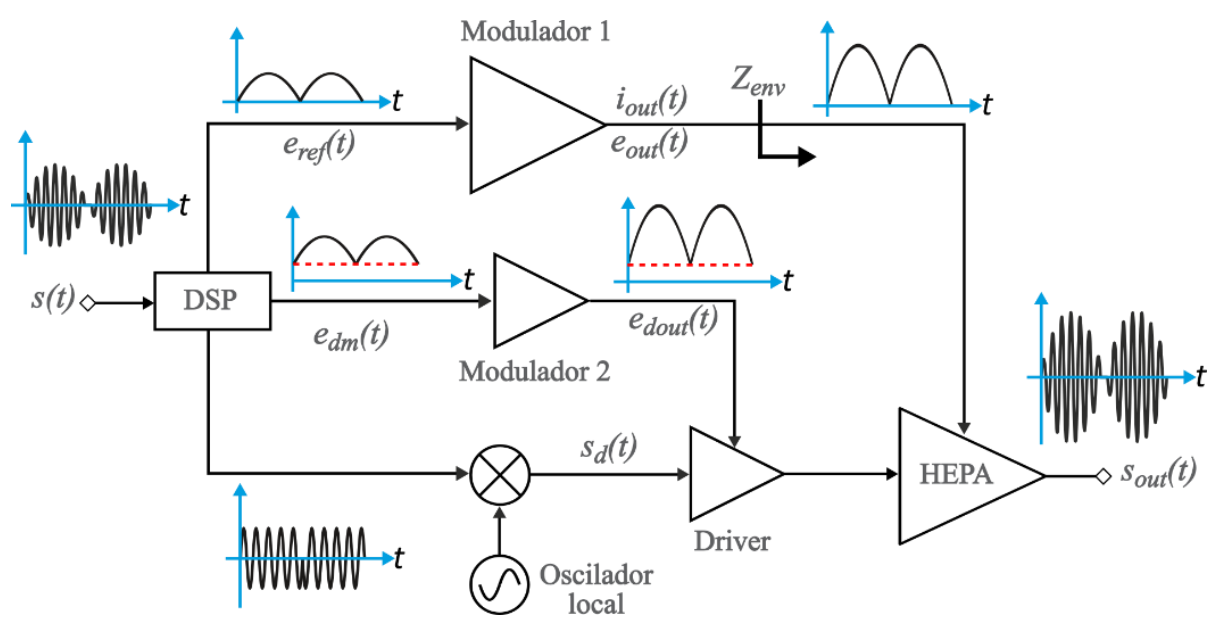

Figura 4.47 Esquema de un sistema EER con modulación parcial de excitación.

La técnica de modulación parcial de driver requiere de un nivel de excitación mínimo para paliar los inconvenientes de la arquitectura EER. Es este trabajo se propone un sistema que no requiere ese nivel de excitación mínimo y también es capaz de mitigar los inconvenientes de la arquitectura EER.

La técnica propuesta combina la técnica de modulación de excitación del amplificador de RF con la adaptación de la señal de referencia de envolvente a niveles bajos de señal. Ello permite mejorar el funcionamiento del sistema a bajos niveles de excitación incrementando el rango dinámico del amplificador y reduciendo el feedthrough, además de mejorar la linealidad del sistema.

\subsubsection{Propuesta técnica EER híbrida}

Normalmente, el nivel de excitación del amplificador de RF en un sistema EER no puede ser cero debido a limitaciones técnicas en el amplificador de envolvente. La carga presentada en el puerto de alimentación del amplificador de RF se incrementa en los instantes donde la señal de RF presenta cortes por cero o a niveles muy bajos de envolvente causando una subida o baja brusca de la tensión de salida del amplificador de envolvente.

El sistema EER híbrido presentado en este trabajo acepta condiciones de excitación nulas o muy bajas gracias al funcionamiento en modo mixto del amplificador de RF. El esquema de este sistema propuesto se muestra en la Figura 4.48. 


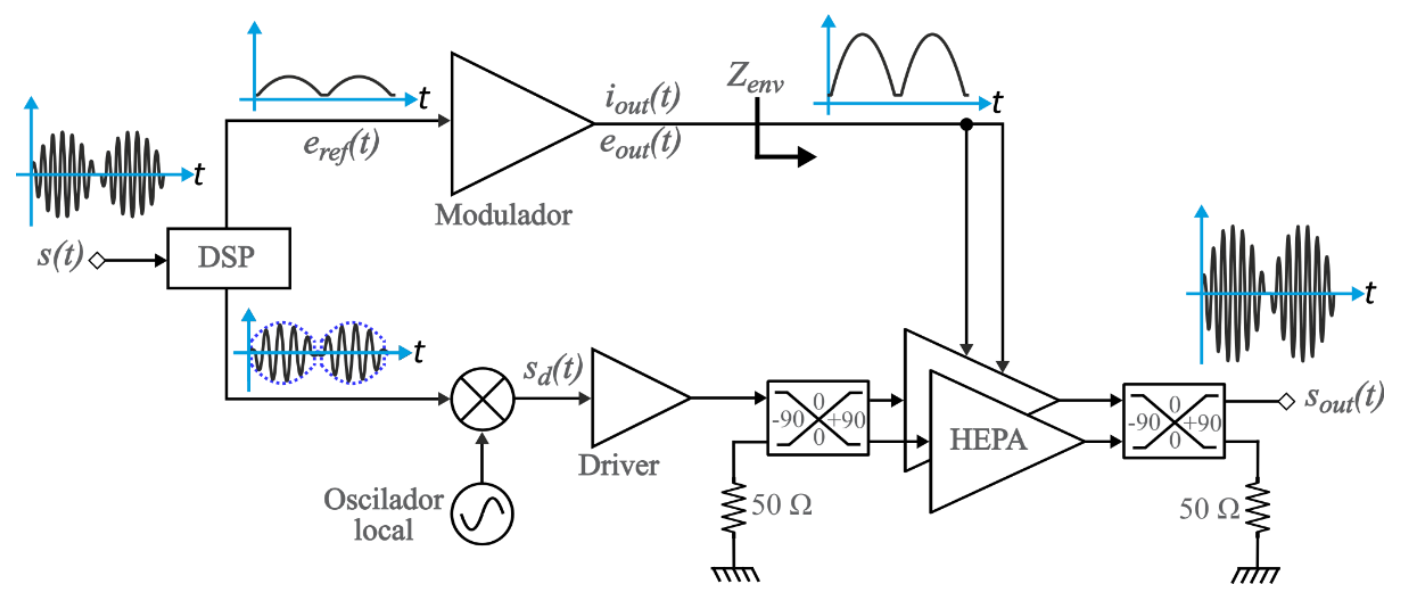

Figura 4.48 Esquema del sistema EER híbrido propuesto.

Como se puede comprobar es el mismo esquema que un sistema EER tradicional donde cambian las formas de onda de entrada al amplificador de envolvente y al driver de RF. Estas formas de onda se generan con relativa facilidad a través de técnicas de procesado digital de la señal que son menos costosas y más flexibles comparadas con el uso de circuitos electrónicos.

Con el fin de solventar los problemas de distorsión en un transmisor EER procedentes del amplificador de envolvente conmutado a bajos niveles de señal, se optó por hacer trabajar al amplificador de RF en dos modos de funcionamiento diferentes: el amplificador trabaja en modo de conmutación (clase E subóptima) a altos niveles de envolvente y en modo lineal (clase A) a bajos niveles de envolvente, como se describe en [168].

La señal de RF de entrada al sistema $\mathrm{s}(\mathrm{t})$ está compuesta por la información de envolvente e(t) y por la información de fase $\varphi(t)$, definida en la Ecuación 4.2.

$$
s(t)=e(t) \cdot \exp (j \varphi(t))
$$

A partir de técnicas de procesado digital de la señal se puede generar la señal de excitación del amplificador de RF normalizada como:

$$
\overline{s_{d}}(t)=\overline{e_{d}}(t) \cdot \sin \left(\omega_{c} t+\varphi(t)\right)
$$

donde $\omega_{c}$ es la pulsación de la frecuencia portadora, $\overline{e_{d}}(t)$ es la envolvente normalizada de la señal de excitación de RF y $e_{\max }$ es la amplitud máxima de la información de envolvente.

$\overline{e_{d}}(t)$ que queda definida en función de la zona de trabajo del amplificador de $\mathrm{RF}$, tal y como se muestra en la Ecuación 4.4. A niveles de envolvente por encima de un 
nivel $E_{k}$ fijado previamente, la envolvente de la señal de excitación será la envolvente de referencia con un nivel de excitación adicional $E_{0}$, que hace que el amplificador de RF entre siempre en modo de saturación y ajusta la ganancia entre el modo lineal y el modo conmutado. En este caso, la impedancia de entrada en el puerto de alimentación del amplificador de RF $z_{\text {env }}(t)$ se mantiene constante. Cuando la envolvente es inferior a $E_{k}$, la envolvente de la señal de excitación es la misma que la de la señal de envolvente original.

$$
\overline{e_{d}}(t)= \begin{cases}\frac{E_{0}+e(t)}{e_{\max }+E_{0}} & \text { si } e(t)>E_{k} \\ \frac{e(t)}{e_{\max }+E_{0}} & \text { si } e(t) \leq E_{k}\end{cases}
$$

La envolvente de entrada del amplificador de envolvente $e_{r e f}(t)$ también es definida en función de la zona de trabajo como se puede ver en la Ecuación 4.5. Si la envolvente de la señal es mayor que $E_{k}$, la envolvente de referencia $e_{\text {ref }}(t)$ se corresponde con la envolvente de la señal $e(t)$. En cambio, a niveles de envolvente iguales o inferiores a $E_{k}$, la envolvente de referencia es constante de valor $E_{K}$. De esta manera, el amplificador trabaja en la zona lineal proporcionando alta linealidad a niveles bajos de señal y también $z_{\text {env }}(t)$ constante.

$$
e_{\text {ref }}(t)= \begin{cases}e(t) & \text { si } e(t)>E_{k} \\ E_{k} & \text { si } e(t) \leq E_{k}\end{cases}
$$

La envolvente de salida del amplificador de envolvente $e_{\text {out }}(t)$ básicamente es la envolvente de referencia multiplicada por la ganancia del amplificador de envolvente GEA, tal y como se define en la Ecuación 4.6.

$$
e_{\text {out }}(t)=G_{E A \cdot} \cdot e_{\text {ref }}(t)
$$

Combinando ambos modos de funcionamiento se obtiene una señal de salida $s_{\text {out }}(t)$ que idealmente es una copia exacta de la señal de entrada pero amplificada con la ganancia del sistema EER GEeR, tal y como se muestra en la Ecuación 4.7.

$$
s_{\text {out }}(t)=G_{E E R} \cdot e(t) \cdot \sin \left(\omega_{c} t+\varphi(t)\right)
$$

La Figura 4.49 muestra las formas de onda medidas normalizadas de la tensión de salida del amplificador de envolvente $e_{\text {out }}(t)$, de la corriente de salida del amplificador de envolvente $i_{\text {out }}(t)$ y de la tensión de excitación de entrada del amplificador de RF $\overline{S_{d}}(t)$ y la impedancia de puerto de envolvente $Z_{e n v}$ instantánea para una señal de prueba de dos tonos aplicada al sistema EER propuesto. $e_{\text {out }}(t)$ como $i_{\text {out }}(t)$ siguen la envolvente de la señal mientras se mantenga por encima del valor $E_{k}$ funcionando como un sistema EER normal, pero serán constantes cuando la envolvente de la señal sea menor o igual que $E_{k}$ (lo que se corresponde con niveles de excitación 
bajos) trabajando en clase A. La forma de onda de excitación de entrada $\overline{S_{d}}(t)$ se caracteriza por mantener saturado al amplificador de RF en la zona de envolventes altas añadiendo un nivel de excitación extra $E_{0}$, pero cuando el nivel de envolvente está por debajo de $E_{k}$ la señal de excitación se modula con el fin de igualar la ganancia en potencia en la zona conmutada con la ganancia en potencia de la zona lineal.

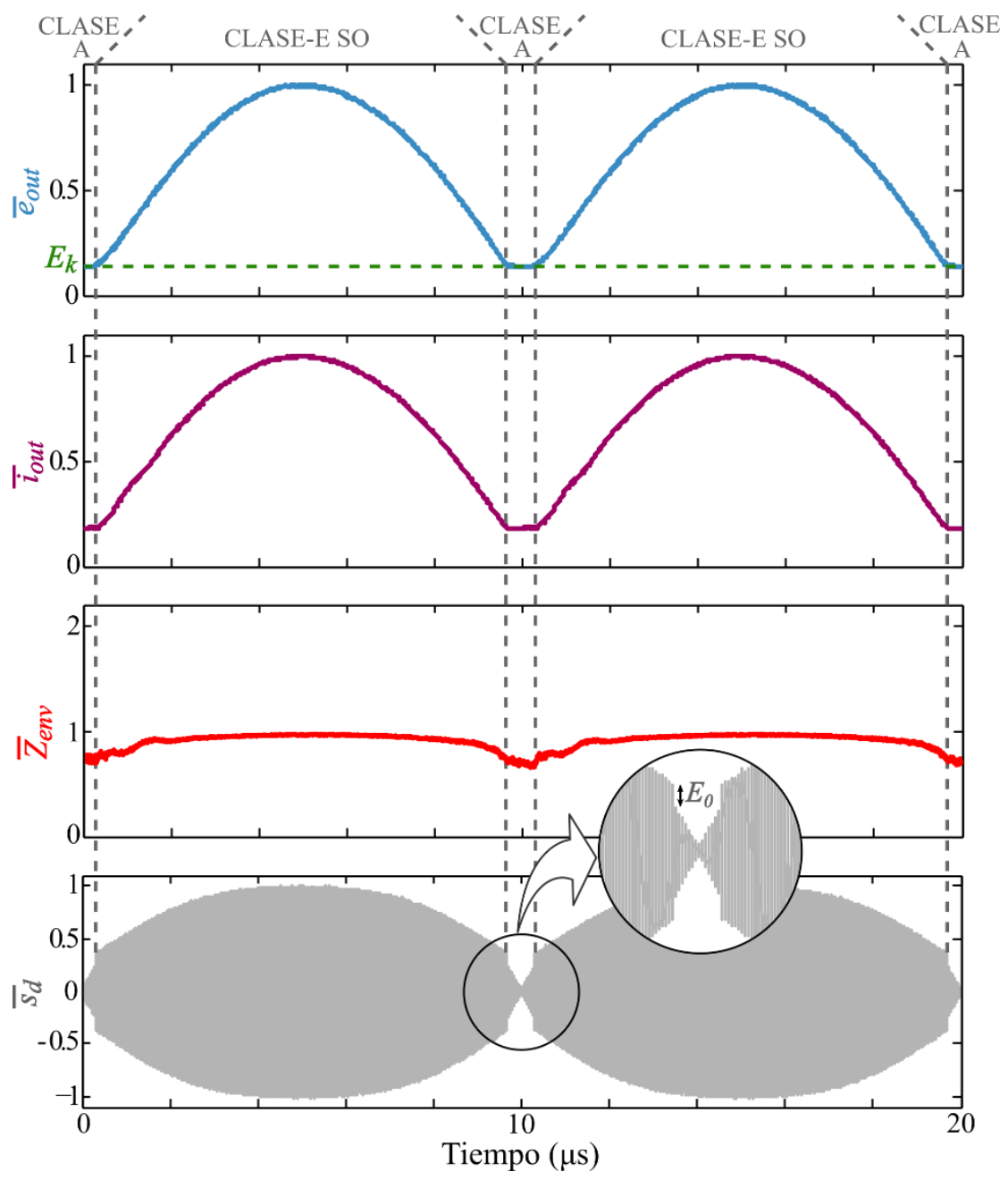

Figura 4.49 Formas de onda medidas del sistema EER híbrido para una señal de prueba de dos tonos.

Recordar que la aplicación de la modulación de driver propuesta por Raab [83] requiere de un nivel de excitación alto a la entrada del amplificador de RF. Ello conlleva que, a niveles bajos en envolvente, se malgaste una cantidad importante de potencia de excitación que conlleva la reducción de la ganancia en potencia y de la PAE del sistema. Además, produce el efecto feed-through debido a las capacidades parásitas, haciendo que se incremente la distorsión por intermodulación. 
La técnica EER híbrida propuesta en [168] minimiza los productos de intermodulación a la vez que incrementa la PAE y ganancia en potencia del sistema. La Figura 4.50 presentada en [168] muestra la simulación de la mejora de los productos de intemodulación de tercer y quinto orden con respecto a la técnica de modulación de excitación propuesta por RAAB [83] para diferentes valores de $E_{k}$ y $E_{0}$. Destacar que un valor $E_{k}=0$ indica un funcionamiento completo en modo de conmutación y que un valor $E_{k}=1$ se corresponde con un modo de funcionamiento completamente lineal [168]. También se presenta el rendimiento de drenador del sistema en función de la anchura de la región donde el amplificador de RF trabaja en su zona lineal, o lo que es lo mismo, en función del valor de $E_{k}$. Los productos de intermodulación son óptimos para ciertos valores de $E_{k}$, pero valores de $E_{k}$ bajos proporcionan un rendimiento de drenador mayor debido a que la zona de funcionamiento lineal es menor.

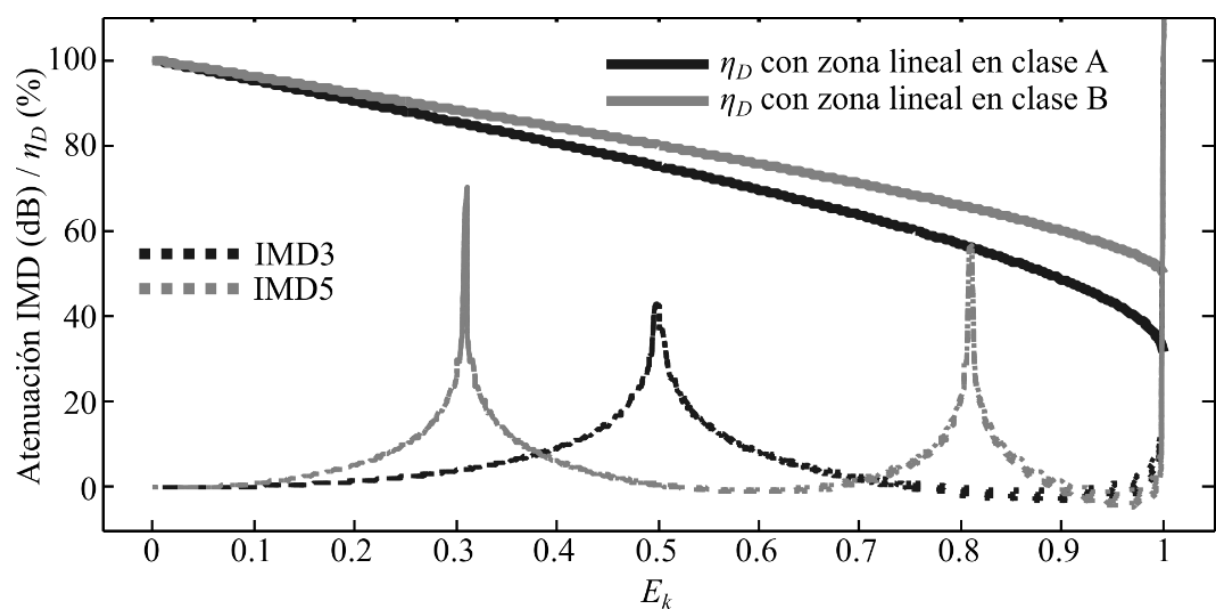

Figura 4.50 Productos de intermodulación normalizados con respecto a la técnica de modulación de excitación de Raab [83] $\left(E_{k}=0\right)$ para diferentes valores de $E_{0}$ y rendimiento de drenador de la técnica de modulación propuesta en este trabajo.

Finalmente, este amplificador necesita implementar técnicas complementarias tal como predistorsión digital con el objetivo de alcanzar niveles de linealidad parecidos a los conseguidos con amplificadores clase $\mathrm{AB}$ con las mismas características de potencia y frecuencia. El método aplicado para obtener las curvas de predistorsión es el mismo que se explicó en el Capítulo 3 para el cálculo de las curvas de predistorsión del amplificador clase E en banda L.

A modo de recordatorio, la técnica empleada consiste en alimentar al amplificador de potencia conmutado con una señal triangular que abarque todo el rango 
dinámico de tensiones, es decir, desde 0 hasta $28 \mathrm{~V}$. En este caso, esta señal triangular será la envolvente de referencia del amplificador de envolvente.

Aplicando este método al amplificador se obtiene la señal de salida en el dominio del tiempo que se muestra en la Figura 4.51. Como se puede observar, a tensiones de alimentación muy próximas a $0 \mathrm{~V}$ el transistor entra en corte. Esto es una característica que se suele dar en los sistemas EER en las zonas donde las señales a amplificar presentan cortes por $0 \mathrm{~V}$.

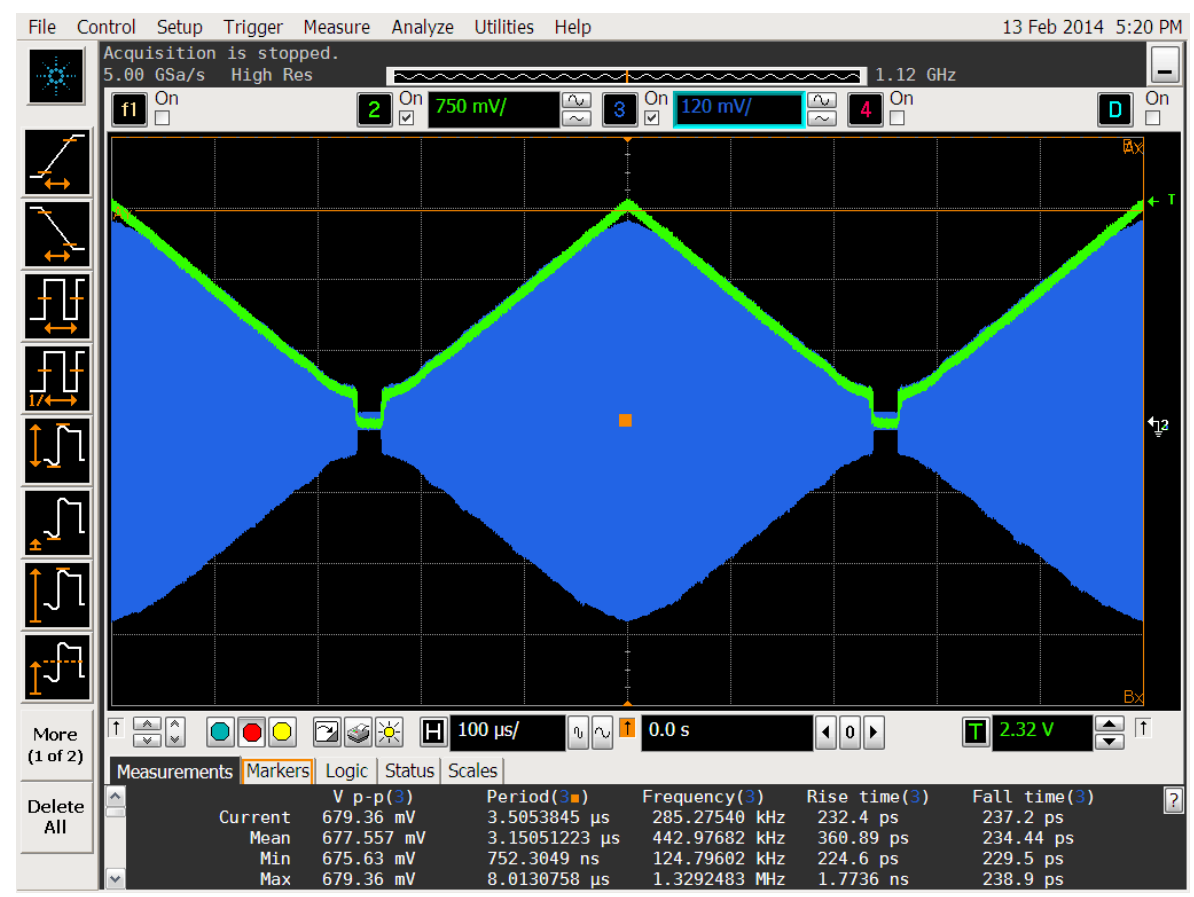

Figura 4.51. Caracterización de la distorsión del transmisor a partir de una señal triangular de baja frecuencia.

Esta señal de salida puede ser demodulada bien empleado un demodulador analógico o bien capturando la traza de la señal temporal para posteriormente ser demodula por software, por ejemplo, usando Matlab. Ambos métodos fueron implementados y se obtuvieron resultados prácticamente idénticos. En la Figura 4.52 se muestra las señales de salida demoduladas analógicamente usando el analizador vectorial de señal (VXA) instalado en el N9020A de Agilent. 
Ch1 AM Main Time

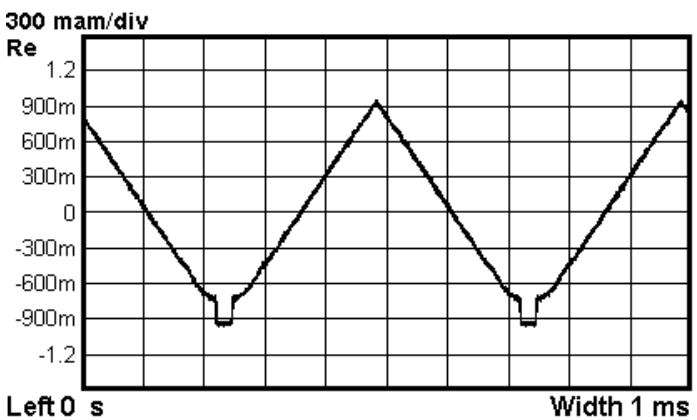

Ch1 PM Main Time

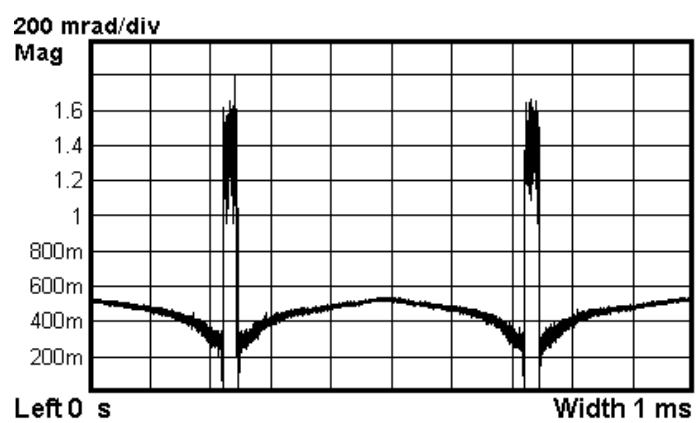

Figura 4.52. Demodulación en amplitud (izq) y en fase (drch) de la señal triangular aplicada al transmisor siendo la frecuencia portadora $1300 \mathrm{MHz}$.

La conversión $\mathrm{V}_{\mathrm{dd}}-\mathrm{PM}$ tiene un efecto significativo a niveles altos de envolvente (cuando el amplificador de RF trabaja en modo de conmutación). A niveles bajos de envolvente, en la región $e(t) \leq E_{k}$, donde el amplificador trabaja en su zona lineal, tanto la conversión $\mathrm{V}_{\mathrm{dd}}-\mathrm{AM}$ (Figura 4.53) como $\mathrm{V}_{\mathrm{dd}} \mathrm{PM}$ (Figura 4.54) deben ser corregidas [169] [170]. En este trabajo no se han tenido en cuenta los efectos memoria que se puedan producir.

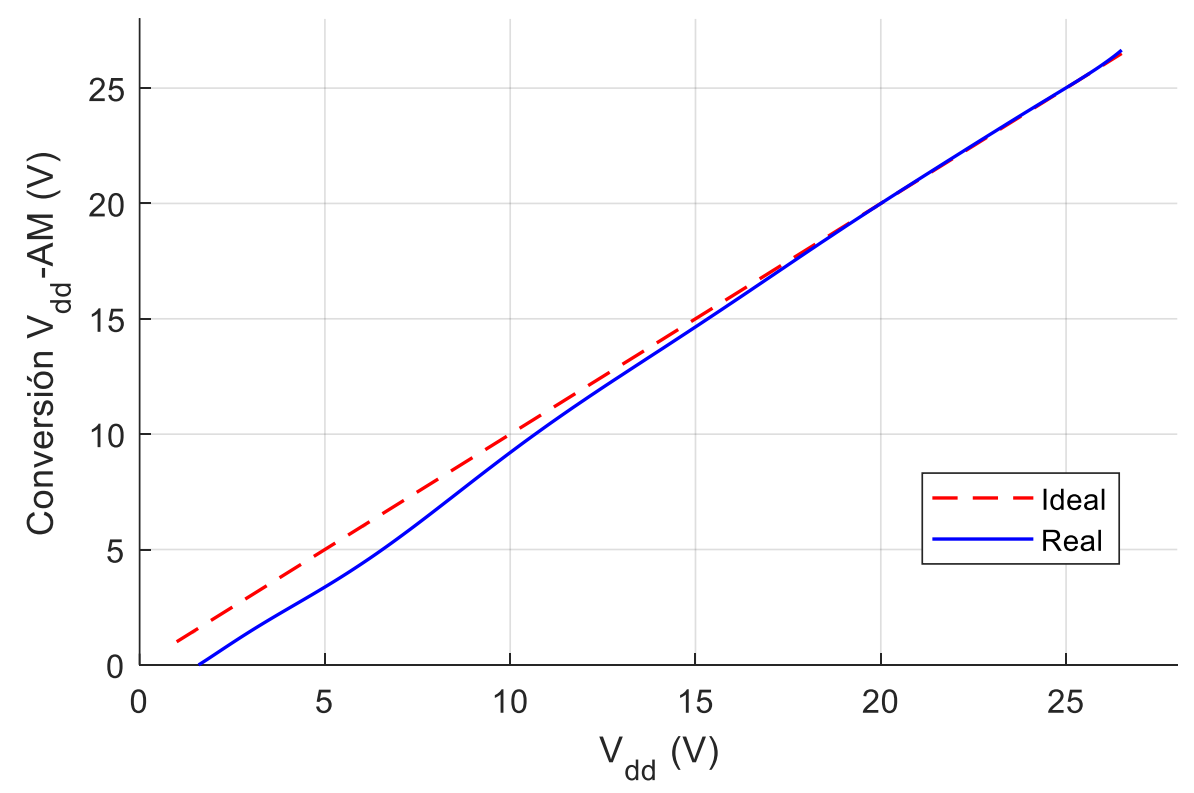

Figura 4.53 Conversión Vdd-AM del sistema EER a 1200 MHz. 


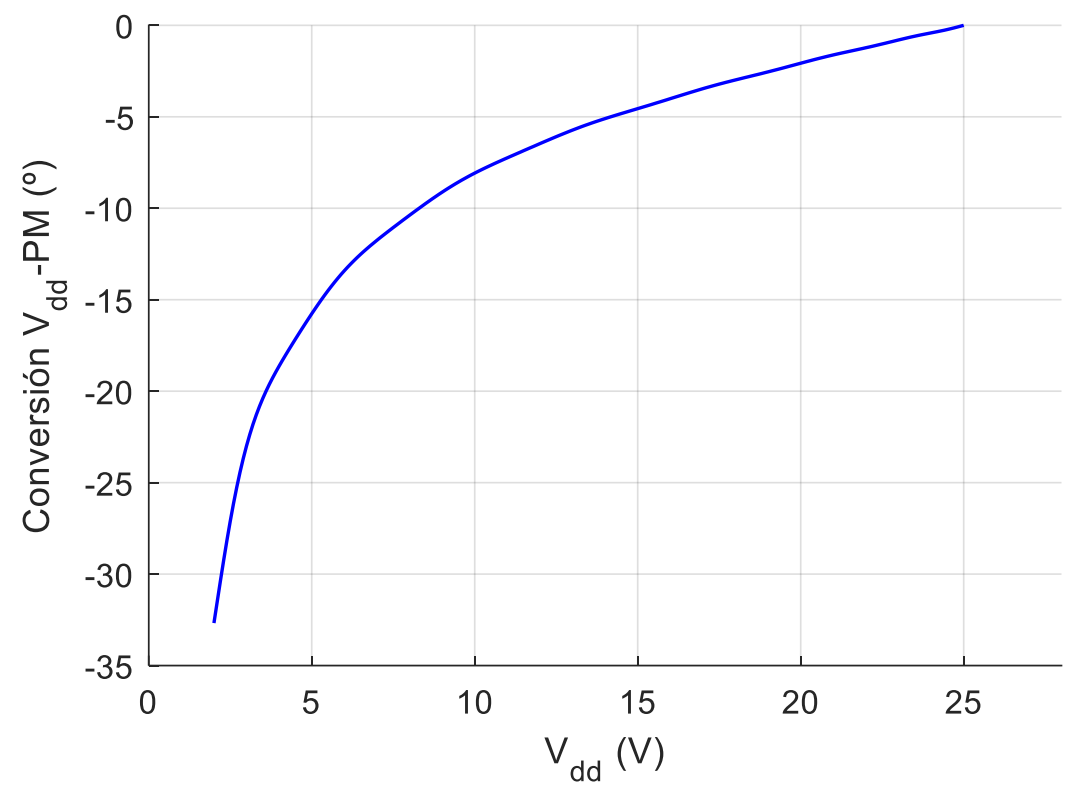

Figura 4.54 Conversión $\mathrm{V}_{\mathrm{dd}}-\mathrm{PM}$ del sistema EER a $1200 \mathrm{MHz}$.

\subsubsection{Prototipo sistema EER}

La Figura 4.55 muestra una fotografía del prototipo montado. En ella se puede distinguir el amplificador de envolvente que alimenta al amplificador de potencia de RF clase E subóptimo.

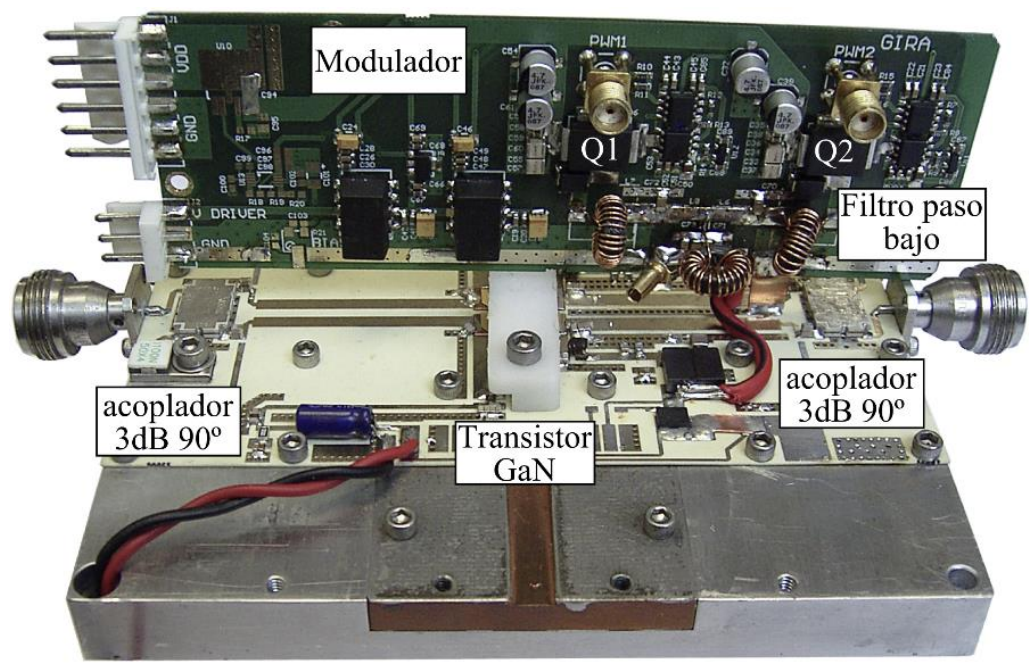

Figura 4.55 Fotografía del prototipo de transmisor EER.

Con el fin de caracterizar el transmisor, se realizaron medidas de linealidad y de rendimiento. Una de las señales de prueba que mejor caracteriza este tipo de 
arquitecturas de linealización es una señal de dos tonos. La principal característica de esta señal es que presenta cruces por cero, lo que permite poner a prueba las limitaciones e inconvenientes de trabajar a bajos niveles de tensión con un sistema EER. Además, se realizó la comparación del sistema EER híbrido propuesto con respecto al sistema EER convencional propuesto por Kahn, con la peculiaridad de que en ambas implementaciones fue aplicada la predistorsión digital en banda estrecha.

Las características de la señal de prueba de dos tonos es la siguiente:

- Frecuencia del tono de $50 \mathrm{kHz}(100 \mathrm{kHz}$ de ancho de banda de RF)

- Frecuencia de portadora $1200 \mathrm{MHz}$.

- Tensión de alimentación $28 \mathrm{~V}$.

- Potencia de salida media de $47.8 \mathrm{dBm}$.

Las señales de envolvente y de excitación utilizadas por estas dos arquitecturas han sido generadas a través de Matlab y posteriormente cargadas a un generador de funciones arbitrario y a un generador de señal vectorial. La señal de salida será capturada con un analizador de espectros, un analizador de señal y un osciloscopio digital con el fin de obtener la traza de la señal y posteriormente analizarla usando Matlab.

Un punto importante en el sistema es el filtro de reconstrucción. En [162] se hace un estudio exhaustivo de diferentes tipos de filtros y diferentes parámetros para el diseño de este filtro de reconstrucción. Para esta prueba de dos tonos, debido a que el tono es de baja frecuencia, se ha implementado un filtro paso bajo a la salida del convertidor de envolvente con las siguientes características:

- Tipo: Legendre

- Orden: 4

- Frecuencia de corte: $1 \mathrm{MHz}$

- Resistencia de fuente: $0 \Omega$.

- Resistencia de fuente: $5 \Omega$.

En la Figura 4.56 se muestran los valores del filtro diseñado y la respuesta en frecuencia del mismo. Es necesario comentar que la frecuencia de conmutación del convertidor es de $4 \mathrm{MHz}$, por lo que utilizando este filtro siendo la frecuencia de corte 
1 Mhz se obtiene una atenuación de más de $50 \mathrm{~dB}$. Además, la elección de una frecuencia de corte 10 veces mayor que el ancho de banda de RF de la señal de prueba hace que el filtro presente un retardo de grupo constante.

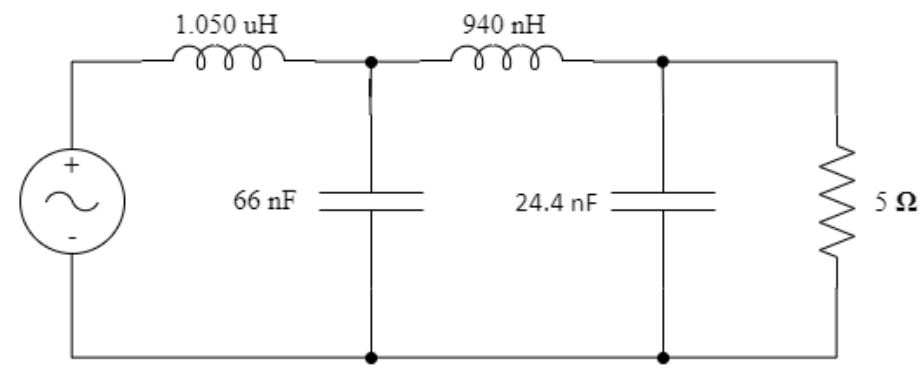

(a)

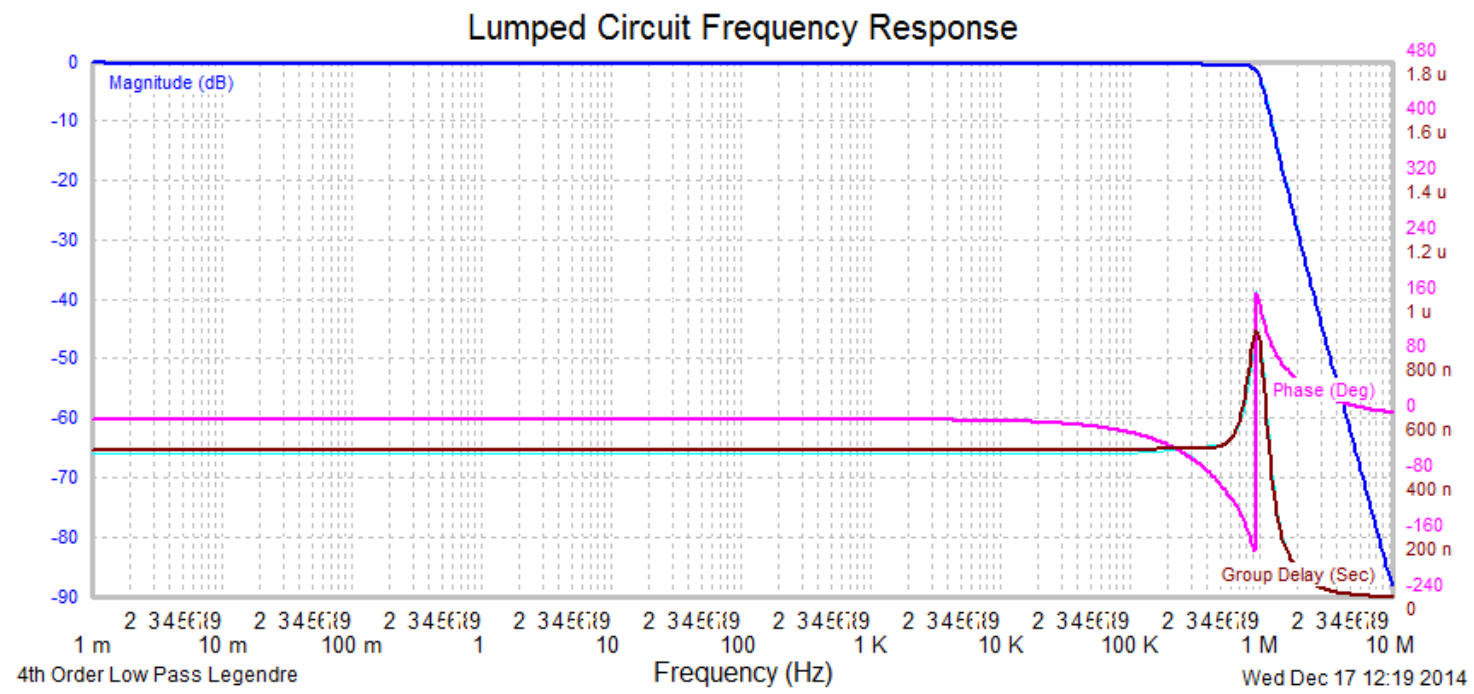

(b)

Figura 4.56. Filtro paso bajo diseñado (a) y respuesta en frecuencia (b) empleado en la prueba de dos tonos.

En las Figura 4.57 y Figura 4.58 se puede ver la forma de onda medida con el osciloscopio digital tanto para el caso de un sistema EER convencional como para el sistema EER híbrido propuesto, respectivamente. En ella se puede observar que en el caso de un sistema EER convencional la forma de onda de la tensión de salida del amplificador de envolvente presenta un desvanecimiento a partir de un determinado nivel de envolvente bajo, lo que provoca una fuerte distorsión a la salida del sistema. Sin embargo, en el caso de sistema EER híbrido propuesto, a partir de un determinado nivel de envolvente (concretamente cuando $E_{k}=0.2$ en este caso) se mantiene un nivel de alimentación fijo y la distorsión que se produce en esta zona es muchísimo menor debido al funcionamiento en clase A que presenta. 

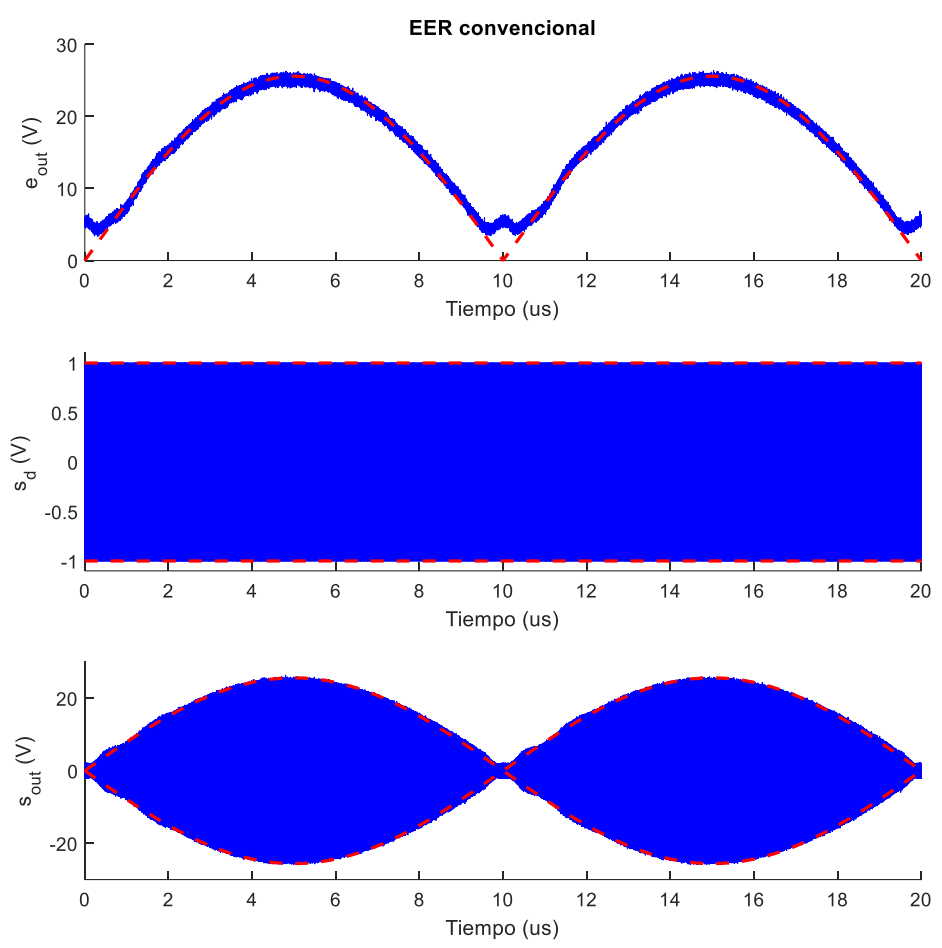

Figura 4.57. Formas de onda de un sistema EER convencional (azul) para una señal de prueba de dos tonos comparadas con las formas de onda de envolvente ideales (rojo).
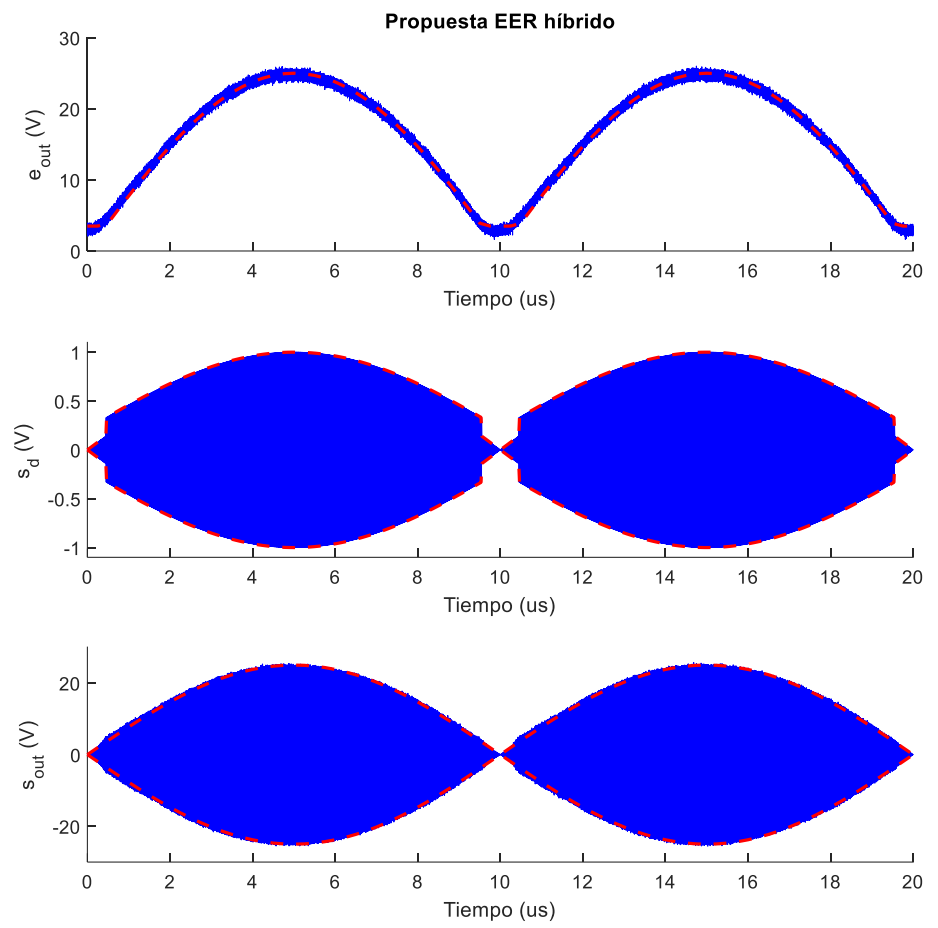

Figura 4.58. Formas de onda del sistema EER híbrido propuesto (azul) para una señal de prueba de dos tonos comparadas con las formas de onda de envolvente ideales (rojo). 
La Figura 4.59 presenta la medida del espectro de la salida del sistema para la señal de dos tonos. Como se puede observar, mejoras de más de $10 \mathrm{~dB}$ a IMD3 y de más de 4 dB a IMD5 han sido conseguidas empleando la técnica EER híbrida propuesta en comparación con la técnica EER convencional.

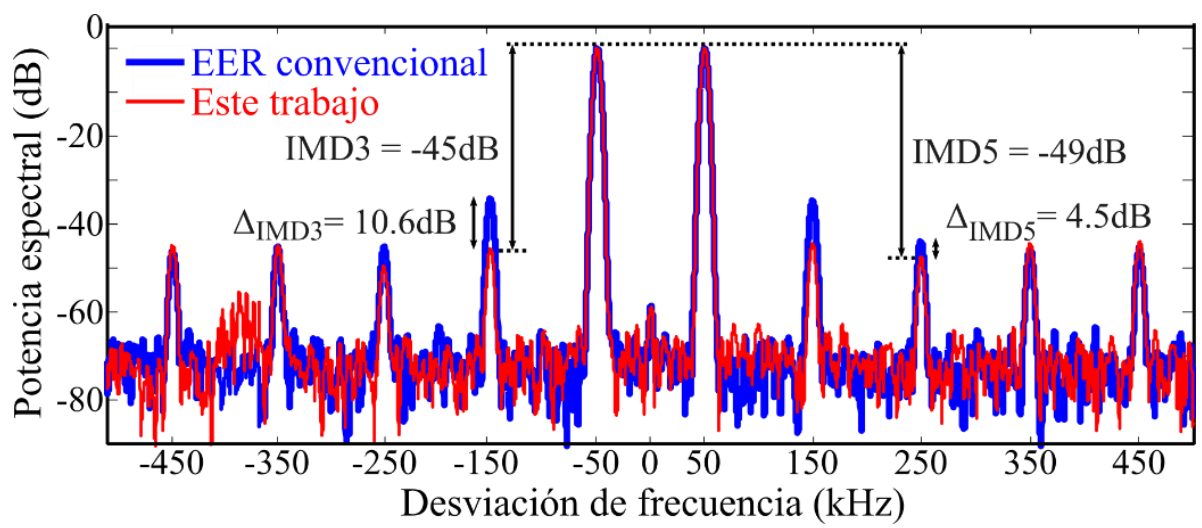

Figura 4.59. Medida del espectro de un sistema EER convencional y del sistema EER híbrido propuesto para una señal de prueba de dos tonos.

La Tabla 4.1 resume las medidas de funcionamiento más significantes del sistema para la prueba de dos tonos, realizando la comparación entre ambas implementaciones. Como punto de partida para poder realizar la comparación, se fijó la misma potencia de salida para ambas implementaciones. De la comparación se puede concluir que en la técnica EER híbrida al existir zonas donde el amplificador de RF trabaja en modo lineal presenta un menor rendimiento de drenador como era de esperar, pero mejora la ganancia de sistema debido a que se reduce la potencia de excitación de entrada. Esto se traduce en que la PAE del sistema EER híbrido solamente experimenta un decremento del 2\% comparado con el sistema EER convencional.

De la misma manera, también se realizaron pruebas con señales digitales. A continuación, se muestra la caracterización del funcionamiento de ambos sistemas para una señal OFDM con modulación 16QAM. Esta señal de prueba presenta una PAR de $8.5 \mathrm{~dB}$ y un ancho de banda de $1 \mathrm{MHz}$. 
Tabla 4.1 Resultados experimentales de un sistema EER convencional y del sistema EER híbrido propuesto para una señal de prueba de dos tonos.

\begin{tabular}{|c|c|c|}
\hline Parámetro & EER convencional & EER híbrido propuesto \\
\hline Potencia de salida & $47.8 \mathrm{dBm}$ & $47.9 \mathrm{dBm}$ \\
\hline Rendimiento de drenador & $75 \%$ & $70.5 \%$ \\
\hline PAE & $69.5 \%$ & $67.5 \%$ \\
\hline Ganancia de potencia & $11.4 \mathrm{~dB}$ & $13.9 \mathrm{~dB}$ \\
\hline IMD3@ @ $\Delta \mathrm{f}=100 \mathrm{kHz}$ & $-34.4 \mathrm{~dB}$ & $-45 \mathrm{~dB}$ \\
\hline IMD5@ @ $\Delta \mathrm{f}=100 \mathrm{kHz}$ & $-44.5 \mathrm{~dB}$ & $-49 \mathrm{~dB}$ \\
\hline
\end{tabular}

Para esta señal es necesario modificar el filtro paso bajo de reconstrucción que se empleó con la señal de prueba de dos tonos. En este caso, la frecuencia de conmutación del amplificador de envolvente es de $10 \mathrm{MHz}$. Los parámetros de diseño del filtro son los siguientes:

- Tipo: Legendre

- Orden: 4

- Frecuencia de corte: $4 \mathrm{MHz}$

- Resistencia de fuente: $0 \Omega$.

- Resistencia de fuente: $5 \Omega$.

En la Figura 4.60 se muestran los valores del filtro diseñado y la respuesta en frecuencia del mismo. A pesar de que la frecuencia de corte del filtro es solamente 4 veces mayor que el ancho de banda de la señal de RF de prueba, también se mantiene un retardo de grupo constante.

La Figura 4.61 presenta la medida del espectro de la salida del sistema para la señal OFDM con predistorsión digital aplicada en ambos casos. Como era de esperar, la propuesta de sistema EER híbrido mejora el ACPR con respeto al sistema EER convencional. 


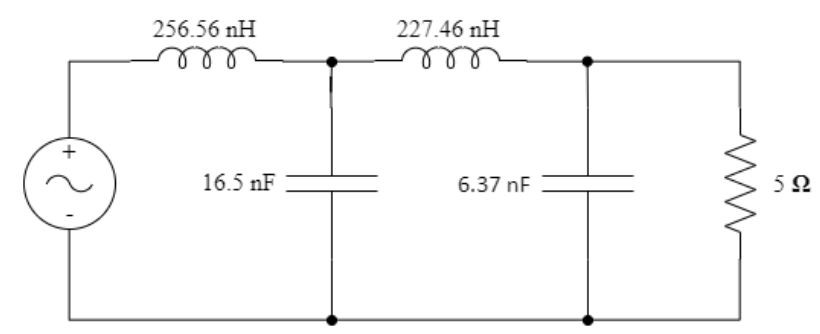

(a)

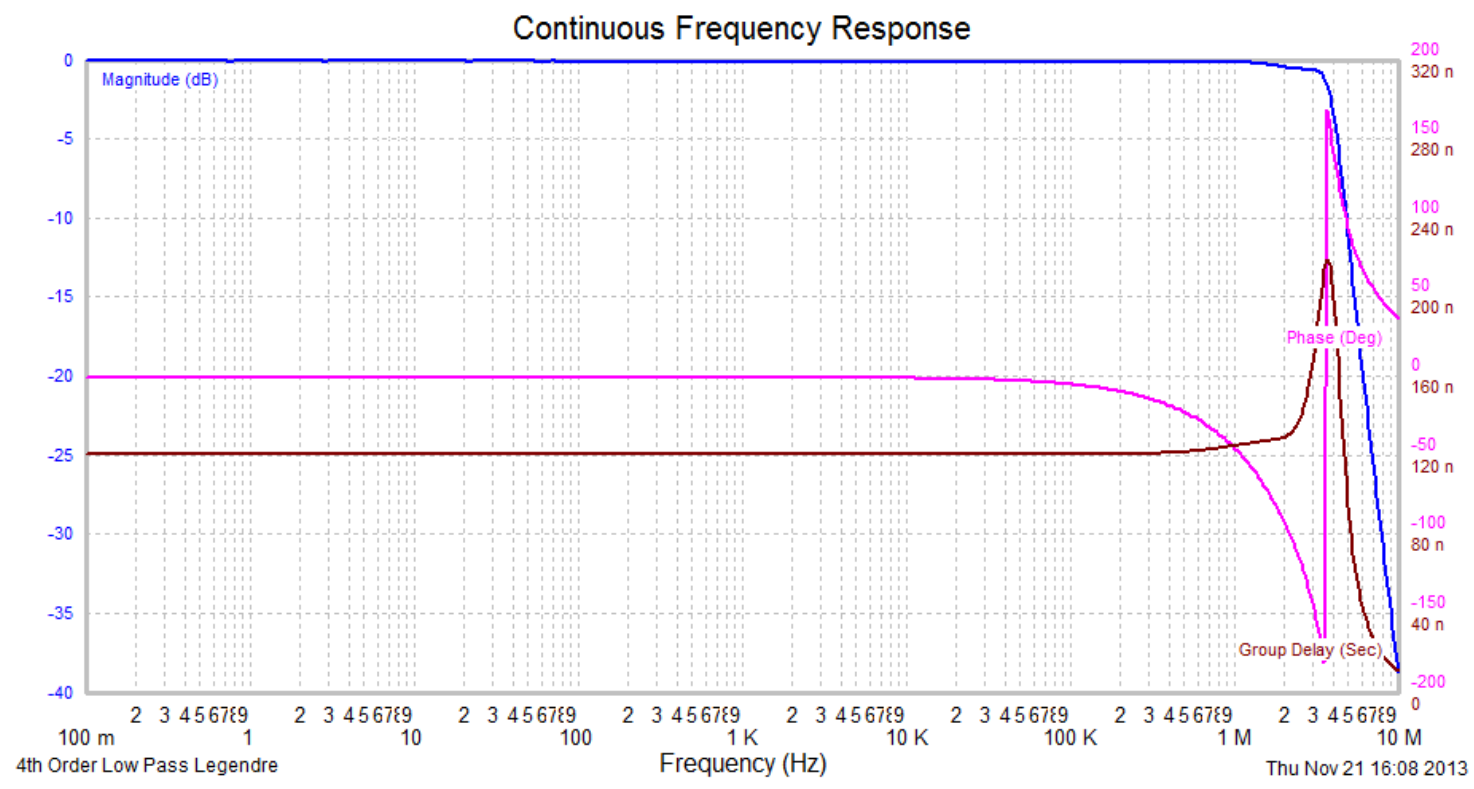

(b)

Figura 4.60 Filtro paso bajo diseñado (a) y respuesta en frecuencia (b) empleado en la prueba OFDM.

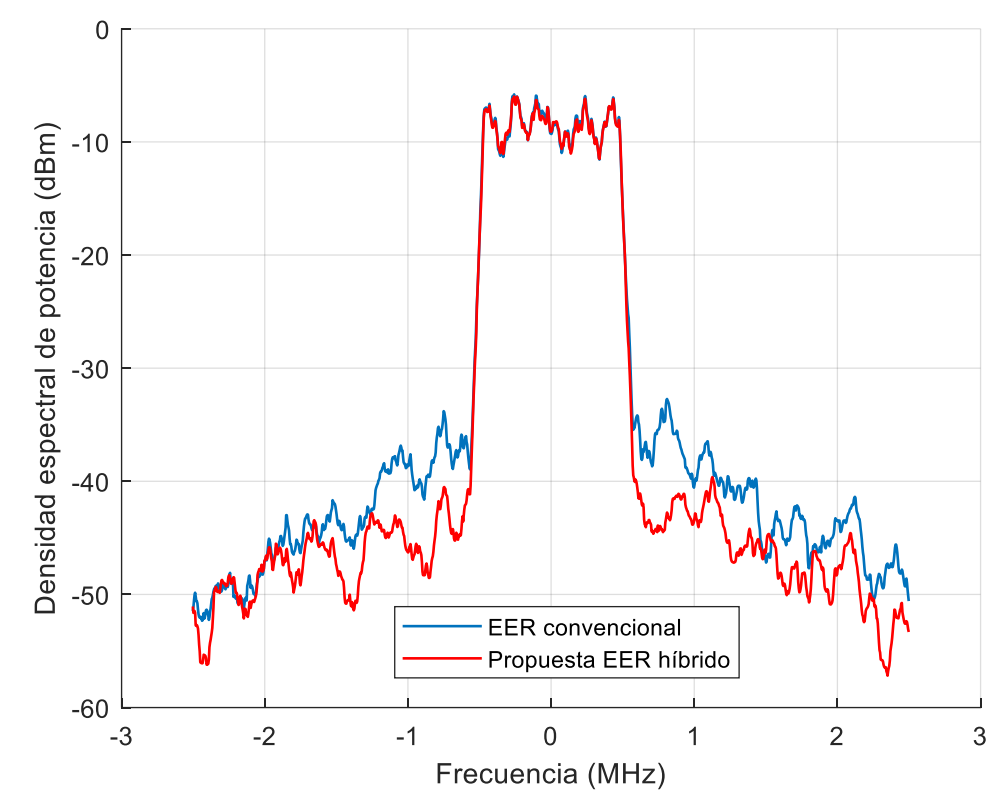

Figura 4.61. Medida del espectro de un sistema EER convencional y del sistema EER híbrido propuesto para una señal de prueba OFDM (con predistorsión digital). 
Al ser una señal digital, una medida característica que se puede realizar es la medida del EVM (Figura 4.62). Por ejemplo, las especificaciones del estándar 802.11 indican que el EVM necesario debe ser menor del 5\% [171]. En este caso, una mejora del $7 \%$ ha sido conseguida implementando el sistema EER híbrido propuesto con respecto al sistema EER convencional.

EER convencional

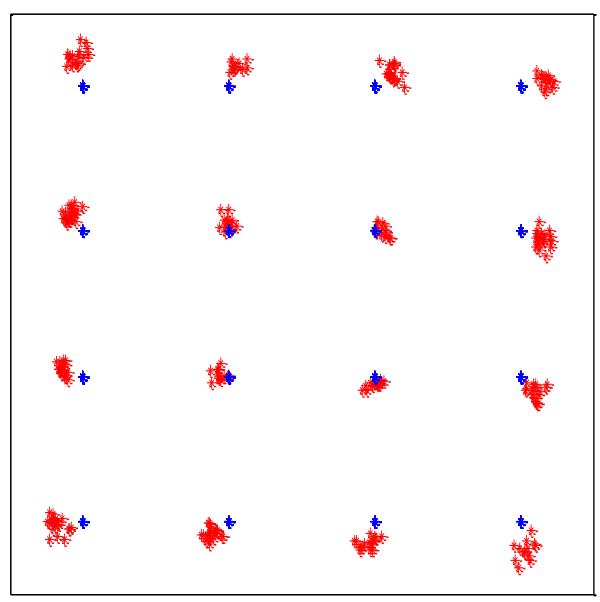

EER híbrido propuesto

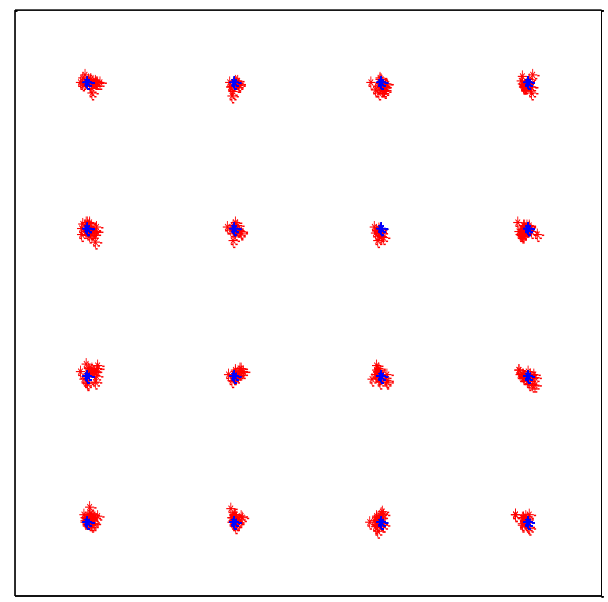

Figura 4.62. Medida del EVM para el sistema EER convencional (9.95\%) y para el sistema EER híbrido propuesto $(2.86 \%)$ para una señal de prueba OFDM (con predistorsión digital).

La Tabla 4.2 resume las medidas de funcionamiento más significantes del sistema para la prueba OFDM, realizando la comparación entre ambas implementaciones. Como punto de partida para poder realizar la comparación, se fijó la misma potencia de salida para ambas implementaciones. De la comparación se puede concluir que existe una mejora significativa en la linealidad del sistema, dando como resultado una mejora del EVM de un $7 \%$ y en los niveles de ACPR de más de $5 \mathrm{~dB}$. El rendimiento medio se reduce con respecto a la señal de dos tonos debido a las variaciones en la amplitud de la envolvente que hacen que el rendimiento del amplificador de envolvente se reduzca. Por otro lado, la potencia media de excitación en el caso del sistema EER híbrido propuesto es menor que en el sistema EER convencional para obtener la misma potencia de salida, lo que se traduce en una mayor ganancia de potencia y en no tener una gran influencia en la PAE del sistema. 
Tabla 4.2 Resultados experimentales de un sistema EER convencional y del sistema EER híbrido propuesto para una señal de prueba OFDM.

\begin{tabular}{ccc}
\hline Parámetro & EER convencional & EER híbrido propuesto \\
\hline Potencia de salida & $45.6 \mathrm{dBm}$ & $45.6 \mathrm{dBm}$ \\
\hline Rendimiento de drenador & $38.3 \%$ & $33.9 \%$ \\
\hline PAE & $33.1 \%$ & $30.6 \%$ \\
\hline Ganancia de potencia & $11.1 \mathrm{~dB}$ & $13.2 \mathrm{~dB}$ \\
\hline EVM & $9.98 \%$ & $2.86 \%$ \\
ACPR L & $-34.4 \mathrm{~dB}$ & $-45 \mathrm{~dB}$ \\
ACPR R & $-44.5 \mathrm{~dB}$ & $-49 \mathrm{~dB}$ \\
\hline \hline
\end{tabular}

En conclusión, el sistema EER híbrido propuesto solventa las limitaciones de linealidad del sistema EER convencional sin repercutir significativamente en el rendimiento. Además, al necesitar de menor potencia de excitación, mejorar la ganancia del sistema y la PAE. En modulaciones digitales, la mejora en la linealidad permitiría cumplir los requisitos espectrales de la mayoría de las aplicaciones, siempre teniendo en cuenta que es necesario aplicar predistorsión digital al sistema. Además, otra ventaja del sistema EER híbrido propuesto es que el alineamiento temporal entre la información de fase y de amplitud no debe ser tan preciso como en el caso de un sistema EER convencional. 


\section{Capítulo 5}

\section{Conclusión}

Resumen, conclusiones, contribuciones y trabajos futuros 


\section{Capítulo 5}

\section{Conclusión}

\section{Resumen, conclusiones, contribuciones, publicaciones y trabajos futuros}

\section{CONTENIDO}

5.1 Resumen y conclusiones

5.2 Contribuciones y publicaciones

5.3 Trabajos futuros

La motivación de esta tesis doctoral tiene como punto de partida la evolución de los nuevos sistemas de comunicaciones con el fin de incrementar la tasa de transmisión de datos y aprovechar el limitado espectro de frecuencias. Este tipo de señales presentan una serie de características que hace que su amplificación sea un reto para los diseñadores de transmisores de radiofrecuencia en búsqueda de ahorro de costes, tamaño y precio.

En esta tesis se han propuesto diferentes soluciones enfocadas a resolver esta problemática. Se han diseñado diferentes transmisores de alto rendimiento basados en las arquitecturas estudiadas. Además, el empleo de una nueva tecnología de semiconductor también ha sido un reto a tratar en esta investigación.

En este último capítulo se va a resumir la consecución de los objetivos perseguidos, las conclusiones extraídas de la investigación y las contribuciones científicas que aporta. Estas contribuciones han sido avaladas mediante la publicación de numerosos artículos y ponencias que serán listados. Por último, líneas de investigación futuras serán propuestas en función de los resultados, inconvenientes o retos encontrados durante la realización de esta tesis doctoral. 


\subsection{Resumen y conclusiones}

La evolución de los sistemas de comunicación inalámbricos en búsqueda de comunicaciones mucho más eficientes provoca que cambien las características de las señales a transmitir por los sistemas de telecomunicación. Por ello, el primer paso ha sido investigar en el estado del arte de las características de dichas señales. La principal tendencia en esta evolución es la mejora de la tasa de transmisión de datos y la eficiencia espectral a consta de presentar relaciones pico-medio cada vez más altas, lo que implica una reducción en el rendimiento de los amplificadores de potencia.

La investigación de nuevas técnicas de amplificación y/o arquitecturas capaces de mejorar el rendimiento de los sistemas es un campo en continuo desarrollo. Muchos investigadores buscan la manera de obtener mayores potencias de salida, rendimiento, ganancia o cualquier otro parámetro de ser optimizable a partir de las técnicas básicas de diseño de amplificadores y transmisores. De ahí que otro punto importante en el desarrollo de esta tesis haya sido el estudio de las técnicas y métodos de diseño, así como de las nuevas arquitecturas que van apareciendo cada día en la literatura. Muchas veces estás nuevas arquitecturas se basan en combinar unas técnicas con otras, dando lugar a combinaciones que pueden conseguir los objetivos planteados.

No solo se pueden obtener mejorar a partir de nuevas técnicas de amplificación, sino que el dispositivo activo es el elemento más importante dentro de un sistema de comunicaciones. La continua investigación en nuevos semiconductores capaces de presentar características adecuadas para determinadas aplicaciones es otro punto importante. En esta tesis se ha realizado un estudio del arte de las tecnologías de semiconductores para las aplicaciones que aquí se plantean. Como conclusión, la tecnología GaN aporta tanto las ventajas del Si (alta potencia) como las ventajas del GaAs (alta frecuencia), lo que le hace un sería candidato a desbancar a los amplificadores de potencia de alta frecuencia diseñados con Si o GaAs. Es más, actualmente se están empezado a sustituir equipos por nuevos amplificadores basado en la tecnología GaN. El único inconveniente de los dispositivos de GaN es que es una tecnología emergen y hay una cierta reticencia por parte de las empresas en proceder a la sustitución de sus viejos sistemas por temas de fiabilidad y precio. Sin embargo, con el paso de los años estos inconvenientes irán poco a poco desapareciendo, provocando ventajas competitivas. 
Tras el estudio del arte, el siguiente paso fue buscar en el mercado dispositivos de $\mathrm{GaN}$ que cumplieran las características de alta potencia y alta frecuencia para el diseño de los amplificadores presentados. En el momento en el que comenzó esta tesis doctoral, los dispositivos de GaN acababan de aparecer en el mercado y eran escasos. Además, los fabricantes aún no disponían de modelos fiables que ayudaran en el diseño de los amplificadores mediante verificación por simulación.

Debido a ello, otros métodos de diseño tuvieron que ser empleados. En esta ocasión, el método load pull fue empleado para el diseño e implementación de un amplificador clase F en banda L. Es un método totalmente empírico que requiere de una especial destreza a la hora de calibrar el banco de medidas. El método load pull trata de buscar las impedancias de carga que hacen que el dispositivo maximice sus parámetros de rendimiento, potencia, ganancia o cualquier otro parámetro deseado. En la realización de esta tesis se realizaron diferentes prototipos de amplificador clase $\mathrm{F}$ utilizando este método. Las medidas de caracterización del dispositivo hicieron posible la implementación de un tercer prototipo que conseguía mejorar el funcionamiento con respecto a otros trabajos.

A medida que pasaba el tiempo, nuevos dispositivos de GaN iban apareciendo y los fabricantes ofrecían herramientas para poder diseñar amplificadores de potencia. Por ello, se procedió al diseño de otro amplificador de potencia de alto rendimiento en banda $L$ a partir de la técnica de síntesis de la admitancia de carga para un amplificador clase E. Sin embargo, la amplificación clase E demanda unos altos requisitos a los dispositivos que a día de hoy hace que no se puedan implementar amplificadores clase $\mathrm{E}$ óptimos a altas frecuencias. La frecuencia crítica determina el funcionamiento óptimo del dispositivo en clase E.

A pesar de ello, es posible implementar amplificadores en clase E con estos dispositivos trabajando fuera de su punto óptimo. La idea de clase E subóptima estudiada por Raab ofrecen unas prestaciones muy buenas en comparación con otras clases de amplificación. La investigación en este campo conllevo el diseño, simulación e implementación de dos amplificadores que empleaban esta clase subóptima: uno en banda L y otro en banda S. Tras los resultados, la principal conclusión es que la topología clase E subóptima es una solución que permite proporcionar alto rendimiento 
a altas frecuencias compitiendo con otras topologías como clase $\mathrm{F}$ o clase $\mathrm{F}^{-1}$ para la misma banda de frecuencia y potencia.

No obstante, los amplificadores diseñados son altamente no lineales. Esto no les hace adecuados para la amplificación de las señales de comunicaciones modernas que presentan alta PAPR. Estos amplificadores si tienen su importancia en otro tipo de aplicaciones como puede ser RADAR donde las señales a transmitir no presentan cambios en la envolvente. En este tipo de aplicaciones, estos amplificadores no lineales contribuyen con altos rendimiento y altas potencias, lo que ayuda a reducir el tamaño y el coste de los sistemas.

Otras aplicaciones donde los amplificadores de alto rendimiento pueden tener cabida es en el diseño de transmisores de alto rendimiento basados en alguna arquitectura de linealización. Entre ellas, ET y EER son dos prometedoras arquitecturas que se adaptan perfectamente a señales de alta PAPR.

Estas arquitecturas fueron implementadas utilizando los amplificadores de alto rendimiento implementados anteriormente. Dos transmisores de alto rendimiento fueron diseñados: el primero estaba compuesto por el amplificador clase F presentado en esta tesis junto con un convertidor buck multifase diseñado por el Centro de Electrónica Industrial de la Universidad Politécnica de Madrid; y el segundo constaba del amplificador clase E subóptimo en banda L propuesto en esta tesis y un convertidor buck multifase diseñado por el Grupo de Ingeniería de Radio de la Universidad Politécnica de Madrid.

El primer transmisor fue diseñado en arquitectura EER y en ET, de tal manera que se pudieron comparar los resultados obtenidos. Las medidas realizadas a los transmisores indicaban que el convertidor multinivel introduce distorsión en la señal de salida debido principalmente a sus transiciones, hecho que se hace más patente a medida que aumenta el ancho de banda. A pesar de ello, se aprecia una mejora significativa en el rendimiento del sistema debida al convertidor multinivel, por lo que la aplicación de predistorsión al sistema podría ayudar a mejorar la linealidad sin variar las características de rendimiento.

Comparando el funcionamiento de este primer transmisor en ET y EER, se concluye lo siguiente: el aumento del ancho de banda o la PAPR de la señal de prueba 
degrada considerablemente el rendimiento y la linealidad que puede ofrecer el amplificador de envolvente. Se han obtenido mejores resultados de linealidad con la configuración EER con respecto a la configuración ET, debido principalmente a la influencia del regulador lineal que asiste al convertidor multinivel. Sin embargo, el rendimiento del transmisor ET es mayor que el del transmisor EER. Esto se debe a que el regulador lineal introduce importantes pérdidas en el transmisor EER. Por todo ello, la integración que puede conseguir un mejor funcionamiento del transmisor es ET, ya que a pesar de tener menor linealidad puede ser corregida con otros métodos de fácil implementación y poco costosos como puede ser predistorsión digital con procesado digital de la señal.

Partiendo de los beneficios e inconvenientes obtenidos en la implementación del transmisor EER y ET, se procedió a la realización de otro transmisor. En este caso, la arquitectura que se eligió fue EER pero, con el fin de solventar alguno de los inconvenientes de esta técnica, se propuso una arquitectura híbrida. Esta arquitectura se caracteriza por hacer trabajar al amplificador de RF en dos modos de funcionamiento diferentes: el amplificador trabaja en clase E subóptima a niveles de envolvente altos y en clase A a bajos niveles de envolvente. Esto se consigue procesando digitalmente las señales de envolvente y de fase de tal manera que en cada instante el amplificador de RF trabaje en un modo diferente en función de la envolvente. Además, es este caso se empleó predistorsión digital con el fin de obtener niveles de linealidad parecidos a los obtenidos con amplificadores lineales para la misma banda de frecuencia y potencia.

Esta arquitectura híbrida fue comparada con la arquitectura EER convencional, de tal manera que se pudo comprobar que la técnica propuesta solventa las limitaciones de linealidad de un sistema EER convencional sin repercutir significativamente en el rendimiento. El empleo de esta arquitectura híbrida con modulaciones digitales permitiría cumplir los requisitos de la mayoría de las aplicaciones, siempre teniendo en cuenta la necesaria aplicación de predistorsión digital. Otra ventaja de este sistema EER híbrido propuesto es que el alineamiento temporal entre la información de amplitud y de fase no es tan crítico como en el caso de un transmisor EER convencional. 


\subsection{Contribuciones y publicaciones}

Toda la teoría y técnicas descritas en esta tesis tienen como objetivo el diseño de transmisores lineales de alto rendimiento. Este objetivo se engloba dentro de dos áreas de contribución: diseño de amplificadores de potencia de alto rendimiento y diseño de transmisores lineales de alto rendimiento. Numerosas publicaciones y ponencias avalan la calidad de estas contribuciones. A continuación, se hace un resumen de las contribuciones aportadas en esta tesis doctoral, así como las publicaciones certifican esas contribuciones:

\section{1) Diseño de amplificadores de alto rendimiento:}

Los efectos parásitos del dispositivo y el encapsulado juegan un papel importante en el diseño de los amplificadores de potencia de alto rendimiento. Un método para la extracción de estos parámetros ha sido explicado en el Capítulo 3. Partiendo de una modelo de interno del transistor, se propuso que polarizando el dispositivo de una determinada manera es posible obtener algunos de los parámetros del dispositivo interno que influyen en el diseño de los amplificadores de potencia, como son las capacidades intrínsecas no lineales del transistor. Una vez se dispone de un modelo del dispositivo, es posible mapear las impedancias de carga que hacer trabajar al dispositivo en el modo que se desea.

El diseño de amplificadores de potencia de alto rendimiento depende en gran medida de las técnicas de diseño empíricas debido a la complejidad y al comportamiento no lineal de los dispositivos de alta potencia para altas frecuencias como se describe en el Capítulo 3. En esta tesis se propone una técnica load pull multiarmónica sencilla y de bajo coste con la que controlar las impedancias a fundamental y a armónicos con el fin de obtener un funcionamiento en alto rendimiento. Este método fue verificado mediante el diseño de un amplificador clase $\mathrm{F}$ en banda $\mathrm{L}$ capaz de entregar una potencia de salida de hasta $45 \mathrm{~W}$ con un rendimiento del $70 \%$ controlando únicamente los dos primeros armónicos. La principal ventaja de este diseño es que es fácil de reproducir ya que no emplea elementos concentrados críticos y presenta un 
tamaño reducido. A continuación, se realiza un listado de las publicaciones o ponencias realizadas correspondientes a este diseño:

- "Amplificador de potencia clase F a $1.64 \mathrm{GHz}$ con control de armónicos”. Universidad Carlos III de Madrid. 6-9 septiembre 2011. XXVI Simposium Nacional de la Unión Científica Internacional de Radio (URSI 2011). Simposium nacional.

- "L-Band Class-F High Efficiency Power Amplifier based on GaNHEMT Technology". 5th Annual Meeting, Centro de Electrónica Industrial (CEI), Universidad Politécnica de Madrid. Póster. 22-23 marzo 2012.

- "Amplificador de potencia clase $\mathrm{F}$ en banda L basado en tecnología GaN". Centro de congresos de Elche, Elche. 12-14 septiembre 2012. XXVII Simposium Nacional de la Unión Científica Internacional de Radio (URSI 2012). Simposium nacional.

- "High Efficiency Broadband GaN RF \& Microwave Amplifiers". 6th Annual Meeting, Centro de Electrónica Industrial (CEI), Universidad Politécnica de Madrid. Póster. 14-15 marzo 2013.

Otra técnica de diseño empleada en esta tesis doctoral está basada en la síntesis de la admitancia de carga descrita en el Capítulo 3. Esta técnica permite hallar la carga necesaria a fundamental y a armónicos para el funcionamiento en clase $\mathrm{E}$ nominal. Sin embargo, los transistores empleados (CGH40180PP y CGH40010P) no pueden trabajar en el modo óptimo debido a las limitaciones de las capacidades internas y a la frecuencia de trabajo. Por ello, se propuso el diseño de los amplificadores en un modo subóptimo capaz de competir con otras topologías de amplificación. Está técnica de diseño fue verificada mediante el diseño de dos amplificadores clase E subóptimos. El diseño en banda L es capaz de entregar una potencia de salida de $180 \mathrm{~W}$ con un rendimiento del $85 \%$ desde $900 \mathrm{MHz}$ hasta $1500 \mathrm{MHz}$; el diseño en banda S ofrece una potencia de salida de $14 \mathrm{~W}$ con un rendimiento del $79 \%$ en la banda de $2100 \mathrm{MHz}$ a $2600 \mathrm{MHz}$. Las publicaciones y ponencias que validan dichos resultados se muestran a continuación: 
- F. J. Ortega-Gonzalez, D. Tena-Ramos, M. Patiño-Gomez, J. M. PardoMartin and D. Madueño-Pulido, "High-Power Wideband L-Band Suboptimum Class-E Power Amplifier," in IEEE Transactions on Microwave Theory and Techniques, vol. 61, no. 10, pp. 3712-3720, Oct. 2013.

- "Amplificador de potencia de alto rendimiento clase E en banda S". Escuela Politécnica Superior, Universidad Autónoma de Madrid. 5-7 septiembre 2016. XXXI Simposium Nacional de la Unión Científica Internacional de Radio (URSI 2016). Simposium nacional.

Un banco de medidas automático fue implementado para la caracterización de los amplificadores diseñados. El banco de pruebas presentado en el Capítulo 3 controla los equipos de medida a través de Matlab y permite realizar las medidas de una manera rápida y sencilla. Esto evita el sobrecalentamiento de los dispositivos que en muchas ocasiones provocan errores en las medidas debido a los efectos memoria.

\section{2) Diseño de transmisores lineales de alto rendimiento:}

La linealización de amplificadores de potencia de alto rendimiento es una de las aportaciones aportada por esta tesis doctoral. En el Capítulo 4 se ha presentado el diseño y la integración de varios transmisores basados en la técnica ET y EER.

Dentro de esta área, la primera contribución es la integración del amplificador de potencia clase $\mathrm{F}$ diseñado en el Capítulo 3 junto con un amplificador de envolvente basado en un convertidor buck de 8 fases asistido en serie por un regulador lineal. Tanto la arquitectura ET como EER fueron implementadas sin la aplicación de predistorsión digital. Ambas emplearon como señal de prueba una modulación 64QAM con diferentes anchos de banda. La arquitectura EER obtuvo un rendimiento medio del $27 \%$ para señales hasta $4800 \mathrm{kHz}$, y unos niveles de ACPR por encima de los 30dB. La arquitectura ET fue capaz presenta un rendimiento del $33 \%$ para señales hasta $4800 \mathrm{kHz}$, con unos niveles de ACPR por encima de los $28 \mathrm{~dB}$. Una publicación avala estos resultados: 
- M. Vasić et al., "The Design of a Multilevel Envelope Tracking Amplifier Based on a Multiphase Buck Converter," in IEEE Transactions on Power Electronics, vol. 31, no. 6, pp. 4611-4627, June 2016.

De la misma manera, el Capítulo 4 presenta la integración del amplificador clase E subóptimo diseñado en el Capítulo 3 junto con un amplificador de envolvente basado en un convertidor buck multifase. En este caso, una novedosa arquitectura EER híbrida fue propuesta, donde el amplificador de RF trabaja en un modo mixto en función de la amplitud de la envolvente de la señal. Este modo mixto solventa alguno de los inconvenientes de la arquitectura EER como son los niveles bajos o nulos de envolvente. La arquitectura EER híbrida propuesta mantiene el mismo circuito que para un transmisor EER convencional y a través del procesado digital de las señales de envolvente y de fase de entrada se consigue variar la clase del funcionamiento del amplificador de RF. Además, con el fin de cumplir con la mayoría de los requisitos de linealidad de los sistemas de comunicaciones, las medidas han sido realizadas aplicando predistorsión digital a la señal de entrada. Para una señal de prueba de dos tonos, el transmisor es capaz de entregar una potencia de salida de $47.9 \mathrm{dBm}$ con un rendimiento del $70.5 \%$ mejorando en $10 \mathrm{~dB}$ los niveles de IMD3 y en 5dB los niveles de IMD5 con respecto a un transmisor EER convencional con predistorsión digital. Usando una OFDM con modulación 16QAM como señal de prueba, el transmisor puede entregar una potencia de salida de $45.6 \mathrm{dBm}$ con un rendimiento del 30.6\% y unos niveles de ACPR de más 45 dB. Además, el EVM mejora en un $7 \%$ con respecto a un transmisor EER convencional con predistorsión digital. Estas contribuciones han sido publicadas y expuestas en:

- "High efficiency EER transmitter". 7th Annual Meeting, Centro de Electrónica Industrial (CEI), Universidad Politécnica de Madrid. Póster. 27-28 marzo 2014.

- "High efficiency linear EER amplifier for the L-band". 8th Annual Meeting, Centro de Electrónica Industrial (CEI), Universidad Politécnica de Madrid. Póster. 16-17 abril 2015.

- "Nueva técnica de modulación de excitación para sistemas EER". Escuela Técnica Superior de Ingenieros Industriales y de 
Telecomunicación, Universidad Pública de Navarra. 2-4 septiembre 2015. XXX Simposium Nacional de la Unión Científica Internacional de Radio (URSI 2015). Simposium nacional.

- "A new drive modulation applied to an L-band EER transmitter". Centro de congresos Ciutat D’Elx, Elche. 6-8 julio 2016. Seminario Anual de Automática, Electrónica Industrial e Instrumentación (SAAEI 2016). Congreso internacional.

- D. Tena-Ramos, F. J. Ortega-González and M. Patiño-Gómez, "Hybrid Envelope Elimination and Restoration Technique for Enhancing the Linearity of Switchmode Envelope Amplifiers," in IEEE Microwave and Wireless Components Letters, vol. 27, no. 2, pp. 186-188, Feb. 2017.

Un banco de medidas fue implementado para la generación y caracterización de los transmisores diseñados. El banco de pruebas presentado en el Capítulo 4 es capaz de generar las señales de prueba con las que caracterizar los transmisores ET y EER y obtener las medidas de los equipos a través de Matlab. La principal característica de este bando de medidas es que permite de una manera rápida y sencilla generar las señales de referencia necesarias y ajustar el retardo entre el camino de fase y el camino de envolvente ágilmente. La captura de las señales permite su demodulación posterior con el fin de obtener los parámetros que caracterizan el funcionamiento del transmisor, como puede ser el EVM.

\subsection{Trabajos futuros}

La gran variedad de áreas en las que tiene cabida este trabajo deja abierta algunas cuestiones en referencia al diseño de ampliadores de potencia de alto rendimiento, de técnicas de procesado de señal y de integración de sistemas. El continuo avance en las comunicaciones inalámbricas conlleva nuevos requisitos de diseño, generando nuevos desafíos a los investigadores. A continuación se describen algunas de las vías más interesantes para futuras investigaciones. 


\section{Dispositivo pre-adaptados a frecuencias armónicas:}

Hoy en día la fabricación de circuitos integrados es una técnica habitual para determinadas aplicaciones, aún más importante a frecuencias muy altas. De la misma manera que se puede realizar la adaptación a los armónicos con módulos de pre-adaptación externos al dispositivo, una buena vía de investigación es la inclusión de esos circuitos de pre-adaptación dentro del dispositivo, con el fin de generar las impedancias armónicas deseadas para la clase de amplificación diseñada. De esta manera, el diseñador del amplificador únicamente debería diseñar la red a la frecuencia fundamental. La principal limitación es el ancho de banda en el cuál el dispositivo ofrecería ese comportamiento.

\section{Predistorsión digital para el transmisor ET/EER:}

La aplicación de predistorsión digital a los sistemas es un buen método para mejorar la linealidad de los sistemas. Una de las vías de investigación futuras más interesante es la aplicación de predistorsión digital al transmisor compuesto por el amplificador clase $\mathrm{F}$ y el convertidor multifase asistido por el regulador lineal. La conversión AM-PM y AM-AM puede ser corregida aplicando técnicas de predistorsión. Estas técnicas de predistorsión pueden ser estáticas (los valores de compensación se guardan en una tabla) o dinámicas (se crea un lazo de predistorsión que compara la señal de salida con la señal de entrada y aplica la compensación correspondiente). La primera opción tiene el inconveniente de no poder variar la compensación en función de parámetros como la temperatura.

\section{Corrección de los efectos memoria del transistor:}

Los efectos memoria generan distorsiones en el amplificador de RF causada por variaciones en el tiempo de las características del dispositivo. Por ejemplo, el funcionamiento del dispositivo puede cambiar en función de la temperatura, de la humedad o simplemente por envejecimiento. Típicamente, uno de los métodos para la corrección de estos efectos memoria es la retroalimentación. Por ello, 
como vía de investigación futura se puede diseñar un lazo de retroalimentación que controle la distorsión debida a los efectos memoria.

\section{Amplificador de envolvente híbrido:}

Hasta la fecha, muchas son los trabajos donde el amplificador de envolvente se compone de un convertidor buck asistido por un regulador lineal. Ello mejora la linealidad de la señal de envolvente a costa de una reducción importante en el rendimiento del amplificador de envolvente.

En este trabajo se ha propuesto una técnica en la que el amplificador de RF es capaz de trabajar en dos modos diferentes en función del nivel de envolvente: trabaja en alto rendimiento para niveles de envolvente altos y en modo lineal para niveles de envolvente bajos, donde el rendimiento es pobre. Ello genera una mejora en la linealidad pero reduce ligeramente el rendimiento dado que el modo lineal solo entra en funcionamiento en ciertos instantes.

La vía de investigación que se propone es traspasar este modo de funcionamiento al amplificador de envolvente en una arquitectura EER. De esta manera, el convertidor buck solo entraría en funcionamiento para niveles de envolvente altos y un amplificador lineal se encargaría de los niveles bajos. Este comportamiento implica que el convertidor buck debe cumplir unos requisitos de linealidad altos, ya que toda la información de envolvente será proporcionada por el amplificador de envolvente.

\section{Dispositivos de bandgap amplio:}

Como se ha podido demostrar en los resultados obtenidos en esta tesis doctoral con el uso de GaN, el empleo de este tipo de dispositivos es muy favorable para el incremento del rendimiento: presenta una resistencia de conducción más pequeña y, además, el valor de las capacidades internas también es inferior al de otros dispositivos. Esto los hace adecuados para aumentar la velocidad de conmutación reduciendo las pérdidas e incrementando la frecuencia de funcionamiento. 


\section{Bibliografía}

[1] L. Larson, P. Asbeck and D. Kimball, "Next generation power amplifiers for wireless communications - squeezing more bits out of fewer joules," 2005 IEEE Radio Frequency integrated Circuits (RFIC) Symposium - Digest of Papers, Long Beach, CA, USA, 2005, pp. 417-420.

[2] S. Mclaughlin et al., "Techniques for improving cellular radio base station energy efficiency," in IEEE Wireless Communications, vol. 18, no. 5, pp. 10-17, October 2011.

[3] L. M. Correia et al., "Challenges and enabling technologies for energy aware mobile radio networks," in IEEE Communications Magazine, vol. 48, no. 11, pp. 66-72, November 2010.

[4] G. Koutitas and P. Demestichas, "A Review of Energy Efficiency in Telecommunication Networks," Telfor Journal, vol. 2, 2010.

[5] M. Liu, L. Liu, X. Wu, L. Teng, J. Cao, and S. Qin, "A Research on the Telecommunication Base Station Power Consumption Investment Analysis and Optimized Configuration Method for Hybrid Energy Power," in Telecommunications Energy Conference 'Smart Power and Efficiency' (INTELEC), Proceedings of 2013 35th International, 2013, pp. 1-6

[6] OPERA-NET 2: Optimising Power Efficiency in Mobile Radio Networks 2. Disponible en https://www.celticplus.eu/project-opera-net2/ (2020)

[7] K. Li, "Mobile communications 2008: Green thinkingbeyond TCO consideration," Whitepaper IN0804307WHT, InStat, May 2008

[8] O. Blume, D. Zeller and U. Barth, "Approaches to energy efficient wireless access networks," 2010 4th International Symposium on Communications, Control and Signal Processing (ISCCSP), Limassol, 2010, pp. 1-5.

[9] Z. Wang, Envelope Tracking Power Amplifiers for Wireless Communications: Artech House, 2014.

[10] Y. -. Wu, M. Moore, A. Saxler, T. Wisleder and P. Parikh, "40-W/mm Double Field-plated GaN HEMTs," 2006 64th Device Research Conference, State College, PA, USA, 2006, pp. 151-152.

[11] R. Coffie et al., "p-capped GaN-AlGaN-GaN high-electron mobility transistors (HEMTs)," in IEEE Electron Device Letters, vol. 23, no. 10, pp. 588-590, Oct. 2002.

[12] M. A. Khan et al., "Insulating gate III-N heterostructure field-effect transistors for high-power microwave and switching applications," in IEEE Transactions on Microwave Theory and Techniques, vol. 51, no. 2, pp. 624-633, Feb. 2003. 
[13] J. C. Zolper, "Wide Bandgap Semiconductor Microwave Technologies: From promise to practice," 1999 IEDM Tech. Dig., pp. 289-392, Dec 1999.

[14] Y. -. Wu, B. P. Keller, S. Keller, D. Kapolnek, S. P. Denbaars and U. K. Mishra, "Measured microwave power performance of AlGaN/GaN MODFET," in IEEE Electron Device Letters, vol. 17, no. 9, pp. 455-457, Sept. 1996.

[15] N. J. Kolias, C. S. Whelan, T. E. Kazior and K. V. Smith, "GaN technology for microwave and millimeter wave applications," 2010 IEEE MTT-S International Microwave Symposium, Anaheim, CA, 2010, pp. 1222-1225.

[16] Syed, A., "Large Signal Physical Simulations of Si LDMOS Transistor for RF Application", Ph.D. dissertation, Dept. of Physics, Linköping University, 2004

[17] Trew, R. J., "Wide Bandgap Semiconductor Transistors for Microwave Power Amplifiers," IEEE Microwave Magazine, Vol. 1, March 2000, pp. 46-54

[18] Eastnan, L. F., and U.K. Mishra, "The Toughest Transistor Yet," IEEE Spectrum, Vol. 18, No. 3, May 2002, pp. 28-33

[19] Nagy, W., "Linearity Characteristics of Microwave-Power GaN HEMTs," IEEE Transactions on Microwave Theory and Techniques, Vol. 51, No. 2, February 2003, pp. 660-664

[20] Wu, Y. F., et al., “30-W/mm GaN HEMTs by Field Plate Optimization,” IEEE Electron Device Letters, Vol. 25, No. 3, March 2004, pp. 117-119

[21] U. K. Mishra, P. Parikh and Yi-Feng Wu, "AlGaN/GaN HEMTs-an overview of device operation and applications," in Proceedings of the IEEE, vol. 90, no. 6, pp. 1022-1031, June 2002.

[22] R. Gaska, J. Yang, A. Osinsky, M. Asif Khan and M. S. Shur, "Novel high power AlGaN/GaN HFETs on SiC substrates," International Electron Devices Meeting. IEDM Technical Digest, Washington, DC, USA, 1997, pp. 565-568.

[23] U. K. Mishra, L. Shen, T. E. Kazior and Y. Wu, "GaN-Based RF Power Devices and Amplifiers," in Proceedings of the IEEE, vol. 96, no. 2, pp. 287-305, Feb. 2008.

[24] B. Heying, et al., "Reliable GaN HEMTS for High Frequency Applications", 2010 IEEE MTT-S International Microwave Symposium Digest, pp. 1218-1220, May 2010

[25] M. Dammann et al., "Reliability status of GaN transistors and MMICs in Europe," 2010 IEEE International Reliability Physics Symposium, Anaheim, CA, 2010, pp. 129-133.

[26] F. Yamaki et al., "Ruggedness and reliability of GaN HEMT," 2011 6th European Microwave Integrated Circuit Conference, Manchester, 2011, pp. 328331. 
[27] W. Saito, "Reliability of GaN-HEMTs for high-voltage switching applications," 2011 International Reliability Physics Symposium, Monterey, CA, 2011, pp. 4E.1.1-4E.1.5.

[28] G. Gauthier, Y. Mancuso and F. Murgadella, "KORRIGAN - A Comprehensive Initiative for GaN HEMT Technology in Europe", Proceedings European Microwave Week, October 2005, Paris, France

[29] P. Duême et al., "Overview of the KORRIGAN project", Proceedings WOCSDICE 2009, Malaga, Spain

[30] G. Gauthier, F. Reptin, «KORRIGAN: Development of GaN HEMT Technology in Europe», CS MANTECH Conference, April 24-27, Vancouver, British Columbia, Canada, pp. 49-51 (2006)

[31] K. Hirche et al., "GaN Reliability Enhancement and Technology Transfer Initiative (GREAT2)", Abstract of ESA/ESTEC Contract No. 21.499/08/NL/PA, 2008

[32] H. Blanck, J. Splettstöser, D. Floriot, "GaN technology for RF electronics: Development Status in Europe, " in Technical Digest of IEEE CSIC Symposium, 2009, pp. 22-25

[33] Monografías del SOPT. Tecnología de semiconductores GaN y SiC. Edicion 2011. Ministerio de defensa. 2011

[34] R. Thompson, et al., "Performance of theAlGaN HEMT Structure with a Gate Extension," IEEE Transactions on Electron Devices, vol. 51, no. 2, pp. 292-295, Feb. 2004

[35] Dave Aichele, et. al, "Compact L- and S-Band GaN High Power Amplifiers", RFMD, Resource Center - White papers - Nov 2010.

[36] Pat Hindle , "Future RF Markt Opportunities for GaN", Microwave Journal, June 2012

[37] I.A. Rumyancev, "Efficient Amplification Techniques for Signals with High PAPR", IEEE NW Russia Young Researchers in Electrical and Electronic Engineering Conference, pp. 139-142, 2015

[38] F. H. Raab, P. Asbeck, S. Cripps, P. B. Kenington, Z. B. Popovic, N.Pothecary, J. F. Sevic, and N. O. Sokal, "Power amplifiers and transmitters for RF and microwave", IEEE Trans.Microw. Theory Tech., vol. 50, no. 3, pp. 814-826, Mar. 2002

[39] S. Cripps, Advanced Techniques in RF Power Amplifier Design. Artech House Publishers, 2002

[40] V.Camarchia, V. Teppati, S. Corbellini, and M. Pirola, "Microwave measurements part II non-linear measurements," Instrumentation Measurement Magazine, IEEE, vol. 10, pp. 34-39, June 2007 
[41] A Comparison of Harmonic Tuning Methods for Load Pull Systems Author: Gary Simpson, MSEE Director of Technical Development in Engineering, Maury Microwave Corporation 2009

[42] J.M. Cusack, S.M. Perlow, B.S. Perlman, Automatic load contour mapping for microwave power transistors. IEEE Trans. Microw. Theory Tech. 22, 11461152, 1974.

[43] F. Secchi, R. Paglione, B. Perlman, J. Brown, A computer controlled microwave tuner for automated load pull.RCA Rev. 44(4), 566-583 (1983)

[44] F. Microwaves, "Application note database." Disponible en : http://focusmicrowaves.com/ (2020)

[45] Maury Application note library. Disponible en:: http://www.maurymw.com/MW_RF/Application_Notes_Library.php (2020)

[46] F. Deshours, E. Bergeault, F. Blache, J.-P. Villotte, B. Villeforceix, Experimental comparison of load-pull measurement systems for nonlinear power transistor characterization. IEEE Trans. Instrum. Meas. 57(11), 1251-1255 (1997)

[47] M.S. Hashmi, F.M. Ghannouchi, P.J. Tasker, K. Rawat, Highly reflective loadpull. IEEE Microw. Mag. 11(4), 96-107 (2011)

[48] Sokal, N. O. and "Class E _ a new class of high-efficiency tuned single-ended switching power amplifiers," IEEE J. Solid-State Circuits, vol. SC- 26 10, no. 3, pp. 168-176, June 1975

[49] T. Mader, E. W. Bryerton, M. Markovic, M. Forman, and Z. Popovic, "Switched-mode high-efficiency microwave power amplifiers in a free-space power-combiner array," Microwave Theory and Tecniques, IEEE Transactions on, vol. 46, pp. 1391-1398, Aug. 2002

[50] Andrei Grebennikov., Switchmode RF and Microwave Power Amplifiers, Academic Press, 2nd edition, 2012.

[51] A. Grebennikov, "Class E High-Efficiency Power Amplifiers: Historical Aspect and Future Prospect,' Applied Microwave \& Wireless, vol. 14, pp. 64-71, July 2002, pp. 64-72, August 2002

[52] T. Mury and V. F. Fusco, "Series-L/parallel-tuned class-E power amplifier analysis," 2005 European Microwave Conference, Paris, 2005, pp. 4 pp.-.

[53] T. Mury and V. F. Fusco, "Inverse Class-E Amplifier With Transmission-Line Harmonic Suppression," in IEEE Transactions on Circuits and Systems I: Regular Papers, vol. 54, no. 7, pp. 1555-1561, July 2007.

[54] Tyler, A new High-efficiency High Power Amplifier, Marconi Review 1958 
[55] D. M. Snider, "A theoretical analysis and experimental confirmation of the optimally loaded and overdriven RF power amplifier," in IEEE Transactions on Electron Devices, vol. 14, no. 12, pp. 851-857, Dec. 1967.

[56] F. H. Raab, "An introduction to class-F power amplifiers," RF Design, vol. 19, no 5, pp. 79-84, May 1996.

[57] F. H. Raab, "Class-E, Class-C, and Class-F power amplifiers based upon a finite number of harmonics," in IEEE Transactions on Microwave Theory and Techniques, vol. 49, no. 8, pp. 1462-1468, Aug. 2001.

[58] A.M Sandman, "Class S, a novel approach to amplifier distorsion", Wireless World, Semptember 1982

[59] S. C. Cripps, RF Power amplifiers for wireless communications, Artech House, 2nd edition, 2006.

[60] M. Nielsen and T. Larsen, "A transmitter architecture based on delta-sigma modulation and switch-mode power amplification." IEEE Transactions on Circuits and Systems II: Express Briefs, vol. 54, no. 8, pp.735-739, Aug 2007

[61] R. N. McKenzie and W. T. Ng, "A Distortion Suppression Technique for a Digital Class D Audio Power Amplifier with Pulse Density Modulation," 2018 International Conference on Audio, Language and Image Processing (ICALIP), Shanghai, 2018, pp. 315-320

[62] H. Matsumura et al., "E-band Impulse Radio Transceiver with 2-Bit PulsePosition Modulation," 2018 48th European Microwave Conference (EuMC), Madrid, 2018, pp. 1513-1516.

[63] V. Saari, P. Juurakko, J. Ryyndnen and K. Halonen, "13.5 MHz class-S modulator for an EER transmitter," Proceedings Norchip Conference, 2004., Oslo, Norway, 2004, pp. 253-256

[64] S. C. Cripps, P. J. Tasker, A. L. Clarke, J. Lees and J. Benedikt, "On the Continuity of High Efficiency Modes in Linear RF Power Amplifiers," in IEEE Microwave and Wireless Components Letters, vol. 19, no. 10, pp. 665-667, Oct. 2009

[65] P. Colantonio, "High efficiency class e design methodologies", Gallium Arsenide and Other Semiconductor Application Symposium, Paris 2005

[66] Kim, Saturated power amplifire optimized for efficiency using self-generated harmonic current and voltaje, MTTS, 2011

[67] J. Moon, J. Lee, R. S. Pengelly, R. Baker and B. Kim, "Highly Efficient Saturated Power Amplifier," in IEEE Microwave Magazine, vol. 13, no. 1, pp. 125-131, Jan.-Feb. 2012

[68] Park, I., Powers, E.J. and Xu, G., "Parallel Adaptive Predistorsion for RF Power Amplifier Linearization," Global Telecommunications Conference, vol. 1, pp. 82-86, November 1997 
[69] Qiu, J., Abe, K., Antonsen, T. M., Danly, B.G., Levush, B. and Myers, R.E., "Linearizability of TWTAs Using Predistortion Techniques," IEEE Transaction on Electron Devices, vol. 52, pp. 718-727, May 2005

[70] Jecklen, E. G., Ghannouchi, F. M. and Sawan M. A., "A New Adaptive Predistortion Technique Using Software-Defined Radio and DSP Technologies Suitable for Base Station 3G Power Amplifiers," IEEE Transactions on Microwave Theory and Techniques, vol. 52, pp. 2139-2147, September 2004

[71] Abed, K. H., Kazimierczuk, M.K., Nerurkar, S. B. and Senadeera, M. P., "Linearization Techniques in Power Amplifiers for $1.9 \mathrm{GHz}$ Wireless Transmitters," Midwest Symposium on Circuits and Systems, vol. 2, pp. 11031106, August 2005

[72] Doherty, W. H., "A new high efficiency power amplifier for modulated waves," Proc. Of the Institute of Radio Engineers, vol. 24, pp. 1163-1182, September 1936

[73] Peter B.Kenington, High-Linearity RF Amplifier Design. Artech House, 2000

[74] W. C. E. Neo, J. Qureshi, M. J. Pelk, J. R. Gajadharsing and L. C. N. de Vreede, "A Mixed-Signal Approach Towards Linear and Efficient \$N\$-Way Doherty Amplifiers," in IEEE Transactions on Microwave Theory and Techniques, vol. 55, no. 5, pp. 866-879, May 2007.

[75] D. Cox, "Linear Amplification with Nonlinear Components," in IEEE Transactions on Communications, vol. 22, no. 12, pp. 1942-1945, December 1974.

[76] Chireix, H., "High Power Outphasing modulation," Proc. Of the Institute of Radio Engineers, vol. 23, pp. 1370-1392, November 1935

[77] H. S. Black, Translating system. U.S. Patent 1686792, October, 1928

[78] Seidel, H., "A feedforward experiment applied to an L-4 Carrier System Amplifier," IEEE Transactions on Communications Technology, vol. COM-19, pp. 320-325, June 1971

[79] Olver, T. E., Andrews, D. C., Abrams, B. S. and Barr, E. E., "Linear wideband HF power amplifier using adaptive feedforward cancellation," Proc. Of the IEEE Military Communications Conference, MILCOM, vol. 1, pp. 21.6/1-8, 1982

[80] M. S. Ltd, RF Device Data, Vol.II. U.S. Patent 1686792, 5th edition., 1988

[81] N. Pothecary, Feedforward Linear Power Amplifiers. Norwood, MA, Artech House, 1999

[82] Kahn, L. R., "Single-sideband Transmission by Envelope Elimination and Restoration", IEEE Proc. of the IRE, vol. 40, no. 7,pp. 803-806, July 1952

[83] Raab, F. H., "Drive Modulation in Kahn-Technique Transmitters," IEEE MTT-S International Microwave Symposium Digest, vol. 2, pp. 811-814, 1999 
[84] Raab, F. H., "Intermodulation Distortion in Kahn-Technique Transmitters," IEEE Transactions on Microwave Theory and Techniques, vol. 44, pp. 22732278, December 1996

[85] Weiss, M. D., Raab, F. H. and Popovic, Z. V., "Linearity of X-Band Class-F Power Amplifiers in High-Efficiency Transmitters," IEEE Transactions on Microwave Theory and Techniques, vol. 49, pp. 1174-1179, June 2001

[86] L.E. Barton, "A Plate Modulation Transformer for Broadcasting Stations", University of Arkansas, Bulletin Number 8, Engineering Experiment Station, May 1930

[87] P. Draxler, S. Lanfranco, D. Kimball, C. Hsia, J. Jeong, J.van de Sluis, and P. Asbeck, "High efficiency envelope tracking LDMOS power amplifier for WCDMA, IEEE MTT-S Int'1 Microwave Symp. Dig pp.1534-1537, 2006

[88] D. F. Kimball, J. Jeong, C. Hsia, P. Draxler, S. Lanfranco,W. Nagy, K. Linthicum, L. E. Larson, and P. M. Asbeck,"High-efficiency envelope-tracking W-CDMA base-station amplifier using GaN HFETs," IEEE Trans. Microw. Theory Tech., vol. 54, pp. 3848-3856, 2006

[89] D. Tena et. al, "Amplificador de potencia clase F a $1.64 \mathrm{GHz}$ con control de armónicos". Universidad Carlos III de Madrid. 6-9 septiembre 2011. XXVI Simposium Nacional de la Unión Científica Internacional de Radio (URSI 2011). Simposium nacional

[90] RF Micro Devices, "RF3931 Datasheet," Disponible en: https://datasheet.octopart.com/RF3931-RFMD-datasheet-10884927.pdf (2020)

[91] "The First in a Family of Versatile High Power GaN Tansistors", RF Micro Devices, Mcicrowave Journal, Jan 2010

[92] Hoversten, M. Roberg, and Z. Popovic, "Harmonic load pull of high-power microwave devices using fundamental-only load pull tuners," ARFTG Microwave Measurement Symp. Dig., Anaheim, CA, USA, pp. 1-4, May 2010

[93] L. Sang, Y. Xu, Y. Chen, Y. Guo, and R.-M. Xu, "Large Signal Equivalent Circuit Model for Package AlGaN /GaN HEMT," Progress In Electromagnetics Research Letters, Vol. 20, 27-36, 2011

[94] Yuk, K. S., G. R. Branner, and D. J. McQuate, "A wideband multiharmonic empirical large-signal model for high-power GaN HEMTs with self-heating and charge-trapping effects," IEEE Transactions on Microwave Theory and Techniques, Vol. 57, No. 12, 3322-3332, Dec. 2009

[95] Zhao Bo-Chao, Lu Yang, Han Wen-Zhe, Zheng Jia-Xin, Zhang Heng-Shuang, Ma Pei-jun, Ma Xiao-Hua, Hao Yue. X-band inverse class-F GaN internallymatched power amplifier. Chinese Physics B, 2016

[96] Jarndal, A. and C. Kompa, \An accurate small-signal model for AlGaN-GaN HEMT suitable for scalable larger-signal model construction," IEEE Microwave Wireless Components Letter, Vol. 16, No. 6, 333-335, Jun. 2006 
[97] Lu, J., Y. Wang, L. Ma, and Z. Yu, \A new small-signal modeling and extraction method in AlGaN/GaN HEMTs," Solid-state Electronics, Vol. 52, No. 1, 115120, Jan. 2008

[98] J. Moon, S. Jee, J. Kim, J. Kim and B. Kim, "Behaviors of Class-F and Class-F ${ }^{-1}$ Amplifiers," in IEEE Transactions on Microwave Theory and Techniques, vol. 60, no. 6, pp. 1937-1951, June 2012.

[99] Minicircuits. "ZNBT-60-1W datasheet”, Disponible en: https://www.minicircuits.com/pdfs/ZNBT-60-1W+.pdf (2020)

[100] Minicircuits. "ZFBT-4R2GW-FT datasheet", Disponible en: https://ww2.minicircuits.com/pdfs/ZFBT-4R2GW-FT+.pdf (2020)

[101] G. Engen and C. Hoer, "Thru-reflect-line: An improved technique for calibrating the dual six-port automatic network analyser," Microwave Theory and Techniques, IEEE Transactions on, vol. 27, pp. 987-993, Dec. 1979.

[102] Sta, "In-fixture microstrip device measurement using TRL* calibration," Application Note 8720-2, Agilent Technologies, June 2000

[103] “TRL calibration", Microwaves 101. Disponible en: https://www.microwaves101.com/encyclopedias/trl-calibration (2020)

[104] S. Pajic, Narisi Wang, P. M. Watson, T. K. Quach and Z. Popovic, "X-band twostage high-efficiency switched-mode power amplifiers," in IEEE Transactions on Microwave Theory and Techniques, vol. 53, no. 9, pp. 2899-2907, Sept. 2005

[105] F. H. Raab, "Maximum efficiency and output of class-F power amplifiers," Microwave Theory and Techniques, IEEE Transactions, vol. 49, no 6, pp. 11621166, Jun 2001

[106] D. Schmelzer and S. Long, "A GaN HEMT class F amplifier at $2 \mathrm{GHz}$ with greater than 80\% PAE," Solid-State Circuits, IEEE Journal of, vol. 42, no. 10, pp. 2130-2136, Oct 2007

[107] D. Y.-T. Wu, S. Boumaiza, "10W GaN Inverse Class F PA with Input/Output Harmonic Termination for High Efficiency WiMAX Transmitter," Wireless and Microwave Technology, IEEE Conference of, no. 1, Apr 2009

[108] Y. Y. Woo, Y. Yang, and B. Kim, "Analysis and experiments for high-efficiency class-F and inverse class-F power amplifiers," Microwave Theory and Techniques, IEEE Transactions on, vol. 54, no. 5, pp. 1969-1974, May 2006

[109] D. M.Pozar. Microwave Engineering. Wiley. 1998.

[110] Y. Wang and P. Wang, "A high-efficiency power amplifier using GaN HEMTs," 2011 International Conference on Electronics, Communications and Control (ICECC), Ningbo, 2011, pp. 1815-1817. 
[111] D. Kessler and M. K. Kazimierczuk, "Power losses and efficiency of class E power amplifier at any duty cycle," IEEE Trans. Circuits Syst. I, Reg. Papers, vol. 51, no. 9, pp. 1675-1689, Sep. 2004

[112] A. Mediano and P. Molina, "Frequency limitation of a high-efficiency class E tuned RF power amplifier due to a shunt capacitance," in IEEE MTT-S Int. Microw. Symp. Dig., Anaheim, CA, USA, 1999, pp. 363-366

[113] T. Suetsugu andM. K. Kazimierczuk, "Maximum operating frequency of class E amplifier at any duty cycle," IEEE Trans. Circuits Syst. II, Exp. Briefs, vol. 55, no. 8 , pp. 768-770, Aug. 2008

[114] T. Suetsugu and M. K. Kazimierczuk, "Design procedure of class E amplifier for off-nominal operation at 50\% duty ratio," IEEE Trans. Circuits Syst. I, Reg. Papers, vol. 53, no. 7, pp. 1468-1476, Jul. 2006

[115] F. J. Ortega-Gonzalez, D. Tena-Ramos, M. Patiño-Gomez, J. M. Pardo-Martin and D. Madueño-Pulido, "High-Power Wideband L-Band Suboptimum Class-E Power Amplifier," in IEEE Transactions on Microwave Theory and Techniques, vol. 61, no. 10, pp. 3712-3720, Oct. 2013

[116] A. Grebennikov, N. O. Sokal, and M. J. Franco, Switchmode RF and Microwave Power Amplifiers, 2nd ed. New York, NY, USA: Academic, 2012

[117] M. K. Kazimierczuk and W. A. Tabisz, "Class C-E high-efficiency tuned power amplifier," in IEEE Transactions on Circuits and Systems, vol. 36, no. 3, pp. 421-428, March 1989.

[118] Wolfspeed, "CGH40180PP data Sheet," Disponible en: https://www.wolfspeed.com/media/downloads/403/CGH40180PP.pdf (2020)

[119] N. O. Sokal and R. Redl, "Power transistor output port model," RF Design, vol. 10, no. 6, pp. 45-48, 50, 51, 53, Jun. 1987

[120] F. Raab, "Idealized operation of the class E tuned power amplifier," in IEEE Transactions on Circuits and Systems, vol. 24, no. 12, pp. 725-735, December 1977.

[121] T. Mury and V. Fusco, "Exploring figures of merit associated with the suboptimum operation of class-E power amplifiers," in IET Circuits, Devices \& Systems, vol. 1, no. 6, pp. 401-407, December 2007.

[122] F. H. Raab, "Suboptimum operation of class-E power amplifiers," in Proc. RF Techn. Expo, Santa Clara, CA, USA, Feb. 1989.

[123] T. Suetsugu and M. K. Kazimierczuk, "Off-nominal operation of class E amplifier at any duty ratio," IEEE Trans. Circuits Syst. I, Reg. Papers, vol. 54, no. 6 , pp. $1389-1397$, Jun. 2007

[124] J. H. Kim, G. D. Jo, J. H. Oh, Y. H. Kim, K. C. Lee, J. H. Jung, and C. S. Park, "High-efficiency envelope-tracking transmitter with optimized class $\mathrm{F}$ amplifier 
and 2-bit envelope amplifier for 3G LTE base station," IEEE Trans. Microw. Theory Techn., vol. 59, no. 6, pp. 1610-1621, Jun. 2011

[125] N. Tuffy, L. Guan, A. Zhu, and T. J. Brazil, "A simplified broadband design methodology for linearized high-efficiency continuous class-F power amplifiers," IEEE Trans. Microw. Theory Techn., vol. 60, no. 6, pp. 1952-1963, Jun. 2012

[126] V. Carrubba, J. Lees, J. Benedikt, P. J. Tasker, and S. C. Cripps, "A novel highly efficient broadband continuous class-F RFPA delivering $74 \%$ average efficiency for an octave bandwidth," in IEEE MTT-S Int. Microw. Symp. Dig., Baltimore, MD, USA, Jun. 2011, pp. 1-4

[127] N. O. Sokal, "High Efficiency Tuned Switching Amplifier, United States Patent, 1975

[128] M. Kazimierczuk, "Effects of the collector current fall time on the class E tuned power amplifier," in IEEE Journal of Solid-State Circuits, vol. 18, no. 2, pp. 181-193, April 1983.

[129] C. H. Avratoglou and N. Voulgaris, "A new method for the analysis and design of the class E power amplifier taking into account theQ_Lfactor," in IEEE Transactions on Circuits and Systems, vol. 34, no. 6, pp. 687-691, June 1987.

[130] F. J. Ortega-Gonzalez, "Load-pull wideband class-E amplifier," IEEE Microw. Wireless Compon. Lett., vol. 17, no. 3, pp. 235-237, Mar. 2007

[131] A. V. Grebennikov, "Class E with parallel circuit-a new challenge for highefficiency RF and microwave power amplifiers," in IEEE MTT-S Int.Microw. Symp. Dig., Seattle, WA, USA, vol. 3, pp. 1627-1630

[132] F. J. Ortega-Gonzalez, "High efficiency power amplifier driving methods and circuits: Part I,” Microw. J., vol. 47, pp. 22-38, Apr. 2004

[133] I. Kim, Y. Y. Woo, J. Kim, J. Moon, J. Kim and B. Kim, "High-Efficiency Hybrid EER Transmitter Using Optimized Power Amplifier," in IEEE Transactions on Microwave Theory and Techniques, vol. 56, no. 11, pp. 25822593, Nov. 2008.

[134] N. Wang, X. Peng, V. Yousefzadeh, D. Maksimovic, S. Pajic, and Z. Popovic, "Linearity of -band class-E power amplifiers in EER operation," IEEE Trans. Microw. Theory Techn., vol. 53, no. 3, pp. 1096-1102, Mar. 2005

[135] Y. Funahashi, T. Kato, T. Tango, A. Yamaoka, K. Yamaguchi, and Y. Tanabe, "An analysis of performance degradation of a memoryless DPD due to frequency response of an envelope amplifier in an EER power amplifier," in Proc. Eur.Microw. Conf., Paris, France, Sep. 2010, pp. 723-726

[136] Grebennikov, Power Combiners, Impedance Transformers and Directional Couplers: Part II. High Efficiency Electronics. 2008. 
[137] S. Rochette, O. Vendier, D. Langrez, J. Cazaux, M. Kuball, M. Buchta,and A. Xiong, "A high efficiency $140 \mathrm{~W}$ power amplifier based on asingle GaN HEMT device for space applications in -band," in 7thEur. Microw. Integr. Circuits Conf., Manchester, U.K., Sep. 2006, pp.127-130

[138] A. Al Tanany, A. Sayed, and G. Boeck, "Broadband GaN switch modeclass E power amplifier for UHF applications," in IEEE MTT-S Int.Microw. Symp. Dig., Boston, MA, USA, Jun. 2009, pp. 761-764.

[139] E. Cipriani, P. Colantonio, F. Di Paolo, F. Giannini, R. Giofre, R. Diciomma,B. Orobello, and M. Papi, "A highly efficient octave bandwidthhigh power amplifier in GaN technology," in Microw. Integr.Circuits Conf., Manchester, U.K., Oct. 2011, pp. 188-191

[140] F. Y. Ng-Molina, T. M. Martin-Guerrero, C. Camacho-Peñalosa,J. A. Garcia, and J. Mata-Contreras, "GaN transistor-based class Epower amplifier for the low -band," in Integr. Nonlinear Microw.Millimetre-Wave Circuits Workshop, Vienna, Austria, Apr. 2011, pp.1-4

[141] C. Florian, A. Musio, A. F. Scappaviva, R. P. Paganelli, and M. Feudale, "Design of and band class E power amplifiers with GaAspHEMT technology for space SAR," in IEEE Int. RF Microw. Conf.,Seremban, Negeri Sembilan, Malaysia, Dec. 2011, pp. 47-350.

[142] C. Kenle and D. Peroulis, "Design of highly efficient broadband class-Epower amplifier using synthesized low-pass matching networks," IEEETrans. Microw. Theory Techn., vol. 59, no. 12, pp. 3162-3173, Dec.2011.

[143] S. Rochette, O. Vendier, D. Langrez, J. Cazaux, M. Kuball, M. Buchta,and A. Xiong, "A high efficiency $140 \mathrm{~W}$ power amplifier based on asingle GaN HEMT device for space applications in -band," in 7thEur. Microw. Integr. Circuits Conf.,Amsterdam, The Netherlands,Oct.2012, pp. 127-130.

[144] A. Grebennikov, "High-Efficiency Class-E Power Amplifier With Shunt Capacitance and Shunt Filter," in IEEE Transactions on Circuits and Systems I: Regular Papers, vol. 63, no. 1, pp. 12-22, Jan. 2016.

[145] Wolfspeed, "CGH40010P datasheet." Disponible en : https://www.wolfspeed.com/media/downloads/317/CGH40010-Rev4_0.pdf (2020)

[146] T. He and U. Balaji "Design of a Class F Power Amplifier, "PIERS Online., vol. 6, no. 2, pp. 141-144, 2010

[147] J. Wang, Y. Xu, and X. Zhu, "Digital predistorted inverse class-F GaN PA with novel PAPR reduction technique," presented at the IEEE Int. Microw. Symp., Baltimore, 2011, paper TU4G-2

[148] P. A. Godoy, S. Chung, T. W. Barton, D. J. Perreault, and J. L. Dawson, “A highly efficient $1.95-\mathrm{GHz}, 18-\mathrm{W}$ asymmetric multilevel outphasing transmitter for wideband applications," IEEE MTT-S Int. Microw. Symp. Dig., Baltimore, USA, pp. 1-4, Jun. 2011 
[149] L. Ma, F. You and X. Hou, "An Output Match Design Method for High Efficiency and Broadband Class-J PA," IEEE Topical Conference on Power Amplifiers for Wireless and Radio Applications (PAWR),Newport Beach, USA, pp. 43-45, Jan. 2014

[150] W. L. Pribble, J. M. Milligan and R. S. Pengelly, "High Efficiency Class-E Amplifier Utilizing GaN HEMT Technology," IEEE Radio and Wireless Symposium, 2006.

[151] Pablo Fernández Miaja, Mejoras en convertidores CC/CC para aplicaciones de Envelope Tracking, Tesis Doctoral, Universidad Oviedo, 2012.

[152] O. García, M. Vasić, P. Alou, J. Á. Oliver and J. A. Cobos, "An Overview of Fast DC-DC Converters for Envelope Amplifier in RF Transmitters," in IEEE Transactions on Power Electronics, vol. 28, no. 10, pp. 4712-4722, Oct. 2013.

[153] M. C. W. Hoyerby and M. A. E. Andersen, "Ultrafast Tracking Power Supply With Fourth-Order Output Filter and Fixed-Frequency Hysteretic Control," in IEEE Transactions on Power Electronics, vol. 23, no. 5, pp. 2387-2398, Sept. 2008.

[154] A. Soto, J. A. Oliver, J. A. Cobos, J. Cezon and F. Arevalo, "Power supply for a radio transmitter with modulated supply voltage," Nineteenth Annual IEEE Applied Power Electronics Conference and Exposition, 2004. APEC '04., Anaheim, CA, USA, 2004, pp. 392-398 Vol.1.

[155] M. Rodriguez, P.F. Miaja, A. Rodriguez, J. Sebastian, "Multilevel converter for Envelope Tracking in RF power amplifiers", IEEE Energy Conversion Congress \& Exposition ECCE 2009

[156] P. Miaja, M. Rodríguez, A. Rodríguez, J. Sebastián, "A linear assisted DC/DC converter for Envelope Tracking and Envelope Elimination and Restoration application", IEEE Energy Conversion Congress \& Exposition ECCE 2010

[157] P. M. Cheng, M. Vasić, O. Garcia, J. A. Oliver, P. Alou and J. A. Cobos, "Design of envelope amplifier based on interleaved multiphase buck converter with minimum time control for RF application," 2011 IEEE Energy Conversion Congress and Exposition, Phoenix, AZ, 2011, pp. 1279-1283.

[158] V. Yousefzadeh, E. Alarcon and D. Maksimović, "Band separation and efficiency optimization in linear-assisted switching power amplifiers," 2006 37th IEEE Power Electronics Specialists Conference, Jeju, 2006, pp. 1-7.

[159] M. Vasić et al., "The Design of a Multilevel Envelope Tracking Amplifier Based on a Multiphase Buck Converter," in IEEE Transactions on Power Electronics, vol. 31, no. 6, pp. 4611-4627, June 2016.

[160] P. Cheng, M. Vasić, O. García, J. Á. Oliver, P. Alou and J. A. Cobos, "Minimum Time Control for Multiphase Buck Converter: Analysis and Application," in IEEE Transactions on Power Electronics, vol. 29, no. 2, pp. 958-967, Feb. 2014. 
[161] K. Morris, K. Chen and M. Beach, "Reducing feed-through effect within envelope elimination and restoration transmitters," in Electronics Letters, vol. 43, no. 16, pp. 875-877, 2 August 2007.

[162] J. Sebastian, P. Fernandez-Miaja, F. J. Ortega-Gonzalez, M. Patiño, M. Rodriguez, "Design of Two-Phase Buck Converter With Fourth-Order Output Filter for Envelope Amplifier of Limited Bandwidth", IEEE Transactions on Power Electronics, vol. 29, pp. 5933-5948, Nov 2014

[163] D. Díaz, O. García, J.A. Oliver, P. Alou, Z.Pavlovic, J. A. Cobos, "The Ripple Cancellation Technique Applied to a Synchronous Buck Converter to Achieve a Very High Bandwidth and Very High Efficiency Envelope Amplifier", IEEE Transactions on Power Electronics, Vol. 29, no. 6, pp. 2892 - 2902, June 2014

[164] G. B. Yundt, "Series- or Parallel-Connected Composite Amplifiers", IEEE Transactions on Power Electronics, Vol. PE-1, no. 1, pp. 48 - 54, January 1986

[165] Funk, G.D., and Johnston, R.H.: 'A linearized 1 GHz class E amplifier'. IEEE 39th Midwest Symp. on Circuits and Systems, Iowa, USA, August 1996, Vol. 3, pp. $1355-1358$

[166] J. Sebastian, P. Fernandez-Miaja, A. Rodriguez, and M. Rodriguez, "Analysis and design of the output filter for buck envelope amplifiers," IEEE Trans. Power Electron., vol. 29, no. 1, pp. 213-233, Jan. 2004

[167] F. H. Raab, "Power-conserving drivemodulation method for envelopeelimination-and-restoration (EW transmitters," Patent Pending, Green Mountain Radio Research Company, Colchester, VT, 1998

[168] D. Tena-Ramos, F. J. Ortega-González and M. Patiño-Gómez, "Hybrid Envelope Elimination and Restoration Technique for Enhancing the Linearity of Switchmode Envelope Amplifiers," in IEEE Microwave and Wireless Components Letters, vol. 27, no. 2, pp. 186-188, Feb. 2017

[169] J. C. Pedro, J. A. García, and P. M. Cabral, "Nonlinear Distortion Analysis of Polar Transmitters," IEEE Trans. on Microwave Theory and Tech., Vol. 55, No. 12, pp. 2757 - 2765 Dec. 2007

[170] N. Wang, X. Peng, V. Yousefzadeh, D. Maksimovic, S. Pajic, Z. Popovic, "Linearity of X-band class-E power amplifiers in EER operation", IEEE Trans. Microw. Theory Tech., vol. 53, no. 3, pp. 1096-1102, March 2005

[171] F. Wang et al., "An Improved Power-Added Efficiency 19-dBm Hybrid Envelope Elimination and Restoration Power Amplifier for 802.11g WLAN Applications," in IEEE Transactions on Microwave Theory and Techniques, vol. 54, no. 12, pp. 4086-4099, Dec. 2006. 
